\title{
Book of Abstracts ESMRMB 2021 Online 38th Annual Scientific Meeting 7-9 October 2021
}

Thursday 7/10

Scientific Session S1

Thursday $7 / 10$

14:00-15:30

Clinical and preclinical non-brain imaging

Scientific Session S2

Thursday $7 / 10$

15:30-17:30

Clinical and preclinical brain imaging

Scientific Collaborative Session C1

Thursday $7 / 10$

15:30-17:30

B-Q Minded: European Training Network on Q-MRI

\section{Friday 8/10}

Scientific Session S3

Friday $8 / 10$

$13: 30-15: 30$

Acquisition and reconstruction

Scientific Session S4

Friday $8 / 10$

15:30-17:30

Functional, perfusion, diffusion and spectroscopy

Scientific Collaborative Session C2

Friday $8 / 10$

15:30-17:00

HYBRID: European Training Network on Hybrid Imaging

\section{Saturday 9/10}

Scientific Session S5

Saturday 9/10

13:30-15:00

Hardware, materials and techniques 
Scientific Session S6

Saturday $9 / 10$

15:00-17:00

Image analysis

Scientific Collaborative Session C3

Saturday 9/10

15:00-16:30

NOVA-MRI: European Training Network on Novel Applications in 19F MRI 


\section{S1.01.}

DW- and DCE-MRI and super-resolution ultrasound imaging for monitoring breast tumour response to radiotherapy

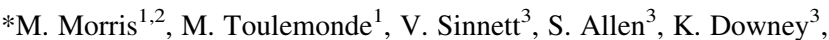
N. Tunariu 2,3, C. Lucy ${ }^{2,3}$, L. Gothard ${ }^{2,3}$, G. Hopkinson ${ }^{3}$, E. Scurr ${ }^{3}$, E. Harris ${ }^{2,3}$, M. Tang ${ }^{1}$, N. Somaiah ${ }^{2,3}$, M. Blackledge ${ }^{2}$

${ }^{1}$ Imperial College London, Department of Bioengineering, London, $G B$;

${ }^{2}$ The Institute of Cancer Research, Division of Radiotherapy and Imaging, London, GB;

${ }^{3}$ The Royal Marsden NHS Foundation Trust, London, GB

Patients with locally advanced/recurrent breast cancer require radiotherapy (RT) for local control. Image-based stratification of patient dose could improve tumour sensitivity to RT. Tumours are associated with high cellularity and chaotic and hyperpermeable microvasculature; our hypothesis is that super-resolution ultrasound (SRUS) ${ }^{1}$ combined with dynamic-contrast enhanced and diffusion-weighted MRI (DCE/DW-MRI) will enable unprecedented quantification of tumour microvessel dynamics and detection of early resistance/relapse. 6 patients with locally advanced breast cancer underwent bilateral breast MR-imaging pre- and $2 \mathrm{w}$ post-RT as part of a clinical trial assessing the effectiveness of intra-tumoral $\mathrm{H}_{2} \mathrm{O}_{2}$ injections as a radiosensitiser. $3 / 6$ patient also received a follow-up scan $6 \mathrm{~m}$ postRT (study ongoing). Axial DW- and 3D isotropic DCE-MRI were acquired using $14 \mathrm{~b}$ values from 0 to $1200 \mathrm{~s} / \mathrm{mm}^{2}$ and variable flip angle acquisition $\left(3^{\circ}\right.$ and $\left.16^{\circ}\right)$ respectively. Contrast injection was performed with a power injector (Dotarem, $0.1 \mathrm{mmol} / \mathrm{kg}$ at rate $3 \mathrm{ml} /$ s), with post-contrast dynamic images acquired at $16^{\circ}$ only. A temporal resolution of $12.2 \mathrm{~s}$ is achieved using TWIST view-sharing (central region $33 \%$, sampling density $33 \%$ ), to quantify contrast dynamics while maintaining high spatial resolution to capture intratumoral heterogeneity. Changes in T1 calculated using a variable flipangle model ${ }^{2}$ are converted into contrast concentration values. Tumour volumes of interest were delineated and approved by a consultant radiologist, from which mean ADC (apparent diffusion coefficient) values were calculated, alongside mean AUC90 (area under curve for first $90 \mathrm{~s}$ ) from DCE-MRI uptake curves. SRUS maps of the tumour microvasculature were generated from high frame-rate contrast enhanced (Sonovue contrast agent) US acquisitions at the same time-points.

Preliminary analysis confirmed that breast tumour microvasculature can be imaged using SRUS with remarkable clarity, and changes in microvascular structure can be visually detected post-RT (Fig. 1). There was an increase in mean ADC after RT for all patients apart from one ( $p$ value $=0.04$; Fig. 2). After $6 \mathrm{~m}$, ADC measurements remained elevated. Mean AUC90 values showed no significant change post-RT across the small population ( $\mathrm{p}$ value $=0.23$; Fig. 3 ), but individual effects may be visible upon further interogation. Figure 1 (SRUS) and 3 (ADC) is patient 3 pre- and $2 \mathrm{w}$ post-RT.
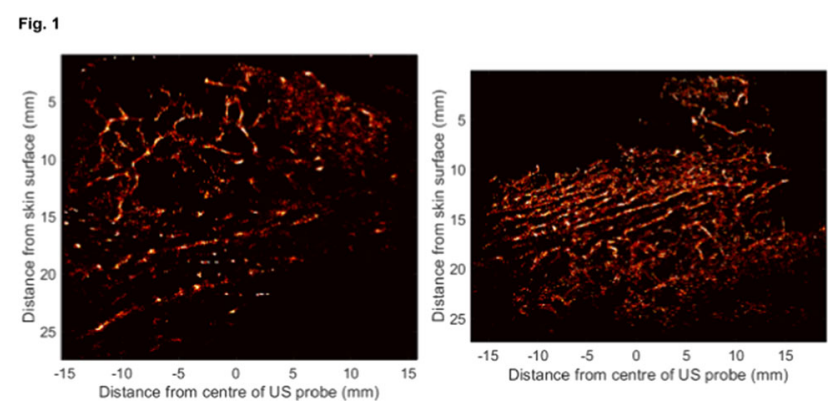
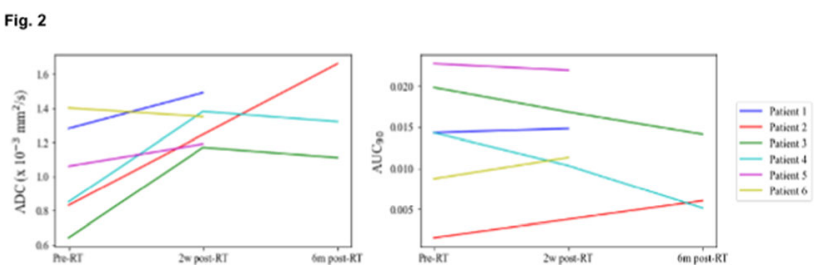

Fig. 3

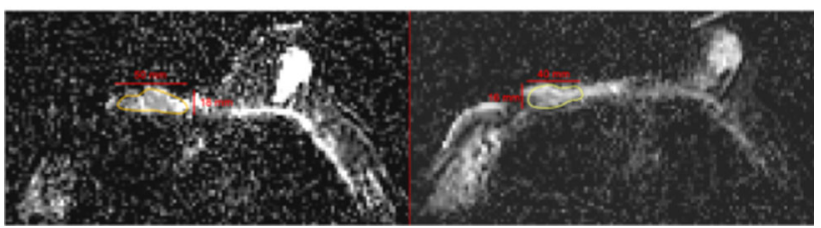

Initial pilot data shows that radiation induced changes can be detected by MRI/SRUS as early as $2 \mathrm{w}$ post-RT. Future analysis will include investigation of intravoxel incoherent motion (IVIM) modelling ${ }^{3}$ and correlating quantitative measurements from SRUS and MRI, to provide early response/resistance biomarkers for RT.

\section{References:}

${ }^{1}$ Christensen-Jeffries, K et al. (2020) Ultrasound Med Biol 46(4).

${ }^{2}$ Fram, E. K., Herfkens, R. J., Johnson, G. A., et al., (1987) Magn Reson Imaging, $\mathbf{5}(3)$

${ }^{3}$ Sigmund, E. E., Cho, G. Y., Kim, S., et al., (2011) Magn Reson Med, 65(5).

\section{S1.O2.}

\section{Low-field lung MRI at $0.55 \mathrm{~T}$ using bSTAR}

\author{
*G. Bauman ${ }^{1,2}$, O. Bieri ${ }^{1,2}$ \\ ${ }^{1}$ University Hospital of Basel, Department of Radiology / Division \\ of Radiological Physics, Basel, CH; \\ ${ }^{2}$ University of Basel, Department of Biomedical Engineering, Basel, \\ $\mathrm{CH}$
}

Introduction: Despite physical and technical challenges associated with thoracic MRI, several imaging techniques such as ultra-short echo time or balanced steady-state free precession (bSSFP) have shown promising results for the assessment of both lung morphology and function $(1,2)$. Only recently, bSTAR imaging was introduced for morphological chest imaging at $1.5 \mathrm{~T}(3,4)$. The technique is based on a half-radial dual-echo balanced steady-state free precession kernel using an interleaved Archimedean spiral k-space trajectory and allows for single breath-hold or free-breathing chest scans. In this work we assess bSTAR imaging on a commercially available lowcost $0.55 \mathrm{~T}$ clinical MR scanner and test its feasibility in a group of healthy volunteers.

Methods: Experiments were performed on a $0.55 \mathrm{~T}$ whole-body MRscanner (MAGNETOM Free.Max, Siemens Healthineers) using a 6-channel chest array coil and a 6-channel spine coil. Five healthy volunteers were scanned using breath-hold and free-breathing bSTAR. All scans were performed with: TE1/TE2/TR $=0.08 / 2.1 /$ $2.2 \mathrm{~ms}$, field-of-view $=34 \times 34 \times 34 \mathrm{~cm}^{3}, 100$ us hard RF pulse, flip angle $\mathrm{a}=25^{\circ}$, bandwidth $=1002 \mathrm{~Hz} /$ pixel yielding $1.3 \mathrm{~mm}$ isotropic resolution. Breath-hold scans (BH-bSTAR) were performed in $23 \mathrm{~s}$ using 10,000 half-projections and 4 interleaves, while the free- 
breathing scans (FB-bSTAR) were acquired in 5:30 min using 150,000 half-projections and 300 spiral interleaves. BH-bSTAR and FB-bSTAR images were reconstructed using FISTA algorithm (5). Retrospective respiratory gating was used to extract data acquired at the end-expiratory phase of the respiratory cycle. For comparison, BH-bSTAR acquisitions were performed at $1.5 \mathrm{~T}$ (MAGNETOM AvantoFit, Siemens Heathineers) as described in (3).

Results: Figure 1 shows exemplary chest images in axial and coronal projections acquired using BH-bSTAR and FB-bSTAR in healthy subjects. Furthermore, maximum intensity projection images were generated for improved visualization of the vasculature. A comparison between BH-bSTAR scans performed at $0.55 \mathrm{~T}$ and $1.5 \mathrm{~T}$ is shown in Fig. 2.

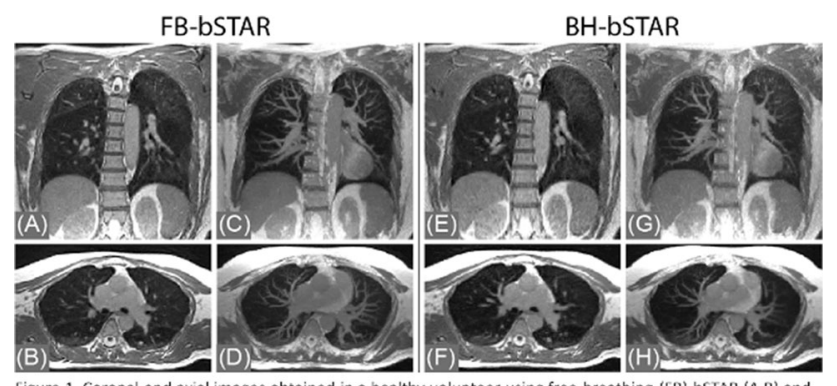

Figure 1. Coronal and axial images obtained in a healthy volunteer using free-breathing $(F B)$-bSTAR $(A, B)$ and breath-hold (BH)-bSTAR (E,F) acquisition. Maximum intensity projection reconstructions were performed with $15 \mathrm{~mm}$ slice thickness for $\mathrm{FB}-\mathrm{bSTAR}(\mathrm{C}, \mathrm{D})$ and $\mathrm{BH}-\mathrm{bSTAR}(\mathrm{G}, \mathrm{H})$ scans at corresponding slice locations.

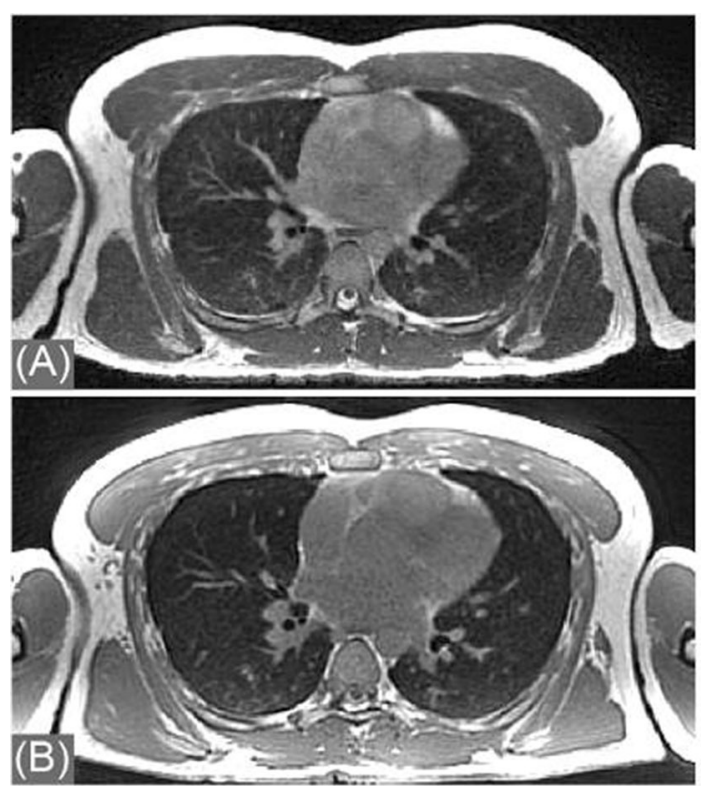

Figure 2. Comparison between breath-hold bSTAR acquisition performed at $0.55 \mathrm{~T}$ (A) and 1.5T (B) in a healthy volunteer. Images show corresponding anatomical location in axial projection. Both scans were reconstructed with $1.4 \mathrm{~mm}$ isotropic resolution.

Discussion: In the recent years, low-field MR systems have regained popularity due to improved hardware, signal post-processing quality and significantly reduced running costs as compared to standard 1.5$3 \mathrm{~T}$ MR systems. In this work we have shown that for lung MRI a similar image quality can be achieved on a low-cost commercially available $0.55 \mathrm{~T}$ clinical system with limited gradient specifications as compared to a high-end 1.5 T MR-system. In conclusion, we have demonstrated excellent prospects for clinical lung imaging using 3D bSTAR in a group of healthy volunteers at $0.55 \mathrm{~T}$.

\section{References:}

1. Johnson KM et al. Magn Reson Med 2013;70:1241-1250.

2. Haye et al. J Magn Reson Imaging 2015 Sep;42(3):602-9.

3. Bauman G \& Bieri O. Magn Reson Med 2020 Jul;84(1):237-246.

4. Bauman G \& Bieri O. Proceedings of 29th ISMRM, p. 3231.

5. Beck A \& Teboulle M. SIAM J Imaging Sci. 2009;2:183-202.

\section{S1.03.}

Investigation of left-atrial flows using a 3D radial based self-gated respiratory motion corrected 4D Flow MRI sequence

\author{
S. Boccalini ${ }^{1}$, M. Mousseaux ${ }^{2}$, P. Chevalier ${ }^{2}$, P. Douek ${ }^{3,1}, * M$. \\ Sigovan $^{3}$ \\ ${ }^{1}$ Hospices Civils de Lyon, Departement of Radiology, Lyon, FR; \\ ${ }^{2}$ Hospices Civils de Lyon, Department of Cardiac Rythm, Lyon, FR; \\ ${ }^{3}$ CREATIS, CREATIS Laboratory, Villeurbanne, FR
}

Introduction: Atrial fibrillation (AF) is a strong risk factor for embolic stroke likely due to prothrombotic alterations in left atrium (LA) blood flow. Four-dimensional velocity mapping MRI (4D Flow) allows quantification of LA and pulmonary veins (PV) flows. However, in the context of $\mathrm{AF}$, standard 4D Flow leads to quantification errors from the inherent averaging of MRI [1].

Our goal was to develop a respiratory motion corrected free-running $3 \mathrm{D}$ radial based $4 \mathrm{D}$ Fow sequence and to investigate the $\mathrm{AF}$ related variability of LA blood flows.

Methods: We implemented a free-running interleaved 3D radial velocity mapping sequence on a 1.5 T Philips Ingenia (Philips, Best, The Netherlands), based on a spiral phyllotaxis pattern [2]. Imaging was performed in 6 paroxysmal AF patients, 2 permanent $\mathrm{AF}$, and 5 age matched volunteers as follows: TE/TR $3.5 / 7.5 \mathrm{~ms}, \mathrm{FA}=6^{\circ}$, VENC $=70-96 \mathrm{~cm} / \mathrm{s}, 2.2-2.8 \mathrm{~mm}$ isotropic voxel for an acquisition time of $8-12$ min.

The respiratory self-gated signal was obtained from the k-space center as previously proposed [3], and used to bin the data in 5 respiratory phases. The $3 \mathrm{D}$ translation motion of the heart between end-expiration and all other phases was estimated and used to correct the phase of the k-space data. Finally, the corrected data was binned into cardiac phases using the ECG signal and a fixed temporal resolution. This strategy reduces the velocity errors related to averaging over variable RR durations.

Images were reconstructed offline using a compressed sensing algorithm implemented in Matlab (The Mathworks, Inc, Natick, MA). 4D Flow data analysis was performed with PIE Medical (The Netherlands).

Results: Qualitatively, respiratory motion correction improved the sharpness of the PVs and modified slightly the flow pattern in the LA (Fig. 1). Streamline visualization demonstrated significant differences in flow patterns, specifically a physiological vortex for healthy subjects, and absence of vortex and lower overall LA velocities for AF patients (Fig. 2). 


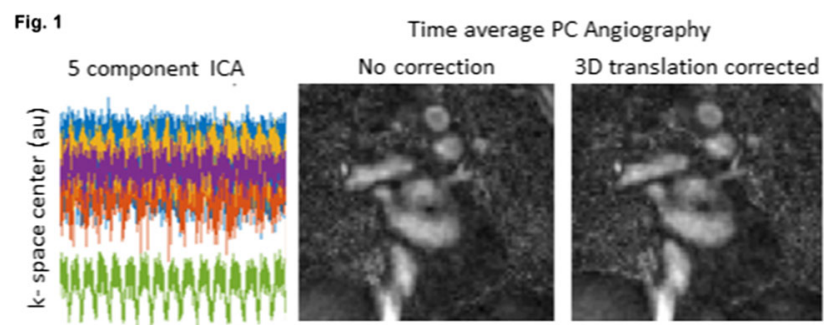

Fig. 2

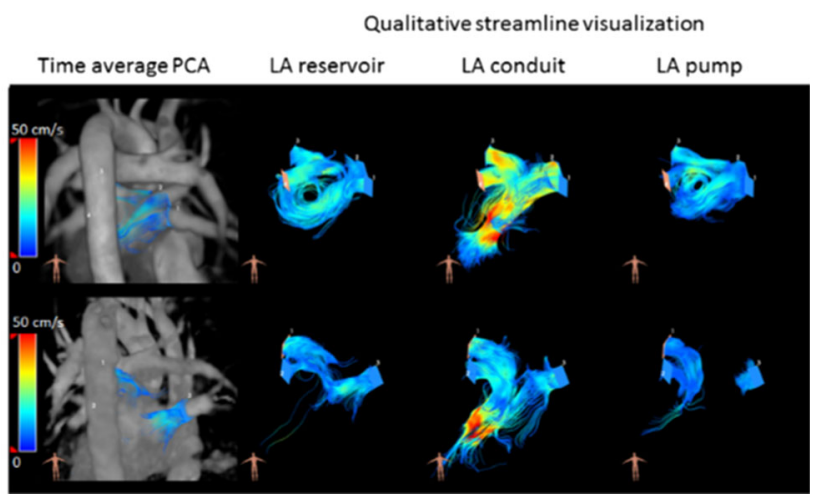

Discussion: We present here preliminary results demonstrating the feasibility of intra-atrial flow assessment using a 3D radial based 4D Flow MRI with respiratory motion correction. The initial results will be completed by an ongoing quantitative analysis of the LA flows (vorticity, vortex size). Particular interest will be paid to the impact of respiratory motion correction on these quantitative descriptors.

LA flows are related to the entire cardiac circulation. Thus an added advantage of the 3D radial 4D Flow is the capability to measure the hemodynamic parameters of the entire heart in the same amount of time as a standard Cartesian 4D Flow covering only the LA, albeit with stronger under-sampling.

Funding: ANR-18-CE19-0025-01

\section{References:}

1. Markl M, et al. (2016) Int J Cardiovasc Imaging 32:807-815.

2. Ma LE, et al. (2020) Radiol Cardiothorac Imaging 2:e200219.

3. Qi H, et al. (2019) Magn Reson Med 82:1331-1342.

\section{S1.04.}

\section{High temporal-resolution dynamic MRI} for the assessment of brown adipose tissue metabolism during mild-cold exposure in young healthy adults

\author{
*A. S. D. Sardjoe Mishre ${ }^{1,2}$, M. E. Straat ${ }^{2}$, B. Martinez-Tellez ${ }^{2}$ \\ M. R. Boon ${ }^{2}$, O. Dzyubachyk ${ }^{3}$, A. G. Webb ${ }^{1}$, P. C. N. Rensen ${ }^{2}$, \\ H. E. $\operatorname{Kan}^{1}$
}

${ }^{1}$ Leiden University Medical Center, Department of Radiology / C.J. Gorter Center for High Field MRI, Leiden, NL;

${ }^{2}$ Leiden University Medical Center, Department of Endocrinology, Leiden, $N L$;

${ }^{3}$ Leiden University Medical Center, Department of Radiology, Leiden, $N L$
Introduction: Brown adipose tissue (BAT) is considered as a potential therapeutic target against cardiometabolic diseases ${ }^{1}$. Activated BAT combusts intracellular fatty acids leading to a reduction in fat fraction $(\mathrm{FF})^{2}$. Both cold exposure and pharmacological stimuli can activate BAT, but the short-term dynamics of BAT activation are not fully explored. Therefore, we aimed to develop a MRI protocol for assessing supraclavicular BAT (scBAT) during cold exposure, with a high temporal resolution and also including non-rigid image registration to reduce variability due to respiratory motion.

Methods: Five healthy volunteers $(25.0 \pm 3.0$ years; BMI $21.8 \pm 2.4 \mathrm{~kg} / \mathrm{m}^{2}$ ) underwent a standardized cooling procedure for BAT activation using a water-circulating blanket inside the MRI suite. After $10 \mathrm{~min}$ at thermoneutrality $\left(32^{\circ} \mathrm{C}\right)$, the subject was moved into the scanner: after a further $10 \mathrm{~min}$ at thermoneutrality the temperature was set to $18{ }^{\circ} \mathrm{C}$ for one hour. Subjects reported thermal perceptions at thermoneutrality and every $15 \mathrm{~min}$ during cooling using a thermal perception scale ranging from thermoneutral (1) to extreme cold (10). Images were acquired at $3 \mathrm{~T}$ using a 16-channel array coil and a 3D gradient-echo 12-point Dixon sequence $(\mathrm{TR}=$ $12 \mathrm{~ms}, \mathrm{TE}_{1}=1.12 \mathrm{~ms}, \Delta \mathrm{TE}=0.87 \mathrm{~ms}, \mathrm{FA}=3^{\circ}, 2.1 \mathrm{~mm}$ isotropic resolution, FOV of $400 \times 229 \times 134 \mathrm{~mm} 3$, a breath-hold duration of $16 \mathrm{~s}$, mDIXON QUANT scanner reconstructions). The acquisition time per scan was 1.03 min to yield a total of 70 scans. For analysis, we co-registered the first-echo magnitude images of each dynamic to the first thermoneutral scan (reference scan) using Elastix ${ }^{3}$ (Fig. 1). The FF of the scBAT depot was obtained by coarsely delineating the depot on the reference scan, and voxels below $30 \%$ FF were excluded in the reference scan and in every registered dynamic (Fig. 1$)^{4}$. The FF of the ROIs in the trapezius muscle was used as a reference. FF differences were calculated by subtracting the average FF of each dynamic from the average FF of the 10 thermoneutral scans. To determine the variability, we fitted a linear function through all scBAT FF dynamics and computed the mean squared error (MSE) of residuals.

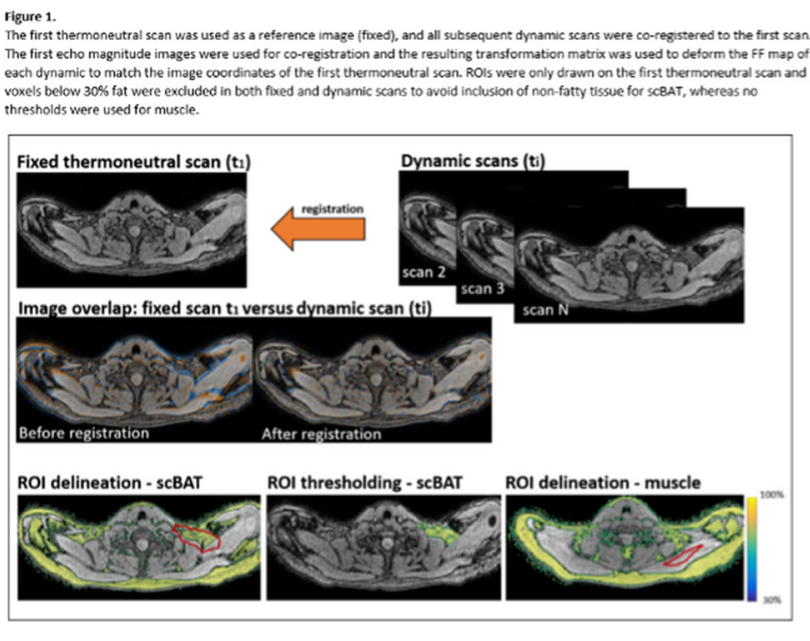

Results: All subjects were able to adhere to the protocol. Reported thermal scores and MSE values are provided in Table 1. Averaged FF differences are shown for scBAT and skeletal muscle in Fig. 2A, B. We found a gradual scBAT FF decrease during cooling, whereas no changes were observed in muscle. We found an overall MSE of $0.09 \pm 0.02 \%$ along the scBAT FF dynamics (Table 1 ). 


\section{rigure 2}

Ayeraged $\mathrm{FF}$ changes plotted as a function of time for scBAT and muscle. FF differences were obtained from co-registered data and

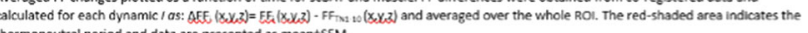
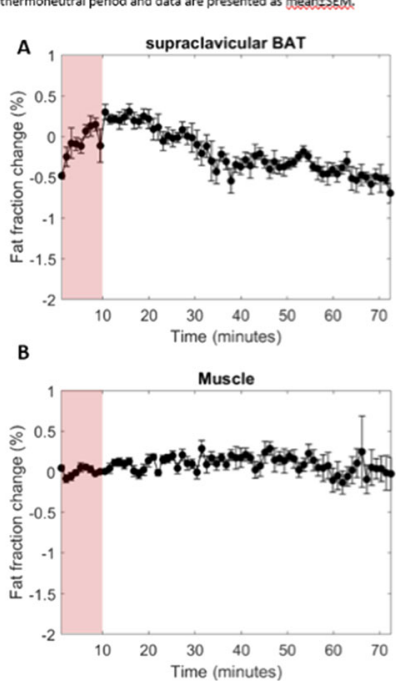

Fig. 3

Table 1. Reported thermal perception scores ranging from thermoneutral (1) to extreme cold (10) during thermoneutrality (TN) and coling Mern squared errors as a function of scBAT drnomiss are also represented.

\begin{tabular}{l|lllll|l} 
& $\begin{array}{l}\text { SCORE } \\
1 \text { (TN) }\end{array}$ & $\begin{array}{l}\text { SCORE 2 } \\
\text { (COOLING) }\end{array}$ & $\begin{array}{l}\text { SCORE 3 } \\
\text { (COOLING) }\end{array}$ & $\begin{array}{l}\text { SCORE 4 } \\
\text { (COOLING) }\end{array}$ & $\begin{array}{l}\text { SCORE 5 } \\
\text { (COOLING) }\end{array}$ & MSE SCBAT \\
\hline $\begin{array}{l}\text { SUBJECT } \\
\text { A }\end{array}$ & 1 & 4 & 4 & NA & NA & $0.10 \%$ \\
$\begin{array}{l}\text { SUBJECT } \\
\text { B }\end{array}$ & 1 & 3 & 3 & 4 & 4 & $0.07 \%$ \\
$\begin{array}{l}\text { SUBJECT } \\
\text { C }\end{array}$ & 2 & 3 & 3 & 3 & 2 & $0.07 \%$ \\
$\begin{array}{l}\text { SUBJECT } \\
\text { D }\end{array}$ & 2 & 4 & 3 & 2 & 3 & $0.08 \%$ \\
$\begin{array}{l}\text { SUBJECT E } \\
\text { AVERAGE }\end{array}$ & 1.5 & 3 & 3 & 5 & 5 & $0.11 \%$ \\
& & & & & & $0.09 \pm 0.02 \%$
\end{tabular}

Discussion: We showed the feasibility of obtaining 1-min resolution data of scBAT FF dynamics during mild-cooling. We found an overall variability of less than $0.1 \%$, which over 60 dynamics should enable the detection of decreasing trends in scBAT FF dynamics.

\section{References}

1. Berbeé JFP, et al., Nat Commun., 2015.

2. Karampinos DC, et al., Handb Exp., 2019.

3. Klein S, et al., IEEE Trans Med Imag., 2010 4.Abreu-Vieira G, Front Endocrinol., 2020.

\section{S1.O5.}

\section{Repeatability of relaxation time measurements with MRF in knee articular cartilage: phantom and in vivo study}

*S. Assili ${ }^{1}$, V. Casula ${ }^{2,1}$, E. Panfilov ${ }^{1}$, M. A. Cloos ${ }^{3}$, R. Lattanzi ${ }^{4}$, M. T. Nieminen ${ }^{2,1,5}$

${ }^{1}$ University of Oulu, Research Unit of Medical Imaging, Physics and Technology, Oulu, FI;

${ }^{2}$ University of Oulu, Medical Research Center, Oulu, FI;

${ }^{3}$ University of Queensland, Centre for Advanced Imaging, Brisbane, $A U$;

${ }^{4}$ New York University Grossman School of Medicine, Center for Advanced Imaging Innovation and Research, New York, NY, US; ${ }^{5}$ Oulu University Hospital, Department of Diagnostic Radiology, Oulu, FI
Introduction: Magnetic resonance fingerprinting (MRF) is a novel MR data acquisition paradigm that enables simultaneous estimation of multiple quantitative MRI (qMRI) parameters, such as T1 and T2 relaxation times. Moreover, MRF is claimed to provide higher robustness to noise and be less prone to acquisition errors compared to traditional qMRI sequences ${ }^{1}$. However, the repeatability of MRF based T1 and T2 maps still needs to be determined for various clinical applications, such as qMRI of the knee cartilage. The aim of this study was to assess the intra-scanner variability of a MRF sequence ${ }^{2}$ as a fast multiparametric quantitative imaging technique for assessment of femoral and tibial knee articular cartilage (AC) sub-regions. Methods: Nine Nickel-doped agarose gel phantoms ${ }^{3}$ with T1 (300-1000 ms) and T2 (25-100 ms) within AC tissue range (Fig. 1) and four volunteer knee-subjects (two females, age $=30 \pm 5$ years) underwent 3 T-MRI (Siemens MAGNETOM Vida, 18-channel Tx/ Rx knee coil) using a recently proposed 2D-MRF sequence (TR/ $\mathrm{TE}=7.5 / 3.5 \mathrm{~ms}, \mathrm{FA}=60^{\circ}, 0.6 \times 0.6 \times 3.0 \mathrm{~mm}^{3}, 6$ radial spokes each consisting of 1000 time points $)^{2}$. To assess the intra-scanner repeatability of the MRF sequence, the measurements in phantom and in vivo were repeated three times, each after repositioning. Femoral and tibial cartilage segmentation into 16 sub-regions (Fig. 2A) was performed manually for single slices from both lateral and medial knee compartments. Mean values of T1 and T2 relaxation times were calculated for all phantoms and sub-regions. Intra-scanner repeatability was quantified using the root mean square coefficient of variation (RMS-CV).

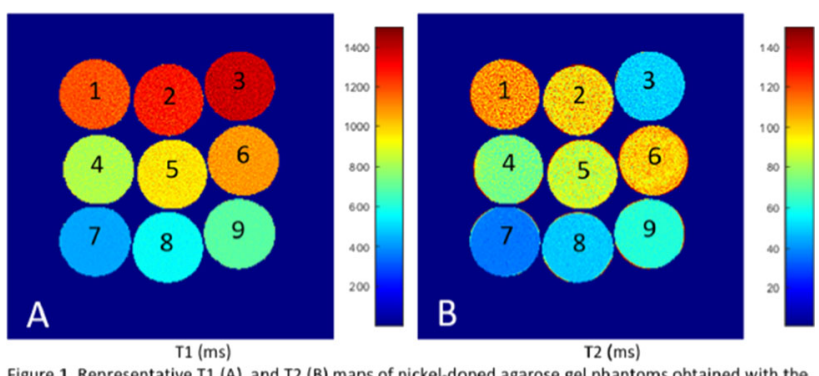

Figure 1. Representative $\mathrm{T}_{1}(\mathrm{~A})$, and $\mathrm{T} 2$ (B) maps of nickel-doped agarose gel phantoms obtained with the MRF sequence. The phantoms with higher agarose concentrations have lower $\mathrm{T} 1$ and $\mathrm{T} 2$ relaxation times.
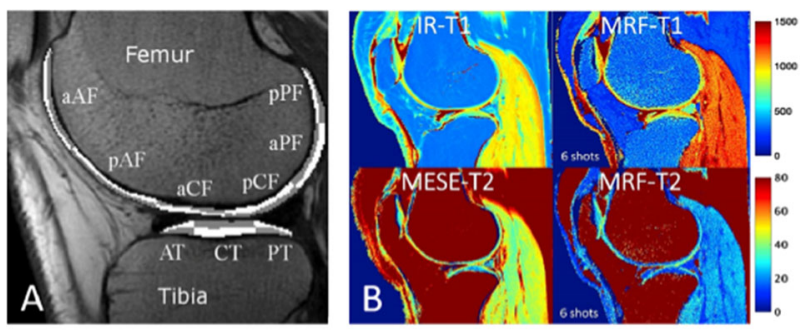

Figure 2. (A) Nomenclature of femorotibial cartilage sub-regions. ROIs are abbreviated as $x Y Z$, here $x$. (a)nterior, (P)osterior; Y - (A)nterior, (C)entral, (P)osterior; Z - (F)emoral, (T)ibial cartilage. (B) Representative $\mathrm{T} 1$ and $\mathrm{T} 2$ relaxation time maps from a right knee joint of 34-year-old female obtained with the MRF sequence (9:01 $\mathrm{min})$. For comparison, conventional qMRI T1 map (IR Turbo SE, 8 TIs $=50-3900 \mathrm{~ms}$, $\mathrm{TR} / \mathrm{TE}=4060 / 8.6 \mathrm{~ms}, 20: 48 \mathrm{~min}$ ), and T2 map (MESE, 18slices, $\mathrm{TR}=1680 \mathrm{~ms}, 5 \mathrm{TEs}=13-69 \mathrm{~ms}, 6 \mathrm{~min}$ ) are $\mathrm{TR} / \mathrm{TE}=4060 / 8$.
presented.

Results: The results demonstrated excellent repeatability of MRF in phantom with RMS-CVs of $0.7 \%$ and $1.8 \%$ for $\mathrm{T} 1$ and $\mathrm{T} 2$ relaxation times, respectively. For in vivo analysis, the average intra-scanner RMS-CVs for lateral and medial compartments were $~ 3 \%$ and $2 \%$ for $\mathrm{T} 1$, and $\sim 8 \%$ and $\sim 8 \%$ for T2, respectively (Fig. 3). 


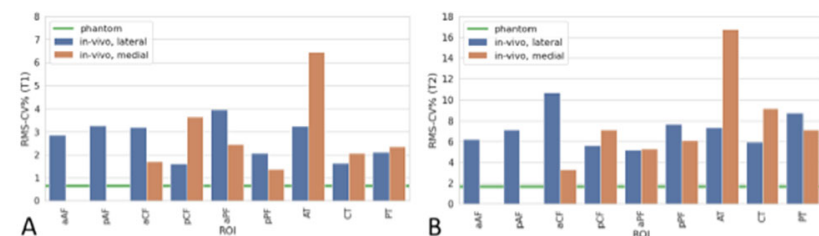

Figure 3. Intra-scanner root mean square-coefficients of variations (RMS-CVs) of T1 (A) and T2 (B) relaxation times for femorotibial cartilage sub-regions from lateral and medial compartments ( $n=4$ knees). The green line denotes the RMV-CV in phantom.

Discussion: T1 and T2 measurements with MRF in phantoms were highly repeatable. For in vivo, the RMS-CVs over 16 sub-regions were generally less than $3 \%$ and $8 \%$ for $\mathrm{T} 1$ and $\mathrm{T} 2$, respectively. Repeatability was lower in some sub-regions (particularly, AT), presumably due to challenges in segmentation and imperfect slice repositioning. Our results for in vivo are in good agreement with the previous studies ${ }^{2},{ }^{4}$ over all cartilage. Our results demonstrated high level of repeatability of MRF sequence to quantitatively assess subregions in knee AC.

\section{References:}

1. Ma, D. et al. Magnetic resonance fingerprinting. Nature (2013).

2. Cloos, M. A. et al. Rapid Radial T1 and T2 Mapping of the Hip Articular Cartilage With Magnetic Resonance Fingerprinting. $J$. Magn. Reson. Imaging (2019).

3. Christoffersson, J. O. et al. Nickel-doped agarose gel phantoms in MR imaging. Acta radiol. (1991).

4. Sharafi, A. et al. MR fingerprinting for rapid simultaneous T1, T2, and $\mathrm{T} 1 \rho$ relaxation mapping of the human articular cartilage at $3 \mathrm{~T}$. Magn. Reson. Med. (2020).

\section{S1.06.}

Paramagnetic contrast enhancement in MRI imaging of liver using an original hepatotropic high-affinity complex-2-(2-carboxymethyl-(4-hexa-decyloxyphenylcarbamoyl-methyl))-aminoethyl-(4-hexadecyloxyphenyl-carbamoyl methyl)-aminoacetic acid with Manganese(II)-GDOF-Mn-DTPA

*O. Y. Borodin ${ }^{1,2}$, M. L. Belyanin ${ }^{3}$, A. S. Podyablonskii ${ }^{3,2}$, M. V. Belousov ${ }^{3}$, V. D. Filimonov ${ }^{3}$, *W. Y. Ussov ${ }^{1,3}$,

N. L. Shimanovskii ${ }^{4}$

${ }^{I}$ Tomsk National Medical Research Center, Laboratory of Tomography, Tomsk, $R U$;

${ }^{2}$ Tomsk Regional Cancer Institute, Department of Diagnostic Radiology, Tomsk, $R U$;

${ }^{3}$ Tomsk Polytechnic University, Tomsk, RU;

${ }^{4}$ N.I. Pirogov National Research Medical University, Moscow, $R U$

Introduction: Design of new organ-specific paramagnetic contrast agents for routine employment in clinical MRI studies is highly necessary because of very little number of such agents currently in use, in particular in liver studies.

Purpose: There was studied the dependence of specific contrast properties of the paramagnetic complex 2-(2-carboxymethyl-(4-hexadecyloxyphenyl-carbamoyl-methyl))-aminoethyl-(4-hexadecyl-oxyphenyl-carbamoyl methyl)-aminoacetic acid (Fig. 1) labelled with manganese-GDOF-Mn-DTPA (Fig. 2) -in magnetic resonance imaging from the administered dose of the drug.
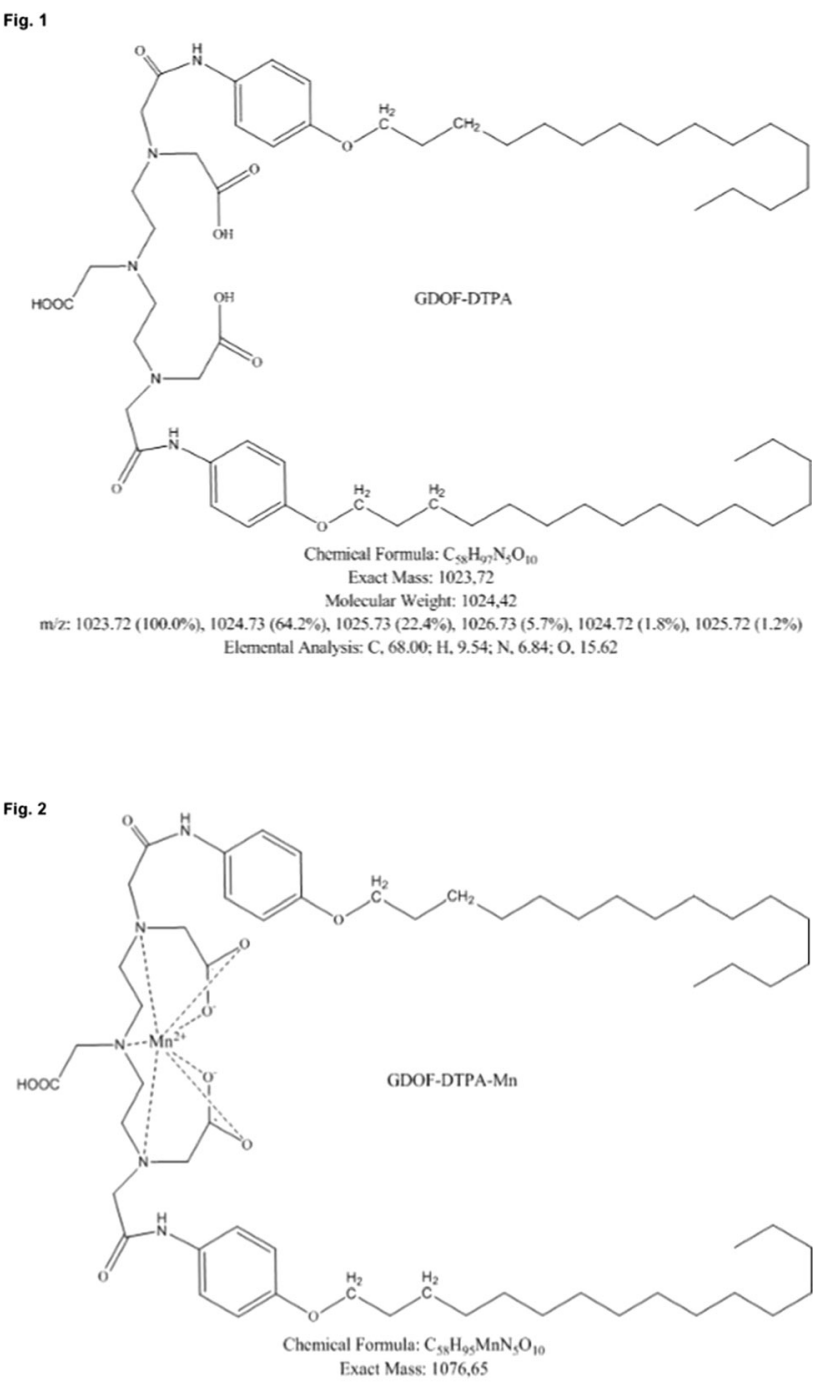

Material and methods: The GDOF-Mn-DTPA was obtained and purified by original synthesis technology in the N.M.Kizhner research Center of the National Research Tomsk Polytechnic University. The dynamics of changes in the liver contrast ratio over time at different dosages of the contrast compound GDOF-Mn-DTPA was evaluated, and changes in the T1 relaxation time of liver and kidney tissue of laboratory animals (Wistar rats, more than $300 \mathrm{~g}$ body weight) at different dosages of GDOF-Mn-DTPA were calculated.

Results: Visual analysis of contrast-enhanced MRI scans with GDOF-Mn-DTPA already at a dose of $0.025 \mathrm{mmol} / \mathrm{kg}$ reliably visualized the accumulation of paramagnet in the liver (Fig. 3), while further concentration of the drug in the bile ducts of animals was noted, with the actual absence of visually detectable kidney contrast. When evaluating the $\mathrm{T} 1$ relaxation time for the liver and kidneys, a persistent decrease in the T1 time for liver tissue was obtained for doses of $0.1,0.05$ and $0.025 \mathrm{mmol} / \mathrm{kg}$, in particular for $0.025 \mathrm{mmol} /$ $\mathrm{kg}$ from the initial $760(747-755) \mathrm{ms}$ to $488(474-505) \mathrm{ms}(\mathrm{p}<0.02)$. On the contrary, the obtained values of $\mathrm{T} 1$ relaxation time for kidney tissue showed no significant accumulation of the paramagnetic contrast compound GDOF-Mn-DTPA to the renal parenchyma at a dosage of $0.025 \mathrm{mmol} / \mathrm{kg}$ or lower. GDOF-Mn-DTPA showed a high degree of hepatoselectivity, with a pronounced reduced excretion through the kidneys. 


\section{Fig. 3}

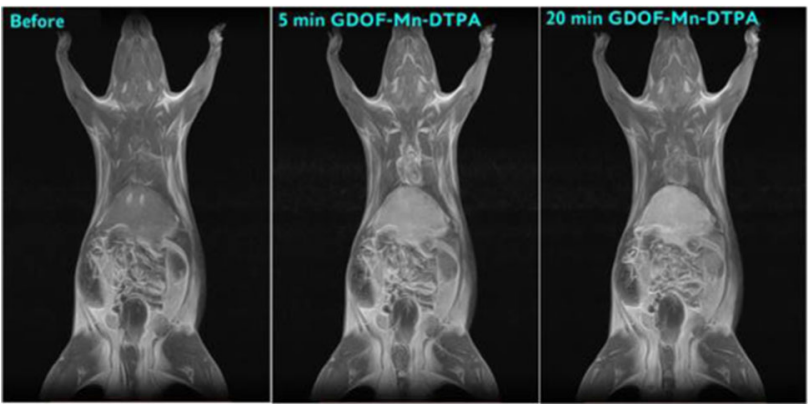

Conclusion: The GDOF-Mn-DTPA complex is a stable compound with a high degree of selective contrast of the hepatic parenchyma, with minimal or no renal excretion, a reliable basis for a hepatoselective contrast agent for imaging and functional studies of the liver with MRI and clinical use in the near future. 


\section{S2.01.}

\section{Combined ${ }^{23} \mathrm{Na}$ MRI and ${ }^{1} \mathrm{H}-\mathrm{MRSI}$ compared to depth electrode recordings in focal epilepsy}

*M. Azilinon ${ }^{1,2,3}$, J. Scholly ${ }^{2,4}$, W. Zaaraoui ${ }^{1,2}$, P. Vioult ${ }^{1,2}$, T. Roussel ${ }^{1,2}$, M. El-Mendili ${ }^{1,2}$, F. Bartolomei ${ }^{3,4}$, J. P. Ranjeva ${ }^{1,2}$, V. Jirsa ${ }^{3}$, M. Guye G $^{1,2}$

${ }^{1}$ Aix-Marseille University, Aix Marseille University-CNRS, Marseille, $F R$;

${ }^{2}$ University Hospitals of La Timone, Center for Magnetic Resonance in Biology and Medicine, Marseille, FR;

${ }^{3}$ Aix-Marseille University, French National Institute of Health and Medical Research (Inserm), Marseille, FR;

${ }^{4}$ University Hospitals of La Timone, Epileptology Department, Marseille, FR

Introduction: A better in vivo characterisation of ionic and metabolic alterations could be key for localising the regions responsible for seizures (i.e. the epileptogenic zone (EZ)) in drug-resistant focal epilepsy for surgical perspective. In this study we combined, for the first time, ${ }^{23} \mathrm{Na}$ MRI and ${ }^{1} \mathrm{H}-\mathrm{MR}$ Spectroscopic Imaging ( $\left.{ }^{1} \mathrm{H}-\mathrm{MRSI}\right)$ and compared changes probed by this multimodal approach to invasive electrophysiological recordings (using stereotactic-EEG (SEEG)), looking for neuroglial alterations deciphering EZ from the rest of the brain.

Methods: We performed a multi-echo density adapted 3D projection reconstruction pulse sequence at $7 \mathrm{~T}\left({ }^{23} \mathrm{Na} \mathrm{MRI}\right)$ and a 3D-EPSI acquisition at $3 \mathrm{~T}\left({ }^{1} \mathrm{H}-\mathrm{MRSI}\right)$ in 21 patients with SEEG and 25 healthy controls. After data processing described in [1], we obtained ${ }^{23} \mathrm{Na}$ concentrations and $\mathrm{T}_{2} *$ short and long components of the signal. After data processing of 3D-EPSI described in [2] we obtained NAA, Cho and $\mathrm{tCr}$ 3D maps. All measures were extracted from spherical ROIs around bipolar contact of SEEG electrodes and normalized against controls using Z-score. We performed a group comparison for each imaging metrics across all bipolar SEEG-based ROIs categories (see Fig. 1) between patients and healthy controls, using a bootstrap t-test. Then we performed a cost-sensitive logistic regression to predict EZ using backward stepwise selection of MRI measures as predictors.

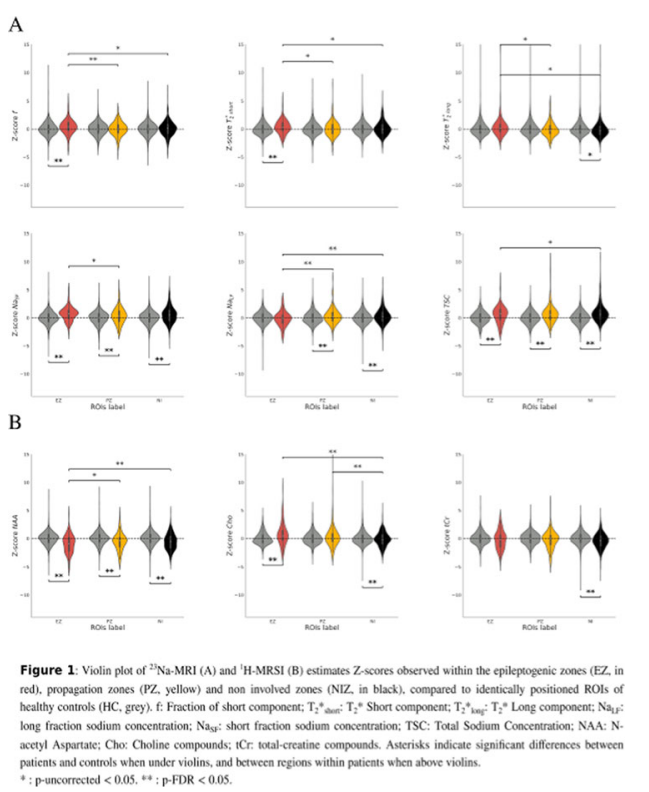

Results: Group comparison showed significant increase of the short signal fraction (f) and $\mathrm{T}_{2}{ }_{\text {short }}$ in EZ, and of the long fraction concentration $\left(\mathrm{Na}_{\mathrm{LF}}\right)$ outside the EZ (Fig. 1). Short fraction concentration $\left(\mathrm{Na}_{\mathrm{SF}}\right)$ and total concentration, were overall higher in patients NAA was significantly lower in all zones in patients, particularly in EZ compared to other zones. The stepwise regression procedure did not identify any feature for the ${ }^{1} \mathrm{H}$-MRSI and the ${ }^{23} \mathrm{Na}-\mathrm{MRI}$ model. However, for the mixed model, $\mathrm{T}_{2}{ }_{\text {short }}, \mathrm{Na}_{\mathrm{LF}}, \mathrm{Na}_{\mathrm{SF}}$ and NAA were identified and were also used for the shuffled model. The mixed model exhibited a significantly better AUC and MCC than the shuffled one (Fig. 2).
A

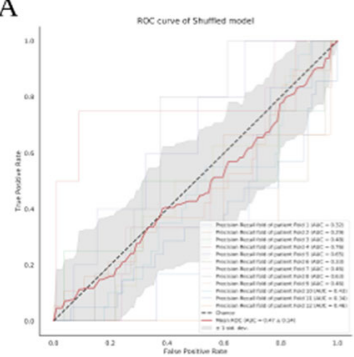

B

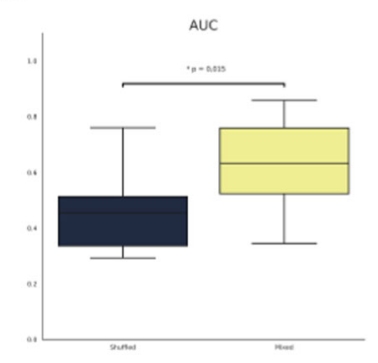

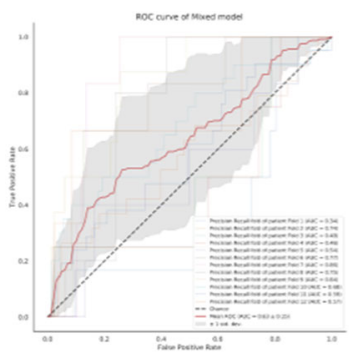

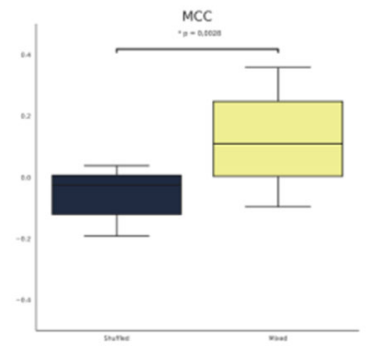

Figure 2: Cost-sensitive logistic regression analysis of Z-scores of ${ }^{23} \mathrm{Na}-\mathrm{MRI}$ and 'H-MRSI estimates as predictors of the epileptogenic zone (EZ). Cross validated ROC plots of the shuffled (left) and mixed model ( ${ }^{23} \mathrm{Na}$ MRI + 'H-MRSI) (right) (A) and area under the curve (AUC) and Matthews correlation coefficient (MCC) of shuffled and the mixed model (B).

Discussion: The association of $\mathrm{Na}_{\mathrm{SF}}$ and $\mathrm{T}_{2}{ }^{*}$ short increases with NAA decrease leads to the hypothesis of both energetic failure and cell viability accompanying ionic homeostasis, reflecting hyperexcitability. Conversely, increased $\mathrm{Na}_{\mathrm{LF}}$ in regions outside the EZ is more likely linked to structural changes as described by morphometry or diffusion MRI in the literature or vascular contribution. This unique combination is a useful tool to study the complex neuroglial changes in epilepsy and for presurgical evaluation.

\section{References:}

1. Ridley, B. et al. Distribution of brain sodium long and short relaxation times and concentrations: a multi-echo ultra-high field ${ }^{23} \mathrm{Na}$ MRI study. Sci. Rep. 8, 4357 (2018)

2. Lecocq, A. et al. Whole-brain quantitative mapping of metabolites using short echo three-dimensional proton MRSI: 3D- ${ }^{1} \mathrm{H}-\mathrm{MRSI}$ Covering the Whole Brain. J. Magn. Reson. Imaging 42, 280-289 (2015)

S2.02.

Longitudinal rs-fMRI and graph theoretical analysis reveal brain network changes in the GAERS rat model of absence epilepsy

*L. Hebbelmann ${ }^{1}$, L. Wachsmuth ${ }^{1}$, H. Lambers ${ }^{1}$, C. Faber ${ }^{1}$, A. Lüttjohann ${ }^{2}$, T. Budde ${ }^{2}$ 
${ }^{1}$ University Hospital Münster, Translational Research Imaging Center (TRIC), Münster, DE;

${ }^{2}$ University of Münster, Department of Physiology I, Münster, DE

Introduction: The Genetic Absence Epilepsy Rats from Strasbourg (GAERS) resemble many features of non-convulsive childhood absence epilepsy. Rats experience seizures during $10-15 \%$ of their time in quiet wakefulness. Seizures lead to short periods of impaired consciousness and behavioral arrest ${ }^{1}$. Whether learning and attention deficits in humans ${ }^{2}$ and rodents ${ }^{3}$ represent comorbidities or consequences of frequently occurring absences remains unclear. We performed longitudinal resting-state (rs-)fMRI and used graph theory analysis ${ }^{4}$ to characterize differences in brain networks of GAERS and nonepileptic controls (NEC) and to detect potential longterm brain network alterations.

Methods: Rs-fMRI under low dose Isoflurane was performed with GE-EPI (TR $1 \mathrm{~s}$, TE $18 \mathrm{~ms}$, resolution $0.32 \times 0.35 \mathrm{~mm}^{2}, 1.2 \mathrm{~mm}$ slices, 12 slices, 30 min scan time) at $9.4 \mathrm{~T}$ (Bruker Biospec) in 12 GAERS and 12 NEC at 6 time points between 3 (seizure prevalence $100 \%$ ) and 8 months. MagnAN (Biocom, Germany) ${ }^{5}$ was used to register data to an anatomical rat atlas template and to calculate crosscorrelation matrices with a multi-seed correlation approach (MSRA). Gephi ${ }^{6}$ was used to calculate and display brain network community structure. Network-based statistics (NBS) ${ }^{7}$ were applied to compare connection strength between GAERS and NEC at each timepoint and between timepoints within strains.

Results: Functional groups gathered in largely similar communities in NEC and GAERS at all timepoints (Fig. 1). In NEC few statistically stronger connections were observed, primarily between sensory input and limbic regions (Fig. 2A). GAERS formed a lot of stronger connections than NEC at all timepoints. Particularly, many connections were found between cortical regions and between limbic regions/ basal ganglia and cortical regions (Fig. 2B).
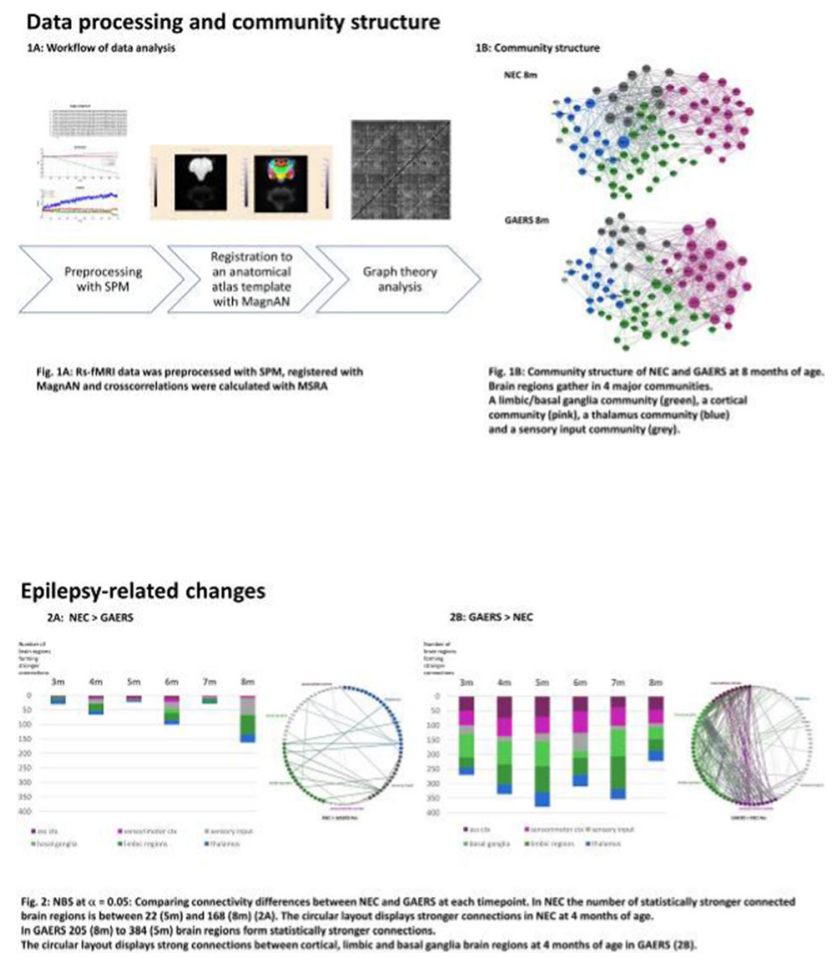

With age, the number of stronger connections increased in NEC (Fig. 3A) and GAERS (Fig. 3B), reaching a peak at 7 months. In NEC, stronger connections were observed in sensory input regions
(Fig. 3A). In GAERS, foremost the number of connections between cortical, limbic regions and basal ganglia (Fig. 3B) increased.

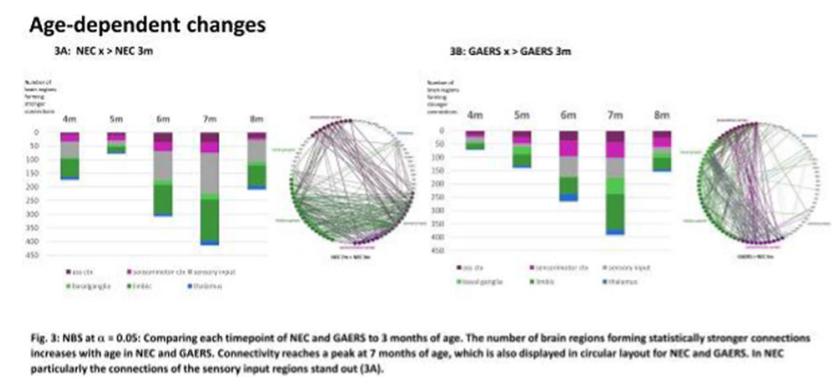

Discussion: Rs-fMRI and graph theoretical analysis are suitable to detect brain network alterations. Overall community structure was similar in NEC and GAERS at all time points, indicating preserved functionality. NBS revealed prominent network differences between GAERS and NEC irrespective of age. Cortical segregation and strong connectedness between cortical and limbic/basal ganglia regions were observed in GAERS. Increasing numbers of significant connections with age compared to young rats of both strains most likely represent the rising level of network complexity during brain maturation. Of note, the number of connections formed by sensory input regions increased with age in NEC and remained low in GAERS, suggestive for impaired development of sensory perception and cognition.

\section{References (PMID):}

1. 26068173

2. 21421063

3. 26742792

4. 19819337

5. 29875622

6. 24914678

7. 20600983

\section{S2.03.}

\section{Correction of the haemodynamic artefact in fluorescence recordings using multimodal BOLD fMRI measurements}

\author{
${ }^{*}$ H. Lambers ${ }^{1}$, L. Wachsmuth ${ }^{1}$, C. Faber ${ }^{1}$ \\ ${ }^{1}$ University Hospital Münster, Clinic for Radiology, Münster, DE
}

Introduction: Combining fMRI and fluorescence recordings has become an important tool for probing brain activation and function. However, the increase of blood volume caused by the haemodynamic response leads to artefacts in fluorescence recordings, since blood absorbs fluorescence light. A correction method for these artefacts is needed. The changes in the absorption can be modelled by an absorption term $\left(\mathrm{e}^{-\Delta \mu(\mathrm{t})}\right) .^{1} \mathrm{We}$ extended this model by including a proportionality between changes in the absorption coefficient and the BOLD response $(\Delta \mu=b \cdot B O L D)$. We present an fMRI-based method for the correction of the haemodynamic artefact (Fig. 1). 


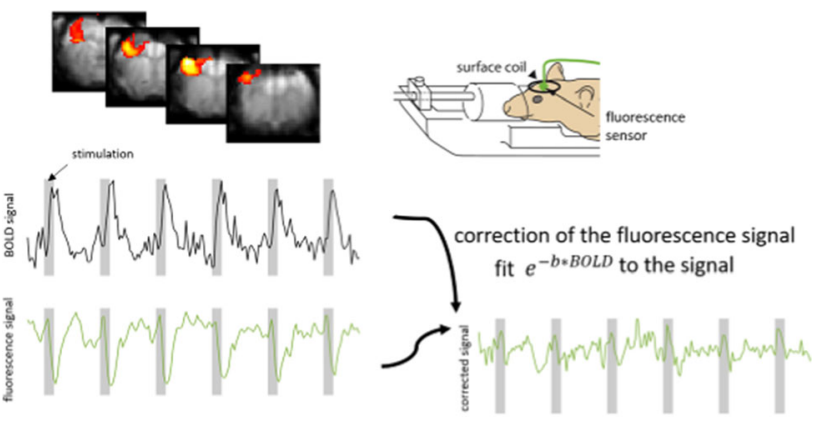

fig. 1: Principle of the fMRI-based correction method: The BOLD response detected with $f M R I$ can be used for the correction of the haemodynamic artefact in a simultaneously performed fluorescence recording.

Methods: AAVs encoding the ratiometric sensors Twitch- $2 \mathrm{~B}^{2}$ or Laconic $^{3}$ (FRET sensors, consisting of two fluorophores: donor and acceptor) were injected in the forelimb region (S1Fl) of Fischer rats $(\mathrm{n}=4, \mathrm{n}=3)$. After at least 4 weeks, fluorescence recordings and fMRI were simultaneously performed under medetomidine sedation upon electrical paw stimulation (paradigm: $5 \mathrm{~s}$ on, $25 \mathrm{~s}$ off, $1 \mathrm{~ms}$ pulses, $1.5 \mathrm{~mA}, 9 \mathrm{~Hz}$ ). For fMRI single shot GE-EPI measurements $(\mathrm{TR}=1 \mathrm{~s})$ were performed at $9.4 \mathrm{~T}$ using a $2 \mathrm{~cm}$ surface coil. For fluorescence recordings the sensor expressing region was illuminated $(458 \mathrm{~nm})$ using an optical fiber $(\Phi: 400 \mu \mathrm{m})$. The fluorescence light was separated into donor and acceptor signals. Data were analyzed using MATLAB: The BOLD response of an S1Fl covering ROI was extracted using a voxel-wise U-test. ${ }^{4}$ The ratio of the fluorescence signals was calculated (Twitch: acceptor/donor ${ }^{2}$, Laconic: donor/acceptor $\left.^{3}\right)$. The absorption term $e^{-b \cdot B O L D}$ was fitted to the fluorescence signals by adjusting the parameter $b$. For the correction, fluorescence signals were divided by this term. Data were checked for a response to the stimulation using a U-test.

Results: The haemodynamic artefact was corrected in fluorescence recordings of 4 Laconic (Fig. 2) and 6 Twitch (Fig. 3) datasets. Before correction, stimulation- and rest-periods of all time courses (donor, acceptor and ratio) showed significant differences $(\mathrm{p}<0.01)$. After correction, no stimulus response was detected with Laconic. With Twitch we detected significant activation $(\mathrm{p}<0.05)$ in donor and ratio signals in 5 datasets.

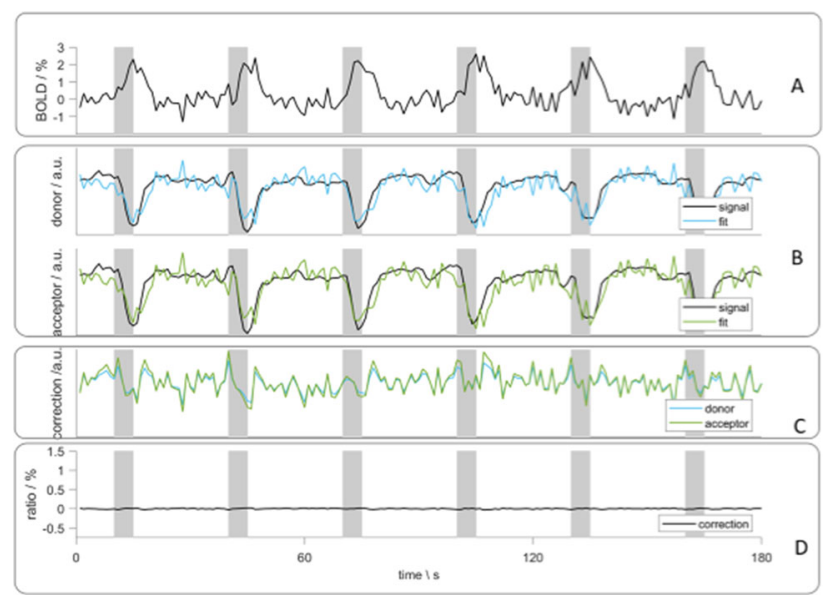

fig. 2: Time courses of an exemplary Laconic experiment. The BOLD response (A) was used for fitting the fluorescence signals (B). The fits were used for the correction of the artefact (C) and the ratio of the corrected signals was calculated (D).

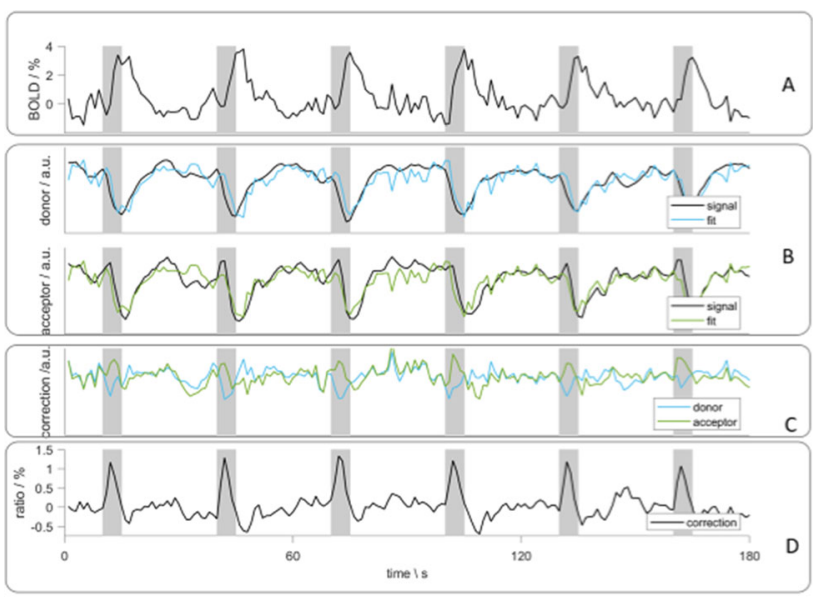

fig. 3: Time courses of an exemplary Twitch experiment. The BOLD response (A) was used for fitting the fluorescence signals $(\mathrm{B})$. The fits were used for the correction of the artefact (C) and the ratio of the corrected signals was calculated (D).

Discussion: Laconic is a lactate sensor and Twitch is a calcium sensor. At a stimulation duration of $5 \mathrm{~s}$ we do not expect a lactate but a calcium response. Our corrected data are consistent with this expectation. Thus, the presented correction method removes the artefact and leaves the true response. So far, light-based correction methods were presented ${ }^{1}$. For ratiometric sensors the calculation of the ratio of donor and acceptor signals represents such a method. However, a stimulus response was detected in the Laconic-ratio. Thus, the artefact is not completely eliminated by the light-based method, while the fMRI-based method presented in this work eliminates the haemodynamic artefact.

\section{References:}

${ }^{1}$ PMID:27574312.

${ }^{2}$ PMID:24390440.

${ }^{3}$ PMID:23469056.

${ }^{4}$ PMID:31846759.

\section{S2.04.}

\section{Relationship between blood pressure} and cerebrovascular reactivity

\author{
${ }^{*}$ S. Moia ${ }^{1}$, G. Chen ${ }^{2}$, M. Termenon ${ }^{1}$, E. Uruñuela ${ }^{1}$, R. Stickland ${ }^{3}$, \\ C. Caballero-Gaudes ${ }^{1}$, M. Bright ${ }^{3,4}$ \\ ${ }^{1}$ Basque Center on Cognition, Brain and Language, San Sebastian, \\ $E S$; \\ ${ }^{2}$ National Institute of Mental Health, Scientific and Statistical \\ Computing Core, Bethesda, MD, US; \\ ${ }^{3}$ Northwestern University, Physical Therapy and Human Movement \\ Sciences/Feinberg School of Medicine, Chicago, IL, US; \\ ${ }^{4}$ Northwestern University, McCormick School of Engineering \\ (Biomedical Engineering), Evanston, IL, US
}

Introduction: Cerebrovascular Reactivity (CVR) is the ability of vessels to respond to vasoactive stimuli. It can be measured using a Breath-Holding (BH) task during a functional MRI sequence ${ }^{1}$. CVR is also sensitive to systemic changes in blood pressure, to the point that such changes were suggested as CVR-inducing stimuli ${ }^{2}$, and that not taking into account subject pressure can bias inter-subject results interpretation $^{3,4}$. To our knowledge, the impact of pressure on local CVR in individuals has not been reported in the literature yet. This study aims to examine the link between pressure and CVR measures based on a precision functional mapping study of BH-induced CVR. 
Methods: 7 healthy volunteers had 10 MRI sessions (3 T Siemens PrismaFit) 1-week apart, at the same time of day. A BH task ${ }^{5}$ was administered at the end of each session (ME-fMRI data, TR $=1.5 \mathrm{~s}, 5$ echoes, $2.4 \times 2.4 \times 3 \mathrm{~mm}^{3}$ ). $\mathrm{CO}_{2}$ levels were measured using a nasal cannula with gas analyzer. A T1-w MP2RAGE was also collected in each session. Diastolic and systolic blood pressure were acquired before each MRI session with the subject in a supine position after a few minutes of rest, once for each arm. Then, each measurement was averaged between arms and pulse pressure (PP) and mean arterial pressure (MAP) were derived for each session. Pulse was also recorded. CVR maps were obtained from the optimally combined signal (optcom) following the steps in ${ }^{6}$, normalized to the MNI152 template (2.5 mm isotropic) and smoothed ( $5 \mathrm{~mm}$ FWHM). A linear mixed effect model was set up using $3 \mathrm{dLMEr}^{7}$, considering the effects of MAP, PP, and pulse on CVR. As group differences were observed between females and males, the interaction with sex was also considered, resulting in the following model:

$\mathrm{CVR} \sim \operatorname{sex} *(\mathrm{MAP}+\mathrm{PP}+$ pulse $)+(1 \mid$ ses $)$

$$
+((\mathrm{MAP}+\mathrm{PP}+\text { pulse }) \mid \text { subj }) \text {. }
$$

The same model was run using vascular lag maps as dependent variable. The results of both models were FDR corrected $(\mathrm{q}<0.05)$. Results: MAP, PP, and sex showed significant local relations to CVR (see Fig. 1). MAP showed a positive impact on CVR in multiple regions of the cerebellum and cortical gray matter, except regions in the frontal lobe. PP showed a positive impact on CVR in the left insula, the right auditory cortex, the middle temporal gyrus and the medial superior frontal gyrus. At the group level, females showed a lower CVR response compared to males across most of the gray matter, in agreement with previous literature ${ }^{8}$. No significant relation was found for interactions, nor an impact of any variable on haemodynamic lag.

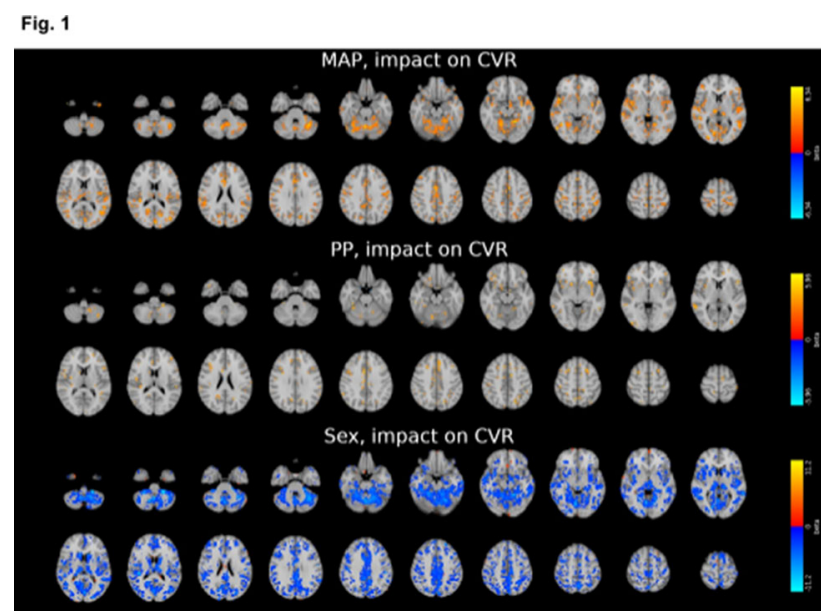

Discussion and conclusion: Measuring blood pressure before collecting CVR data with fMRI should be considered, especially if differences in spatial distributions of CVR are of interest.
S2.05.

Changes in intracranial compliance in patients with long-term ventriculomegaly and normotensive hydrocephalus according to PC-MRI data

*O. Bogomyakova ${ }^{1}$, Y. Stankevich ${ }^{1}$, L. Vasilkiv $^{1}$, A. Savelov ${ }^{1}$, A. Tulupov ${ }^{1}$

${ }^{I}$ Russian Academy of Sciences, International Tomography Center, Novosibirsk, $R U$

Introduction and purpose: To study the changes in intracranial compliance in patients with normotensive hydrocephalus and ventriculomegaly according to phase contrast magnetic resonance imaging.

Materials and methods: A control group (15 people without signs of pathology) and a group of patients (10 people with clinical and radiological signs of normotensive hydrocephalus, 10 people with asymptomatic ventriculomegaly) were formed. On a Philips "Ingenia" $3.0 \mathrm{~T}$ using phase-contrast MRI and the Q-Flow method, the volume-velocity characteristics of hemo- and CSF dynamics were studied at the some intracranial levels. An assessment of arterial inflow, venous outflow, intracranial compliance index and the CSF dynamics in the control and patients groups was made. The volume flow was assessed for the following structures: brain aqueduct, foramen magnum, internal carotid and basilar arteries, superior sagittal and straight sinuses. Comparison of the measured characteristics between groups of patients and controls was carried out using nonparametric methods of analysis.

Results: In the groups of patients with normotensive hydrocephalus, there was a 1.5 times decrease in intracranial compliance $(\mathrm{p}<0.01)$, a 1.5 times decrease in venous outflow in the straight and superior sagittal sinuses $(\mathrm{p}<0.05)$, an increase in the stroke volume of CSF flow at the level of the brain aqueduct by 3 times $(\mathrm{p}<0.01)$, with a change in the prevailing direction of flow to the caudo-cranial direction. In patients with ventriculomegaly, there was a 1.5 times decrease in the stroke volume of the cerebrospinal fluid at the level of the foramen magnum $(p<0.05)$. At the same time, an increase in venous outflow at the level of the straight sinus is determined by 1.2 times $(p<0.05)$. There was a tendency towards a decrease in the index of intracranial compliance.

Discussion and conclusion: Thus, in patients with asymptomatic long-term ventriculomegaly, there is an increase in venous outflow in the absence of significant changes in the parameters of CSF dynamics as one of the compensatory mechanisms. However, such patients are at the group of risk, since there is a tendency to impairment of the functional intracranial relationship. In patients with normotensive hydrocephalus, a significant change in the compliance of the intracranial space and a decrease in venous outflow are determined, which indicates a violation of cerebrospinal fluid dynamics and changes at the level of the brain parenchyma (decreased plasticity of the brain substance).

\section{References:}

1. Kastrup, MRI (2001).

2. Panerai, Physiol Meas (1998).

3. Dumville, Stroke (1998).

4. Hetzel, Stroke (1999).

5. Bright \& Murphy, Neuroimage (2013).

6. Moia, Neuroimage (2021).

7. Chen, Neuroimage (2013).

8. Kassner, J Magn Reson Imaging (2010). 

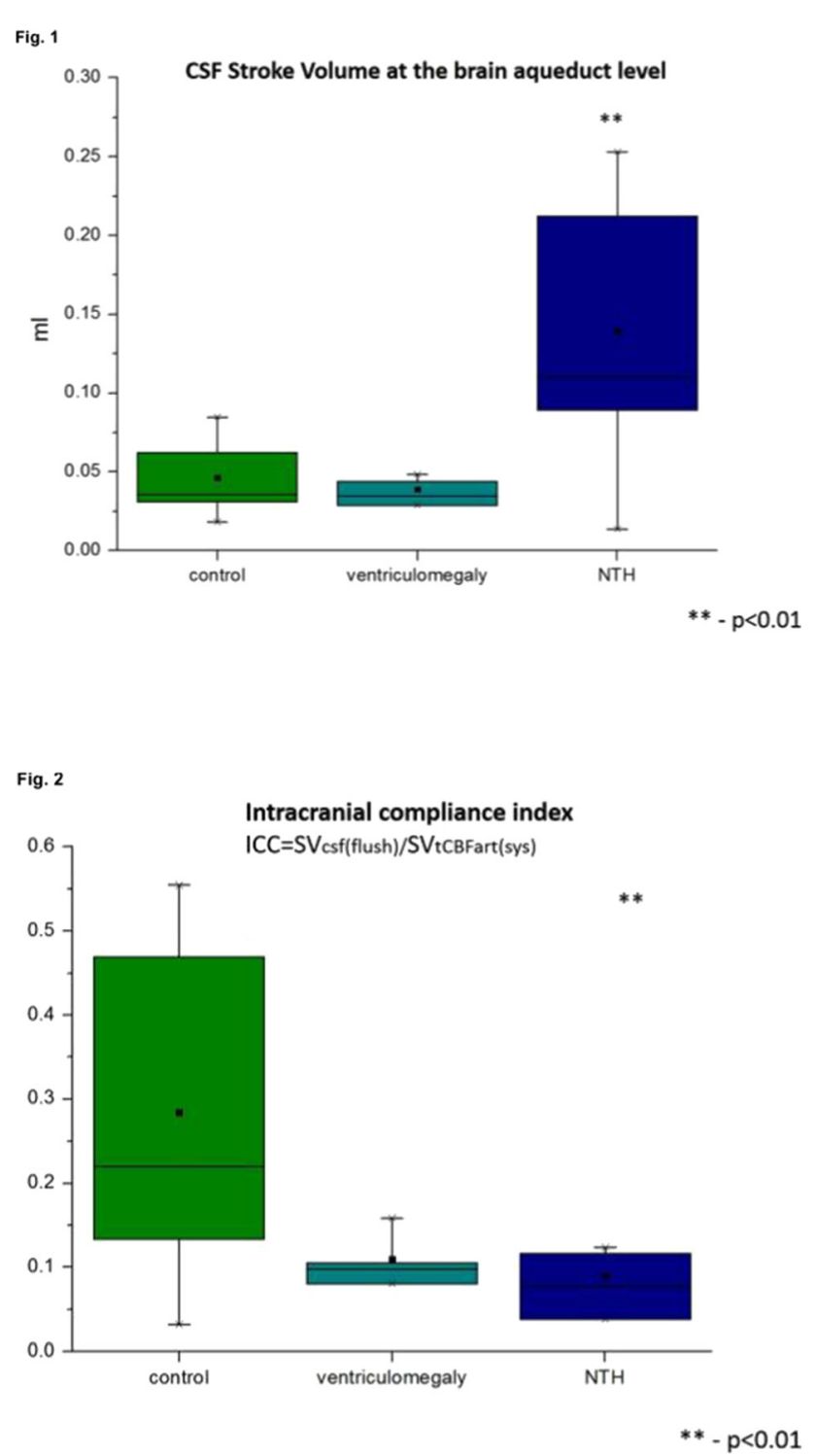

We thanks the Russian Science Foundation (project No. 19-7520093).

\section{S2.06.}

\section{White matter GABA + and GABA-levels: multivendor study}

*P. Menshchikov ${ }^{1,2}$, S. Kartashov ${ }^{3}$, A. Manzhurtsev ${ }^{1,4}$, M. Ublinskiy ${ }^{1,4}$, A. Yakovlev $^{1}$, D. Kupriyanov ${ }^{3}$, T. Akhadov ${ }^{1}$, N. Semenova ${ }^{1,4}$

${ }^{1}$ Clinical and Research Institute of Emergency Pediatric Surgery and Traumatology (CRIEPST), Moscow, $R U$;

${ }^{2}$ LLC Philips, Moscow, $R U$;

${ }^{3}$ Kurchatov Institute, Moscow, RU;

${ }^{4}$ Russian Academy of Sciences, Emanuel Institute of Biochemical Physics, Moscow, $R U$

Monitoring of GABA concentrations in the white matter regions could be useful. For instance, white matter contains functional
GABAB that responds to an anoxic efflux of GABA by recruiting a convergent intracellular mechanism involving protein kinase $\mathrm{C}$ (PKC). The net result of this receptor-mediated cascade is an increase in resistance to anoxia, which presumably allows CNS white matter to tolerate better a common class of ischemic. Moreover, WM GABA is potentially seen as a biomarker of stroke recovery. The main aim of this study was to quantify GABA $+\mathrm{MM}$ and pure GABA levels in WM-riched regions using different vendors MRI scanner.

17 health volunteers underwent MRI examinations on two MRI scanners-3.0 T Philips Achieva dStream 3.0 T Siemens Magnetom Verio. GABA + MM and GABA concentrations were measured in WM separately with MEGA-PRESS (TE/TR $=68 / 2000 \mathrm{~ms}$; ON/ $\mathrm{OFF}=1.9 / 7.5 \mathrm{ppm})$ and MEGA-PRESS with symmetrical suppression of MM signal $(\mathrm{TE} / \mathrm{TR}=80 / 2000 \mathrm{~ms}, \mathrm{ON} / \mathrm{OFF}=1.9 / 1.5 \mathrm{ppm})$. All $1 \mathrm{H}$ MRS voxels $50 \times 17 \times 29 \mathrm{~mm}$ in size were placed in the left and right dorsolateral pre-frontal area (Fig. 1). GABA $+/ \mathrm{tCr}$ and $\mathrm{GABA}-/ \mathrm{tCr}$ ratios were quantified using GANNET. In addition, GABA-to-tCr ratios were corrected for vendor-related editing efficiency of GABA and co-edited MM signal [1].

Fig. 1

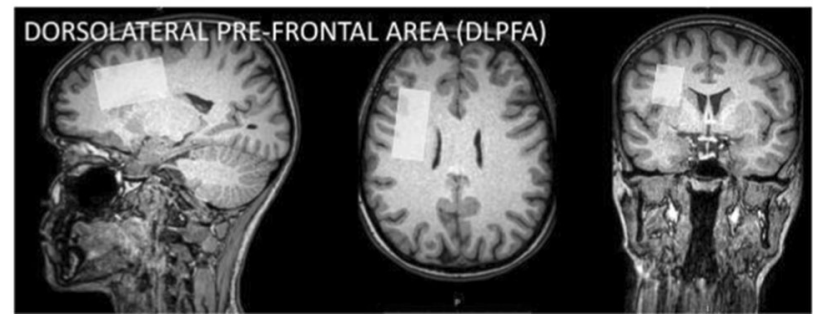

Statistical analysis revealed significantly reduced $(\mathrm{p}<0.05)$ uncorrected $\mathrm{GABA}+/ \mathrm{tCr}$ ratios from Siemens as compared to Philips for both left and right hemispheres (Fig. 2). Left and right corrected GABA + levels did not show any significant changes for both Philips and Siemens data. GABA- levels do not differ from each other before and after correction (Fig. 3). Mean WM:GM:CSF ratio was 0.76:0.22:0.02.
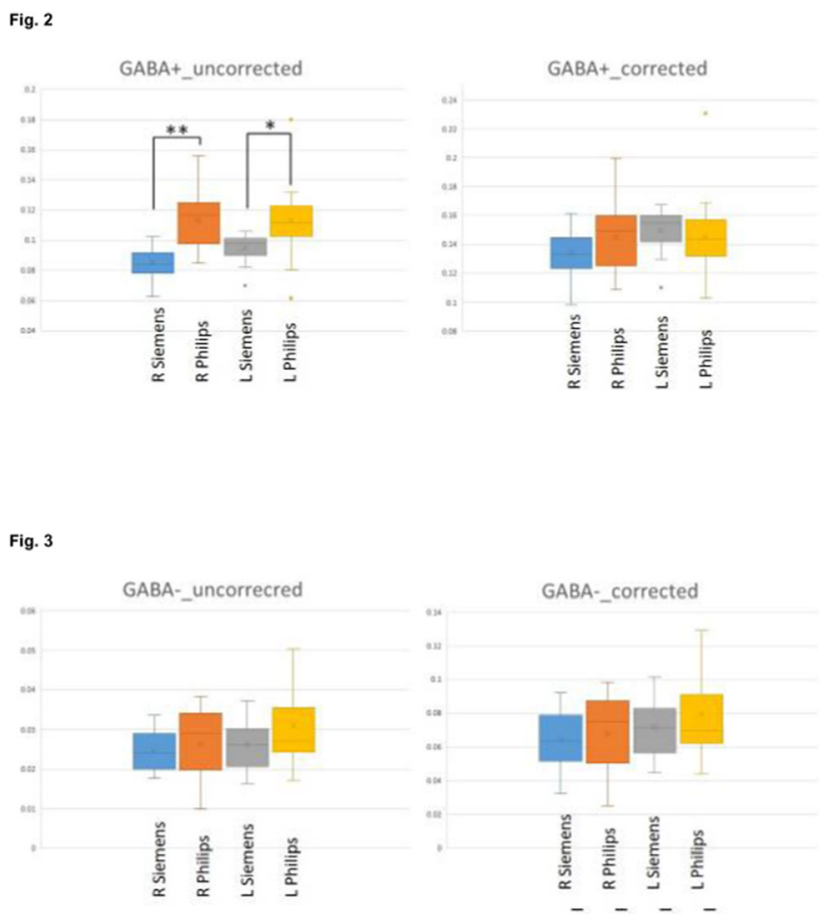
For the first time GABA + and GABA- were quantified and compared between different MRI scanners and hemispheres. The main finding of the study is that both GABA + and GABA - concentrations are equal in the right and left hemispheres of the normal brain. This fact allows using of contralateral GABA concentration for ischemic and stroke evaluation in the situation of the one-sided lesion. Uncorrected GABA $+/ \mathrm{tCr}$ ratios depend on the Manufacturer in a similar way that was obtained previously in GM-riched right sensorimotor cortex [1]. Thus, comparison of GABA + concentrations between different vendors should be at least accompanied by editing efficiency and MM co-editing corrections or using a Multi-vendor standardized MEGA-PRESS pulse sequence [2]. Both uncorrected and corrected GABA - values did not show significant changes depending on the vendor. The difference in MM contamination might not have an influence on signal intensity and should not be corrected. Moreover, since the Siemens sequence produced the least amount of MM contamination its influence on the GABA + signal might be strong. The second reason for no difference between Siemens and Philips GABA-levels is a large spread of GABA-values.

\section{References:}

1) Harris et.al 2017.

2) Saleh et al. 2019.

This work was supported by grant RFBR 19-29-10040.

\section{S2.07.}

\section{Estimation of cortical creatine kinase activity} by dynamic ${ }^{31} \mathrm{P}$ brain MRI in healthy volunteers at $7 \mathrm{~T}$

\author{
*R. Porciuncula Baptista ${ }^{1}$, F. Mauconduit ${ }^{1}$, A. Vignaud ${ }^{1}$, C. Rabrait- \\ Lerman $^{1}$, F. Boumezbeur ${ }^{1}$ \\ ${ }^{I}$ Paris-Saclay University, Gif-sur-Yvette, FR
}

Introduction: Energy metabolism plays a crux role in brain function and its chronic deficits have been associated with many neurodegenerative diseases ${ }^{1}$. In vivo phosphorus-31 $\left({ }^{31} \mathrm{P}\right) \mathrm{NMR}$ spectroscopy allows the estimation of ATPase and creatine kinase (CK) activity using saturation transfer (ST) approaches ${ }^{2}$. However, long acquisition times (TA) limit its use to a few regions of interest (ROI). Here, we propose and evaluate an interleaved frequency selective ${ }^{3}$ (FS) 3D imaging protocol to quantify the $\gamma$-adenosine triphosphate ( $\gamma \mathrm{ATP}$ ) and phosphocreatine ( $\mathrm{PCr}$ ) concentrations and estimate the $\mathrm{CK}$ reaction flux $\left(V_{C K}\right)$ in various cortical ROI in a TA compatible with clinical applications.

Methods: 3 Healthy volunteers ( $2 \mathrm{M} / 1 \mathrm{~F}, 26 \pm 3$ years) were scanned on a whole-body $7 \mathrm{~T}$ MRI (Siemens Healthineers, Erlangen, DE) using a ${ }^{1} \mathrm{H} /{ }^{31} \mathrm{P}{ }^{1} \mathrm{Tx} /{ }^{8} \mathrm{Rx}$ phased array volume antenna ${ }^{4}$. Four $\mathrm{PCr}$ and $\gamma$ ATP images were acquired using a FS SPGR sequence ${ }^{5}$ combined to a $\gamma$ ATP ST module (Fig. 1). Anatomical references were also acquired. To control for the effective FA applied for excitation and saturation, as TA forbids acquisition of individual $\mathrm{B}_{1}{ }^{+}$maps, a template in vivo $\mathrm{B} 1+$ map was calculated from a cohort apart $(3 \mathrm{M} /$ $3 F, 29 \pm 6$ y.o., Fig. 2). Images were corrected for $\mathrm{B}_{1}{ }^{+}$inhomogeneities following registration to the template space and segmented using nipype ${ }^{7}$. Five ROIs were defined from the Harvard-Oxford Atlas (Fig. 3) and both signals were averaged over each ROI. Calibration of concentrations were performed using two external references (PBS at $25 / 50 \mathrm{mM})^{8}$, differential and relaxation effects 9 being accounted for, using experimental or literature ${ }^{10}$ data. To estimate the individual kinetic constant of the $\mathrm{CK}$ reaction $(\mathrm{PCr}+$ $\mathrm{ADP} \leftrightarrow \gamma \mathrm{ATP}+\mathrm{Cr}$ ) (and then $V_{C K}=[\mathrm{PCr}] k_{f}$ ) from the ${ }^{31} \mathrm{P}$ images, magnetizations for the different saturation values were simulated using the Bloch-McConnell Eqs. ${ }^{9,11}$. To compensate for the Rician noise, its average value was subtracted, estimated from an acquisition with a $\mathrm{FA}=0^{\circ}$. Then, $k^{f}$ was determined using the "least squares minimization" algorithm.
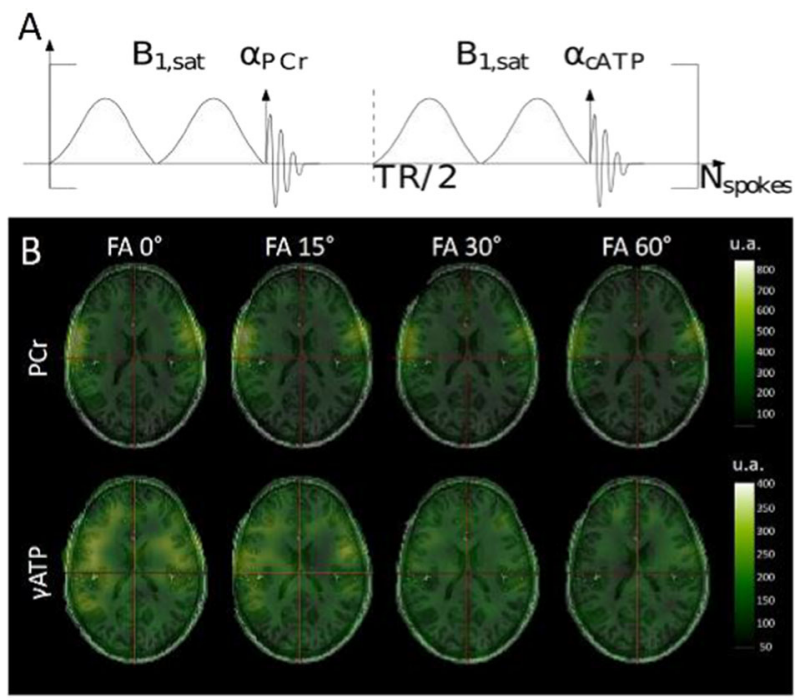

Figure 1: (A) Chronogrom of our interleoved frequency-selective $1 \delta_{p o}=0, \delta_{\text {yat }}=-2.5 \mathrm{pPm}$ ) SPGR sequence (TE/TR=5/250 ins, $F A=25^{\circ}$, res $=12.5 \mathrm{~mm}$ iso, TA=15 min, 3600 spokes). The yATP saturation module consists in two $40 \mathrm{~ms}$ Gaussia registered to their sant
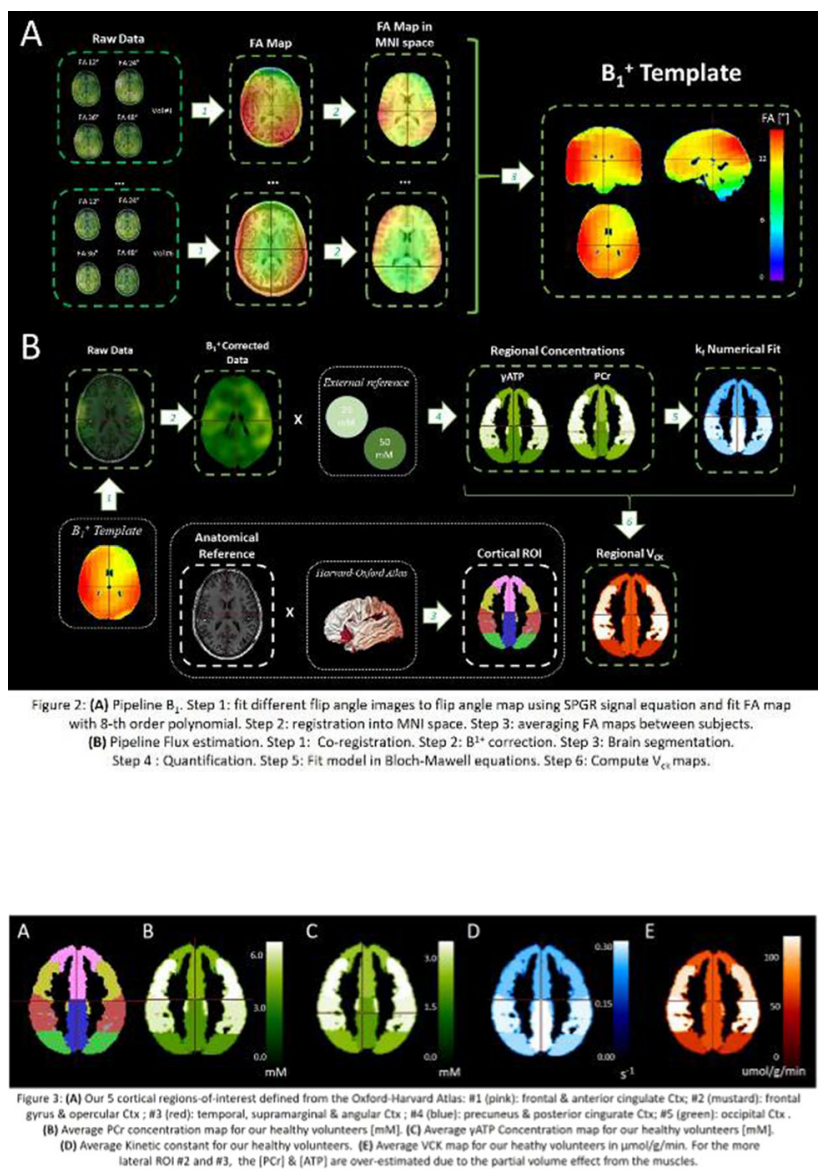
Results/discussion: Figure 3 shows our results. Both concentrations, $k_{f}$ and $V_{C K}$ values are all consistent to the literature ${ }^{2}$ for ROIs $1,4,5$. Our method would benefit from the addition of saturation bands for the muscles. Lastly, we managed to set-up a sensitive and time-efficient $3 \mathrm{D}$ dynamic ${ }^{31} \mathrm{P}$ MRI protocol exhibiting a relatively high spatial resolution (theoric $12.5 \mathrm{~mm}$ iso). Future work will include the application of our method to Alzheimer"s Diseases patients.

This work received financial support from Leducq Foundation (large equipment ERPT program, NEUROVASC7T project). CEA.

\section{References:}

${ }^{1}$ Zhu et al. Front. Aging Neurosci. 2018

${ }^{2}$ Zhu et al. Neuroim. 2012.

${ }^{3}$ Rink et al., MRM, 2015.

${ }^{4}$ Avdievich. Appl. Magn. Reson. 2011.

${ }^{5}$ Coste et al., ISMRM 2018.

${ }^{6}$ Boada et al., MRM. 1997.

${ }^{7}$ Gorgolewski et al., Front. Neuroinform. 2011.

${ }^{8}$ Soher et al., MRM, 1996.

${ }^{9}$ Lei et al., MRM, 2003.

${ }^{10}$ Ren et al., NMR in biomed, 2015.

${ }^{11}$ Bottomley et al., MRM, 2002.

\section{S2.08.}

Joint multi-field $T_{1}$ quantification for fast field-cycling imaging identifies ischaemic stroke at magnetic field strength below $20 \mathrm{mT}$

*L. Broche ${ }^{1}$, O. Maier ${ }^{2}$, M. Bödenler ${ }^{2,3}$, R. Stollberger ${ }^{2,4}$, J. Ross ${ }^{1}$, V. Mallikourti ${ }^{1}$, M. J. MacLeod ${ }^{5}$, H. Scharfetter ${ }^{2}$

${ }^{1}$ University of Aberdeen, Aberdeen Biomedical Imaging Centre (ABIC), Aberdeen, GB;

${ }^{2}$ Graz University of Technology, Institute of Medical Engineering, Graz, AT;

${ }^{3}$ FH JOANNEUM University of Applied Sciences, Institute of eHealth, Graz, AT;

${ }^{4}$ BioTechMed-Graz, Graz, AT,

${ }^{5}$ University of Aberdeen, Institute of Medical Sciences, Aberdeen, GB

Introduction: Fast Field-Cycling (FFC) imaging ${ }^{1}$ is a novel modality that measures variations of $\mathrm{T}_{1}$ relaxation with the magnetic field strength, exploiting novel biomarkers based on $\mathrm{T}_{1}$ dispersion that are invisible to other imaging modalities. Here we report the results of the PUFFINS study that aimed at characterising acute and sub-acute strokes with FFC imaging. We also propose a purpose-made reconstruction method ${ }^{2}$ that exploits the high spatial redundancy of FFC images data by using total generalised variation $\left(\mathrm{TGV}^{3}\right)$ regularisation over all images, combined with a mono-exponential model of magnetisation behaviour to provide $\mathrm{T}_{1}$ maps directly.

Methods: 30 patients gave informed consent to participate to the PUFFINS study from $02 / 2018$ to $02 / 2020$ (ethics approved by NoSREC, number 16/NS/0136) and were scanned by FFC-imaging within 24-96 h of presentation using a field-cycled inversion recovery spin echo sequence with three to six evolution fields typically ranging from $0.2 \mathrm{~T}$ to $0.2 \mathrm{mT}, 2$ to $4 \mathrm{~mm}$ in-plane resolution, $290 \mathrm{~mm}$ FOV, $10 \mathrm{~mm}$ slice thickness, 16 to $24 \mathrm{~ms}$ TE and no averaging. Total scan duration was approximately $40 \mathrm{~min}$.

Multi-field $\mathrm{T}_{1}$ quantification was first performed using a standard curve fitting approach and later using the joint TGV regularisation algorithm after validation on simulated test datasets. The position of the stroke found from FFC images was compared with CT and MRI images for validation.
Results: The TGV-based method showed excellent noise filtering while maintaining sharp image features, clearly outperforming the reference approach on simulated images (Fig. 1). Patient data also showed significant improvements in visual appearance over all fields (Fig. 2) and exhibited visible contrast at $20 \mathrm{mT}$ and below, with lower fields showing the most pronounced contrast with brain tissues with a maximum at the lowest field used. The infarct region measured by FFC corresponded with CT and $3 \mathrm{~T}$ MRI images. The in-vivo $\mathrm{T}_{1}$ dispersion profiles obtained from the $\mathrm{T}_{1}$ images showed profiles for the stroke area that differed from other brain tissues in the low-field regime (Fig. 3), likely due to modifications of water diffusion due to cell damage.
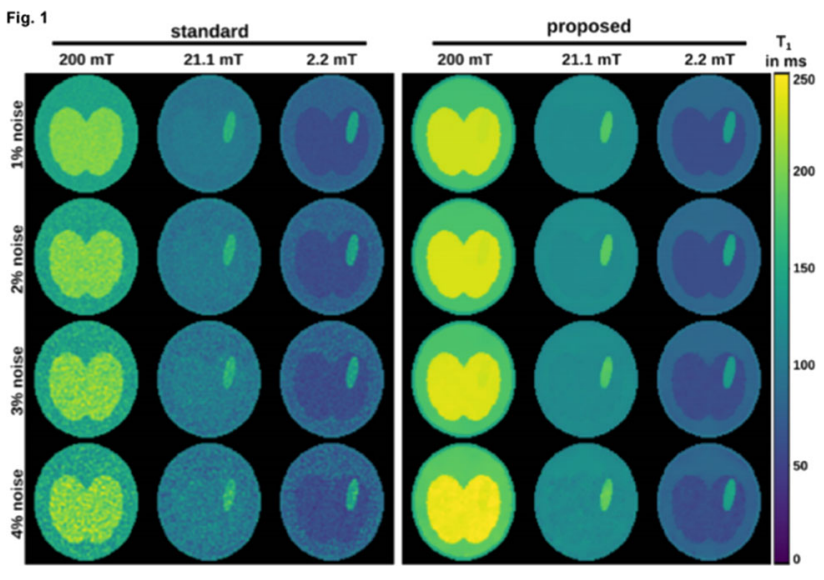

Fig. 2

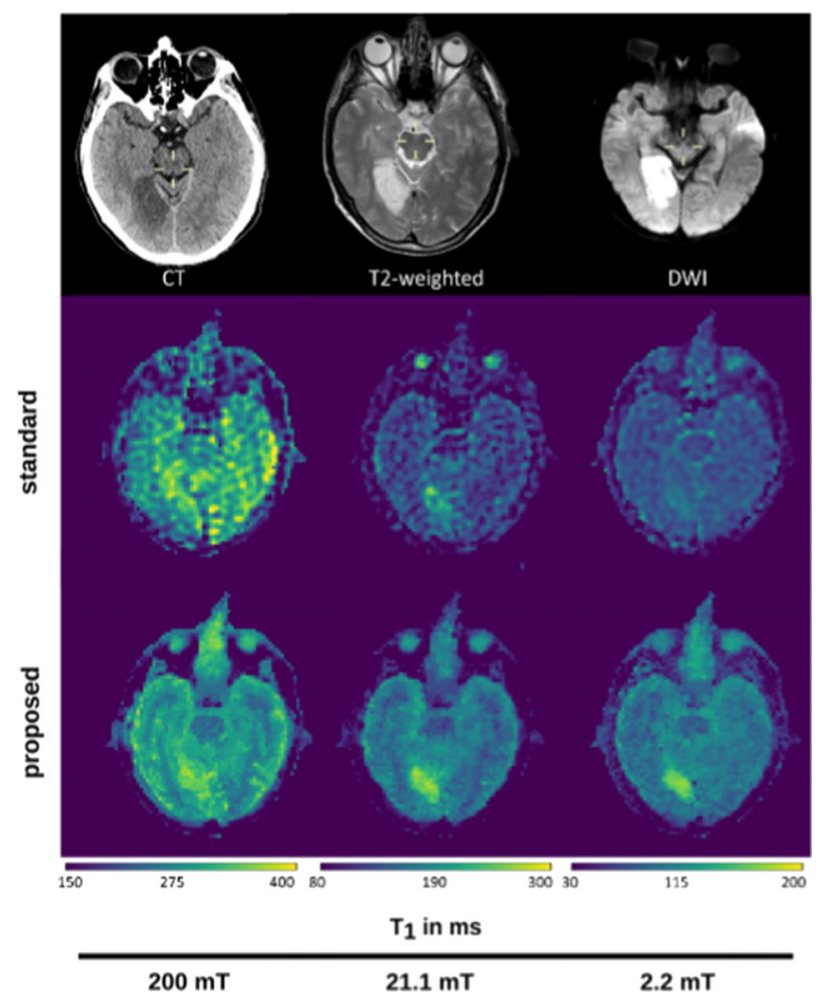


Fig. 3
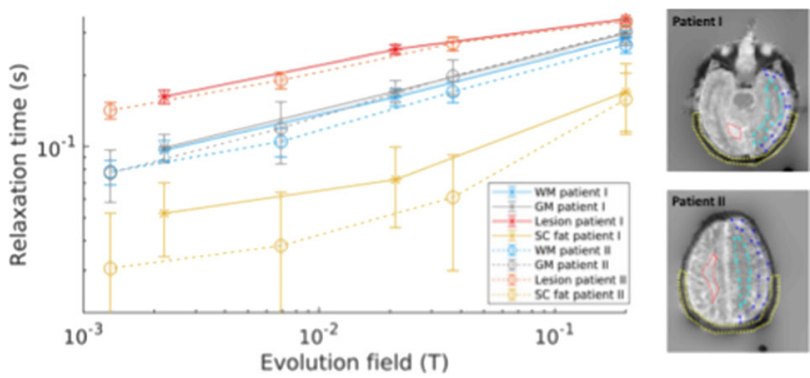

Discussion: This is the first-ever in-vivo measurement of $T_{1}$ dispersion of stroke, which was made possible by the joint TGV regularisation method. This processing tool is available online ${ }^{4}$.

The results demonstrated the potential for low fields in this clinical field but also the limitations of $\mathrm{T}_{1}$ contrast above $20 \mathrm{mT}$. We hope these results will help guiding the design process of dedicated lowfield devices and we are currently starting the second stage of the PUFFINS study to explore potential FFC biomarkers for the differentiation of stroke types and mimics.

\section{References:}

1. Broche, L. M. et al. Sci. Rep. 9, 10402 (2019).

2. Bödenler, M. et al. Magn. Reson. Med. In print,

3. Knoll, F. et al. Magn. Reson. Med. 65, 480-491 (2011).

4. Maier, O. et al. J. Open Source Softw. 5, 2727 (2020).

\section{S2.09.}

\section{High-resolution imaging at low-fields: how far can we} go?

\author{
*O. Bieri ${ }^{1,2}$, G. Bauman ${ }^{1,2}$ \\ ${ }^{1}$ University Hospital of Basel, Department of Radiology / Division \\ of Radiological Physics, Basel, CH; \\ ${ }^{2}$ University of Basel, Department of Biomedical Engineering, Basel, \\ $\mathrm{CH}$
}

Introduction: The development of superconducting magnets with higher and higher field strength has been driven by the promise of exploring unprecedented details and anatomical richness with MRI, especially in the field of neuroscience. Following this line of thought, it was recently shown that $0.44 \mathrm{~mm}$ isotropic images can be achieved with a 3D MPRAGE in a 40 min scan at $7 \mathrm{~T}$ offering a SNR of about 50 and 20 for brain tissue and CSF, respectively (1). Contrary in this work, we explore brain resolution limits on a low-cost clinical lowfield system.

Methods: Imaging was performed on a $0.55 \mathrm{~T}$ low-field MR-system (MAGNETOM Free.Max, Siemens Healthineers) with a gradient system offering $26 \mathrm{~T} / \mathrm{m}$ at $45 \mathrm{~T} / \mathrm{m} / \mathrm{s}$ and using a 12-channel head coil. To explore resolution boundaries for brain MRI, we used a recently proposed radial bSSFP sequence, termed bSTAR (2), offering highest sampling efficiency $(\eta)$. For a TR $=3.9 \mathrm{~ms}$, a hard RF pulse $=600$ us, a bandwidth $=631 \mathrm{~Hz} /$ pixel, for a field-of-view $=256 \times 256 \times$ $256 \mathrm{~mm}^{3}, \eta=0.81$, and scanning was completed within 9:45 min using 150,000 half-radial projections. For brain tissue at this field strength, T1/T2 7-8 (3), and scanning was performed a flip angle $=40^{\circ}$. This setting offers a nominal isotropic resolution of $0.66 \mathrm{~mm}$ that can be subsequently regridded to lower resolutions to explore SNR. The reconstruction was performed using FISTA (4).
Results: Exemplary bSTAR images with $0.66 \mathrm{~mm}$ isotropic resolution are shown in Fig. 1, offering an SNR of 25 and 63 for white matter and CSF, respectively. Especially fluid-like structures, such as the inner-ear (cf. Fig. 2), benefit from the exceptional bSSFP signal properties. Generally, with decreasing resolution, SNR is increased as expected; see Table 1. Overall, sub-millimeter brain MRI with bSTAR can be performed in less than $5 \mathrm{~min}$.
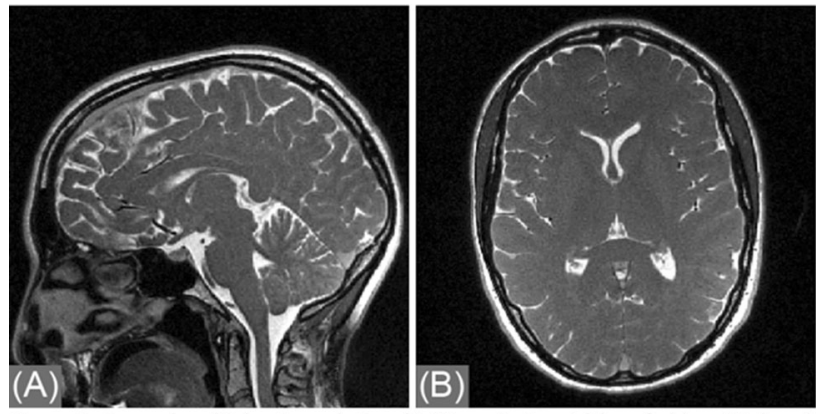

Figure 1: Exemplary highest resolution (0.66mm isotropic) low-field sagittal and axial brain images

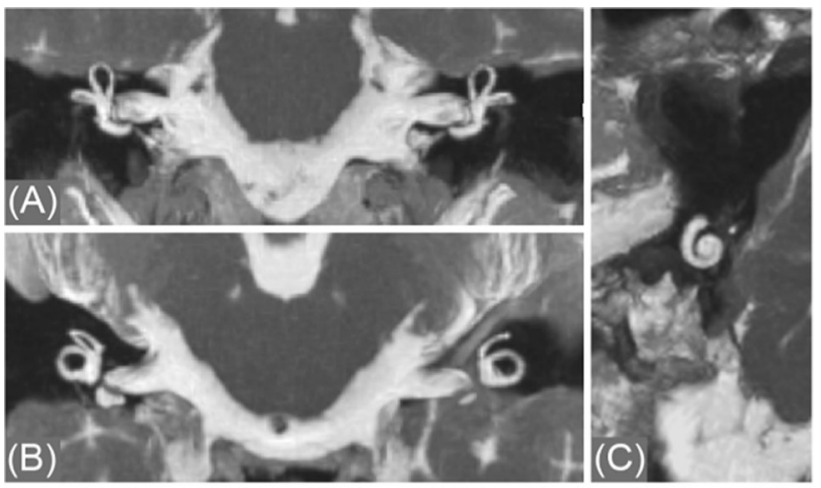

Figure 2: Inner-ear image in coronal (A) and coronal-oblique (B) and sagittal-oblique $(\mathrm{C})$ projection using $0.66 \mathrm{~mm}$ isotropic resolution bSTAR imaging at low-field.

\begin{tabular}{|l|l|l}
\hline Resolution [mm] & SNR (white matter) & SNR (CSF) \\
\hline 0.66 & 25 & 63 \\
\hline 0.83 & 49 & 115 \\
\hline 0.96 & 79 & 201 \\
\hline
\end{tabular}

Table 1: SNR values of tissue and CSF using bSTAR.

Discussion: Among all sequences, bSSFP not only offers the highest SNR per unit time (5), but also its steady state and thus its signal increases with decreasing field strength. Thus, the combination of maximum SNR with highest sequence efficiency, as proffered by bSTAR, can be used to yield an estimate of the maximum achievable resolution. Here, we have shown that for clinically tolerable scan times of less than about $10 \mathrm{~min}$, this limit is far beyond the typical desire for sub-millimeter voxel sizes. Moreover, at low fields, bSSFP imaging does not suffer from banding-artifacts and can be combined with different magnetization preparation schemes to modify its intrinsic T2/T1 contrast. In conclusion, especially at low fields, bSSFP-based imaging surpasses expected resolution limits thus offering excellent prospects for broad clinical use and applicability. 
References:

1. Stucht et al. PLoS One. 2015 Jul;10(7):e0133921.

2. Bauman G \& Bieri O. MRM. 2020 Jul;84(1):237-246.

3. Zhu J et al. Proc. 22nd ISMRM, 2014, p.3208.

4. Beck A \& Teboulle M. SIAM J Imaging Sci. 2009;2:183-202.

5. Scheffler et al. Eur Radiol. 2003 Nov;13(11):2409-18. 


\section{S3.01.}

\section{An open source triggered CEST module for Bruker systems for reliable CEST MRI with efficient motion artifact mitigation}

*S. Mueller ${ }^{1,2}$, R. Pohmann ${ }^{1}$, R. Chiaffarelli ${ }^{3,4}$, S. H. L. Hoffmann ${ }^{3,4}$, A. F. Martins ${ }^{3,4}$, K. Scheffler ${ }^{1,2}$, M. Zaiss ${ }^{1,5}$

${ }^{1}$ Max Planck Institute for Biological Cybernetics, High-field Magnetic Resonance Center, Tübingen, DE;

${ }^{2}$ Eberhard Karls University Tübingen, Department of Biomedical Magnetic Resonance, Tübingen, DE;

${ }^{3}$ Eberhard Karls University Tübingen, Cluster of Excellence iFIT

(EXC 2180), Tübingen, DE;

${ }^{4}$ Eberhard Karls University Tübingen, Werner Siemens Imaging Center/Department of Preclinical Imaging and Radiopharmacy, Tübingen, DE;

${ }^{5}$ University Hospital Erlangen, Department of Neuroradiology, Erlangen, $D E$

Introduction: Chemical exchange saturation transfer (CEST) MRI is an outstanding tool to investigate biological questions. However, motion artifacts are a major problem as CEST experiments are performed over several minutes. This issue was highlighted for CEST MRI in humans ${ }^{1}$ but lacks attention in animal studies. Moreover, many animal experiments employ xenografts in the abdomen, where motion is even more severe and rigid body motion correction is not feasible. We demonstrate such artifacts exemplarily and propose to mitigate motion artifacts using a module that includes triggered acquisition after reaching and keeping a CEST steady-state (SS) saturation. This enables reliable snapshot ${ }^{2}$ CEST MRI triggered to breathing cycle of the animal and is provided as open source library. Methods: The idea is to perform CEST MRI in a snapshot like manner in SS. Instead of a continuous wave $(\mathrm{CW})$ preparation of fixed duration, quasi- $\mathrm{CW}$ allows to extend the duration of the preparation as long as necessary to reach a certain phase within the breathing cycle (Fig. 1A). Both quasi-CW and $\mathrm{CW}$ preparation yield consistent results (Fig. 1BC). Frequency offsets for CEST preparation are read from a text file or are equally spaced within a certain range. Parameters such as pulse duration, inter-pulse delay, $\mathrm{B}_{1}$ and recovery times are adjusted via the extended graphical user interface. The CEST module is contained in source code files, which are independent of the image readout's (RO) source code. They get included into the existing RO by a simple \#include command. Additionally, some minor modifications need to be done manually to ensure full functionality of the RO. A template that shows how to modify the existing RO accordingly is provided. All source code is available via gitlab.com/ SebMue/cest_module_for_bruker. The proposed CEST module was used with Bruker's FISP RO in an egg white phantom and in the abdomen of a healthy rat. Experiments were performed on Bruker's $14.1 \mathrm{~T}$ (phantom) and $7 \mathrm{~T}$ BioSpec systems (animal). Animal experiments were performed in accordance with the local ethics committee.
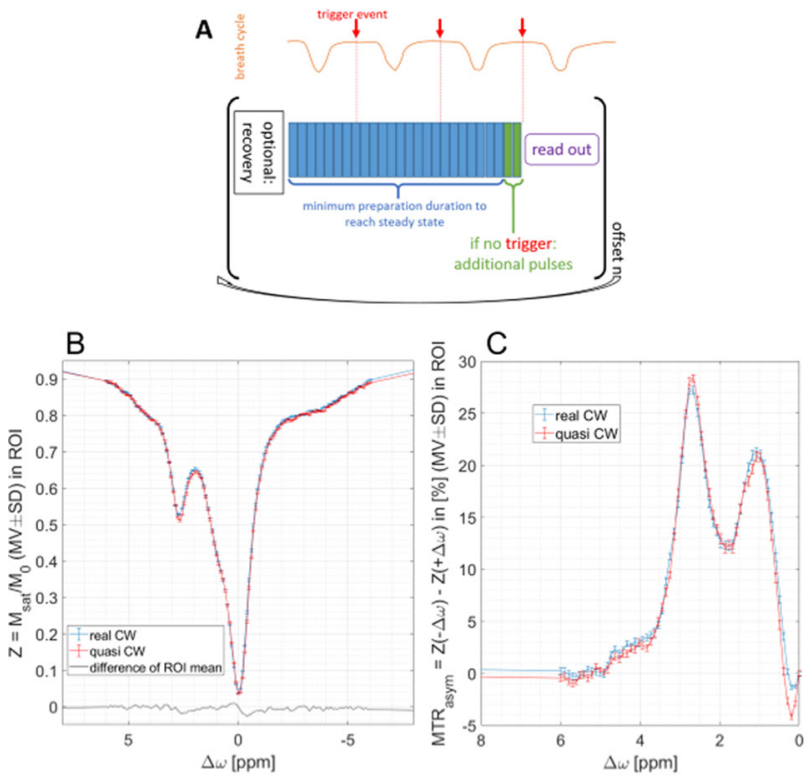

Figure 1: (A) Sequence diagram showing the basic idea behind the proposed CEST MRI SBB. After an optional recovery time, a minimum quasi-CW preparation is applied to reach steady state CEST preparation. Depending on the breath cycle, additional pulses are added before the entire $k$-space is acquired for the $n^{\prime \prime \prime}$ offset in a snapshot 2 like manner. The read out can be chosen independently of the CEST SBB, as only minor manual programming is required. (B) Z-spectra acquired in egg white at $B_{0}=14 \mathrm{~T}$ with either 5 seconds continuous wave (CW) or pulsed "quasi CW" (duty cycle DC $=\mathrm{t}_{\mu} /\left(\mathrm{t}_{\mu}+\mathrm{t}_{\mu}\right)>99 \%$ ). (C) Resulting $\mathrm{MTR}_{\mathrm{wam}^{3}}{ }^{3}$ for spectra shown in (B). Nominal $\mathrm{B}_{1}=2.5 \mu \mathrm{T}$, for the quasi $\mathrm{CW}$ approach pulse duration of $\mathrm{t}_{\mathrm{p}}=10$ ms was used with an inter-pulse delay $\mathrm{t}_{i 1}<50 \mu$ s. No substantial differences between $\mathrm{CW}$ and quasi $\mathrm{CW}$ are observed. Data were corrected for inhomogeneity in $\mathrm{B}_{0}$ using linear interpolation based on field maps acquired using WASAB!

Results: Figure 2 shows how severe motion can affect time series data. By using a trigger, tSNR increases significantly (factor of 1.7, median). This allows more reliable and reproducible quantification of CEST effects in vivo (Fig. 3). Additionally, retrospective motion correction should be performed.
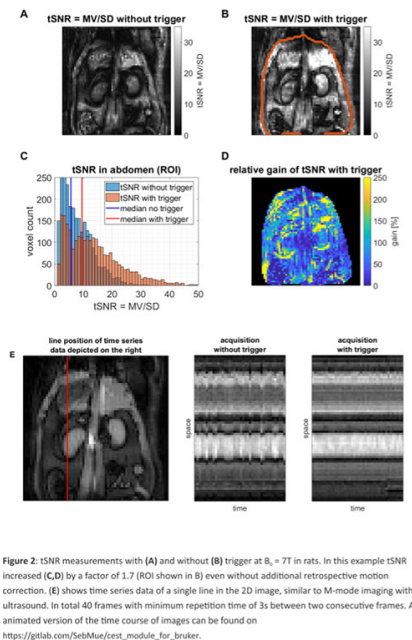
A

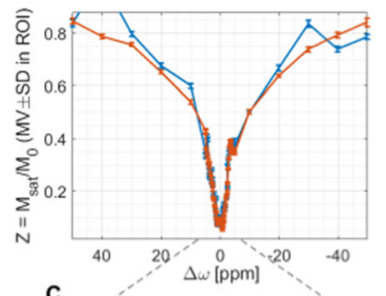

C

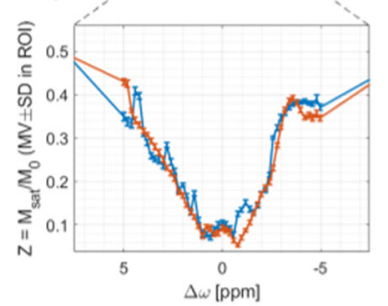

Figure 3: Z-spectra (A) and resulting MTR asymmetry (B) acquired with the proposed SBB with and without trigger to breath. Subplots $(C, D)$ show the same data but within a more narrow frequency range. Data acquired at $B_{0}=7 T$ in rats (in accordance with the local ethics committee) and corrected for $B_{a}$ inhomogeneity based on WASABI field maps. Data shows average over a ROI in the kidney. CEST preparation: at least $6 \mathrm{~s}$ quasi $\mathrm{CW}$ saturation at $B_{1}=2.5 \mu T$ (minimum: $n_{t}=133$ pulses of duration $\left.t_{t}=45 \mathrm{~ms}, D C=t_{2} /\left(t_{n}+t_{n}\right)>99 \%\right)$. Especially for far off-resonant ( $>30 \mathrm{ppm}$ ) saturation, MTR without trigger.

Discussion: It was demonstrated, how severe motion artifacts bias CEST MRI in animals. With the proposed open source module, this issue was significantly reduced. The modular source code requires only minimal manual programming to include it into an existing RO. We hope the proposed open source module is useful for other research groups, and will facilitate improved CEST experiments in animal models.

\section{References:}

${ }^{1}$ Zaiss, JMR, https://doi.org/10.1016/j.jmr.2018.11.002

${ }^{2}$ Zaiss, NMRB, https://doi.org/10.1002/nbm.3879

${ }^{3}$ Guivel-Scharen, JMR, https://doi.org/10.1006/jmre.1998.1440

${ }^{4}$ Schuenke, MRM, https://doi.org/10.1002/mrm.26133

\section{S3.02.}

\section{Addressing inadvertent MT effects in $7 \mathrm{~T}$ brain T1 mapping by simple pulse length scaling}

\author{
*K. Pine ${ }^{1}$, L. Edwards ${ }^{1}$, G. Helms ${ }^{2}$, N. Weiskopf ${ }^{1,3}$ \\ ${ }^{I}$ Max Planck Institute for Human Cognitive and Brain Sciences, \\ Department of Neurophysics, Leipzig, DE; \\ ${ }^{2}$ Lund University, Department of Medical Radiation Physics, Lund, \\ $S E$; \\ ${ }^{3}$ Leipzig University, Felix Bloch Institute for Solid State Physics, \\ Leipzig, DE
}

Introduction: $T_{1}$ mapping by the variable flip angle method (VFA) is known to be affected by inadvertent magnetization transfer (MT) effects from the excitation radiofrequency pulse. These effects can be moderated by "controlled saturation magnetization transfer" $(\mathrm{CSMT})^{1}$ or more simply by varying the pulse duration such that $\mathrm{B}_{1}{ }^{+}$RMS is kept constant between acquisitions ${ }^{2}$. We show that also at $7 \mathrm{~T}$, incidental MT can be addressed by scaling the excitation pulse length to maintain constant $\mathrm{B}_{1}{ }^{+}$RMS.

Methods: Bloch simulations were used to examine the effect of pulse length scaling on the bound pool.
A healthy experienced volunteer was scanned on a $7 \mathrm{~T}$ MR system (MAGNETOM Terra, Siemens Healthineers, Erlangen, Germany) using a multi-echo 3D gradient echo sequence (RF- and gradientspoiled) at an isotropic resolution of $0.8 \mathrm{~mm}$ (TR $25 \mathrm{~ms}, 8$ equally spaced echoes TE 2.8.. $18.9 \mathrm{~ms}$, FA 8 and 25 degrees). The image pairs for $\mathrm{T}_{1}$ mapping with variable flip angles were acquired three times with different on-resonance excitation pulses of sinc window shape and lengths: (a) equal (both $560 \mu \mathrm{s})^{3}$, (b) scaled linearly for constant $\mathrm{B}_{1}{ }^{+}$peak $(180,560 \mu \mathrm{s})^{4}$ and (c) scaled quadratically for constant $\mathrm{B}_{1}{ }^{+}{ }_{\text {RMS }}(140,1368 \mu \mathrm{s})^{2} . \mathrm{B}_{1}{ }^{+}$was mapped by the SE/STE EPI method ${ }^{5}$.

Quantitative maps were calculated with the hMRI-toolbox ${ }^{6}$ within the SPM12 framework (https://www.fil.ion.ucl.ac.uk/spm/software/ spm12/) in MATLAB (Mathworks, Natick, USA). The same toolbox was used to compute maps of tissue probability.

Results: Simulations (Fig. 1) demonstrate the expected variations in bound-pool saturation and steady-state signal in the three regimes (equal pulse lengths, constant $\mathrm{B}_{1}{ }^{+}$peak , constant $\mathrm{B}_{1}{ }^{+}$RMS $)$. In vivo results (Fig. 2) reveal that constant $\mathrm{B}_{1}{ }^{+}$RMs scaling leads to tighter clustering of grey and white matter $\mathrm{T}_{1}$ values, suggesting lower spatial bias. An example slice shown in Fig. 3 also shows lower spatial bias and a sharper white matter/grey matter interface.
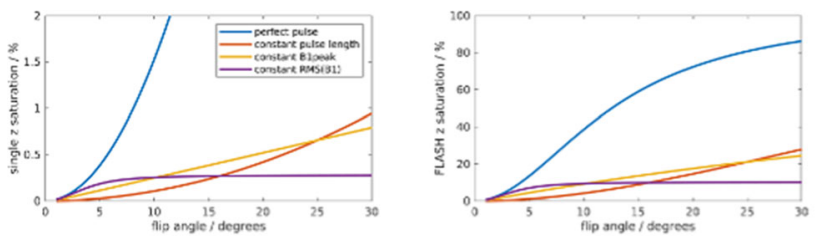

Figure 1: Bloch simulations showing saturation behaviour of the bound pool after a single RF excitation (left; assuming rectangular pulses and $\mathrm{T}_{2}=20 \mu \mathrm{s}$ ) and in the steady state (right; assuming $\mathrm{T}_{1}=1 \mathrm{~s}, \mathrm{TR}=25 \mathrm{~ms}$ and perfect spoiling). In the steady state, the constant $B_{1}{ }^{*}$ Rms gives a constant saturation over a broad range of flip angles. The 'perfect pulse' case approximates the saturation of the free pool.
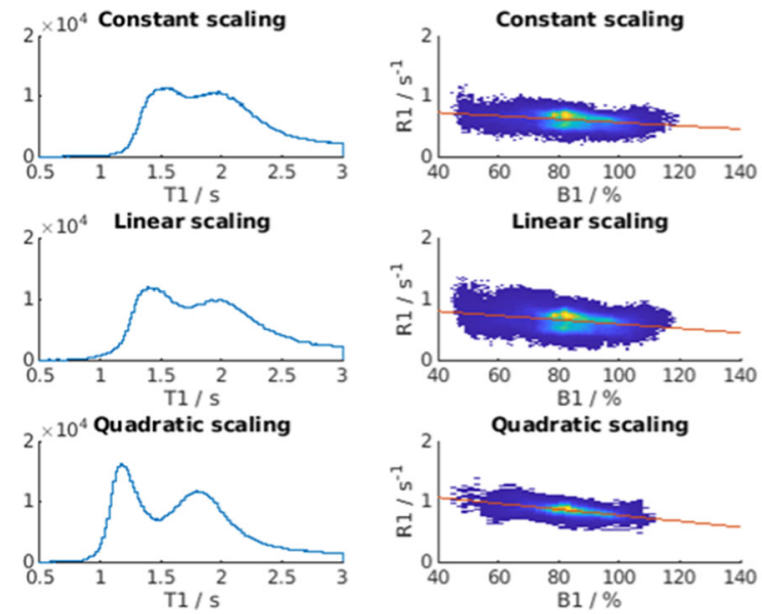

Figure 2: Histograms over a brain mask for relatively well-shimmed $\left(\Delta B_{0}= \pm 100 \mathrm{~Hz}\right.$ ) voxels (left), and scatter plot of $R_{1}$ versus measured $B_{1}{ }^{+}$for white matter voxels (right) show narrower histograms in the quadratic scaling case. Residual dependence of $\mathrm{R}_{1}$ on $\mathrm{B}_{1}{ }^{+}$due to higher order $\mathrm{B}_{1}{ }^{+}$effects are observed in all cases. 


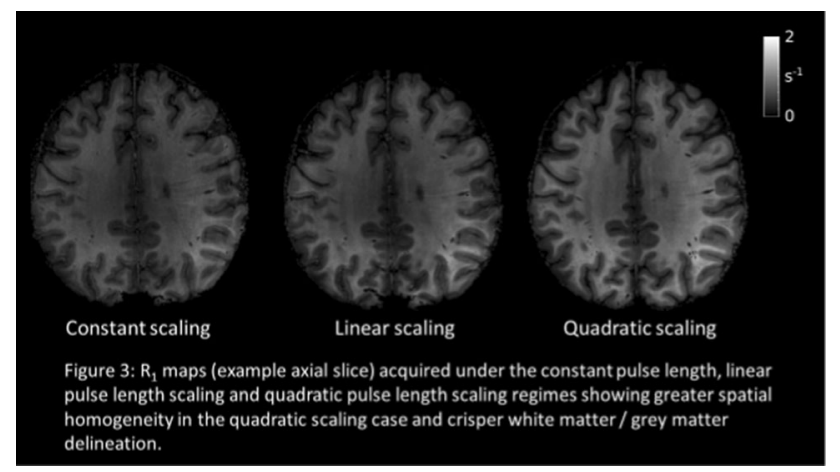

Discussion: Given that CSMT is not provided on the standard MRI product platforms, the approach of scaling the RF pulse lengths to maintain constant $\mathrm{B}_{1}{ }^{+}$RMS is a simple and attractive option to moderate inadvertent $\mathrm{MT}$ effects in $\mathrm{T}_{1}$ mapping and appears advantageous at $7 \mathrm{~T}$. Other reasons may speak for scaling by $\mathrm{B}_{1}{ }^{+}$peak (e.g. to avoid consequences of gain non-linearity in the transmit RF chain) or having equal lengths (e.g. excitation spectrum control [3]). Despite keeping $\mathrm{B}_{1}{ }^{+}$RMS temporally constant, higher order spatial $\mathrm{B}_{1}$ inhomogeneity effects, the altered RF excitation bandwidth and other sources of bias remain and could be addressed in future, for example by parallel RF transmission.

\section{References:}

1. Teixeira RP AG et al. (2019) Magn Reson Med 81:907-920.

2. Helms G (2021) In Proc. 29th ISMRM; 3073.

3. Olsson H et al. (2020) Magn Reson Med 84(3);1347-1358.

4. Lutti A et al. (2013) In Proc. 21st ISMRM; 2478.

5. Lutti A et al. (2012) PLOS ONE 7(3); e32379.

6. Tabelow K et al. (2019) Neuroimage 194, 191-210.

\section{S3.03.}

Submillimeter whole brain magnetization transfer ratio imaging at low field

\author{
*R. Afshari ${ }^{1,2}$, G. Bauman ${ }^{1,2}$, O. Bieri ${ }^{1,2}$ \\ ${ }^{1}$ University of Basel, Department of Biomedical Engineering, Basel, \\ $\mathrm{CH}$; \\ ${ }^{2}$ University Hospital of Basel, Department of Radiology / Division \\ of Radiological Physics, Basel, $\mathrm{CH}$
}

Introduction: Balanced steady-state free precession (bSSFP) has shown excellent prospects and sensitivity for rapid whole-brain magnetization transfer (MT) imaging $(1,2)$ but can be hampered by the presence of local off-resonances leading to signal voids (banding artifacts); especially at high field strength. Thus, bSSFP MT imaging might benefit from overall reduced main magnetic field inhomogeneities at low fields. In this work, we explore the potential of highresolution whole-brain bSSFP-MT imaging on a low-cost commercial $0.55 \mathrm{~T}$ clinical system.

Methods: MRI was performed on a commercially available $0.55 \mathrm{~T}$ low-field MR-system (MAGNETOM Free.Max, Siemens Healthineers, Erlangen, Germany) using a 12-channel head coil. Brain images were acquired with a 3D half-radial dual-echo bSSFP method (3), using a flip angle of $40^{\circ}, 725 \mathrm{~Hz} / \mathrm{px}$ bandwidth, 80,000 radial half-projections, 408 samples per half-projection for a FOV of $256 \times 256 \times 256 \mathrm{~mm}^{3}$, yielding an isotropic resolution of $0.87 \mathrm{~mm}$. MT-sensitization was achieved by a modulation of the RF pulse duration (1). For the non-MT-weighted scan $\left(S_{\text {nonMT }}\right)$ the duration of the hard $\mathrm{RF}$ pulse $\left(\mathrm{T}_{\mathrm{RF}}\right)$ was $800 \mu \mathrm{s}$ and $\mathrm{TE} 1 / \mathrm{TE} 2 / \mathrm{TR}=0.43 / 2.9 /$
$3.5 \mathrm{~ms}$, whereas the MT-weighted volume $\left(\mathrm{S}_{\mathrm{MT}}\right)$ was acquired a $\mathrm{T}_{\mathrm{RF}}$ of $200 \mu$ s yielding TE1/TE2/TR $=0.13 / 2.7 / 2.9 \mathrm{~ms}$. Scan time was 3:52 $\mathrm{min}$ for the MT-weighted and 4:40 min for the non-MTweighted scan. Magnetization transfer ratio (MTR) images in percentage units $(\mathrm{pu})$ were calculated from $\mathrm{MTR}=100 \times\left(\mathrm{S}_{\mathrm{nonMT}^{-}}\right.$ $\left.\mathrm{S}_{\mathrm{MT}}\right) / \mathrm{S}_{\text {nonMT }}$.

Results: As expected, bSSFP imaging is essentially free of banding artifacts at low field and MT-effects are strongly modulated by the RF pulse duration (Fig. 1). Corresponding MTR images are shown in Fig. 1-C. For the proposed setting, average MTR values for WM and GM are 33 pu and 26.7 pu (see ROI in Fig. 1). [a.u.]

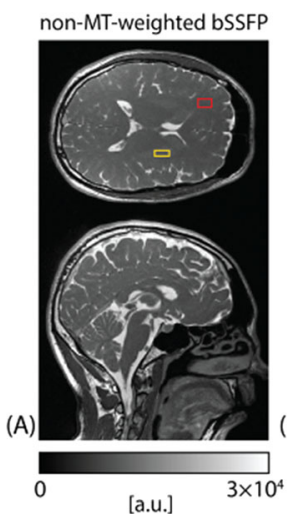

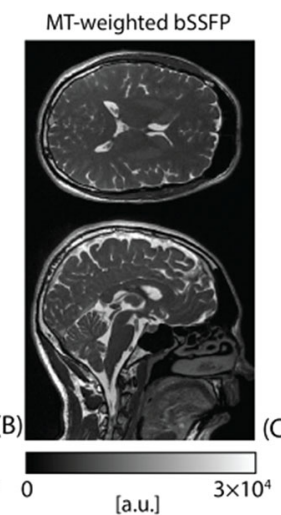

[a.u.]

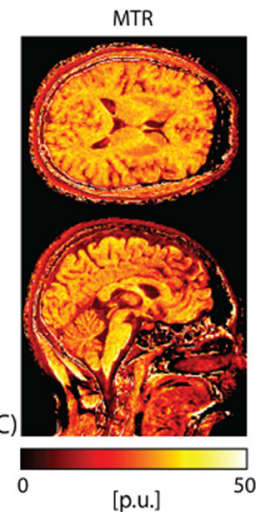

[p.u.]
Figure 1: Exemplary axial and sagittal view of non-MT-weighted bSSFP (A), MT-weighted bSSFP (B), and the corresponding MTR images(C).

Discussion: Surprisingly, the proposed method provides enough signal-to-noise to yield submillimeter isotropic whole brain MTR images at $0.55 \mathrm{~T}$ in a clinically acceptable scan time. This is particularly due to the high sequence efficiency proffered by the half-radial dual-echo approach in combination with the increased T2/T1 ratio with decreasing field strength offering increased bSSFP signal. Moreover, any possible residual off-resonance-related MTR variations are successfully mitigated at low field. Future work will explore different RF pulse prolongation settings to maximize MT sensitivity. In conclusion, bSSFP-based MT-sensitized imaging offers excellent prospects for broad clinical translation and application in low fields.

\section{References:}

1. Bieri O. \& Scheffler K. MRM 2007;58:511-518.

2. Amann M. et al. Neuroimage. 2015;108:87-94.

3. Bauman G. \& Bieri O. MRM. 2020;84:237-246.

\section{S3.04.}

\section{Sub-millisecond 2D MRI of the vocal fold oscillation using single point imaging with rapid encodin (SPIRE)}

\footnotetext{
*J. Fischer ${ }^{1}$, A. C. Özen ${ }^{1,2}$, S. Ilbey ${ }^{1}$, L. Traser ${ }^{3}$, M. Echternach ${ }^{4}$, B. Richter ${ }^{3}$, M. Bock ${ }^{1}$

${ }^{1}$ Albert-Ludwig-University of Freiburg, Departement of Radiology, Freiburg im Breisgau, DE;

${ }^{2}$ German Cancer Research Center (DKFZ), German Consortium for Translational Cancer Research Partner Site Freiburg, Heidelberg, DE;

${ }^{3}$ Albert-Ludwig-University of Freiburg, Freiburg Institute for Musicians' Medicine, Freiburg im Breisgau, DE;

${ }^{4}$ Ludwig Maximilian University of Munich, Division of Phoniatrics and Pediatric Audiology / Department of Otorhinolaryngology, Munich, $D E$
} 
Introduction: The position of the vocal folds in the larynx limits optical examinations to a top down view in laryngoscopy. MR does provide the free choice of imaging plane, however, the rapid oscillation of $100 \mathrm{~Hz}$ and higher is too fast for conventional acquisitions. We have shown that the use of short PE gradients enables sub-millisecond temporal resolution when a one-dimensional periodic motion is aligned with PE direction [1]. Here, we show how extending PE to the second in-plane direction allows for dynamic imaging of the vocal folds oscillation in the coronal view with sub-millisecond temporal resolution.

Methods: The proposed method uses single point imaging [2] to encode k-space and each pair of PE gradient moments is applied as fast as possible, such that encoding time $t_{\mathrm{PE}}$ decreases towards $\mathrm{k}$-space center (Fig. 1). To achieve a spatial resolution below $1 \mathrm{~mm}$, a rectangular FOV $(60 \mathrm{~mm} \times 41 \mathrm{~mm})$ and a matrix size of $64 \times 44$ were chosen, resulting a maximum gradient duration of $\mathrm{t}_{\mathrm{PE}}=660 \mu \mathrm{s}$. Two transverse saturation bands were applied in SI direction to avoid oversampling artefacts (Fig. 2). Unwanted motion of the larynx was measured by acquiring interleaved projection navigators in both inplane directions. Saturation bands and navigators were applied about every $100 \mathrm{~ms}$. With $\mathrm{a}=10^{\circ}, \mathrm{TE}=1.2 \mathrm{~ms}, \mathrm{TR}=2.45 \mathrm{~ms}$ and 24 repetitions, the total acquisition time was about $3 \mathrm{~min}$. The volunteer was asked to phonate at $160 \mathrm{~Hz}$ and rebreathe at will. An electroglottogramm (EGG) was acquired during the MR-measurement with two MR-safe electrodes [3], to obtain the oscillation phase of the vocal folds during each phase encoding gradient. With this information, the acquired MR-data are gated in one of $10 \mathrm{k}$-spaces. Prior to reconstruction, motion was estimated using the navigator data by calculating the phase only cross correlation to a reference line. A spatial shift is then corrected using the Fourier shift theorem by multiplying a constant phase to the k-space point. Dynamic images are reconstructed with a total variation constraint using BART [4].
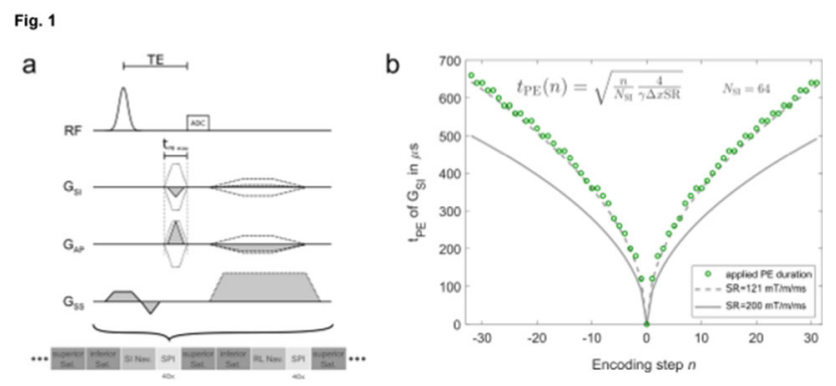

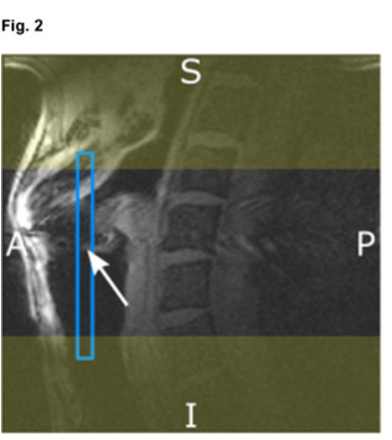

sagittal

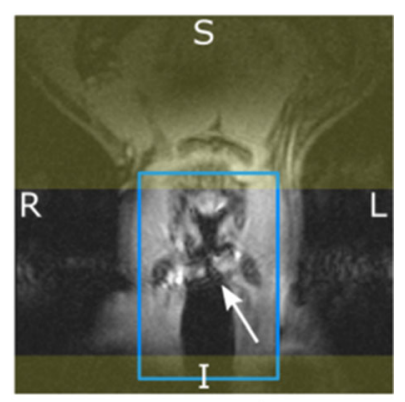

coronal
Results: About $28 \%$ of the acquired data is rejected, mainly because no phonation was detected by the EGG. During all accepted acquisitions the mean oscillation frequency was $165.13 .4 \mathrm{~Hz}$. The individual reconstructed frames are shown in Fig. 3, each with a temporal resolution of $606 \mu \mathrm{s}$, as well as the mean EGG signal during each frame. Consistent with the low EGG signal, the vocal folds are opened in the first two images. In frames 3-7 the vocal folds are closed and an upwards motion can be seen after which both sides separate again.
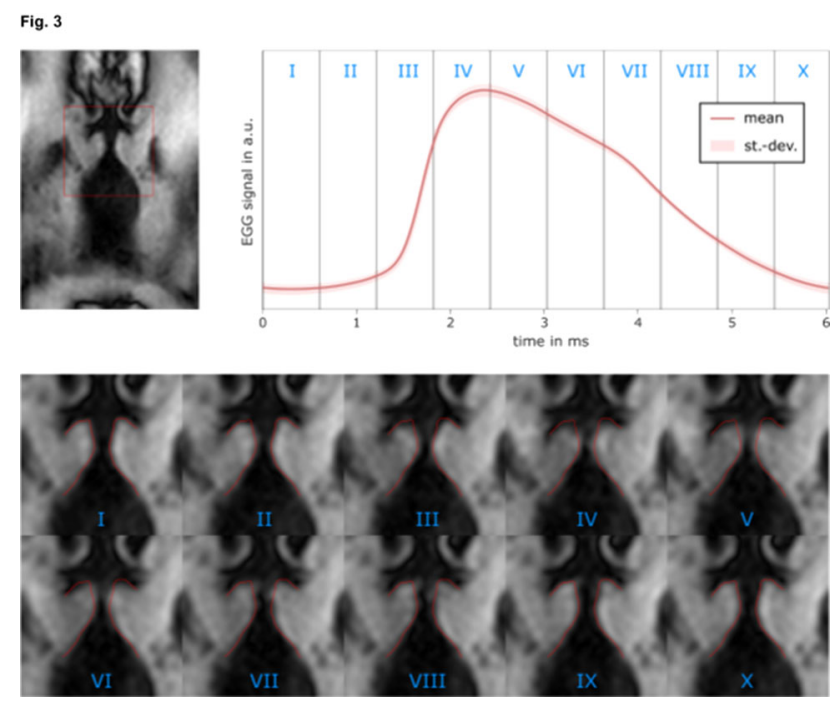

Discussion: For the first time, dynamic 2D MR images of the oscillating vocal folds are presented. The dynamic image information correlates well with the oscillation phases from the EGG-signal, and the open and closed phase can be clearly distinguished in the SPIRE images.

\section{References:}

[1] https://doi.org/10.1002/mrm.27982.

[2] https://doi.org/10.1016/0378-4363(85)90087-7.

[3] https://doi.org/10.1002/mrm.26037.

[4] https://doi.org/10.5281/zenodo.592960.

\section{S3.05. \\ QSM streaking artifact reduction using non-uniform Fourier transformation}

\author{
*P. Fuchs ${ }^{1}$, K. Shmueli ${ }^{1}$ \\ ${ }^{1}$ University College London, Department of Medical Physics \\ and Biomedical Engineering, London, $G B$
}

Introduction: Quantitative susceptibility mapping (QSM) aims to reconstruct local susceptibility variations from global phase perturbations. These are related through a convolution with a unit dipole field and inverting this is a major QSM research area. Initially, direct methods were proposed $[1,2]$, followed by iterative methods $[3,4]$ and, more recently, by machine learning approaches [5].

Here we investigate a novel approach to directly solve the inverse problem. This is an ill posed problem due to pointwise division by zeros in the dipole kernel (D), with low values of D leading to streaking artifacts. The problem is often regularised by thresholding $\mathrm{D}^{-1}$ [1], known as Thresholded K-space Division (TKD), or by applying Tikhonov terms $[3,6]$. We propose an adjusted sampling pattern in frequency space. By using a non-uniform Fourier Transform (nuFT) to avoid sampling areas where D is small, we aim to achieve considerable streaking artifact reduction. 
Methods: To adjust the sampling pattern, we drew frequency space coordinates for the nuFT from a probability distribution that resembles D: low values in D correspond to low probabilities of sampling these values and more samples where $\mathrm{D}^{-1}$ is well defined. The nonuniformly sampled $\mathrm{D}^{-1}$ was then thresholded and used as in TKD. The nuFT was implemented using the BART toolbox[8]. All computations were performed using MATLAB on an Intel i9-10920x (12 core) CPU with $64 \mathrm{~Gb}$ of RAM. We compared our method to TKD [1], and a non-linear Total Variation regularised method (nlTV), using default settings (threshold 2/3) and open source code [6,7], on local 3D GRE field maps of the brain acquired at high $(1 \mathrm{~mm}$ isotropic) resolution in vivo as described in [10]. The direct methods were $4 \times$ oversampled in $\mathrm{k}$-space (zero padded) and the results were corrected for underestimation [2].

Results: Figures 1 and 2 show a comparison of susceptibility maps between the different methods. A difference image between the standard TKD reconstruction and proposed nuFT-based susceptibility map is presented in Fig. 3 and shows primarily streaking artifacts (e.g. see arrows). The nuFT reconstructions were computed in less than $4 \mathrm{~min}$, TKD in seconds, and nlTV in $2 \mathrm{~min}$.
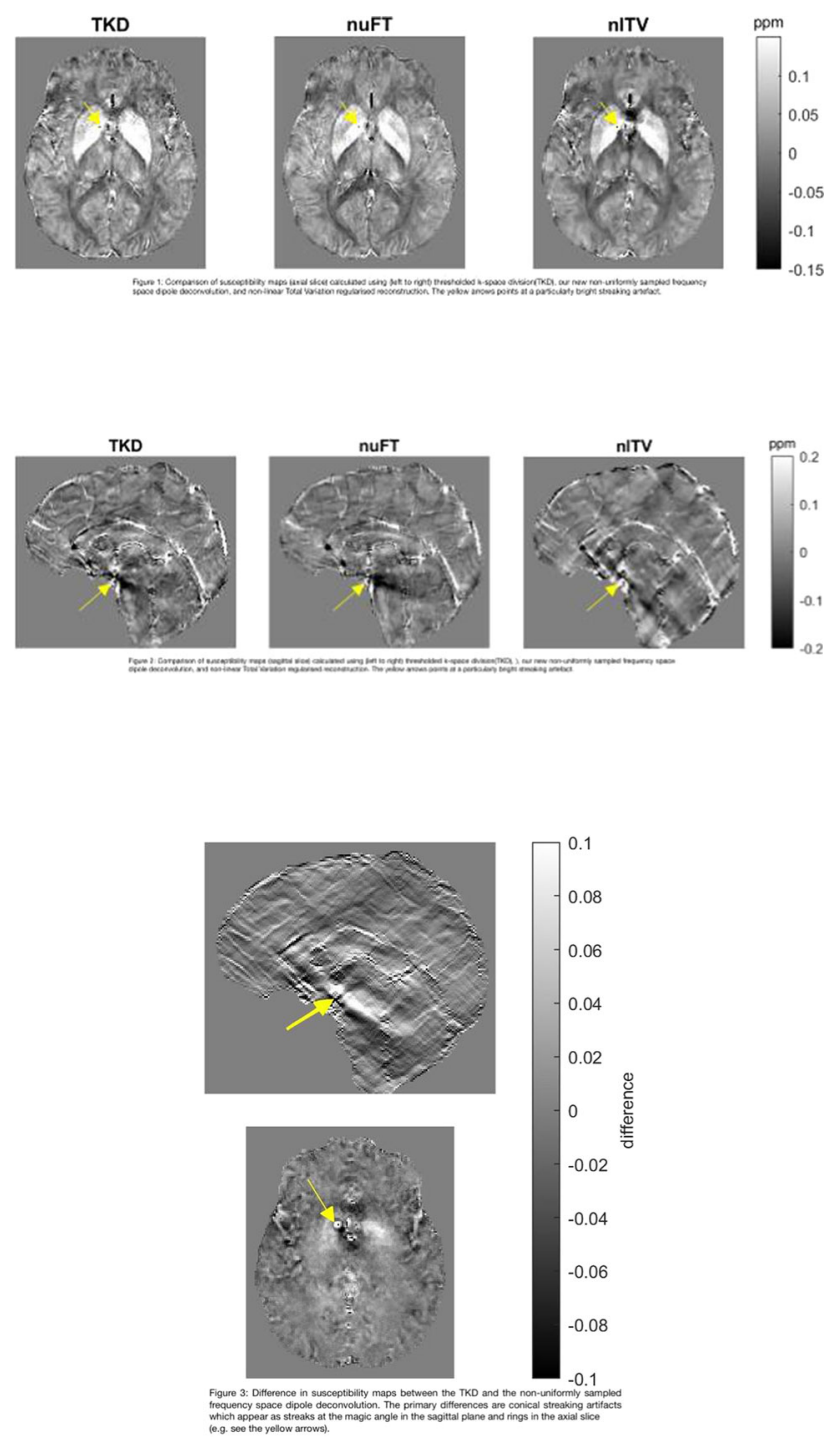

Discussion: Here, we investigated the effect of non-uniform sampling of frequency space for QSM. This nuFT approach resulted in fewer streaking artefacts without using extra explicit regularisation. It also provides detailed control and insight into the kernel $\left(D^{-1}\right)$ in Fourier space and could be combined with compressed sensing techniques [11] to further improve QSM reconstruction.

\section{References:}

[1] Shmueli, Magn. Res. Med., 2009.

[2] Schweser, Magn. Res. Med.,2012.

[3] Karsa, ISMRM 2019.

[4] Milovic, Magn. Res. Med., 2018.

[5] Lai, MICCAI 2020.

[6] https://xip.uclb.com/product/mri_qsm_tkd

[7] https://gitlab.com/cmilovic/FANSI-toolbox

[8] https://zenodo.org/record/4570601

[10] Karsa, Magn. Res. Med, 2018.

[11] De Leeuw den Bouter, J. of Phys. Comm., 2021.

\section{S3.06.}

Multi-parametric quantitative mapping of R1, R2*, PD, and MTsat is reproducible when accelerated with compressed SENSE

\author{
*R. Berg ${ }^{1}$, T. Leutritz ${ }^{2}$, N. Weiskopf ${ }^{2}$, C. Preibisch ${ }^{1}$ \\ ${ }^{I}$ Technical University of Munich, Department of Neuroradiology, \\ Munich, DE; \\ ${ }^{2}$ Max Planck Institute for Human Cognitive and Brain Sciences, \\ Department of Neurophysics, Leipzig, DE
}

Introduction: Conventional clinical routines use qualitative magnetic resonance imaging (MRI) to assess diseased tissue. Multi-parametric mapping (MPM) of R1, R2*, PD, and MTsat is a quantitative MRI method that promises better comparability across sites and time as well as a higher sensitivity to systemic tissue changes. For clinical applications of MPM, the required sequences need to be accelerated and their accuracy be verified. Therefore, this study investigates the reproducibility and repeatability of MPM using sequences accelerated with Compressed SENSE.

Methods: Five healthy subjects were scanned three times on a Philips 3 T Ingenia Elition. MPM comprised B1 mapping and gradient echo sequences with $\mathrm{T} 1 \mathrm{w}$ : TR $=18 \mathrm{~ms}, \alpha=25^{\circ}$; PDw: TR $=18 \mathrm{~ms}$, $\alpha=4^{\circ}$; MTw: TR $=48 \mathrm{~ms}, \alpha=6^{\circ}, \alpha_{\mathrm{MT}}=220^{\circ}, \mathrm{t}_{\mathrm{MT}}=8 \mathrm{~ms}, \mathrm{f}_{\mathrm{MT}}=$ $1000 \mathrm{~Hz}$; with six echoes each (TE1/DTE $=2.4 / 2.4 \mathrm{~ms}$ ) and $1 \times 1 \times 1 \mathrm{~mm}^{3}$ resolution. In each scan session, three different imaging accelerations were used: standard SENSE (AP: 2, RL: 1.25, $20 \mathrm{~min}$ ) and Compressed SENSE (CS) with acceleration factors $\mathrm{CS}=4(15: 40 \mathrm{~min})$ and $\mathrm{CS}=6(10: 30 \mathrm{~min})$. Quantitative parameter maps (qMaps) were calculated using the in vivo histology (hMRI) toolbox $^{1,2}$ correcting for $\mathrm{B} 1$ bias $^{3}$ and insufficient RF spoiling ${ }^{4,5}$. Coefficient-of-variation $(\mathrm{CoV})$ maps were calculated for each quantitative parameter across both scan session ("repeatability") and accelerations within the same session ("reproducibility" with different acceleration parameters) and compared quantitatively in wholebrain gray matter (GM) and white matter (WM).

Results: R1, R2*, PD, and MTsat parameter maps appeared visually similar across different accelerations (Fig. 1A) and scan sessions (Fig. 2A). Repeatability-based $\mathrm{CoV}$ values of qMaps acquired with the same acceleration across scan sessions were comparable for SENSE, CS $=4$, and CS $=6$ (Fig. 1B). Likewise, reproducibilitybased $\mathrm{CoV}$ values of qMaps acquired in the same scan session with different accelerations were comparable for scans $\mathrm{A}, \mathrm{B}$, and $\mathrm{C}$ (Fig. 2B). In GM and WM, subject-mean reproducibility-based CoVs 
(green) were comparable to repeatability-based CoVs (blue) for R1 and PD and even slightly decreased for R2* and MTsat (Fig. 3).

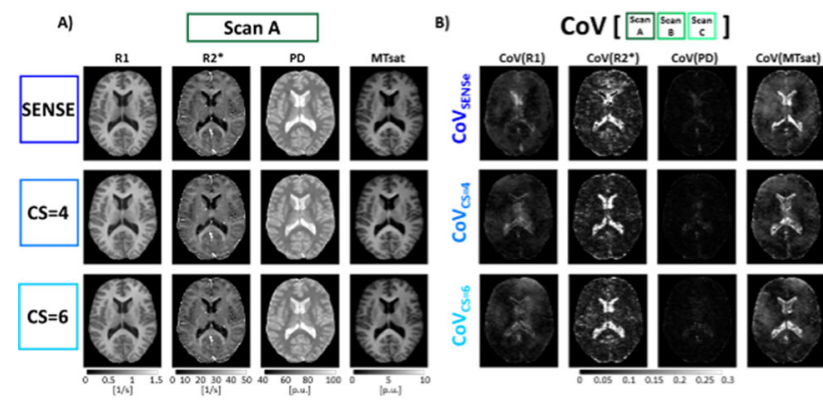

Figure 1: Repeatability evaluation: Exemplary maps of quantitative parameters $(A)$ and coefficients of variation ( $\mathrm{COV}$ ) (B) from a healthy volunteer for different acceleration methods. The quantitative parameter maps in A) were acquired in Scan A with different accelerations and the CoV maps were calculated from the
scans with the same acceleration method acquired in different scan sessions (Scan A, Scan B, and Scan C).

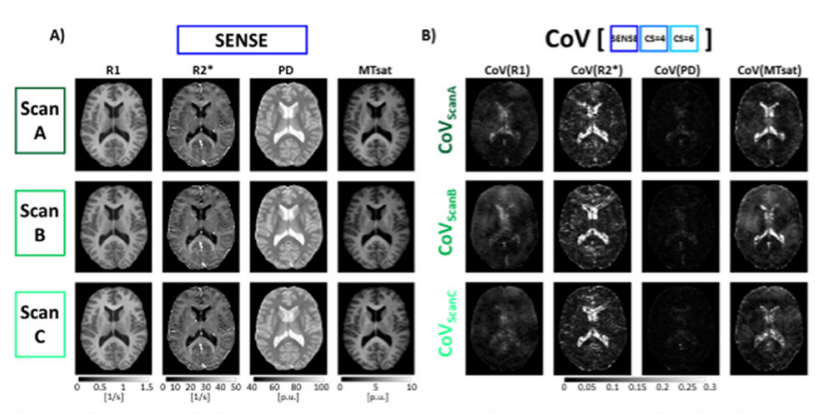

Figure 2: Reproducibility evaluation: Exemplary maps of quantitative parameters (A) and coefficients of variation (COV) maps $(B)$ from the healthy volunteer shown in Fig. 1 for different scan sessions. The quantitative parameter maps in A) were acquired with SENSE in different scan sessions and the CoV maps were calcula.
the three sequences within the same scan session and with different accelerations (SENSE, CS $=4$, CS=6).
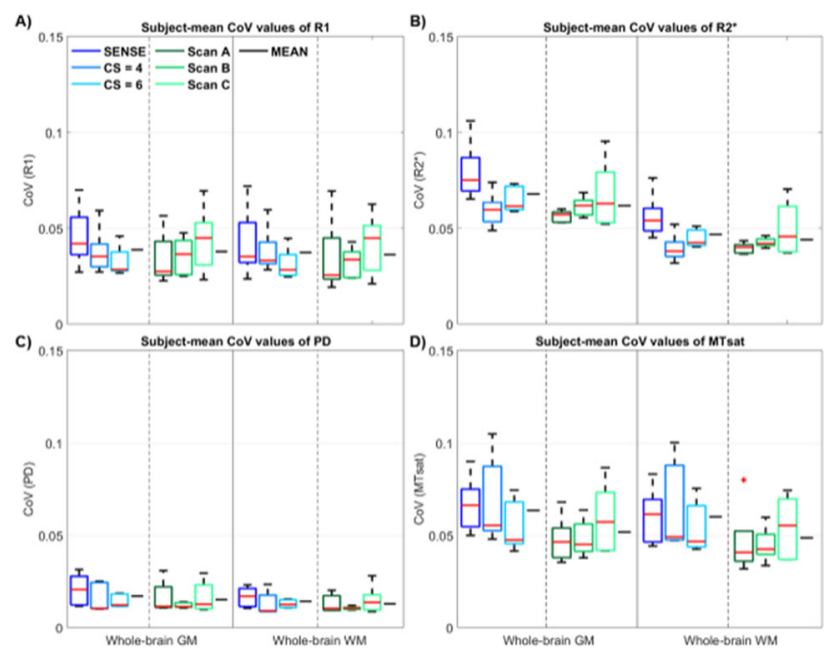

Figure 3: Boxplots of coefficients of variation (CoV) of quantitative parameter maps from the repeatability (blue) and reproducibility (green) measurements. In each panel, the subject-mean CoV values of R1 (A), R2* (B), PD (C), and MTsat (D) are shown for whole-brain GM and whole-brain WM segmentations. The average or

Discussion: Both reproducibility-based and repeatability-based $\mathrm{CoV}$ values of all qMaps agree well with those from a previous study ${ }^{6}$. Average R1 and PD values showed similar variability across either scan sessions or accelerations. Moreover, average R2* and MTsat values were even more stable (lower $\mathrm{CoV}$ ) across accelerations than scan sessions. Together with the high visual similarity between
qMaps acquired with different accelerations, this suggests that the highest investigated acceleration, CS with acceleration factor of 6 , provides accurate MPM results. As this allows to considerably reduce scan duration, CS $=6$ facilitates MPM in clinical routines.

\section{References:}

1. Tabelow 2019 NeuroImage

2. Weiskopf 2015 CurrOpinNeurol

3. Yarnykh 2007 MRM

4. Preibisch 2009 MRM

5. Baudrexel 2018 MRM

6. Leutritz 2020 HumBrainMapp

\section{S3.07.}

Measurement of hyperpolarized xenon (129Xe) ventilation MR image quality in healthy and asthma patients with varied compressed sensing sampling *M. Tavakkoli ${ }^{1,2}$, S. Svenningsen

${ }^{1}$ McMaster University, Department of Electrical and Computer Engineering, Hamilton, $C A$

${ }^{2}$ St. Joseph's Healthcare, Imaging Research Centre, Hamilton, CA; ${ }^{3}$ St. Joseph's Healthcare, Firestone Institute for Respiratory Health, Hamilton, $C A$

Introduction: Computed Tomography (CT) has been the standard imaging technique to evaluate lung structure. However, due to its ionizing radiation and lack of functional information, novel Magnetic Resonance Imaging (MRI) methods are of high interest. Alternatively, hyperpolarized noble gas MRI has shown great promise. This approach requires breathing in hyperpolarized xenon-129 $\left(\mathrm{HP}^{129} \mathrm{Xe}\right)$ and subsequent breath-holding during acquisition. In patients with severe disease, such as severe asthma, breath-holding is often difficult [2,3]. Accelerated imaging using Compressive Sensing (CS) can help [4]. CS aims to recover good quality images from undersampled $\mathrm{k}$-space permitting faster data acquisition. CS is now becoming routine for 1H-MRI. However, its application in $\mathrm{HP}^{-129} \mathrm{Xe}$ lung ventilation imaging has not been optimized. The goal of the current work is to assess CS sampling schemes and their effect on image Signal to Noise Ratio (SNR), Structural Similarity Index Measure (SSIM) and resolution (i.e. Point-Spread Function, PSF).

Methods: Ten subjects ( $n=5$ healthy and $n=5$ severe asthma) were scanned using a GE MR750 $3 \mathrm{~T}$ scanner, acquiring fully sampled 3D multi-slice $\mathrm{HP}^{129} \mathrm{Xe}$ lung ventilation images $(10 \mathrm{~s}$ breath-hold, $128 \times 80$ (PExFE) and 16 slices) [6]. Using fully sampled data, 200 masks were pseudo-randomly generated [5] each at 7 different sampling rates, $15 \%, 25 \%, 35 \%, 45 \%, 55 \%, 65 \%$ and $75 \%$. The Parallel Imaging Compressive Sensing (PICS) command from the Bart toolbox [7], with L1 wavelet optimization and 100 iterations, was employed to reconstruct undersampled data. SNR, SSIM and PSF of each were subsequently compared.

Results: Example HP- ${ }^{129} \mathrm{Xe}$ ventilation images are shown (Fig. 1). The ratio of the main lobe to the standard deviation of the PSF sidelobes is considered the incoherence value reported for each image. The mean and standard deviation of each SNR, SSIM and incoherence values of all 5 healthy data are reported in Table 1; the lowest and highest sampling ratios $(15 \%, 75 \%)$ are depicted in Fig. 2 in the right column. The exact computation for the asthmatic data also can be found in Table 1 and the left column of Fig. 2. 


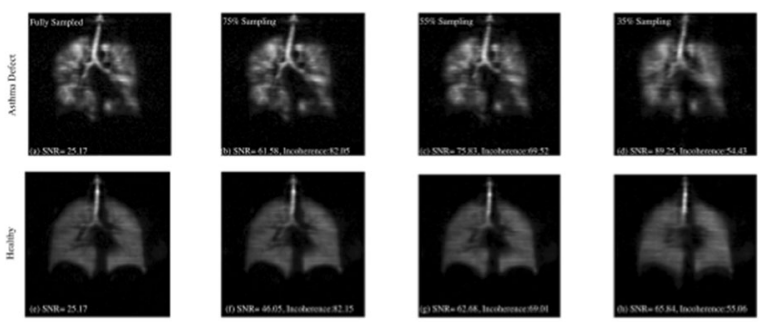

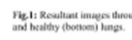

Fig. 2

\begin{tabular}{|c|c|c|c|c|c|c|}
\hline $\begin{array}{l}\text { Sampliog } \\
\text { Rate }\end{array}$ & $\begin{array}{l}\text { SNR } \\
\text { (Asthma) }\end{array}$ & $\begin{array}{l}\text { SNR } \\
\text { (Healthy) }\end{array}$ & $\operatorname{SSIM}$ (Asthma) & SSIM (Hcalltyy) & Incobrencece (Asthma) & Incobererence (Heallthy) \\
\hline $15 \%$ & $19.23 \pm 7.45$ & $1332 \times 4.02$ & $0.058=0.0076$ & $0.061 \pm 0.0072$ & $37.60 \pm 0.5705$ & $37.15 \div 1.2948$ \\
\hline $25 \%$ & $28.40 \pm 9.97$ & $17.59 \approx 5.98$ & $0.106=0.0116$ & $0.11=0.0100$ & $46.82=0.2941$ & $46.79=1.0010$ \\
\hline $35 \%$ & $33.90=11.38$ & $19.62 \div 7.94$ & $0.157 \pm 0.0137$ & $0.161 \pm 0.0135$ & $54.40 \neq 0.1060$ & $54.4 \div 0.1591$ \\
\hline $45 \%$ & $38.79 \pm 14.51$ & $20.61=7.50$ & $0.219=0.0171$ & $0.218 \pm 0.0167$ & $61.94=0.1520$ & $61.82=0.1170$ \\
\hline $59 \%$ & $45.53 \pm 17.74$ & $21,43 \pm 8.51$ & $0.293 \pm 0.0238$ & $0.287 \pm 0.0209$ & $68.71=0.1695$ & $68.60 \div 0.1060$ \\
\hline $65 \%$ & $54.45 \pm 20.74$ & $25.25 \pm 9.91$ & $0.906 \pm 0.0282$ & $0.396 \pm 0.0249$ & $75.21 \pm 0.2007$ & $75.06 \pm 0.0949$ \\
\hline $75 \%$ & $59.56 \pm 20.81$ & $28.16 \simeq 10.88$ & $0.529 \simeq 0.0345$ & $0.513 \approx 0.0317$ & $81.24=0.2312$ & $81.05 \simeq 0.0913$ \\
\hline
\end{tabular}

Fig. 3
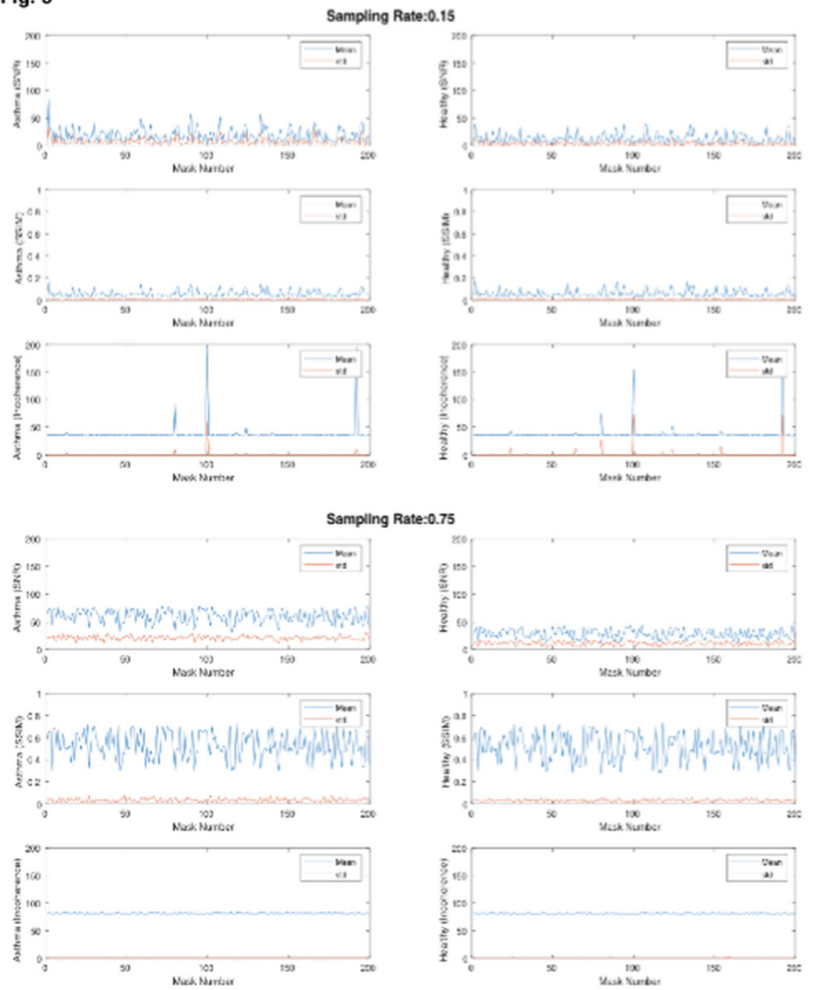

Fig.2: Mean \pm standard deviation of SNR, SSIM and incoherence value of (un)healthy patients for the lowest versus highest sampling ratio $(15 \%, 75 \%)$

Discussion: With greater undersampling, the degree of incoherence and SSIM decreased, while SNR increased (expected due to CS reconstruction noise filtering [4]). Higher SNR in asthma data was noted and is likely the result of the same amount of $\mathrm{HP}_{-}{ }^{129} \mathrm{Xe}$ gas being distributed over less lung volume due to ventilation defects, compared to healthy. Most importantly, there was a high degree of variation in results from each of the 200 masks of each undersampling rate. The greater the undersampling the greater the variation in SNR and SSIM on both healthy and asthma groups (Table 1). Incoherence also decreases for healthy, yet not for asthma lungs. These results indicate there is an optimized rate and pattern of undersampling. This needs thorough investigation to allow optimal CS accelerated $\mathrm{HP}^{129} \mathrm{Xe}$ lung ventilation imaging.

\section{S3.08.}

\section{Iterative RAKI with complex-valued convolution for improved image reconstruction with limited scan- specific training samples}

\author{
*P. Dawood ${ }^{1,2}$, M. Blaimer ${ }^{3}$, F. Breuer ${ }^{3}$, P. R. Burd ${ }^{4}$, P. M. Jakob ${ }^{1}$, \\ J. Oberberger ${ }^{2}$ \\ ${ }^{1}$ Julius Maximilian University of Würzburg, Department \\ of Experimental Physics 5, Würzburg, DE; \\ ${ }^{2}$ Julius Maximilian University of Würzburg, Department of Internal \\ Medicine I, Würzburg, DE; \\ ${ }^{3}$ Fraunhofer Institute for Integrated Circuits IIS, Development Center \\ $X$-ray Technology (EZRT), Würzburg, DE; \\ ${ }^{4}$ Julius Maximilian University of Würzburg, Department \\ of Theoretical Physics and Astrophysics, Würzburg, DE
}

Introduction: RAKI [1] is a scan-specific machine learning approach for k-space interpolation. It has demonstrated superior reconstruction quality in comparison to the standard parallel imaging approach GRAPPA [2]. However, RAKI may reveal severe artefacts in standard 2D imaging given a reduced amount of auto-calibration-signal (ACS) for high accelerations due to its increased parameter space. We propose iRAKI, an iterative extension of RAKI with complex-valued Convolutional Neural Network $(\mathrm{CNN})[3,4]$ to improve its reconstruction quality with only a limited number of ACS.

Methods: iRAKI:An initial GRAPPA reconstruction is performed using the fully sampled central k-space region as original ACS to obtain synthetic ACS of increased size for RAKI training [5], and to assign an increased filter size to the first hidden layer in the CNN [6] (Fig. 1). Synthetic ACS is extracted from $\mathrm{N}=100$ central lines of the initial GRAPPA k-space reconstruction. Subsequent iterations follow in which the CNN weights are transferred, and further optimized using $\mathrm{N}^{\prime}=100$ central lines from the RAKI reconstruction of the previous iteration as ACS (orig. ACS re-inserted at each iteration). The learning rate $\eta$ is decreased by $\Delta \eta$ after each iteration (initial value $\left.\eta_{0}=0.05\right)$ and we set $\Delta \eta=0.003$ for $\mathrm{R}=4(0.004$ for $\mathrm{R}=5$, R: undersampling rate). The total iteration number amounts to $\eta_{0} / \Delta \eta$. Complex ReLU [7] is chosen as activation, and the mean-squarederror as cost function. The final optimized CNN interpolates the multi-coil subsampled k-spaces simultaneously, which are transformed via FFT and combined via RSS. 


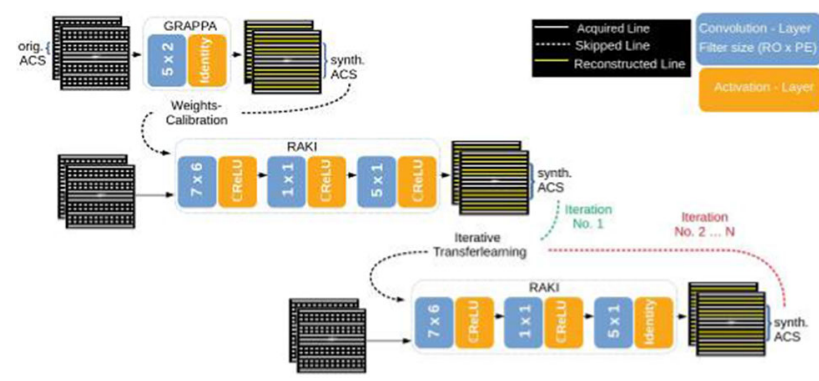

Figure 1: The general principle of IRAKI and its data stream. GRAPPA is assigned a filter size $5 \times 2$ while the first hidden layer in RAKI is assigned a filter size 7x6, given an increased synthetic ACS from the
initial GRAPPA reconstruction (Dimension: Readout $x$ Phase-encoding). The complex Rectifier Linear Unit ( $\mathbb{C R e L U}$ ) is chosen as activation function in RAKI.

In vivo data: $2 \mathrm{D}$ anatomical brain imaging with 16 coils acquired on healthy volunteers at $3 \mathrm{~T}$ (Magnetom Skyra, Siemens Healthineers) were used for evaluation. One set (ref. to as neuro1) was acquired using TSE with TR/TE $=500 / 10 \mathrm{~ms}$, FOV $=220 \times 193 \mathrm{~mm}^{2}$, matrix size $256 \times 224$. Another set (ref. to as neuro2) was performed using FLASH with TR/TE $=250 / 2.90 \mathrm{~ms}, \mathrm{FOV}=230 \times 230 \mathrm{~mm}^{2}$, matrix size $320 \times 320$.

Results: Using only 18 ACS lines (i.e. $8 \%$ of total Phase-encoding lines), iRAKI improves RAKI by removing severe artefacts encountered in the neuro 1 dataset for $\mathrm{R}=4$ (Fig. 2 top) and by enhancing the suppression of noise-amplification regarding GRAPPA in neuro2 dataset (Fig. 2 bottom), also indicated by its SSIM and NMSE metrics (Fig. 3) for $\mathrm{R}=5$, too.
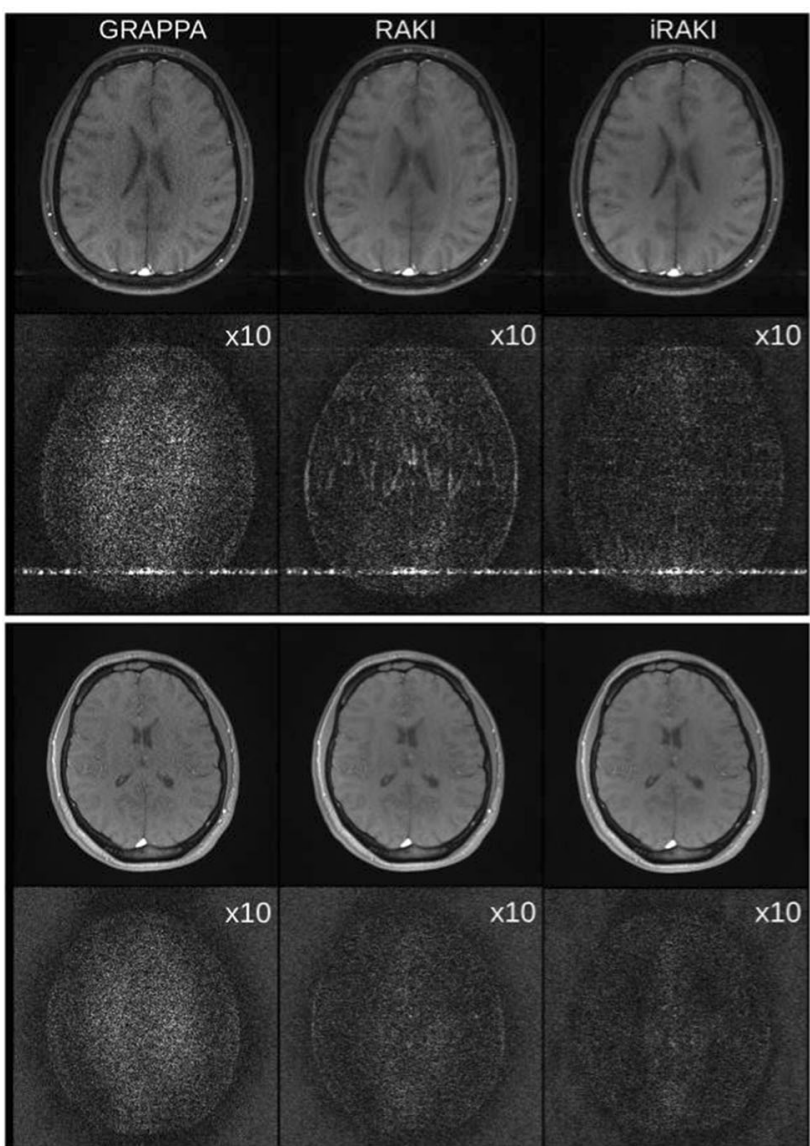

Figure 2: GRAPPA (left), RAKI (center) and iRAKI (right) in comparison (R=4) for neuro1 (top) and neuro2 dataset (bottom). Difference images w.r.t. the fully sampled reference image are depicted below and scaled for display. The artefacts in the posterior region of the neuro1 data set can be attributed to pulsative flow.
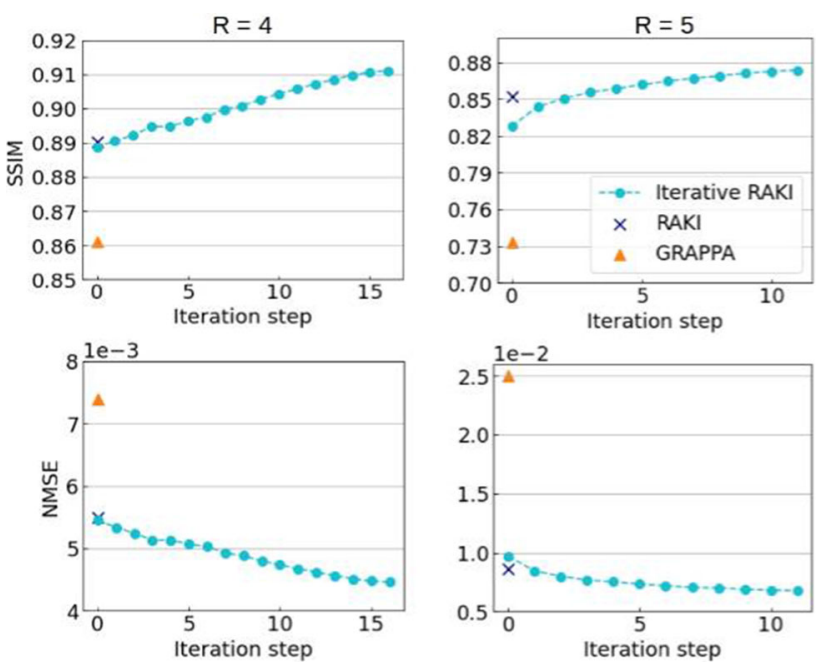

Figure 3: The Structural Similarity Index Measure (SSIM) in the top row, and the Normalized Mean Squared Error (NMSE) in the bottom row of neuro2 dataset reconstructions for 4-fold (left column) and 5-fold undersampling (right column) w.r.t. the fully sampled reference image.

Discussion: The iterative procedure with learning-rate decay reduces the over-all reconstruction error in iRAKI and leads to convergence towards improved data consistency for the shown imaging experiments at four and fivefold acceleration.

\section{References:}

[1] Akçakaya et al. MRM. 2019.

[2] Griswold et al. MRM. 2002.

[3] Cole et al. MRM. 2021.

[4] Virtue et al. IEEE(ICIP), 2017.

[5] Dawood et al. Proc. of ISMRM 2021 \#1961.

[6] Nencka et al. MRM. 2020.

[7] Trabelsi et al. Proc.of ICLR, 2018.

Acknowledgements: The authors thank the data lab DataSphere@JMUW for informative discussions and the Ger. Federal Ministry of Education and Research for funding VIP+ 03VP04951.

S3.09.

\section{Deep learning based retrospective motion correction ensuring data consistency during inference}

\author{
*T. Küstner ${ }^{1}$, D. Nickel $^{2}$, K. Hammernik ${ }^{3}$, D. Rueckert ${ }^{3}$, S. Gatidis ${ }^{1}$ \\ ${ }^{1}$ University Hospital Tübingen, Medical Image and Data Analysis \\ (MIDAS.lab) / Department of Diagnostic and Interventional \\ Radiology, Tübingen, DE; \\ ${ }^{2}$ Siemens Healthcare GmbH, Erlangen, DE; \\ ${ }^{3}$ Technical University of Munich, Munich, DE
}

Introduction: Motion is the main extrinsic source for imaging artifacts in MRI which can strongly deteriorate image quality and thus impair diagnostic accuracy. Numerous motion correction strategies have been proposed to mitigate these artifacts $^{1-4}$. We have recently proposed a deep learning (DL) framework ${ }^{5,6}$ (MedGAN) to perform motion correction without prior motion knowledge. In this work we reformulate the motion correction as an image reconstruction task ${ }^{7}$ which allows to involve data consistency during inference. The proposed MedGAN motion correction is trained and evaluated on a 
cohort of 350 patients for non-rigid motion correction in $\mathrm{T} 2 \mathrm{w}$ abdominal imaging.

Material and methods: We formulate motion correction as an image reconstruction problem with randomly undersampled high-frequencies. We assume that i) motion can be modelled as sparse outliers in $\mathrm{k}$-space ${ }^{8}$ and ii) non-rigid motion can be represented as a superposition of local translational displacements ${ }^{9}$. The motion-affected $\mathrm{k}$-space $y_{m}(k)$ is thus a composition of the motion-free $\mathrm{k}$-space $y(k)$ and the k-space modulated by linear phase drifts along phase-encoding directions $k_{y}, k_{z}$ described by the local translations $u_{t}$ in a neighborhood $W$ at a random high-frequency given by the sampling mask $\Phi$ (Fig. 1B).

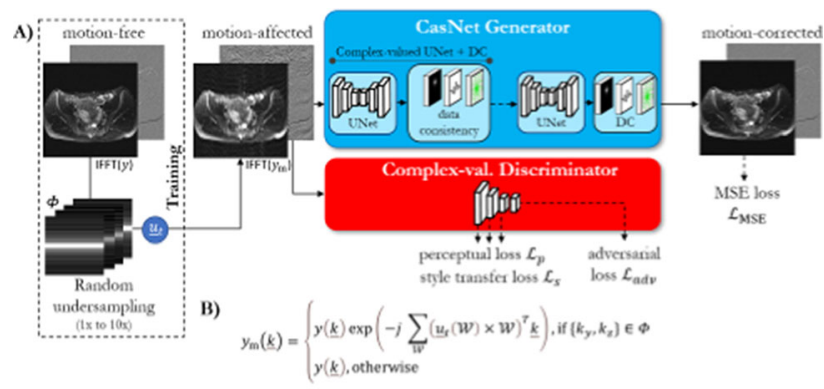

Fig. 1: A) Proposed MedGAN for motion cortection formulated as an image reconstruction task. It consists of a Cosk generator with six concatenated UNets and intermittent proximal gradient data consistencies, and trainable discriminators for

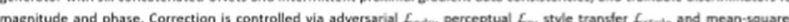
ag ias and

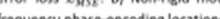

The proposed MedGAN in Fig. 1A contains a generator consisting of six cascaded UNets with intermittent proximal gradient data consistency and receives a complex-valued (magnitude and phase) motionaffected image as input. The MedGAN is trained adversarial with a patch-based magnitude/phase discriminator and with perceptual, style transfer and mean-squared-error loss ${ }^{5}$. During training, variabledensity Gaussian and Poisson-Disc undersampling masks are generated with $1 \times$ to $10 \times$ acceleration. Training is conducted for 50 epochs via ADAM (learning rate scheduler 0.001, batch size 8). MedGAN is trained on 330 patients and tested on 20 patients (informed and written consent), imaged with an axial T2w BLADE: TE/ $\mathrm{TR}=104 / 7560 \mathrm{~ms}, \quad \mathrm{ETL}=51, \quad$ GRAPPA $2 \mathrm{x}, \quad \mathrm{FA}=130^{\circ}$, $0.9 \times 0.9 \times 4 \mathrm{~mm}^{3}$ resolution.

Results and discussion: Figure 2 shows two patients with mild and strong simulated motion to investigate data consistency. Figure 3 depicts two patients with real respiratory motion. Motion-corrected images can be obtained with close resemble to motion-free case. The current study has limitations: Only 2D MR images of a single sequence type were corrected, i.e. through-plane motion correction is limited.

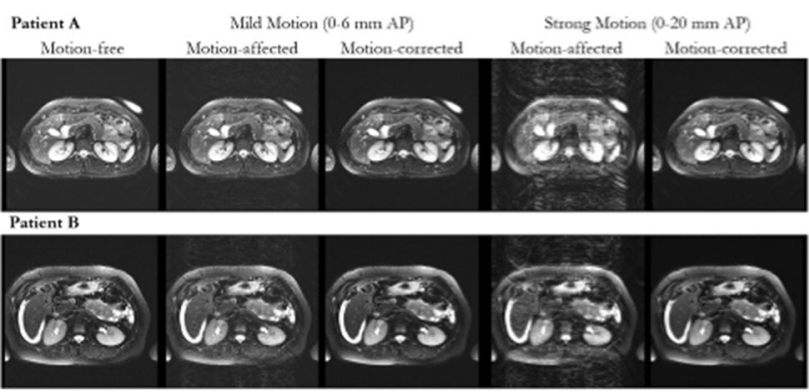

ig. 2: Retrospectively simulated translational motion in anterior-posterior direction for mild $(0-6 \mathrm{~mm})$ and strong $10-20 \mathrm{~mm}$ displacement. Motion-free, motion-affected (simulated) and motion-corrected images are shown. Good performance for changing motion impact and high consistency of motion-corrected to ground-truth can be observed.

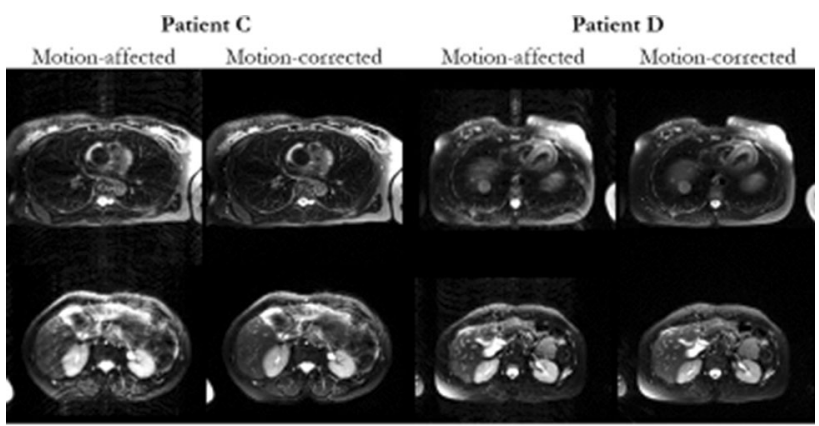

Fig. 3: Real respiratory and pulsation motion observed in two exemplary patients for failing respiratory triggering during acquisition. Motion obstructed anatomy can be recovered with ensured data consistency during correction.

Conclusion: Retrospective motion correction can be formulated as an image reconstruction with the proposed MedGAN providing high quality and motion-free images ensuring data consistency during inference.

\section{References:}

1. Zaitsev et al. JMRI 2015

2. McClelland et al. Medical image analysis 2013

3. Maclaren et al. MRM 2013

4. Atkinson et al. IEEE TMI 1997

5. Küstner et al. MRM 2019

6. Armanious et al. arXiv 2018

7. Oksuz et al. MICCAI 2018

8. Hedley et al. IEEE TMI 1991

9. Gilliam et al. ISBI 2016 


\section{S4.01.}

\section{Assessment of functional QSM in advanced experimental paradigms for neuroimaging research}

*M. Lancione $e^{1,2}$, M. Costagli ${ }^{2,3}$, G. Handjaras ${ }^{1}$, M. Tosetti ${ }^{2,4}$, E. Ricciardi ${ }^{1}$, P. Pietrini ${ }^{1}$, L. Cecchetti ${ }^{1}$

${ }^{1}$ IMT School for Advanced Studies Lucca, MoMiLab, Lucca, IT; ${ }^{2}$ IRCCS Stella Maris, Laboratory of Medical Physics and Magnetic Resonance, Pisa, IT;

${ }^{3}$ University of Genoa, Department of Neuroscience, Rehabilitation, Ophthalmology, Genetics, Maternal and Child Sciences (DINOGMI), Genoa, IT;

${ }^{4}$ Imago 7 Research Foundation, Pisa, IT

Introduction: Functional Quantitative Susceptibility Mapping (fQSM) allows the measuring of time-varying magnetic susceptibility with potentially higher spatial specificity than conventional fMRI. The use of fQSM in combination with General Linear Model (GLM) and On/Off paradigms has been previously assessed ${ }^{1,2,3}$, yet the evaluation of potentials and limitations of this method in advanced experimental paradigms and analyses is still missing. Moreover, voxels with positive fQSM response, i.e., of the same sign as fMRI, require further investigation. To test the usefulness of fQSM and to characterize its activations, we used 7 T MRI, tonotopic mapping, and both univariate (GLM and population Receptive Field; pRF) and multivariate (Representational Similarity Analysis; RSA) analyses. Methods: We acquired complex-valued GRE-EPI datasets at $7 \mathrm{~T}$ $(\mathrm{TE} / \mathrm{TR}=21.3 \mathrm{~ms} / 2.5 \mathrm{~s}$, voxel size $=1.8 \mathrm{~mm}$ isotropic $)$ on 9 subjects passively listening to pure tones of 10 frequency bands (100$6400 \mathrm{~Hz}, 7.5$ s-blocks interleaved by 7.5/10 s-rest, 8 trials per band). fMRI and fQSM data were processed and analyzed via GLM (Fig. 1). Positive fQSM responses were anatomically localized via gray/white matter (GM/WM) segmentation performed on $1 \mathrm{~mm}$-MP2RAGE image. Tonotopic maps were computed via $\mathrm{pRF}$ and the cortical representation of distinct frequency bands was evaluated via RSA and Multidimensional Scaling (MDS).

A

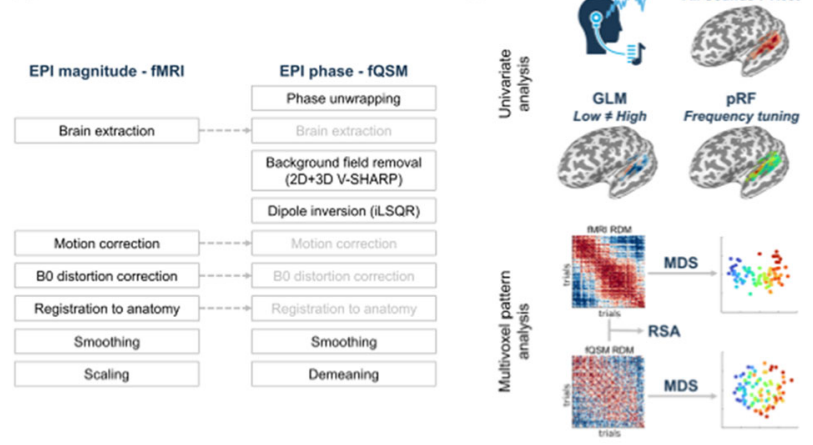

Figure 1: Graphical summary of the reconstruction and preprocessing pipelines (panel A) and of the analysis that were performed on the fMRI and fQSM dataset (panel B). In panel A, gray arrows indicate operations performed on the EPI magnitude and then applied to the phase dat

Results: The fQSM tone-responsive area was $15 \pm 9 \%$ of the active Primary Auditory Cortex (PAC) observed with fMRI and had higher inter-subject variability (Fig. 2A/B). The ratio of active voxels belonging to $\mathrm{GM}$ was higher $(\mathrm{p}<0.01)$ for fQSM $(73 \pm 8 \%)$ than fMRI $(65 \pm 4 \%)$. Positive fQSM responses were found in $14 \pm 10 \%$ of active voxels, mainly in WM (42\%) rather than GM (12\%). pRF revealed that fQSM and fMRI tonotopic maps were significantly correlated (Pearson's $r=0.22 \pm 0.11, \mathrm{p}<0.001$ ). Although fMRI maps were smoother, voxel tunings in fQSM were more equally represented across the whole frequency range $(\mathrm{p}<0.05)($ Fig. $2 \mathrm{C} / \mathrm{D})$. RSA results showed correspondence between response patterns of
fMRI and fQSM $\left(r=0.49 \pm 0.15, \mathrm{p}<10^{-5}\right)$ (Fig. 3A). In MDS analysis, trials of the same stimulus condition clustered along the first dimension for both fMRI and fQSM (Fig. 3B).

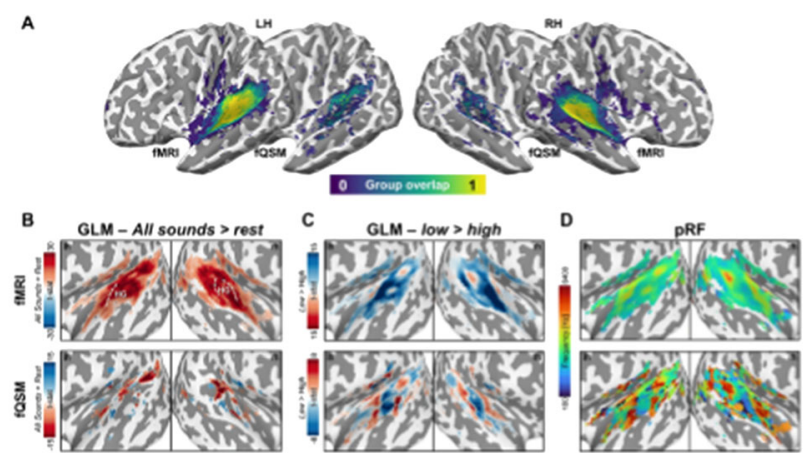

Figure 2: Panel A shows the grouptevel protablistic PAC ROls. the ROI compulod via 1OSM was smaller and showed less inter-subject overlao than the RMRI ROL. Panels B disolays tone-responsive voxels for a represertative mapsects for AMRI (top) and fOSM (bothom). The dottod ines
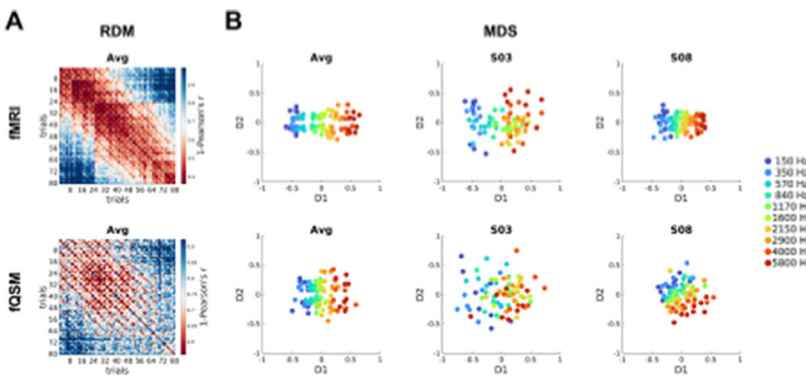

figure 3: Panel A shows the group suerage RDAls computad via the cerrelation distance bettacen astivation patterns in PAC

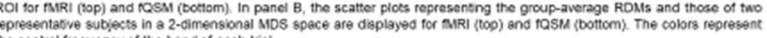

Discussion: fQSM had lower sensitivity but higher spatial specificity than fMRI. Positive fQSM responses were mainly in WM and do not hamper the interpretation of GLM results. Even though fMRI and fQSM tonotopic maps were comparable, the representation of frequency tunings was more balanced in fQSM. Despite being noisier than fMRI, fQSM activation patterns can be used to reconstruct the fine-grained topographical mapping of PAC. Overall, fQSM results are complementary to conventional fMRI. Also, its quantitative nature and higher spatial specificity support its use in longitudinal and multicentric studies and pre-surgical mapping.

\section{References:}

1 Chen et al., J Neurosci Met 2013.

2 Balla et al., Neuroimage 2014.

3 Ozbay et al., Neuroimage 2016.

\section{S4.02. \\ Preliminary investigation of APT- and glutamate- weighted CEST at $7 \mathrm{~T}$ for gadolinium-free imaging of high-grade gliomas}

*B. Schmitz Abecassis ${ }^{1}$, J. A. F. Koekkoek ${ }^{2,3}$, J. de Bresser ${ }^{1}$, L. Dirven ${ }^{2,3}$, M. J. B. Taphoorn ${ }^{2,3}$, M. J. P. van Osch ${ }^{1}$, E. Ercan ${ }^{1}$ 
${ }^{1}$ Leiden University Medical Center, Department of Radiology, Leiden, $N L$;

${ }^{2}$ Leiden University Medical Center, Department of Neurology,

Leiden, $N L$;

${ }^{3}$ Haaglanden Medical Center, Department of Neurology, The Hague, $N L$

Introduction: Current diagnosis and monitoring of gliomas include the assessment of conventional MR imaging, and gadolinium contrast enhancement (GdCE) on $\mathrm{T}_{1}$ weighted $\left(\mathrm{T}_{1} \mathrm{w}\right)$ images. However, the administration of gadolinium-based contrast agents (GBCA) is invasive and of concern due to gadolinium deposition in the brain ${ }^{1}$. A noninvasive alternative to GdCE is warranted to minimize these associated risks. Chemical exchange saturation transfer (CEST) is an MRI technique that can provide endogenous contrast from metabolites and proteins. Previous studies have shown that amide proton transfer (APT) and nuclear overhauser effect (NOE) CEST contrasts are increased and decreased respectively in high-grade gliomas $(\mathrm{HGG})^{2,3}$. More recently, glutamate-weighted CEST (GluCEST) contrast has investigated metabolic changes in diffuse gliomas at $7 \mathrm{~T}^{4}$. However, the relationship between GdCE, APT, NOE, and GluCEST contrasts in HGG patients remains yet to be studied. In this preliminary work, we compare APT, NOE and GluCEST contrasts with GdCE contrast in HGG patients at $7 \mathrm{~T}$.

Methods: We prospectively included two pre-operative patients: one 48 years old male patient and one 60 years old female patient, both with a glioblastoma. CEST scans were performed on a 7TMRI scanner, whereas anatomical scans were retrospectively obtained from clinical imaging at $3 \mathrm{~T}$. Image acquisition parameters can be found in Table1. Image post-processing and analysis details are shown in Fig. 1.

\begin{tabular}{|c|c|c|c|c|c|}
\hline \multicolumn{6}{|l|}{$\begin{array}{l}\text { Fig. } 1 \\
\text { Table } 1\end{array}$} \\
\hline Imaging parameters & $\begin{array}{c}\text { APT \& NOE } \\
\text { CEST }\end{array}$ & GluCEST & DREAM $B_{1}$ map & 3D $T_{1}-W l+G d$ & $T_{2}$-FLAIR \\
\hline Field strength & \multicolumn{3}{|c|}{$\pi$} & \multicolumn{2}{|c|}{$3 T$} \\
\hline TR/TE (ms) & $3.3 / 1.82$ & $3.3 / 1.82$ & $8.0 / 1.97$ & $9.91 / 4.67$ & $11000 / 125$ \\
\hline Fov & $\begin{array}{c}246 \times 246 \mathrm{x} \\
32 \mathrm{~mm}^{3}\end{array}$ & $\begin{array}{c}246 \times 246 \times 32 \\
\mathrm{~mm}^{3}\end{array}$ & $\begin{array}{c}246 \times 246 \times 32 \\
\mathrm{~mm}^{3}\end{array}$ & $\begin{array}{c}220 \times 175 \times 16 \\
\mathrm{~mm}^{3}\end{array}$ & $\begin{array}{c}220 \times 175 \times 27 \\
\mathrm{~mm}^{3}\end{array}$ \\
\hline Voxel size & $2 \times 2 \times 4 \mathrm{~mm}^{3}$ & $2 \times 2 \times 4 \mathrm{~mm}^{3}$ & $1.5 \times 1.5 \times 1.5 \mathrm{~mm}^{3}$ & $1 \times 1 \times 1 \mathrm{~mm}^{3}$ & $5 \times 5 \times 5 \mathrm{~mm}^{3}$ \\
\hline $\begin{array}{c}\mathrm{RF} B \text {, amplitudes } \\
\mathrm{RF} \\
\text { duration/interval/repetition }\end{array}$ & $0.8 \mu \mathrm{T} \& 1.3 \mu \mathrm{T}$ & $3.52 \mu \mathrm{T}$ & - & - & - \\
\hline (ms) & $45 / 45 / 24$ & $50 / 50 / 10$ & - & ${ }_{0.1 \mathrm{ml} \text { prebolus }}$ & - \\
\hline $\begin{array}{c}\text { Contrast } \\
\text { Total scan time }\end{array}$ & $\dot{03.24 \mathrm{~min}}$ & $05: 06 \mathrm{~min}$ & $00: \dot{09} \min$ & $\begin{array}{c}0.1 \mathrm{~mm} \text { prebolus } \\
0.2 \mathrm{ml} \text { per } \mathrm{kg} \\
02.57 \mathrm{~min}\end{array}$ & 02:12 min \\
\hline
\end{tabular}

Results and discussion: Figure 2 shows CEST maps and the corresponding GdCE $\mathrm{T}_{1} \mathrm{w}$ and $\mathrm{T}_{2}$-FLAIR anatomical images from both patients. Patient 1, who has a clear GdCE lesion on the post gadolinium $\mathrm{T}_{1} \mathrm{w}$ image, exhibits a higher $\mathrm{APT}_{\mathrm{AREX}}$ contrast (Fig. 2D) and lower $\mathrm{NOE}_{\mathrm{AREX}}$ contrast (Fig. 2E) in the area corresponding to the GdCE. This is in accordance with previous studies where increased APT and decreased NOE contrast were found for HGG at $7 \mathrm{~T}^{2,3}$. On the other hand, patient 2, who did not have any GdCE, exhibits lower APT (Fig. 2I) and NOE contrast (Fig. 2J).This suggests that APT contrast relates to the tumor physiology portrayed by contrast enhancement. Interestingly, GluCEST contrast was increased for patient 2, who did not have any GdCE. This is in accordance with a previous study where GluCEST contrast was observed in diffuse gliomas, but contrarily to their observations of increased GluCEST contrast in the presence of $\mathrm{GdCE}^{4}$. In conclusion, our preliminary observations suggest a relationship between tumor enhancement, APT and GluCEST contrast in HGG. These highlight the potential of CEST to be used as an alternative imaging method to GBCAs. Future work is in progress to verify our findings in a larger group of patients, as well as to optimize image quality and data analysis to allow quantitative measurements.

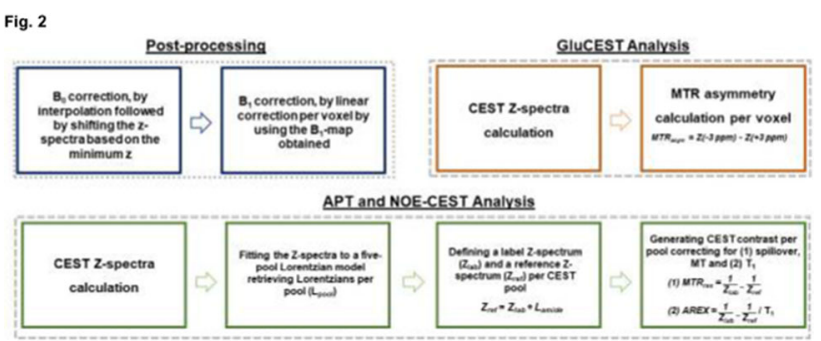

Figure 1. Image analysis workflow. Post-processing performed for Glutamate-weighted CEST (GluCEST) and APT. CEST scans are shown in dark blue. The GluCEST analysis to assess glutamate-weighted contrast and five-poo
Lorentzian analysis to obtain APT

Fig. 3
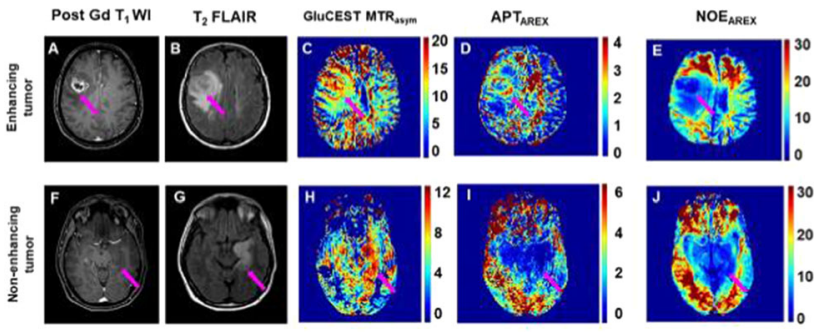

Figure 2. A,F. Post Gadolinium (Gd) T-weighted images (WI) and B.G, $T_{2}$-FLAIR anatomical images are displayed

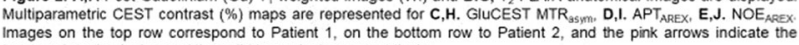
Images on the top row correspond to Patient 1, on the botton
tumang lesion and the solid tumor lesion, respectively.

\section{References:}

1. Kanda T et al. Radiology. 2014.

2. Zaiss $\mathrm{M}$ et al. Neuroimage 2015.

3. Heo HY et al. J Magn Res Imag. 2016.

4. Neal A et al. NeuroImage Clin. 2019.

Funding: Medical Delta Cancer 3.0 \& NWO grant \#16862

\section{S4.03.}

\section{MR vascular fingerprinting (MRVF) of hybrid gradient-spin echo DSC for characterization of microvasculature in gliomas}

*K. Venugopal ${ }^{1}$, F. Arzanforoosh ${ }^{1}$, D. van Dorth ${ }^{2}$, M. Smits ${ }^{1}$, M. J. P. van Osch $^{2}$, J. A. Hernandez-Tamames ${ }^{1}$, E. Warnert ${ }^{1}$, D. H. J. Poot ${ }^{1}$

${ }^{1}$ Erasmus Medical Center, Department of Radiology and Nuclear Medicine, Rotterdam, NL;

${ }^{2}$ Leiden University Medical Center, Department of Radiology, Leiden, $N L$

Introduction: Dynamic Susceptibility Contrast (DSC) imaging commonly uses GRE-EPI to trace the first passage of GBCA to measure cerebral blood volume $(\mathrm{CBV})^{1}$. Vessel architecture imaging (VAI) is an emerging DSC variant with combined GRE/SE-EPI where the sensitivity of SE to smaller vessel radii (R) is exploited. We propose a $\mathrm{MRVF}^{2}$ approach to disentangle VAI biomarkers: $\mathrm{rCBV}, \mathrm{R}$ and permeability $(\mathrm{k})$ during bolus injection. In glioma patients we compared MRVF to a conventional vessel size imaging (VSI) technique ${ }^{3}$. 
Methods: Hybrid EPI (HEPI, a fast acquisition technique combining GRE and SE) DSC were acquired from 3 patients with confirmed diagnosis of glioma. Dynamic images (TR/TE $\mathrm{GRE} / \mathrm{TE}_{\mathrm{SE}} 1.5 \mathrm{~s} / 20 \mathrm{~ms} /$ $70 \mathrm{~ms}$, 15 slices, $3 \mathrm{~T}$, MR750, GE) were acquired during bolus injection of $7.5 \mathrm{ml}$ of GBCA (Gadovist, Bayer, GE) after a preload of equal size. We imported the HEPI sequence as played out on the scanner into a Bloch based DCE simulation tool ${ }^{4}$ that simulates CA extravasation and MR signal. A dictionary was created by simulating 5 vessels with $\mathrm{k}=[0,1.8,4.8]^{*} 10^{-3} \mathrm{~s}^{-1}, 30$ logarithmically spaced values of $\mathrm{R}=[5,100] \mu \mathrm{m}$ and $\mathrm{rCBV}=[0.5,10] \%^{3,4}$. For each dictionary atom the simulation included $400 \mathrm{~s}$ to cover preload and delay, followed by $100 \mathrm{~s}$ of the main bolus and HEPI acquisition. The dictionary was matched to the patient data by allowing a separate scaling factor for the GRE and SE to compensate for baseline (T2) differences between dictionary and in-vivo signals. The parameters obtained were compared with those estimated using the VSI method ${ }^{3}$. Results: The k, R and rCBV values of the best dictionary match to the in-vivo data are shown in Table 1 for 3 voxels in white matter, grey matter and tumor. Non-zero $\mathrm{k}$ values are obtained only for the enhancing tumor voxels. For the voxels of patient 1, Fig. 1 shows the GRE and SE time series and dictionary match. Figure 2 shows for each patient a slice of the T2 FLAIR with the corresponding parametric maps obtained by the two methods. It is observed that both techniques can differentiate normal and tumor tissues. The enhancing tumor in patient 3 is also seen as hyper-intense area in its rCBV maps while those obtained for VSI are comparatively lower, which needs further investigation.

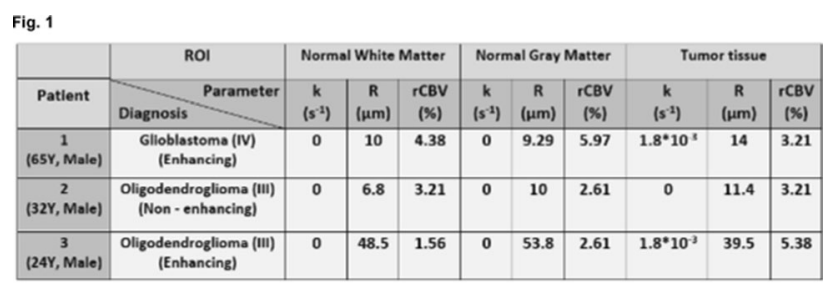

Table 1: $k, R$ and rCBV volues obtained from the best match of the HEPI signals in a voxel in the normal white matter, grey matter, and tumor regions for the 3 sets of patient dota

Fig. 2
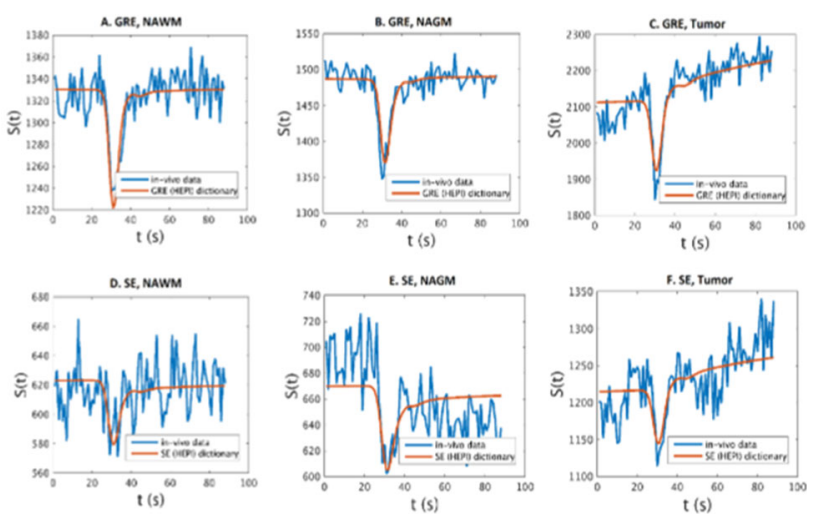

Figure 1. GRE and SE atoms of the HEPI dictionary (red) with the best match to the signols from Normal Appearing White Matter (NAWM), Grey Matter (NAGM) and tumor voxels of GRE and SE images, respectively, of patient 1 (blue)

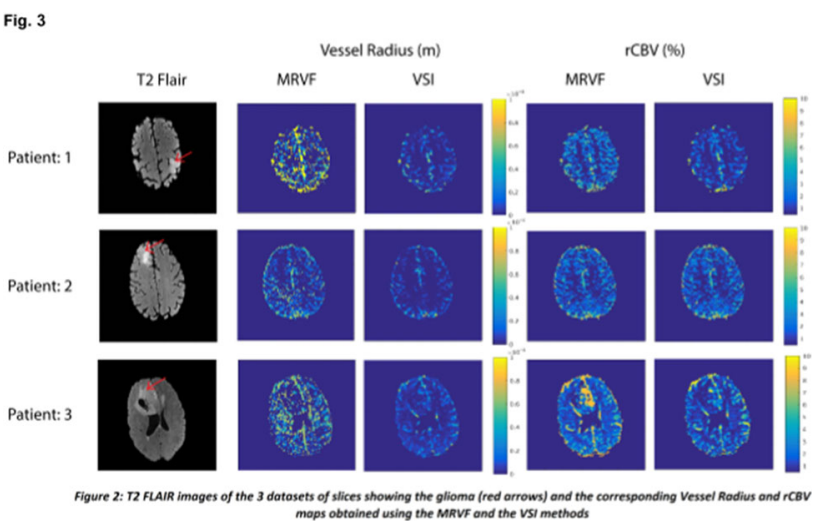

Discussion: By dictionary matching of HEPI-data a good match was found between simulated and in-vivo data. We compared the quantitative maps of R and rCBV from MRVF to conventional VSI values and found similar patterns, although also quantitative differences. The dictionary should be expanded by more densely sampling $\mathrm{k}$ and $\mathrm{R}$ values to improve the matching. First-pass DSC-HEPI MRVF has the advantage that it is faster compared to MRVF measuring asymmetric $\mathrm{SE}$ signals during static pre/post-contrast phases ${ }^{2}$. Further research will study whether permeability is essential for the dictionary for DSC with preload.

\section{References:}

${ }^{1}$ Shiroishi et al., JMRI, 2015.

${ }^{2}$ Christen et al., Neuroimage, 2014.

${ }^{3}$ Kellner et al., JMRI, 2015.

${ }^{4}$ Pannetier et al., Plos one, 2013.

\section{S4.04.}

Remarkable myogenic effect of ultrasound combined with microbubbles on the brain perfusion in rats

*W. Labriji ${ }^{1}$, C. Simoncini ${ }^{1}$, C. Cirillo ${ }^{1}$, C. Lafon $^{2}$, L. Hirschler $^{3}$, J. Warnking ${ }^{3}$, E. L. Barbier ${ }^{3}$, I. Loubinoux ${ }^{1}$, F. Desmoulin ${ }^{1,4}$

${ }^{I}$ University of Toulouse, Toulouse Neuro-Imaging Center (ToNIC), Toulouse, FR;

${ }^{2}$ University of Lyon, LabTAU / Centre Léon Bérard, Lyon, FR;

${ }^{3}$ Grenoble Alpes University, Institute of Neuroscience, Grenoble, FR; ${ }^{4}$ University of Toulouse, Toulouse, FR

Introduction: The use of ultrasound with microbubbles (US + MB) for Blood Brain Barrier (BBB) opening is an increasingly common method in clinical and preclinical research. However, its effects on cerebral perfusion have been little investigated. The aim of this study was to evaluate changes in brain perfusion following US $+\mathrm{MB}$ in rat brain using pseudo-continuous arterial spin labelling (pCASL) [1], a non-contrast-based method, and Dynamic Susceptibility Contrast (DSC), a Gadolinium based method.

Methods: The protocol included 10 female Wistar rats, 3 of them were used as control group. A catheter was placed on the rat tail vein. Prior to sonication, animals were imaged in a $7 \mathrm{~T}$ MRI scanner (Bruker Biospec 70/20) for phase optimization acquisitions of the pCASL sequence. Outside the magnet, the animals received an injection of $200 \mu \mathrm{L}$ of microbubbles (Sonovue) and were exposed to unfocused US using a flat transducer with an acoustic pressure of $0.3 \mathrm{MPa}$ applied at the right hemisphere during $2 \mathrm{~min}$. A series of pCASL acquisitions $(\mathrm{TE}=15 \mathrm{~ms} ; \mathrm{TR}=2600 \mathrm{~ms} ;$ PLD $=300 \mathrm{~ms}$ ) were then carried out every $4 \mathrm{~min}$ to assess Cerebral Blood Flow (CBF) after BBB opening. ADC maps were computed from the DTI 
Bruker protocol $\left(\mathrm{TE}=20 \mathrm{~ms} ; \mathrm{TR}=2.5 \mathrm{~s} ; \mathrm{b} \sim 0\right.$ and $800 \mathrm{~s} / \mathrm{mm}^{2}$ in 3 directions). Following the pCASL acquisitions, T1 maps were acquired, prior and after the injection of $300 \mu \mathrm{L}$ of $\mathrm{Gd}$ (Dotarem, $0.5 \mathrm{mmol} / \mathrm{mL}$ ), using a Multi-Inversion Time sequence (FAIR-EPI, $\mathrm{TE}=13.5 \mathrm{~ms}$; TR $=10 \mathrm{~s}$ ). Three animals from the group underwent DSC acquisition during Gd injection $(\mathrm{TE}=10 \mathrm{~ms}$; $\mathrm{TR}=500 \mathrm{~ms}$; $\mathrm{NR}=300$ ). CBF maps were computed using MP3 [2].

Results/discussion: Following US + MB, CBF maps show, first, a decrease at certain regions of the exposed hemisphere (Fig. 1) up to $40 \%$, then, a progressive recovery. The volume with CBF affected by $\mathrm{US}+\mathrm{MB}$ is different from the one showing a BBB opening ( $\mathrm{Gd}$ extravasation) albeit both volumes displayed similar CBF evolution patterns (Fig. 2) with a recovery after 50 min confirmed by the DSCCBF maps. ADC maps between the exposed region of interest and its contralateral remain unchanged. These findings suggest that the transmural pressure increase consecutive to the US + MB leads to $\mathrm{BBB}$ opening but can also produce a local myogenic response. Indeed, the myogenic response is a vasoconstriction in response to an increase in transmural pressure (i.e. the pressure across the vessel wall) [3]. Moreover, pCASL-CBF measurements in our experiment were not affected by the BBB disruption in agreement with the generalized model assuming a rapid water exchange [4]. This approach is promising to assess the local vasoconstrictive capacity implied in the cerebral autoregulation and estimate the potent cerebral vascular reserve (CVR). Further investigations using small volume focusing US transducers are needed to build CVR maps.
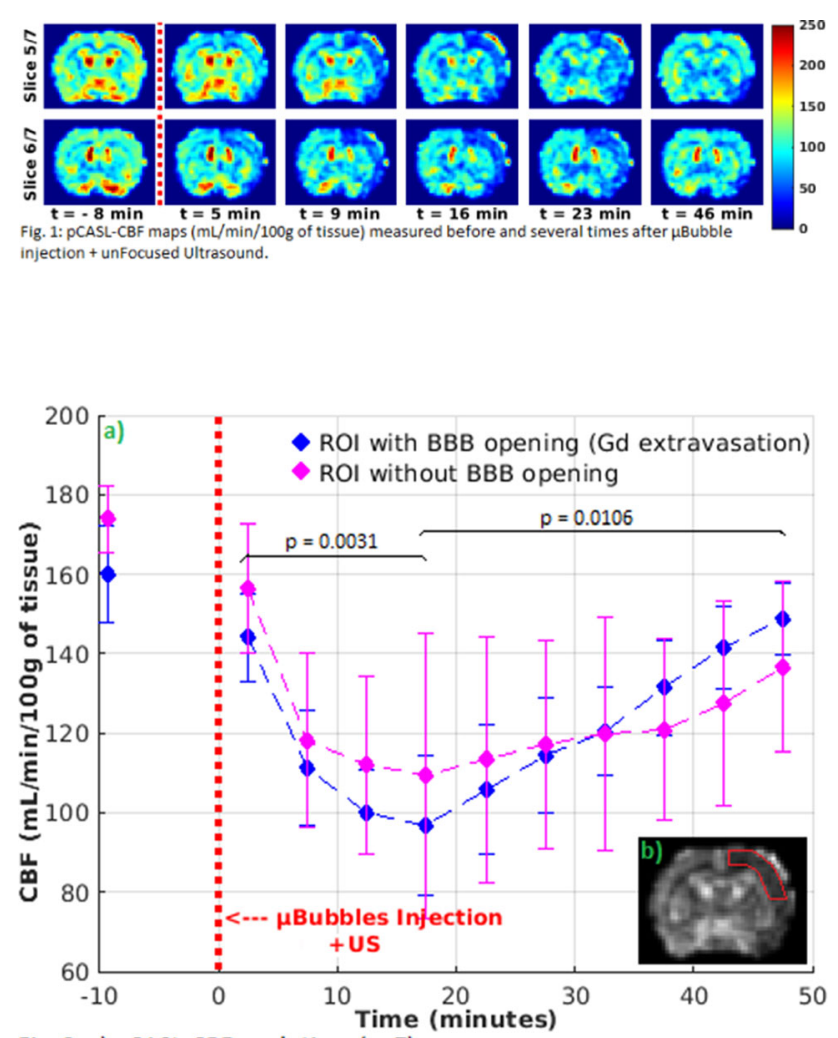

Fig. 2: a) pCASL-CBF evolution $(n=7)$

b) $\mathrm{CBF}$ map with the cortical region of interest used for $\mathrm{CBF}$ estimation.

\section{References:}

[1] Hirschler L, et al. MRM, 2018.

[2] C. Brossard, et al. Frontiers in Neuroinfo, 2020.

[3] D. Lidington, et al. JCBFM, 2018.

[4] Buxton, et al. MRM, 1998.

\section{S4.05.}

Considering intra-voxel transit times is a must for robust determination of blood brain barrier integrity using arterial spin labeling

\author{
*M. A. Buck ${ }^{1,2}$, A. Mahroo ${ }^{1}$, J. Huber ${ }^{1}$, M. Günther ${ }^{1,2,3}$ \\ ${ }^{1}$ Fraunhofer Institute of Digital Medicine (MEVIS), Bremen, DE; \\ ${ }^{2}$ University of Bremen, Faculty 01 (Physics, Electrical Engineering), \\ Bremen, DE; \\ ${ }^{3}$ mediri GmbH, Heidelberg, DE
}

Introduction: Arterial Spin Labeling (ASL) is a non-invasive method which can be used to measure the water exchange through the blood brain barrier (BBB) to obtain its functionality and integrity using a multi-echo ASL sequence (multi-TE) [1]. The imaging voxels consist of the intravascular and extravascular space as well as the arteries. In this abstract a new three-compartment model (3CM) is presented which introduces an intra-voxel transit time ITT between the arteries and vascular space which has been neglected so far in the twocompartment model (2CM) [2].

Methods: Theory: Compartment 1 contains the signal of the blood in the arteries before it enters the intravascular space (compartment 2) after ITT. There, the blood water exchanges with tissue water (compartment 3) via the BBB (Fig. 1).

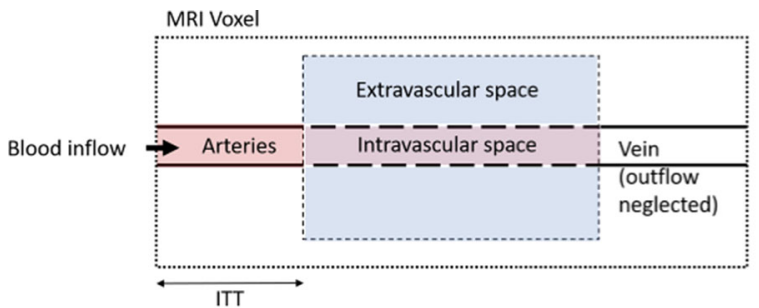

Figure 1: Schematic structure of the MRI voxel as assumed by the three-compartment model. The labeled blood flows during the intra-voxel transit time ITT through the arteries (compartment 1) into the intravascular space (compartment 2) where the exchange to the brain tissue/ extravascular space (compartment 3 ) takes place.

Simulation: 3D-data were simulated for TI $=0-4000 \mathrm{~ms}$ and $\mathrm{TE}=0$ $500 \mathrm{~ms}$ for different exchange times (Texch) (100-1000 ms) using the $3 \mathrm{CM}$ with following parameters: $\mathrm{M} 0=1 ; \mathrm{f}=60 \mathrm{ml} / 100 \mathrm{~g} / \mathrm{min}$; alpha $=1 ;$ ATT $=500 \mathrm{~ms} ; \quad$ ITT $=300 \mathrm{~ms} ; \quad$ BL $=1800 \mathrm{~ms} ;$ T1b $=$ $1700 \mathrm{~ms} ; \mathrm{T} 2 \mathrm{~b}=150 \mathrm{~ms} ; \quad \mathrm{T} 1 \mathrm{tis}=1100 \mathrm{~ms} ; \mathrm{T} 2 \mathrm{tis}=80 \mathrm{~ms}$. The simulated signal at eight inflow times (TI) $(100,600,1000,1400$, $1800,2200,2600,3000 \mathrm{~ms})$ and six echo times (TE) $(19,57,95,133$, $171,209 \mathrm{~ms}$ ) were then used to determine the quantified perfusion values and the exchange time with the $2 \mathrm{CM}$.

Results: Figure 2 shows the simulated data and the fit of the $3 \mathrm{CM}$ and $2 \mathrm{CM}$ for exchange time Texch $=100 \mathrm{~ms}$ exemplarily for $\mathrm{TE}=50 \mathrm{~ms}$ and $200 \mathrm{~ms}$. It can be seen that for lower TE values the $2 \mathrm{CM}$ can describe the signal similarly to the $3 \mathrm{CM}$, but with clearly overestimated Texch. For higher TE values the signal curves cannot be reproduced properly with the $2 \mathrm{CM}$. 
a)
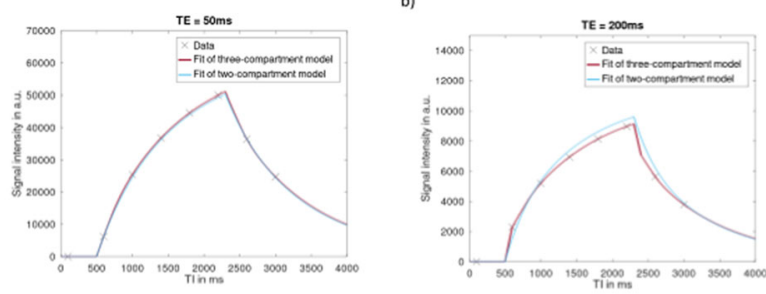

(a) smaller TE value (50ms) the signal curve of the fitted $2 \mathrm{CM}$ is nearly identical to the signal curve of the $3 \mathrm{CM}$, but the Texch value
estimated by the $2 \mathrm{CM}$ is overestimated ( $321 \mathrm{~ms}$ ) while the $3 \mathrm{CM}$ estimates Texch: $100 \mathrm{~mm}$. (b) for a higher TE value $(200 \mathrm{~ms})$ it can be estimated by the $2 \mathrm{CM}$ is overesstimated (321ms) while the $3 \mathrm{CM}$ estimates Texch $100 \mathrm{~ms}$. (b) for a higher If value $(200 \mathrm{~ms}$ ) it can be

In Fig. 3a the Table shows the calculated Texch and $\mathrm{f}$ values for different simulations with varying Texch. It can be clearly seen that the $2 \mathrm{CM}$ overestimates Texch in all cases. The observed relative standard deviation to the simulation value is in the range of $220 \%$ for Texch $=100 \mathrm{~ms}$ and decreases with increasing Texch to $35 \%$ (Fig. 3b).

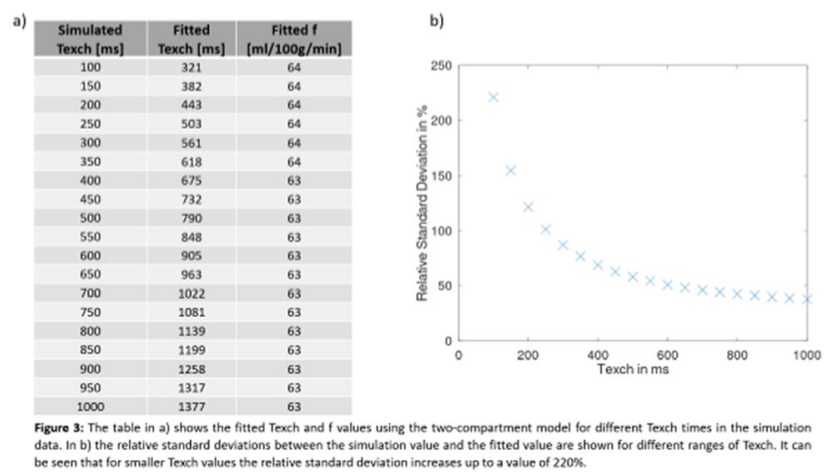

Discussion and conclusion: The signal of the arteries and ITT are not explicitly considered in the $2 \mathrm{CM}$. This explains the huge difference in the calculated Texch for the simulated data. Figure 2 shows that the $2 \mathrm{CM}$ can model the signal curve nearly as well as the $3 \mathrm{CM}$ for lower TE times, but overestimates Texch up to $220 \%$. Especially, for small Texch the 2CM would compensate the ITT missing in the model with an apparently higher Texch. As a leakage of BBB would normally be seen in a decrease of Texch [3,4], using the $2 \mathrm{CM}$ instead of $3 \mathrm{CM}$ (where the ITT is explicitly modelled) disguises the drop in Texch. This points out how important it is to incorporate ITT for a robust and correct detection of the BBB integrity.

\section{References:}

[1] Mahroo et al., Proc. ISMRM 2021, 1854.

[2] Gregori et al., JMRI 2013, 37.

[3] Farrall et al., Neurobiol. Aging 2009, 30.

[4] Wells et al., MRM 2021, 85.

\section{S4.06.}

\section{Does hypocapnia affect oxygen induced changes in brain $\mathrm{T} 1$ times at $3 \mathrm{~T}$ ?}

\author{
*A. McCabe ${ }^{1,2}$, D. McGrath ${ }^{3}$, N. Blockley ${ }^{3,4}$, R. Panek ${ }^{2,5}$ \\ ${ }^{1}$ University of Nottingham, Department of Clinical Oncology, \\ Nottingham, GB; \\ ${ }^{2}$ University of Nottingham, School of Medicine, Nottingham, GB; \\ ${ }^{3}$ National Institute of Health Research (NIHR), Nottingham \\ Biomedical Research Centre (BRC), Nottingham, GB;
}

${ }^{4}$ University of Nottingham, School of Life Sciences, Nottingham, GB; ${ }^{5}$ University of Nottingham, Department of Medical Physics \& Clinical Engineering, Nottingham, GB

Introduction: Breathing high concentration oxygen causes $T_{1}$ shortening due to the paramagnetic effect of molecular oxygen. However, during an hyperoxic challenge in healthy individuals, $\mathrm{pCO}_{2}$ reduces resulting in contraction of vascular smooth muscle and decreased cerebral blood flow. This can reduce oxygen delivery and be a confounding factor in hyperoxia challenge MRI ${ }^{1}$. We sought to measure in-vivo hyperoxic $\Delta \mathrm{T} 1$ times with and without control of end tidal $\mathrm{pCO}_{2}\left(\mathrm{PETCO}_{2}\right)$ in various brain regions.

Methods: 7 healthy volunteers were scanned at $3 \mathrm{~T}$ (Philips Ingenia) and $\mathrm{T}_{1}$ estimated via the variable flip angle method (3D spoiled GRE, $\mathrm{FA}=3^{\circ} / 18^{\circ}, \quad \mathrm{TE} / \mathrm{TR}=0.896 / 2.7 \mathrm{~ms}, \quad$ matrix: $80 \times 80, \quad \mathrm{FoV}:$ $240 \times 240 \mathrm{~mm}, 37 \times 3.5 \mathrm{~mm}$ slices, 300 dynamics, $2.95 \mathrm{~s}$ each). Oxygen was delivered 4 min into the acquisition using a sequential gas delivery breathing circuit with flow rates adjusted to control $\mathrm{PETCO}_{2}$ (RespirActTM, Thornhill Research). Two runs were performed; with and without control of $\mathrm{PETCO}_{2}$.

Images were corrected for motion and segmented into Grey Matter (GM), White Matter (WM) and Cerebrospinal Fluid (CSF) using SPM12 ${ }^{2}$. Lobar segmentation was performed by co-registering the MNI atlas ${ }^{3}$. Lateral ventricles (LV) were identified manually. $\Delta \mathrm{T} 1$ times were defined as difference between hyperoxic (6:15-8:45 min) and initial normoxic period $(2: 30 \mathrm{~min})$ and normalised to $\triangle \mathrm{PETO}_{2}$.

Results: 2 acquisitions were excluded due to technical issues.

Figure 1 shows an example $\mathrm{T}_{1}$ time series and gas trace. Table 1 displays baseline $\mathrm{T}_{1}$ and mean $\Delta \mathrm{T} 1$. All changes, except $\mathrm{PETCO}_{2}$ control LV CSF, are significant at $\mathrm{p}<0.05$ level. Non-lateral ventricle CSF (non-LVCSF) shows the greatest $\Delta \mathrm{T}_{1}$ with and without PETCO $_{2}$ control. All GM regions show similar $\Delta \mathrm{T}_{1}$ (Fig. 2).

Mean $\pm \mathrm{SD} \quad \mathrm{PETCO}_{2}$ was $38.3 \pm 1.8 \mathrm{mmHg}$ with control and $33.7 \pm 5.4 \mathrm{mmHg}$ without.

\begin{tabular}{|c|c|c|c|c|}
\hline & $\begin{array}{l}\text { Initial } T,(m s) \\
\text { and standard } \\
\text { deviation }\end{array}$ & $\begin{array}{c}\mathrm{CO}_{2} \text { control } \\
\text { Normalised } \Delta \\
(m s / m m H g) \\
\text { mean and } \mathrm{Cl}\end{array}$ & $\begin{array}{c}{\mathrm{No} \mathrm{CO}_{2} \text { control }}_{\text {Normalised } \Delta \mathrm{T}_{1}} \\
(\mathrm{~ms} / \mathrm{mmHH}) \\
\text { mean and } \mathrm{Cl}\end{array}$ & $\begin{array}{c}p \text {-value for } \\
\text { difference in } \\
\Delta T,(m s)\end{array}$ \\
\hline Grey Matter & $\begin{array}{l}1565 \\
(107)\end{array}$ & $\begin{array}{c}-0.034 \\
(-0.038 \text { to }-0.030)\end{array}$ & $\begin{array}{c}-0.053 \\
(-0.057 \text { to }-0.049)\end{array}$ & $<0.0001$ \\
\hline White Matter & $\begin{array}{l}1265 \\
(83)\end{array}$ & $\begin{array}{c}0.006 \\
(0.003 \text { to } 0.008)\end{array}$ & $\begin{array}{c}-0.011 \\
(-0.013 \text { to }-0.008)\end{array}$ & $<0.0001$ \\
\hline $\begin{array}{l}\text { Lateral } \\
\text { ventricle CSF }\end{array}$ & $\begin{array}{l}2603 \\
(271)\end{array}$ & $\begin{array}{c}-0.008 \\
(-0.027 \text { to } 0.011)\end{array}$ & $\begin{array}{c}-0.029 \\
(-0.044 \text { to }-0.014)\end{array}$ & 0.9192 \\
\hline $\begin{array}{l}\text { Non lateral } \\
\text { ventricle CSF }\end{array}$ & $\begin{array}{c}2112 \\
(79)\end{array}$ & $\begin{array}{c}-0.267 \\
(-0.277 \text { to }-0.257)\end{array}$ & $\begin{array}{c}-0.211 \\
(-0.219 \text { to }-0.203)\end{array}$ & $<0.0001$ \\
\hline
\end{tabular}

Table 1: Regional $T_{1}$ times and hyperoxic change $\left(\Delta \mathrm{T}_{1}\right)$ normalised to change in end tidal $\mathrm{pO}_{2}$ with and without active end tidal $\mathrm{CO}_{2}$ control. $\mathrm{P}$-values determined from a paired $\mathrm{t}$-test. $\mathrm{Cl}=95 \%$ confidence interval. CSF = Cerebrospinal Fluid. 
Fig. 2
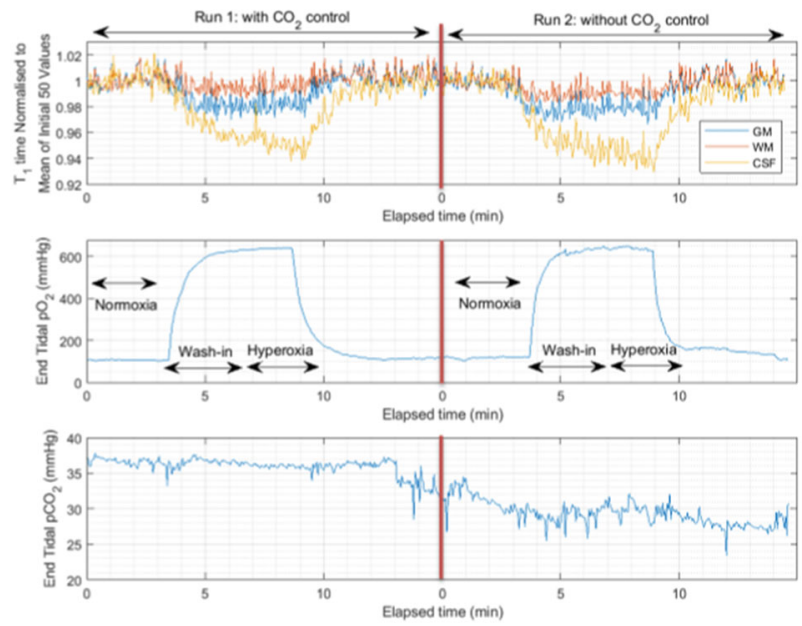

Figure 1: Example of $\mathrm{T}_{1}$ time series (normalised to mean of initial 50 values) with corresponding gas traces

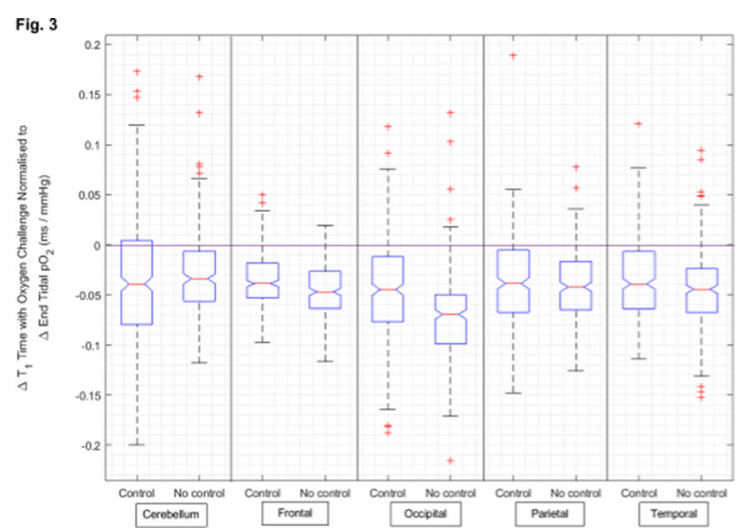

Figure 2: Notched box plots showing regional grey matter changes of normalised $T_{1}$ with and without end tidal $\mathrm{CO}_{2}$ control

Discussion: Although GM and WM regions showed statistically significant $\Delta \mathrm{T}_{1}$ with hyperoxic challenge, the magnitude was relatively small compared to non-LVCSF which shows an absolute $\Delta \mathrm{T}_{1}$ in the region of $140 \mathrm{~ms}$. This is in keeping with previous studies that only found hyperoxic challenge $\Delta \mathrm{T}_{1}$ significant in non-LVCSF${ }^{4}$.

$\mathrm{PETCO}_{2}$ control results in smaller $\Delta \mathrm{T}_{1}$ for both GM and WM. Using a two compartment model ${ }^{5}$ we suggest that a reduction in blood volume due to uncontrolled $\mathrm{PETCO}_{2}$ results in a relative increase in tissue volume fraction, increasing $\Delta \mathrm{T}_{1}$ due to the shorter $\mathrm{T}_{1}$ of tissue compared to blood. In non-LVCSF, $\Delta \mathrm{T}_{1}$ is greater with $\mathrm{PETCO}_{2}$ control which may be due to the lower blood volume in that space and partial volume effects.

In conclusion, hypocapnia does affect oxygen induced brain $T_{1}$ changes at $3 \mathrm{~T}$.

\section{References:}

1. Moreton et al. NeuroImage. Clin 11: 667-77 (2016)

2. SPM12 Software-Statistical Parametric Mapping. Accessed June 2021. https://www.fil.ion.ucl.ac.uk/spm/software/spm12

3. Mazziotta et al. Proc R Soc Lond B Biol Sci 356, 1293-1322 (2001)

4. Bhogal et al. Cereb Blood Flow Metab 37 (4): 1461-69 (2017)

5. Schwarzbauer et al. Magn Reson Med 29 (5): 709-12 (1993)
S4.07.

\section{Modelling temporal dynamics of cerebrovascular reactivity using BOLD and ASL imaging}

\author{
${ }^{*}$ J. Pinto ${ }^{1}$, N. Blockley ${ }^{2}$, J. W. Harkin ${ }^{3}$, D. P. Bulte ${ }^{1}$ \\ ${ }^{1}$ University of Oxford, Institute of Biomedical Engineering, Oxford, \\ $G B$; \\ ${ }^{2}$ University of Nottingham, School of Life Sciences, Nottingham, GB; \\ ${ }^{3}$ Poole Hospital NHS Foundation Trust, Poole, GB
}

Introduction: Cerebrovascular reactivity (CVR) reflects the mechanism of cerebral blood vessels to adjust their calibre and cerebral blood flow (CBF) in response to vasoactive stimuli (e.g., hypercapnia $)^{1,2}$. This parameter has been shown to be impaired in several pathologies. In this work, we compared CVR and CBF parameters derived from BOLD and multi-PLD ASL, using different modelling strategies and focusing on temporal features.

Methods: 10 healthy subjects $(5 \mathrm{M}, 20.4 \pm 0.8$ years old) were studied on a $3 \mathrm{~T}$ Siemens Prisma scanner, with a multiPLD pCASL sequence (2D multi-slice GE-EPI, TR/TE 4100/14 ms, PLDs 250/500/750/1000/1250/1500 ms), acquired during normocapnia/hypercapnia ( $\sim 7 \mathrm{~min}$ each), and a pCASL acquisition with a double-excitation (DEXI) readout during 5/4 interleaved blocks of air/hypercapnia ( $9 \mathrm{~min}, \mathrm{TR} / \mathrm{TE} 1 / \mathrm{TE} 26100 / 14 / 35 \mathrm{~ms}), \quad$ from where BOLD data was extracted. Hypercapnia was achieved using the RespirAct Gen 3 (Thornhill Medical, $\triangle$ PETCO $_{2} \sim 8 \mathrm{mmHg}$ ).

All data was analysed using FSL and MATLAB. ASL data was modelled using a standard kinetic model (BASIL) and adding a gamma-shaped dispersion kernel $^{3}\left(\mathrm{M}_{\text {disp }}\right)$ and/or intravascular arterial component ${ }^{4} \quad\left(\mathrm{M}_{\text {art }}\right)$. BOLD model fitting was performed using a GLM approach (box-car regressor convolved with gamma function, std $6 \mathrm{~s}$, mean lag $15 \mathrm{~s}$ ), yielding $\mathrm{CVR}_{\mathrm{BOLDWBopt}}(\mathrm{CVR}=$ $\triangle \mathrm{CBF} / \triangle \mathrm{PETCO}{ }_{2}$ ). Additional GLMs with time-shifted regressors (10:30 s, in steps of $1 \mathrm{~s}$ ) were performed in order to account for different temporal BOLD dynamics across the brain, selecting the shift that provides the best fit for each voxel (Delay BolDVoxopt, $\mathrm{CVR}_{\text {BOLDVoxopt }}$ ).

Statistical testing included voxelwise group analysis (FSL's randomise), paired T-tests and linear regression fitting (Matlab fitlm function).

Results: When comparing BOLD optimisation strategies, we observed significant differences across all regions tested (Fig. 1). Voxelwise analysis showed significant differences across the brain, in particular where delays were larger (e.g., WM, occipital area). Significant correlations were only observed when comparing multi-PLD ASL CVR (Fig. 2) and BOLD CVR with delay optimisation in GM (Fig. 3). No significant correlations were observed for bolus arrival time and/or Delay BOLDVOXopt $_{\text {. }}$

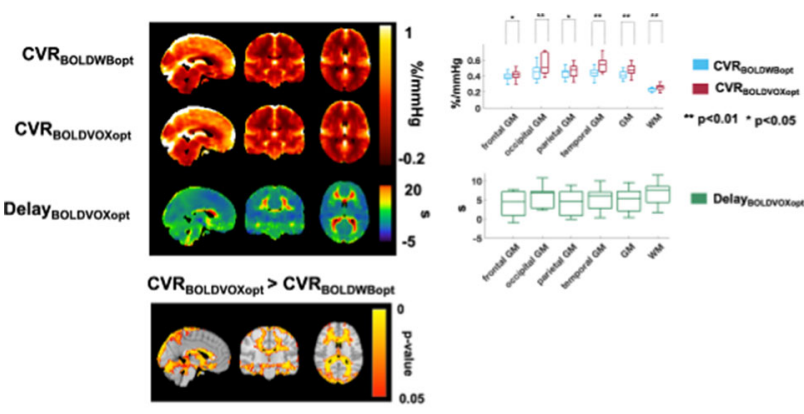

Figure 1. Representations of average CVR 0 ace and Delay BOLD data with and without delay optimisation, as well the regions that yielded statistically significant differences between the two optimisation strategies (bottom left) ( $p<0.05$, threshold-free cluster enhancement). Regional average 


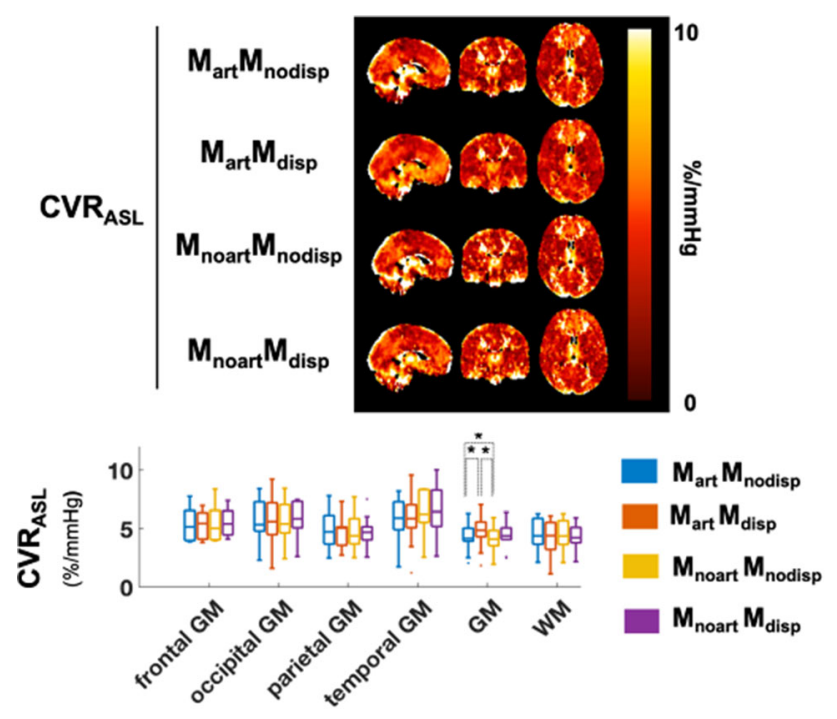

Figure 2. Representations of average $\mathrm{CVR}_{\mathrm{ASL}}$ maps across subjects for each modelling strategy (top). Regional average $\mathrm{CVR}_{\mathrm{ASL}}$ values (in $\% / \mathrm{mmHg}$ ) obtained for each ASL model strategy tested.
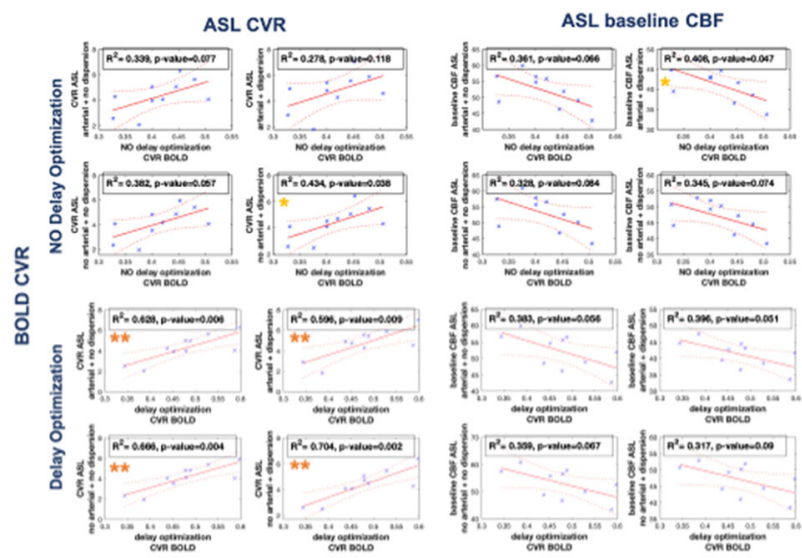

*tik $p<0.01, p<0.05$

Figure 3. Pearson correlation between BOLD CVR (with and without delay optimisation) and multiplePLD ASL CVR and baseline CBF, obtained with the four different ASL modelling strategies (arterial component and dispersion), across GM.

Discussion: Our results suggest that BOLD delay-optimisation can influence CVR quantification in specific brain regions, improving BOLD CVR correlation with multi-PLD ASL CVR in GM, regardless of the ASL modelling strategy used. Additionally, there is a trend towards a significant correlation between baseline CBF and BOLD CVR. Further work is still required in order to fully understand the physiological meaning of CVR temporal dynamics.

Acknowledgements: EPSRC grants EP/G004277/1, EP/K025716/1, EP/S021507/1.

\section{References:}

1. Liu, P. et al., Neuroimage 187, 104-115(2019).

2. Pinto, J. et al. Frontiers in Physiology 11 1711(2021).

3. Chappell, M. A. et al. Magn. Reson. Med. 69, 563-70(2013).

4. Chappell, M. A. et al. Magn. Reson. Med. 63, 1357-65(2010).

\section{S4.08.}

\section{Combined RS-fMRI and calcium recordings show stabile brain states in mice after switching anesthetic regimen}

\author{
*B. Pradier ${ }^{1,2}$, L. Wachsmuth ${ }^{1}$, N. Nagelmann ${ }^{1}$, D. Segelcke ${ }^{2}$, \\ E. Pogatzki-Zahn ${ }^{2}$, C. Faber ${ }^{1}$ \\ ${ }^{1}$ University Hospital Münster, Clinical Radiology, Translational \\ Research Imaging Center, Münster, DE; \\ ${ }^{2}$ University Hospital Münster, Department of Anesthesiology, \\ Intensive Care and Pain Medicine, Münster, DE
}

Introduction: The popularity of resting-state (RS) functional (f)MRI is rising in mouse models with still open questions on the effect of anesthetics on brain state and neuronal network dynamics. Recently, the combination of low-dose Isoflurane (ISO) and Medetomidine (MED) has become an advocated anesthetic regimen in rats ${ }^{1}$. However, uncertainty resides over how fast relevant parameters adapt. Therefore, we investigated the brain state and -networks of mice during the transition from a single anesthetic (ISO) to a combined anesthetic regimen (ISO/MED) using a multimodal approach with RS-fMRI and calcium recordings.

Methods: 12 female C57/BL6J mice were used in this study to investigate brain states under different anesthetic conditions. MRI measurements were conducted on a 9.4 T Bruker Biospec 94/20 small animal scanner using a CryoProbe (Bruker Biospin). Functional MR acquisitions were recorded with a GE-EPI sequence (TR/TE:1000/ $18 \mathrm{~ms}, 0.5 \mathrm{~mm}$ slice thick., FOV $28 \times 26$, matrix $80 \times 80$ ). The first RS-fMRI was acquired under $1 \%$ ISO anesthesia, subsequently, MED infusion $\left(0.1 \mathrm{mg} / \mathrm{kg}, 0.2 \mathrm{mg} / \mathrm{kg}^{*} \mathrm{~h}\right)$ was started and ISO reduced to $0.2 \% .3$ further RS-fMRI scans were acquired following MED bolus. Results: Acute calcium transients occur at $<0.5 \mathrm{~Hz}$ (1A, green) under ISO in S1HL. Shortly after MED infusion their frequency increases towards $0.5-1.0 \mathrm{~Hz}(1 \mathrm{~A}, \mathrm{~B})$ and become constant after $35 \mathrm{~min}$ (1C). A spectral analysis of spontaneous BOLD activity in S1HL consistently shows decreased low-frequency events throughout the change of anesthetic regimen (1D). RS-network analysis show constant network efficiency and clustering throughout different anesthetic conditions $(2 \mathrm{~A}, \mathrm{~B})$. Connectivity strength and coherence within functional groups was also not affected (2C,D). Further, analysis of functional connectivity (FC) shows significantly altered components of ISO and ISO/MED $45 \mathrm{~min}$ with ISO/MED25 min, respectively (Fig. 4), thereby indicating that the transitional period between anesthetic conditions is especially vulnerable to changes in FC. 


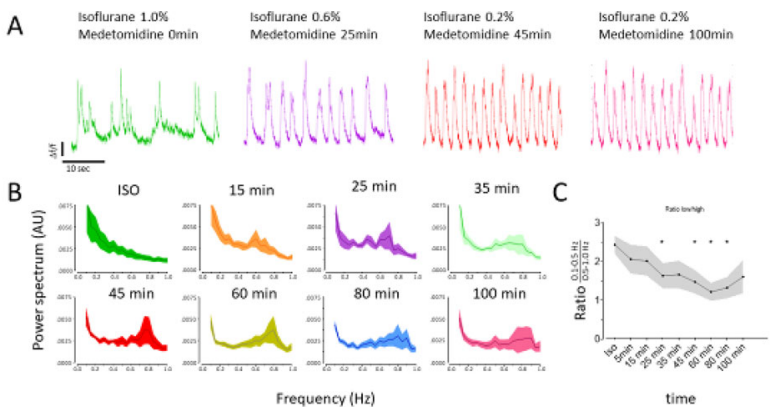

D

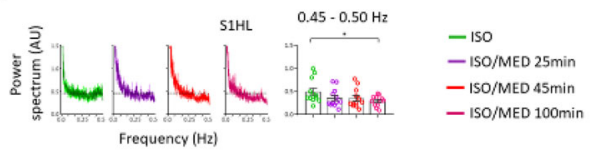

Calcium recordings show different brain states in $\mathrm{S} 1 \mathrm{HL}$ depending on anesthetic condition. (A) Under ISO anesthesia, calcium transients show frequent UP.DOWN transitions (green) while changing to a persistent state at 25 (purple), 45 (red), and 100 (pink) minutes after switching to ISO/MED anesthesia. (B) Fourier-transformed calcium transients show an emerging peak between $0.5 \mathrm{~Hz}$ and $1.0 \mathrm{~Hz} 15$ to 100 minutes after switching anesthetic regimens. (C) Frequencies of calcium transients between $0.1 \cdot 0.5 \mathrm{~Hz}$ decreased $(p=0.002$ ), while frequencies from $0.5 \cdot 1.0 \mathrm{~Hz}$ increased $(p=0.027$. (D) Averaged power spectra obtained from BOLD activity during RS-fMRI in S1HL before (ISO, green) and after the 100 min - pink; * $\left.{ }^{p}<0.05\right)$.

Figure1
A

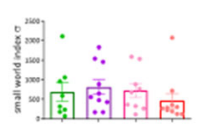

B
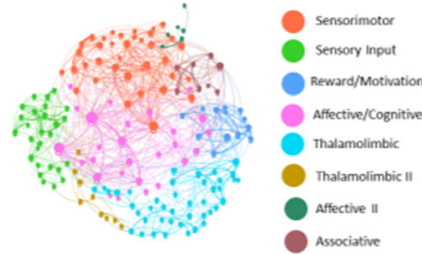

C

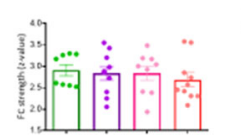

W 150 iso/MED 25min

D

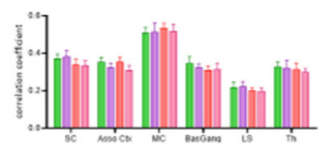

W ISO/MED 45min
Global network parameters were not affected by different anesthetic regimens. (A) Measures for network efficiency and segregation like small-world index ( $p=0.5)$ were not significantlychanged by switching from ISO to ISO/MED. (B) Clustering did not change after change in anestheticcondition. Representative cluster structure under ISO condition. We detected eight communities: sensorimotor, thalamolimbict, thalamolimbicll, affective/cognitive, sensory input. reward/motivation, affective and associative. (C) Global functional
functional groups was not changed by different anesthetic regimen.

Figure2
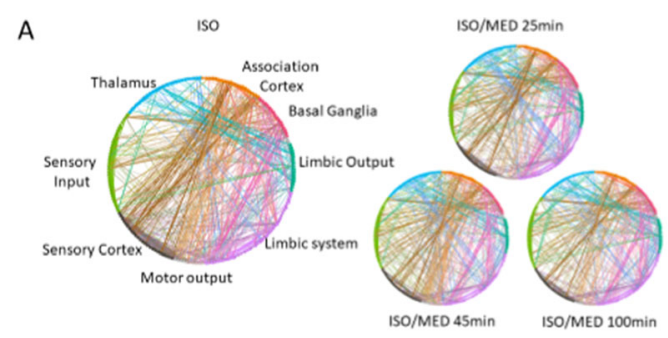

B

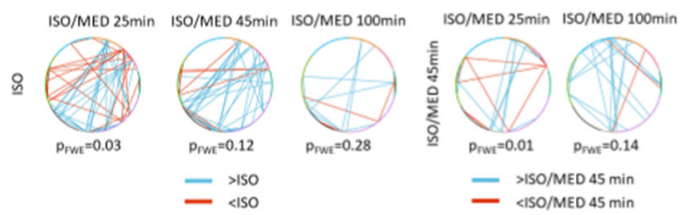

Changes in functional connectivity $(\mathrm{FC})$ were mostly related to a decrease in 150 concentration. (A) Circular and averaged network representation for each group. Each dot represents a brain region, color codes functional groups, and lines represent functional connectivity based on pearson's correlation coefficient. (B) Statistical differences in FC,
comparing ISO vs. later time points (left panel) and ISO/MED $45 \mathrm{~min}$ vs. ISO/MED $25 \mathrm{~min}$ and ISO/MED $100 \mathrm{~min}$ (right panel) using a permutation-based network statistic.

Figure3

Discussion: Calcium and RS-fMRI recordings clearly show a brain state with slow-wave activity under ISO (UP-DOWN transitions) that rapidly changes to a persistent state upon switching the anesthetic regimen to ISO/MED in the S1HL. These changes were paralleled by alterations in the pattern of FC. Specifically, the coupling between the sensory cortex, association cortex, and the limbic system changed during the transition period. Therefore, we recommend a 40-min waiting period after switching from a single anesthetic regimen with ISO to combined ISO/MED anesthesia when studying the somatosensory cortex with RS-fMRI in mice.

\section{References:}

1. van Alst et al. Anesthesia differentially modulates neuronal and vascular contributions to the BOLD signal. Neuroimage (2019).

2. Kreitz, $\mathrm{S}$ et al. New analysis of RS connectivity and graph theory reveals distinctive short-term modulations due to whisker stimulation in rats. Front. Neurosci. (2018).

\section{S4.09.}

\section{Test-retest reliability of resting-state correlations between the default mode network fMRI activity and EEG features}

*M. Xavier ${ }^{1}$, I. Esteves ${ }^{1}$, F. Ayres-Ribeiro ${ }^{1}$, A. L. Giraud ${ }^{2}$, S. Sadaghiani ${ }^{3}$, J. Wirsich $^{4}$, P. Figueiredo ${ }^{1}$

${ }^{1}$ University of Lisbon, Lisbon, $P T$;

${ }^{2}$ University of Geneva, Department of Neuroscience, Geneva, CH;

${ }^{3}$ University of Illinois at Urbana-Champaign, Beckman Institute I

Department of Psychology, Urbana, IL, US;

${ }^{4}$ University of Geneva, Department of Clinical Neurosciences / EEG and Epilepsy Unit, Geneva, $\mathrm{CH}$

Introduction: Simultaneous EEG-fMRI acquisitions are used to exploit the highly complementary characteristics of the two modalities. The default mode network (DMN) is robustly identified in resting-state fMRI studies, while its electrophysiological correlates remain incompletely understood. Previous work on the topic focused on finding patterns of correlation between the fMRI DMN and EEG features that were consistent between subjects [1], but reliability 
studies assessing the consistency of these correlations within subjects are lacking. Here we investigate the test-retest reliability of restingstate correlations between the activity of the DMN measured by fMRI and multiple EEG features.

Methods: EEG-fMRI data was acquired from 23 healthy subjects, on a Siemens 3 T MR scanner, during 3 sessions of 10 min eyes-closed rest [2] with GRE-EPI (TR/TE $=2000 / 50 \mathrm{~ms}, 3 \mathrm{~mm}$ isotropic). EEG was acquired with a 62-channels MR-compatible system (BrainAmpMR).

The fMRI DMN was identified by group-level ICA, followed by template matching [3]. Its activity time-series was extracted as the fMRI signal of interest.

From the EEG time-frequency spectrum, the following features were derived: i) Univariate features: root mean squared frequency (RMSF), total power (TP) and band-specific power; and ii) Functional connectivity (FC) features: a dynamic FC network was built for each frequency band by estimating the imaginary part of coherency (ICoh) between each channel pair, and the weighted node degree (WND) was then computed for each channel. Bands considered: $\delta(1-4 \mathrm{~Hz}), \theta$ $(4-8 \mathrm{~Hz}), \alpha(8-12 \mathrm{~Hz}), \beta(12-30 \mathrm{~Hz})$. The resulting EEG feature time-series were convolved with a set of double-gamma hemodynamic response functions (HRF), with overshoot delays of 10, 8, 6, 5, 4 and $2 \mathrm{~s}$.

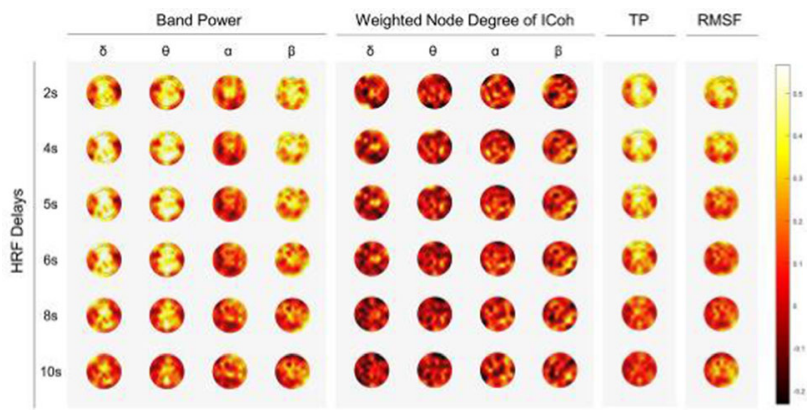

Fig. 1. Topographic representation of the intraclass correlation coefficient (ICC) values for each of the EEG metrics (left to right: band-specific power, weighted node degree of the band-specific imaginary part of coherency (ICoh) network, total power (TP) and root mean squared trequency (RMSF). Rows corrospond to EEG delays. which wore introducod by convolving each EEG feature with a set of hemodynamic response functions (HRFs) with overshoot delays of 2, 4, 5, 6, 8 and 10 seconds.

Pearson"s correlation was computed between the time-series of each EEG feature and the fMRI DMN time-series. The test-retest reliability of the correlations was evaluated with the intraclass correlation coefficient (ICC)[4], which estimates measurement consistency across the 3 sessions.

Results: The estimated ICC values are presented in Figs. 2 and 3. EEG-DMN correlations were significantly more reliable for univariate than for FC features. Correlations were also significantly less reliable for alpha than for the remaining frequency bands, and less reliable for the temporal than the remaining lobes. Regarding delays, reliability was highest at $4 \mathrm{~s}$.
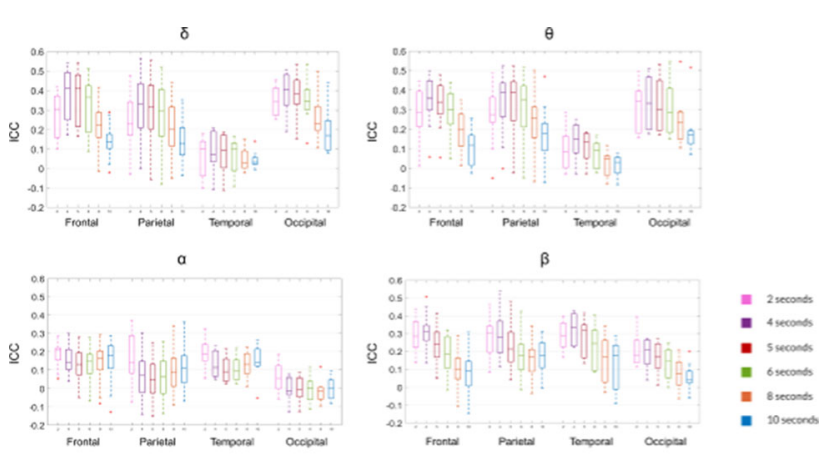

Fig. 2. Comparison of the interclass correlaston coeffidient (ICC) values for across EEG delays - 2, 4, 5, 6, 8 and $10 \mathrm{~s}$ - and channel poles -

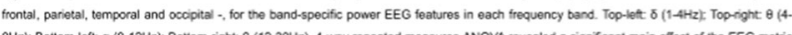
8Hz). Bottom-left a (8-12Hz); Bottom-right $\beta$ (12-30Hz), 4-way ropeated measures ANOVA revoalod a signifcent main effoct of the EEG metic

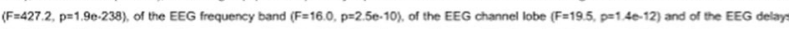

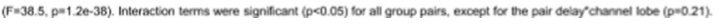

Fig. 3

Table 1. Intraclass correlation coefficient (ICC) values (means \pm standard deviation) for each of the EEG metrics: bandspecific power, weighted node degree of the band-specific imaginary part of coherency network (WND-ICoh), total power (TP) and root mean squared frequency (RMSF). Results in respect to the EEG features convolved with a hemodynamic response function with a $2 \mathrm{~s}$ overshoot delay. Values in parentheses indicate the percentage of chamels with ICC $>0.4$. ICC values can be classified in terms of reliability according to the criteria proposed by Clochett et al. (5): poor (<0.4), fair $(0.41-0.59)$, good $(0.60-0.74)$ and excellent $(<0.75)$

\begin{tabular}{|c|c|c|c|c|c|}
\hline \multirow[b]{2}{*}{ Metric } & \multirow[b]{2}{*}{ Band } & \multicolumn{4}{|c|}{$\operatorname{ICC}(2,1)$} \\
\hline & & Frontal & Parietal & Temporal & Oociptal \\
\hline \multirow{4}{*}{ Band Power } & Delta & $0.367 \pm 0.125(53)$ & $0.314 \pm 0.152\langle 41\rangle$ & $0.0876 \pm 0.101\langle 0\rangle$ & $0.391 \pm 0.101(50)$ \\
\hline & Theta & $0.353 \pm 0.109(35)$ & $0.346 \pm 0.136(48)$ & $0.141 \pm 0.089(0)$ & $0.335 \pm 0.134(50)$ \\
\hline & Alpha & $0.155=0.069(0)$ & $0.075 \pm 0.110(0)$ & $0.131 \pm 0.069(0)$ & $-0.002 \pm 0.063(0)$ \\
\hline & Beta & $0.314 \div 0.092(18)$ & $0.288=0.113(17)$ & $0.326 \pm 0.083(38)$ & $0.190=0.082(0)$ \\
\hline \multirow{4}{*}{ WND-ICoh } & Dolta & $0.054 \div 0.124(0)$ & $0.027 \pm 0.138(0)$ & $0.053 \pm 0.096(0)$ & $-0.067 \pm 0.098(0)$ \\
\hline & Theta & $0.009=0.101(0)$ & $0.061 \pm 0.114(0)$ & $-0.018 \div 0.076(0)$ & $-0.008 \pm 0.081(0)$ \\
\hline & Apha & $\cdot 0.003 \pm 0.073(0)$ & $0.093 \approx 0.120(0)$ & $0.068 \pm 0.119(0)$ & $0.060=0.101(0)$ \\
\hline & Beta & $0.037 \pm 0.113(0)$ & $0.083 \pm 0.142(0)$ & $0.022 \pm 0.156(0)$ & $0.166 \pm 0.100(0)$ \\
\hline TP & - & $0.359 \pm 0.078(41)$ & $0.305=0.115(24)$ & $0.164=0.067(0)$ & $0.226 \pm 0.100(13)$ \\
\hline RMSF & . & $0.287 \div 0.073(12)$ & $0.216 \pm 0.105(10)$ & $0.229=0.052(0)$ & $0.106: 0.081(0)$ \\
\hline
\end{tabular}

Discussion: In evaluating the test-retest reliability of the resting-state correlations between the fMRI DMN and EEG features, we found significant effects with respect to the EEG metric, frequency band, channel lobe and delay. Despite global mean values of reliability being poor (ICC $<0.04$ ), several EEG features showed fair reliability $(0.41<$ ICC $<-0.59)[5]$.

\section{References:}

[1] K. Nishida et al. Neuropsychobiology 2015.

[2] Sadaghiani et al. J Neurosci 2010.

[3] Smith et al. Proc Natl Acad Sci USA 2009.

[4] McGraw et al. Psychol Meth 1996.

[5] Cicchetti. J Clin Exp Neuropsy 2010. 


\section{S5.01.}

\section{A receiver coil-based method for reduction of electromagnetic interference on a $50 \mathrm{mT}$ permanent magnet system}

\author{
*J. Parsa ${ }^{1,2}$, B. de $\operatorname{Vos}^{1}$, A. G. Webb ${ }^{1}$ \\ ${ }^{1}$ Leiden University Medical Centre, Department of Radiology, Leiden, \\ $N L$; \\ ${ }^{2}$ Percuros B.V., Leiden, $N L$
}

Introduction: In recent years low field MRI has received much attention due to its low cost and portability ${ }^{1-3}$, as well as the possibility of its use in unconventional locations without an RF shielded room. However, this means that it is necessary to cancel any electromagnetic interference (EMI) created by equipment close to the scanner. Many different methods to reduce EMI for MRI and NMR have been explored in the past ${ }^{4-7}$. In this work, we have used an MRinactive RF coil, which detects only the EMI. A noise cancellation system has been implemented on a Halbach-based low field MRI system operating at $2.15 \mathrm{MHz}^{1}$. Images of a leg phantom have been acquired with and without EMI.

Methods: A solenoid was used as the transmit coil (Fig. 1). Two receive saddle coils ( $\mathrm{Rx}$ coils) with orthogonal magnetic fields were designed and simulated by using a sum of sinusoidal function over the desired cylinder. The outer saddle (EMI detector) coil with a $17.3 \mathrm{~cm}$ diameter and the inner saddle coil with a $15.6 \mathrm{~cm}$ diameter were positioned such that $B_{1}$ aligned and perpendicular with $B_{0}$, respectively. Both $\mathrm{Rx}$ coils have the same length of $20.5 \mathrm{~cm}$ (Fig. 1). Because of the close proximity, coupling between Tx and Rx coils is strong, detuning circuitry was used on each coil for this purpose. The $90^{\circ}$ rotation between the two saddle coils resulted in intrinsic decoupling. The two receive coils were connected to a $180^{\circ}$ power combiner with $0.2 \mathrm{~dB}$ insertion loss, $0.5^{\circ}$ phase unbalance, and $25 \mathrm{~dB}$ inner-channel isolation. A battery-operated drill motor was used as a broadband EMI source. Body providing a path by which EMI is transmitted into the imaging field-of-view, images were acquired with a leg phantom consists of water doped with $1.5 \mathrm{~g} / 1$ $\mathrm{NaCl}$ and $1.3 \mathrm{~g} / \mathrm{l} \mathrm{H} 2 \mathrm{KO} 4 \mathrm{P}$ with $90 \mathrm{~cm}$ length and $11 \mathrm{~cm}$ diameter. A turbo spin-echo sequence was used.
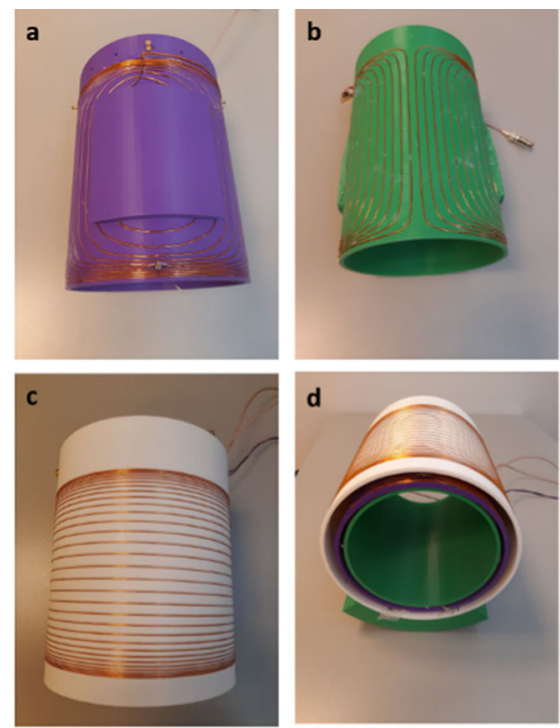

Figure 1. Signal (a) and EMI (b) receiver coils. (c) transmit solenoid coil. (d) The three coils are placed co centrically. Feeding the outputs of the two receiver coils through a $180^{\circ}$ power combiner is used for EM reduction.
Results: The simulated $B_{1}$ fields of the two saddles are shown in Fig. 2: the magnetic field vectors of both $\mathrm{Rx}$ coils are almost orthogonal over the cylindrical region of interest of $10 \times 16 \mathrm{~cm}$. Reflection coefficients $\left(S_{11}\right)$ for each of $\mathrm{Rx}$ coils and coupling between two Rx coils were lower than $-22 \mathrm{~dB}$, which are the same as measurement data with $\sim 1 \%$ error. Fig. 3 shows image data with and without EMI. The results in Fig. 3c-e show $\sim 80 \%$ reduction in EMI.
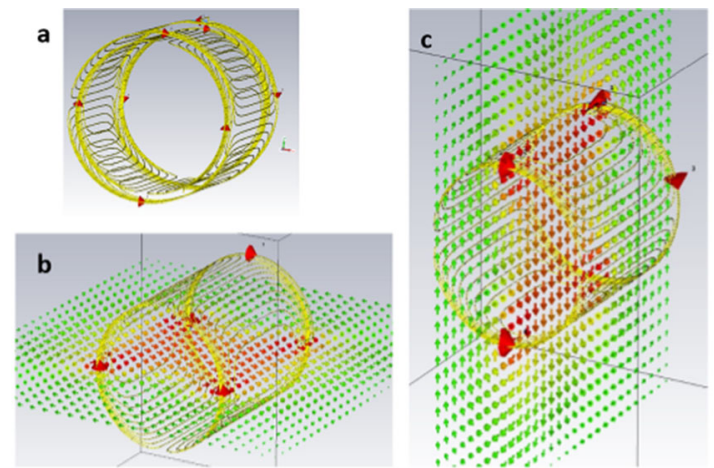

Figure 2. Two receiver coils inside each other (a). 2D-plot arrow of the magnetic field $\left(\mathrm{B}_{1}\right)$ at the center of signal (b) and EMI (b) coils.

a
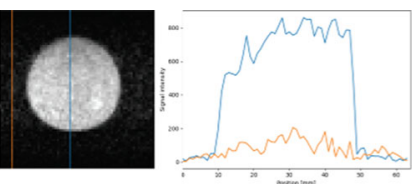

b
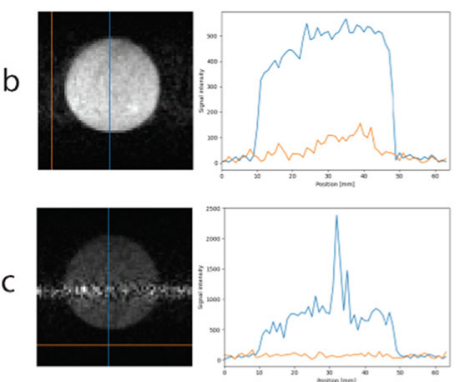

d
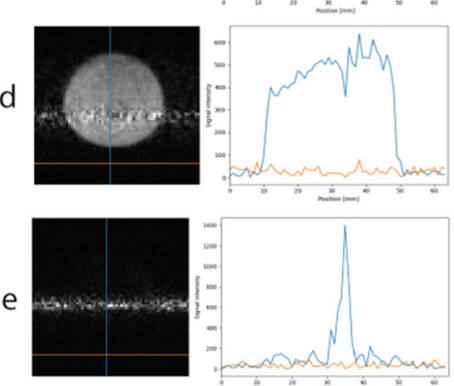

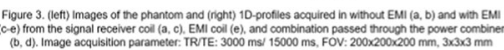


Conclusion: Significant reduction in EMI has been achieved using the new design which does not require external sensors. This method of EMI reduction results in an intrinsically reduced SNR compared to an image acquired without EMI, due to the Johnson noise added from the EMI coil. EMI reduction is also affected by the slightly different sensitivities of the two receiver coils which have similar but not identical diameters. If two independent receive channels could be used, then a weighting function could be introduced to correct for this, and improve the EMI.

\section{References:}

${ }^{1}$ O'Reilly (2020).

${ }^{2}$ Hyperfine (2021).

${ }^{3}$ Cooley (2021).

${ }^{4}$ Brosnan (1988).

${ }^{5}$ Trushkin (1994)

${ }^{6}$ Dalgaard (2012).

${ }^{7}$ Srinivas (2021).

\section{S5.02.}

\section{Flexible split-coil design for various field strengths}

\author{
*R. Kowal ${ }^{1,2}$, E. Pannicke ${ }^{1,2}$, M. Ehses ${ }^{1,2}$, G. Rose ${ }^{1,2}$, O. Speck ${ }^{2,3}$ \\ ${ }^{I}$ Otto von Guericke University, Institute for Medical Engineering, \\ Magdeburg, DE; \\ ${ }^{2}$ Otto von Guericke University, Research Campus STIMULATE, \\ Magdeburg, DE; \\ ${ }^{3}$ Otto von Guericke University, Department of Biomedical Magnetic \\ Resonance, Magdeburg, DE
}

Introduction: Receive coils play an essential role in MR imaging as the initial step in the receiving chain to pick up the RF-signal. They are usually tuned to the Larmor frequency by discrete capacitors placed on the circumference of the coil. An alternative to this is the use of Split-Coils consisting of partially overlapping microstrip transmission lines $[1,2]$ to eliminate the need for rigid solder joints along the coil thus providing flexibility for non-flat surfaces. At the same time unconventional manufacturing methods such as printing or cutting plotters become practical, along with the possibility of integrating coils into fabrics. In this work, the applicability of the design will be further investigated to determine whether its adjustment by overlap can be used for various clinical field strengths $(0.55-3 \mathrm{~T})$.

Methods: A Split-Coil is simulated by full-wave method (CST, Dassault Systèmes). Figure 1 shows a top view as well as a cross section of the coil with the overlaps $\varphi$ and $\varphi_{\text {port }}$, center radius $b=67.5 \mathrm{~mm}$ and trace width $w=5 \mathrm{~mm}$. A dielectric is chosen with a height of $h=0.1 \mathrm{~mm}$ and rel. permittivity of $\varepsilon_{\mathrm{r}}=3$. The coil is loaded with tissue imitating medium (rel. permittivity $\varepsilon_{\mathrm{r}}=80$, conductivity $\sigma=0.46 \mathrm{~S} / \mathrm{m}$ ) on one side at a distance of $5 \mathrm{~mm}$. The front and back side traces are symmetrical. For validation, an exemplary coil was built and compared with simulations based on its input impedance considering de-embedding. A matching capacitor $C_{\mathrm{M}}$ is connected in series to compensate the imaginary part of the input impedance. This is done discretely during post-processing to focus on the coil tuning but can be realized in practice by additional overlapping traces to form $C_{\mathrm{M}}$.

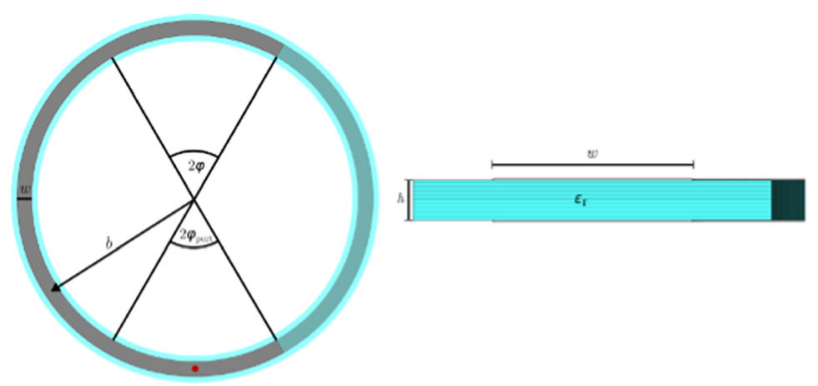

Figure 1 Modeled double-overlap Split-Coil for simulation. Left: Top view of the coil with traces in gray, dielectric in blue and port in red. Right: Cross section with overlapping traces separated by the dielectric.

Results: The validation measurement, shown in Fig. 2, demonstrates high agreement with less than $2 \mathrm{MHz}$ deviation in the inductive range between the extrema. The coil shown in Fig. 1 can be tuned to the specified target frequencies by varying only its overlaps. Figure 3 shows the $S_{11}$ curves for the coils, as well as their $\mathrm{B}_{1}{ }^{+}$distributions. Higher quality factors and more homogenous field strengths are seen with lower frequency. The required overlaps for tuning and the matching capacitance are listed in Table 1.
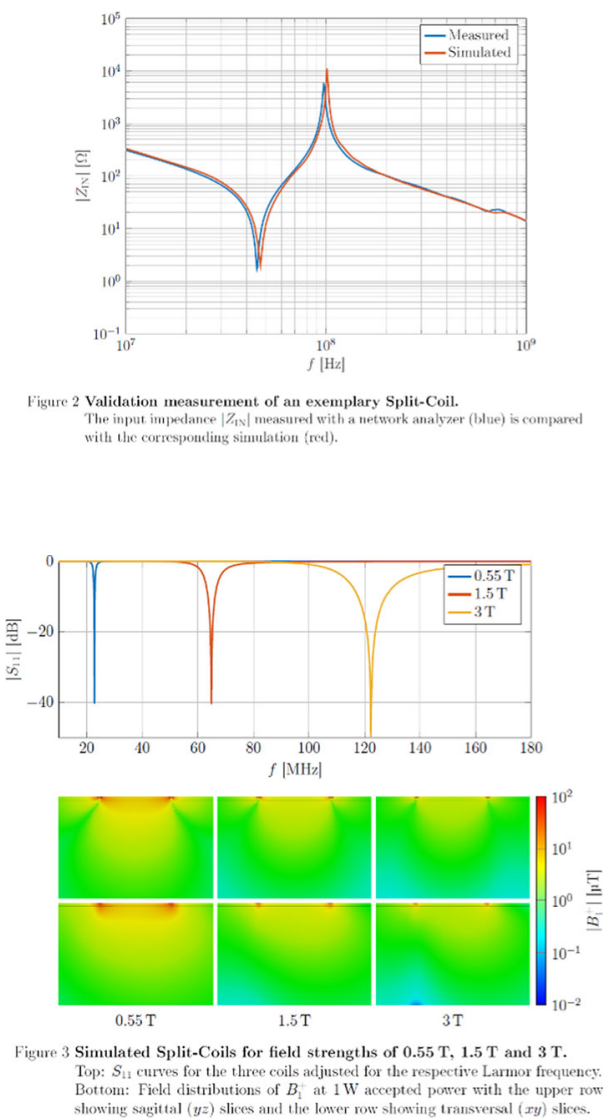

Discussion: This work demonstrated frequency adjustment by overlap of Split-Coils. The simulations were based on validation measurements with real coils. The coils can be tuned with uniform geometry only by varying the two overlaps to field strengths of 0.55 , 
1.5 and 3 T. Simulations of the $\mathrm{B}_{1}{ }^{+}$-fields show typical sensitivity profiles. This increases the range of application for Split-Coils to clinically relevant field strengths and can be expanded upon by varying the size of the coil, trace or dielectric for further optimization.

\section{References:}

[1] Pannicke et al., EMBC, 2021 (submitted).

[2] Corea et al., Nature Communications, 2016.

\section{S5.03.}

\section{Overcoming bandwidth limitations of high $Q$ coils for low field MRI using high input impedance preamplifiers}

\author{
*T. O’Reilly ${ }^{1}$, Z. Abdulrazaq ${ }^{1}$, A. G. Webb ${ }^{1}$ \\ ${ }^{1}$ Leiden University Medical Center, Department of Radiology, Leiden, \\ $N L$
}

Introduction: Coil losses dominate over sample losses in low field MRI [1] which means minimizing coil losses (and maximizing the Q factor) is vital in order to maximize SNR at low field. A high Q brings challenges too, the coil bandwidth is given by $\mathrm{f}_{0} / \mathrm{Q}$ which for low field can be much less than the imaging bandwidth. Commonly a resistor is used to lower the $\mathrm{Q}$ factor $[2,3]$ which reduces $\mathrm{SNR}(\mathrm{SNR} \propto \sqrt{ } \mathrm{Q})$. Previous works has shown that an impedance-mismatched preamplifier can reduce the coupling between array coil elements without introducing additional losses[4]. In this work we show that a high impedance preamplifier can overcome the bandwidth limitations of high Q coils at low field without sacrificing SNR.

Methods: Two $15 \mathrm{~cm}$ long, $15 \mathrm{~cm}$ diameter solenoids with 45 turns of copper wire solenoidal RF coils were constructed. The high $\mathrm{Q}$ conFiguration was constructed using $1.5 \mathrm{~mm}$ diameter copper wire, the "standard" imaging coil used $0.8 \mathrm{~mm}$ diameter wire and an $8 \Omega$ resistor. The Q factors of the high Q and standard coils, measured as $2 \mathrm{f}_{0} /(\Delta \omega 3 \mathrm{~dB})$ of the $\mathrm{s}_{11}$ parameter, were 452 and 179 giving a bandwidth of $9.5 \mathrm{kHz}$ and $24 \mathrm{kHz}$ respectively.

The preamplifier is a two stage preamplifier based on a On Semiconductor 2SK3557 JFET with $30 \mathrm{~dB}$ gain with the output impedance matched to $50 \Omega$ (see Fig. 2). The coil is impedance matched to $50 \Omega$ and connected directly to the gate of the JFET which has a very high $(\sim 1.2 \mathrm{k} \Omega)$ gate to drain impedance. An active T/R switch based on the Analog Devices HMC545AETR was used to interface the coil to the RF power amplifier and preamplifier.
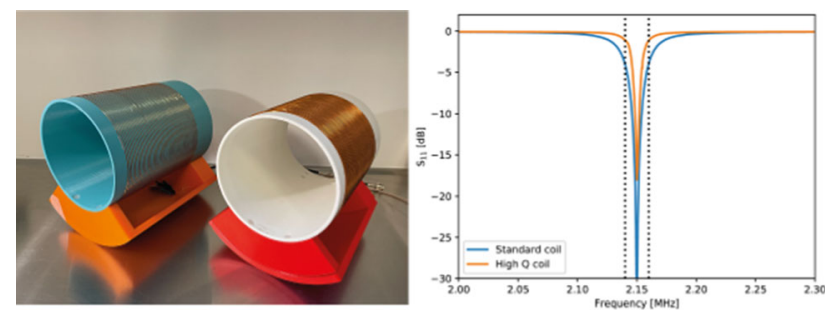

Figure 1. Left) Image of the standard imaging coil (blue) and the high Q factor coil (white), both coils are $15 \mathrm{~cm}$ long $15 \mathrm{~cm}$ diameter with 45 turns of copper wire. Right) The $S_{\text {"I }}$ parameters of the two coils. The standard coil has a bandwidth of $24 \mathrm{kHz}$, the high Q coil of $9.5 \mathrm{kHz}$. The black lines represent an imaging bandwidth of $30 \mathrm{kHz}$, showing that the high Q coil has almost no sensitivity at the edges of that bandwidth.

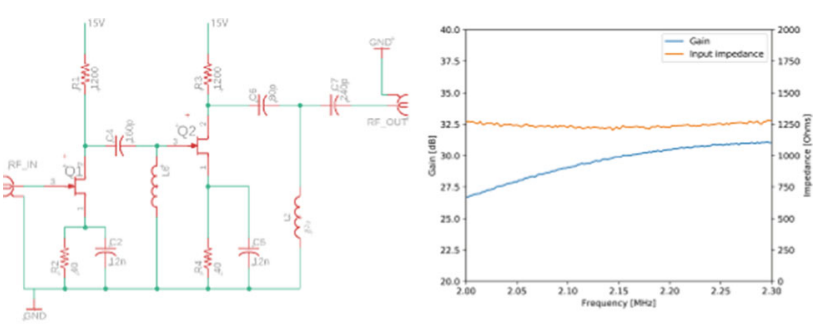

Figure 2. Left) Circuit diagram of the high impedance preamplifier based on the On Semiconductor $25 \mathrm{~K} 3557 \mathrm{JFET}$ Right) Gain and impedance around the $2.15 \mathrm{MHz}$ operating frequency. The noise figure of the preamplifier is

Data were acquired using a Halbach based MRI scanner operating at $2.15 \mathrm{MHz}$ [3], using a 3D turbo spin echo sequence on a $15 \times 15 \times 30 \mathrm{~cm}$ cylindrical phantom with the following parameters: Field of view: $200 \times 200 \times 300 \mathrm{~mm}$, resolution: $2 \times 2 \times 5$ $\mathrm{mm}$, TR/TE: $200 \mathrm{~ms} / 15 \mathrm{~ms}$, echo train length $=6$, acquisition bandwidth: $30 \mathrm{kHz}$, scan duration: $3 \min 20 \mathrm{~s}$.

Results: Figure 2 shows the gain and input impedance of the preamplifier. The noise Figure of the preamplifier is around $3 \mathrm{~dB}$. Figure 3 shows 4 images acquired with the two coils connected to a $50 \Omega$ preamplifier and the high input impedance preamplifier. Signal loss away from the center is reduced for the high $\mathrm{Q}$ with the high impedance preamp while maintaining the SNR benefit over the standard coil connected to the same preamp.

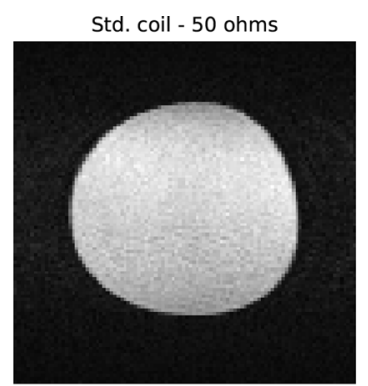

High Q - 50 ohms

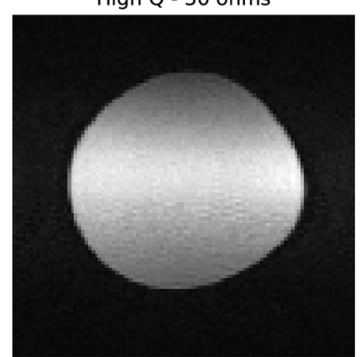

Std. coil - High Z preamp

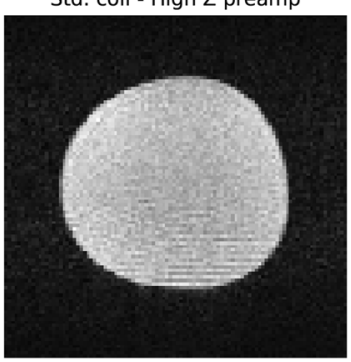

High Q - High Z preamp

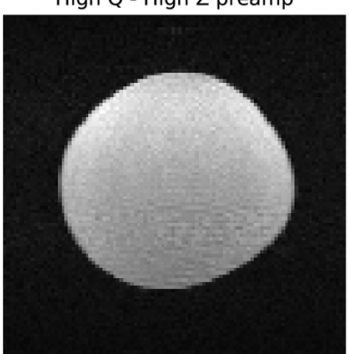

Figure 3. Phantom images acquired with a $30 \mathrm{kHz}$ acquisition bandwidth, the frequency encoding direction is indicated by the white arrow. The High Q coil shows clear signal loss towards the edges of the field of view. The signal loss disappears when the coil is connected to a high impedance preamplifier. The high Q coil has an SNR improvement of around $80 \%$ over the standard imaging coil.

Discussion: In this work we have shown that a high input impedance preamplifier can be used to overcome the low bandwidth associated with high Q factor coils at low field. Improvements to the currently high noise Figure of the preamp and further increasing the $\mathrm{Q}$ factor of the coil can yield significantly higher SNR compared to current approaches of coil construction at low field. Impedance mismatched preamplifier have also shown promise at reducing inter-element coupling in low field coil arrays [5] and will be investigated in future work. 


\section{References:}

1. Gilbert (2008)

2. Shen (2020)

3. O"Reilly (2020)

4. Roemer (1990)

5. Sánchez-Heredia (2018)

\section{S5.04.}

\section{Spherical dielectric resonators improve SNR and SAR} of RF surface coils at $3 \mathrm{~T}$

\author{
C. Rizza ${ }^{1}$, E. Palange ${ }^{2}$, A. Galante ${ }^{3,4,5}, *$ M. Alecci ${ }^{3,4,5}$ \\ ${ }^{1}$ University of L'Aquila, Department of Physical and Chemical \\ Sciences, L'Aquila, IT; \\ ${ }^{2}$ University of L'Aquila, Department of Industrial and Information \\ Engineering and Economics, L'Aquila, IT, \\ ${ }^{3}$ University of L'Aquila, Department of Life, Health \\ and Environmental Sciences, L'Aquila, IT; \\ ${ }^{4}$ University of L'Aquila, Institute for Superconductors, L'Aquila, IT; \\ ${ }^{5}$ National Institute for Nuclear Physics (INFN), Gran Sasso National \\ Laboratory, L'Aquila, IT
}

Introduction: In the past 20 years metamaterial (MM) science has allowed the design of novel RF coils suitable to improve MRI applications [1-3]. Moreover, the use of high-performance dielectric materials has been widely used in the context of EPR, NMR, and MRI with several functions, including dielectric resonators (DRs) and RF shimming pads [4-5]. Only recently the two paths converged with DRs proposed as an add-on RF hardware tool, useful to mimic a negative permeability $\mathrm{MM}$ and enhance SNR/SAR of single or multiple channel RF surface coils [6-9]. Here, we show that a smallsize high-permittivity homogeneous dielectric sphere, selected to operate at a specific Mie resonance, can accurately mimic the EM field outside the sphere produced by magnetic localized surface plasmons, generally supported by a negative permeability sphere of the same diameter.

Methods: To confirm the analytical result, valid for the equivalence of lossless $\mathrm{MM}$ and dielectric, full-wave EM calculations at $3 \mathrm{~T}$ $(127.7 \mathrm{MHz})$ were done taking as a model a low-losses $[\tan \delta=$ $\operatorname{Im}\left(\varepsilon_{\mathrm{DR}}\right) / \operatorname{Re}\left(\varepsilon_{\mathrm{DR}}\right)=5 \times 10^{-3}$ ] DR sphere (diameter of $6.8 \mathrm{~cm}$; tuned at the first resonant mode, $\mathrm{L}=1$ ) having a permittivity of $\varepsilon_{\mathrm{DR}} \cong$ $1200+\mathrm{i} 48\left(\mu_{\mathrm{DR}}=1\right)$ positioned in front of a standard circular RF coil ( $8.4 \mathrm{~cm}$ diameter), this being at $2 \mathrm{~mm}$ from a large cylindrical sample (diameter and length $25 \mathrm{~cm} ; \varepsilon_{\text {SAMPLE }} \cong 64+\mathrm{i} 100$; $\left.\mu_{\text {SAMPLE }}=1\right)$.

Results: Our combined DR sphere, RF coil, and sample EM model show a strong mutual coupling with an observed maximum SNR enhancement of about 2.7 , and a reduction of the peak SAR $10 \mathrm{~g}$ of about $22 \%$, within $2 \mathrm{~mm}$ from the sample surface. Higher-order modes of the DR sphere, having the same $\varepsilon_{\mathrm{DR}}$, will give a better improvement $(\mathrm{L}=3: \mathrm{SNR}=3.3$, diameter $\mathrm{DR} 12.4 \mathrm{~cm})$, although with a reduced penetration depth within the sample. Our theoretical model shows that the $\mathrm{L}=1 \mathrm{DR}$ sphere is equivalent to a MM sphere having $\mu_{\mathrm{MM}} \cong-1.98+\mathrm{i} 0.40, \varepsilon_{\mathrm{MM}}=1$.

Discussion: These numerical results at $3 \mathrm{~T}$, seem to anticipate useful applications of DRs for UHF MRI applications. It is worth noting that there are practical advantages in using a DR sphere instead of a negative permeability $\mathrm{MM}$ one, thanks to the availability of low losses dielectrics, such as ceramics based on lead zirconate titanate [5]. Our approach holds at extremely near-field scales since it is not based on an effective medium theory and the considered structure does not exhibit spatial subwavelength inhomogeneities as for MM based on macroscopic arrays of LC resonators.

\section{References:}

[1] Wiltshire MCK, et al., Science 291, 849 (2001).

[2] Freire MJ, et al., Appl Phys Lett 93, 231108 (2008).

[3] Lezhennikova K, et al., Phys Rev Appl 13, 064004 (2020).

[4] Webb AG, Conc Magn Reson B 38, 148 (2011).

[5] Rupprecht S, et al., Magn Reson Med 79, 2842 (2018).

[6] Rizza C, et al., Phys Rev Appl 12, 044023 (2019).

[7] Rizza C, et al., Phys Rev Appl 14, 034040 (2020).

[8] Rizza C, et al., J Phys D Appl Phys 54, 165108 (2021).

[9] Rizza C, et al., WO 2021/014354 A1 (2021).

S5.05.

MRI acoustic noise: limits of the linear timeindependent model

\author{
*P. Šiuryte ${ }^{1}$, J. Tourais ${ }^{1}$, S. Weingärtner ${ }^{1}$ \\ ${ }^{1}$ Delft University of Technology, Department of Im \\ aging Physics, Delft, NL
}

Introduction: With up to $130 \mathrm{~dB}$ sound pressure level (SPL), acoustic noise is one of the main sources of patient discomfort in MRI [1]. In the current commercial systems, the SPL of a scan noise $p(t)$ is estimated by using linear time-invariant models, as a function of the gradient input derivative $g^{\prime}(t)$. In frequency domain, this is expressed via the transfer function $H(f)=P(f) / G^{\prime}(f)$ for each gradient coil [2-3]. In this work, the model and the limits of the linearity assumptions are revisited.

Methods: Pulse sequences using triangular gradient pulses were used on a $3 \mathrm{~T}$ system (Philips Ingenia) in four different modes: $\mathrm{X}, \mathrm{Y}, \mathrm{Z}$ single gradient inputs and simultaneous triple coil input. Data was averaged over 5 pulses and includes $21 \mathrm{ramp}$ up times $t_{s l}=0.10,0.12$, $\ldots 0.50 \mathrm{~ms}$ and 27 amplitudes $g_{A}=1,2, \ldots 27 \mathrm{mT} / \mathrm{m}$ with a repetition time $\mathrm{TR}=0.5 \mathrm{~s}$. All noise signals were recorded at $44.1 \mathrm{kHz}$ sampling rate using an optical fibre microphone (Phonoptics) and filtered to $0.3-6 \mathrm{kHz}$.

To study the linearity of the scaling, root mean square (RMS) sound pressure was calculated for all $g_{A}$ and $t_{s l}$ in the four modes. The correlation coefficient with the best linear fit was then estimated for all $t_{s l}$. To study the linearity of superposition, noise power from multiple gradient coils (4th mode) was compared to the individual gradient coils.

Results: Signal scaling plots in Fig. $1\left(t_{s l}=0.1,0.3,0.5 \mathrm{~ms}\right)$ show that RMS sound pressure response is fairly linear at low $g_{A}$ (below 15 $\mathrm{mT} / \mathrm{m}$ ) but becomes noticeably saturated as the system slew rate limit is approached. The system deviates from the linear model when rampup times below $0.2 \mathrm{~ms}$ are used, resulting in the overestimation of the sequence SPL (Fig. 2).

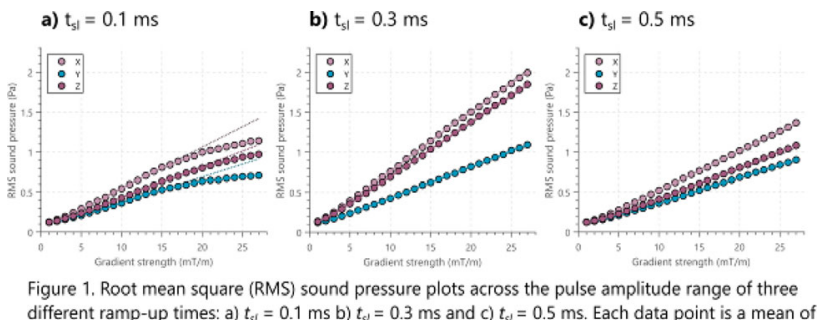
. An -up times: a) $\left.t_{\mathrm{sl}}=0.1 \mathrm{~ms} \mathrm{~b}\right) t_{s \mathrm{~s}}=0.3 \mathrm{~ms}$ and c) $t_{\mathrm{sl}}=0.5 \mathrm{~ms}$. 5 inputs. An interpolated linear fit for $<15 \mathrm{mT} / \mathrm{m}$ amplitude pulses is also plotted in the dashed lines. 
a) Corr.coef. with linear fit

b) $27 \mathrm{mT} / \mathrm{m}$ pulse deviation
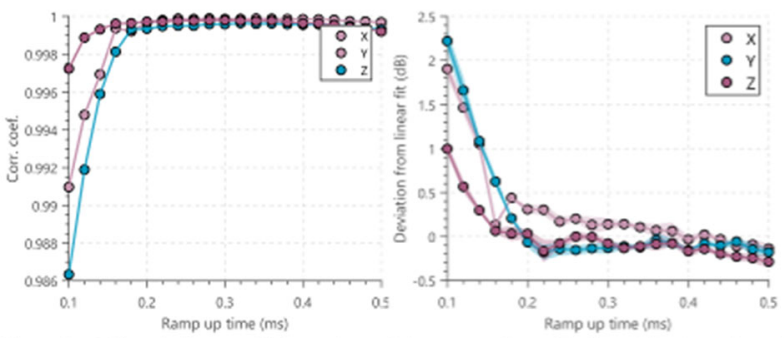

Figure 2. a) Correlation coefficient plots of the curves from Fig. 1 and the best linear fit, evaluated for each ramp-up time td. b) SPL overestimation in $\mathrm{dB}$ for $27 \mathrm{mT} / \mathrm{m}$ pulse using a linear model, with standard deviation indicated by the shaded area.

Minor deviations in the noise estimation are observed when gradients are played in superposition (Fig. 3). An overall increasing overestimation compared to the measured noise $P_{\text {meas }}$ is observed for increasing $g_{A}$ and at certain ramp-up times (Fig. 3a). In contrast, when the transfer function $H(f)$ is estimated from simultaneously played gradients as an input, minimal difference in SPL is observed (Fig. 3b). a) $P=G \cdot\left(H_{X}+H_{Y}+H_{Z}\right)$ error $(\mathrm{dB})$

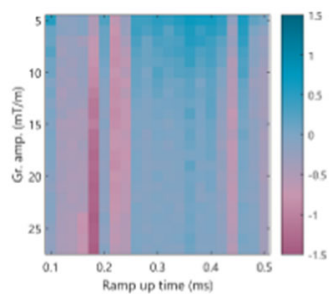

b) $P=G \cdot H_{X Y Z}$ error $(\mathrm{dB})$

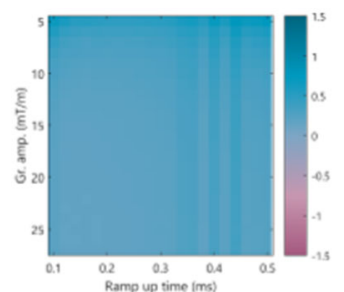

Figure 3. Simultaneous coil noise estimation error (dB) using the transfer function method: a) Superposition of three individual gradient components and b) SPL estimation error under the assumption of a unified transfer function for identical gradient inputs (negative dB values

Discussion: The linear time-invariant model shows substantial overestimation at high gradient amplitudes and high slew rates for single gradients. Notably, the $\mathrm{Z}$ gradient has an overall higher correlation coefficient compared to $\mathrm{X}$ and $\mathrm{Y}$, which could be attributed to different coil geometry. Additional overestimation occurs as gradients are played out simultaneously, showing that single gradients may also induce a substantial force in the neighbouring coils. More advanced models may help to better characterize the realistic noise burden and aid the development of silent MRI sequences.

\section{References:}

[1] M.J.McJury, Journal of Magnetic Resonance Imaging (2021).

[2] R.A. Hedeen, W.A. Edelstein, Magnetic Resonance in Medicine, 37:7-10 (1997).

[3] Z.Wu et al., Magnetic Resonance in Medicine 71:1613-1620 (2014).

\section{S5.06.}

\section{Magneto-stimulation limits in medical imaging applications with rapid field dynamics}

*J. Alonso ${ }^{1}$, D. Grau-Ruiz ${ }^{1,2}$, J. P. Rigla ${ }^{2}$, E. Pallás ${ }^{1}$, J. M. Algarín ${ }^{1}$, J. Borreguero ${ }^{1}$, R. Bosch ${ }^{1}$, G. López ${ }^{1}$, E. Díaz ${ }^{2}$, F. Galve ${ }^{1}$,

C. Gramage ${ }^{1}$, J. M. González ${ }^{2}$, R. Pellicer ${ }^{1}$, A. Ríos ${ }^{2}$, J. M. Benlloch ${ }^{1}$

${ }^{I}$ Technical University of Valencia, MRILab / Institute for Molecular Imaging and Instrumentation (i3M), Valencia, ES;

${ }^{2}$ Tesoro Imaging S.L., Valencia, ES
Introduction/methods: Peripheral Nerve Stimulation (PNS) constrains the clinical performance of Magnetic Resonance and Particle Imaging (MRI and MPI) systems. Extensive magneto-stimulation studies have been carried out recently in the field of MPI [1], where typical operation frequencies range from single to tens of kilo-hertz. PNS literature is scarce for MRI in this regime, which can be relevant to low-field dedicated MRI setups [2], and where the resonant character of MPI coils prevents studies of broad-band excitation pulses. At a more fundamental level, stimulation models known to be accurate for magnetic excursion times above hundreds of microseconds [3] have been less thoroughly tested for shorter timescales. Some authors even question the validity of these models at extreme frequencies [4]. All in all, there is a general lack of PNS data relevant to medical imaging applications exploiting rapid magnetic field dynamics.

We have constructed an apparatus designed for PNS threshold determination on a subject's limb (Fig. 1), which allows for fast narrow and broad-band excitation pulses (excursion times $<40 \mathrm{us}$ ), and can be conFigured for different spatial magnetic field strength distributions.

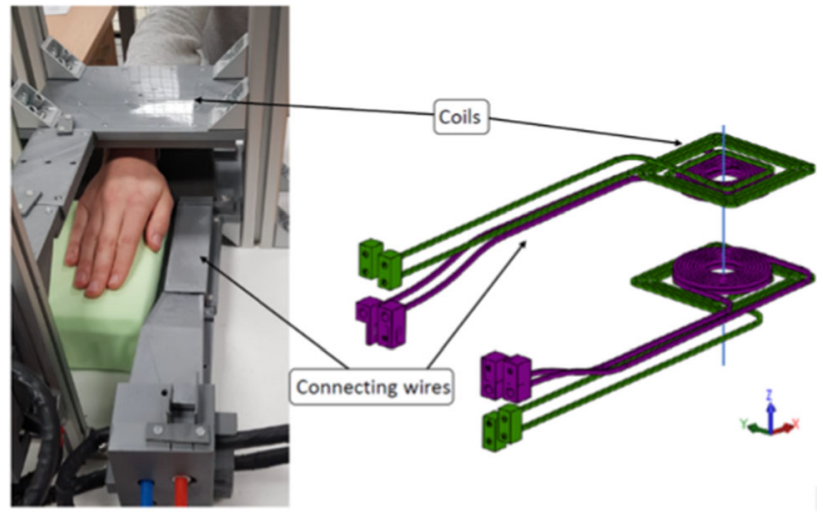

Figure 1a) Photograph of PNS measurement setup. b) Coil design, consisting on two pairs of coils.

Results/discussion: From measurements on 51 volunteers (Fig. 2 left and middle), we observe that PNS limits coincide for sinusoidal (biphasic narrow-band) and triangular (biphasic broad-band) excitations, and are slightly lower for trapezoidal (monophasic broad-band) pulses (not shown in the Figure). The observed dependence on pulse frequency/rise-time is compatible with traditional stimulation models where nervous responses are characterized by a rheobase and a chronaxie [3].

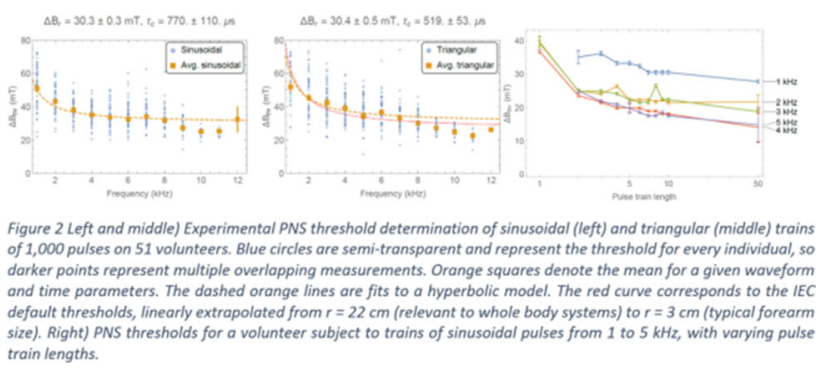

In a second set of experiments (Fig. 2 right), we have confirmed thresholds increase significantly as trains transition from tens to a few pulses. By changing the polarity of the coils in our setup, we also looked at the influence of the spatial distribution of magnetic field strength on PNS effects. We find that thresholds are higher in an approximately linearly inhomogeneous field (relevant to MRI) than in a rather homogeneous distribution (as in MPI). 
Finally, in Fig. 3 we report the results of testing the influence of the arm position on which nerve fibers are triggered, as well as the strength of their response. As shown in the Figure, the location and intensity of the stimulation depends strongly on the exact subject position and field conFiguration.

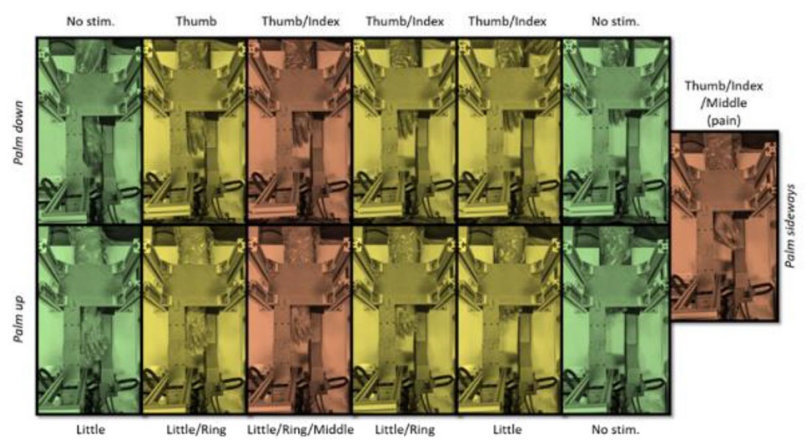

Figure 3 Photographs of the positions where a volunteer was subject to stimuli. In the top (bottom) row the hand pain is facing down (Up), while the photograph on the left is with the palm facing sidewoys. The color code roughly indicates the intensity of the stimulation: green corresponds to no or mild stimuiation, yellow corresponds to moderate intensity and red corresponds to strong stimulation. The volunteer only reported pain with the palm facing sideways. The label on each photogroph denote the fingers where the volunteer felt stimuiated. All pulse trains were sinusoidal $(5 \mathrm{kHz})$ 1,000 pulses long, and with an amplitude of 125

Outlook: Given the large intersubject variability of PNS sensitivity, we propose employing a versatile low-cost system (such as presented here) for fast offline determination of a subject's limits prior to medical scanning, and then using this information to boost clinical imaging while preserving the patient's safety.

\section{References:}

[1] Saritas 2013, https://doi.org/10.1109/TMI.2013.2260764

[2] Grau 2020, arxiv.org/abs/2012.06232

[3] Irnich 1989, https://doi.org/10.1109/iembs.1989.95583

[4] Weinberg 2012, https://doi.org/10.1118/1.3702775 


\section{S6.01.}

\section{Fast $\mathrm{T} 1$ and $\mathrm{T} 2 *$ mapping of tissues with very short transverse relaxation times and application for tissue segmentation of the knee}

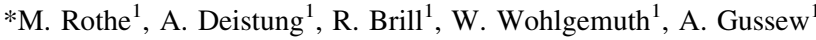 \\ ${ }^{1}$ Martin Luther University Halle-Wittenberg, Clinic and Polyclinic of Radiology, Halle (Saale), DE}

I: Ultra-short echo-time (UTE) imaging enables mapping of relaxation parameters in tissues with short transverse relaxation times. Recently, Krämer et al. ${ }^{1}$ demonstrated that a bivariate histogram of the longitudinal (T1) and effective transverse relaxation times (T2*) allows to segment different knee tissues within a total acquisition time (TA) of 22-63 min, using a triple echo method for T2* mapping and either variable flip angle or variable repetition time method for T1 mapping ${ }^{1}$. We provide a fast approach for high-resolution, isotropic T2* and T1 mapping within 9 min by using a double echo sequence ${ }^{2}$ for $\mathrm{T} 2 *$ mapping and a mixed approach relying on two flip angles (FA) and two repetition times (TR) for T1 mapping. Exemplarily, this approach has been applied to segment various tissues types of the knee.

M: Measurements were performed on a $3 \mathrm{~T}$ MR-scanner (MAGNETOM Skyra, Siemens Healthcare, Erlangen, Germany) using a 15-ch. transmit/receive knee coil and a prototypical 3D spoiled gradient echo UTE sequence with stack of spirals readout. Two measurements with an isotropic spatial resolution of $0.8 \mathrm{~mm}$ were performed (M1: TE1/ TE2 $=0.04 / 4.92 \mathrm{~ms}$, TR1 $=9.92 \mathrm{~ms}, \mathrm{FA} 1=16^{\circ}, \mathrm{TA}=6 \mathrm{~min} ; \mathrm{M} 2$ : $\left.\mathrm{TE} 1=0.04 \mathrm{~ms}, \mathrm{TR} 2=4.45 \mathrm{~ms}, \mathrm{FA} 2=3^{\circ}, \mathrm{TA}=3 \mathrm{~min}\right)$. The $\mathrm{T} 2 *$ map was calculated based on the two echoes obtained in M1. A T1 look-up-Table (LUT) to allocate the ratio of two gradient echo signals with identical TE has been computed using discrete $1 \mathrm{~ms}$ steps ranging from 1 to $2500 \mathrm{~ms}$. T1 was obtained by applying the LUT to the ratio of the first echo of M1 and M2 (Fig. 2). The proposed T1 and $\mathrm{T} 2 *$ mapping was compared with the inversion recovery method and multi-echo gradient-echo method, respectively, by measuring plasticine and solutions with different agar and Gadolinium concentrations (Fig. 1). The $\mathrm{T} 1$ and $\mathrm{T} 2 *$ maps were converted into a bivariate histogram to identify tissue specific $\mathrm{T} 1 / \mathrm{T} 2 *$ clusters by using previous literature values 2 based on which the $\mathrm{T} 1$ and $\mathrm{T} 2 *$ thresholds determined various tissues.
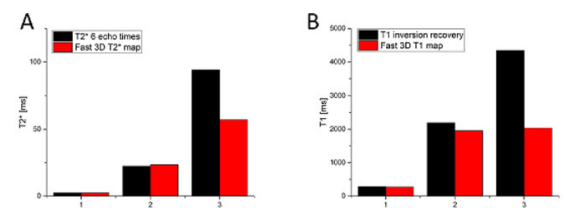
phantom compartments by using the proposed fast mapping technigue (black bars) and conventional reference methods (red proposed fast mapping lechnique (black bars) and the inversion recovery method with 12 inversion times ranging between 30 and $2500 \mathrm{~ms}$. Reference $\mathrm{T2}^{*}$ values were obtained from a multi-echo gradient-echo experiment $\left(\mathrm{TE}_{1.6}=\right.$ $0.04 / 2.46 / 4.92 / 9.84 / 19.68 / 39.36 \mathrm{~ms})$. In the $T 2^{\star}$ experiment (A), the fast relaxation phantom compartment (1) contained a tube of plasticine, whereas compartments two and three contained solutions with decreasing agar concentrations. While the $T 2^{*}$ values of rast relaxating probes ( 1 and 2 ) are comparable between the two approaches, the above 50 ms because of the shortlongest echo time In the T1 experiment $(B)$ specific T1 values were adjusted by doping aqueous solutions with varying concentrations of Gadolinium. For the short T1 compartment (1), the T1 value of the proposed fast mapping technique is comparable with the one of the reference method. With longer adjusted $\mathrm{T}$ values, however, the last $T 1$ mapping technique exhibits increased divergence wi respect to the reterence TI values because the chosen repettions times and flip angles e fast T1 mapping approach were optimized for a look up table in the range of 1 $2500 \mathrm{~ms}$
R: Workflow, images and $\mathrm{T} 1 / \mathrm{T} 2 *$ maps of a healthy volunteer are shown in Fig. 2. The bivariate histogram, presented in Fig. 3, reveals tissue specific clusters. Applying the histogram-based classification allows differentiation of bone marrow and patellar tendons (Fig. 3BD).
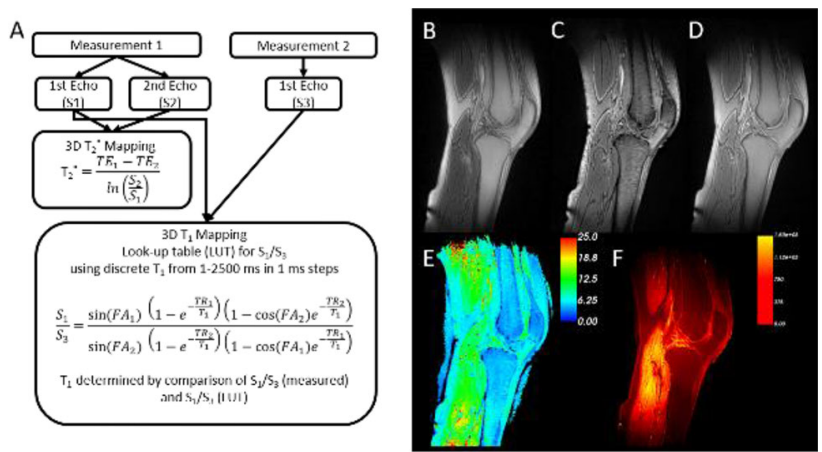

Fig2: Workflow to calculate $\mathrm{T}^{*}$ and T1 maps (A). Acquired images of the two different UTE scans showing the knee in sagittal view (B-D). Measurement 1 (M1): TE $=0.04 \mathrm{~ms}$, TR=9.92ms, FA=16 ${ }^{\circ}$ (B), measurement 1 (M1) $T E=4.92 \mathrm{~ms}, T R=9.92 \mathrm{~ms}, F A=16^{\circ}(\mathrm{C})$, measurement $2(\mathrm{M} 2)$ : $T E=0.04 \mathrm{~ms}, T R=4.45 \mathrm{~ms}, F A=3^{\circ}(\mathrm{D})$. The lower row shows the corresponding T2* (calculated based on (B) and (C)) and T1 (calculated based on (B) and (D)) map in $(E)$ and $(F)$, respectively.
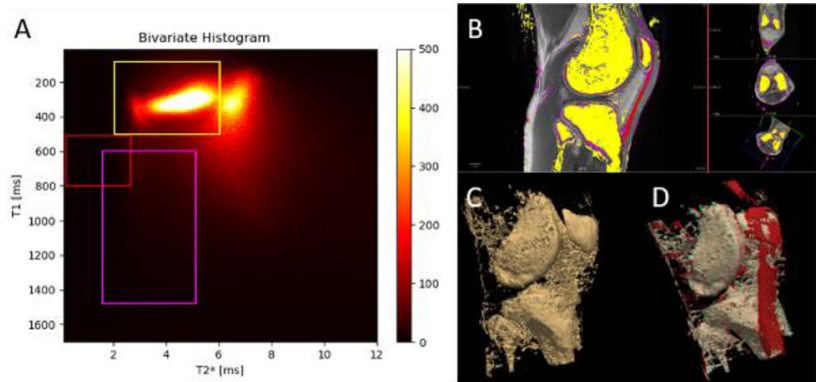

Fig3: T1 and T2*-based segmentation of the knee. (A) Bivariate histogram generated from $\mathrm{T}^{*}$ and T1 map data with three regions of interest (bone marrow=yellow, patellar tendons=red, bone=purple). Three segmented tissues types are shown as an overlay in (B). A 3D-visualization of the bone marrow (yellow label in (B)) is shown in (C). In (D), the patellar tendons (brown) were added to the bone marrow.

D: $\mathrm{T} 1$ and $\mathrm{T} 2 *$ values of the knee tissue as well as pixel count peaks of the bivariate histogram obtained in a clinically appropriate TA of $9 \mathrm{~min}$ are in line with findings by Krämer et al. ${ }^{1}$. The 3D-visualization of bone marrow and the patellar tendons shows segmentation of these tissues opening the door to quantitative lesion characterization in bone or tendons. We intend to apply our approach to a larger cohort (controls and patients) to optimize segmentation thresholds for the knee and validate the outcome with manual segmentations. Our approach might also be applied for other joints such as the ankle, elbow or shoulder.

\section{References:}

1 Krämer M Magn Reson Imaging. 2019;63:29-36.

2 Krämer M Z Med Phys. 2020;30(1):51-59. 
S6.02.

Estimation of a robust multi-scanner radiomic signature to predict the response to neoadjuvant chemotherapy in breast cancer using MRI

\author{
*M. J. Saint Martin ${ }^{1}$, C. Malhaire ${ }^{1,2}$, P. $\mathrm{Akl}^{3}$, D. Sebbag $\mathrm{Sfez}^{2}$, \\ F. Frouin ${ }^{1}$, F. Orlhac ${ }^{1}$ \\ ${ }^{1}$ Paris-Saclay University, LITO, Orsay, FR; \\ ${ }^{2}$ Curie Institute, Department of Radiology, Paris, FR; \\ ${ }^{3}$ Hospices Civils de Lyon, Department of Radiology, Lyon, FR
}

Introduction: Neoadjuvant chemotherapy (NAC) can be proposed to patients with locally advanced breast cancer to reduce tumors sizes before surgery and improve breast conservation rates. Predicting the response to NAC using preoperative MRI could improve the delivery of a personalized therapy. Radiomic models, based on features extracted from lesions, have shown promises in predicting pathological complete response (pCR) but as radiomic features (RF) values depend on each scanner, models are weakly transferable. This work intends to develop a robust multi-scanner radiomic model to predict pCR to NAC.

Methods: A retrospective study included 103 breast cancer patients, of wich 49 responders, scanned before NAC using T2 and T1weighted DCE modalities. Twenty-five were acquired on a GE Optima MR450w (1.5 T) machine with an 8-channel coil and 78 on a Siemens MAGNETOM Aera (1.5 T) machine with an 18-channel coil $(n=19)$ or with a 16-channel Sentinelle coil $(n=59)$. Based on [1], 3D images were corrected for bias field, spatially resampled using $\mathrm{B}$-spline interpolation and $\mathrm{z}$-score normalized with mean values and standard deviation computed in the breast outside the tumors manually segmented. A total of $2010 \mathrm{RF}$ were extracted from each lesion using Pyradiomics. For robustness, RF were harmonized between the 3 MR coils using ComBat [2]. For 30 patients, lesions were segmented by two radiologists and two-way intraclass correlation coefficients (ICC) calculated between RF extracted from each radiologist's region. RF selection included a three-step procedure: 1) RF with an ICC $>0.8 ; 2$ ) lower bound of AUC of ROC curve $>0.5 ; 3$ ) Boruta algorithm after the z-score normalization of RF. Random forest models were then trained and evaluated using 10 repetitions of leave-one-out cross-validation on 3 experiments: T1-based RF (E1), T2-based RF (E2), T1 plus T2-based RF (E3). Performances of the models were evaluated using the Youden Index ( $\mathrm{Y}=$ sensitivity + specificity-1) to predict pCR. Finally, a permutation test was conducted 100 times, by randomly assigning a response label to each patient while respecting the proportion of responders to non-responders in each scanner.

Results: Regarding RF selection, 946 T1-RF and 884 T2-RF displayed an ICC $>0.8$ between the two radiologists' segmentations. The harmonization by ComBat led to a greater number of RF selected by univariate analysis (Fig. 1) and better performances for each experiment (Fig. 2). The best predictive model (E3) yielded a Youden $\mathrm{Y}=0.49 \pm 0.02$ (Fig. 2), outperforming any predictive models trained on reshuffled response labels in permutation tests (Fig. 3, $p<0.001)$.
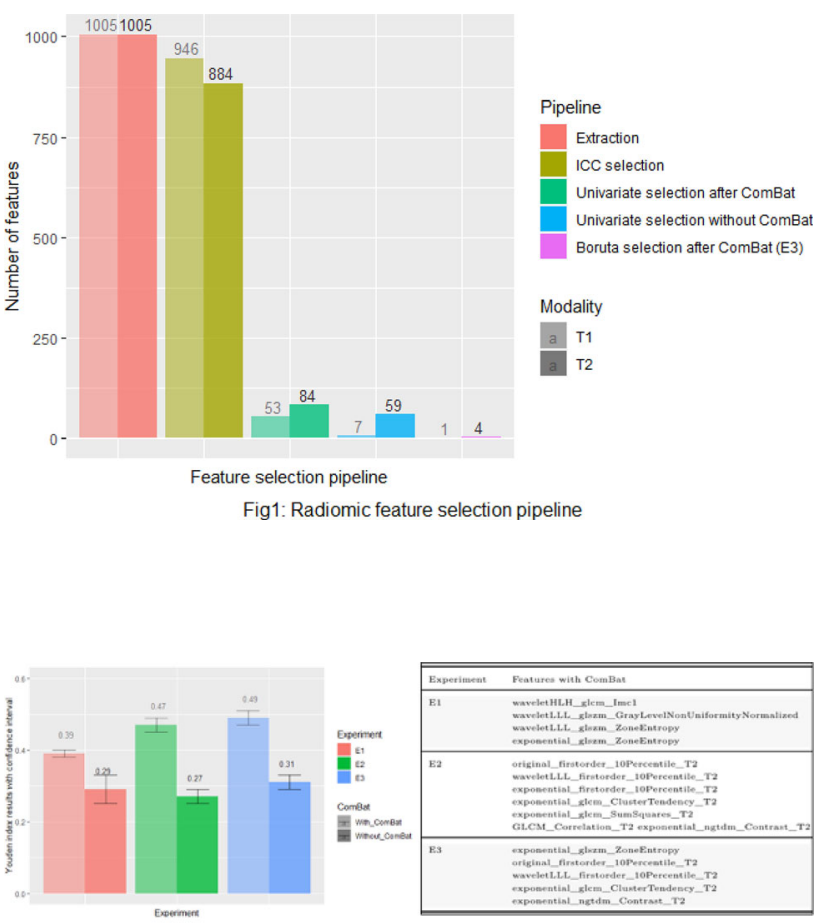

Fig2: Predictive models Youden index and features

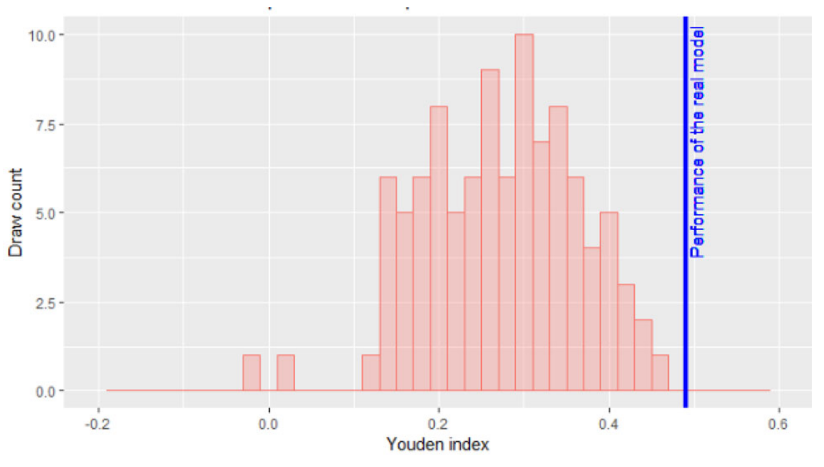

Fig3: Youden Index of 100 models trained to predict random label vectors in permutation experiment

Discussion: ICC selection spotted features robust to slight variations in segmentation shapes while harmonization by ComBat enabled models to be robust to the different scanners. The combined pipeline ensured the construction of a robust and transferable model to predict pCR.

\section{References:}

[1] Saint Martin et al. MAGMA 2021.

[2] Orlhac et al. Eur Radiol 2021. 
S6.03.

DdoS: dynamic dual-channel U-Net for improving deep learning based super-resolution of abdominal dynamic MRI

*C. Sarasaen ${ }^{1,2}$, S. Chatterjee ${ }^{1,3,4}$, A. Nürnberger ${ }^{3,4,5}$, O. Speck ${ }^{1,5,6,7}$

${ }^{1}$ Otto von Guericke University, Department of Biomedical Magnetic Resonance, Magdeburg, DE;

${ }^{2}$ Otto von Guericke University, Institute for Medical Engineering, Magdeburg, DE;

${ }^{3}$ Otto von Guericke University, Faculty of Computer Science, Magdeburg, DE;

${ }^{4}$ Otto von Guericke University, Data and Knowledge Engineering Group, Magdeburg, DE;

${ }^{5}$ Otto von Guericke University, Center for Behavioral Brain Sciences, Magdeburg, DE;

${ }^{6}$ German Center for Neurodegenerative Diseases (DZNE),

Magdeburg, DE;

${ }^{7}$ Leibniz Institute for Neurobiology, Magdeburg, DE

Introduction: The trade-off between spatial and temporal resolution in dynamic MRI is a hindrance for MR-guided interventions, which require high temporal resolution while visualizing details. Deep learning based super-resolution (SR) has shown promising results in dealing with this trade-off [1]. Nevertheless, the available temporal information of dynamic MRI has not been exploited in this prior work. The potential of improving the reconstruction quality of dynamic MRIs by incorporating the temporal information has been demonstrated recently [2-4]. This work extends the previous work by ameliorating the previous model [2] a the dual-channel (static + dynamic images) super-resolution approach, termed DDoS (Dynamic Dual-channel of SuperRes).

Methods: An artificial dynamic dataset was generated by applying random elastic deformations [5] to the publicly available CHAOS dataset (T1 in- and opposed phase) [6]. Then, a low resolution dataset was simulated by performing in-plane undersampling $[7,8]$ taking only the centre of the k-space of each slice. A modified U-Net model [1] was trained with dual-channel input, consisting of the low-resolution image of the current time point $\left(\mathrm{LR}_{-} \mathrm{TP}_{\mathrm{n}}\right)$ and the high resolution image of the previous time point $\left(\mathrm{HR} \_\mathrm{Tp}_{\mathrm{n}-1}\right)$, see Fig. 1. This training strategy tries to let the network learn the spatio-temporal relationship over time points, with the help of perceptual loss [9] using a perceptual loss network [10], and was minimised using Adam optimiser for 18 epochs. $3 \mathrm{D}$ patches of the volumes were created for training with a patch size of $24^{3}$, and with a stride of 6 for the slice dimension and 12 for the rest. For testing, a stride of 3 was used for all dimensions with the same patch size. A 3D abdominal dynamic MRI data was acquired at 3 T Siemens Magnetom Skyra [GRE, T1w Flash 3D, TR: $2.23 \mathrm{~ms}$, TE: $10.93 \mathrm{~ms}$, voxel size: $1.09 \times 1.09 \times 4.0 \mathrm{~mm}$ ] and was also undersampled in the same manner and were used for testing.

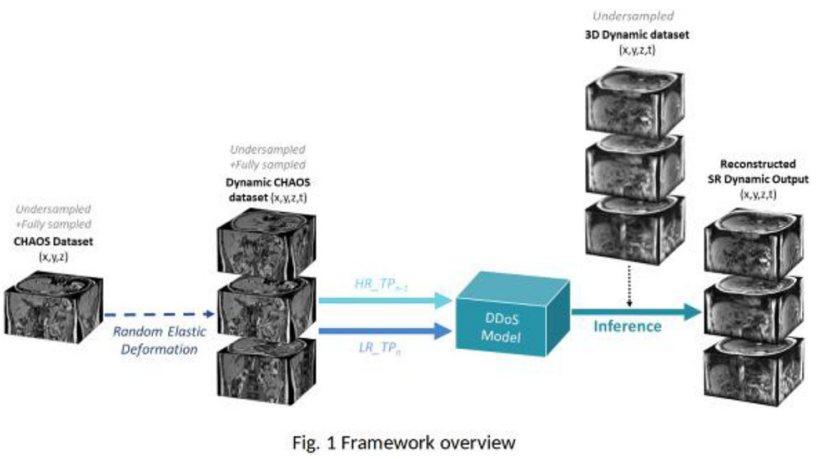

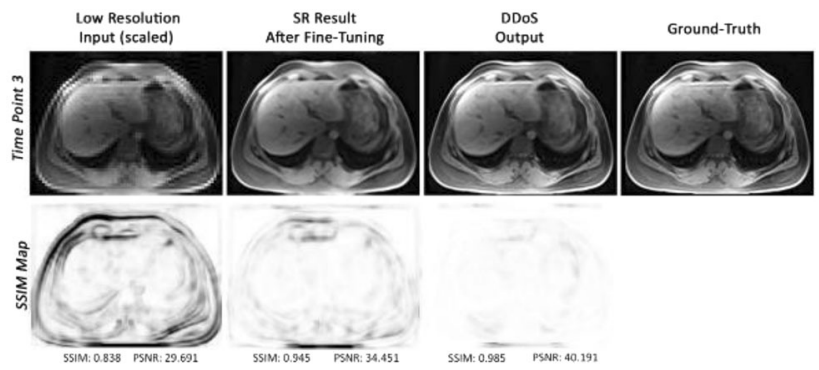

Fig. 2 An example results and SSIM map of one time point for undersampled $6.25 \%$ of centre $\mathrm{k}$-space. From left to right, compared low resolution input, SR result after fine-tuning, DDoS output and its corresponding ground-truth.

Results and discussion: The highest undersampling (only $6.25 \%$ of centre k-space per slice) explored in the former work [1], was applied to evaluate the improvements with the proposed approach. Figure 2 shows the comparison of low-resolution input (for the lowest resolution examined), SR result after subject specific fine-tuning [1], DDoS results and its corresponding ground-truth images. The average SSIM value while comparing the results against the ground-truth improved from $0.949 \pm 0.003$ (for SR result after fine-tuning [1]) to $0.979 \pm 0.004$ and the average PSNR value improved from $34.647 \pm 0.240$ to $39.411 \pm 0.536$. One can observe qualitatively that the results of the DDoS model are more similar to the groundtruth compared to the result of SR after fine-tuning. In conclusion, this research illustrates that by incorporating the temporal information in a super-resolution approach [1] using the DDoS model can improve the reconstruction quality, and may be extended for application during real-time interventions due to the fast inference speed.

\section{References:}

[1] Sarasaen et al. Fine-tuning deep learning model parameters for improved super-resolution of dynamic MRI with prior-knowledge, arXiv preprint arXiv:2102.02711 (2021).

[2] Rasch et al. Dynamic MRI reconstruction from undersampled data with an anatomical prescan. Inverse problems 34(7), (2018): 074001.

[3] Kofler et al. Spatio-temporal deep learning-based undersampling artefact reduction for $2 \mathrm{D}$ radial cine MRI with limited training data. IEEE transactions on medical imaging 39(3), (2019): 703-717.

[4] Küstner et al. CINENet: deep learning-based 3D cardiac CINE MRI reconstruction with multi-coil complex-valued 4D spatio-temporal convolutions. Scientific reports 10(1), (2020): 1-13.

[5] Pérez-García et al. TorchlO: a Python library for efficient loading, preprocessing, augmentation and patch-based sampling of medical images in deep learning. arXiv preprint arXiv: 2003.04696 (2020).

[6] Kavur et al. CHAOS challenge-combined (CT-MR) healthy abdominal organ segmentation. Medica Image Analysis (69), (2021).

[7] soumickmj/MRUnder: Zenodo. doi:10.5281/zenodo.3901455: CenterRatioMask (undersampling type 5).

[8] Chatterjee et al. ReconResNet: Regularised Residual Learning for MR Image Reconstruction of Undersampled Cartesian and Radial Data. arXiv preprint arXiv:2103.09203 (2021).

[9] Johnson et al. Perceptual losses for real-time style transfer and super-resolution. European conference on computer vision. Springer, Cham, (2016).

[10] Chatterjee et al. DS6, Deformation-aware Semi-supervised Learning: Application to Small Vessel Segmentation with Noisy Training Data. arXiv preprint arXiv:2006.10802 (2020).

\section{S6.04.}

\section{Noise removal in line-scanning fMRI}

*C. Passarinho ${ }^{1,2}$, L. Raimondo ${ }^{3,2}$, J. Heij ${ }^{3,2}$, T. Knapen ${ }^{3,2}$, S. Dumoulin ${ }^{2,4}$, J. Siero ${ }^{2,5}$, W. van der Zwaag ${ }^{2}$

${ }^{1}$ University of Lisbon, Lisbon, $N L$;

${ }^{2}$ Spinoza Centre for Neuroimaging, Amsterdam, NL;

${ }^{3}$ Vrije Universiteit Amsterdam, Amsterdam, NL; 
${ }^{4}$ Utrecht University, Utrecht, $N L$;

${ }^{5}$ University Medical Center Utrecht, Department of Radiology, Utrecht, $N L$

Introduction: By sacrificing spatial coverage of the brain outside the region of interest, line-scanning (LS) fMRI allows extremely high spatial and temporal resolution ${ }^{1}$, resulting in a more accurate characterization of the HRF across cortical layers. The small voxel size and very short TR $(\sim 100 \mathrm{~ms})$ lead to substantial amounts of thermal noise and the small area being scanned increases the sensitivity of LS to motion. In this work, we present a LS denoising technique based on Noise reduction with Distribution Corrected (NORDIC) $\mathrm{PCA}^{2,3}$

Methods: Four healthy volunteers were scanned at $7 \mathrm{~T}$ (Philips) using a 32-channel receive head coil (Nova Medical). The acquisition was based on a modified 2D ME-GRE sequence, with line resolution $250 \mu \mathrm{m}, \mathrm{TR}=105 \mathrm{~ms}, \mathrm{TE} 1 / \Delta \mathrm{TE}=6 / 8 \mathrm{~ms}, 3200$ time points, array size 720 and line thickness $2.5 \mathrm{~mm}$. Fat suppression was performed using SPIR. Two OVS pulses $(7.76 \mathrm{~ms})$ were used to suppress the signal outside the line of interest in the visual cortex (Fig. 1). The phase-encoding gradient perpendicular to the line was turned off ${ }^{4}$. One run of functional data was acquired for each volunteer with block design visual task, consisting of a $20 \mathrm{~Hz}$ flickering checkerboard (10 s ON/OFF, $\mathrm{n}=16)$. Offline reconstruction using Matlab and Gyrotools included a denoising step based on NORDIC prior to coil combination. A singular value decomposition (SVD) of the data was submitted to hard thresholding to eliminate all components indistinguishable from zero-mean gaussian noise ${ }^{2}$, followed by a tSNR and coil sensitivity-weighted sum of squares coil combination. Reconstruction with and without denoising were compared (tSNR, t-values and PSC) within a 10-voxel ROI for 4 thresholds $(20,50,70$ or $90 \%$ of singular values were set to zero for noise removal).
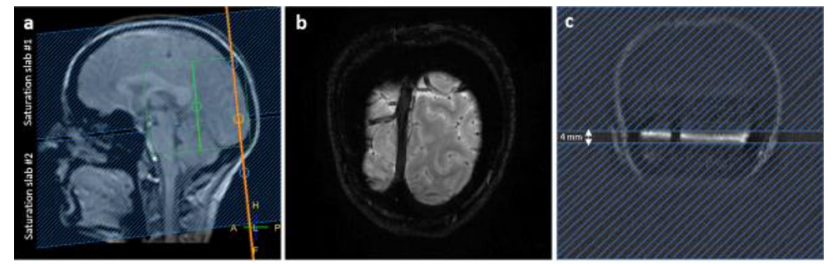

Figure 1 - (a) Sngital view of the placement of the line of interes (orange) and saturation stabs (blue), (b) acquired slice and (e) placemeat of tbe

Results: Figure 2 shows LS-fMRI data for the original (panel a) and denoised data, after the removal of 50\% (panel b) and 90\% (panel c) of the SVD components. Figure $2 \mathrm{~d}$ shows the resulting timecourses averaged over the 10-voxel ROI. Figure 3 shows the tSNR and PSC for the different thresholds for an example subject and the distribution of t-values across the line for all four subjects. Overall, more voxels with higher $\mathrm{t}$-values were found in all volunteers.

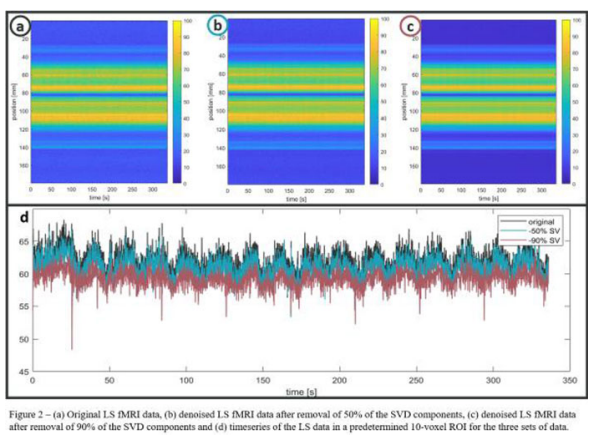

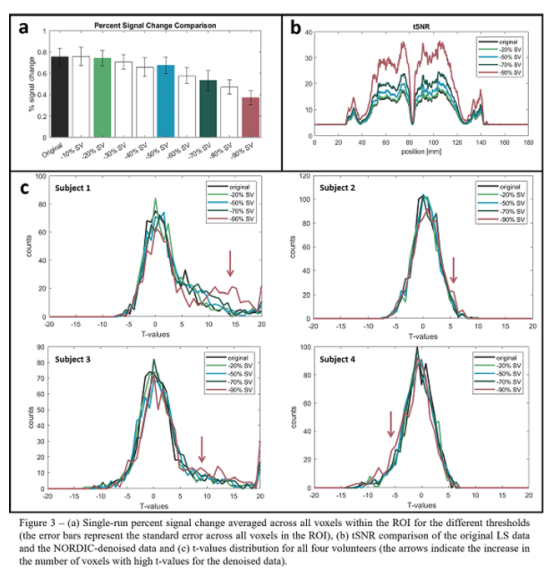

Discussion: Our results reveal that NORDIC PCA improves the quality of LS-fMRI data, making its application more feasible. The signal outside the brain was visibly reduced for both thresholds, while the BOLD response to the visual task was maintained in the time series (Fig. 2). By removing the thermal noise, NORDIC facilitates the removal of cardiac and respiratory components ${ }^{3}$, thus being valuable for physiological noise removal. These outcomes can be further enhanced by assessing an ideal numerical threshold and by optimizing the line placement during planning 5 .

\section{References:}

1. Yu, X., et al.Nat. Methods 11, 55-58 (2014).

2. Moeller, S. et al.Neuroimage 226, (2021).

3. Vizioli, L. et al.bioRxiv (2020).

4. Raimondo, L. et al. ISMRM (2021).

5. Heij, J. et al. ISMRM (2021).

\section{S6.05.}

Rational approximation of the Ernst equation for dual angle R1 mapping revisited: beyond the small flip-angle assumption

\author{
L. Edwards ${ }^{1}, *$ K. Pine ${ }^{1}$, G. Helms ${ }^{1,2}$, N. Weiskopf ${ }^{1,3}$ \\ ${ }^{I}$ Max Planck Institute for Human Cognitive and Brain Sciences, \\ Department of Neurophysics, Leipzig, DE; \\ ${ }^{2}$ Lund University, Department of Medical Radiation Physics, Lund, \\ $D E$; \\ ${ }^{3}$ Leipzig University, Felix Bloch Institute for Solid State Physics / \\ Faculty of Physics and Earth Sciences, Leipzig, DE
}

Introduction: Dual flip angle R1 mapping-collecting two FLASH volumes with different flip angles ( $\alpha 1$ and $\alpha 2$ ) and (potentially) different repetition times (TR1 and TR2)-is a workhorse of proton density (PD) and R1 mapping in the in vivo human brain [1] . An efficient method for computing R1 and PD from the two volumes, implemented in the hMRI toolbox (hmri.info), uses a rational approximation of the Ernst equation derived under the assumption of short TR and small $\alpha[1,2]$. However, the assumption of small $\alpha$ can break down at $7 \mathrm{~T}$, where B1 inhomogeneities are large [3]. Here we present and validate a modified rational approximation that is valid even for large $\alpha$. Methods: The derivation is presented in Fig. 1. First, we make a halfangle tangent substitution into the Ernst equation (Eq. (1)) to simplify the trigonometric functions of $\alpha$ without approximation (Eq. (4)) [4]. We then make the assumption TR is small such that R1 TR is small. Because the Ernst equation is a rational function, we use rational Padé approximants [5] to expand around TR $=0$, then solve for R1 and PD. 
Starting from the Ernst equation

$$
S(\alpha, \mathrm{TR})=\mathrm{PD} \sin (\alpha) \frac{1-\exp (-\mathrm{R} 1 \mathrm{TR})}{1-\cos (\alpha) \exp (-\mathrm{R} 1 \mathrm{TR})}
$$

we follow [4] in making half-angle tangent substitutions using the identities

$$
\sin (\alpha)=\frac{\tau}{1+\langle\tau / 2)^{2}} ; \quad \cos (\alpha)=\frac{1-(\tau / 2)^{2}}{1+(\tau / 2)^{2}}
$$

where

$$
\tau=2 \tan (\alpha / 2)
$$

to give

$$
S(\tau, \mathrm{TR})=\mathrm{PD} \tau \frac{1-\exp (-\mathrm{R} 1 \mathrm{TR})}{1-\exp (-\mathrm{R} 1 \mathrm{TR})+(\tau / 2)^{2}(1+\exp (-\mathrm{R} 1 \mathrm{TR}))},
$$

which is equivalent to Eq. (5) in [4].

The [1/1] Padé approximant [5] of Eq. (4) around R1 TR $=0$ is:

$$
S(\tau, \mathrm{TR}) \approx \mathrm{PD} \tau \frac{\mathrm{R} 1 \mathrm{TR}}{\tau^{2} / 2+\mathrm{R} 1 \mathrm{TR}} .
$$

The left panel of Fig. 2 shows that Eq. (5) gives a good approximation of the signal. Note that Eq. (5) differs from the result of simply inserting $\exp (-\mathrm{R} 1 \mathrm{TR}) \approx 1-\mathrm{R} 1 \mathrm{TR}$ into Eq. (4), which does not approximate the signal well (Fig- 2).

Eq. (5) has the same form as the small angle approximation result Eq. (A.5) in [1] (with T instead of $\alpha$ ), so we can immediately write our final result

$$
\mathrm{R} 1 \approx \frac{1}{2} \frac{\left(S_{1} \tau_{1} / \mathrm{TR} 1\right)-\left(S_{2} \tau_{2} / \mathrm{TR} 2\right)}{\left\langle S_{2} / \tau_{2}\right)-\left(S_{1} / \tau_{1}\right)}
$$

and

$$
\mathrm{PD} \approx S_{2} S_{1} \frac{\left(\mathrm{TR} 2 \tau_{1} / \tau_{2}\right)-\left(\mathrm{TR} 1 \tau_{2} / \tau_{1}\right)}{S_{1} \mathrm{TR} 2 \tau_{1}-S_{2} \mathrm{TR} 1 \tau_{2}}
$$

from Eqs. (A.6) and (A.7) in [1]

Figure 1: Derivation of the [1/1] Padé rational approximation and solution for a dual flip-angle experiment.

We tested the method in simulations and on a $7 \mathrm{~T}$ in vivo dataset. To demonstrate that the result is non-obvious, the simulations also compared the result of simply inserting the linear expansion of $\exp (-\mathrm{R} 1 \mathrm{TR})$ into Eq. (4).

Results and discussion: Figure 2 shows the benefits of the novel approximation for a simulated $7 \mathrm{~T}$ acquisition. Figure 3 shows these benefits also extend to a typical experimental dataset.

Formally, our result is the specific dual-angle case of an $\mathrm{N}$ flip angle result suggested (but not evaluated) in [6]. The left panel of Fig. 2 shows that it is important to always evaluate the numerical accuracy of such results, as substituting linear approximations into rational functions is not guaranteed to give good results.

We retained the assumption of short TR. While a closed form exact solution without approximation is possible when TR1 = TR2 [4], this is not generally applicable. Nonlinear fitting can be used to estimate R1 and PD from Eq. (1), but will be very slow.
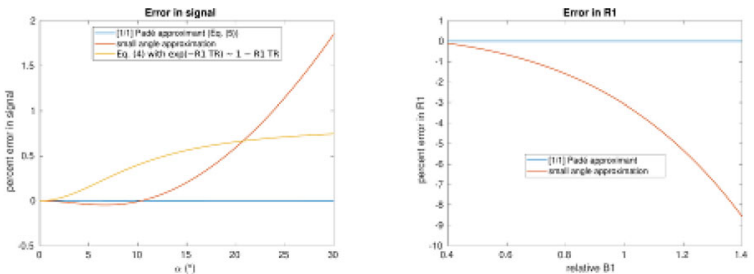

Figure 2: Relative errors in approximations from simulations. Data simulated using the Ernst equation (Eq. (1)) and typical $7 \mathrm{~T}$ parameters: $\mathrm{TR}=23.5 \mathrm{~ms}, \mathrm{RI}=0.7 \mathrm{~s}^{-1}$. For simplicity. $\mathrm{PD}=1$. Left: Errors in the signal grow large when using either the small angle approximation or expansion of the exponentials in Eq. (4), but remain close to zero for the Padé approximant (Eq. (5)), showing that the Padé approximant is numerically better behaved. Right: Errors in the signal correspond to errors in R1 estimates which scale with B1 inhomogeneity. Nominal flip angles: $\alpha 1=8^{\circ}, \alpha 2=28^{\circ}$. Flip angles were chosen to be close to optimal for estimation of R1 following [4]. The B1 range covers a typical range in human brain at $7 \mathrm{~T}$. Because the third signal model gave a poor representation of the signal (left panel), it is omitted in the right panel.

We omitted magnetization transfer and imperfect spoiling in our analysis, which also increase with increasing flip angle [7, 8]. However, Fig. 3 implies that our method still removes a significant amount of bias.
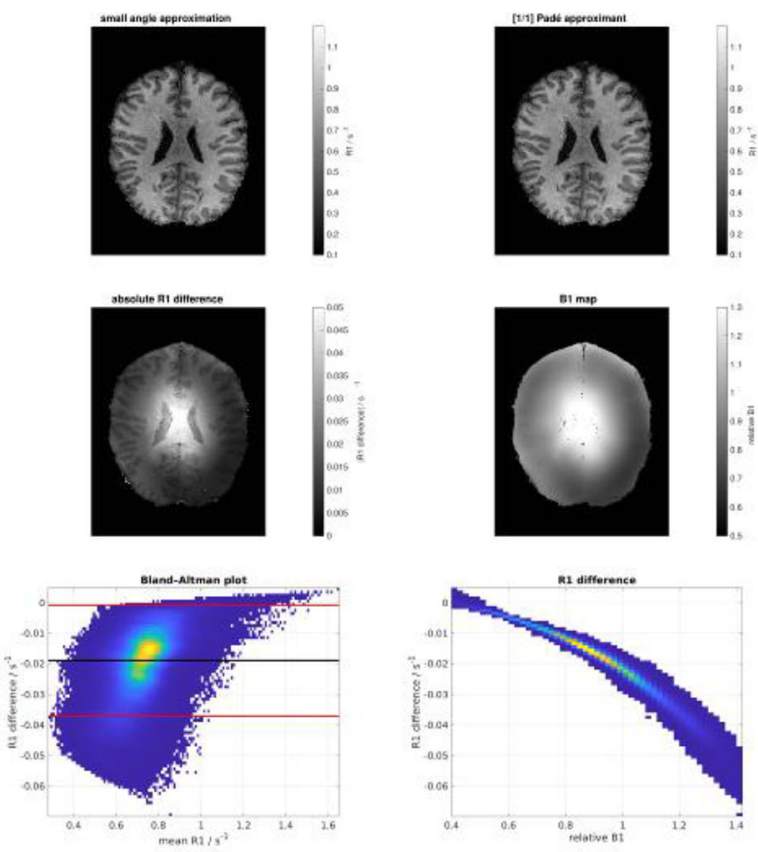

Figure 3: In vivo results. The subject gave written informed consent before scanning. Pacameters: Siemens Terra 7T MRI scanner, $0.65 \mathrm{~mm}$ isotropic resolution FLASH acquations, TR $=2355$ as, 6 ogr TR = 23.5 m, 6 equ corrected using a SE/STE-EPI BI map [3]. R1 and PD estimated with the hMRI toolbox [1] and a version modified to use Eq. (6). The R1 maps are superficially similar (top row), but thei difference (middle-left) shows a pattern reflecting B1 inhomogeneity (middle-right; interpolated to R1 space). The Bland-Altman plot of R1 over a white matter mask (bottom-left) shows the small-angle approximation is biased relative to the Padé approximant (black line: mean difference, red lines: mean difference \pm 1.96 standard deviations of the difference). Plotted as a function of B1 (bottom-right), we see that the white matter R1 estimates follow the theoretical prediction of Fig. 2

This method has been implemented into the hMRI toolbox (hmri.info) and will be included in an upcoming release. This will allow others to efficiently extract more precise R1 estimates.

\section{References:}

[1] K. Tabelow et al. Neuroimage, 2019.

[2] G. Helms et al. Magn. Reson. Med., 2008. Erratum: Magn. Reson. Med. 63:1136 (2010).

[3] A. Lutti et al. PLOS ONE, 2012.

[4] H. Dathe and G. Helms. Phys. Med. Biol., 2010.

[5] W. H. Press et al. Numerical Recipes in $\mathrm{C}++$ : The Art of Scientific Computing. 2002.

[6] G. Helms et al. Magn. Reson. Med., 2011.

[7] A. G. Teixeira et al. Magn. Reson. Med., 2019.

[8] N. Corbin and M. F. Callaghan. Magn. Reson. Med., 2021.

\section{S6.O6.}

\section{QC Flex: a flexible quality control tool for large MRI} cohorts, presented for FreeSurfer segmentations

\section{${ }^{*}$ S. Eggenreich ${ }^{1}$, S. Ropele ${ }^{1}$, L. Pirpamer ${ }^{1}$ \\ ${ }^{I}$ Medical University of Graz, Department of Neurology, Graz, AT}

Purpose of the software: The accurate segmentation of brain tissue plays an important role in neuroscience for several purposes. The assessment of global and regional tissue labels, for example, has become a crucial application in several neurodegenerative diseases ${ }^{1,2}$ and also in normal ageing, ${ }^{3}$ to study subject specific volumetric changes or to perform regional quantitative analyses. Although 
advanced brain segmentation tools, such as FreeSurfer, ${ }^{4}$ have become more robust, segmentation errors still might occur. Therefore, manual quality assurance is still indispensable, which however becomes difficult and time consuming at a larger scale. Here, we present a tool for fast, explorative and interactive quality assessment optimally suited for larger cohorts, combining the visualization of a segmentation output along with the numeric outcome of the resulting dataset.

Materials and methods: QCFlex was developed under Python 3.9.5 with the Python packages Numpy, Matplotlib, pandas, Pillow, qimage2ndarray. The GUI was built with PyQT5 and can be distributed as an executable. A single table file builds the basis of variables to explore and the location of corresponding image paths. The interactive image viewer displays a precomputed high resolution 2D image of the resulting segmentations, which can be inspected in detail by zooming into the region of interest. The integrated comment feature allows the annotation of images and storage of those comments in the table file for later use. The interactable scatterplots, depicting two selectable outcome-measures (such as brain volume and gray matter volume), allow an instant overview of the data distribution. This feature facilitates the detection of outliers, which can be further inspected by clicking on the datapoint. Buttons to annotate the images with Pass/Fail further supports the user's ability to assess the overall quality of the calculations, while still being able to keep track of outliers.

Features illustrated at the exhibit: The tool will be presented based on a precomputed dataset of brain segmentations processed by FreeSurfer (v.6). Moreover, we will explain, how QC Flex can be adapted for different purposes, where imaging data is used to generate numeric outcome measures and where quality assessment can be performed by a $2 \mathrm{D}$ image.

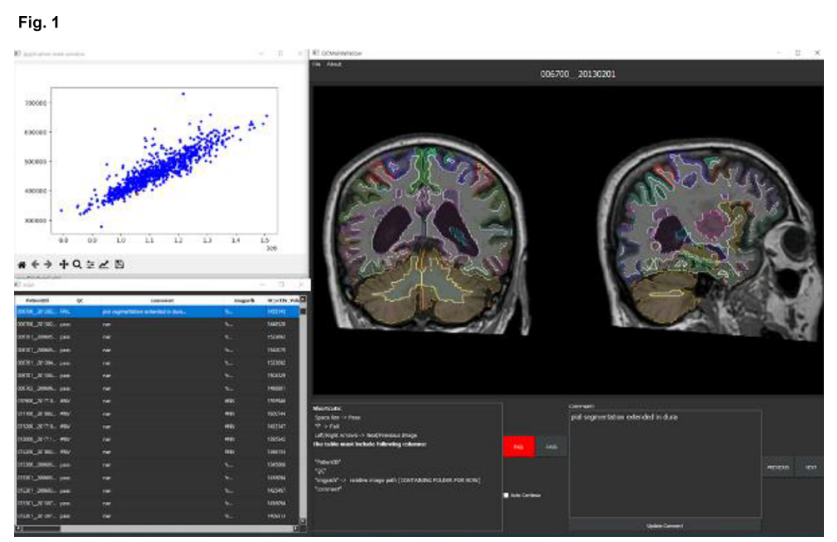

\section{References:}

1. Popescu, V. et al. Grey Matter Atrophy in Multiple Sclerosis: Clinical Interpretation Depends on Choice of Analysis Method. PLoS One 11, e0143942 (2016).

2. Brain volumes and their ratios in Alzheimer's disease on magnetic resonance imaging segmented using Freesurfer 6.0. Psychiatry Research: Neuroimaging 287, 70-74 (2019).

3. Dickie, D. A. et al. Variance in brain volume with advancing age: implications for defining the limits of normality. PLoS One 8, e84093 (2013).

4. FreeSurfer. Neuroimage 62, 774-781 (2012).
S6.07.

Automated correspondence and registration between CEST and histological mouse brain sections for in vivo virus tracking

*S. Piluso ${ }^{1,2}$, N. Souedet ${ }^{1}$, J. Flament ${ }^{1}$, M. Gaudin ${ }^{1}$, C. Jan ${ }^{1}$, G. Bonvento ${ }^{1}$, C. Clouchoux ${ }^{2}$, T. Delzescaux ${ }^{1}$

${ }^{I}$ CEA Molecular Imaging Research Center, Fundamental Research Division, Fontenay-aux-Roses, FR;

${ }^{2}$ WITSEE-NEOXIA, Paris, FR

Introduction: In vivo monitoring of transgene expression in the brain after gene therapy remains a complex task. Chemical Exchange Saturation Transfer (CEST) is an emerging imaging modality that could be used to achieve this goal [1] . An Adeno-Associated Virus (AAV) expressing an arginine rich-protein reporter (polyARG) has been designed, with frequency-selective contrast as well as tdTomato (a fluorescent protein). To validate the use of the CEST reporter (polyARG), we need precise co-localization of the AAV with anatomical ground truth (histological slices series). In this abstract, we propose an automated method to identify and register the best histological slice candidate corresponding to a single CEST image.

Methods: An AAV-PolyARG-tdTomato was injected in the left striatum of 2 wild-type mice. For each mouse, a single CEST slice $\left(0.13 \times 0.13 \times 1 \mathrm{~mm}^{3}\right.$ resolution $)$, as well as its corresponding anatomical MRI $(A)$, were acquired in the striatum. Acquisition of 25 anatomical MRI sections $(R)$ was performed around the CEST signal acquisition location $\left(0.07 \times 0.07 \times 0.3 \mathrm{~mm}^{3}\right.$ resolution $)$. Finally, 12 histological slices $(T)$ were cut and digitized in the same region $\left(0.03 \times 0.03 \times 0.2 \mathrm{~mm}^{3}\right.$ resolution $)$. To identify the best $T_{b}$ candidate among $T$, matching $A$, and given intensity bias in $A$, we chose to first identify the best $R_{b}$ section in $R$, and use it as a reference to identify $T_{b}$. After preprocessing, we explored every possible 2D-2D registrations between all $R$ sections and $A$ using BlockMatching [2], with first rigid and then affine registration, increasing degrees of freedom. For each pair of registered sections, Normalized Mutual Information (NMI) [3] quantified their similarity at every registration step (rigid, affine). Finally calculating the maximum of an equal contribution of both rigid and affine NMI values enabled the identification of $R_{b}$ corresponding to $A$ (Fig. 1). Same steps were subsequently applied to identify $T_{b}$ corresponding to $R_{b}$.

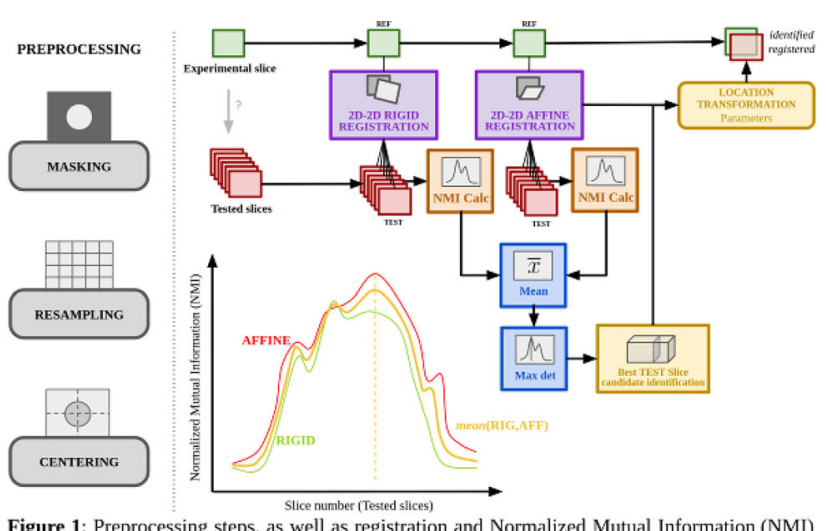
processing steps of the proposed method.

Results: Results show that one single maximum NMI value stands out in both cases for $T_{b}$ identification (Fig. 2). Paired image results were visually validated by experts and are presented in Fig. 3. 

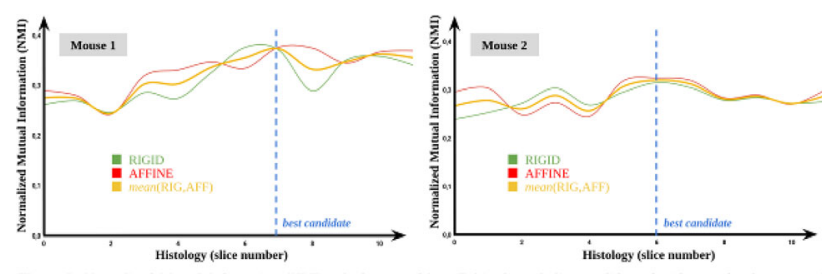

Figure 2: Normalized Mutual Information (NMI) calculation and best $T_{\text {s }}$ histological slice candidate identification by the propose methed for the bes $R$ MRI section

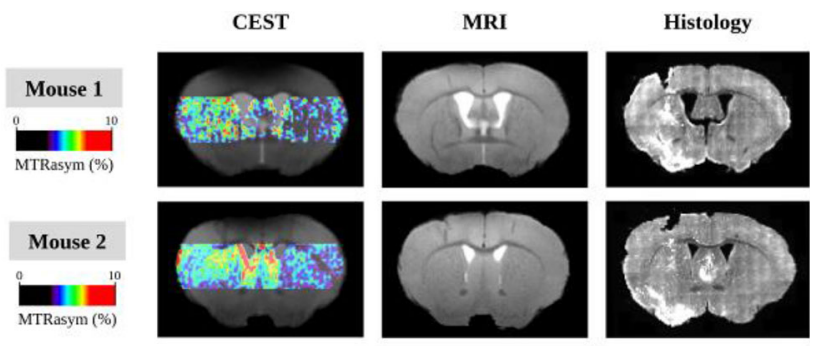

Figure 3: CEST signal, MRI corresponding registered anatomical section $\left(R_{b}\right)$, and histological corresponding registered slice $\left(T_{b}\right)$ for the two mouse brains.

Discussion: The proposed method demonstrated its ability to automatically identify and register the best histological slice corresponding to the CEST image. These results enable the evaluation of the AAV co-localization between a CEST image and its ground truth in the brain. This generic method makes it possible to associate one or several ground truth histological slices to a limited brain region covered by the CEST image.

\section{References:}

[1] Gilad et al., "Artificial reporter gene providing MRI contrast based on proton exchange", Nature biotechnology, 25(2), 217-219, 2007.

[2] Ourselin et al., "Reconstructing a 3D structure from serial histological sections", Image and Vision Computing, 19(1-2), 25-31, 2001.

[3] Pluim et al., "Mutual information matching in multiresolution contexts“, Image and Vision Computing, vol 19, 45-52, 2001.

\section{S6.08.}

\section{ExploreASL GUI: processing large arterial spin labeling datasets without programming skills}

\author{
*M. Pasternak ${ }^{1}$, V. Keil ${ }^{2}$, P. Clement ${ }^{3}$, N. Pinter ${ }^{3,4}$, M. Dijsselhof ${ }^{2}$, \\ M. Stritt ${ }^{2,5}$, J. Petr ${ }^{2,6}$, H. J. Mutsaerts ${ }^{2}$ \\ ${ }^{1}$ University of Toronto, Institute of Medical Science, Toronto, CA; \\ ${ }^{2}$ Amsterdam University Medical Center, Department of Radiology \\ and Nuclear Medicine, Amsterdam, NL; \\ ${ }^{3}$ Ghent University, Department of Radiology and Nuclear Medicine, \\ Ghent, US; \\ ${ }^{4}$ Dent Institute, Buffalo, NY, US; \\ ${ }^{5}$ mediri GmbH, Heidelberg, DE; \\ ${ }^{6}$ Helmholtz Center Dresden-Rossendorf, Institute \\ of Radiopharmaceutical Cancer Research, Dresden, DE
}

Introduction: While several arterial spin labelling (ASL) processing pipelines are freely available, programming skills are usually needed to efficiently process large datasets (1), deterring clinicians from analyzing ASL data. Leaving data unanalysed reduces the speed of progress in medical research and this unavailability keeps research restricted to a select few specialized academic institutions. These hurdles are addressed by ExploreASL, an ASL processing pipeline that analyzes ASL data on an individual and population level, and is free for non-commercial purposes (2). To enable its use for researchers without any coding knowledge, a graphical user interface (GUI) was developed in collaboration with clinical radiologists to specifically address their needs (3).

Methods: This GUI wraps around ExploreASL (4), which can be downloaded for Linux, MacOS, and Windows (3), either as Matlab code, as compiled executable or as a Docker image. The GUI is divided into four steps (Fig. 1):

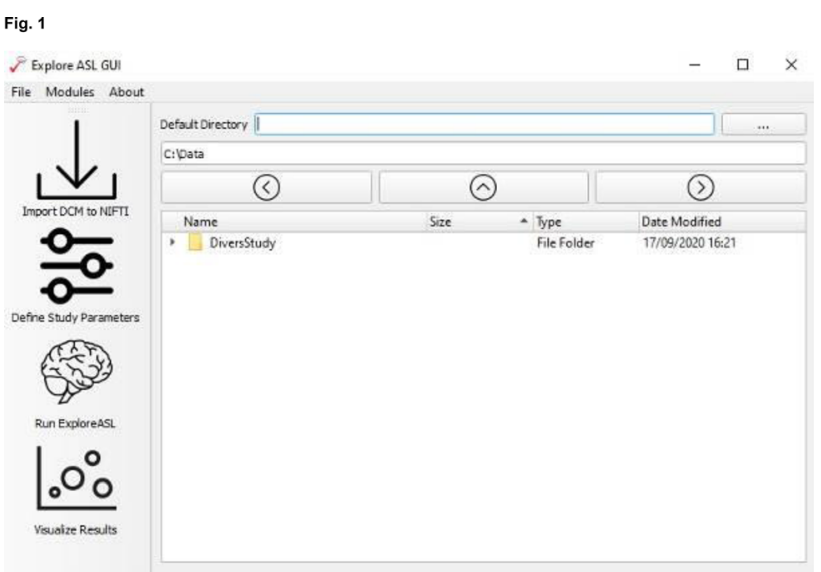

1. DICOM conversion

Step 1 will create directories needed for processing and save the data as NIfTI.

2. Defining Processing Settings

Step 2 allows for precise definition of study parameters (sequence type, labelling method, M0 map availability, etc.), subject exclusion, ASL sequence parameters, and options for processing methods and masks.

3. Running ExploreASL

Step 3 runs ExploreASL and its structural, ASL, and population processing modules. It can easily be parallelized to workstation capacities, and processing steps can be selected and deselected at will, as well as a data rerun.

4. Visualizing the Derived Data

Step 4, enables the user to create graphical presentations of the processed dataset, including options for different atlases, partial volume statistics, and parameters such as the coefficient of variation (Fig. 2). 


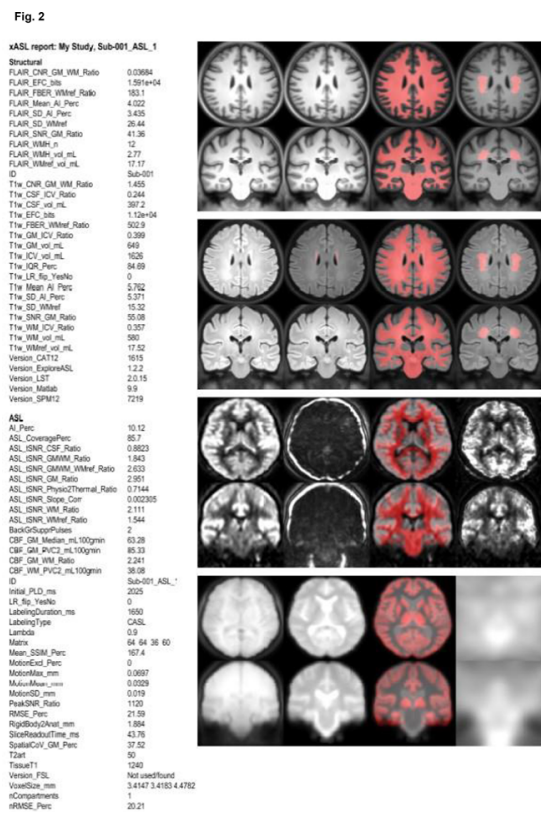

The GUI launches an offline dashboard application (Fig. 3). Users can select various plot types, interactively set variables of interest, subset the data, and receive hover feedback. Users may also click on data points to load in corresponding images.

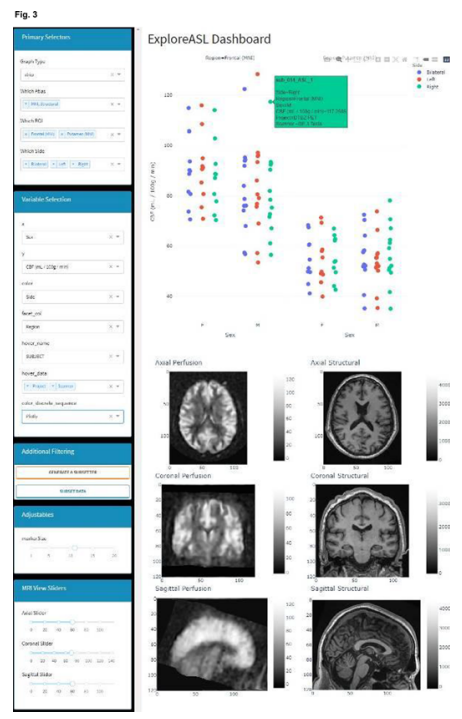

Results: First experience with two programming-naïve clinical radiologists showed that successful data processing could be achieved, supported by online tutorials and manuals. The GUI was considered user friendly after the online material had been studied. Data processing quality was comparable with standard processing in ExploreASL. The clinical users considered the GUI a game-changer for their scientific work.

Discussion: The ExploreASL GUI empowers clinicians to process their scientific ASL data. Thereby, it may democratize and accelerate medical research. The ExploreASL GUI is a multi-institution ongoing freeware project, developed by developers and users.

\section{References:}

1. https://www.osipi.org/task-force-1-1

2. Neuroimage. 2020 Oct 1;219:117031.

3. https://github.com/MauricePasternak/ExploreASL_GUI

4. https://github.com/ExploreASL/ExploreASL/releases/

\section{S6.09. \\ Correcting signal intensity bias in ${ }^{19} \mathrm{~F}$ MR imaging of inflammation by statistical modelling}

\author{
*L. Starke ${ }^{1}$, T. Niendorf ${ }^{1}$, S. Waiczies ${ }^{1}$ \\ ${ }^{1}$ Max Delbrück Center for Moleculare Medicine (Helmholtz. \\ Association), Berlin Ultrahigh Field Facility, Berlin, DE
}

Introduction: Labeling cells with ${ }^{19} \mathrm{~F}$ nanoparticles (NPs) continues to elicit interest for non-invasive ${ }^{19} \mathrm{~F}$ MRI of inflammation. We reported strong bias despite Rician Noise Bias Correction (RNBC) in conventional, magnitude reconstruction ${ }^{19} \mathrm{~F}$ MRI in a mouse model of multiple sclerosis (EAE). ${ }^{1}$ Here we propose a statistical model to correct this bias for quantitative applications of ${ }^{19} \mathrm{~F}$ MR.

Methods: We examine 11 in vivo datasets from 5 mice partially scanned on multiple days and ex vivo datasets from 5 different mice. PFCE nanoparticles were administered daily from 5d following EAE induction. $^{2}$

3D-RARE ${ }^{19}$ F-MRI: TR $=800 \mathrm{~ms}, \mathrm{TE}=4.4 \mathrm{~ms}, \mathrm{ETL}=40, \mathrm{FOV}=$ $(45 \times 16 \times 16) \mathrm{mm}^{3},(140 \times 40 \times 40)$ matrix. $32 \mathrm{~m}$ and $80 \mathrm{~m}$ (in vivo) or $128 \mathrm{~m}$ (ex vivo) scans were used as test and Reference data. Noise level $\sigma$ was estimated in a background region. Images were thresholded at SNR $=3.5$ and $<3$ connected signal voxel features were removed as outliers. $S_{\mathrm{t}}$ and $S_{\mathrm{r}}$ denote the test and Reference signal after RNBC. ${ }^{3}$

For true, but unknown signal $S^{*}$ and measured signal $S_{\mathrm{m}}$, the forward model is given by Rician distribution $\operatorname{Ri}\left(S_{\mathrm{m}} ; S^{*}, \sigma\right)$. We model $S^{*}$ as drawn iid from log-normal prior distribution $\operatorname{LN}\left(S^{*}, \theta\right)$ (Fig. 1A). Parameters $\theta$ are estimated separately for each image by maximizing the likelihood of the measured signal under the marginal distribution $\pi_{\sigma \theta}\left(S_{\mathrm{m}}\right) \propto \int \operatorname{Ri}\left(S_{\mathrm{m}} ; S^{*}, \sigma\right) \mathrm{LN}\left(S^{*}, \theta\right) \mathrm{d} S^{*}$ (Fig. 1B). We compute the corrected SI as the posterior mean following Bayes theorem (Fig. 1C). Estimation bias $S_{\mathrm{t}}-S_{\mathrm{r}}$ and $S_{\mathrm{c}}-S_{\mathrm{r}}$ is shown for single voxels and features.
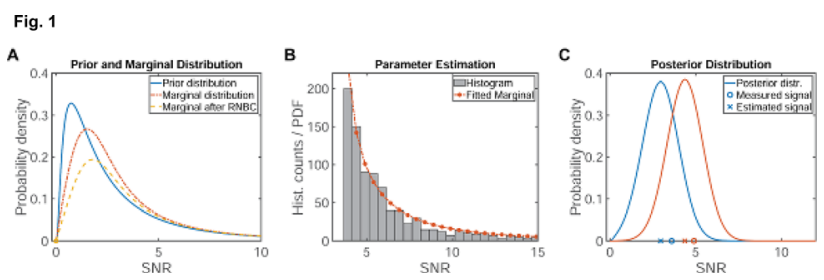

Results: In an example in vivo dataset, the SI is overestimated at all signal levels by on average $0.5 \sigma$ (Fig. $2 \mathrm{~A}$ ). The correction removes this bias without altering random spread. The same holds true when averaging over signal features. No difference between smaller and larger features is noticeable (Fig. 2B). In contrast to the original, the corrected image contains a balance of overestimated and underestimated voxels (Fig. 2C). 


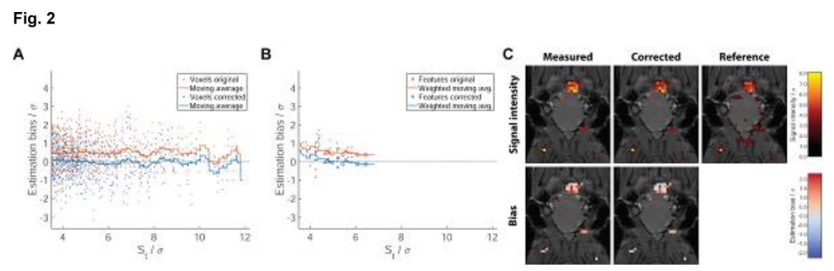

Averaged over all datasets the overestimation in uncorrected in vivo data is slightly larger than in the example (Fig. 3A/C). Again, the average bias in the corrected data is close to 0 . Analysis of the ex vivo data yields similar results (Fig. 3B/D).

Fig. 3
A
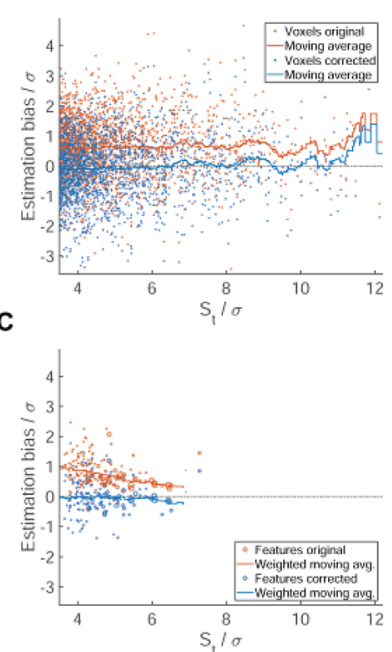

B
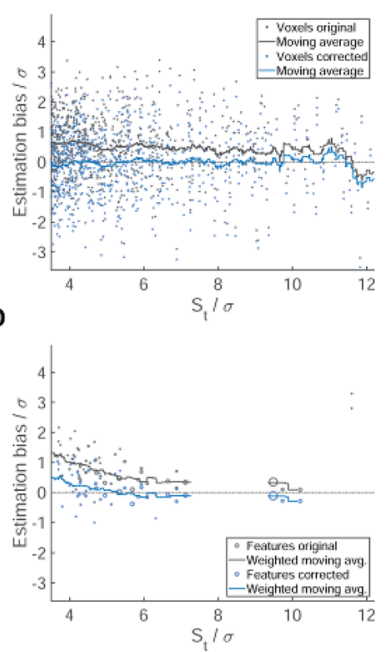

Discussion: Systematic overestimation in low SNR MRI of ${ }^{19} \mathrm{~F}-\mathrm{NPs}$ can be explained and corrected by assuming that SIs are drawn from a heavily skewed distribution, e.g. a log-normal. The effect size is below the random uncertainty for individual voxels, but overestimation in uncorrected data and effectiveness of model-based correction persist when averaging over ROIs. Thus, correction is essential for quantitative conclusions.

The correction can be applied without the need to change data acquisition protocols. It remains to be seen whether similar effects occur in other low SNR MRI applications, such as quantification of fluorinated drugs.

\section{References:}

1. Starke L, et al. Magn Reson Med. 2020

2. Waiczies S, et al. Scientific reports. 2017

3. Henkelman RM. Med Phys. 1985. 


\section{C1.01.}

\section{A recurrent inference machine framework to reduce the impact of residual misregistration in quantitative MRI}

\section{*E. Ribeiro Sabidussi ${ }^{1}$, S. Klein ${ }^{1}$, D. H. J. Poot ${ }^{1}$ \\ ${ }^{1}$ Erasmus Medical Center, Department of Radiology and Nuclear Medicine, Rotterdam, $N L$}

Introduction: In Quantitative MRI, parameters are estimated from weighted images that are often misaligned by patient motion. This induces errors in the quantitative maps. Routinely, an image registration step is performed prior to mapping, however, residual misalignment might remain. We propose a Recurrent Inference Machine (RIM) based method to estimate $\mathrm{T}_{2}$ maps while ignoring small misalignments in weighted images. The method is evaluated with simulated and in-vivo data.

Methods: Theory: A RIM is a neural network that learns an iterative optimization scheme to find a regularized solution to an inverse problem [1] (Fig. 1). Our RIM uses the gradient of the likelihood function $\mathrm{L}=(\boldsymbol{\kappa}, \sigma \mid \mathbf{S})$, given the signal model $\mathbf{S}=\mid \mathbf{A} \exp \left(-\tau_{\mathrm{n}} /\right.$ $\left.\mathbf{T}_{\mathbf{2}}\right) \mid+\eta(\sigma)$, where $\tau_{\mathrm{n}}$ is the $n^{\text {th }}$ echo time, and $\mathbf{\kappa}=\left(\mathbf{A}, \mathbf{T}_{\mathbf{2}}\right)^{\mathrm{T}}$, with $\mathbf{T}_{\mathbf{2}}$ the transverse relaxation time and $\mathbf{A}$ related to the proton density. $\eta(\sigma)$ is a Gaussian noise with standard deviation $\sigma$.

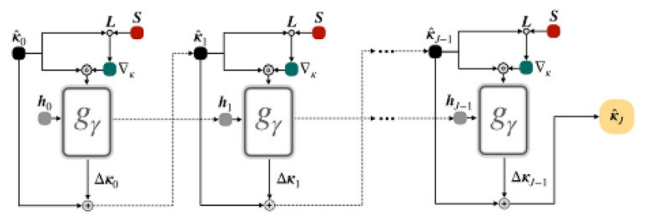

Figure 1: The RIM framework. The RNN portion of the framework, $\mathrm{g}_{\gamma}$, receives the current estimat $\hat{\kappa}_{j}$ and the gradient of $L\left(\nabla_{\kappa}\right)$ as (concatenated) inputs, and it outputs an update to the paramete estimate $\hat{\kappa}_{j}$ at each inference step $j$. Here, $J=6$, and initialization of $\hat{\kappa}$ and hidden states $h$, and the form of $\mathrm{g}_{\gamma}$, are the same as in $[2]$.

Datasets and evaluation: Simulated datasets are denoted $\mathrm{D}^{\mathrm{v}, \sigma}{ }_{\mathrm{d}}$, with $v$ the SNR and $\sigma_{d}$ the standard deviation of deformations: First, motion-free weighted images are created with the procedure described in [2], without acquisition noise. We then simulate motion through 2D translations, rotations and bicubic interpolation. Translation parameters are drawn from $\mathrm{N}\left(0, \sigma_{\mathrm{d}}\right)$ for each image in the set. The rotation parameter is defined as the number of pixels displaced at the border of the image, and is also sampled from $\mathrm{N}\left(0, \sigma_{\mathrm{d}}\right)$. These parameters are normalized to ensure zero-mean motion. Finally, noise is added, with same $\sigma$ as [2]. To train the RIM to ignore misalignments (mRIM), a motion-corrupted dataset was used, with $\sigma_{\mathrm{d}}=0.25$. To train the baseline method (aRIM), we set $\sigma_{\mathrm{d}}=0$.

Nine datasets $\mathrm{D}^{\mathrm{v}, \sigma} \sigma_{\mathrm{d}}$ were created: $\mathrm{D}^{5, \sigma}{ }_{\mathrm{d}}, \mathrm{D}^{25, \sigma}{ }_{\mathrm{d}}$, and $\mathrm{D}^{50, \sigma}{ }_{\mathrm{d}}$, with $\sigma_{\mathrm{d}}=\{0,0.25,0.5\}$. In each, 100 samples were generated with same ground-truth. The RMSE was used as measure of error and its distribution across the (masked) brain is presented. Additionally, the $\mathrm{T}_{2}$ dataset from [2], which was pre-registered but still slightly misaligned, was used to assess performance with in-vivo data.

Results: Figure 2 shows the evaluation with simulated data for different SNR and displacement levels. The proposed method mRIM has lower RMSE than the baseline aRIM for all cases except for dataset $\mathrm{D}^{25,0} . \mathrm{T}_{2}$ maps from the in-vivo dataset are shown in Fig. 3. The mRIM produced a smoother map than aRIM, with better distinction between tissues and shorter $T_{2}$ in vessels.

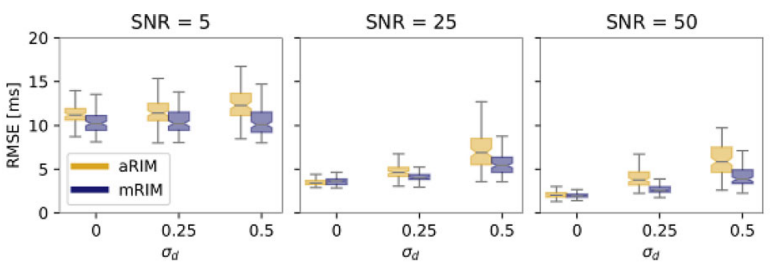

Figure 2: RMSE as function of SNR and $\sigma_{d}$. The boxplot shows the RMSE distribution across all brain pixels.
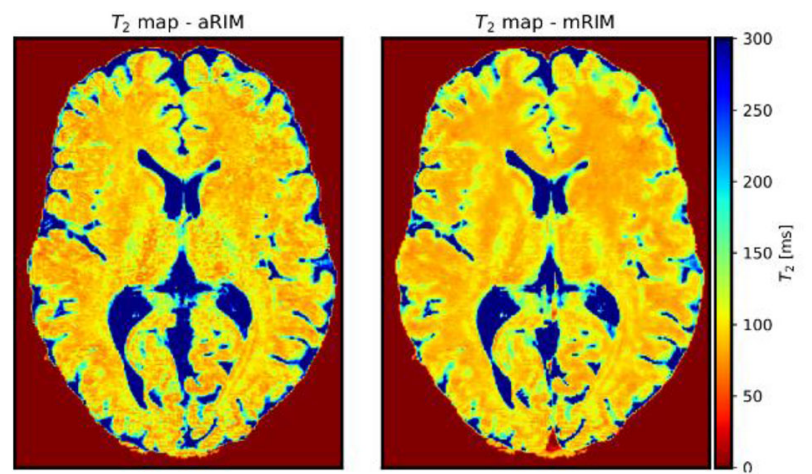

Figure 3: In-vivo $T_{2}$ mapping. Left) aRIM. Right) mRIM.

Discussion: We presented a novel method for MR relaxometry that is able to compensate small misalignment between images. Our method reduces the estimation error compared to a mapping-only baseline. Our results suggest that, with mRIM, small discrepancies between signal model and data can be compensated by the learned prior, improving the quality of estimates.

Acknowledgements: Research Grant: B-Q MINDED (EU H2020 MSCA ETN 764513).

\section{References:}

[1] Putzky et al. arXiv:1706.04008, 2017, 2017.

[2] Sabidussi et al. arXiv:1823.37832 20212021.

\section{C1.02.}

\section{Accelerated multi-shot diffusion weighted imaging with joint estimation of diffusion and phase parameters}

*B. Shafieizargar ${ }^{1}$, B. Jeurissen ${ }^{1}$, D. H. J. Poot $^{2}$, J. Van Audekerke ${ }^{3}$, M. Verhoye ${ }^{3}$, A. J. den Dekker ${ }^{1}$, J. Sijbers ${ }^{1,4}$

${ }^{1}$ University of Antwerp, imec-Vision Lab, Antwerp, BE;

${ }^{2}$ Erasmus Medical Center, Rotterdam, NL;

${ }^{3}$ University of Antwerp, Bio-Imaging Lab, Antwerp, BE;

${ }^{4}$ University of Antwerp, $\mu N E U R O$ Research Centre of Excellence, Antwerp, $B E$

Introduction: Even though multi-shot EPI has great potential for diffusion MRI (dMRI), its long acquisition time compared to singleshot EPI and its sensitivity to shot-to-shot phase inconsistencies limit its application [1]. We propose ADEPT, a framework for Accelerated Diffusion EPI with multi-contrast shoTs. ADEPT provides 1) accelerated dMRI by varying diffusion contrast settings for each shot in a multi-shot acquisition, introducing intra-scan modulation, 2) estimation of the diffusion tensor (DT) parameters directly from the k-q- 
space data, surpassing the intermediate image reconstruction step of conventional dMRI. To account for the phase mismatches between different shots, ADEPT estimates the phase map parameters for each shot jointly with the DT parameter maps. In a simulation study, the performance of ADEPT in terms of the RMSE is evaluated for different acceleration rates and compared to conventional two-step diffusion estimation.

Methods: Intra-scan modulated k-space data is considered, where each shot of a multi-coil ( $n_{c}$ coils), multi-shot $\left(n_{s}\right.$ shots) EPI acquisition is acquired with a different diffusion weighting and the phase map of each shot is assumed to vary linearly (due to rigid coherent bulk motion during the application of the diffusion sensitized gradients) [2]. The diffusion tensors, the complex-valued non-DW image, and the parameters of the linear phase maps of all shots are estimated jointly from the measured k-q-space data using a least-squares estimator (see Fig. 1), adopting a cyclic Block Coordinate Descent approach [3].

\begin{tabular}{|c|c|}
\hline $\boldsymbol{q}_{n, c} \in \boldsymbol{C}^{n_{k} \times 1}$ & K-space data of $\mathrm{n}^{\text {th }}$ shot and $\mathrm{c}^{\text {th }}$ coil \\
\hline$A_{n} \in\{0,1\}^{n_{k} \times n_{v}}$ & Binary sampling operator corresponding to $\mathrm{n}^{\text {th }}$ shot \\
\hline $\mathcal{F} \in C^{n_{v} \times n_{v}}$ & Fourier Transform operator \\
\hline $\boldsymbol{C}_{c} \in \boldsymbol{C}^{\boldsymbol{n}_{\boldsymbol{v}} \times n_{n_{p}}}$ & Diagonal matrix of the $\mathrm{c}^{\text {th }}$ coil sensitivity map \\
\hline$S_{0} \in C^{n_{v} \times 1}$ & Vectorized complex-valued non-DW image \\
\hline$b_{n} \in R^{6 \times 1}$ & Vectorized components of $3 \times 3$ symmetric DW b-matrix \\
\hline $\bar{D} \in R^{6 \times n_{v}}$ & Symmetric diffusion tensors of the vector of all voxels \\
\hline$\phi_{n} \in R^{n_{v} \times 1}$ & Phase map of the $\mathrm{n}^{\text {th }}$ shot \\
\hline
\end{tabular}

with $\overline{\boldsymbol{\theta}}=\left(\boldsymbol{\theta}_{1}^{T}, \ldots, \boldsymbol{\theta}_{n_{5}}^{T}\right)^{T} \in \boldsymbol{R}^{3 n_{s} \times 1}$ the phase map parameter vector of all shots and $\boldsymbol{\theta}_{n}=\left(\theta_{n 0}, \theta_{n 1}, \theta_{n 2}\right)^{T}$ the 3 parameters describing the linear phase map of the $\mathrm{n}^{\text {th }}$ shot.

Fig.1. Joint estimation of diffusion tensor and phase map parameters along with the non-DW image. The diffusion weighted signals of a multi-coil acquisition are modelled in the image space using the DTI model and coil sensitivity maps and transferred to k-space using the Fourier Transform operator. A binary operator is then applied to select specific k-q-space trajectories of each shot in the multi-shot acquisition.

Experiments: Monte Carlo simulations with 20 noise realizations $(\mathrm{SNR}=20)$ of the multi-contrast multi-shot data were performed. A fully sampled multi-channel dataset $\left(n_{c}=8\right)$ was simulated including $1 \mathrm{~b} 0$ and $60 \mathrm{DW}$ (b value $=1.15 \mathrm{~ms} / \mu \mathrm{m}^{2}$ ) images, using ground truth (GT) DT maps estimated from a real dMRI dataset and GT phase parameters drawn from zero-mean Gaussian distributions. This dataset was retrospectively sampled to generate 4-, 8- and 12-shot datasets. The estimation performance of ADEPT was compared to that of a conventional approach in which the diffusion tensor parameters for each voxel are estimated from SENSE reconstructed images of the individual shots (SENSE-re).

Results: Figure 2 shows the estimated Mean Diffusivity (MD) and Fractional Anisotropy (FA) maps for different acceleration factors. Figure 3 shows the RMSE maps of MD and FA estimated with SENSE-re and ADEPT.

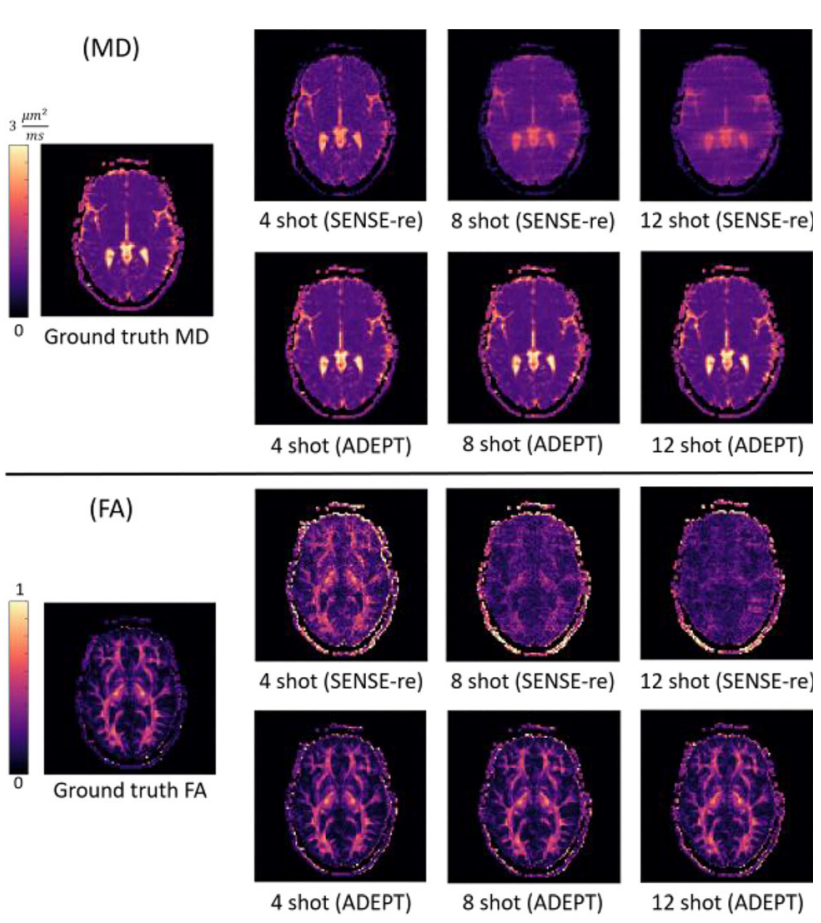

Fig.2. Estimated MD (top) and FA (bottom) maps for different acceleration factors. The ground truth values (left) are shown along with the SENSE-re and ADEPT results.

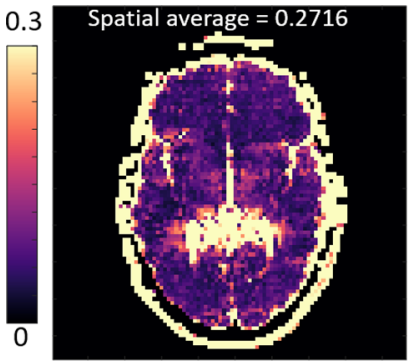

MD RMSE (SENSE-re )

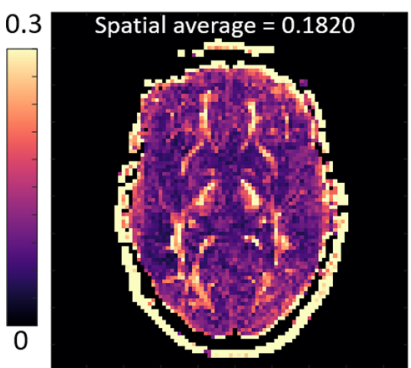

FA RMSE (SENSE-re)

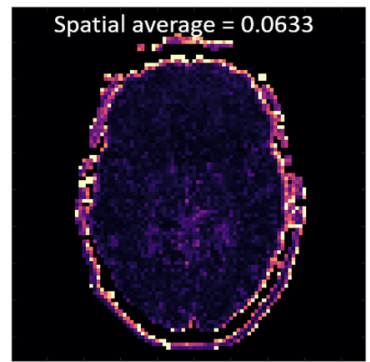

MD RMSE (ADEPT)

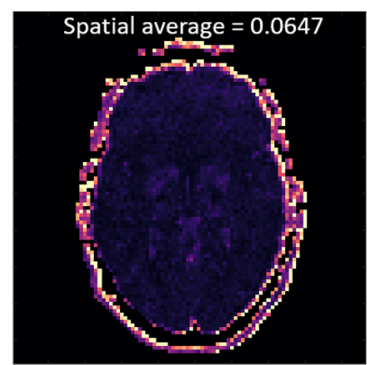

FA RMSE (ADEPT)
Fig.3. RMSE maps of MD (top) and FA (bottom) estimated with SENSE-re (left) and ADEPT (right) for an 8-shot accelerated dataset.

Discussion: Figures 2 and 3 reveal that ADEPT better preserves structural details at high acceleration rates and has a lower RMSE compared to SENSE-re. Further validation of ADEPT using in-vivo rat brain experiments is currently ongoing and we will present the corresponding results by the time of the conference.

Grant: B-Q MINDED (EU H2020 MSCA ETN 764513). 


\section{References:}

[1] M. Mani et al., MRM, 78 (2), 494-50, 2017.

[2] I. Rabanillo et al., Math. Vis., 226249, 19-27, 2019.

[3] A. Beck et al., SIAM J. Optim., 23(4), 2037-2060, 2013.

\section{C1.03.}

\section{High-resolution T2* mapping of the knee based on UTE Spiral VIBE MRI}

${ }^{*}$ C. Smekens ${ }^{1}$, Q. Beirinckx ${ }^{2}$, F. Vanhevel ${ }^{3}$, P. Van Dyck ${ }^{3}$, A. J. den Dekker $^{2}$, J. Sijbers ${ }^{2}$, T. Janssens ${ }^{1}$, B. Jeurissen ${ }^{2}$

${ }^{I}$ Siemens Healthcare NV/SA, Beersel, BE;

${ }^{2}$ University of Antwerp, imec-Vision Lab / Department of Physics,

Wilrijk, BE;

${ }^{3}$ University of Antwerp, Department of Radiology, Edegem, BE

Introduction: T2* mapping using ultra-short echo time (UTE) MRI enables quantitative evaluation of knee structures with short transverse relaxation times. ${ }^{1}$ However, acquisitions with low throughplane resolution are commonly used to obtain $\mathrm{T} 2 *$ maps within reasonable scan time, affecting the estimation accuracy due to partial volume effect. ${ }^{2}$ In this work, a model-based resolution-enhancing reconstruction (RER) method is used to obtain isotropic, high-resolution (HR) T2* maps of the knee from UTE Spiral VIBE MRI with low through-plane resolution within a reasonable scan time.

Methods: 3 volunteers were scanned on a 3 T MR scanner (Prisma Fit, Siemens Healthcare) with a 15-channel knee coil (QED) using 3 $\mathrm{T} 2 *$ mapping protocols based on the accelerated prototypical 3D UTE Spiral VIBE sequence (Table 1$){ }^{3}$ TEs were chosen close to the inphase TEs of water and fat. A longer TR was used for protocols B (Reference) and RER to include more and longer TEs. The RER protocol consisted of five acquisitions with low through-plane resolution rotated around the frequency-encoding direction (Fig. 1). For protocols $\mathrm{A}$ and $\mathrm{B}$, rigid registration was performed in Elastix ${ }^{4}$ and $\mathrm{T} 2 *$ values were estimated voxel-wise using constrained non-linear least-squares fitting of a mono-exponential T2* relaxation model. Model-based RER T2* mapping with joint inter-scan motion estimation was performed as a constrained and regularized non-linear least squares estimation (Fig. 1). ${ }^{5,6}$ Regions-of-interest (ROIs) were drawn in eight knee structures and verified by an MSK radiologist. Finally, medians and ranges of median ROI $\mathrm{T} 2 *$ values were computed.

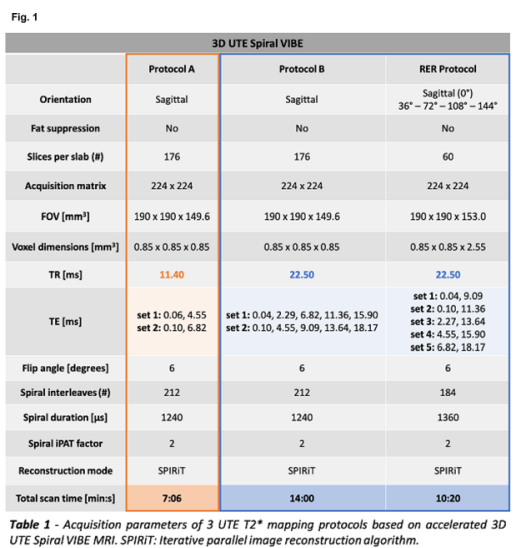

Fig. 2

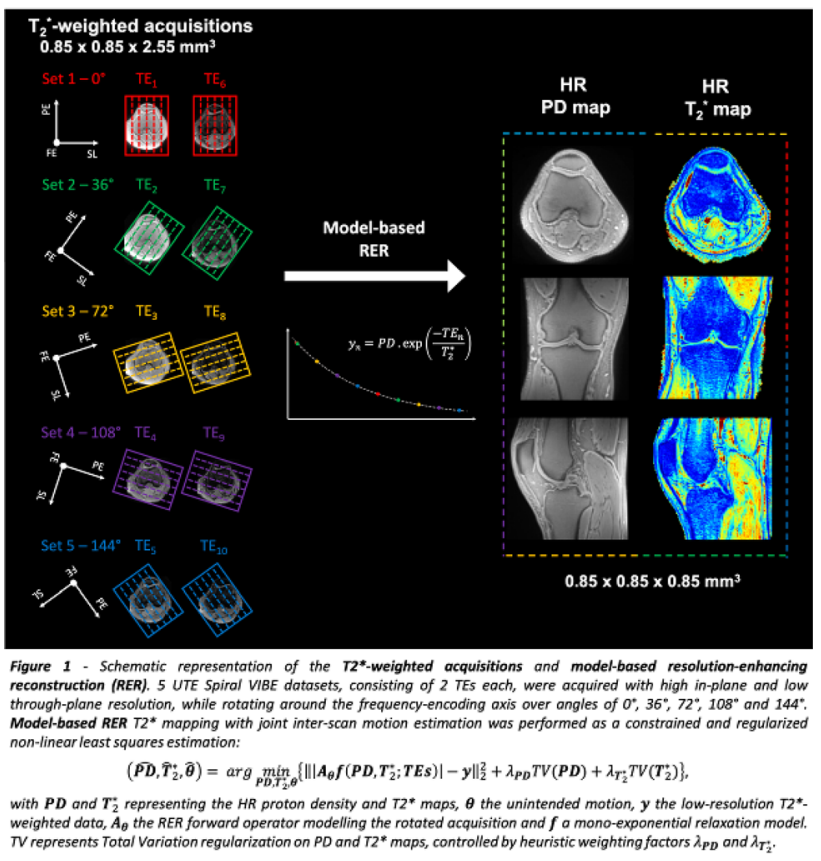

Results: Figure 2A shows representative T2* and PD maps. Protocol A displays the lowest T2* values. Protocols B and RER provide similar T2* maps. Fig. 2B displays the medians and ranges of median ROI T2* values from all subjects computed for all protocols. Median $\mathrm{T} 2 *$ values of protocols B and RER are comparable, while protocol A seems to underestimate $\mathrm{T} 2 *$ values in long $\mathrm{T} 2 *$ structures.

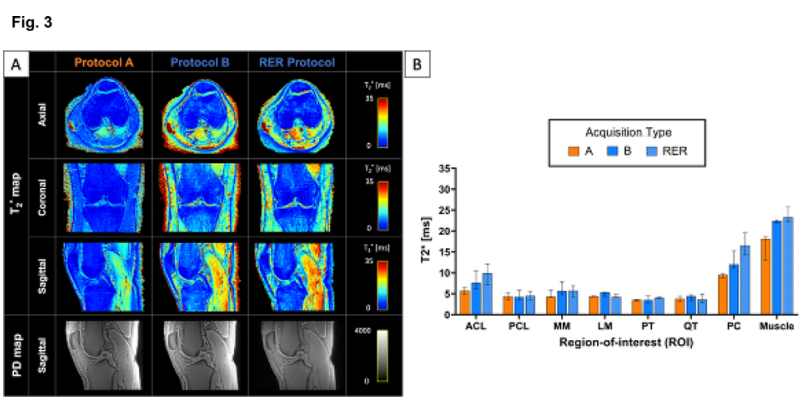

Figure 2-A: Representative $T 2^{*}$ and $P D$ maps from 1 volunteer estimated from 1 short (A) and 2 long (B and RER) $T 2^{*}$ mapping protocols. $B$ : Bar chort representing medians and ranges of the median $\mathrm{ROI} T 2^{*}$ values from all subjects, ROls were manually

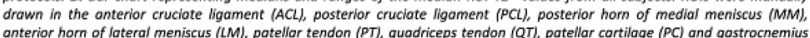
anterior horn of

Discussion: RER UTE T2* mapping provides T2* estimates that are comparable to $\mathrm{T} 2 *$ results obtained with direct 3D UTE T2* mapping, while requiring approximately $25 \%$ less acquisition time. RER UTE T2* mapping thus shows great promise for HR T2* mapping of knee structures within reasonable scan time.

Acknowledgements: Research Grant: B-Q MINDED (EU H2020 MSCA ETN 764513).

\section{References:}

${ }^{1}$ de Mello et al., AJR. 2019.

${ }^{2}$ Chu et al., OJSM. 2019.

${ }^{3}$ Qian et al., MRM. 2008.

${ }^{4}$ Klein et al., IEEE Trans Med Imaging. 2010.

${ }^{5}$ Beirinckx et al., Fundam. Inform. 2020.

${ }^{6}$ Beirinckx et al., Magn Reson Mater Phy. 2019. 


\section{C1.04.}

Outcome prediction of mild traumatic brain injury using support vector machine based on longitudinal MR diffusion imaging from CENTER-TBI

*M. Siqueira Pinto ${ }^{1,2}$, S. Winzeck ${ }^{3,4}$, S. Richter ${ }^{4}$, M. Correia ${ }^{5}$, E. Kornaropoulos ${ }^{4}$, D. Menon ${ }^{4}$, B. Glocker ${ }^{3}$, P. J. Guns ${ }^{6}$, A. J. den Dekker $^{2,7}$, J. Sijbers ${ }^{2,7}$, V. Newcombe ${ }^{4}$, P. Van Dyck ${ }^{1}$

${ }^{I}$ Antwerp University Hospital (UZA), Department of Radiology, Antwerp, BE;

${ }^{2}$ University of Antwerp, Department of Physics / imec-Vision Lab, Antwerp, BE;

${ }^{3}$ Imperial College London, BioMedIA Group / Department of Computing, London, GB;

${ }^{4}$ University of Cambridge, Division of Anaesthesia / Department of Medicine, Cambridge, GB;

${ }^{5}$ University of Cambridge, MRC Cognition and Brain Sciences Unit, Cambridge, GB;

${ }^{6}$ University of Antwerp, Department of Physiopharmacology, Antwerp, BE;

${ }^{7}$ University of Antwerp, $\mu N E U R O$ Research Center of Excellence, Antwerp, BE

Introduction: Mild traumatic brain injury (mTBI) accounts for over $85 \%$ of head injuries, affecting millions worldwide each year, with a third showing persistent functional impairment for months1. We investigated the applicability of Support Vector Machines (SVMs) for prediction of Glasgow Outcome Scale score [GOSE]-based outcome via multicenter longitudinal diffusion MRI.

Methods: Fifty-nine CENTER-TBI2 mTBI patients (15-70 years old, Glasgow Coma Scale (GCS): 13-15) with acute (MR1: 0-4 days post-injury) and subacute (MR2: 9-36 days post-injury) MR scan were analyzed. Patients were divided into groups with good $(\mathrm{n}=35$, 6 -months GOSE $=8)$ and poor $(\mathrm{n}=24$, GOSE $<8)$ outcome. T1w and DTI scans were acquired with similar protocols at 12 centers using $3 \mathrm{~T}$ scanners3. Mean diffusivity (MD) and fractional anisotropy (FA) maps were coregistered to $\mathrm{T} 1 \mathrm{w}$ and projected to a template space. FA, MD and meta-data (age, sex, GCS and injury mechanism) were simultaneously used to predict GOSE-based outcome. SVMs were trained on MR1, MR2, longitudinal MR (MR2-MR1) and/or meta-data via fourfold cross-validation. The $10 \%$ most discriminative WM voxels were identified in each fold. Voxels identified in at least 2 folds were mapped to the JHU atlas4. Relative voxel count was calculated as the percentage of discriminative voxels in the tracts relative to all voxels in the WM structure.

Results: MR1's FA and MD yielded highest mean accuracy $(74.4 \%$, Table 1), outperforming meta-data, longitudinal and subacute MR.

\section{Fig. 1}

Table 1. Average performance of 4 -fold cross-validation. Metrics displayed in $\%$ as mean \pm standard deviation.

\begin{tabular}{|c|c|c|c|c|}
\hline SVM input & Accuracy & Precision & Recall & F1 Score \\
\hline MR1 + meta-data & $74.4 \pm 18.8$ & $74.8 \pm 21.9$ & $71.9 \pm 19.4$ & $72.5 \pm 19.9$ \\
\hline MR2 + meta-data & $61.1 \pm 11.1$ & $63.7 \pm 17.8$ & $58.2 \pm 16.7$ & $57.3 \pm 10.9$ \\
\hline MR2-MR1 + meta-data & $66.0 \pm 8.6$ & $67.9 \pm 14.4$ & $62.0 \pm 12.8$ & $61.8 \pm 8.6$ \\
\hline Meta-data & $71.2 \pm 8.3$ & $70.8 \pm 8.2$ & $71.2 \pm 9.5$ & $70.6 \pm 8.3$ \\
\hline
\end{tabular}

Figure 1 depicts the discriminative voxels in WM, while Fig. 2 shows the voxel counts in identified tracts. Most predictive MR1 voxels were found in the corpus callosum, corona radiata and cerebral peduncles (FA) as well as the superior cerebellar peduncles (MD). The fornix showed discriminative FA and MD at all time points.

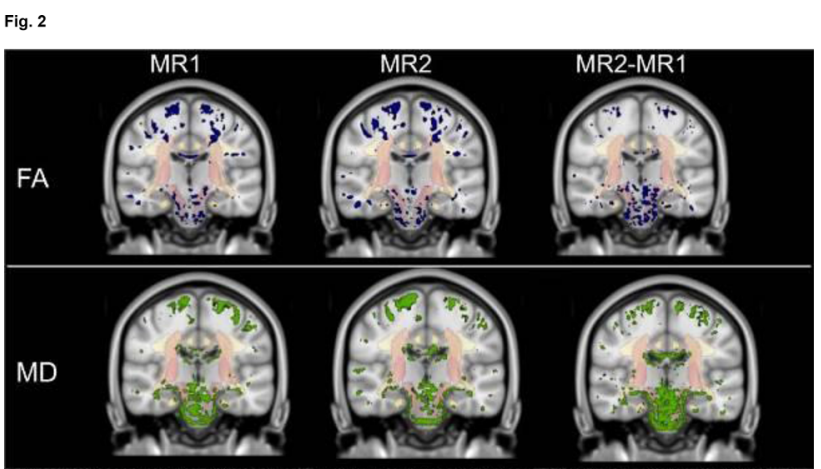

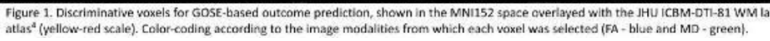

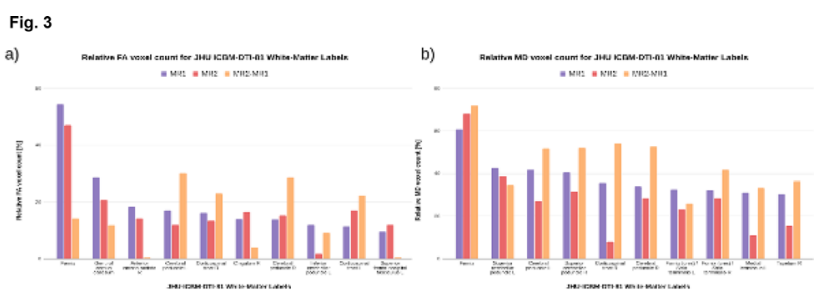

Figure 2. Relative voxel count representing the discriminative voxels trom the SVM analysis, separately shown for FA [a) and MD (b), for specific

Discussion: The SVM analysis predicted clinical outcome based on early diffusion MRI, achieving $74.4 \pm 18.8 \%$ accuracy, supporting the selection of mTBI patients for clinical follow-up. Our results suggest that combining meta-data with acute MR provides better GOSE-based outcome prediction than meta-data combined with either subacute or longitudinal MR, or meta-data only. The cross-sectional findings are coherent with reported analysis 1 . The longitudinal SVM exhibits lower prediction metrics, suggesting that subtraction might not be the most efficient way to exploit longitudinal information. Regions identified by the SVMs were previously reported as structures relevant for outcome after mTBI5. This multicenter diffusion study indicates the potential of GOSE-based outcome prediction using acute MRI.

Grants: B-Q MINDED (EU H2020 MSCA ETN 764513) and CENTER-TBI (EC 602150).

\section{References:}

1-Richter et al., JAMA. 2021; 4(3), e210994.

2-Maas et al., Neurosurgery. 2015;76(1):67-80.

3-CENTER-TBI https://www.center-tbi.eu/project/mri-study-proto cols

4-Mori et al., Neuroimage. 2008; 40(2), 570-582.

5-Wallace et al., Neurosci \& Biobehav Reviews. 2018;92, 93-103.

\section{C1.05.}

Optimal experimental design for the T2-weighted diffusion kurtosis imaging free water elimination model

*V. Anania ${ }^{1,2}$, B. Jeurissen ${ }^{2,3}$, J. Morez $^{2}$, A. E. Buikema ${ }^{2}$, T. Billiet ${ }^{1}$, J. Sijbers ${ }^{2,3}$, A. J. den Dekker ${ }^{2,3}$

${ }^{1}$ University of Antwerp, imec-Vision Lab (IcoMetrix), Leuven, BE;

${ }^{2}$ University of Antwerp, imec-Vision Lab, Antwerp, BE; 
${ }^{3}$ University of Antwerp, $\mu$ NEURO Research Centre of Excellence, Antwerp, BE

Introduction: The two-compartment free water elimination (FWE) diffusion model [1] allows to account for partial volume effects in brain tissue voxels affected by free water contamination. The illconditionedness of the FWE model can be mitigated by explicitly incorporating in the model the $\mathrm{T} 2$ relaxation properties of the tissue and free water compartments [2], $\mathrm{T}_{2}{ }^{\text {tissue }}$ and $\mathrm{T}_{2}{ }^{\mathrm{fw}}$ respectively. By representing the tissue compartment signal with the diffusion kurtosis imaging (DKI) model, we obtain the T2-DKI-FWE model. Due to acquisition time constraints in the clinical practice, the choice of the acquisition scheme remains crucial. Therefore, in this work, we show the potential benefits of using a data acquisition protocol with optimized $\mathrm{b}$ values $(b)$ and echo times (TE).

Methods: The acquisition settings, namely $b$ and TE, were optimized in terms of the Cramér-Rao lower bound (CRLB) variances of the model parameters [3]. During the optimization procedure, the number of uniformly distributed gradient directions was fixed to 120 while $b$ and TE were free to assume any values in the range $0-2 \mathrm{~ms} / \mu \mathrm{m}^{2}$ and $63-121 \mathrm{~ms}$, respectively. Ground truth values for the model parameters were obtained by fitting the T2-DKI-FWE model to a real dataset. Subsequently, 500 white matter voxels were selected with an estimated free water signal fraction $(f)$ between 0.05 and 0.3 . As an optimality criterion, we minimized a weighted sum of the CRLB variances of the model parameters assuming Gaussian distributed data and, in our experiments, $\mathrm{T}_{2}{ }^{\mathrm{fw}}$ was fixed to $1573 \mathrm{~ms}$.

Results: In Fig. 1, a conventional acquisition scheme with fixed $b$-TE combinations is compared to the scheme resulting from the optimization procedure as well as to a more practical clustered version of the optimized scheme. Figure 2 shows that, compared to the conventional scheme, the clustered optimized scheme leads to a higher attainable precision for the diagonal elements of the diffusion tensor and for all kurtosis tensor elements. However, the conventional scheme results in a lower CRLB of $\mathrm{T}_{2}{ }^{\text {tissue }}$.
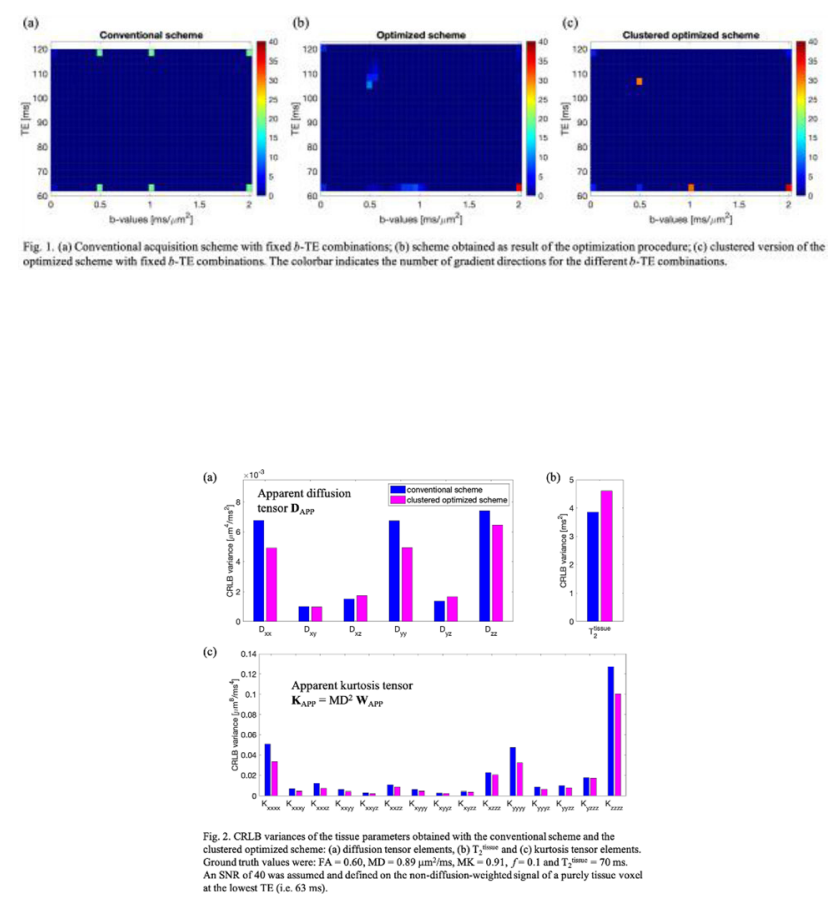

Finally, we assessed the sample variance of fractional anisotropy (FA), mean diffusivity (MD) and mean kurtosis (MK) in single-voxel experiments for different signal-to-noise ratio (SNR) values and Gaussian distributed data: for all 3 metrics, extracted using an unconstrained non-linear least squares estimator, the clustered optimized scheme outperformed the conventional one (Fig. 3).
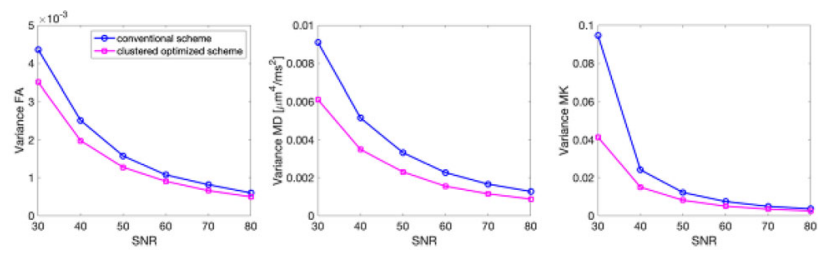

Fig. 3. Sample variances of FA, MD and MK for both the conventional and the clustered optimized scheme and different SNR levels,

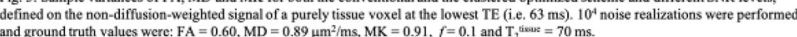

Discussion: This study points out that, given a fixed acquisition time, a higher precision of the DKI parameter estimates and derived metrics can be achieved by using CRLB-optimized $b$-TE combinations. Lower SNR ranges can be addressed by introducing constraints in the fitting procedure.

\section{References:}

[1] Pasternak et al., https://doi.org/10.1002/mrm.22055

[2] Collier et al., ISMRM 2017, p. 1783.

[3] Poot et al., https://doi.org/10.1109/TMI.2009.2037915

Research Grants: B-Q MINDED (EU H2020 MSCA ETN 764513), FWO Belgium (G084217N, 12M3116N).

\section{C1.06.}

\section{B0-inhomogeneity correction for accelerated GRASE scans based on the calibration region}

\author{
*R. Byanju ${ }^{1}$, S. Klein ${ }^{1}$, A. Cristobal-Huerta ${ }^{1}$, J. A. Hernandez- \\ Tamames $^{1}$, D. H. J. Poot ${ }^{1}$ \\ ${ }^{1}$ Erasmus Medical Center, Rotterdam, NL
}

Introduction: GRASE allows acceleration of MR acquisitions by acquiring gradient echoes next to a spin echo (SE) ${ }^{1}$. However, the standard GRASE acquisition and reconstruction causes some imaging artifacts. In earlier work $^{2}$, we showed that by explicitly modeling the gradient echoes and SEs in a 3D-GRASE scan in a subspace constrained reconstruction (SCR) method, we could reconstruct images with less artifacts from highly undersampled GRASE acquisition. Figure 1 shows the difference between the conventional and proposed GRASE scans. However, with realistic B0 variations the required number of subspace components is large, limiting the acceleration factor. Here, we propose compensation of the majority of the B0 variation based on a calibration region, allowing a reduction of the number of components in the SCR and hence higher acceleration factor. We compare the performance of the proposed compensation technique to B0-inhomogeneity correction in conventional GRASE scans. 


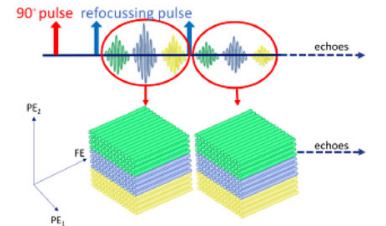

(a) Conventional GRASE (GRASE-CONV) scans where the gradient echoes and spin echoes are used to sample the same k-space for every refocussing pulse. The images can be reconstructed using pulse. The images can be reconstructed using
inverse Fourier transform. Conventional B0-inhomogeneity correction is applied to each frequency encoding (FE) line.

Figure 1: Two strategies for GRASE scans shown for GRASE factor of 1. Each cylinder represents a FE line. Gradient echoes are shown with green and yellow colour and spin echo by blue.

Methods: A fully sampled scan of ISMRM model $130^{3}$ phantom was performed using a $3 \mathrm{~T}$ GE Discovery MR750 scanner with scan settings as in ${ }^{4}$, except for repetition time set to $1800 \mathrm{~ms}$ due to SAR limits. The total scan time was $5 \mathrm{~h}: 23 \mathrm{~m}$. In this scan, every k-space position was sampled for every echo allowing retrospective generation of both the conventional GRASE-CONV and proposed GRASESCR. For GRASE-CONV B0-inhomogeneity correction was applied to each frequency encoding line using the reference echo $\operatorname{train}^{1}$. GRASE-SCR used a pseudo-random sequence generated by the Halton sequence with a $12 \times 12$ fully sampled central region (FSCR). We use the FSCR to generate low-resolution images by direct reconstruction. Coil combined images $\mathrm{S}_{-} \mathrm{CAL}_{\mathrm{j}, \mathbf{x}}$ where $\mathbf{x}$ is spatial index and $\mathrm{j}$ indexes echoes, were generated using coil maps drived using the ESPIRiT technique as implemented in the BART toolbox ${ }^{5}$. Using the phase $\Phi_{\mathrm{j}, \mathbf{x}}=\angle \mathrm{S}-\mathrm{CAL}_{\mathrm{j}, \mathbf{x}}$, which accounts for the majority of the phase changes due to $\Delta \mathrm{B} 0$, we reconstruct $S-R M_{j, x}$ with the $\mathrm{SCR}^{2}$ where $\mathrm{S}-\mathrm{REM}_{\mathrm{j}, \mathbf{x}}=\mathrm{S}_{\mathrm{j}, \mathbf{x}} \Phi_{\mathrm{j}, \mathbf{x}}$. As $\mathrm{S}-\mathrm{REM}_{\mathrm{j}, \mathbf{x}}$ has lower range of $\Delta \mathrm{B} 0$ than that of $\mathrm{S}_{\mathrm{j}, \mathbf{x}}$, reconstruction requires less number of subspace components.

Results/discussion: Figure $2 \mathrm{a}-\mathrm{c}$ shows comparison between $\Delta \mathrm{B} 0$ estimated from calibration region and the actual $\Delta \mathrm{B} 0$. Figure $2 \mathrm{~d}$ shows the energy in each components after singular value decomposition for the range of $\Delta \mathrm{B} 0$ expected with and without compensation. Figure 3 a shows the comparison between GRASECONV and GRASE-SCR (first SE) where the blurring effect seen in GRASE-CONV is not present in GRASE-SCR, showing it can produce better images than GRASE-CONV. Figure $3 b$ compares results from fully sampled fast SE scan and accelerated GRASE-SCR where the difference is mostly contained in the first echo while the rest of the images are similar showing potential for acceleration.

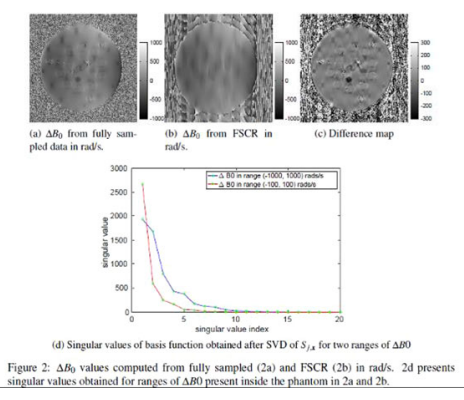

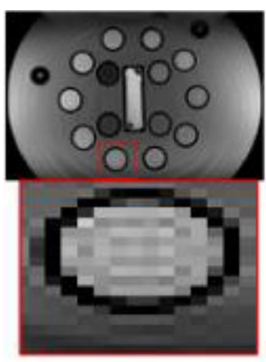
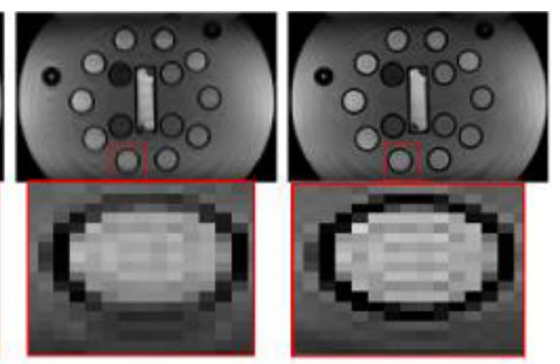

$(\mathbf{a})$
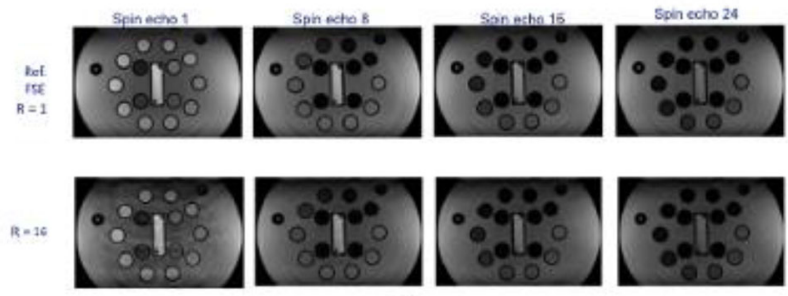

Figure 3: (a) Left: first echo of the fast spin echo re constructed using inverse Fourier transform. Center. first echo of GRASE-CONV. Right: first spin echo of the GRASE-SCR with $R=3$. (b) Comparison between reference fast spin echo and GRASE-SCR for $R=16$ for echoes $1,8,16$ and 24 . The vertical direction is phase encoding direction while horizontal direction is frequency encoding direction.

\section{Research Grant: EU H2020 MSCA ETN 764513}

${ }^{1}$ Cristobal-Huerta et al., MRM 48, 2018.

${ }^{2}$ Byanju et al., proc. ESMRMB 2020.

${ }^{3}$ Jiang et al., MRM 78, 2017.

${ }^{4}$ Piredda et al., MRM 85, 2021.

${ }^{5}$ Uecker et al., proc. ISMRM Workshop 2016.

\section{C1.07.}

\section{Rotated or shifted sets of multi-slice MR images for super-resolution reconstruction? A Bayesian answer}

${ }^{*}$ M. Nicastro ${ }^{1}$, B. Jeurissen ${ }^{1,2}$, Q. Beirinckx ${ }^{1}$, C. Smekens ${ }^{3}$, D. H. J. Poot $^{4}$, J. Sijbers ${ }^{1,2}$, A. J. den Dekker ${ }^{1,2}$

${ }^{1}$ University of Antwerp, Department of Physics / imec-Vision Lab, Antwerp, BE;

${ }^{2}$ University of Antwerp, $\mu$ NEURO Research Centre of Excellenc, Antwerp, BE;

${ }^{3}$ Siemens Healthcare NV/SA, Beersel, BE,

${ }^{4}$ Erasmus Medical Center, Department of Radiology and Medical Informatics, Rotterdam, $N L$

Introduction: In clinical MRI, direct acquisition at isotropic high resolution (HR) and with high signal-to-noise ratio (SNR) is often infeasible due to the long scan time required. Multi-slice super-resolution reconstruction (MS-SRR) can reduce this limitation by reconstructing an HR 3D isotropic image from a series of MS images with an anisotropy factor (AF, defined as the ratio between image slice thickness and in-plane resolution) exceeding $1^{1}$. The multi-slice images are conventionally acquired either with parallel orientations, shifted by different sub-pixel distances in the through-plane direction ${ }^{2}$ or rotated around the phase encoding axis by different angles ${ }^{3}$. In this simulation study, both MS-SRR acquisition protocols were compared using the Bayesian Mean Squared Error ${ }^{4}$ (BMSE) of the maximum a posteriori (MAP) estimator as performance criterion.

Methods: A Gaussian Markov random field ${ }^{5}$ prior distribution was adopted for the HR image to be reconstructed and its hyperparameters 
were estimated from a training dataset of realistically simulated T2-w brain images $^{6}$ (Fig. 1). The bias and variance components of the BMSE were calculated separately to allow evaluation of both accuracy and precision. The acquisition schemes were compared for different combinations of $\mathrm{AF}$ and number of acquired MS images, while keeping the scan time and the spatial resolution of the reconstructed image fixed for all protocols. An isotropic HR MS acquisition protocol $(\mathrm{AF}=1)$ requiring the same scan time was additionally used as a reference. The results of the BMSE analysis were validated numerically by means of Monte Carlo simulations. Bias, standard deviation and root MSE (RMSE) maps were estimated for each protocol from 100 noise realizations.

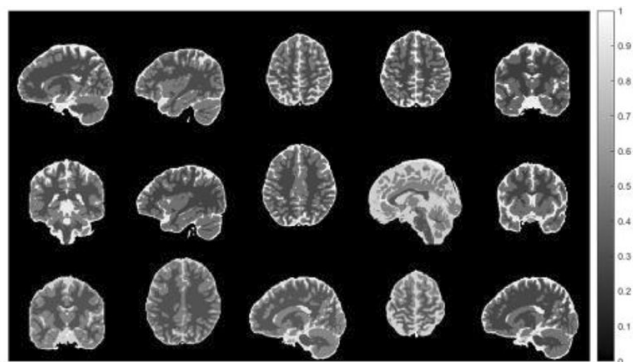

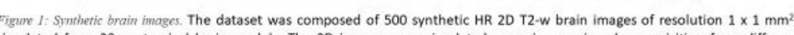
smutem 20 anatomical brain models. The $2 D$ images were simulated assuming a spin-echo acquisition from different acquisition planes (sagittal, transverse and coronal), and with different T2 contrasts

Results: Results (Fig. 2, Fig. 3) show that MS-SRR based on rotated multi-slice images outperforms MS-SRR based on shifted multi-slice images in terms of accuracy and precision of the reconstructed image. Compared to the HR reference, MS-SRR based on rotated multi-slice images shows a significant improvement in precision, especially for higher $\mathrm{AF}$, at the cost of a slight increase in bias.

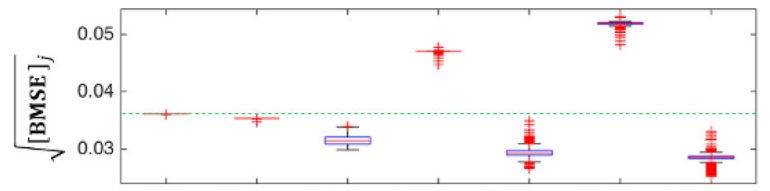

HR SRsh $_{\mathrm{AF} 2}$ SRrot $_{\mathrm{AF} 2} \mathrm{SRsh}_{\mathrm{AF} 4}$ SRrot $_{\mathrm{AF} 4} \mathrm{SRsh}_{\mathrm{AF} 6}$ SRrot $_{\mathrm{AF} 6}$

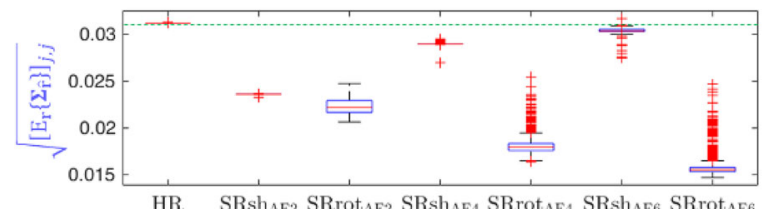

HR SRsh $_{\mathrm{AF} 2}$ SRrot $_{\mathrm{AF} 2}$ SRsh $_{\mathrm{AF} 4}$ SRrot $_{\mathrm{AF} 4}$ SRsh $_{\mathrm{AF} 6}$ SRrot $_{\mathrm{AF}}$

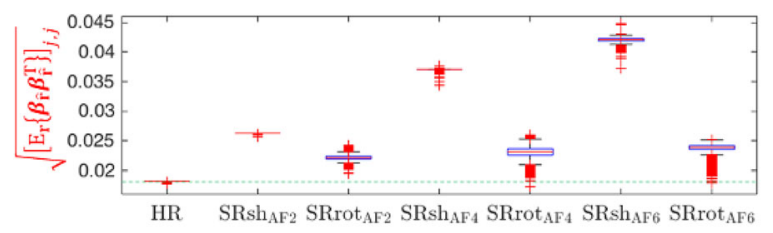

$$
\begin{array}{rlr}
{[\text { BMSE }]_{j}=\left[\mathrm{E}_{\mathbf{r}, \tilde{s}}\left\{(\hat{\mathbf{r}}-\mathbf{r})(\hat{\mathbf{r}}-\mathbf{r})^{\mathrm{T}}\right\}\right]_{j, j}} & \boldsymbol{\Sigma}_{\hat{\mathbf{r}}}=\operatorname{cov}(\hat{\mathbf{r}}) \\
=\left[\mathrm{E}_{\mathrm{r}}\left\{\mathrm{\Sigma}_{\hat{\mathbf{r}}}\right\}\right]_{j, j}+\left[\mathrm{E}_{\mathbf{r}}\left\{\boldsymbol{\beta}_{\hat{\mathbf{r}}} \beta_{\hat{\mathbf{r}}}^{\mathrm{T}}\right\}\right]_{j, j} & \boldsymbol{\beta}_{\hat{\mathbf{r}}}=\operatorname{bias}(\hat{\mathbf{r}}) \\
\text { Precision term Accuracy term } &
\end{array}
$$

Figure 2: BMSE results. $\mathbf{r}$ : ground truth image. $\tilde{\mathbf{s}}$ : acquired LR images. $\hat{\mathbf{r}}$ : SRR MAP estimate. HR: isotropic high-resolution multi-slice acquisition (AF=1). SRsh: SR protocol where the LR images were simulated uniformly shifted in the slice-encoding axis direction. SRrot: SR protocols where the LR images were simulated rotated around the phase-encoding axis, with rotation angles uniformly distributed. The AF value is reported as subscript for each SR protocol.

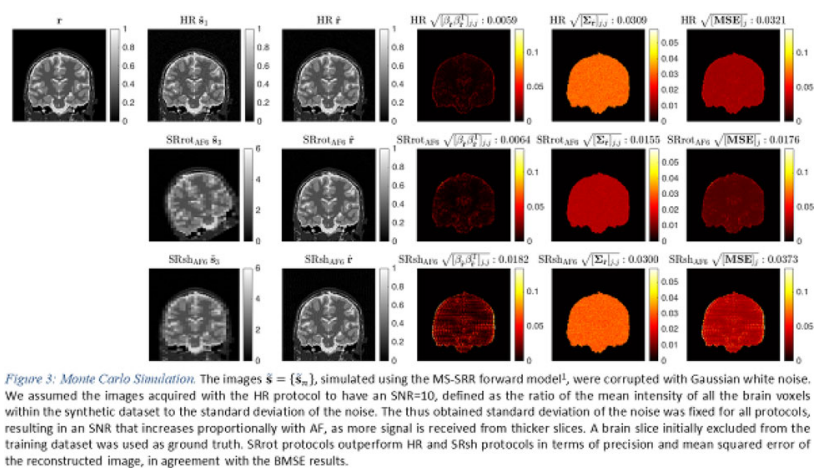

Discussion: The difference in terms of BMSE suggests that adopting the rotated acquisition scheme in an MS-SRR experiment can lead to a substantially reduced scan time compared to the shifted acquisition scheme and the reference scheme, while preserving the same MSE of the estimated SRR image. Validation of the BMSE results on real data will be subject of future work.

Research Grant: B-Q MINDED (EU H2020 MSCA ETN 764513).

\section{References:}

1. Poot, D.H.J. et al. MICCAI (2010), pp. 615-622.

2. Greenspan, H. et al. Magn Reson Imaging, 20(5) (2002), pp. 437-446.

3. Shilling, R.Z. et al. IEEE ICIP (2008), pp. 2240-2243.

4. Van Trees, H.L. (2001). John Wiley \& Sons, Inc.

5. Bardsley, J. IPI, 7(2) (2013), pp. 397-416.

6. http://www.bic.mni.mcgill.ca/brainweb/

\section{C1.08.}

\section{Decoding multiple sclerosis EDSS disability scores from MRI using deep learning}

*R. Paolella ${ }^{1,2}$, E. de la Rosa ${ }^{1,3}$, D. M. Sima ${ }^{1}$, D. Dive ${ }^{4}$, F. DurandDubief $^{5}$, D. Sappey-Marinier ${ }^{5}$, B. Jeurissen ${ }^{2}$, J. Sijbers ${ }^{2}$, T. Billiet ${ }^{1}$

${ }^{1}$ University of Antwerp, imec-Vision Lab (IcoMetrix), Leuven, BE;

${ }^{2}$ University of Antwerp, imec-Vision Lab, Antwerp, BE;

${ }^{3}$ Technical University of Munich, Department of Computer Science, Munich, BE;

${ }^{4} \mathrm{CHU}$ de Liège, Liège, BE;

${ }^{5}$ University of Lyon, Biomedical Imaging Research Lab, Lyon, FR

Introduction: Multiple sclerosis (MS) is an autoimmune disease that affects the brain and spinal cord. Symptoms of MS are various and can be of different severity. There is currently no cure for MS, but many treatments can slow down the symptoms' progress, resulting in less disability over time.

Disease progression monitoring and clinical decision making often rely on the expanded disability status scale (EDSS). Unfortunately, EDSS is manually assessed and suffers from poor reliability, repeatability, and high inter-rater variability. Therefore, automatic and objective EDSS scoring using MRI information could potentially help to monitor disease progression reliably and optimize treatment. This work is the first step towards the prediction of future EDSS in MS patients from MRI.

Methods: We used multi-center and multi-scanner FLAIR and T1 MRI data from 316 MS patients. EDSS score was established within 89 days before or after the MRI scan for each patient. The distribution of EDSS was strongly unbalanced with 240 patients belonging to the 
class of EDSS $\leq 4$. Icobrain [1] was used to preprocess the images and obtain the volumetric quantification of grey matter, white matter, whole brain, lateral ventricles, T1 hypointense and FLAIR hyperintense lesions.

EDSS was automatically scored with three different approaches: 1) a random forest regression (RF) model based on the brain volumes; 2) a deep learning (DL) model based on pure MRI images with the two modalities T1 and FLAIR as channels; and 3) a DL model based on the combination of pure MRI and brain volumes. The DL model used consists of 4 consecutive 3D convolutional blocks, followed by 2 fully connected layers.

The performance was assessed using the mean squared error (MSE), mean absolute error (MAE) and Pearson $\rho$ correlation computed on the concatenated results of the test folds in a fivefold cross-validation experiment. In addition, the accuracy and the percentage of correct binary classification were calculated considering 2 classes split according to an EDSS threshold of 4.

Results: The results of the proposed automatic EDSS estimation methods are summarised in Table 1 .

\section{TABLE 1}

\begin{tabular}{lcllll}
\hline & Methods & MSE & MAE & $\rho$ & Accuracy \\
\hline 1. & $\begin{array}{c}\text { Deep Learning } \\
+\end{array}$ & 2.19 & 1.12 & 0.57 & 0.85 \\
& $\quad \begin{array}{l}+ \\
\text { Brain biomarkers }\end{array}$ & & & & \\
\hline 2. & Deep Learning & 2.3 & 1.16 & 0.53 & 0.83 \\
\hline 3. & Random Forest Regression & 2.24 & 1.18 & 0.54 & 0.78 \\
\hline
\end{tabular}

Overall, these results indicate a slight improvement in the EDSS decoding performance of the DL + biomarkers model compared to the other two methods.

The percentages of patients with correct classification per method using the EDSS threshold 4 are shown in Fig. 1.

$$
\text { Fig. } 2
$$

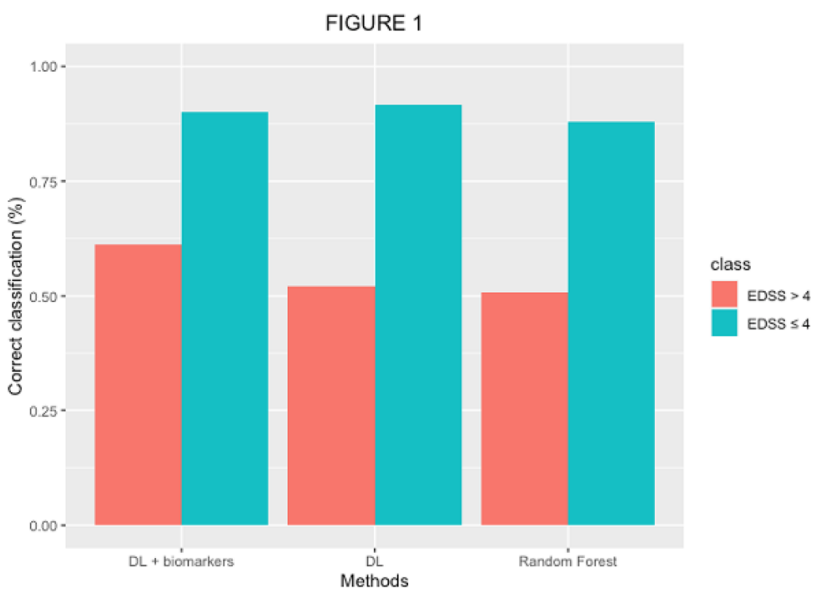

Discussion: These findings suggest that machine learning methods are, to a certain extent, able to learn relations between MRI-based brain volumes and clinical disability measured by EDSS. MRI-based EDSS score might also capture complementary information on disease activity compared to the clinically measured EDSS. Understanding such differences is a prerequisite to predicting future disability progression in MS.

\section{Reference:}

[1] Smeets D., et al., https://doi.org/10.1002/brb3.518.

Research Grant: EU H2020 MSCA ETN 764513 (B-Q MINDED) and 765148 (TRABIT).

\section{C1.09.}

Water content mapping employing super-resolution reconstruction with acquisitions in three orthogonal orientations

*D. Thomas ${ }^{1,2}$, A. M. Oros-Peusquens ${ }^{1}$, D. H. J. Poot ${ }^{3}$, N. J. Shah ${ }^{4,1,5,6}$

${ }^{1}$ Jülich Research Centre, Institute of Neuroscience and Medicine (INM-4), Jülich, DE;

${ }^{2}$ RWTH Aachen University, Aachen, DE;

${ }^{3}$ Erasmus Medical Center, Department of Radiology and Nuclear Medicine, Rotterdam, NL;

${ }^{4}$ Jülich Aachen Research Alliance (JARA), Department

of Translational Brain Medicine, Aachen, DE;

${ }^{5}$ Jülich Research Centre, Institute of Neuroscience and Medicine (INM-11), Jülich, DE;

${ }^{6}$ RWTH Aachen University, Department of Neurology, Aachen, DE

Introduction: Tissue water content is tightly regulated in the healthy brain, and even small changes are indicators of pathology [1]. Techniques to precisely measure water content in the human brain with MRI have been previously developed. Short, clinically relevant, 2D acquisitions for water content mapping have been proposed [2] but using non-isotropic voxels and slice gaps for whole-brain coverage. We aim to use 2D imaging while also achieving high isotropic resolution by combining data from several short scans using superresolution reconstruction (SRR) [3].

Methods: For Super-resolution reconstruction- water content (SRR$\mathrm{H}_{2} \mathrm{O}$ ) mapping (SRR- $\mathrm{H}_{2} \mathrm{O}$ ), three low resolution (LR), multiple-echo spoiled gradient echo (mGRE) images were acquired in orthogonal orientations with slice thickness $=2 \mathrm{~mm}$, in-plane resolution 0.75 $\mathrm{mm} \times 0.75 \mathrm{~mm}, \mathrm{TR}=3270 \mathrm{~ms}, \mathrm{FA}=15 \mathrm{deg}$. These LR images were then reconstructed to one high resolution (HR) mGRE image with isotropic resolution $(0.75 \mathrm{~mm})^{3}$, using a conjugate-gradient based SRR method [3]. The original method was modified and adapted here for water content mapping (Fig. 1). The developed technique was validated using a carrageenan phantom[4] (Fig. 2). The $(0.75 \mathrm{~mm})^{3}$ isotropic resolution $\mathrm{H}_{2} \mathrm{O}$ map obtained from the SRR-reconstructed data was compared to the $0.75 \mathrm{~mm} \times 0.75$ $\mathrm{mm} \times 1.5 \mathrm{~mm} \mathrm{H}_{2} \mathrm{O}$ map obtained using the "long-TR" method. The acquisition times of both the methods were approximately the same. In vivo results were obtained from a healthy volunteer (male, 26 years). All measurements were carried out using 3 T PRISMA scanner, Siemens, Erlangen, Germany.

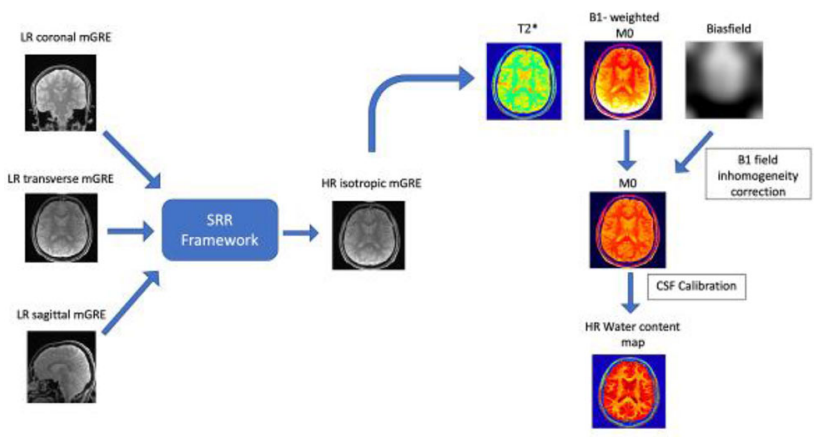

Fig1: Schematic depiction of the super-resolution reconstruction water content (SRR- $\mathrm{H}_{2} \mathrm{O}$ ) mapping technique. First an
isotropic high-resolution mGRE image is reconstructed from three orthogonal low-resolution datasets and then $\mathrm{H}_{2} \mathrm{O}$ mapping performed. 


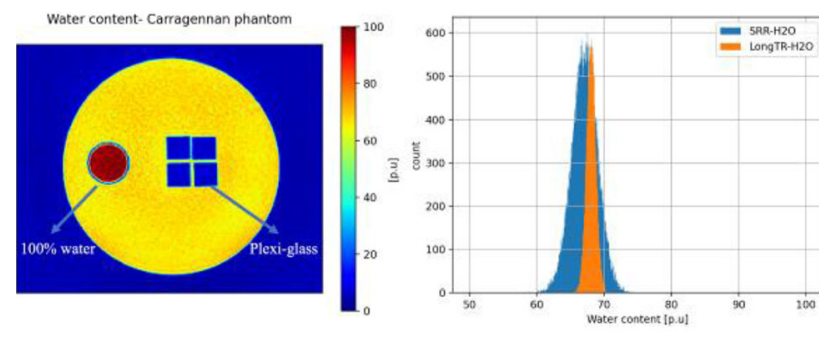

Fig2: Left: Carrageenan phantom to evaluate the improvement in resolution using the proposed method. Right: The histogram plots of the proposed technique ( $\left(\mathrm{SRR}-\mathrm{H}_{2} \mathrm{O}\right)$ and the gold standard (long-TR- $\mathrm{H}_{2} \mathrm{O}$ ) technique

Results: Figure 2 shows the $\mathrm{H}_{2} \mathrm{O}$ map of the carrageenan-agarose phantom obtained from the proposed SRR- $\mathrm{H}_{2} \mathrm{O}$ method. The $\mathrm{H}_{2} \mathrm{O}$ values for a selected ROI are compared between the SRR and longTR method by evaluating their histogram distributions. In Fig. 3, The $\mathrm{H}_{2} \mathrm{O}$ histograms of both methods show the usual bimodal distribution of WM and GM values, with the proposed method showing mean \pm std, WM: $69.73 \pm 3.42$ p.u, GM: $84.23 \pm 4.72$ p.u. and the long-TR method showing mean \pm std, WM: $70.73 \pm 3.12$ p.u, GM: $83.52 \pm 4.52$ p.u.

Whole brain water content maps (Resolution: $0.75 \mathrm{~mm}$ isotropic)
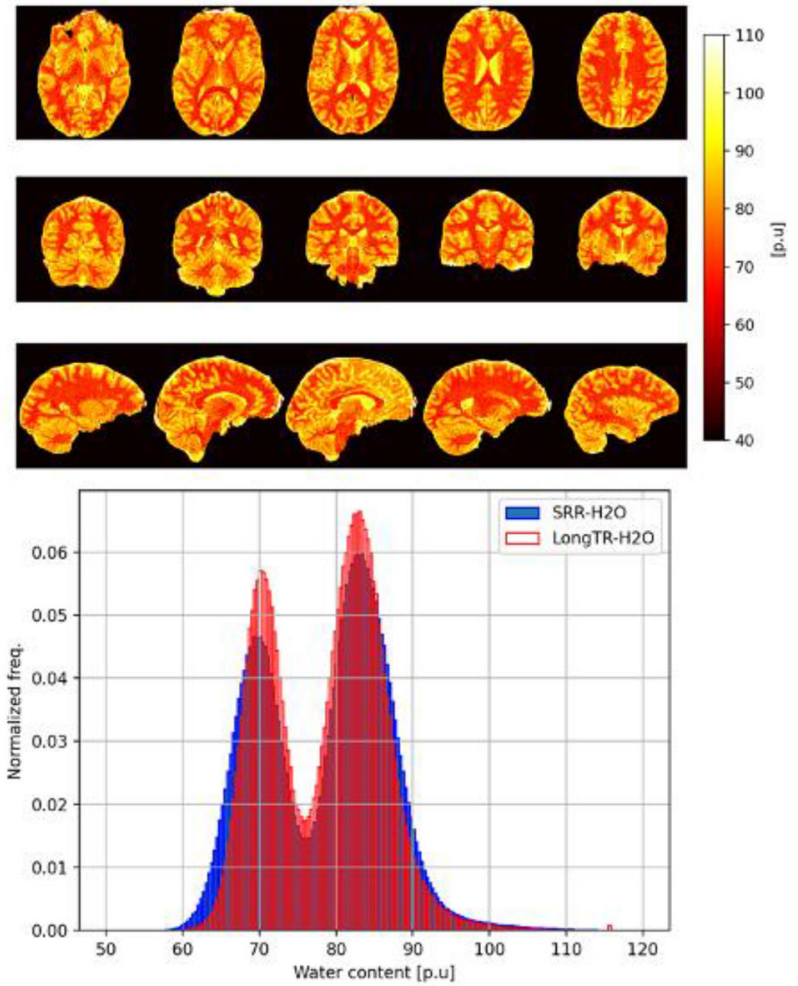

Fig3: Upper figure: Different slices of the whole brain $\mathrm{H}_{2} \mathrm{O}$ maps (in percentage units) generated with the proposed. Lower figure: Histogram plots of the SRR- $\mathrm{H}_{2} \mathrm{O}$ and long-TR- $\mathrm{H}_{2} \mathrm{O}$ values. The peak at around $70 \%$ is for the WM and that around $83 \%$ is for the GM.

Discussion: The broader distribution of values for the SRR- $\mathrm{H}_{2} \mathrm{O}$ in Fig. 2 is due to the smaller voxel volume (half the volume of longTR $-\mathrm{H}_{2} \mathrm{O}$ ) of the reconstructed data as well as to the 2.5 times lower SNR of the original LR images acquired with a low flip angle. The in vivo SRR- $\mathrm{H}_{2} \mathrm{O}$ values correspond well with the literature values and with the values obtained using the long-TR method. In summary, a technique for obtaining high-resolution, whole-brain $\mathrm{H}_{2} \mathrm{O}$ maps with isotropic voxel size using SRR has been developed. It is anticipated that this proof of concept will be of particular interest at higher field strengths, where the resolution can be further improved using the increased SNR.

\section{References:}

[1] H. Neeb et al., NeuroImage, 2006.

[2] A.-M. Oros-Peusquens et al., Front. Neurol, 2019

[3] D. Poot et al., MICCAI, 2010.

[4] K. Yoshimura et al., MRM, 2003.

Research Grant: B-Q MINDED (EU H2020 MSCA ETN 764513).

\section{C1.010.}

\section{EPIFANI for ultrafast B1-corrected T1 and PD mapping}

\author{
${ }^{*}$ M. A. Zampini ${ }^{1,2}$, J. Sijbers ${ }^{2,3}$, M. Verhoye ${ }^{2,3}$, R. Garipov ${ }^{1}$ \\ ${ }^{I}$ MR Solutions Ltd, Guildford, GB; \\ ${ }^{2}$ University of Antwerp, Bio-Imaging Lab/Department of Biomedical \\ Sciences, Wilrijk, BE; \\ ${ }^{3}$ University of Antwerp, $\mu N E U R O$ Research Centre of Excellence, \\ Wilrijk, $B E$
}

Introduction: The longitudinal relaxation time $\left(\mathrm{T}_{1}\right)$ is a clinically relevant biomarker in neuroradiology. Many $\mathrm{T}_{1}$ methods have been developed in the past decade, among which Variable flip angle with Actual Flip angle Imaging (VAFI) ${ }^{1}$ is widely employed. VAFI relies on the acquisition and fitting of Actual Flip angle Imaging (AFI $)^{2}$ and SPoiled GRadient echo (SPGR) signals and simultaneously corrects for the inhomogeneities of the excitation field $\mathrm{B}_{1}$, whose knowledge is of cardinal importance for quantitative imaging at high magnetic field $^{3}$. Here we introduce Echo Planar Imaging Fast Actual Nutation Imaging (EPIFANI), an EPI implementation of VAFI for simultaneous mapping of $\mathrm{T}_{1}, \mathrm{~B}_{1}$, and proton density (PD) with $2 \mathrm{D}$ ultrafast acquisitions.

Methods: EPIFANI was developed by including a 2D EPI readout to acquire data from both an AFI and SPGR sequence (Fig. 1). Voxelwise mapping of $\mathrm{T}_{1}, \mathrm{~B}_{1}$, and $\mathrm{PD}$ was performed by minimizing the residual sum of squares of the signal intensities and the model signal for AFI and SPGR ${ }^{1}$. Simulations to assess the effects on parameter estimation of noise, $\mathrm{T}_{2}{ }^{*}$ induced signal decay, and misalignment among even and odd k-space lines were performed. Data were acquired on an MR Solutions 4.7 $\mathrm{T}$ preclinical scanner in a single shot with flip angle $\mathrm{FA}=60^{\circ}, \mathrm{n}=\mathrm{TR}_{2} / \mathrm{TR}_{1}=1000 \mathrm{~ms} / 200 \mathrm{~ms}$, matrix size $=128 \times 128$, FOV $=40 \times 40 \mathrm{~mm}^{2}$, slice thickness $=1 \mathrm{~mm}-$ SPGR acquisitions with $\mathrm{FA}=[15,25,35]^{\circ}, \mathrm{TR}=200 \mathrm{~ms}-\mathrm{NEX}=3$, and an optimized RF phase increment with high spoiling gradient areas. Steady-state was reached by the use of a preparation pulse ${ }^{4}$. A homogeneous agar gelatin phantom and a mouse head were scanned with a quadrature coil with $38 \mathrm{~mm}$ ID. A 14 points Inversion Recovery ( $\mathrm{TR}=10 \mathrm{~s}, \mathrm{TI} \in[0.05,4] \mathrm{s}$ ) was performed on a gelatine phantom to compute reference $T_{1}$ values. 


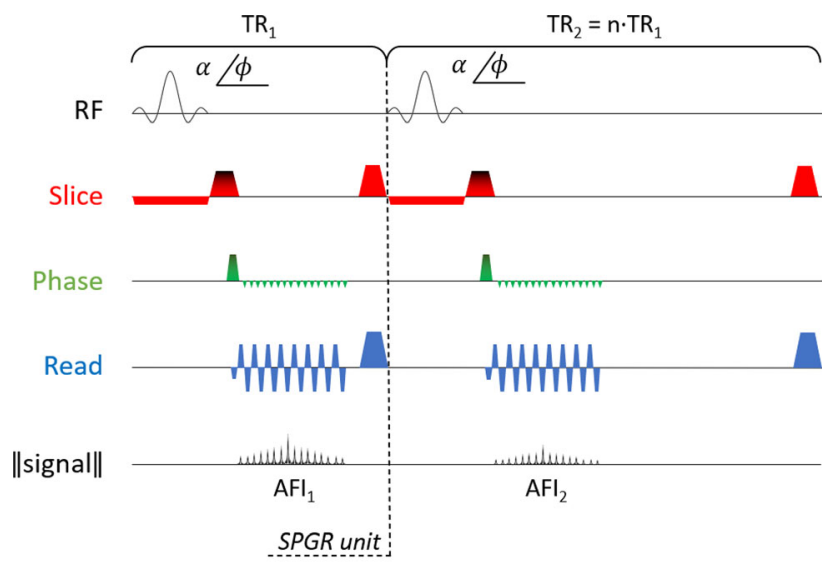

Figure 1: Pulse sequence diagram of EPIFANI.

\section{Results.}

- Simulations: maps of $\mathrm{T}_{1}$ and $\mathrm{B}_{1}$ reliably report the original input values, while PD maps show signal intensity variations provided by both $\mathrm{T}_{2}{ }^{*}$ decay and lines misalignment in k-space (see Fig. 2). For $\mathrm{T}_{2}^{*}<41 \mathrm{~ms}$ median inaccuracies in $\mathrm{T}_{1}$ greater than $10 \%$ arise. This suggests the use of multi-shot acquisitions for very short $\mathrm{T}_{2}{ }^{*} \mathrm{~s}$.
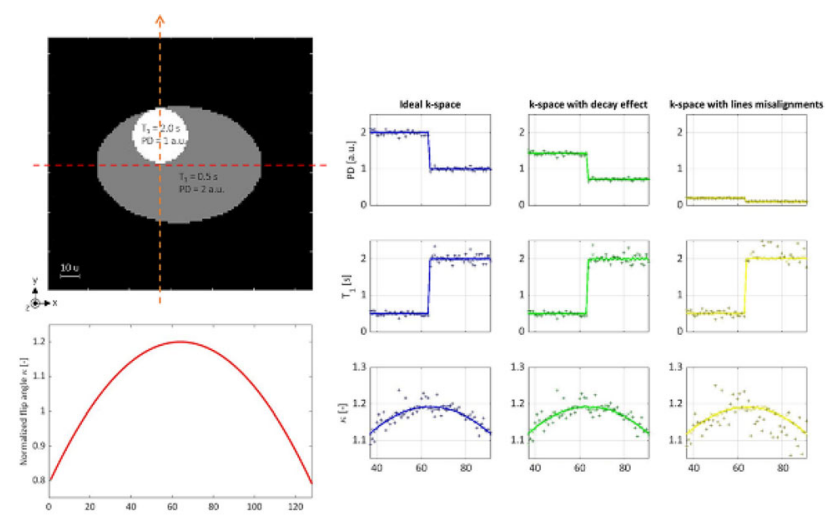

Figure 2: Simulations for EPIFANI mapping. Iop left image: simulated phantom with the respective I1 and PD values Bottom left imase- profle of the normalized flip angle along the horizontal red line (radial symmetry around the center is assumed. Bight image: fitted values for the case of an ideal $k$-space, as well as considering decay $T^{*}=0.3 \mathrm{~s}$ )

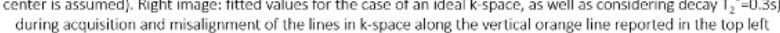
figure. Continuous lines refer to an ideal (noiseless) scenace along the vertical orange line reported in the top left with standard deviation of 0.001 and 0.05 , respectively, added to both real and imaginary channels.

- Acquisitions: with a total acquisition time of $51 \mathrm{~s}$ for a single slice image EPIFANI provided mean $\mathrm{T}_{1}$ values (standard error, SE) of $2.056(0.02) \mathrm{s}$ with respect to $2.054(0.001) \mathrm{s}$ (IR) for the gelatine phantom.

For in vivo data, an example of AFI image and $\mathrm{T}_{1}$ map is reported in Fig. 3. Median values for EPIFANI for cortical grey matter $T_{1}$ values are in line with literature ${ }^{5}$.
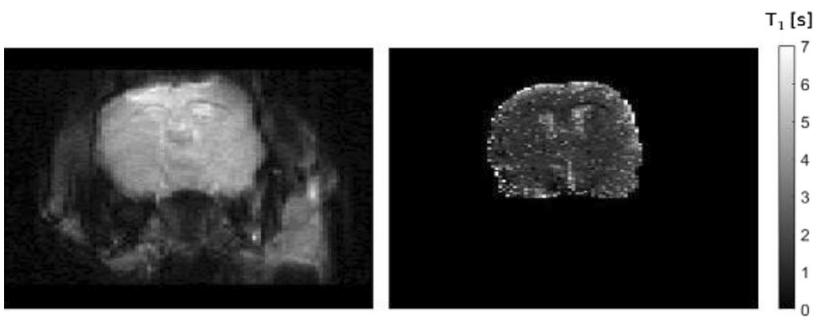

Figure 3: Example of $\mathrm{AFI}_{1}$ image (left) and $\mathrm{T}_{1}$ map (right) for a mouse head scanned at 4.7T.

Discussion: EPIFANI allows the acquisition of ultrafast and accurate $\mathrm{T}_{1}$ and $\mathrm{B}_{1}$ maps. Future developments will focus on the investigation of multi-shot EPIFANI, the implementation of distortion correction methods, and the estimation of their impact on parametric maps. Funding: B-Q MINDED (EU H2020 MSCA ETN 764513)

\section{References:}

1. $10.1002 / \mathrm{mrm} .23199$.

2. $10.1002 / \mathrm{mrm} .21120$.

3. $10.1002 /$ jmri.25692.

4. 10.1007/s10334-020-00876-y.

5. $10.1002 / \mathrm{mrm} .21313$.

\section{C1.011.}

\section{Myocardium numerical phantom at micro-scale: application in MRI simulation}

\author{
*M. Lashgari ${ }^{1}$, N. Ravikumar ${ }^{1}$, I. Teh ${ }^{2}$, J. Schneider ${ }^{2}$, A. Frangi ${ }^{1,2}$ \\ ${ }^{1}$ University of Leeds, School of Computing and School of Medicine, \\ Leeds, GB; \\ ${ }^{2}$ University of Leeds, School of Medicine, Leeds, GB
}

Introduction: MRI biophysical models are formulated based on certain assumptions, derived from biological information of the underlying tissue, to interpret MRI signals. Hence, there is a need for the numerical phantoms to validate these assumptions. Moreover, numerical phantoms can be used to analyse the sensitivity of MRI measurements to changes in tissue microstructure and the use of different pulse sequences [1]. The aim of this study is to develop a numerical phantom for myocardial microstructure, which incorporates the native probability distribution functions (PDFs) of cardiomyocyte $(\mathrm{CM})$ shape parameters, regional curvature, ventricular twist and collagen into the phantom.

Methods The proposed method receives the PDFs of CMs volume $(V)$, length $(L)$, major-axis $(A)$, and minor-axis $(B)$ PDFs, reported in literature [2], as the inputs (Fig. 1a). Using the PDFs of the $A$ and $B$ of the cross-section of the CMs, 2D ellipses are then packed [3] (Fig. 1b) and subsequently transformed into polygons to mimic sheetlet crosssections (Fig. 1c). Next, the 3D structure of a small section of a sheetlet is formed based on the length and volume PDFs of CMs, as illustrated in Fig. 1d. In subsequent steps, several such sheetlet sections are combined vertically to generate a complete sheetlet (Fig. 1.e). Finally, several sheetlets are concatenated horizontally and transformed by applying rotation, twisting, and bending transformations, shown in Fig. $2 \mathrm{a}-\mathrm{d}$, to generate a realistic myocardial wall. Finally, a cube is extracted from this wall to mimic a voxel of MRI (Fig. 2e). 

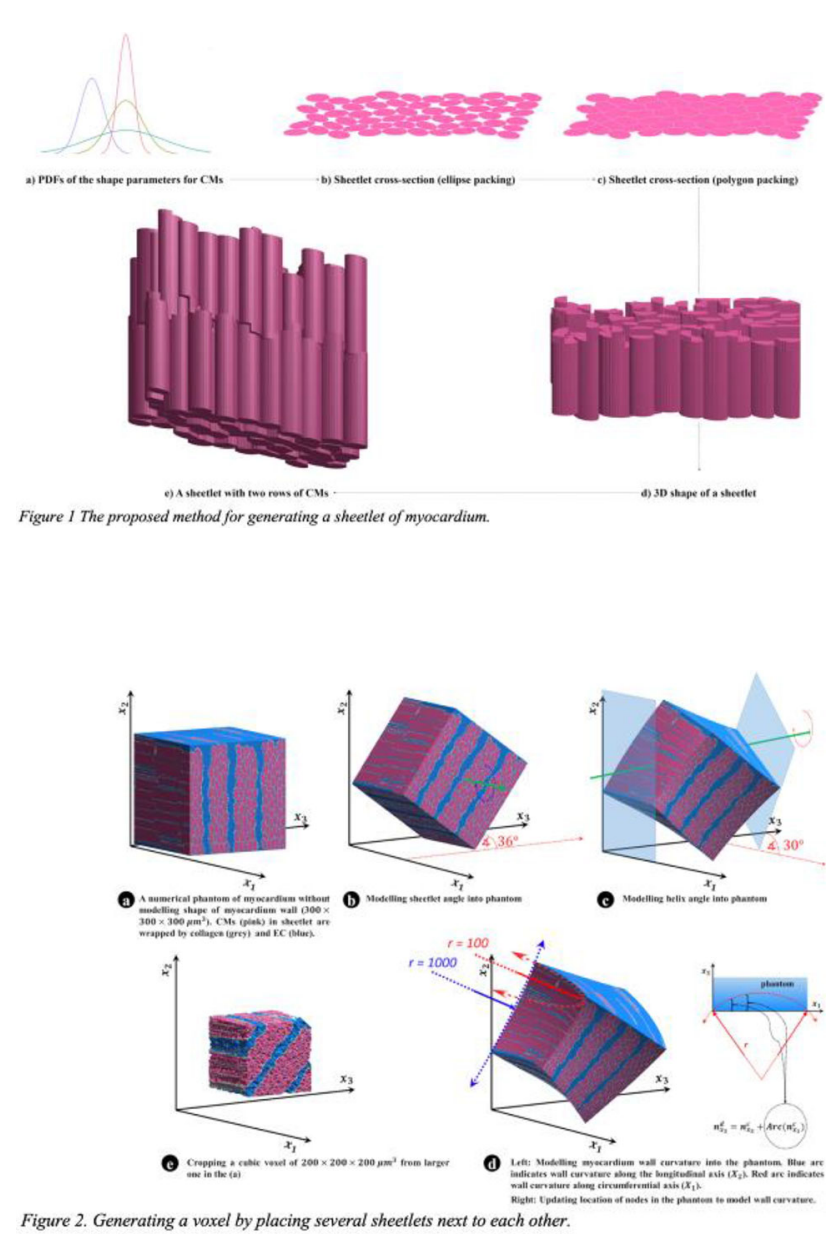

Figure 2. Generating a voxel by placing several sheetlets next to each other.

A two-step statistical test evaluates how inputs PDFs are preserved following the phantom generation. First, using a virtual morphometry, output PDFs of 12,000 virtual CMs are measured. The ShapiroWilk test [4] is used to test the normality of the input and output PDFs of $V, L, A$, and $B$. Finally, the significance of the difference between the input and output PDFs is tested using a t-test [5] for parameters that follow a normal distribution, and a U-test [5] for those that do not follow a normal distribution.

Results: The resulting $\mathrm{p}$ values of the test, shown in Table 1, indicate no significant difference between the distribution of $V(\mathrm{p}=0.76)$, $L(\mathrm{p}=0.99)$, and $A(\mathrm{p}=0.09)$ and $B(\mathrm{p}=0.19)$ of the virtual CMs and the reference values reported in literature [2].

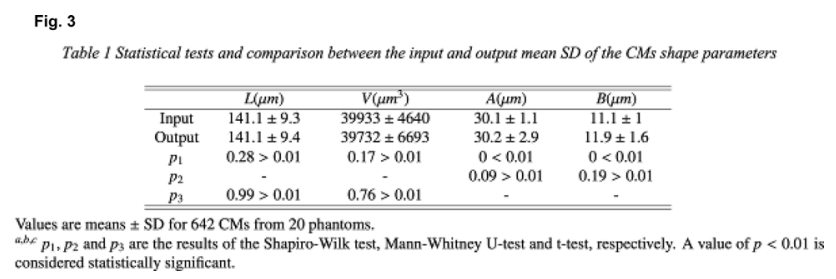

Discussion: The result demonstrates that the proposed method generates a numerical phantom for myocardial microstructure with realistic CMs. Future work will focus on validating this phantom respect similarity between packing of $\mathrm{CMs}$ in the virtual tissue slab and actual tissue using histology images.

\section{References:}

[1] Fieremans, Els, et al., Neuroimage 182 (2018).
[2] Gerdes, A. M. et al., Manual of research techniques in cardiovascular medicine. (2014).

[3] Ilin, Dmitrii N. et al., Granular Matter 18.3 (2016).

[4] Ahad, Nor Aishah, et al. Sains Malaysiana 40.6 (2011).

[5] Nayak, Barun K. et al., Indian journal of ophthalmology 59.2 (2011).

\section{C1.012.}

\section{Towards accelerating cardiac DTI with undersampled 3D spiral acquisitions and a compressed sensing reconstruction based on joint sparsity}

\author{
*S. Golshani ${ }^{1,2}$, I. Teh ${ }^{2}$, N. Ravikumar ${ }^{1,2}$, J. Schneider ${ }^{2}$, A. Frangi ${ }^{1,2}$ \\ ${ }^{1}$ University of Leeds, School of Computing and School of Medicine, \\ Leeds, GB; \\ ${ }^{2}$ University of Leeds, Institute of Cardiovascular and Metabolic \\ Medicine, Leeds, GB
}

Introduction: Cardiac diffusion tensor imaging (cDTI) is impacted by significant challenges including lengthy acquisition times. Undersampled spiral acquisition schemes have recently gained renewed interest ${ }^{1}$, as their realization is more compatible with current scanner hardware, and they lead to incoherent aliasing artifacts wellsuited to reconstructions with compressed sensing (CS). The purpose of this study is to investigate the feasibility of an efficient acquisition/ reconstruction strategy for accelerated high-resolution cardiac DTI based on the combination of a 3D variable-density stack-of-spirals trajectory and a joint CS reconstruction that incorporates group sparsity priors in both gradient and wavelet domains.

Methods: Ground-truth DTI data were acquired from an ex-vivo rat heart on a $7 \mathrm{~T}$ preclinical Bruker scanner (BioSpec 70/20USR) using a 3D PGSE sequence, with diffusion gradients applied in 27 directions $\left(b=1000 \mathrm{~s} / \mathrm{mm}^{2}\right.$, RES $\left.=100 \mu \mathrm{m}^{3}\right)$. A 3D variable-density spiral sampling with golden-angle ordering of the interleaves was designed with the same specifications (Fig. 1) and used to simulate spiral data from complex k-space measurements of the ground-truth data. A joint CS reconstruction method, which exploits the structural similarity among diffusion-weighted images (DWIs) acquired at different directions in both 3D total variation and 3D wavelet-transform domains, was adapted to jointly recover the non-Cartesian data ${ }^{2}$. Retrospectively undersampled data were obtained through randomly skipping the interleaves for each DWI to further ensure the incoherence and reconstructed using the proposed approach. For comparison, a basic disjoint CS method was also implemented. DTI metrics (MD and FA) were derived and compared with the reference.

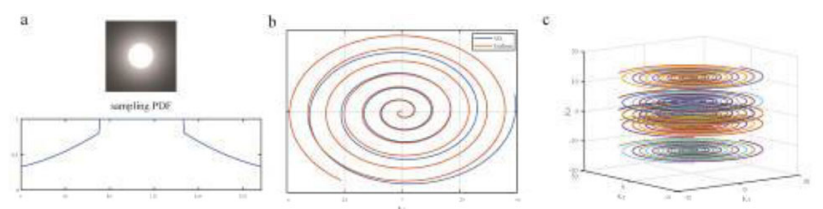

Fig. 1. Proposed sampling scheme. (a) The probability density function (PDF) based on a polynomial of order 2 , used for generating variable density sampling more adapted to signal energy, with the central $20 \%$ of the k-space fully sampled. (b) Comparison of the designed variable-density spiral trajectory with a uniform-density spiral trajectory. (c) The schematic of the resulting 3D stack-of-spirals trajectory used for the retrospective undersampling in 3D $\mathrm{k}$-space (three golden-angle ordered interleaves are shown for each plane).

Results/discussion: Reconstructed images and the corresponding error maps of one diffusion direction with the proposed method at varying acceleration factors are depicted in Fig. 2. Plots comparing the mean RMSE, SSIM, and signal to error ratio (SER) within the ROI (3D myocardium) over all DWIs obtained from conventional CS 
and the proposed method (PJGS) are also displayed. Figure 3 represents maps of FA and MD obtained from the reference and $10 \times-$ accelerated data with respective RMSE values. Conventional CS results in over-smoothed images at high undersampling rates which is reflected in the larger bias for resulting FA and MD maps while the PJGS provides images with superior quality and leads to lower errors.

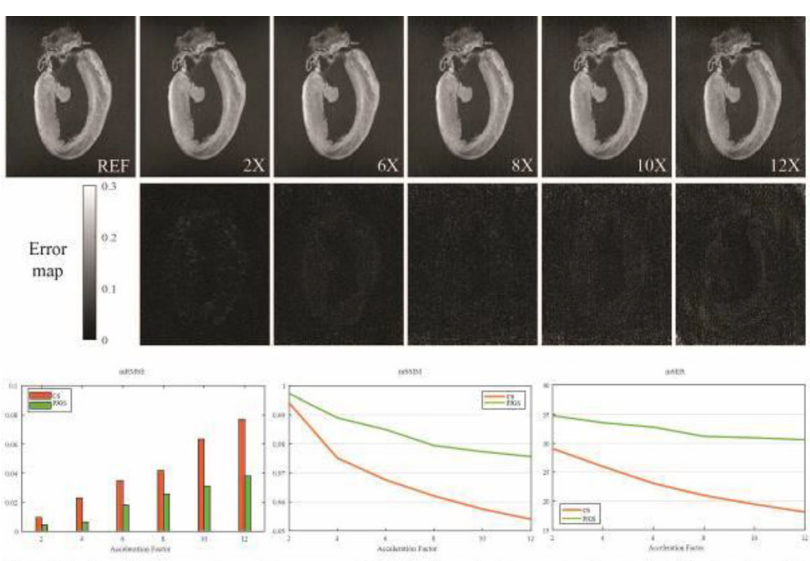

Fig. 2. Performance comparison of the proposed framework against the acceleration factor $(R=2$ to $R=12$ ). Top panel: Reconstructed images and the corresponding error maps of one randomly chosen diffusion direction with the proposed method (PJGS) at varying acceleration factors. The reference image is shown on the leftmost with the proposed method (PJGS) at varying acceleration factors. The reference image is shown on the leftmos top column. The PJGS reconstruction successfully removes the incoherent undersampling artifacts even a
acceleration factors as high as 10 . Artifacts become visible only in the strongly undersampled images $(R=12$ acceleration factors as high as 10 . Artifacts become visible only in the strongly undersampled images ( $R=12$,
with a minimum effect on the region of interest (i.e., the myocardium). Bottom panel: Quantitative metrics with a minimum effect on the region of interest (i.e., the myocardium). Bottom panel: Quantitative metrics comparing the reconstruction performance of the PJGS method with that of conventional CS. RMSE, SSIM and SER values were calculated within the ROI (3D myocardium tissue), considering the correspondin ground-truth images as the reference and aver
ity of the PJGS method is further established.

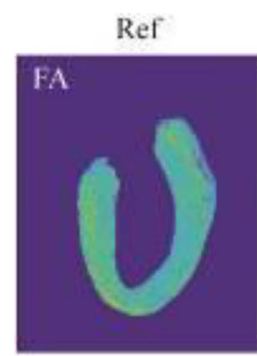

Conventional CS

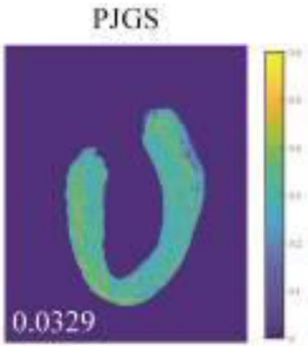

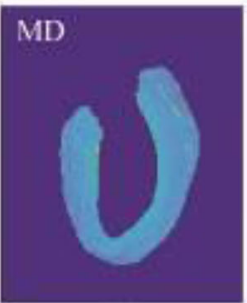

(a)
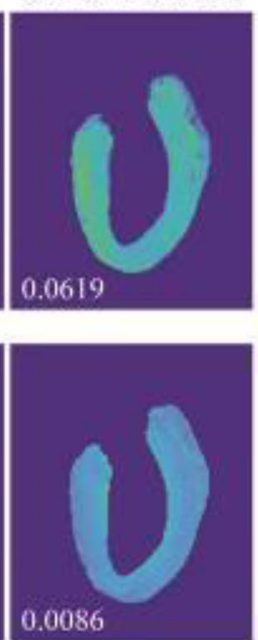

(b)

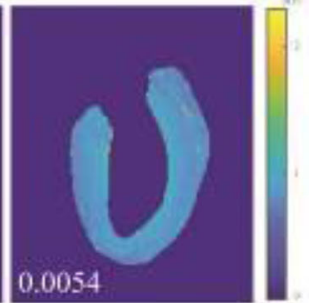

(c)
Fig. 3, Reconstructed ex-vivo FA and MD maps obtained from the ground-truth DTI data (a) and simulated data on the $10 \mathrm{x}$-accelerated $3 \mathrm{D}$ spiral trajectory, using conventional CS (b) and the PJGS (c) reconstructions. The RMSE values with respect to the reference images are shown at the bottom right. Note that for the non-weighted (b0) image only mild undersampling with 2 -fold acceleration was performed. The PJGS images exhibit preserved quality with minimal artifacts as the structural correlation in DWIs is utilized, leading to less biased DTI metrics.
Conclusion: Results demonstrated that the proposed framework yields robust reconstruction performance with well suppressed artifacts in recovering DTI metrics, even at high acceleration factors. This could lead to significant reduction in the scan time required for acquisition of each DWI, from $2.15 \mathrm{~h}$ to roughly $10.26 \mathrm{~min}$ with similar resolution.

Acknowledgement: Research Grant: B-Q MINDED (EU H2020 MSCA ETN 764513).

\section{References:}

1. Gorodezky et al. Magn Reson Med, 81:1580-1594 (2019).

2. Huang et al. Magn Reason Imaging, 32:1344-1352 (2014).

\section{C1.013.}

\section{Performance comparison of monopolar and bipolar readout gradients for $\mathbf{T} 2 *$ quantification}

\author{
*S. Shin ${ }^{1,2}$, S. D. Yun ${ }^{1}$, N. J. Shah ${ }^{1,3,4,5}$ \\ ${ }^{1} J u ̈ l i c h$ Research Centre, Jülich, DE; \\ ${ }^{2}$ RWTH Aachen University, Aachen, DE; \\ ${ }^{3}$ Jülich Research Centre, Institute of Neuroscience and Medicine \\ (INM-11), Jülich, DE; \\ ${ }^{4}$ Jülich Aachen Research Alliance (JARA), Aachen, DE; \\ ${ }^{5}$ RWTH Aachen University, Department of Neurology, Aachen, DE
}

Introduction: To quantify $\mathrm{T} 2 *$, multiple echoes are usually acquired with a multi-echo gradient-echo sequence using either monopolar or bipolar readout gradients. The bipolar readout gradients have a shorter echo spacing time compared to the monopolar readout gradients, enabling the collection of a larger number of echoes within the same scan time, with potentially more accurate $\mathrm{T} 2 *$ quantification. ${ }^{1,2}$ Nonetheless, comparison of the readout gradients for T2* quantification has not been addressed to date. In this work, the performance of the readout gradients has been investigated at $3 \mathrm{~T}$. The bipolar readout gradients provide more precise $\mathrm{T} 2 *$ maps and show robustness under a relatively lower SNR condition.

Methods: Experiments were performed on a $3 \mathrm{~T}$ MRI scanner (PRISMA, Siemens Halthineers). The monopolar and bipolar readout gradients were optimised to have the shortest echo spacing time under the given imaging parameters: $\mathrm{FOV}=240 \times 240 \mathrm{~mm}^{2}$, matrix $=$ $240 \times 240$, slice thickness $=1 \mathrm{~mm}$, \# of slices $=11, \mathrm{TR}=1200$ $\mathrm{ms}, \mathrm{TE} 1=3.84 \mathrm{~ms}, \Delta \mathrm{TE}=2.92 \mathrm{~ms}$ for monopolar $(32$ echoes) and $1.47 \mathrm{~ms}$ for bipolar ( 64 echoes) readout gradients. The flip angle was changed from $75^{\circ}$ to $15^{\circ}$ in $20^{\circ}$ decrements for phantom experiments and $76^{\circ}$ for in vivo experiments. The parallel imaging acceleration factor (AF) was also varied from 1 to 3 . Magnitude fitting was performed with a mono-exponential function, considering Rician noise distribution. $^{3}$

Results: Figures 1 and 2 show the calculated T2* maps from the monopolar and bipolar readout gradients. In Fig. 1, each column and row displays the result for a different set of flip angle and AF combinations. Visual inspection suggests that bipolar readout gradients give less variation than monopolar readout gradients, particularly with a lower flip angle and higher AF (see the area indicated by yellow arrows). In Fig. 2, as with the phantom results, T2* maps from the bipolar readout gradients look less noisy than those from the monopolar readout gradients. The mean and standard deviation of the calculated $\mathrm{T} 2 * \mathrm{~s}$ are presented in Fig. 3 . The results obtained with the bipolar readout gradients show a smaller standard deviation than those from the monopolar readout gradients. 

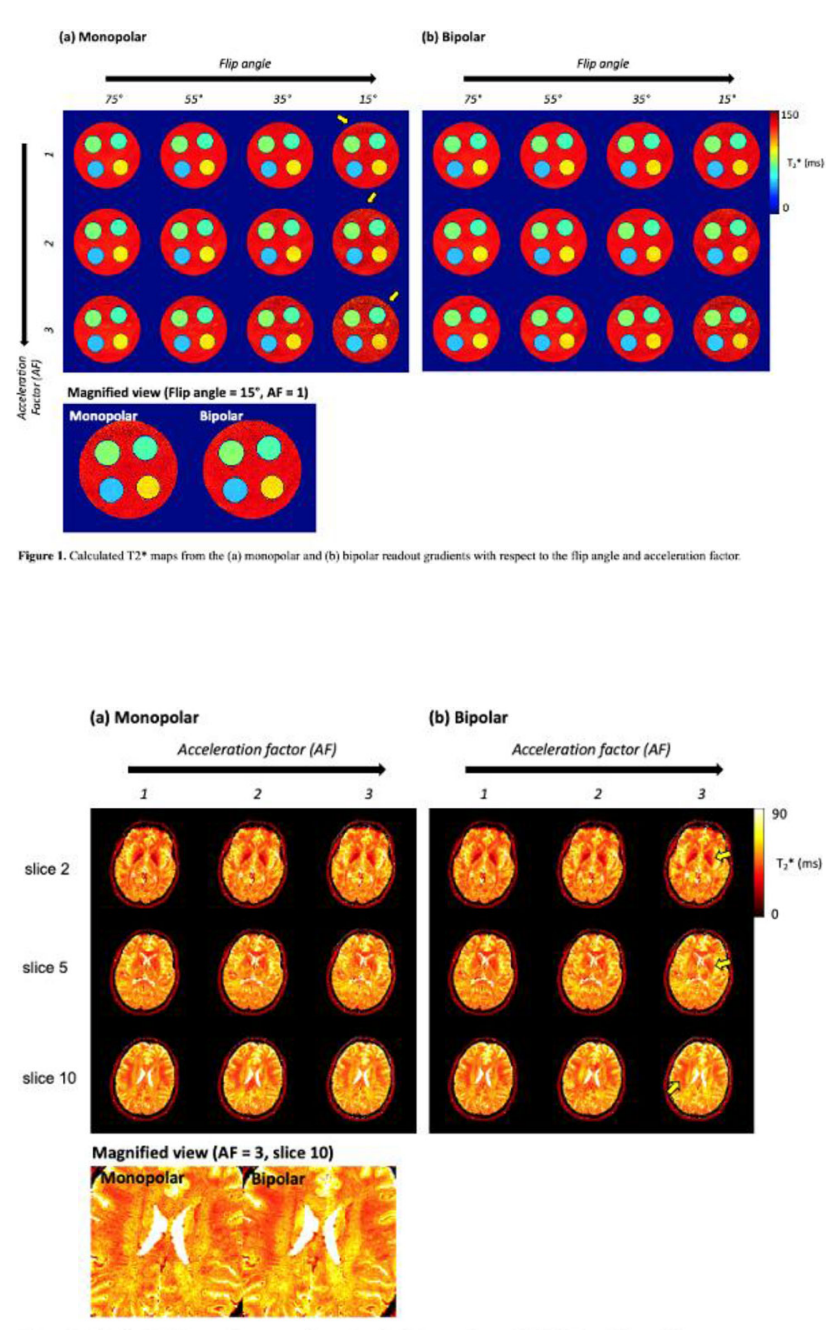

Figure 2. Caleulated $\mathrm{T}^{*}$ maps from in vivo data using the (a) monopolar and (b) bipolar readout gradients.

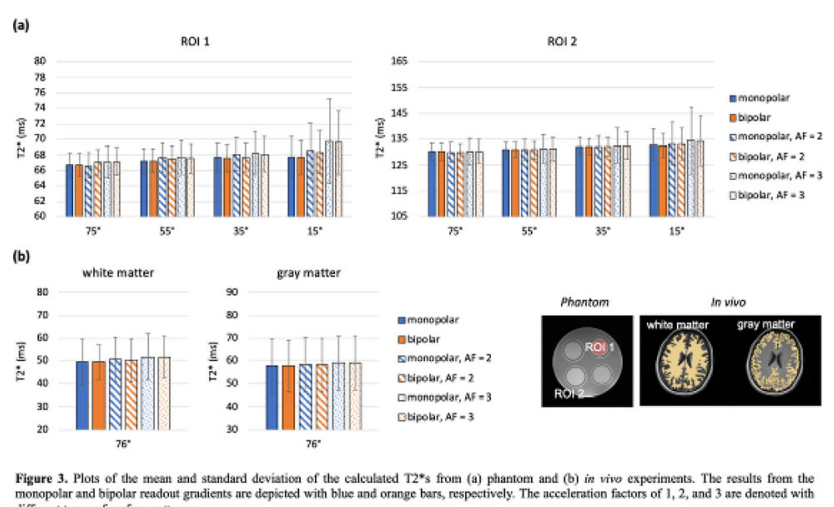

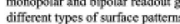

Discussion: An increase in the mean and standard deviation was observed for a larger AF and smaller flip angle for both the monopolar and bipolar readout gradients. The mean $\mathrm{T} 2 *$ was comparable between the two readout gradients. However, in the case of standard deviation, the bipolar readout gradients were less sensitive to the changes in the SNR. It can be seen that the bipolar readout gradients show greater robustness against noise, which was mainly due to the use of a larger number of echoes for $\mathrm{T} 2 *$ quantification.

Research Grant: B-Q MINDED (EU H2020 MSCA ETN 764513).

\section{References:}

[1] Dierkes $\mathrm{T}$, et al. International Congress Series. 2004;1265:181-185.

[2] Yablonskiy D. Intl. Soc. Mag. Reson. Med. 8 (2000).

[3] Sijber J, et al. Int J Imag Syst Tech 1999;10:109-114.

\section{C1.014.}

Effect of varying DWI b values on prostate lesion segmentation accuracy and robustness

\author{
*M. Postigo Fliquete ${ }^{1}$, J. Tol ${ }^{1}$, V. Fortunati ${ }^{1}$, A. van Engelen ${ }^{1}$ \\ ${ }^{1}$ Quantib, Rotterdam, $N L$
}

Introduction: Multiparametric MRI plays a key role in PCa diagnosis, where DWI obtained using high $b$ values are vital for interpretation [1]. The PI-RADS guidelines state that DWI obtained using b values $\geq 1400 \mathrm{~s} / \mathrm{mm}^{2}$ should be used [2], so consistency in $\mathrm{b}$ values amongst centers cannot be expected in clinical practice. This may hamper performance of algorithms trained using specific $b$ value DWIs.

Moreover, previous studies concluded that computed instead of acquired DWI could be used effectively for PCa detection [3]. We investigated prostate lesion segmentation accuracy by training a segmentation network using acquired DWI (aDWI) or computed DWI (cDWI) at different b values.

Methods: The dataset comprised of 414 single-center $1.5 \mathrm{~T}$ mpMRI scans (Siemens, AvantoFit). For each study, lesions were manually segmented and PI-RADS scored by one radiologist, out of a pool of radiologists. An additional distinction was made for PI-RADS 3 lesions, since biopsy may be appropriate on factors besides mpMRI [2]. Lesions with a PI-RADS $\geq 3$ were grouped between high (PIRADS 4-5, biopsied), mild (PIRADS 3, biopsy requested by radiologist) and low (PIRADS 3, not biopsied).

Our lesion segmentation method is based on 3D UNet architecture [4] with three input images: T2w, ADC and DWI. Models were trained and validated in a fivefold cross-validation setup with either aDWI or cDWI with $b$ values of 800 or $1500 \mathrm{~s} / \mathrm{mm}^{2}$ to investigate the model robustness against varying $b$ values. cDWI were calculated from ADC and b800 scans using a mono-exponential fit $[1,3]$.

Classification performance was evaluated with sensitivity (SE) and false positive rate (FPR) at a threshold of 0.5 and FROC curves. Segmentation performance was determined by the Dice Similarity Coefficient (DSC), computed for the true positive (TP) lesions. A prediction was considered TP with at least one voxel overlaps with the reference lesion and false positive (FP) otherwise.

Results.

The DWI train/infer combinations and results are shown in Table 1. Figure 1 illustrates FROC curves for each combination. Figure 2 shows an example of combination outputs. 


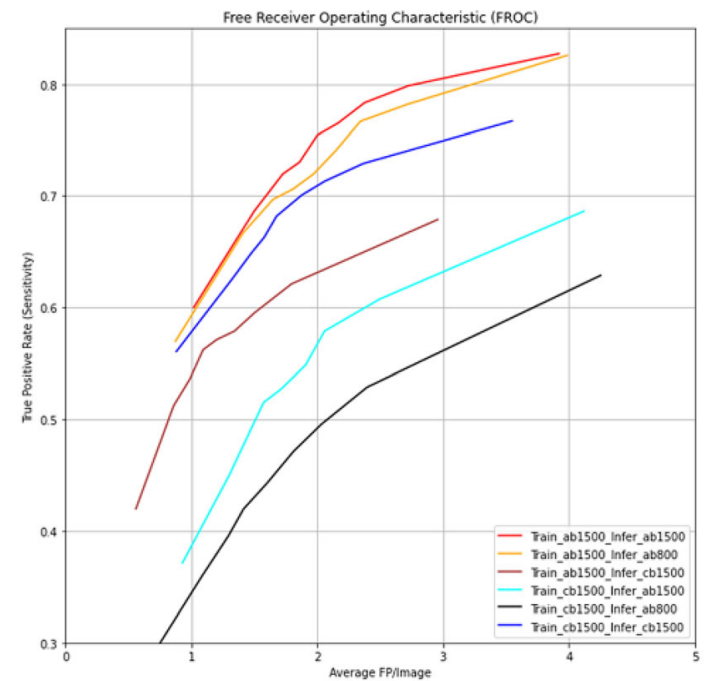

Figure 1. FROC curves for the different train/infer combinations.
Discussion and conclusion: Best results are obtained with ab1500/ ab1500 (acquired b1500) train/infer combination ( $\mathrm{SE}=0.9 / 0.74 / 0.62$ for high/mild/low), followed by ab1500/ab800. Similarity between both FROCs demonstrates robustness against the specific $b$ value used during inference, regardless of the $b$ value used for training. This is not the case for cb1500 (computed b1500), for which performance is lower. We therefore conclude that lesion segmentation networks are less sensitive to different measured $b$ values, than to computed higher $\mathrm{b}$ values.

Research Grants: B-Q MINDED (EU H2020 764513).

\section{References:}

1-Rosenkrantz et al., Academic Radiology. 2016.

2-Barentsz et al., European urology. 2015.

3-Verma et al., Abdominal Radiology. 2016.

4-Çiçek et al., 3D U-Net. 2016.
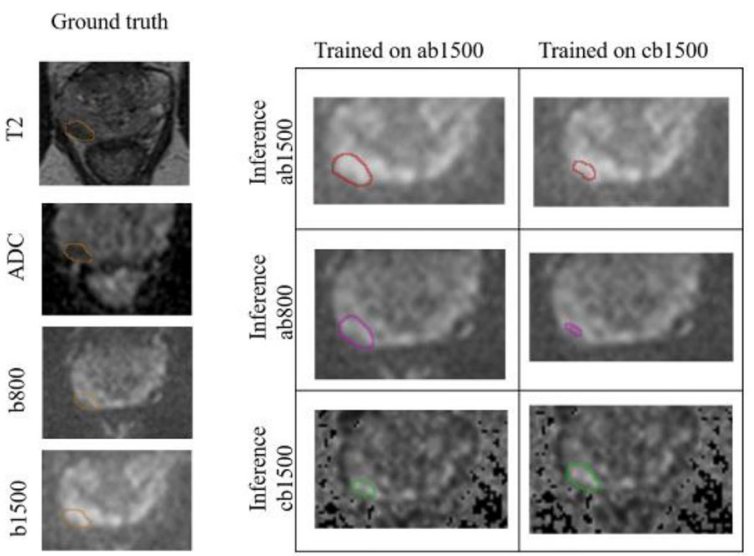

Figure 2. Examples of the lesion segmentation output for the different training/inference combinations in comparison to the ground truth segmentation for a given slice.

Fig. 3

\begin{tabular}{|c|c|c|c|c|c|c|c|c|c|c|c|c|c|c|}
\hline & \multicolumn{7}{|c|}{ Training on ab 1500} & \multicolumn{7}{|c|}{ Truining on cb1500 } \\
\hline & \multicolumn{2}{|c|}{ high } & \multicolumn{2}{|c|}{ mild } & \multicolumn{2}{|c|}{ low } & \multirow{2}{*}{ FPR } & \multicolumn{2}{|c|}{ high } & \multicolumn{2}{|c|}{ mild } & \multicolumn{2}{|c|}{ low } & \multirow{2}{*}{ FPR } \\
\hline & $\mathrm{SE}$ & DSC & SE & DSC & SE & DSC & & $\mathrm{SE}$ & DSC & SE & DSC & SE & DSC & \\
\hline $\begin{array}{l}\text { Inferred on } \\
\text { ab1500 }\end{array}$ & 0.9 & 0.53 & 0.74 & 0.37 & 0.62 & 0.36 & 2.0 & 0.71 & 0.45 & 0.53 & 0.23 & 0.35 & 0.27 & 1.71 \\
\hline $\begin{array}{c}\text { Inferred on } \\
\text { ahsooo }\end{array}$ & 0.88 & 0.52 & 0.68 & 0.37 & 0.58 & 0.35 & 1.97 & 0.63 & 0.43 & 0.41 & 0.22 & 0.27 & 0.26 & 1.6 \\
\hline $\begin{array}{l}\text { Inferred on } \\
\text { ch1500 }\end{array}$ & 0.75 & 0.48 & 0.42 & 0.36 & 0.33 & 0.35 & 1.51 & 0.85 & 0.44 & 0.66 & 0.17 & 0.53 & 0.17 & 1.68 \\
\hline
\end{tabular}

Table 1. Results from doing lesion segmentation inference on different b-values from different trained model ab1500, ab800 and cb 1500 stands for acquired bl500, acquired b800 and computed b1500, respectively. The prostate lesion labels are high (PI-RADS 4-5, biopsied), mild (PIRADS 3, biopsy requested by radiologist) and low (PIRADS 3, not biopsied). FPR stands for False Positive Rate per study, SE stand for Sensitivity and
DSC is Dice Score Coefficient. 
C2.01.

Standard quality control for clinical PET/MRI systems: a european perspective

\author{
*A. Valladares ${ }^{1}$, T. Beyer ${ }^{1}$, I. Rausch ${ }^{1}$ \\ ${ }^{1}$ Medical University of Vienna, Vienna, AT
}

Aim: Hybrid imaging systems play an essential role in the non-invasive characterization of oncological diseases. Therefore, stringent quality control $(\mathrm{QC})$ procedures are required to ensure the proper functionality of the systems and to gain accurate quantitative results. Here, we evaluate existing QC procedures for combined PET/MRI systems in clinical routine.

Materials and methods: Eight highly-experienced European imaging sites (Fig. 1) were surveyed about locally implemented PET/MRI QC procedures. The survey was based on existing recommendations on QC for stand-alone PET and MRI systems. In addition, relevant vendor-specific information on QC measures was collected.

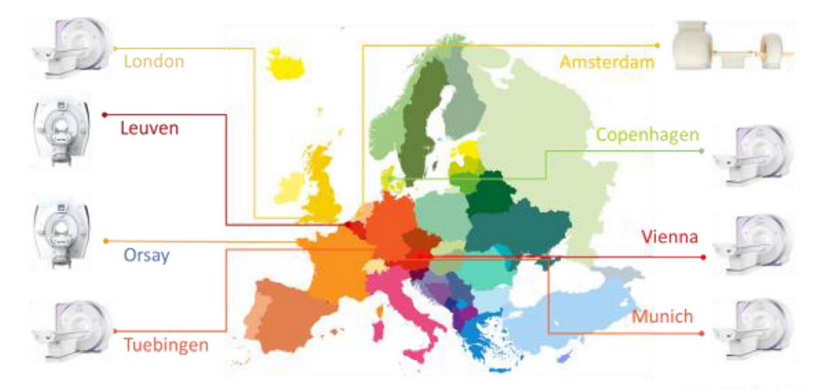

HYBRID

FIG 1. European PET/MR sites with extensive clinical experience participating in the HYBRID consensus effort.

Results: In total, five Siemens, two GE, and one Philips PET/MRI systems were reported. For all the systems, the centres reported performing the PET daily QC as implemented by the vendor. However, we found moderate to high variations across other tests and testing frequencies for the PET and MRI components of the systems. Aiming at addressing this significant variability across sites and vendors, we propose a consensus on $\mathrm{QC}$ procedures. This consensus includes for the PET component: a daily QC as suggested by the local vendor, a quarterly cross-calibration measurement including the assessment of uniformity, and a yearly image quality (IQ) test for the PET component of the systems. For the MRI component, monthly coil checks and a quarterly MR IQ test, including the assessment of signal-to-noise ratios and artefacts that could affect clinical scans and image quality. Figure 2 summarizes the reached consensus.

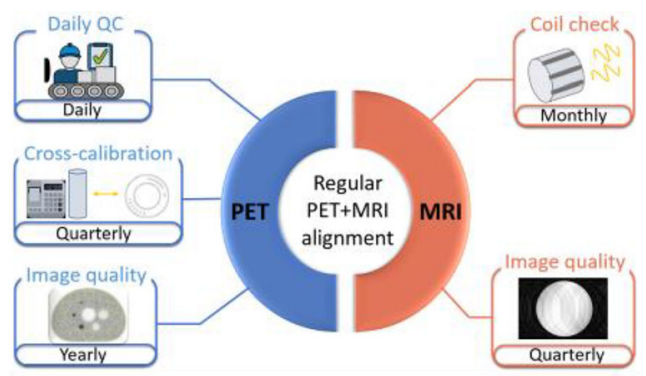

FIG 2. HYBRID consensus recommendations for standard quality control measures for dual-modality PET/MR imaging systems detailing the modality (PET and MR) and frequency of the tests.

Conclusion: We observed significant variations in daily QC procedures across PET/MR imaging sites. Therefore, in this work, we propose a consensus on minimum QC procedures for clinical routine.
This consensus may potentially help assure high-quality examinations across sites.

Acknowledgements: The authors thank all the HYBRID members that contributed to the study: Ronald Boellaard, Zacharias Chalampalakis, Claude Comtat, Sahar Ahangari, Adam E. Hansen, Johan Nuyts, Michel Koole, Laura DalToso, Paul Marsden, Nicoló Capobianco and Esteban Solari. We also thank Jane Mackewn from King"s College London, Francesco Padormo from Guy"s and St. Thomas" National Health Service Foundation Trust, Ronald Peeters from Universitair Ziekenhuis Gasthuisberg, Sebastian Poth from University Hospital Tuebingen, Jansen Floris and Bill Peterson from GE Healthcare.

This work has received funding from the European Union"s Horizon 2020 research and innovation programme under the MSCA No. 764458.

\section{C2.O2.}

\section{Whole-body parametric PET/MRI}

\author{
Z. Chalampalakis ${ }^{1}$, F. Besson ${ }^{1,2}$, N. Tournier ${ }^{1}$, S. Stute ${ }^{3,4},{ }^{*}$ C. \\ Comtat $^{1}$ \\ ${ }^{I}$ Paris-Saclay University, BioMaps Department, Orsay, FR; \\ ${ }^{2}$ Paris-Saclay University, Department of Biophysics and Nuclear \\ Medicine-Molecular Imaging, Paris, FR; \\ ${ }^{3}$ University Hospital Nantes, Department of Nuclear Medicine, \\ Nantes, FR; \\ ${ }^{4}$ University of Nantes, CRCINA, Nantes, FR
}

Introduction: Hybrid PET/MR imaging will reveal its full clinical potential if we revisit the acquisition paradigm towards fully quantitative multi-parametric imaging and do not replicate what is currently performed on separate PET/CT and MR systems. Admittedly, fully quantitative MR imaging requires more time than in usual clinical practice, but it also offers the opportunity to perform dynamic PET imaging in parallel for parametric PET imaging. PET has the ability to deliver fully quantitative functional information of underlying imaged processes, by use of dynamic imaging and kinetic modelling. Dynamic PET can deliver unique biomarker information complementary to quantitative MRI, which can assist in clinical applications and delivery of precision medicine.

Oncological applications of PET/MRI often require imaging over the whole body for tumour staging but hybrid PET/MR scanners provide only a limited axial field of view. To increase the effective field-ofview, dynamic whole-body (DWB) protocols are used over multiple bed positions, at the cost of limitations in acquisition counts and sampling frequency. Advanced and dedicated processing tools are necessary to deal with these limitations. The objective of this work was to improve whole body multi-parametric imaging for PET/MRI applications by use of direct multi-bed dynamic reconstruction in PET.

Methods: A dynamic reconstruction platform was developed within the open source reconstruction software CASToR ${ }^{1}$, that can support direct reconstruction of multi-bed PET data and MRI-guided regularization. The spectral analysis dynamic model ${ }^{2}$ was used in the reconstruction process, because it does not impose any strong assumptions on the underlying kinetics and provides temporal regularisation of reconstructed data, enabling more flexibility for parametric imaging. The direct dynamic reconstruction was applied and evaluated on simulated PET FDG data and on a first in man whole-body pharmacological PET/MR study, using $\left[{ }^{11} \mathrm{C}\right]$ Glyburide to evaluate the role of OATP transporters in drugs pharmacokinetics ${ }^{3}$. Results: $K_{i}$ maps for FDG and $K_{1}$ maps for Glyburide were calculated. Comparison between regular frame by frame static 
reconstructions followed by post reconstruction parametric modelling and dynamic reconstruction of DWB data showed good agreement with no introduction of bias on evaluated metrics. Furthermore, the use of dynamic reconstruction resulted in noticeable noise reduction of activity and parametric images.

Discussion: We effectively applied DWB protocols for whole-body PET/MR parametric imaging. Findings showed that dynamic PET reconstruction is desirable in DWB parametric imaging to achieve accurate and precise quantification, while providing whole-body parametric images of comparable image noise to regular single bed dynamic protocols.

\section{References:}

${ }^{1}$ Merlin et al. PMB, vol. 63, pp. 185005, 2018.

${ }^{2}$ Cunningham et al. JCBFM, vol. 13, pp. 15, 1993.

${ }^{3}$ Marie et al. Neurology, vol.92, pp. 813, 2019.

\section{C2.03.}

\section{Cross-modal distillation in MRI for managing glioma patients}

${ }^{*}$ M. Rahimpour ${ }^{1}$, K. Goffin ${ }^{1}$, M. Koole ${ }^{1}$

${ }^{1}$ KU Leuven, Department of Imaging and Pathology, Leuven, BE

Introduction: MR imaging for clinical follow-up and pre-operative work-up of patients with glioma generally involves a contrast-enhanced T1-weighted (T1ce) sequence. Although the use of contrast agents (e.g., gadolinium) greatly enhances the visibility of tumor and facilitate the delineations of viable tumor tissue, it exposes the patient to higher risk and increases the scanning time [1]. Therefore, we proposed a cross-modal distillation approach for improved segmentation of the viable tumor using only a non-enhanced T1-weighted (T1w) MRI sequence.

Methods: Cross-modal knowledge and feature distillation ( $K D$ and $F D$ ) refer to the process of training a student model to achieve better performance by learning from the teacher model which has access to a privileged source of data [2]. For $K D$, the softened output of the teacher model was used in addition to the ground truth labels to train the student model such that the predictions by both models were matched. However, the softened outputs do not directly reflect the intermediate feature representations learned by the teacher. Therefore, the concept of $K D$ was extended to a more generic framework called $F D$ where the additional supervision was used to synchronize the intermediate activation maps between the teacher and student models. A deep learning segmentation model based on 3D U-Net was used to implement both teacher and student models. Given that the T1ce sequence is highly informative for segmenting the viable tumor, this sequence was used to train the teacher model, while the T1w sequence was the only data available for the student model during inference. For the evaluations, the BraTS 2018 dataset consisting of 285 multi-sequence MRI and an in-house dataset consisting of 37 subjects with LGG and HGG gliomas were used. Due to the limited size of the in-house dataset, transfer learning was applied.

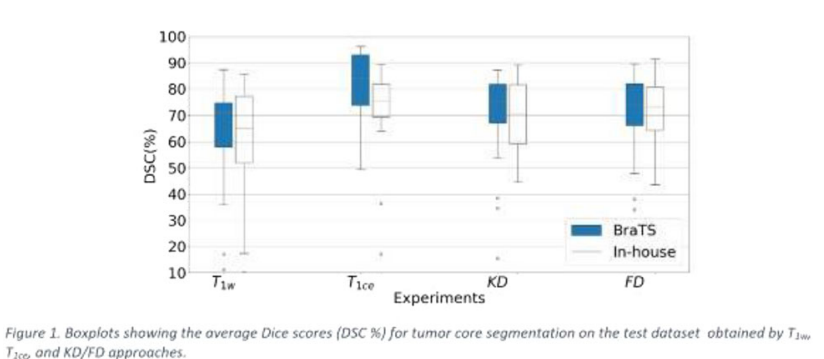

$T_{\text {zee }}$ and KD/FO approaches.

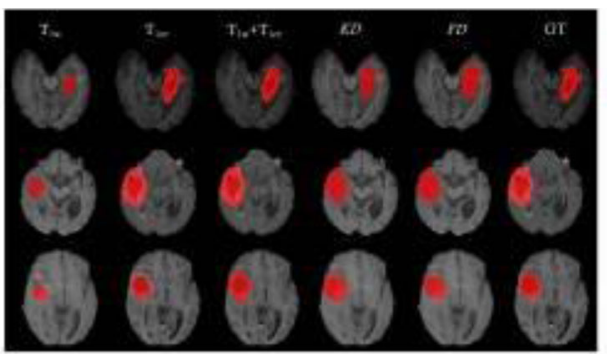

$(a)$

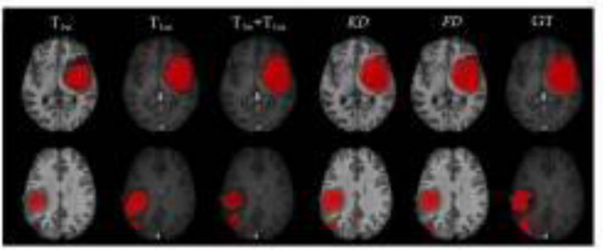

|b|

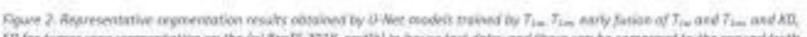

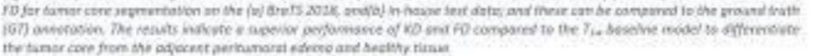

Results: $K D / F D$ approaches improved the segmentation Dice scores up to $8 \%$ compared to the baseline model trained by $\mathrm{T} 1 \mathrm{w}$ only and provided comparable segmentation performance with T1ce-based segmentations. For the clinical evaluations, segmentation results were consistent with the BraTS 2018 evaluation, especially when transfer learning was used to fine-tune the pre-trained models.

Discussion: Segmentation performance of $K D / F D$ using only T1wsequence closely approximated the performance of the model trained by T1ce-sequence. Therefore, this approach provides a valid, clinical alternative for accurate tumor core segmentation when the T1ce-sequence cannot be acquired because of scan time restrictions or safety reasons.

\section{References:}

[1] T. E. Smith, A. Steven, and B. A. Bagert, "Gadolinium deposition in neurology clinical practice, “ Ochsner Journal, vol. 19, no. 1, pp. 17-25, 2019.

[2] M. Rahimpour, J. Bertels, D. Vandermeulen, F. Maes, K. Goffin, M. Koole, "Improving T1w MRI-based Brain Tumor Segmentation Using Cross-modal Distillation,“ Medical Imaging 2021: Image Processing, vol. 11596, 2021. 


\section{C2.04.}

\section{Multi-parametric PET/MRI for radiation treatment planning of catients with cervical cancer}

*S. Ahangari ${ }^{1}$, A. B. $\operatorname{Olin}^{1}$, N. L. Hansen ${ }^{1}$, T. J. Nøttrup ${ }^{2}$, A. K. Berthelsen ${ }^{2}$, A. Loft $^{1}$, I. R. Vogelius ${ }^{2}$, A. Kjaer ${ }^{1,3}$ F. L. Andersen ${ }^{1,4}$, B. M. Fischer ${ }^{1,5,4}$, A. E. Hansen ${ }^{1,4,6}$

${ }^{1}$ Rigshospitalet, Department of Clinical Physiology, Nuclear Medicine and PET, Copenhagen, DK;

${ }^{2}$ Rigshospitalet, Department of Oncology/Section of Radiotherapy, Copenhagen, $D K$;

${ }^{3}$ University of Copenhagen, Cluster for Molecular Imaging, Copenhagen, DK;

${ }^{4}$ University of Copenhagen, Department of Clinical Medicine, Copenhagen, $D K$,

${ }^{5}$ King's College London, PET Centre/School of Biomedical Engineering and Imaging Sciences, London, DK;

${ }^{6}$ Rigshospitalet, Department of Diagnostic Radiology, Copenhagen, $D K$

Introduction: The concept of personalized medicine has brought increased awareness to the importance of inter- and intra-tumor heterogeneity for cancer treatment. Therefore, biological tumor characterization based on functional and molecular imaging might be highly valuable for radiotherapy (RT) planning to allow for an improved target volume definition and an individualized dose prescription within the tumor. The combination of PET and MRI can serve as a one-stop-shop RT planning session and provide measures of e.g., angiogenesis, vascularization and perfusion characteristics, and cellular density.

The aim of this study was to prove the potential and feasibility of multi-parametric hybrid PET/MRI to non-invasively describe tumor heterogeneity prior to chemoradiotherapy.

Methods: Eight patients with histologically proven primary cervical cancer were scanned $30 \mathrm{~min}$ after injection of $200 \mathrm{MBq} 68 \mathrm{Ga}$ NODAGA-E[c(RGDyK)]2. PET data were acquired simultaneously with T1 and T2 weighted TSE sequences, DW-MRI, and DCE-MRI. Standardized uptake value (SUV), apparent diffusion coefficient (ADC), and pharmacokinetic maps obtained with the extended Tofts model (Ktrans, Ve, and Vep) were generated for each dataset.

The primary tumor and a reference structure in the gluteal muscle were manually segmented on T2-weighted images by an experienced radiologist. The anatomical tumor delineation was used for correlation analysis between available pairwise combinations of functional and parametric maps on a voxel and a regional level.

Results: A representative image dataset of functional images and parameter maps of the primary tumor is presented in Fig. 1. MRIderived parameters and SUVmean were significantly different between tumor and reference tissue.

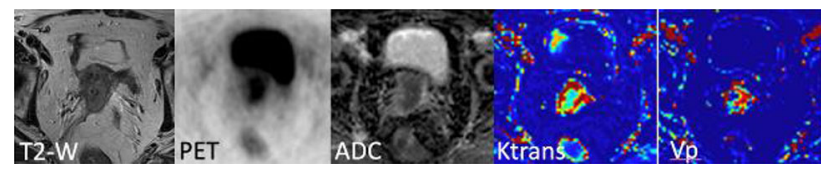

Correlation analysis on the regional level showed a moderate voxelwise correlation between RGD-PET and Ktrans (R2 = 0.3), while the correlation between RGD-PET and ADC map was weak. However, the respective correlation coefficients varied strongly within the patient cohort.

Discussion: The multi-parametric PET/MRI datasets suggests that a combined voxel-wise analysis of PET and MRI-derived parameter maps can contribute to a better characterization of tumor heterogeneity than the modalities alone. Our findings showed a tendency toward higher values of tumor perfusion in areas with more intense RGD uptake [1]. However, according to the literatures, tumor perfusion could also be simulated by FDG uptake and hypoxia, which may occur when the tumor blood flow is inadequate [2,3].

It needs to be stated that the current results are based on small patient numbers, but they might contribute to the future design of individually adapted treatment approaches based on multiparametric functional imaging.

\section{References:}

[1] Metz S, et al. J Nucl Med. 2010;51:1691-1698.

[2] Simoncic U, et al. Med Phys. 2017;44:2358-2368.

[3] Daniel M, et al. Radiotherapy and Oncology. 2017;125:420-425.

\section{C2.05.}

PET/MR imaging of the heart: is it possible to obtain synergistic effects?

*A. Villagran Asiares ${ }^{1}$, T. Vitadello ${ }^{2}$, E. L. Solari ${ }^{1}$, C. Munoz ${ }^{3}$,

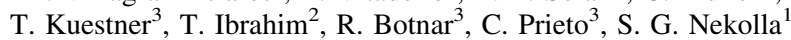

${ }^{1}$ Technical University of Munich, Department of Nuclear Medicine, Munich, DE;

${ }^{2}$ Technical University of Munich, Department of Internal Medicine I, Munich, DE;

${ }^{3}$ King's College London, Division of Imaging Sciences and Biomedical Engineering, London, $G B$

According to the World Health Organization, cardiovascular diseases are the main cause of death in the world, showing the need for substantial contributions by the new medical technologies. In the last decades the value of the information obtained by non-invasive cardiovascular imaging (Echo, MR, SPECT, PET/CT, PET/MR) is considerably increasing in clinical tasks such as diagnosis, prognosis, and therapy monitoring. However, to make significant contributions, it is crucial to mitigate the technical issues associated with cardiovascular imaging, and then to pursue a complete characterization of the disease. Physiological and physical factors such as heart motion during the image acquisition and partial volume effects impact on the quality of the images. Hybrid PET/MR systems allow us to take advantage of the strength of each modality, and due to the simultaneity of the acquisition, to accurately integrate the information to overcome the above issues.

Using FDG PET/MR images of a myocardial viability assessment protocol, different integrative studies were performed in cardiac and respiratory PET gating, PET partial volume correction based on MR derived anatomical references, and PET/MR based contractility recovery prediction after revascularization of chronic coronary total occlusion patients, seeking for parameters validation and potential clinically relevant synergistic effects.

Positive correlations between PET and MR values of morphologic and functional parameters of the left ventricle were found, but with a tendency of underestimation with PET that suggested a non-interchangeability between these modalities in the morphology assessment. Myocardium focused MR image-navigators and PET data-driven methods for heart tracking along the respiratory cycle showed similar results in respiratory motion correction. Hybrid PET/ MR multiparametric assessment presented an improvement beyond the standard analysis based on FDG uptake and LGE for the prediction of contractility recovery after revascularization. However, the prediction based on PET images corrected for partial volume effects was reduced.

Synergistic effects of cardiac PET/MR imaging were observed in myocardial viability assessment protocols, even though further 
studies are needed to evaluate the trade-off between improvement in the information and the added complexity to the complete workflow.

\section{C2.06.}

\section{Segmentation-guided liver registration for CT and MR images}

*X. Tang ${ }^{1}$, E. Jafargholi Rangraz ${ }^{2}$, R. 's Heeren ${ }^{1}$, W. Coudyzer ${ }^{3}$, C. Deroose ${ }^{1,4}$, K. Baete ${ }^{1,4}$, M. Gooding ${ }^{5}$, J. Nuyts ${ }^{1}$

${ }^{1}$ KU Leuven, Department of Imaging and Pathology, Leuven, BE; ${ }^{2}$ Quirem Medical B.V., Deventer, NL;

${ }^{3}$ Universitair Ziekenhuis Leuven, Department of Radiology, Leuven, $B E$;

${ }^{4}$ Universitair Ziekenhuis Leuven, Department of Nuclear Medicine, Leuven, BE;

${ }^{5}$ Mirada Medical Ltd, Oxford, GB

Introduction: Selective internal radiation therapy (SIRT) requires liver registration for $\mathrm{CT}$ and MR images to integrate multi-modality information for precise dose calculation. This study aims at investigating the feasibility of using liver segmentations from convolutional neural network $(\mathrm{CNN})$ and manual landmark segmentations to guide liver registration for $\mathrm{CT}$ and MR images.

Methods: An in-house image-based registration method was extended by incorporating segmentations of livers ( $\mathrm{CNN}$-guided) or both livers and landmarks (CNN\&LM-guided) for guidance. For the segmentation-guided methods, the deformable registration is initialized by an affine one based on segmentations instead of images. The deformable registration uses a combination of mutual information as the image similarity loss function $\left(L_{I}\right)$ and the sum of squared differences as the segmentation similarity loss $\left(L_{S}\right)$. To avoid topologyviolating deformation, the voxels are assumed to be connected via springs, which oppose distance changes. The spring rigidity determines the power of the regularization loss $\left(L_{R}\right)$. Therefore, the final loss function is $w_{I} L_{I}+w_{S} L_{S}+L_{R}$. Liver segmentations were generated by a CNN model in [1].

Twenty SIRT patients were selected for training and testing of the algorithms (see Table 1). Each patient has one Reference CT. Manual liver and landmark (mainly lesions) delineations were used for registration evaluation. The root mean squared distance (RMSD) of mean surface distance between livers and mass center distance between landmarks is used to evaluate each liver registration. The optimal $w I$ and $\mathrm{wS}$ for all registrations were determined by finding the minimal root mean square of RMSDs (RRMSD) through grid searches for the training datasets.

Fig. 1

TABLE 1: The training and test datasets for the registration experiment

\begin{tabular}{cccc}
\hline & \#patients & \# CT & \# MR \\
\hline Training & 10 & 10 & 14 \\
\hline Test & 10 & 10 & 12
\end{tabular}

Results: As shown in Fig. 1, the CNN-guided registration decreases the RRMSD by $3.4 \mathrm{~mm}$ (34\%) compared to the image-based one. The optimal performance of the CNN\&LM-guided registration was achieved when using only affine registration $\left(w_{I} \& w_{S}=0\right)$. The RRMSD is decreased by $1.4 \mathrm{~mm}(21 \%)$ after landmark guidance for the $\mathrm{CNN}$-guided registration.

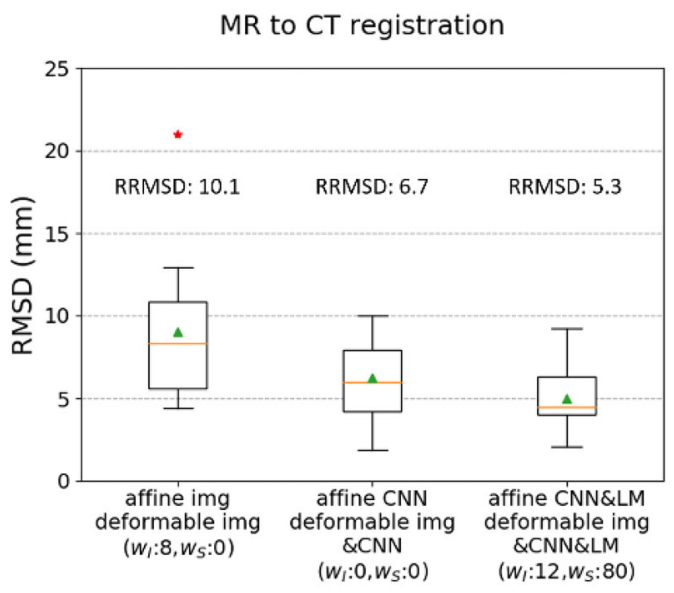

Fig.1 The results of the test datasets for comparison of image-based, CNNguided, and CNN\&LM-guided registrations using the optimal weights. The orange line, green triangle, and red star represent median, mean, and outliers, respectively. The lower and upper bounds of the box represent the first and third quartiles. The whiskers show the range (excl. outliers).

Discussion: The CNN-guided affine registration outperforms the image-based registration, since CNN segmentations help align the liver contours and exclude the disturbance from other organs. The $\mathrm{CNN}$-guided deformable registration degrades the affine registration. This might be caused by segmentation errors in some low-intensity lesion regions of the MR. These regions are deformed due to the surface matching of CNN segmentations. Landmark guidance shows its value of improving the $\mathrm{CNN}$-guided registration. Therefore, we will develop an automatic lesion segmentation to obtain a fully automated segmentation-guided registration.

This study is funded by EU Horizon 2020 research and innovation programme Marie Skłodowska-Curie grant 764458.

\section{Reference:}

[1] Tang X et al. "Segmentation-Guided Multi-Modal Registration of Liver Images for Dose Estimation in SIRT “ to be submitted to Eur J Nucl Med Mol Imaging.

\section{C2.07.}

\section{Prostate cancer staging based on multimodal imaging using deep learning}

*N. Capobianco ${ }^{1,2}$, L. Sibille ${ }^{3}$, M. Chantadisai ${ }^{4,5}$, A. Gafita $^{4}$, T. Langbein ${ }^{4}$, G. Platsch ${ }^{1}$, E. L. Solari ${ }^{4}$, V. Shah ${ }^{3}$, B. Spottiswoode ${ }^{3}$, M. Eiber ${ }^{4}$, W. Weber ${ }^{4}$, N. Navab ${ }^{6}$, S. G. Nekolla ${ }^{4}$

${ }^{1}$ Siemens Healthcare GmbH, Erlangen, DE;

${ }^{2}$ Technical University of Munich, Munich, DE;

${ }^{3}$ Siemens Medical Solutions USA, Inc., Knoxville, TN, DE;

${ }^{4}$ Technical University of Munich, Department of Nuclear Medicine, Munich, DE;

${ }^{5}$ Chulalongkorn University, Faculty of Medicine, Bangkok, DE;

${ }^{6}$ Technical University of Munich, Computer Aided Medical Procedures (CAMP), Munich, DE

Introduction: Multimodal imaging with Prostate Specific Membrane Antigen (PSMA)-ligand PET has shown high accuracy for prostate cancer staging both in PET/MRI [1] and PET/CT [2]. With rising adoption of PSMA-ligand imaging, standardized reporting frameworks and image-derived biomarkers are increasingly employed for reproducible and accurate image assessment. Nevertheless, clinical 
applicability is challenging in cases where manual measurements of numerous suspected lesions are required. In this context, automated image analysis methods are promising to enable replicable, timeefficient, and rigorous evaluation. We developed a deep learning method to support staging in PSMA-ligand imaging.

Methods: 173 patients with confirmed prostate cancer referred to

${ }^{68}$ Ga-PSMA-11 PET/CT were retrospectively analyzed. For each subject, an expert physician segmented regions of elevated tracer uptake, labeled them as physiologic or suspicious for prostate cancer, and assigned them an anatomical location classification. A multi-task convolutional neural network was trained to both classify elevated uptake sites as physiologic or suspicious and assign them an anatomical location, based on the combined PET/CT information. Additional training information was included from 629 patients with lymphoma or lung cancer, imaged with ${ }^{18}$ F-FDG PET/CT and analogously annotated in a former investigation [3], encoding the radiotracer type as network input. A hold-out test set of 52 prostate cancer patients was used exclusively to evaluate the network performance. Moreover, in the test subjects we evaluated the agreement between $\mathrm{N}$ and $\mathrm{M}$ stage assigned based on the network annotations and expert annotations according to the PROMISE miTNM standardized framework [4].

Results: In the hold-out test set of prostate cancer patients, the convolutional neural network yielded $80.4 \%$ average precision [confidence interval (CI): 71.1-87.8], $81.1 \%$ sensitivity (CI: 70.6-90.1), 66.8\% positive predictive value (CI: 60.3-72.7) for identification of suspicious uptake sites; 77\% (CI: 70.0-83.4) accuracy for anatomical location classification of suspicious findings, $81 \%$ agreement for identification of regional lymph node involvement, 77\% agreement for identification of metastatic stage.

Discussion: The evaluated method was able to automatically identify sites of tracer uptake suspicious for prostate cancer and classify their anatomical location in good agreement with the expert assessment. Image quality characteristics may influence the network output and results will require expert supervision for use in clinical context. The investigated method is promising for enabling efficient assessment of cancer stage and tumor burden based on multimodal PSMA-ligand imaging.

\section{References:}

1. Grubmüller B, et al. Clinical Cancer Research. 2018;24:6300-7.

2. Hofman MS, et al. The Lancet. 2020;395:1208-16.

3. Sibille L, et al. Radiology. 2020;294:445-52.

4. Eiber M, et al. Journal of Nuclear Medicine. 2018;59:469-78.

\section{C2.08.}

MRI visible polymers for PET/MR phantoms

\author{
*I. Rausch ${ }^{1}$ \\ ${ }^{1}$ Medical University of Vienna, Center for Medical Physics \\ and Biomedical Engineering, Vienna, AT
}

Introduction: Physical imaging phantoms are essential tools for the evaluation of imaging system performance and to test novel imaging methods. A variety of phantoms exist for the different imaging modalities. For MRI these phantoms are mostly based on liquid-filled compartments or gel-based components as most solids do not present a reasonable imaging signal in standard MRI. This challenges the production of phantoms with controlled MR contrast and use of such phantoms in multi-modality imaging. For example, in PET/MRI phantom studies specific attenuation templates are needed to account for the attenuation of the phantom housing during attenuation correction (AC) as it cannot be visualized using MRI.

To tackle these challenges we investigated the use of MRI visible polymers as phantom building material in PET/MR phantoms. Here we report on results of first tests using a commercially available 3D printable, MR visible material for a PET/MRI phantom and the development of an UV curable resin composition, presenting with an adjustable MRI signal in standard MRI sequences.

Methods: A water fillable phantom was 3D printed with a commercially available, MRI visible polymer (RGD525, Stratasys, USA). A phantom measurement was done in a PET/MRI system and PET reconstructions were performed using a CT based AC as reference and using an MRI based AC incorporating the phantom housing. As an alternative to the commercially available material we introduced oil in variable amounts into a UV curable resin. Thereof, probes were produced and the MRI visibility was tested in a 3 T MRI.

Results: Using a MRI based AC incorporating the phantom housing, average deviations in PET quantification to CT based AC were below $1 \%$. The UV curable rein showed oil content depending MRI signals in standard MRI sequences.

Discussion and conclusion: MRI visible polymers can be used as phantom building material to enable MR based AC in PET/MRI phantom experiments. The UV curable resin was adjustable resulting in different MRI signal intensities, and thus, may be suitable for applications beyond MRI visible phantom housings such as the simulation of tumour heterogeneity. 


\section{C3.01.}

\section{Perfluorocarbons for in vivo use: stabilisation in aqueous environments}

\author{
*N. Larreina ${ }^{1}$, N. Scanu ${ }^{2}$, J. Ruiz-Cabello ${ }^{2}$, M. Carril Garcia ${ }^{3}$, \\ H. Lamb ${ }^{1}$, M. Srinivas ${ }^{4,5}$ \\ ${ }^{1}$ Leiden University Medical Center, Department of Radiology, Leiden, \\ $N L$; \\ ${ }^{2}$ CIC BiomaGUNE, Department of Molecular and Functional \\ Biomarkers, San Sebastian, ES; \\ ${ }^{3}$ University of the Basque Country, Department of Biochemistry \\ and Molecular Biology, Bilbao, ES, \\ ${ }^{4}$ Cenya Imaging B.V., Arnhem, NL; \\ ${ }^{5}$ Wageningen University \& Research, Department of Cell Biology \\ and Immunology, Wageningen, $N L$
}

Introduction: Perfluorocarbons (PFCs) have a long and varied history of clinical use, either as blood substitutes or imaging agents [1]. Their strong hydrophobicity and lipophobicity are a major hindrance to their formulation and manufacturing. Currently, liquid PFCs are gaining interest as imaging agents for fluorine-19 Magnetic Resonance Imaging (19F MRI), particularly for cell tracking [2]. Upscaled production can be difficult, especially at GMP-grade, being the conventional drying method of freeze-drying a rate limiting step [3].

Methods: We reviewed the literature to assess PFC formulation and production techniques, focusing on more efficient upscaled production. We tested some of these techniques with different formulations. Results: High-pressure homogenization, ultrasonication and microfluidization techniques are the most effective methods for PFC encapsulation. While ultrasonication is often an effective and versatile option for small-scale emulsification processes, microfluidization and high-pressure homogenization are capable of producing higher volumes, showing a good reproducibility and size distributions. Stabilization techniques for the aqueous storage of PFC formulations include electrosterical stabilization or the addition of hydrophobic compounds in the majority of the cases. Drying and rehydration processes also involve structural damage risk, so parameters like glass transition temperature of the encapsulating agents or vapour pressure of the PFC need to be considered to choose the right method.

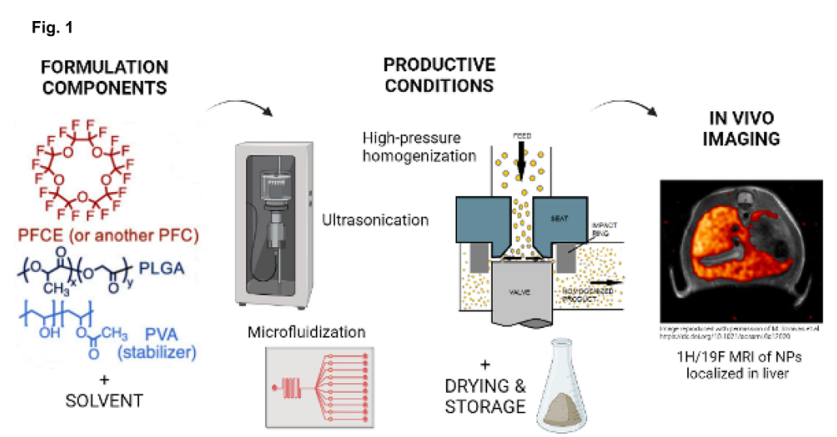

Discussion: The literature and our data show that upscaling and stable formulations are possible if the certain combination of formula, operating parameters and production regime are found. Further research in drying of PFCs is needed to reduce the costs involved with production.

\section{References:}

1. Castro, C. I., \& Briceno, J. C. (2010). Perfluorocarbon-based oxygen carriers: Review of products and trials. In Artificial Organs (Vol. 34, Issue 8, pp. 622-634). 10.1111/j.1525-1594.2009.00944.x
2. Staal, A. H. J., Becker, K., Tagit, O., Koen van Riessen, N., Koshkina, O., Veltien, A., Bouvain, P., Cortenbach, K. R. G., Scheenen, T., Flögel, U., Temme, S., \& Srinivas, M. (2020). In vivo clearance of $19 \mathrm{~F}$ MRI imaging nanocarriers is strongly influenced by nanoparticle ultrastructure. Biomaterials, 261, 120307. 10.1016/ j.biomaterials.2020.120307

3. Hoogendijk, E., Swider, E., Staal, A. H. J., White, P. B., van Riessen, N. K., Glaßer, G., Lieberwirth, I., Musyanovych, A., Serra, C. A., Srinivas, M., \& Koshkina, O. (2020). Continuous-Flow Production of Perfluorocarbon-Loaded Polymeric Nanoparticles: From the Bench to Clinic. ACS Applied Materials \& Interfaces, 12(44), 49335. 10.1021/acsami.0c12020

\section{C3.02.} 19F-nanoparticles: successful platform for in vivo
delivery of fluorinated biomaterials for 19F-MRI

\author{
*A. Mali ${ }^{1}$, E. L. Kaijzel ${ }^{1}$, H. Lamb ${ }^{1}$, L. J. Cruz ${ }^{1}$ \\ ${ }^{1}$ Leiden University Medical Center, Department of Radiology, Leiden, \\ $N L$
}

Introduction: Fluorine-19 Magnetic Resonance Imaging (MRI) features one of the most investigated and innovative techniques for quantitative and unambiguous cell tracking. A high number of magnetically equivalent fluorine atoms are required to gain detectable signals. However, an increased amount of ${ }^{19} \mathrm{~F}$ nuclei induces low solubility in aqueous solutions, making fluorine-based probes not suitable for in vivo imaging applications. In this context, nanoparticle-based platforms play a crucial role, since nanoparticles may carry a high payload of 19F-based contrast agents, increase the imaging agent"s biocompatibility, and provide a highly versatile platform.

Methods:We reviewed the literature in order to investigate the main nanotechnologies employed to date for sensitive 19F-MRI purposes, such as fluorine and theranostic nanovectors, including their design and applications (Fig. 1).

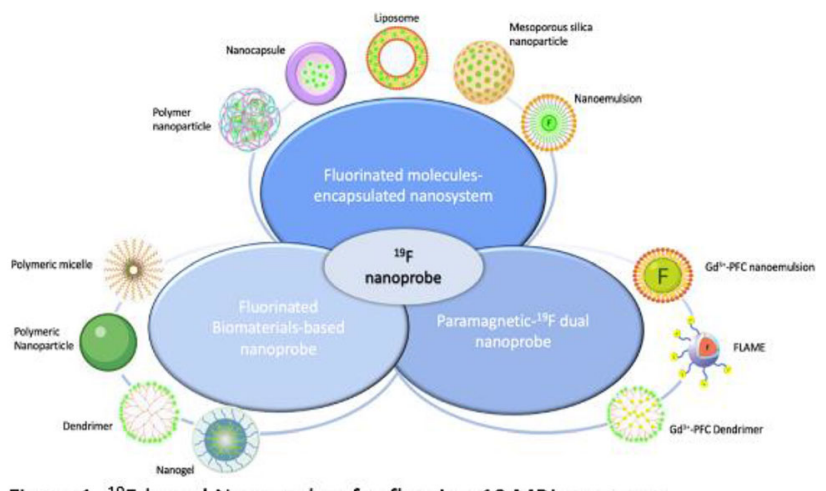

Figure 1. ${ }^{19} \mathrm{~F}$-based Nanoprobes for fluorine-19 MRI purposes.

Results: The most commonly used tracers for 19F-MRI are perfluorocarbons. Nanotechnologies are vital for using perfluorocarbons. Indeed, due to their immiscibility with organic and inorganic solvents, perfluorocarbons need to be emulsified or incorporated into polymer or lipid nanoparticles by vigorously mixing a water solvent and PFCs in the presence of a surfactant, and lastly by applying high pressure homogenization or sonication (Fig. 2). Fluorinated biomaterial-based nanoprobes, such as fluoropolymers, copolymers and lipids are a further pioneering and promising class, characterized by tailorable properties which enable them to be ideal for many biomedical 
applications,such as theranostic purposes or functional imaging in response to specific changes in physiological stimuli. Moreover, they hold a high number of fluorine atoms causing a high ${ }^{19} \mathrm{~F}$-MRI signal, and a low cytotoxicity. In order to address the sensitivity issue of ${ }^{19} \mathrm{~F}$ MRI techniques, other types of nanoprobes have been designed, aimed at improving relaxation properties of PFC molecules. In this regard, the combination of a paramagnetic center (e.g. $\mathrm{Mn}^{2+}, \mathrm{Fe}^{3+}$, $\left.\mathrm{Gd}^{3+}\right)$, with a $19 \mathrm{~F}$ nanoprobe, reduces ${ }^{19} \mathrm{~F}$ T1 values for a rapid image acquisition and increases the signal-to-noise ratio per unit time ${ }^{1}$.

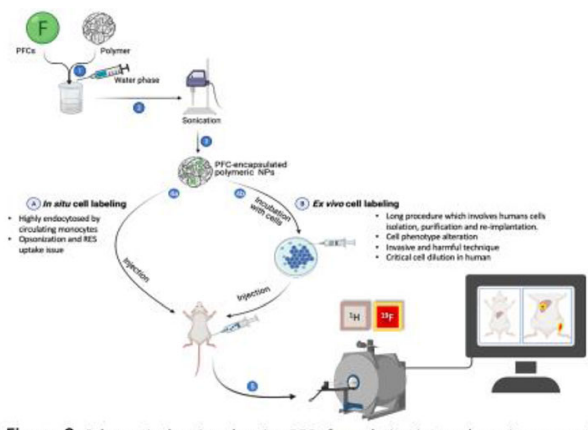

Figure 2. Schematic drawing showing PFCs formulation into polymeric nanoparticles and their application for both $\underline{e x}$ vivo and in situ cell labeling and tracking, including the main drawbacks of the two strategies. Perfluorocarbons and polymers are dissolved in organic solvent, mixed with the water phase containing the emulsifying agent (1), and ultimately nanoparticles are formed by high pressure homogenization or sonication (2). PFC-encapsulated Nanoparticles (3) may either be directly injected in vivo (4a) for tracking cells by ${ }^{19} \mathrm{~F}-\mathrm{MRI}$ or incubated with isolated and purified cells (4b) and, in the final analysis, administered to mice, imaged usin ${ }^{19} \mathrm{~F}$ and ${ }^{\mathrm{H}} \mathrm{H}$-MRI in the same image session. The obtained images are overlaid so as to anatomically localize the nanoparticles.

Discussion: The literature showed that PLGA-based nanoprobes are a very promising ${ }^{19} \mathrm{~F}$ probes developed to date. PLGA nanoparticles have been employed for both small fluorinated molecule encapsulation $^{2}$ and fluorine attachment ${ }^{3}$ in order to synthesize the ${ }^{19} \mathrm{~F}$ copolymer.

\section{References:}

1. Chalmers, Kirsten $\mathrm{H}$ et al. "19F-lanthanide complexes with increased sensitivity for 19F-MRI: optimization of the MR acquisition." Magnetic resonance in medicine (2011).

2. Srinivas, Mangala et al. "Customizable, multi-functional fluorocarbon nanoparticles for quantitative in vivo imaging using 19F MRI and optical imaging." Biomaterials (2010).

3 Neri, G. et al. Fluorinated PLGA Nanoparticles for Enhanced Drug Encapsulation and 19F NMR Detection.Chem.A Eur.J. (2020).

\section{C3.O3.}

\section{MR atherosclerosis imaging using targeted fluorinated probes}

\author{
${ }^{*}$ N. Scanu ${ }^{1}$, M. Carril Garcia ${ }^{2}$, J. Ruiz-Cabello ${ }^{1}$ \\ ${ }^{1}$ CIC BiomaGUNE, Department of Molecular and Functional \\ Biomarkers, San Sebastian, ES; \\ ${ }^{2}$ Basque Center for Biophysics (Biofisika), Biochemistry \\ and molecular biology, Leioa, ES
}

Introduction: Atherosclerosis is a vascular inflammatory disease characterized by the development of fatty plaques in the intimal layer of arteries, hence becoming the underlying cause of strokes and heart attacks. Thereby, it represents one of the most prevalent causes of patient mortality in the western world.

Early diagnosis of inflammation and plaque development in atherosclerosis is yet a challenge, but it can be accomplished using nanotechnology, by developing specific targeting contrast agents to the damaged area. Among common techniques applied in the detection of cardiovascular diseases, 19F MRI presents the unique combination of quantitative non-invasive molecular imaging of biological processes and safety due to lack of ionizing radiation, thus being an optimal candidate for atherosclerosis diagnosis.

Methods: As perfluorocarbons-based contrast agents, we will use several compounds with a single resonance frequency and with high local concentration of fluorine atoms for good signal to noise ratio. Envisaged designs for MRI probes will exhibit the fluorine label attached to polymeric NPs" surface through a water soluble linker, also, we will use fluorine probes (PERFECTA, PFCE) encapsulated inside micelles or liposomes emulsions.

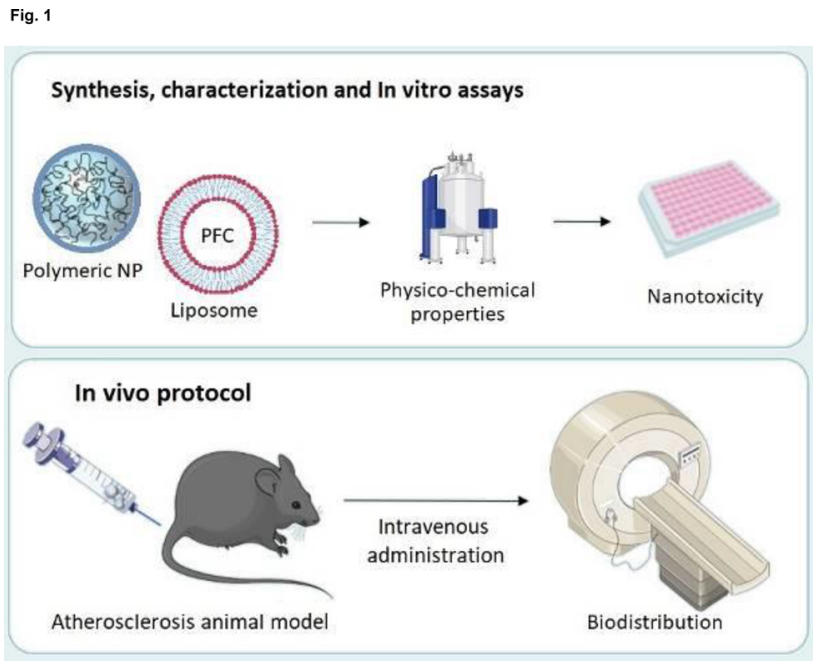

Once generated, physico-chemical characterization of fluorinated formulations can be assessed by $1 \mathrm{H}$ and 19F-NMR, TEM, DLS, whilst cell viability and nanotoxicity will be evaluated by hemolysis, MTT and ROS assays.

Contrast agents targeting can be achieved using different atherosclerosis biomarkers attached to the fluorinatd probes, in order to obtain specific imaging of thrombi.

Afterwards, preclinical experimentation will be conducted by using 19F MRI on high magnetic field (7 T) to evaluate probes biodistribution and in vivo fate. Animal models will be developed by ApoE knockout mice fed with high-fat diet to effectively generate atherosclerotic plaques in the main arteries.

Discussion: 19F-MRI represents a promising platform for early and sensitive diagnosis of atherosclerosis. However, the correct stabilization of fluorine-based formulations in aqueous environments is key for their real application in clinics. Future work in this field will involve strategies to overcome this challenge and develop groundbreaking contrast agents.

\section{References:}

Jia T et al., Front. Cardiovasc. Med. 7:588075, (2020).

Palekar RU et al., Nanomedicine 10, 11 (2015). 


\section{C3.04.}

\section{An editing method for imaging different perfluorocarbons at low magnetic field}

\author{
*J. Parsa ${ }^{1,2}$, A. G. Webb ${ }^{1}$ \\ ${ }^{1}$ Leiden University Medical Centre, Department of Radiology, Leiden, \\ $N L$ \\ ${ }^{2}$ Percuros B.V., Leiden, $N L$
}

Introduction: ${ }^{19} \mathrm{~F}$ is the nucleus with the second highest gyromagnetic ratio. A large number of different applications of in vivo ${ }^{19} \mathrm{~F}$ magnetic resonance have been shown ${ }^{1}$. Several different perfluorocarbons have been used including perfluoro-15-crown-5-ether (PCE) ${ }^{2}$, perfluoropolyether $(\mathrm{PFP})^{3}$, and perfluoro-octylbromide (PFOB $)^{4}$. Recently we showed that it is possible to acquire rapid images of perfluorocarbons on a very low-field system ${ }^{5}$, taking advantage of the very small spectral dispersion of even multi-peak compounds, the short $T_{1}$ relaxation time, and the ability to increase the effective $T_{2}$ in scalar coupled systems using very short interpulse CPMG sequences. Here we extend this work to design sequences which can be used to differentiate between molecules with different scalar coupling behaviour, as well as ones with no scalar coupling.

Methods: The system operating at $50 \mathrm{mT}$ has been described in detail previously ${ }^{5}$, as has the solenoid coil which can image both ${ }^{1} \mathrm{H}$ and ${ }^{19} \mathrm{~F}$ via operation of a mechanical switch. PFOB, fomblin and PCE were studied (Fig. 1). Each sample was placed in a $1.5 \mathrm{~mL}$ cylindrical tube. The $T_{1}$ values were measured via a spectroscopic inversion-recovery sequence, and the $T_{2}$ as a function of inter-pulse delay in a CPMG sequence. The first sequence is a spin-echo with minimum echo time, meaning that essentially a fluorine-density image is obtained. The second sequence is also a spin-echo with a long echo time, which produces very low SNR from PFOB and fomblin samples. The third sequence uses a CPMG preparation module with very short interpulse delay, which results in high signal from PFOB and PCE, but low signal from fomblin due to their different behaviour regarding scalar coupling rephasing. By combining the signal intensities from the three sequences, images can be produced primarily from each of the three species.

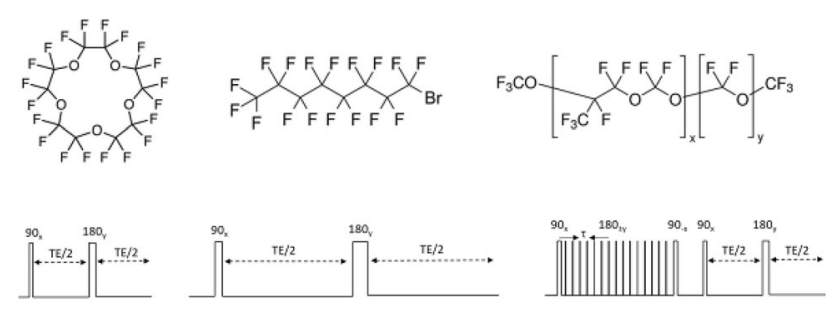

Figure 1. Top row: chemical structures of the three perfluorinated liquids used in this study. (top-left) Perfluoro-crown-ether, (top-centre) perfluoro-octylbromide, and (top-right) fomblin. Bottom row: Three sequences were used to acquire edited ${ }^{19} \mathrm{~F}$ images. (bottom-left) Sequence 1 - spin echo with very short E, (bottom-centre) Sequence 2 - spin echo with long TE, (bottom-right) Sequence 3 - spin-echo with sho $\mathrm{TE}$, preceded by a CPMG magnetization preparation module with short tau and $90^{\circ}$ flip back pulse.

Results: The measured $\mathrm{T}_{1}$ values were $530 \mathrm{~ms}$ (PCE), $720 \mathrm{~ms}$ (PFOB), and $85 \mathrm{~ms}$ (fomblin). Figure 2 shows the apparent $\mathrm{T}_{2}$ values as a function of inter-pulse delay in a CPMG sequence. The strong dependence for PFOB is shown, along with a weaker one for fomblin, and no dependence for PCE. Figure $3 \mathrm{a}-\mathrm{c}$ shows images from three sequences. Figure $3 b$ essentially produces an edited image, with signal from only the PCE, due to the short apparent $\mathrm{T}_{2}$ value of the other two compounds when a long interpulse delay is used. Figure $3 \mathrm{c}$ shows the effect of the CPMG preparation module, resulting in almost complete suppression of the fomblin signal as desired. Figure $3 \mathrm{~d}$, e show the results of subtraction of the relevant images to produce images corresponding to fomblin and PFOB only.

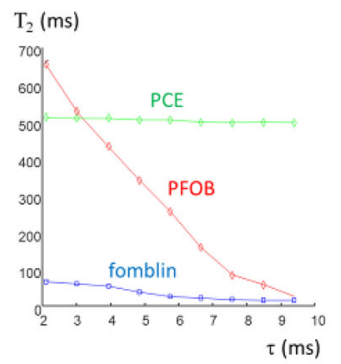

Figure 2. Apparent $\mathrm{T}_{2}$ as a function of inter-pulse delay for a CPMG sequence for all three compounds. (a)
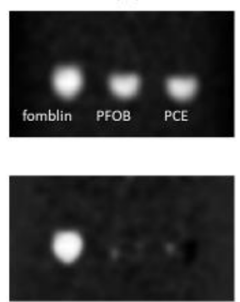

(d) (b)
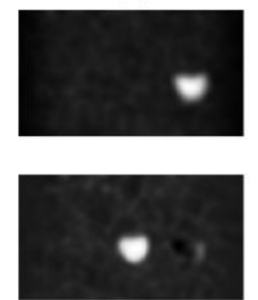

(e) (c)

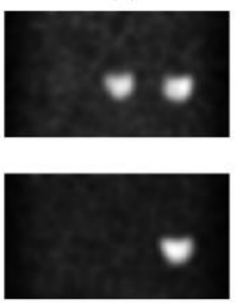

(f)
Figure 3. (top row) Images produced by (a) short TE spin echo sequence, (b) long TE spin echo sequence, (c) CPMG magnetization prepared short TE spin echo sequence. (bottom row) Images for each individual species produced by: (d) fomblin only corresponding to (a-c), (e) PFOB only image corresponding to (c-b), and PCE-only image corresponding to (b).

Conclusion: A simple editing scheme has been designed to differentiate between various perfluorocarbons based upon their scalar coupling properties. The scheme is currently based on spin-echo sequences, but will be extended to more time-efficient turbo-spin echo sequences.

\section{References:}

${ }^{1}$ Waiczies (2019).

${ }^{2}$ Dardzinski (1994).

${ }^{3}$ Jirak (2019).

${ }^{4}$ Giraudeau (2010).

${ }^{5}$ Parsa (2021). 


\section{S1.P1.}

Image registration and subtraction to improve lymphatic vessel conspicuity in dynamic contrastenhanced magnetic resonance lymphangiography in the legs of primary lymphoedema patients

*M. Mills ${ }^{1}$, M. Van Zanten ${ }^{1}$, J. Pearce ${ }^{2}$, B. $\mathrm{Ho}^{2}$, K. Gordon ${ }^{2}$, P. Mortimer ${ }^{1}$, P. Ostergaard ${ }^{1}$, F. A. Howe ${ }^{1}$

${ }^{1}$ St. George's University of London, Department of Molecular and Clinical sciences, London, GB;

${ }^{2}$ St. George's Hospital, Department of Dermatology, London, GB

Introduction: The lymphatic system is pivotal in whole-body fluid homeostasis and immunity. Lymphatic failure causes fluid accumulation within tissue (lymphoedema), with primary lymphoedema (PL) originating from an inherited defect.

Magnetic Resonance lymphangiography (MRL) offers improved spatio-temporal resolution and three-dimensional (3D) depiction of lymphatic vessels (LVs), compared to the $2 \mathrm{D}$ images of the main lymphatic trunks produced with routine lymphoscintigraphy. We investigated whether co-registration and baseline-subtraction of dynamic contrast-enhanced MRL (DCE-MRL) improved LV depiction and diagnostic potential.

Methods: Four PL and four healthy volunteers (HV) legs were imaged with 3D T2 weighted (turbo spin echo, TR/TEeff $=2800$ / $565 \mathrm{~ms}$, voxel $=2 \times 2 \times 3 \mathrm{~mm}^{3}$ ), and pre and post-contrast 3D T1 weighted (spoiled gradient echo, TR/TE/FA $=3.7 / 1.6 \mathrm{~ms} / 12 \mathrm{o}$, voxel $=1 \mathrm{~mm}^{3}$ ) sequences on a $3.0 \mathrm{~T}$ Philips dual Tx Achieva system and 16-element torso coil. Contrast was delivered intradermally to the inter-digital spaces of the feet, after which $\geq 30 \mathrm{~min}$ DCE imaging occurred. Field of view and imaging time varied, however T1 volumes ( $\sim$ ankle to knee) were typically acquired in $3-4 \mathrm{~min}$. DCEMRL datasets were registered to the first post-contrast phase (PC1), using an affine or deformable approach (NiftyReg, Modat et al., 2010), or left unregistered. Default parameters for affine registration, and bending energy (a penalty term promoting smooth deformation $)=10 \%$ and grid spacing $=8$ voxels for elastic registration, were used as previously reported (Borri, 2018). Subtracting PC1 from all subsequent dynamics after registration was also performed.

Results: All PL participants demonstrated torturous and/or enlarged LVs (max diameter $\sim 3 \mathrm{~mm}$ ) without post-processing (Figs. 1A, 2A), while in $2 \mathrm{HV} \mathrm{limbs} \mathrm{LVs} \mathrm{were} \mathrm{clear.} \mathrm{Additional} \mathrm{LVs} \mathrm{emerged}$ post-registration and subtraction in 2 PL participants (Fig. 1B-D) and in HVs previously not depicting LVs (Fig. 3). A single reviewer rated the three pipelines and ranked deformable registration as producing subtraction images of highest quality in $3 \mathrm{PL}$ and $2 \mathrm{HV}$ cases. However, in cases with substantial limb motion (rotation and translation) registration reduced $\mathrm{LV}$ conspicuity (1 PL and $2 \mathrm{HV}$ ). Baseline-subtracted uptake curves were also assessed as being smoother post-registration (Fig. 2).

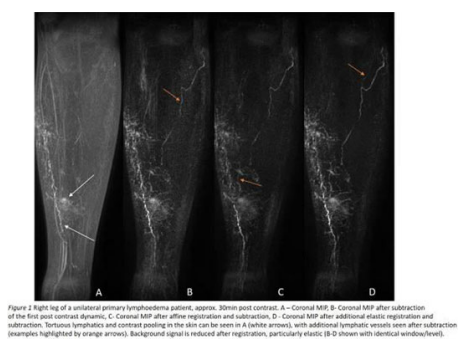

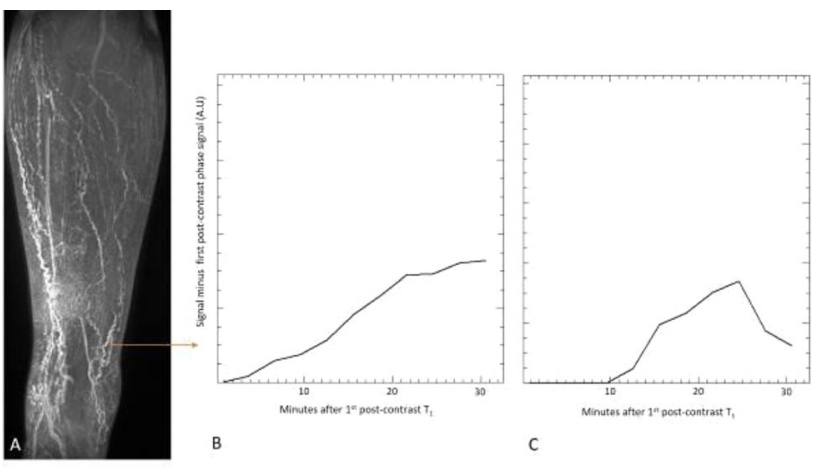

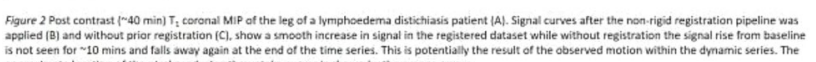

3 not seen for 10 mins and falls aws a agin at the end of the time sefies. This is potentially

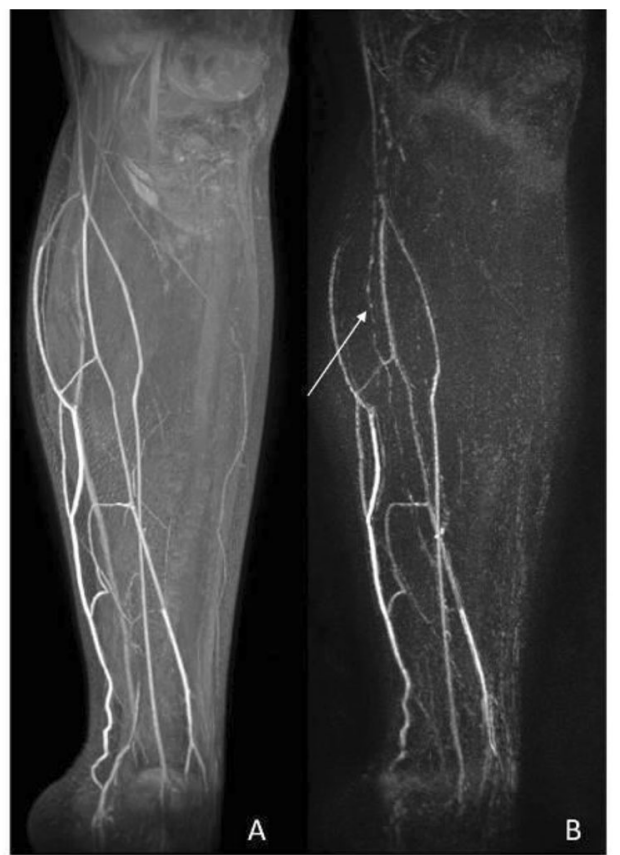

Figure 3 Sagittal T, MIP of the leg of a healthy participant showing only venous structures before further processing (A) and a possible lymphatic vessel (arrow) adjacent to a vein after registration with the elastic registration pipeline and subtraction (B). Images acquired approx. $20 \mathrm{~min}$ post contrast.

Discussion: Baseline subtraction of DCE-MRL images can increase LV conspicuity in both HV and PL participants and may be improved with prior registration, however substantial whole-limb movement may not be sufficiently compensated.

\section{References:}

Borri, M. (2018) Magnetic resonance for breast cancer related lymphoedema: clinical implementation of a novel quantitative imaging and analysis protocol. University of London.

Modat, M. et al. (2010) "Fast free-form deformation using graphics processing units“, Computer Methods and Programs in Biomedicine, 98(3), pp. 278-284. https://doi.org/10.1016/j.cmpb.2009.09.002. 


\section{S1.P2.}

\section{Dflow analysis in vascular pathology of the head and neck}

*M. Del Pópolo ${ }^{1,2,3}$, J. Capó Vera ${ }^{1,2,3}$, N. N. Massaccesi Bove ${ }^{1,2,3}$, T. González Padin ${ }^{1,2,3}$, N. Moyano Brandi ${ }^{1,2,3}$, C. Lisazo ${ }^{1,2,3}$, F. González N. ${ }^{1,4,5}$, R. Isoardi ${ }^{1,4,5}$, P. Ariza ${ }^{1,3}$, D. Fino ${ }^{1,3,5}$

${ }^{1}$ Fundación Escuela de Medicina Nuclear, Department of Magnetic Resonance Imaging, Mendoza, AR;

${ }^{2}$ University of Mendoza, Faculty of Engineering, Mendoza, AR;

${ }^{3}$ Fundacion Argentina para el Desarrollo en Salud, Department of Magnetic Resonance Imaging, Mendoza, AR;

${ }^{4}$ Comisión Nacional de Energía Atómica, GQNYCS, Buenos Aires, $A R$;

${ }^{5}$ National University of Cuyo, Balseiro Institute, San Carlos de Bariloche, $A R$

Introduction: 4DFlow has become an important tool in magnetic resonance (MR) imaging for the clinical evaluation of patients with vascular diseases. This sequence has been specifically designed to extract information from 3D phase-contrast MR (PC), and thus obtain quantitative information of the flow velocity in each cartesian plane. The purpose of this study is to evaluate the performance of this technique in vascular pathologies of the head and neck.

Methodology: This prospective study was approved by the Institutional Review Board. The study comprised 7 patients ( 3 women, 4 men; mean age: 50.9; range: 38-78 years). The MR images were acquired with a $1.5 \mathrm{~T}$ Ingenia (Philips) through a GRE sequence with slice thickness: $3 \mathrm{~mm}$, matrix: $120 \times 120$, ET/RT: $3.42 / 5.28 \mathrm{~ms}$ and a flip angle: $12^{\circ}$. A standard protocol was performed following the medical order (3 patients with a cerebrovascular accident -CVA-, 1 with vestibular schwannoma -VS- and 3 with arteriovenous malformation -AVM-), including the acquisition of 4DFlow.

Two specialist neuro-radiologists segmented the lesions and traced at least two ROIs in each segment to study the vascular flow (Fig. 1). The blood dynamics differences between healthy (HT) and pathological tissue were analyzed taking into account the hemodynamic response function (HRF). Using a Python algorithm (Qt, VTK and SimpleITK), the visualization was integrated into the 3DSlicer platform (v. 4.11) through vectorial DICOM-RGB encoded images.

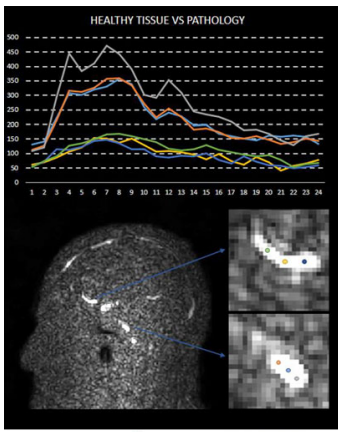

Figure 1: Saccular dilatation at the level of the

Results: A change in the amplitude and behavior of the HRF were evidenced in 2 subjects with CVA, 2 patients with AVM and the subject with VS (Fig. 2). The RGB encoding allowed us to verify the correct directionality of the vascular flow in all HT ROIs, generating turbulent areas with homogeneous speed in the studied pathologies (Fig. 3).

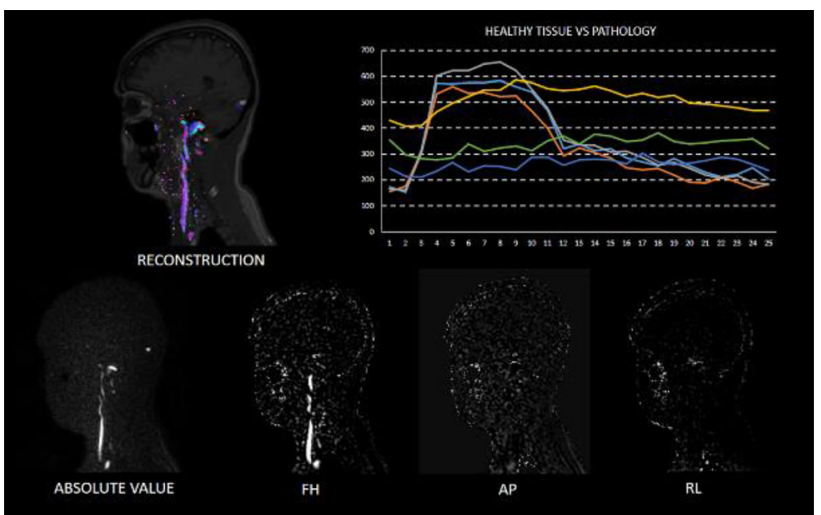

Figure 2: Sequelae of vestibular neurinoma removal. Python recontruction, velocity analysis and components visualization

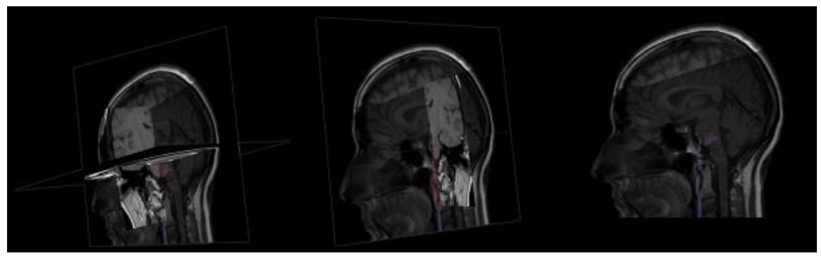

Figure 3: Patient with VS, 40 FLOW proiections using OT VTK and SIMPLE ITK Python libraries.

Conclusions: The 4DFlow is a technique that generates additional information to the anatomical vascular sequences, quantifying information that can be used to determine new standardizations in the evaluation of vascular pathology. Implementing numerical methods like second order Runge Kutta, the pressure exerted by the vascular walls can be quantified through the analysis of temporal changes of the blood velocity, which can be used to assess diseases associated with the elastic properties of the vascular bundle.

\section{References:}

Wymer, David T., et al. "Phase-Contrast MRI: Physics, Techniques, and Clinical Applications." RadioGraphics, vol. 40, no. 1, Jan. 2020, pp. 122-40. DOI.org (Crossref), https://doi.org/10.1148/rg.2020 190039.

Schnell, Susanne, et al. "Four-Dimensional MRI Flow Examinations in Cerebral and Extracerebral Vessels-Ready for Clinical Routine?" Current Opinion in Neurology, vol. 29, no. 4, Aug. 2016, pp. 419-28. DOI.org (Crossref), https://doi.org/10.1097/WCO.000000000000 0341.

\section{S1.P3.}

Prostate index-lesion segmentation using U-NET: impact of T2w and ADC

\section{M. Venancio ${ }^{1}$, A. Guerra ${ }^{2}$, R. Conceição ${ }^{3}$, F. Leite ${ }^{1}, * N$. A. da Silva $^{1}$ \\ ${ }^{1}$ Hospital da Luz, Department of Learning Health, Lisbon, PT; ${ }^{2}$ Hospital da Luz Lisboa, Imaging Department, Lisbon, PT; \\ ${ }^{3}$ University of Lisbon, Lisbon, PT}

Introduction: Multiparametric MRI (mp-MRI) holds promise to characterize lesions, and predict patient outcomes. However, localize/ stage the lesions in mp-MRI is a time consuming process. Automatic 
segmentation would reduce analysis times and would potentiate radiomics analysis. Here we explore the U-Net architecture for lesion segmentation with mp-MRI data and evaluate the impact of mp-MRI data.

Methods: Data Description: Data from 28 patients with biopsy-confirmed tumor were included. Data Acquisition: Data were acquired in a 3 T MR equipment (Siemens Healthineers). MR sequence were: (1) Axial 2D-T2wTSE, (2) DWI b $=50,1000,2000)$, ADC images were calculated within the equipment workflow. Manual Segmentation: Prostate and index lesions were manually segmented by a senior radiologist. Segmentations were performed on T2w and ADC images. Algorithms: Both images (T2w and ADC) were normalized using the Z-score prior to segmentation. We used the U-Net architecture (https://github.com/zhixuhao/unet) where the residual blocks and the multiple-input capabilities were implemented. Models: Two approaches were evaluated: 1-step) segmenting the index-lesion directly from $\mathrm{T} 2 \mathrm{w}$ data and 2-steps) use a cropped prostate and seg ment the index-lesion using ADC and/or T2w data to evaluate the impact of each input. Training/testing: For prostate segmentation, a training/test split of 80/20 was used. For lesion segmentation, fourfold cross-validation was considered. The optimization metric was the DSC for the validation set. Hausdorff Distance (HD) and Mean Square Distance (MSD) were also evaluated.

Results: Automatic prostate segmentation resulted in a DSC of 0.88 , HD of $16.5 \mathrm{~mm}$ and MSD of $2.1 \mathrm{~mm}$ in the validation set. Figure 1 presents a sample slice for prostate segmentation. Regarding the lesion segmentation, using the 1-step, 2-step and 2-step mp-MRI approaches, the average DSC in the validation set were: $0.51,0.71$ and 0.76, respectively. Performing the 2-step resulted in an improvement of $39 \%$ when compared to the 1-step approach. By introducing the mp-MRI information, we obtained an improvement of 10\%. Table 1 summarizes the results and Fig. 2 presents a segmentation result with the proposed mp-MRI model.
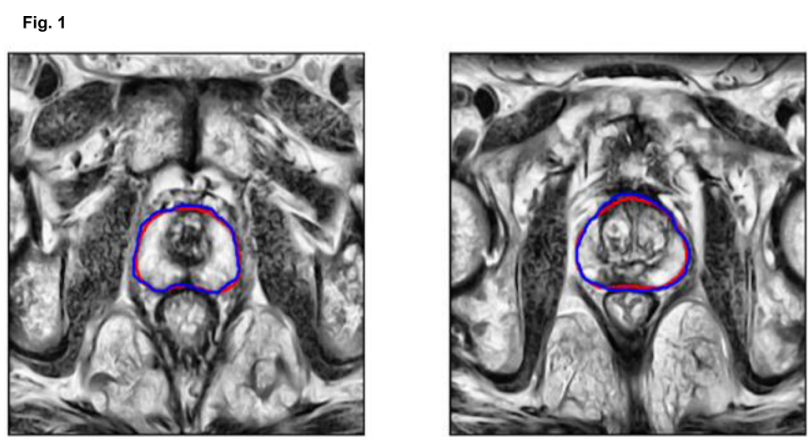

Fig. 2
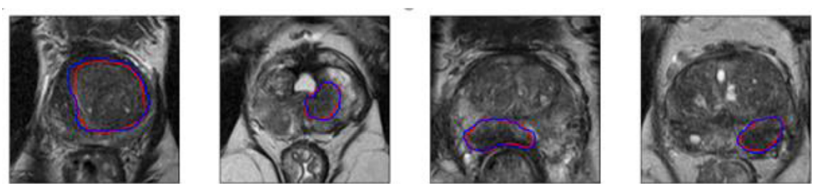

Fig. 3

\begin{tabular}{lccc} 
& 1-step (input T2) & 2-step (input T2) & 2-step (input T2+ADC) \\
\hline DSC & 0,51 & 0,71 & 0,76 \\
HD & $35,6 \mathrm{~mm}$ & $25,3 \mathrm{~mm}$ & $20,2 \mathrm{~mm}$ \\
MSD & $4,9 \mathrm{~mm}$ & $2,5 \mathrm{~mm}$ & $2,1 \mathrm{~mm}$ \\
\hline
\end{tabular}

Discucssion: The model for prostate region segmentation achieved a DSC of 0.88 which is agrees with the state-of-the-art References. The 2-step obtainedbetter results in terms of DSC when compared with 1-step, which highlights the importance restrict the segmentation region. The $\mathrm{T} 2 \mathrm{w}$ index-lesion segmentation improves with the introduction of ADC information in the network, suggesting the potential of mp-MRI. Our work is limited by the factthat we consider the segmentation of a single expert and limited database.

Conclusions: A model to segment prostate index-lesions in mp-MRI was proposed using U-Net. The initial results suggest that mp-MRI information improves index-lesion segmentation and that 2-step approaches improve lesion segmentation results.

\section{S1.P4.}

\section{Characterization of non-contrast CMR features in the polar map for myocardial pathologies}

*N. N. Massaccesi Bove $e^{1,2,3}$, D. Fino ${ }^{1,3,4}$, M. Del Pópolo ${ }^{1,2,3}$, J. Capó Vera $^{1,2,3}$, N. Moyano Brandi ${ }^{1,2,3}$, B. Lima ${ }^{1,3}$, N. Niella ${ }^{1,3}$, F. González

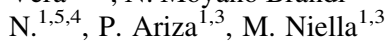

${ }^{1}$ Fundación Escuela de Medicina Nuclear, Department of Magnetic Resonance Imaging, Mendoza, AR;

${ }^{2}$ University of Mendoza, Faculty of Engineering, Mendoza, AR;

${ }^{3}$ Fundación Argentina para el Desarrollo en Salud, Department of Magnetic Resonance Imaging, Mendoza, AR;

${ }^{4}$ National University of Cuyo, Balseiro Institute, San Carlos de Bariloche, AR;

${ }^{5}$ Comisión Nacional de Energía Atómica, GQNYCS, Buenos Aires, $A R$

Introduction: Several pathologies, such as amyloidosis (CA), hypertrophic (HCM) and ischemic (ICM) cardiomyopathies, COVID19, Anderson-Fabry (AFD) and Chagas (ChD) disease, show anomalies in myocardial tissue, which can be analyzed with cardiac magnetic resonance (CMR). The aim of this study was to characterize the CMR pre-contrast features associated with these diseases.

Methods: This retrospective study was approved by the Institutional Review Board. The study comprised 27 healthy controls (HC) (9 women, 18 men; mean age: 40.9; range: $18-78$ years) and 92 patients with cardiac pathologies (CA: 17, HCM: 19, COVID-19: 22, AFD: 3, ChD: 11, ICM: 20). The CMR images were acquired with a $1.5 \mathrm{~T}$ Ingenia (Philips). A standard cardiology protocol was performed, including the acquisition of native $\mathrm{T} 1$ mapping. $\mathrm{T} 1$ values were measured in the 16 myocardial segments for all the subjects (Fig. 1) using the Segment toolbox (Medviso). Left/right atrium areas (LAa/ RAa), left/right ventricular ejection fraction (LVEF/RVEF), left/right end-diastolic/systolic volume (LVEDV, RVEDV, LVESV, RVESV) were measured (with their specific indexation). An ANOVA test was applied to determine the homogeneity of each group per feature. Also, significant differences between $\mathrm{HC}$ and each pathology were evaluated with a two-sample t-test (SPSS v.25). A ROC curve was analyzed to obtain cut-off values for the quantitative variables and $\mathrm{p}<0.05$ was considered as statistically significant. 


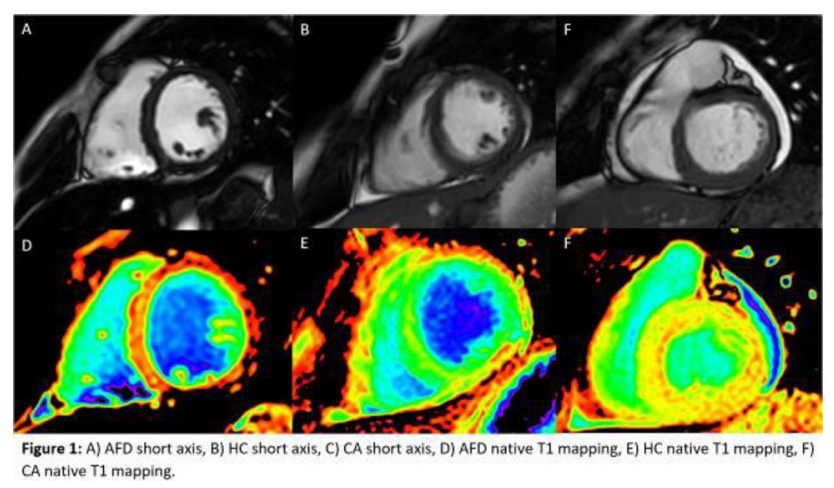

Results: Due to artifacts (partial volume effects), 174/1904 (9.14\%) segments were considered as technical failures and were excluded. Significant differences were found in $\mathrm{T} 1$ values between $\mathrm{HC}$ and all diseases $(\mathrm{p}<0.0001)$. Considering the polar map, all segments of native T1 (HC vs CA, HC vs AFD) had $\mathrm{p}<0.0001$, ICM: 14, HCM: 11, COVID-19: 10 and ChD: 6 (Fig. 2). The LAa measures were obtained at $\mathrm{p}<0.02$ between $\mathrm{HC}$ and CA, ICM, HCM, ChD. For RVEF, significant differences were found between HC and ICM, HCM, ChD. Also, measurements in COVID-19 and AFD pathologies, as well as the RVEDV and LVEDV in all the illnesses, do not show significant differences. The cut-off values for $\mathrm{T} 1$ were, $\mathrm{MCH}$ : 1058 ms, CA: 1093 ms, ChD: 1030 ms, ICM: 1051 ms, COVID-19: 1049 and the cut-off values for LAa were MCH: $21 \mathrm{~cm}^{2}$, CA: $21 \mathrm{~cm}^{2}$, ChD: $20 \mathrm{~cm}^{2}$, ICM: $20 \mathrm{~cm}^{2}$ (Fig. 3).

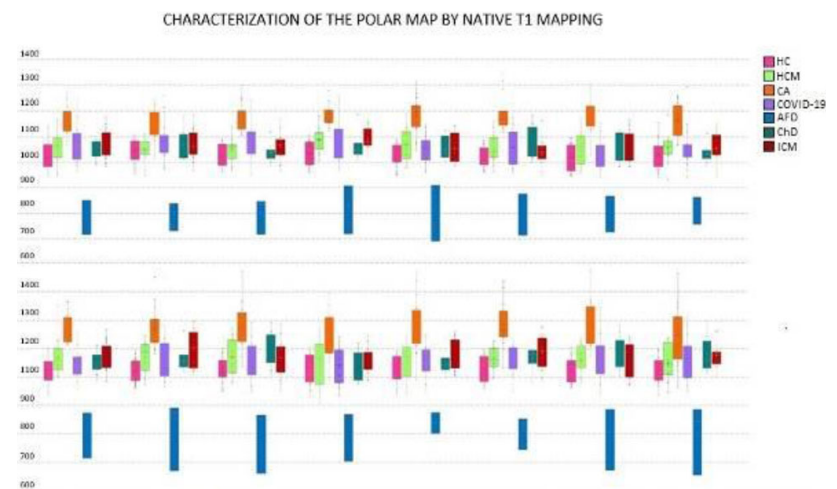

Figure 2: Comparison between HC, MCH, CA, COVID-19, AFD, ChD and ICM for the 16 segments.

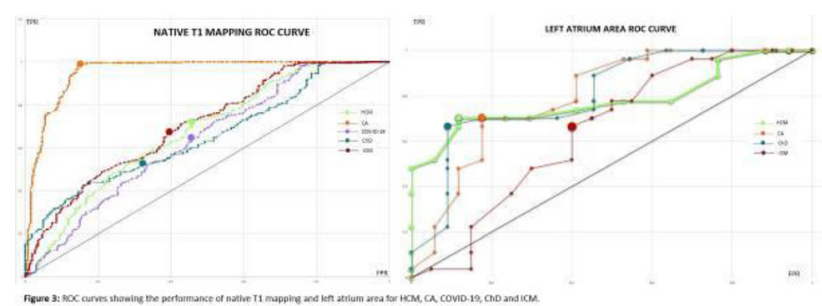

Discussion: T1 mapping, LAa and RVEF were the most representative features to determine several diseases. RVEF was not considered efficient for the detection of different pathologies (ROC). Statistically, the T1 values for AFD are lower than $\mathrm{HC}$, and the CA ones are the highest. AFD was excluded from the T1 ROC curve because of the small sample.

\section{Reference:}

A. Meloni et al., "Myocardial T1 Values at 1.5 T: Normal Values for General Electric Scanners and Sex-Related Differences, “ J Magn Reson Imaging, p. jmri.27639, Apr. 2021, https://doi.org/10.1002/ jmri.27639.

\section{S1.P5.}

The method of extending MRI diagnostics of liver iron overload to other medical centers without constructing new formulas for recalculating $\mathrm{T} 2 *$ in iron concentration using an MR-phantom

*P. Bulanov ${ }^{1,2}$, E. Manzhurtseva ${ }^{2}$, P. Menshchikov ${ }^{3}$, D. Kupriyanov ${ }^{3}$, G. Tereshchenko ${ }^{2}$, G. Novichkova ${ }^{2}$

${ }^{1}$ Lomonosow Moscow State University, Moscow, $R U$;

${ }^{2}$ Dmitry Rogachev National Research Center of Pediatric Hematology, Oncology and Immunology, Moscow, $R U$; ${ }^{3}$ Philips Healthcare, Moscow, $R U$

Introduction: Iron liver overload is a systemic disease that occurs in patients with genetic hemochromatosis, as well as in patients with blood disorders requiring ongoing blood transfusions. For a long time, biopsy was the only method for diagnosing the degree of liver iron overload [1]. However, at present, a non-invasive MRI technique for assessing liver overload by T2* values has become widespread [2]. Despite the advantages of this non-invasive technique, its use implies a correlation between MRI data and biopsy data. Since this correlation can depend on many scan parameters and other factors, it should be performed at every medical center wishing to use this technique reliably. We propose a standardization method that avoids the long and complicated process of constructing a conversion formula between $\mathrm{T} 2 *$ values and iron concentration (LIC). For this, we use the MR-compatible phantom and the previously obtained formula for recalculating the $\mathrm{T} 2 *$ values in the LIC [3].

Materials and methods: The phantom was created on the basis of complex iron oxide nanoparticles, which were obtained in our laboratory in Dmitri Rogachev Pediatric Hematology Center as a result of the Elmore reaction [4]:

$\mathrm{FeSO} 47 \mathrm{H}_{2} \mathrm{O}+2 \mathrm{FeCl}_{3} 6 \mathrm{H}_{2} \mathrm{O}+8 \mathrm{NH}_{3} \mathrm{H}_{2} \mathrm{O}$

$=\mathbf{F e}_{3} \mathbf{O}_{4}+6 \mathrm{NH}_{4} \mathrm{Cl}+\left(\mathrm{NH}_{4}\right) 2 \mathrm{SO}_{4}+23 \mathrm{H}_{2} \mathrm{O}$.

The phantom consisted of 28 tubes and covered the entire range of T2* values found in children with liver iron overload (Fig. 1).

Fig. 1

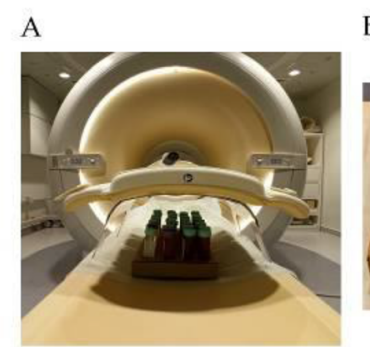

B

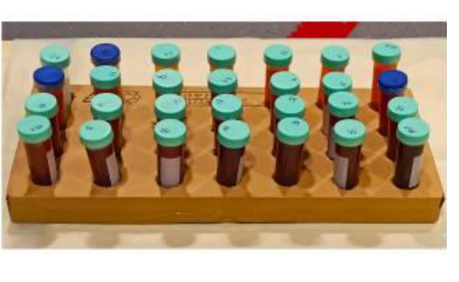

To check the repeatability of the results in our center, the phantom was scanned 6 times on Philips Achieva/3 T and Signa GE/1.5 T in Rogachev Center.

To test the methodology for standardizing the production of $\mathrm{T} 2 *$ cards, the phantom was scanned at two corresponding scanners in other institutions. 
Results and discussion: Repeatability tests showed satisfactory results - the standard deviation was acceptable for determining the overload grade (Fig. 2).

Fig. 2
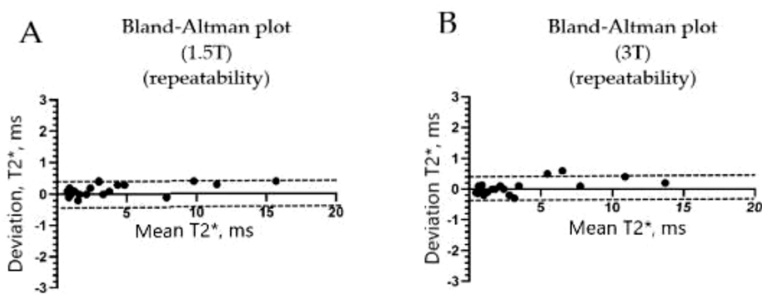

As a result, a method was formulated for standardizing the assessment of liver iron overload using the phantom:

1) Scanning a phantom at another institution.

2) If the results are in the confidence intervals of the reference values obtained on MRIs at the Rogachev Center, then it is possible to use the formula we obtained earlier in a new institution (Fig. 3).

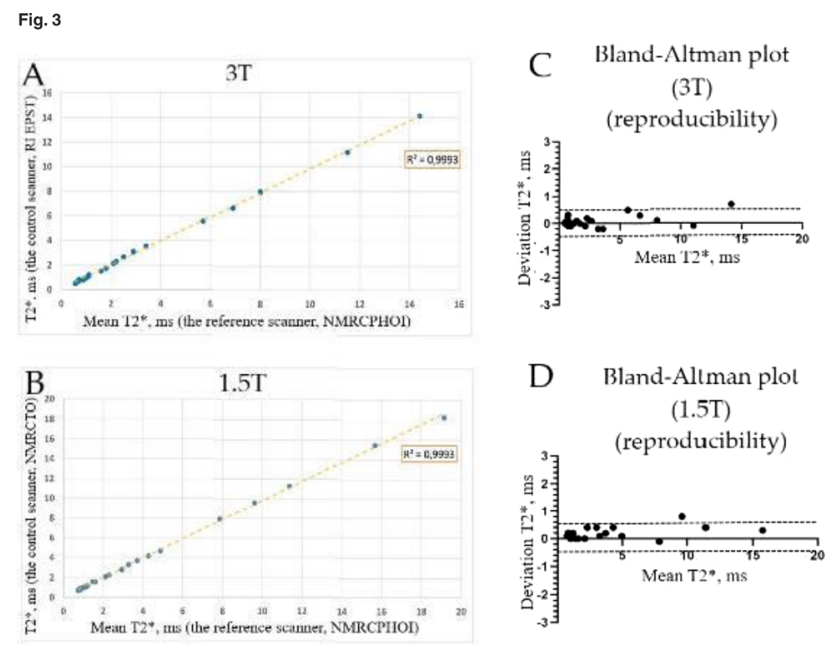

3) If this match is not found, it is possible to calibrate the study parameters until the desired match is reached.

4) If this does not help, it is possible to enter the calibration factors into the conversion formula.

Thus, for a non-invasive assessing of liver iron overload in these medical centers, it is possible to reliably use the previously obtained formula. It is significantly speeds up and simplifies the process of introducing this technique into clinical practice.

\section{References:}

[1] $10.1155 / 2007 / 761029$.

[2] $10.1148 / \mathrm{rg} .2018170079$.

[3] 10.24287/1726-1708-2020-19-3-158-163.

[4] 10.1070/rc2005v074n06abeh000897.

\section{S1.P6.}

Comparison between magnetic resonance elastography and IVIM/KURTOSIS models in multiparametic prostate protocol

*T. González Padin ${ }^{1,2,3}$, J. Capó Vera ${ }^{1,2,3}$, A. Domínguez ${ }^{1,3}$, N. Moyano Brandi ${ }^{1,2,3}$, F. González N. ${ }^{1,4,5}$, M. Noceti ${ }^{1,3}$, L. Plaza $^{1,3}$, R. Boschi ${ }^{1,3}$, R. Isoardi ${ }^{1,4,5}$, P. Ariza ${ }^{1,3}$, D. Fino ${ }^{1,3,5}$

${ }^{1}$ Fundacion Escuela de Medicina Nuclear, Department of Magnetic Resonance Imaging, Mendoza, AR;

${ }^{2}$ University of Mendoza, Faculty of Engineering, Mendoza, AR;

${ }^{3}$ Fundacion Argentina para el Desarollo en Salud, Department of Magnetic Resonance Imaging, Mendoza, AR;

${ }^{4}$ Comisión Nacional de Energía Atómica, GQNYCS, Buenos Aires, $A R$;

${ }^{5}$ National University of Cuyo, Balseiro Institute, San Carlos de Bariloche, AR

Introduction: The intra voxel incoherent motion (IVIM) technique encompasses the measurement of all types of incoherent motion present in an image voxel enhancing signal attenuation in diffusion MR imaging (low b values), such as blood microcirculation and capillary networks (perfusion). This effect is modelled by a biexponential fit and characterized by a diffusion coefficient (D), a pseudodiffusion coefficient $\left(D^{*}\right)$ and the f-IVIM, that represents the flowing blood volume fraction. Similarly, another deviation from normal gaussian distribution is obtained when high $b$ values are used, and a Kurtosis model is also used to increase sensitivity to identify tissue features of intracellular water behavior (perfusion). Another feature associated with prostate lesions analysis is its stiffness. The elastography (MRE) have shown significant differences between prostate carcinoma (PCa) and healthy tissue.The aim of this study is to correlate the results obtained with IVIM/KURTOSIS versus MRE. Methodology: The study was approved by the local Institutional Review Board; magnetic resonance (MR) images were acquired in a $3 \mathrm{~T}$ scanner MR/PET of General Electric (GE) for 16 patients (12 patients with $\mathrm{PCa}$ and 4 with prostatitis); these included diffusionweighted images (DWI), that were obtained with a FOCUS sequence with $15 \mathrm{~b}$ values (from 0 to $3000 \mathrm{~s} / \mathrm{mm}^{2}$, Fig. 1), and MRE-SE (frequency $=60 \mathrm{~Hz}$, amplitude $=70$ ). Two specialist radiologists traced ROIs in healthy (HT) and pathologic tissue. IVIM/KURTOSIS were processed using a Python algorithm (QT, VTK and SimpleITK), while MRE were processed with AW VolumeShare 5 (GE). The triexponential fitting was implemented with the Scipy Python library. Two specialist neuro-radiologists segmented the lesions and traced at least two ROIs in each segment to study the vascular flow (Fig. 1).

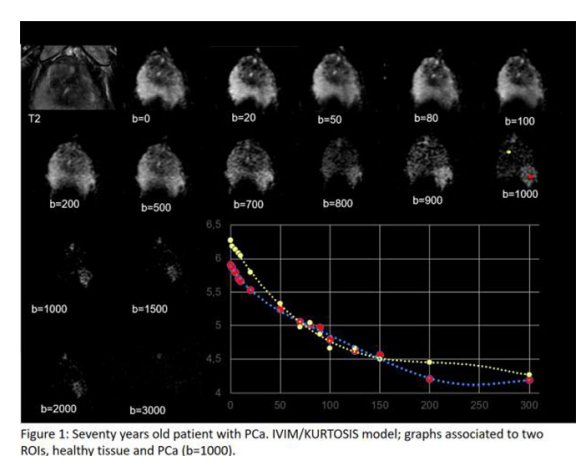


Results: Patients with PCA showed a decrease in the D $(\mathrm{m}=-$ 34.81) and $\mathrm{f}(\mathrm{m}=-71.49)$ coefficients, while the $\mathrm{k}$ coefficient and the stiffness increased $(m=23.93)$ compared with the HT (Fig. 2). A correlation between the coefficient values in the ROIs of HT and PCA was observed (Fig. 3).

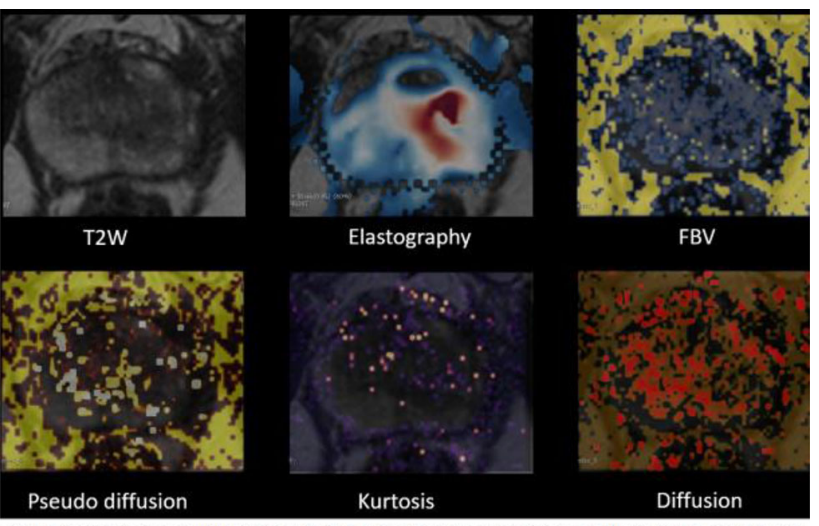

Figure 2: Fifty five years old patient with PCa. Prostate multiparametric maps (D,D*, f-IVIM, K, MRE 0-8 $\mathrm{kPa)}$

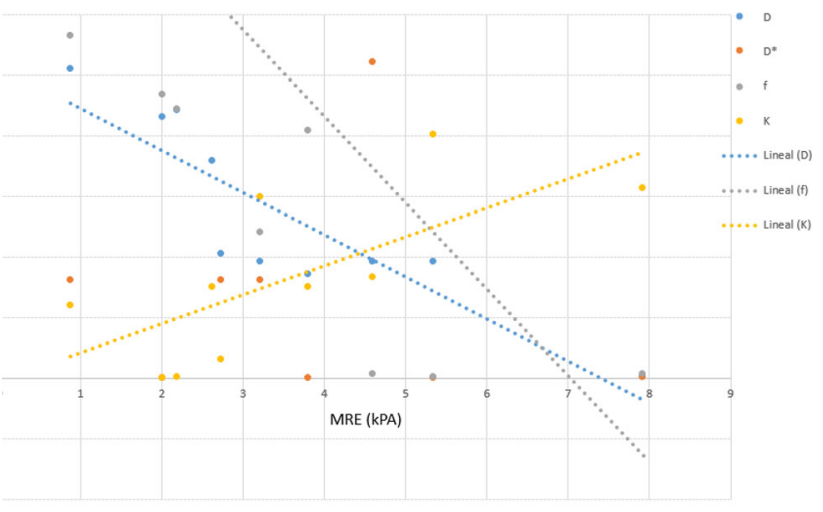

Figure 3: Correlation between MRE values and IVIM/KURTOSIS features.

Conclusión: While the $\mathrm{f}$ and $\mathrm{k}$ coefficients followed the perfusion behavior reported in other studies, as well as the MRE, the D* coefficient did not show a proportional behavior. The curve fitting for prostatitis patients did not generate logic results due to technical failures in the adjustment.

\section{References:}

Liu et al., "Comparative Study of Monoexponential, Intravoxel Incoherent Motion, Kurtosis, and IVIM-Kurtosis Models for the Diagnosis and Aggressiveness Assessment of Prostate Cancer», Front. Oncol., vol. 10, p. 1763, sep. 2020, https://doi.org/10.3389/fonc.2020. 01763.

M. Iima y D. Le Bihan, «Clinical Intravoxel Incoherent Motion and Diffusion MR Imaging: Past, Present, and Future», Radiology, vol. 278, n.o 1, pp. 13-32, ene. 2016, https://doi.org/10.1148/radiol. 2015150244

\section{S1.P7.}

Diagnostic and follow-up MRI of the chest in patients with COVID-19 pneumonia: comparison to CT results

*W. Y. Ussov ${ }^{1}$, N. V. Nudnov ${ }^{2}$, G. A. Ignatenko ${ }^{2,3}$, V. M. Gulyaev ${ }^{1}$, V. O. Vorobyeva ${ }^{2}$, T. A. Bergen ${ }^{4}$, M. B. Pervak ${ }^{3}$, A. V. Dubovaya ${ }^{3}$, G. G. Karmazanovsky ${ }^{5}$

${ }^{I}$ Tomsk National Medical Research Center, Laboratory of Tomography, Tomsk, RU;

${ }^{2}$ Russian Ministry of Health, National Center of Radiology, Moscow, $R U$;

${ }^{3}$ M. Gorky Donetsk National Medical University, Donetsk, RU;

${ }^{4}$ National Research Center, Academician E.N. Meshalkin State Research Institute of Circulation Pathology, Novosibirsk, RU; ${ }^{5}$ National Medical Research Center A.V. Vishnevsky, Institute of Surgery, Moscow, $R U$

Puropose of the study: In the recent time of pandemia of viral pneumonia (VP) caused by COVID-19, we aimed to study the possibility of using chest MRI to image lung damage in this pathology both in primary detection and for follow-up control of recovery.

Material and methods: MRI of the chest in T1-, T2-weighted modes (T1-w, T2-w), also with fat suppression, diffusion-weighted, STIRmodes, in the axial and frontal planes, with breath holding, or with automatic synchronization of acquisition with breathing was carriedout in 47 patients with VP, 32 of them were confirmed by PCR as COVID-19, all did have a clinic of pneumonia. The control group comprised 15 volunteers ( 8 non-smokers, and 7 smokers). In 18 patients, an CT study of the chest was also performed. In 8 patients, MRI of the chest was then performed again, for follow-up control of clinical recovery. There were no deaths among our patients.

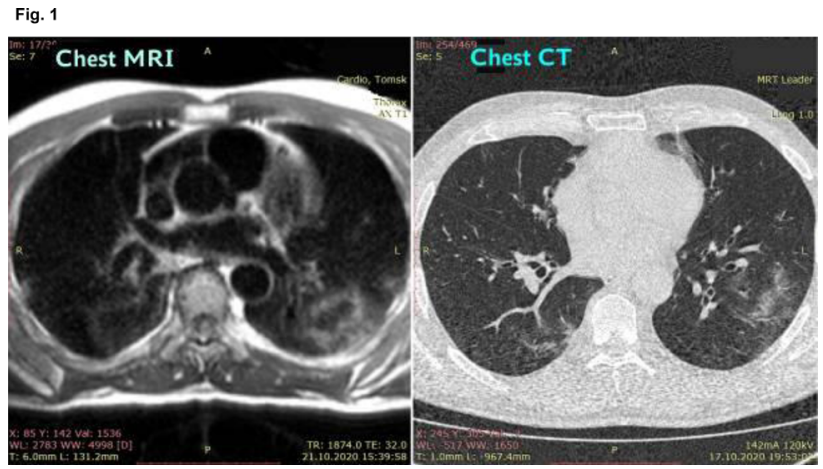

Results: The duration of MRI of the chest was $<25 \mathrm{~min}$ in all cases ( $21 \pm 4 \mathrm{~min}$ on average), and less than $10 \mathrm{~min}$ in the chest CT. In all cases, MRI imaging of the affected area was achieved already using a group of MRI screeninf protocols, comprised axial T1-w and axial and frontal T2-w, did last $<12$ min. 
Fig. 2

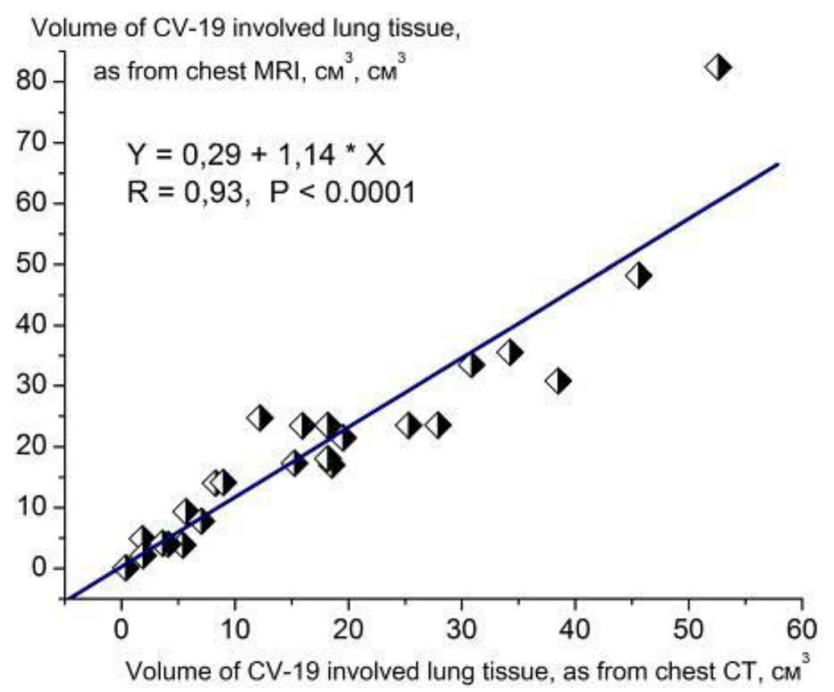

In normal patients without pathology of the lungs, not smoking, the lung was visualized as a diffuse homogeneous air region with a quota of interstitial and vascular space $<4 \%$. In patients-smokers, lung MRI was enhanced in the dorsal parts of both lungs, disorders of airiness, interstitial exudative and fibtosis changes weren't present. In the acute phase of the disease, pulmonary ventilation disorders and interstitial exudative changes that form the morphological basis of lung damage in COVID-19 were visualized as local foci of, corresponding to the location and nature (sub-segmental, segmental, polysegmental) of the pathological focus, both T1-w and T2-w modes.

Fig. 3

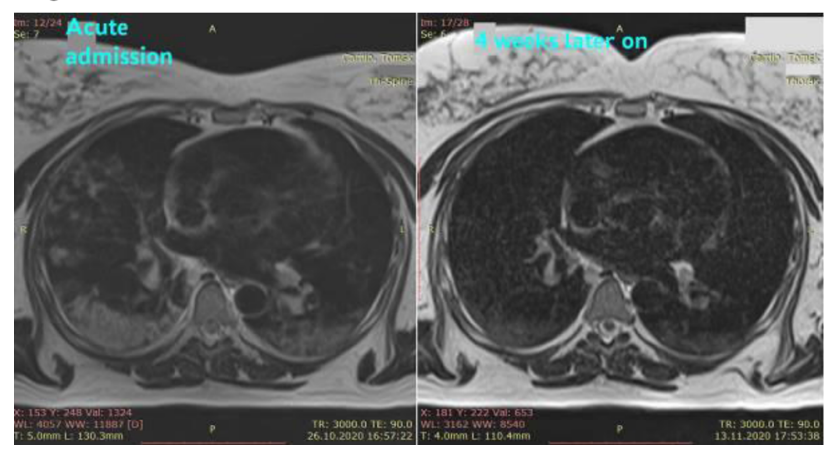

MRI of the chest provided diagnosis of lung pathology in all cases, while the extent of the pathological focus on the MRI image in T2-w was $14-19 \%$ over the CT values. The correlation of the calculated volume of affected lung tissue between $\mathrm{CT}$ and MRI of the chest was as high as $r=0.95(p<0.001)$. The values of the volume of the affected tissue in T1-w and T2-w did not differ from each other in the intergroup comparison and correlated as $r=0.985(\mathrm{p}<0.001)$. MRI in DWI mode showed a sensitivity of $81 \%$ (38/47) in detecting COVID-lung lesions. The volume of pleural effusion, clearly visible on $\mathrm{T} 2-\mathrm{w}$ images, in all our cases did not exceed $100 \mathrm{ml}$. In a prospective follow-up of 8 patients with COVID-19, chest MRI ptovided imaging of recovery process in all cases.

Conclusion: MRI of the chest with respiratory synchronization or with breath-holding can be used for early diagnosis of inflammatory lung lesions in COVID-viral pneumonia and for subsequent follow-up control of therapy.

\section{S1.P8.}

\section{Compression stiffening rate at MR elastography to assess microvascular invasion in patients with hepatocellular carcinomas}

*G. Pagé ${ }^{1}$, P. Garteiser ${ }^{1}$, V. Paradis ${ }^{1}$, R. Sinkus ${ }^{2}$, V. Vilgrain ${ }^{1,3}$, B. Van Beers ${ }^{1,3}$

${ }^{1}$ University of Paris, Center of Research on Inflammation, Paris, FR; ${ }^{2}$ University of Paris, Laboratory for Vascular Translational Sciences, Paris, FR;

${ }^{3}$ University of Paris, Department of Radiology, Paris, FR

Introduction: High tumor solid stress promotes cancer invasiveness, including microvascular invasion [1-5]. The compression stiffening rate measured with MR elastography (MRE) has recently been used to non-invasively assess tumor solid stress in small animals [6]. The aim of our study was to assess if compression MRE using different breath hold modes as stress source [7] could be used to assess cancer invasiveness (i.e. microvascular invasion) in patients with hepatocellular carcinomas (HCC).

Methods: This prospective clinical study, approved by the ethics review committee of our institution, included 41 patients with HCC (median size: $40 \mathrm{~mm}$, interquartile range: [30 mm; $60 \mathrm{~mm}$ ]; $24 \mathrm{HCC}$ with microvascular invasion) who were imaged before tumor surgical resection. The MRI examinations were performed on a 3.0 T Philips Ingenia scanner. MRE was performed with the "eXpresso" " sequence at a frequency of $50 \mathrm{~Hz}(\mathrm{n}=34)$ and $40 \mathrm{~Hz}(\mathrm{n}=7)$ [8], with TR/TE: $89 / 6.9 \mathrm{~ms}$, spatial resolution: $4.2 \mathrm{~mm}^{3}$, FOV: $420 \times 420$ $\mathrm{mm}^{2}$, slices: 8 and 4 temporal steps. Patients were instructed to hold their breath at end inspiration and expiration. Maps of elasticity (G”) and viscosity $(G$ “"”) were calculated by inverting the Helmholtz wave equation.

In addition, T2-weighted MR images were acquired with TR/TE: $531 / 120 \mathrm{~ms}$, spatial resolution: $0.59 \times 0.59 \times 4 \mathrm{~mm}^{3}$ and FOV: $280 \times 376 \mathrm{~mm}^{2}$. Tumor deformation $(\varepsilon)$ maps were calculated by applying a 3D affine registration on the T2 images (Matlab R2020a, Natick, MA). Stress $(\sigma)$ was calculated with the Hooke law, as $\sigma=3 \mathrm{G}_{\text {inspiration. }}$. . Finally, the compression stiffening rate was determined as the linear slope between $G^{*}$ and $\sigma$. Microvascular invasion was determined with histopathological examination.

Results: HCC elasticity was significantly higher at end inspiration than at expiration $(2.8 \pm 1.1 \mathrm{kPa}, 2.1 \pm 0.9 \mathrm{kPa}$, respectively: $\mathrm{t}$ test $\mathrm{p}=0.002$ ), (Fig. 1). At logistic regression, the compression stiffening rate was the only MRE parameter of microvascular invasion: $r_{\text {par- }}$ tial $=-0.42, p=0.007$ (Fig. 2). The compression stiffening rate, in contrast to the visco-elasticity, showed significant diagnostic performance for detecting microvascular invasion as shown by the areas under the receiver operating characteristic curves (AUC): $\mathrm{AUC}_{\mathrm{com}}$ pression stiffening $=0.75, \mathrm{p}=0.002$ versus $\mathrm{AUC}_{\mathrm{G}}=0.55, \mathrm{p}=0.6$, and $\mathrm{AUC}_{\mathrm{G}^{\prime \prime}}=0.57, \mathrm{p}=0.4$ (Fig. 3).

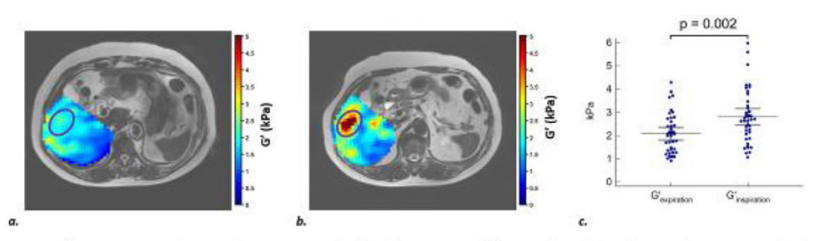

Fig. 1. Elasticity maps obtained at expiration (a.) and inspiration (b.). Purple circle indicates the position of the hepatocellular carcinoma. (c.) Box and scatter plots of elasticity during respiration. Hepatocellular carcinomas have significantly higher elasticity at inspiration than at expiration $(p=0.002)$. 
Fig. 2. Logistic regression of MR elastography parameters to assess microvascular invasion

\begin{tabular}{lccc}
\hline & $\mathbf{r}_{\text {partial }}$ & $\boldsymbol{\beta}$ value & $\mathbf{p}$ \\
\hline Elasticity & & $\begin{array}{c}\text { not included } \\
\text { not included }\end{array}$ & \\
Viscosity & & -13.6 & 0.007 \\
Compression stiffening rate & $-\mathbf{0 . 4 2}$ & - &
\end{tabular}

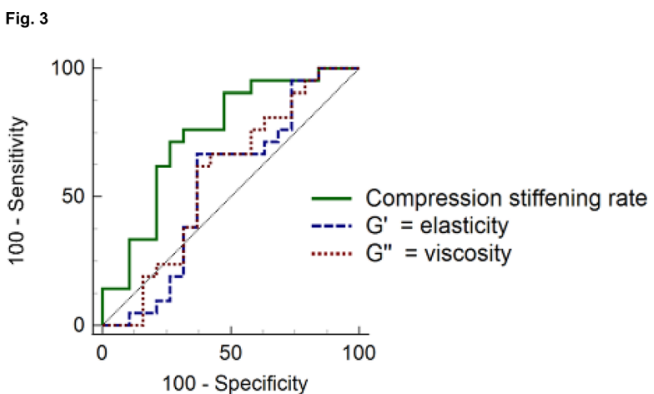

Figure 2. Areas under the receiver operating characteristic curves (AUC) show that compression stiffening rate has high diagnostic performance to diagnose microvascular invasion in hepatocellular carcinomas ( $A \cup C$ compression stiffening rate $=0.75, p=0.002$ ), in contrast to elasticity $\left(\mathrm{AUC}_{\mathrm{G}^{\prime}}=0.55, \mathrm{p}=0.6\right)$ and viscosity $\left(A \cup \mathrm{C}_{\mathrm{G}^{\prime \prime}}=0.57, \mathrm{p}=0.4\right)$ measured at inspiration.

Discussion: In patients with HCC, MRE compression stiffening rate had significantly higher accuracy than visco-elasticity to assess microvascular invasion. These results suggest that compression MRE during forced inspiration and expiration might be useful as a noninvasive clinical method to assess hepatocellular carcinoma invasiveness.

\section{References:}

1. Tse, J.M. et al. PNAS 2012.

2. Kraning-Rush, C.M. et al. PLoS One 2012.

3. Parekh, A. et al. Exp Cell Res 2016.

4. Mierke, C.T. Rep Prog Phys 2019.

5. Nia, H.T. et al. Science $\mathbf{2 0 2 0 .}$

6. Pagé, G. et al. Cancers 2021.

7. Garteiser, P. et al. 27th ISMRM 2019.

8. Garteiser, P. et al. NMR Biomed 2013.

\section{S1.P9.}

\section{Nodal detection in head and neck cancer by USPIO enhanced MRI: Where are the USPIOs in the blood?}

*J. J. van Asten ${ }^{1}$, D. Driessen ${ }^{2}$, E. D. Kers-Rebel ${ }^{2}$, J. H. Kaanders ${ }^{2}$, G. J. Adema ${ }^{2}$, T. W. Scheenen ${ }^{1}$

${ }^{1}$ Radboud University Medical Center, Department of Medical Imaging, Nijmegen, $N L$;

${ }^{2}$ Radboud University Medical Center, Department of Radiation Oncology, Nijmegen, NL

Introduction: In patients with head and neck cancer, accurate detection of lymph node metastases is of great relevance regarding prognosis and treatment $[1,2]$. USPIO-enhanced MRI is a promising imaging method to detect metastatic lymph nodes. After intravenous administration, USPIOs accumulate in cells of the immune system residing within healthy (parts of) lymph nodes [3], attenuating MR signal intensity in T2*-weighted pulse sequences. Suspicious lymph nodes retain MR signal intensity. In the validation process of this technique it is of interest to determine whether phagocytic uptake occurs in the blood or inside the lymph nodes. The aim of the present work is therefore to investigate which blood compartments contain the USPIO particles after infusion.

Methods: Blood samples were collected from five head and neck cancer patients at two different timepoints. The baseline sample was obtained prior to the slow-drip USPIO infusion, the second sample $45 \mathrm{~min}$ after the start of USPIO infusion. The blood was separated into six components: serum (SER), plasma (PLA), full blood (FB), peripheral blood mononuclear cells (PBMC), and granulocytes with and without red blood cells (GRA + RB, GRA-RB). The presence of USPIOs influences the relaxivity of the blood compartments and therefore the transverse relaxation times (T2) were measured, performing the Carr Purcell Meiboom Gill (CPMG) sequence [4,5] with 16 echo time points ranging from 1 to $100 \mathrm{~ms}$, up to 2 to $5000 \mathrm{~ms}$, depending on the a priori estimated $\mathrm{T} 2$. The relaxometry measurements were performed at a Bruker Avance 11.7 Tesla magnet, at room temperature $(298 \mathrm{~K})$. After fourier transform of the 16 free induction decays, the decay curves of the integrated NMR signals of water were fitted with a mono exponential, using the Bruker Topspin software. Shorter T2 relaxation times indicate the presence of USPIOs.

Results: After infusion, significantly shorter T2 times were observed in full blood, serum and plasma indicating the presence of USPIOs, which seem absent in other compartments. The decay of the NMR signal of water is depicted in Fig. 1. Averaged transverse relaxation times of the six blood compartments with and without USPIOs are presented in Table 1.

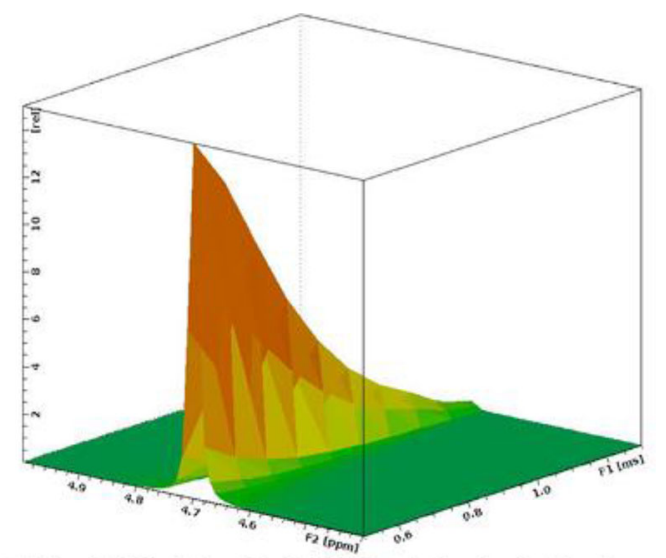

Figure 1. Pseudo $2 D$ of decaying NMR signal of water in blood component.

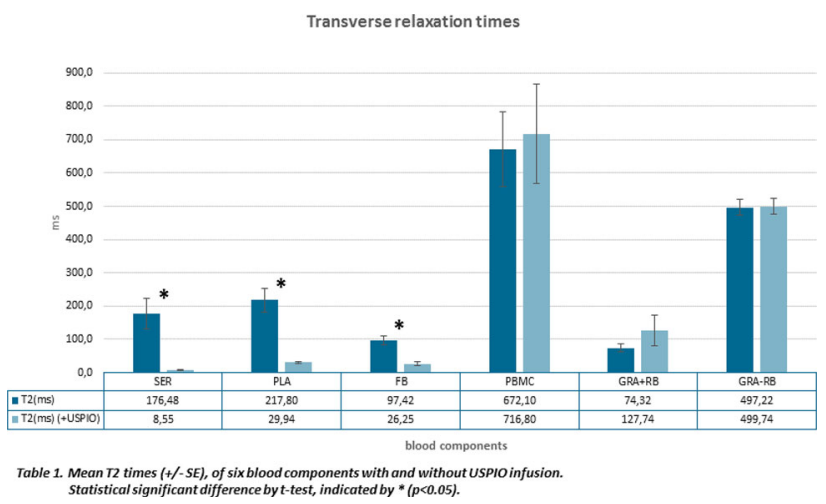


Discussion: Intravenously injected USPIOs are present in the serum and plasma compartments. These data indicate that USPIOs are not phagocytosed by the white blood cells present in the blood within $45 \mathrm{~min}$ after administration, but rather remain in the serum/plasma fraction. The full blood component represents the other compartments all together and therefore also shows significant difference in T2 times between blood with and without USPIOs.

\section{References:}

[1] Snow GB, et al. Clin Otolaryngol Allied Sci. 1982;7(3):185-92.

[2] Leemans CR, et al. Cancer. 1993;71(2):452-6.

[3] Weissleder R, et al. Radiology. 1990;175(2):494-8.

[4] H. Y. Carr and E. M. Purcell, Phys. Rev. 94, 630 (1954).

[5] S. Meiboom and D. Gill, Rev. Sci. Instrum. 29, 688 (1958).

\section{S1.P10.}

\section{Multiparametric MRI evaluation of glioblastoma features in a diet induced obesity mouse model}

\author{
*B. Yagüe Jiménez ${ }^{1}$, M. Donnay Pérez ${ }^{1}$, N. Arias-Ramos ${ }^{1}$, \\ B. Lizarbe ${ }^{1}$, P. López-Larrubia ${ }^{1}$ \\ ${ }^{1}$ Instituto de Investigaciones Biomédicas Alberto Sols (UAM), \\ Madrid, ES
}

Introduction: Obesity is a severe disease, characterized by accumulation of fat in tissues, that induces important alterations in liver, pancreas or brain. It has grown to epidemic proportions, with over 4 million people dying every year as a result of being obese. Besides, obesity is associated with other diseases like diabetes or cancer (1). The influence of overweight in brain tumors, however, is still unclear. On these grounds, an orthotopic glioblastoma mouse model was developed in animals fed with standard diet (SD) and mice submitted to ten weeks of high-fat diet (HFD) feeding prior to intracranial glioma cells injection (2).

Methods: Two cohorts $(\mathrm{n}=12)$ of C57BL6/J male adult mice with HFD (60\% fat) and SD feeding were used. Animals were submitted to GL261 glioma cells intracranial injection 10 weeks after diet diversification. They were constantly supervised, and MRI studies were performed 21-24 days post- intracranial tumor cell injection.

Anatomical images, magnetization transfer (MT), diffusion tensor Images (DTI), T2, and T2* parametric maps were acquired in a $7 \mathrm{~T}$ Bruker Biospec horizontal equipment. Parametric maps were generated using an in-house program developed with MatLab which adjusted the MRI signal to the corresponding mathematical equation. Four regions of interest were manually selected, using ImageJ: i) tumor core, ii) tumor periphery, iii) peritumoral region and iv) contralateral healthy brain. Statistical analysis was performed by twoway ANOVA tests with area and diet as factors and post-hoc multiple comparisons with Bonferroni corrections.

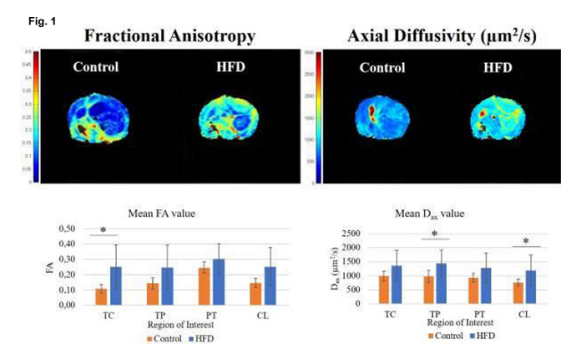

Results: Animals submitted to HFD presented an augmented weight the day of intracranial injection, then, weight tended to decrease till endpoint in both mice cohorts.

Obese mice exhibited significantly higher axial diffusivity (Dax) and fractional anisotropy (FA) ( $p<0.05$ for diet effect). Slightly increased mean diffusivity (MD), T2* and magnetization transfer ratio (MTR); and decreased radial diffusivity (Drad) and T2.

Discussion and conclusion: The results are consistent with the existence of inflammatory proceses, which are more remarkable in the case of the obese mice (3). These preliminary data point to remarkable differences in the assessed MRI parameters suggesting that obesity might influence in glioblastoma"s development.

\section{References:}

1. Apovian, C.M., Obesity: definition, comorbidities, causes, and burden. Am J Manag Care, 2016. 22(7 Suppl): p. s176-85.

2. Miyai M, Tomita H, Soeda A, Yano H, Iwama T, Hara A. Current trends in mouse models of glioblastoma. J Neurooncol 2017 Dec;135(3):423-432.

3. Guadilla I, Lizarbe B, Barrios L, Cerdan S, and López-Larrubia P "Integrative Analysis of Physiological Responses to High Fat Feeding with Diffusion Tensor Images and Neurochemical Profiles of the Mouse Brain “ Int J Obes, 2020. https://doi.org/10.1038/s41366-02100775-9 (in press)

\section{S1.P11.}

Diagnostic value of damping ratio and frequency dispersion coefficient at MRE in nonalcoholic fatty liver disease

*M. Khalfallah ${ }^{1}$, S. Doblas ${ }^{1}$, F. Julea ${ }^{1}$, O. Couture $^{2}$, C. Postic ${ }^{3}$, V. Duvivier ${ }^{4}$, C. Boitard ${ }^{5,6}$, D. Valla ${ }^{7}$, V. Paradis ${ }^{1}$, B. Van Beers ${ }^{1,8}$, P. Garteiser ${ }^{1,9}$

${ }^{1}$ University of Paris, Center of Research on Inflammation, Paris, FR;

${ }^{2}$ Paris Sciences et Lettres University, Paris, FR;

${ }^{3}$ University of Paris, Cochin Institute, Paris, FR;

${ }^{4}$ Sanofi-Aventis R\&D, Discovery Research Metabolism Department, Rueil-Malmaison, FR;

${ }^{5}$ Académie nationale de médecine, Paris, FR;

${ }^{6}$ Académie nationale de pharmacie, Paris, FR;

${ }^{7}$ University of Paris, Paris, FR;

${ }^{8}$ University Hospital Paris Nord, Department of Radiology, Clichy, FR;

${ }^{9}$ University of Paris, French National Institute of Health and Medical Research (Inserm), Paris, FR

Introduction: Nonalcoholic fatty liver disease (NAFLD) is a chronic liver disease that can occur as nonalcoholic fatty liver (NAFL, without or with inflammation at histopathology) or steatohepatitis (NASH) which is characterized by steatosis, lobular inflammation and hepatocellular ballooning ${ }^{(1)}$. Measurements of damping ratio ${ }^{(2)}$, loss modulus $^{(3)}$ and frequency dispersion coefficient ${ }^{(4)}$ have been proposed to assess NASH and liver inflammation. In several rheological models, damping ratio is tied to frequency dispersion, but this relationship has not been precisely assessed in vivo. Here, we evaluated the relationship between damping ratio and frequency dispersion in a mouse model and assessed the value of these mechanical parameters as potential biomarkers of NASH and liver inflammation.

Methods: After Ethics Committee approval, mice fed with normal diet, high fat diet or high fat, choline-deficient diet were examined with MRE at 4, 10 and 16 weeks ( $n=12 /$ time point and group). Liver 3D MRE examinations were performed with a $7 \mathrm{~T}$ Bruker system $\left(300 \mu \mathrm{m}^{3}\right.$, at $200 \mathrm{~Hz}, 400 \mathrm{~Hz}$ and $\left.600 \mathrm{~Hz}\right)$. The damping ratio $\zeta$ was 
calculated at all frequencies, and the frequency dispersion of the shear stiffness $\left(\gamma_{\mathrm{G}^{*}}\right)$ was evaluated as the coefficient of a power law ${ }^{(5)}$. To investigate the relationship between $\zeta, \gamma_{\mathrm{G}^{*}}$ and histological features, rank correlations, logistic regression, Mann-Whitney tests and ROC analysis were used.

Results: Shear stiffness slope $\gamma_{\mathrm{G}^{*}}$ was correlated with $\zeta$ at $200 \mathrm{~Hz}$ only $(\mathrm{p}=0.008, \mathrm{r}=-0.26)$. Both $\gamma_{\mathrm{G}^{*}}$ and $\zeta$ at $200 \mathrm{~Hz}$ and $600 \mathrm{~Hz}$ were solely determined by lobular inflammation (multiple regression $\left.\gamma_{\mathrm{G}^{*}}: \mathrm{p}=0.02, \zeta_{200}: \mathrm{p}=0.01, \zeta_{600}: \mathrm{p}=0.005\right)$ rather than by ballooning or steatosis. Damping ratio at $400 \mathrm{~Hz}$ was not correlated with any histological feature. In contrast to $\zeta$ at any frequency, $\gamma_{G^{*}}$ was an independent determinant of NASH (logistic regression $\mathrm{p}=0.02$, regression coefficient $\mathrm{b}=-1.18$ with $\mathrm{p}=0.02$ ). NAFL vs. NASH group differences were of similar or higher significance for $\gamma_{\mathrm{G}^{*}}$ $\left(\mathrm{p}\left(\gamma_{\mathrm{G}^{*}}\right)=0.03\right) \quad$ than for $\zeta\left(\mathrm{p}\left(\zeta_{200}\right)=0.03, \quad \mathrm{p}\left(\zeta_{400}\right)=0.94\right.$, $\left.\mathrm{p}\left(\zeta_{600}\right)=0.96\right)$ (Fig. 1). For the diagnosis of NASH, the AUC of $\gamma_{\mathrm{G}^{*}}$ $(0.68[0.55-0.80])$ was larger than that of $\zeta$ at each frequency $\left(\zeta_{200}\right.$ : 0.67 [0.53-0.78]; $\zeta_{400}: 0.52$ [0.38-0.65]; $\zeta_{600}: 0.51$ [0.38-0.64]).
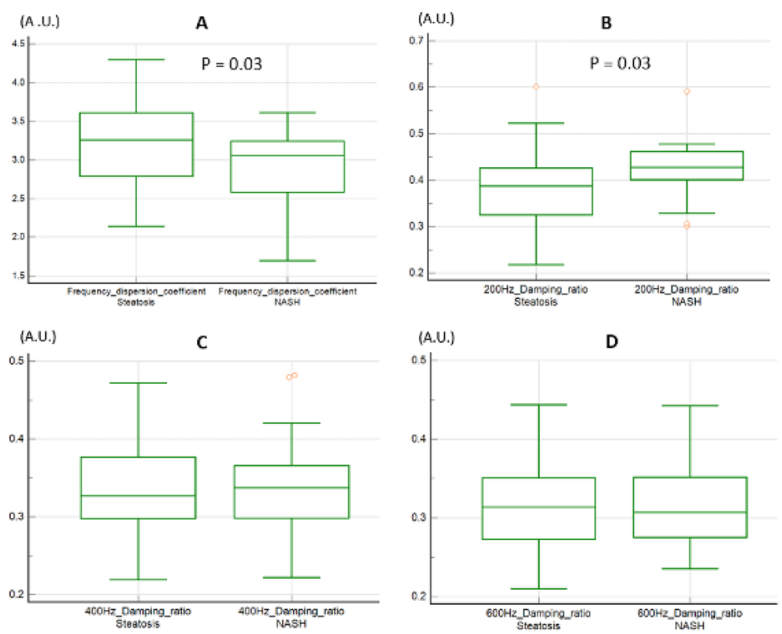

Fig.1 : Boxplots of frequency dispersion coefficient (A), damping ratio at $200 \mathrm{~Hz}$ (B), $400 \mathrm{~Hz}$ (C), and $600 \mathrm{~Hz}(\mathrm{D})$ in NAFL versus NASH. Frequency dispersion coefficient is significantly lower in NASH than in NAFL $(p=0.03)$ (A). Damping ratio is significantly higher in NASH than in NAFL at $200 \mathrm{~Hz}(p=0.03)(B)$, but not at $400 \mathrm{~Hz}(C)$ and $600 \mathrm{~Hz}(D)(p=0.94$ and $p=0.96$, respectively).

Discussion: Our results show that the frequency dispersion coefficient and the damping ratio are determined by lobular inflammation rather than steatosis and ballooning. This may limit their value as biomarkers of NASH and indicate a larger potential for the diagnosis of inflammation. Our study suggests that the diagnostic potential of the damping ratio is not the same at all frequencies, and that the damping ratio at a single frequency may not fully recapitulate the frequency dependence of the viscoelastic parameters.

\section{References:}

(1) Younossi ZM, et al. J Hepatol 2019.

(2) Yin M, et al. Radiology 2017.

(3) Sinkus R, et al. NMR Biomed 2018.

(4) Garteiser P, et al. ISMRM, 2014.

(5) Sinkus R, et al. Magn Reson Imaging 2005.

\section{S1.P12. \\ $\mu$ RIGS: microposition robotics in clinical workflow for MRI guided prostate interventions}

*I. Fomin ${ }^{1,2}$, R. Odenbach ${ }^{1}$, F. Fischbach ${ }^{3}$, E. Pannicke ${ }^{1,2}$, B. Hensen ${ }^{2,4}$, F. Wacker ${ }^{2,4}$, G. Rose Ro $^{1,2}$

${ }^{1}$ Otto von Guericke University, Institute for Medical Engineering, Magdeburg, DE;

${ }^{2}$ Otto von Guericke University, Research Campus STIMULATE, Magdeburg, DE;

${ }^{3}$ Otto von Guericke University, Department of Radiology and Nuclear Medicine, Magdeburg, DE;

${ }^{4}$ Hannover Medical School, Institute for Diagnostic and Interventional Radiology, Hanover, DE

Introduction: Compared to sonography or computed tomography, magnetic resonance imaging (MRI) offers high soft-tissue contrast and allows the measurement of physiological parameters such as temperature non-invasively, as well as multiplanar real-time images for better navigation. These aspects are attractive for controlling and monitoring minimally invasive diagnostics and therapeutics (e.g. biopsy, tumor ablation, brachytherapy). Despite the number of benefits, several disadvantages, such as the constricted space in the MR bore or the non-standardized workflows, make the procedures in clinical practice difficult and limit interventional MRI (iMRI) to relatively small case numbers $[1,2]$. To counteract this, an intuitively operable, MR-compatible, ultra-light and remotely controllable micropositioning system called $\mu$ RIGS has been developed for universal interventions [3]. The aim of this first study is to evaluate the performance of $\mu$ RIGS in the anticipated clinical workflow for a simulated MRI guided prostate intervention.

Methods: The instrument positioning unit (IPU) of $\mu$ RIGS is developed mainly with 3D-printed parts in compact dimensions to achieve five degrees of freedom (DoF) by Bowden cables and a remotely controlled electrical drive unit placed inside the scanner room (SR). For validation, $\mu$ RIGS is set up for an MRI guided pelvis phantom to puncture the prostate (transgluteal access) in a clinical radiology environment (see Fig. 1). Within the anticipated workflow (see Fig. 2) the system and therefore the main puncture is performed from the control room $(\mathrm{CR})$.

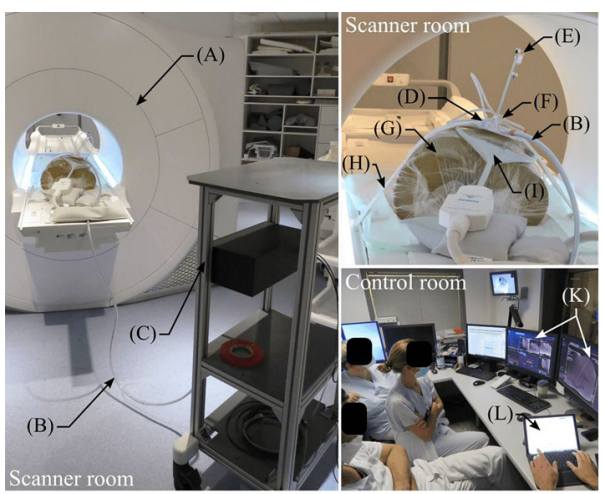

Figure 1: $\mu$ RIGS setup for a phantom test in the department of radiology of the University Hospital in Magdeburg. (A) $1.5 \mathrm{~T}$ Sola system (Siemens Healthineers, Erlangen, Germany). (B) $3 \mathrm{~m}$ long Bowden cables. (C) Electrical drive unit on an MR safe trolley. (D) IPU. (E) $150 \mathrm{~mm}$ long $18 \mathrm{G}$ coaxial puncture needle (Innovative Tomography Products GmbH, Bochum, Germany). (F) Fiducial marker. (G) Pelvis phantom. (H) MR table belts. (I) Single loop coil. (K) Planning data. (L) Graphical user interface communication with (C) via USB cable. 


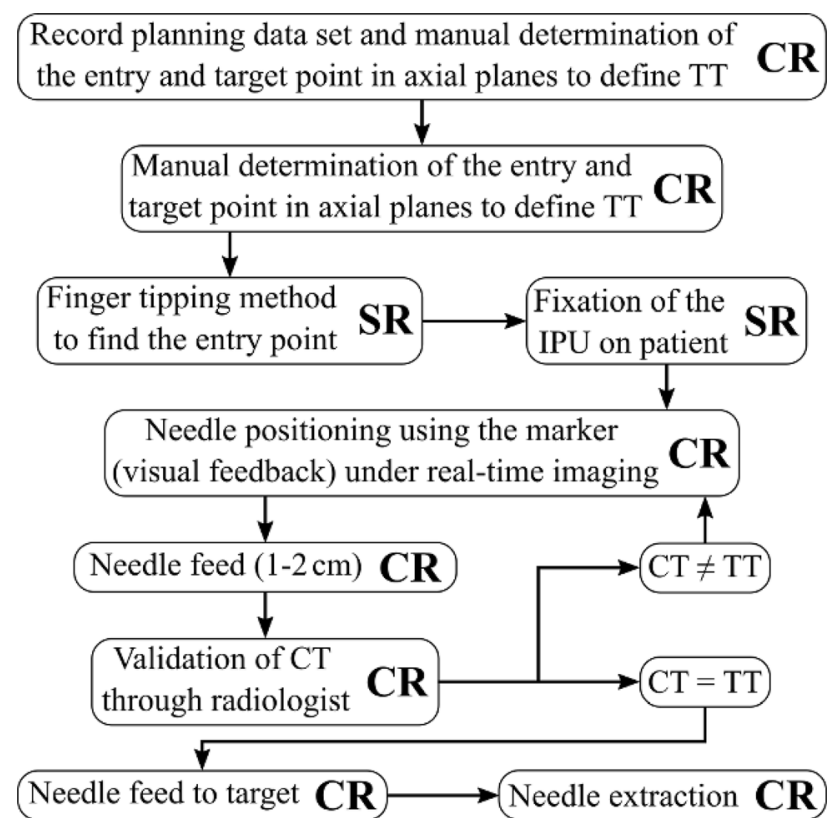

Workflow from control room (CR) or scanner room (SR)

TT: Target trajectory; CT: Current trajectory

Figure 2: Anticipated workflow with the $\mu$ RIGS system to perform a prostate puncture within a clinical environment.

Results: After initial feed (see Fig. 3a), the current trajectory (CT) did not correspond to the target trajectory (TT). This was followed by an iterative angle adjustment with the needle already advanced until the CT matched the TT and immediate verification by the radiologist using the fiducial marker (white) under real-time imaging. Fig. 3b shows the remote feed of the needle along the CT.
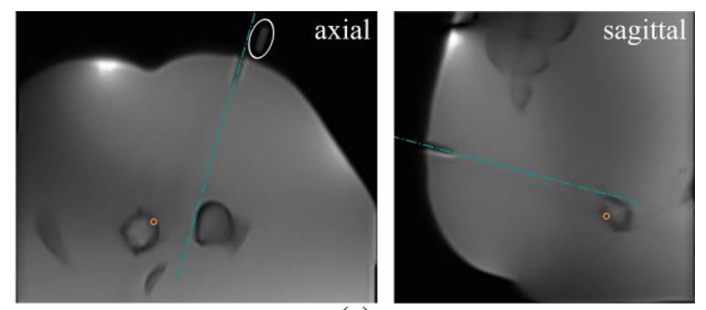

(a)
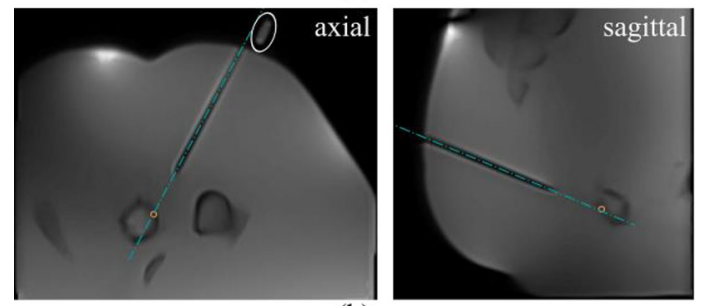

(b)

Figure 3: Slice images during remotely guided puncture in axial and sagittal view. (a) Initial positioning with a needle feed approximately $10 \mathrm{~mm}$. (b) Feed of the needle on CT. (orange) Target point. (turquois) Trajectory on needle artifact. (white) Fiducial marker.
Discussion: This study demonstrated the performance of the $\mu$ RIGS system in a clinical workflow that provides fully remote-controlled needle puncture. Through the iterative angle adjustment, the user has to rethink from the system kinematics relative to the orientation in the slice images. In conclusion, this work has shown the possibility to adjust the instrument position in the MR bore remotely without being present inside the SR. These first results will be used for next $\mu$ RIGS demonstrators, which can guide various instruments at ubiquitous application areas autonomously and more precisely. In future, the complex process of instrument navigation will be taken over by robotic systems to save time and costs as well as to reduce complications during interventions.

\section{References:}

[1] Barkhausen et al., RöFo, 611-623, 2017.

[2] Sequeiros et al., Acta Radiologica, 576-586, 2005.

[3] Fomin et al., CDMBE, 2021 (submitted).

\section{S1.P13.}

\section{Preclinical validation of MRI-guided ultrasound treatment to improve chemotherapy in ovarian cancer}

\author{
*D. Patrucco ${ }^{1}$, F. Garello ${ }^{1}$, C. Furlan ${ }^{1}$, E. Terreno ${ }^{1}$ \\ ${ }^{1}$ University of Turin, Department of Molecular Biotechnology \\ and Health Sciences, Turin, IT
}

Introduction: In cancer research, a challenging goal is to increase the amount of drug that can reach the tumor. Low intensity ultrasound (LIUS) could be an appealing technique, in virtue of their high biocompatibility and good tissue penetration. ${ }^{1}$ The aim of this study is to investigate the ability of LIUS to enhance the tumor delivery of a liposomal formulation of doxorubicin (Doxo), using a clinically approved Gd-based MRI contrast agent (Gadoteridol), co-encapsulated in the liposomes (Gd-Lipo-Doxo), to report on the US-triggered drug release by MRI in a mouse model of ovarian cancer.

Methods: Female athymic nude mice were purchased by Envigo (Milano, Italy) and were inoculated with A2780 cells (human ovarian cancer cell line). Mice tumor growth was monitored by 7 T-MRI. Mice were divided in three groups; i) not treated group ii) group treated with Gd-Lipo-Doxo, and iii) group treated with Gd-LipoDoxo and exposed to US stimulation. The amount of the doxorubicin and $\mathrm{Gd}$ in the tumour was assessed spectrofluorimetrically and by ICP-MS, respectively. Low intensity pulsed US waves were locally applied using $1 \mathrm{MHz}$ piezoelectric transducer (PA, Dorset, UK) in order to induce the temporary permeabilization of the cell membrane and the drug release from the liposome system.

Results/discussion: To optimize the maximal release of agents from liposomes and the permeabilization of the cell membrane (sonoporation) in vitro experiment were performed. Both Gadoteridol and doxorubicin showed a similar ability to be released from the liposomes and be internalized in tumor cells. In the in vivo experiments, the tumour LIUS stimulation resulted in a $\mathrm{T}_{1}$-contrast enhancement in the tumour and in the kidney calix and bladder, thus indicating the effective intratumor release of gadoteridol (and doxorubicin) from the liposomes. The tumour uptake of the Gd-agent and the drug was confirmed by confocal images (Fig. 1) and quantification. Notably, the group of mice treated with Gd-Lipo-Doxo and US-stimulated showed an arrest in the tumor growth. Interestingly, the tumour $\mathrm{T}_{1}$ contrast enhancement measured just after the US treatment showed a positive correlation with the therapeutic outcome after three weeks. 


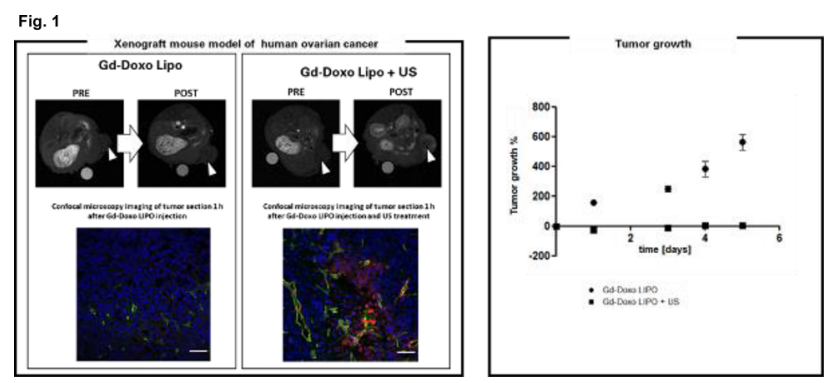

Conclusion: The ultrasound treatment appears a safe and efficient method to improve the chemotherapy efficacy of a liposome-encapsulated doxorubicin in a mouse model of ovarian cancer. All components of the theranostic agents (Lipo, Doxo, and gadoteridol) are already clinically approved (though not in the same system), making this approach suitable for clinical translation. Moreover, MRI seems to be an excellent imaging technique to monitor and predict the therapeutic outcome.

Acknowledgement: We thank the Open Lab of Advanced Microscopy of the University of Torino and AIRC Foundation for 2018 Investigator Grant.

\section{Reference:}

1. Pitt, W. G., Husseini, G. A., \& Staples, B. J. Ultrasonic drug delivery-a general review. Expert opinion on drug delivery, (2004). 1, $37-56$

\section{S1.P14.}

\section{Inter-Observer variability in target volume delineation of uveal melanoma}

*M. Jaarsma ${ }^{1,2}$, L. Klaassen ${ }^{1,2}$, B. Verbist ${ }^{1}$, T. H. K. Vu ${ }^{2}$, Y. Klaver $^{3,4}$, M. Rodrigues ${ }^{3,4}$, T. Ferreira ${ }^{1}$, C. Nabarro ${ }^{1,5}$, G. P. M. Luyten $^{2}$, C. Rasch ${ }^{3}$, M. van Herk ${ }^{6}$, J. W. Beenakker ${ }^{1,2,3}$

${ }^{I}$ Leiden University Medical Center, Department of Radiology, Leiden, $N L$;

${ }^{2}$ Leiden University Medical Center, Department of Ophthalmology, Leiden, NL;

${ }^{3}$ Leiden University Medical Center, Radiation Oncology, Leiden, $N L$; ${ }^{4}$ Holland PTC, Delft, NL;

${ }^{5}$ Ziekenhuis Amstelland, Department of Radiology, Amstelveen, $N L$; ${ }^{6}$ University of Manchester, Division of Cancer Science, Manchester, $G B$

Introduction: Magnetic resonance imaging (MRI) is becoming a valuable tool for the diagnosis ${ }^{1}$ and treatment planning ${ }^{2}$ of uveal melanoma patients. For radiotherapy planning it is important to know the reproducibility of target delineation. The primary aim of this study was to assess the interobserver variation in gross tumour volume (GTV) delineation of uveal melanoma (UM) on MRI. Our secondary aim was to determine the optimal MRI contrast for target volume delineation.

Methods: Six observers delineated the tumours of ten patients. All MRI scans were acquired using the setup as described by Ferreira et $\mathrm{al}^{2}$ and segmented using Big Brother software ${ }^{3}$. The GTV was segmented separately on the T2-weighted (T2w) and the post contrast T1-weighted (cT1w) images. The other sequences (T1w and cT1w or $\mathrm{T} 2 \mathrm{w}$ ) were only used as reference to differentiate the different structures. The volume and variance of the delineations on $\mathrm{T} 2 \mathrm{w}$ and cT1w were compared.
Results: It was observed that on cT1w flat tumour extensions were included more often compared to $\mathrm{T} 2 \mathrm{w}$. The average tumour volume was significantly larger when delineated on $\mathrm{cT} 1 \mathrm{w}$ compared to the $\mathrm{T} 2 \mathrm{w}(\mathrm{p}=0.01)$. The average contour variation was $0.4 \mathrm{~mm}$ on $\mathrm{cT} 1 \mathrm{w}$ and $0.3 \mathrm{~mm}$ on $\mathrm{T} 2 \mathrm{w}$. Greater variability was found at the tumourretinal detachment (RD) interface and at the tumour edge (average 0.5-0.7 $\mathrm{mm}$ ), compared to the tumour-vitreous and tumour-sclera interface (average $0.3-0.4 \mathrm{~mm}$ ).

Discussion: The difference in segmentation volume and observer variation on $\mathrm{cT} 1 \mathrm{w}$ and $\mathrm{T} 2 \mathrm{w}$ has been observed before ${ }^{4}$ and can be partially explained by the interobserver window level differences found in cT1w. The average observer variation of $0.3 \mathrm{~mm}$ and $0.4 \mathrm{~mm}$ is low ( $\sim 0.5$ acquisition voxel) although for small lesions like uveal melanoma it might still be of importance. Finally, even though the variation is higher on cT1w, we recommend to delineate the GTV on cT1w with T2w and T1w as reference, as small tumour extensions appear to be missed on $\mathrm{T} 2 \mathrm{w}$.

\section{References:}

1. Ferreira et al. Cancers (2019)

2. Fleury et al. Medical Physics (2020)

3. Steenbakkers et al. Radiotherapy and Oncology (2015)

4. Kim et al. Neurology (2015)

Figure 1. Delineation results of patient 2. A. In this patient difference in delineation between $\mathrm{cT} 1 \mathrm{w}$ and $\mathrm{T} 2 \mathrm{w}$ can be clearly observed. B. Example of a delineation on cT1w where tumour edge enhancement is often included in the gross tumour volume (arrow). C. Example of a delineation on T2w. D. A different window level led to a larger delineation by this observer.

Fig. 1
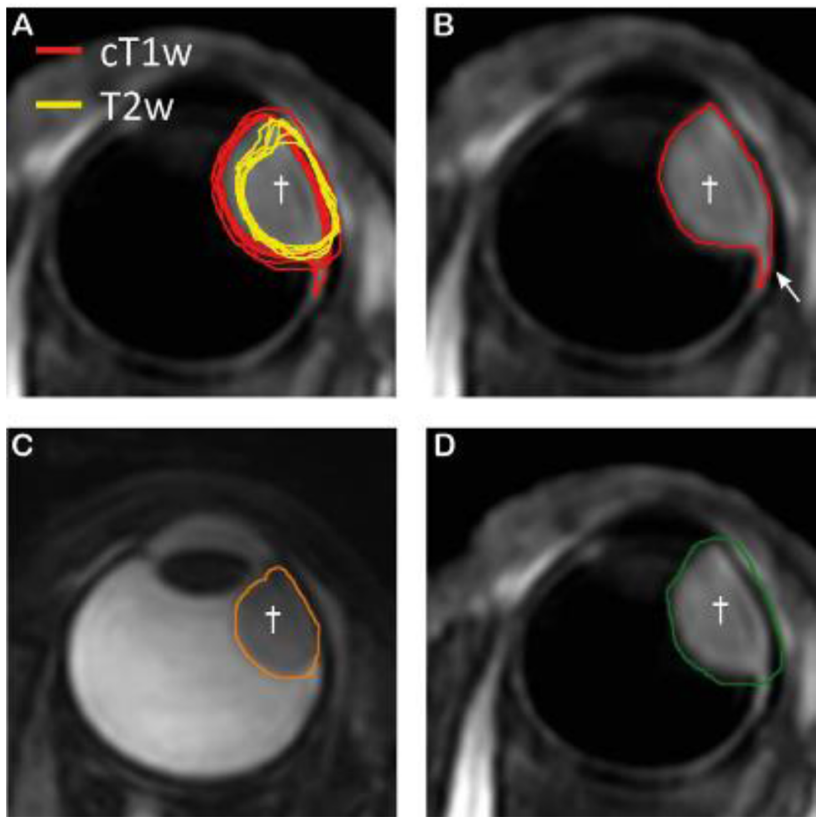

Figure 2. Results. A. For each case edge, base, tumour-vitreous and tumour-RD border were marked to determine the average SD per region. B. Higher variations were found at the tumour-RD interface and at the tumour edge. On cT1w the variability is higher compared to $\mathrm{T} 2 \mathrm{w}$ especially at the edge and tumour-vitreous border. 
Fig. 2
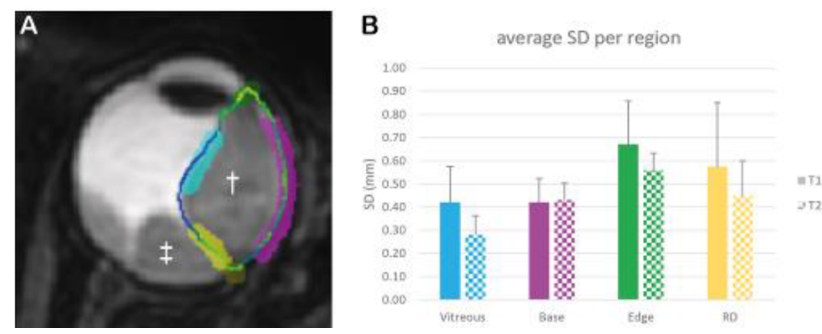

Table 1 The GTV is significantly larger when delineated on cT1w compared to $\mathrm{T} 2 \mathrm{w}$. The median observer variation seems to be larger on cT1w as well.

Fig. 3

\begin{tabular}{|l|l|l|l|l|}
\hline \multicolumn{4}{|l|}{ Volume $\left(\mathrm{cm}^{3}\right)$, average(min-max) } & \multicolumn{2}{l|}{ median SD (mm) } \\
\hline & cT1w & T2w & cT1w & T2w \\
\hline Pt. 1 & $0.32(0.24-0.47)$ & $0.27(0.24-0.32)$ & 0.4 & 0.3 \\
\hline Pt. 2 & $0.73(0.62-0.96)$ & $0.57(0.53-0.64)$ & 0.4 & 0.3 \\
\hline Pt. 3 & $0.31(0.27-0.39)$ & $0.29(0.24-0.34)$ & 0.3 & 0.3 \\
\hline Pt. 4 & $1.01(0.84-1.26)$ & $0.87(0.75-0.95)$ & 0.5 & 0.3 \\
\hline Pt. 5 & $0.64(0.53-0.83)$ & $0.56(0.51-0.59)$ & 0.4 & 0.2 \\
\hline Pt. 6 & $0.26(0.20-0.35)$ & $0.21(0.17-0.23)$ & 0.3 & 0.3 \\
\hline Pt. 7 & $0.29(0.22-0.40)$ & $0.23(0.19-0.30)$ & 0.3 & 0.3 \\
\hline Pt. 8 & $0.16(0.11-0.30)$ & $0.18(0.15-0.25)$ & 0.4 & 0.4 \\
\hline Pt. 9 & $1.79(1.61-2.02)$ & $1.79(1.67-1.96)$ & 0.3 & 0.4 \\
\hline Pt. 10 & $0.18(0.08-0.29)$ & $0.16(0.07-0.22)$ & 0.7 & 0.5 \\
\hline average & $0.57(0.16-1.79)$ & $0.51(0.16-1.79)$ & 0.4 & 0.3 \\
\hline p-value & $0.002 *$ & 0.066 & \\
\hline
\end{tabular}

\section{S1.P15.}

\section{How cafeteria diet induced changes in fat content and metabolic profiles at rats mothers: MRI studies}

${ }^{*}$ T. Zalewski ${ }^{1}$, J. Matuszewska ${ }^{2}$, A. Klimaszyk ${ }^{1,3}$, S. Jurga ${ }^{1}$, J. H. Sliwowska ${ }^{2}$

${ }^{1}$ Adam Mickiewicz University in Poznan, NanoBioMedical Centre, Poznan, PL;

${ }^{2}$ Poznan University of Life Sciences, Laboratory of Neurobiology/ Faculty of Veterinary Medicine and Animal Science, Poznan, PL;
${ }^{3}$ Adam Mickiewicz University in Poznan, Department of Macromolecular Physics, Poznan, PL

Introduction: Consumption of "Western diet" containing high concentrations of sugar and fat contributing to development of obesity. To mimic effects of this diet, in animal studies cafeteria (CAF) diet is used.

We hypothesize that CAF diet given to rats before, during pregnancy, and lactation differently influence metabolic profiles and fat content. Females were exposed to CAF diet for four weeks before pregnancy, during pregnancy, and lactation. Control rats $(\mathrm{C})$ were fed standard chow diet AIN93G. Body composition of females were analyzed.

Methods: MRI experiments were carried out using a preclinical horizontal scanner operating at $9.4 \mathrm{~T}(400 \mathrm{MHz}$ for protons-Agilent) equipped with a $600 \mathrm{mT} / \mathrm{m}$ gradient system. For MRI imaging a 72-mm i.d. quadrature birdcage type coil was used. During the MRI experiment animals were put at a special designed holder and anesthetized with $1.5-2 \%$ isoflurane in a 50/50 air-oxygen mixture. The temperature of the animal was kept at $37{ }^{\circ} \mathrm{C}$. Respiration of the animal was monitoring and used to synchronize MRI experiments

Results: CAF diet in mothers increased food intake during pregnancy and altered body weight and fat content before, during and after pregnancy. There were significant differences in food intake in mothers in the second and third weeks of administration of CAF diet before pregnancy. We have found that during these weeks mothers from $\mathrm{CAF}$ group ate more compared to $\mathrm{C}$ group. Moreover, there was difference in food intake in the second week of pregnancy, when CAF mothers ate more compared to C. Mothers" body weight in CAF diet group was also increased, but reached statistically significant value at 6 after CAF diet exposure. A similar trend was seen at week 7 . Moreover, there were changes in percentage of fat content in animals. While at 1st week fat content was higher in $\mathrm{C}$ compared to CAF females, at week 13th CAF rats had higher fat content compared to controls.

Discussion: We confirmed the hypothesis that mothers cafeteria diet differently influences metabolic profiles and fat content. Besides study of pups, we also examined changes in females before during pregnancy and lactation. We found that dams on CAF diet had increased food intake in the second and third week before pregnancy and in the second week of pregnancy when compared to $\mathrm{C}$ group. These findings are in agreement with data obtained by SanchezBlanco et al., which shown that female rats kept on CAF diet 22 days before pregnancy and during pregnancy had higher food intake than control.

\section{Reference:}

Sanchez-Blanco C, Amusquivar E, Bispo K, Herrera E. Influence of cafeteria diet and fish oil in pregnancy and lactation on pups' body weight and fatty acid profiles in rats. Eur J Nutr. 2016;55(4):1741-53 
S2.P1.

\section{Assessment of diffusion characteristics of intact brain matter in stroke patients}

${ }^{*}$ Y. Stankevich ${ }^{1}$, K. Juravleva ${ }^{1}$, A. Savelov ${ }^{1}$, O. Bogomyakova ${ }^{1}$, L. Vasilkiv ${ }^{1}$, A. Tulupov ${ }^{1}$

${ }^{1}$ Russian Academy of Sciences, International Tomography Center, Novosibirsk, $R U$

Introduction: Stroke is one of the leading causes of death and disability in the world. According the latest data $(1,2)$ it was suggested the diffusion characteristics of the brain change not only in the ischemic focus, but also in the intact white matter. The aim was to study diffusion characteristics changes of the intact (contralateral) hemisphere white matter at the acute and chronic period ischemic stroke vs control group using diffusion-weighted and kurtosisweighted imaging.

Methods: The three groups of the patient at the age 40-70 yo was examined: control $(\mathrm{n}=11)$, acute stroke $(1-3$ days after clinical manifestation, $\mathrm{n}=12$ ), chronic stroke $(>3$-month, $\mathrm{n}=17)$. It was performed on the $3 \mathrm{~T}$ MR-scanner with sequences: 3D T1-TFE (axi), T2-TSE (axi), 3D FLAIR-SPIR (sag), DWI-EPI (axi). DTI sequence was additional to the routine protocol: $\mathrm{b}=0,1000$ and $1500 \mathrm{~s} / \mathrm{mm}^{2}$, TR/TE $10,500 / 73, \quad$ matrix $96 \times 94 \times 25, \quad$ voxel $2.33 \times 2.33 \times 2.33 \mathrm{~mm}, 25$ slices, $13 \mathrm{~min} 55 \mathrm{~s}, 32$ directions of the diffusion gradient, located mainly along the edges of the cube. Based on the this the coefficients of axial, radial, mean diffusion and fractional anisotropy of the intact hemisphere white matter were assessment with a mono-exponential (gaussian model, apparent diffusion coefficient map) and bi-exponential analysis (non-gaussian model, intravoxel incoherent motion model or diffusion kurtosis imaging) of data (3).

Results: The values of the coefficients axial, radial, mean diffusion and kurtosis for the white matter of the intact hemisphere were obtained for each of the groups (Fig. 1). According the analysis of variance the statistically significant difference was founded for the mean diffusion coefficient for the conrol vs acute and control vs chronic stroke groups, as well as for the mean kurtosis coefficient for the control vs acute stroke groups (Table 1).The statistically significant differences between the control vs stroke groups in the mean diffusion coefficient $(p=0.0009)$ and the mean kurtosis coefficient (0.007) in the white matter of the intact hemisphere was confirmed by the Mann-Whitney test.
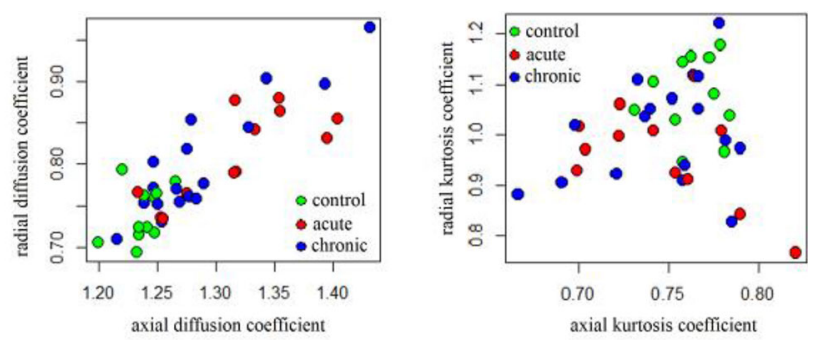

Figure 1. Distribution of the values coefficients mean diffusion and mean kurtosis for the control, acute and chronic strokes groups.

\begin{tabular}{|c|c|c|c|}
\hline groups & $\begin{array}{c}\text { mean diffusion } \\
\text { coefficient }\end{array}$ & $\begin{array}{c}\text { mean kurtosis } \\
\text { coefficient }\end{array}$ & fractional anisotropy \\
\hline $\begin{array}{c}\text { acute vs } \\
\text { control }\end{array}$ & $\mathbf{0 . 0 0 5}$ & $\mathbf{0 . 0 1 2}$ & 0.32 \\
\hline $\begin{array}{c}\text { chronic vs } \\
\text { control }\end{array}$ & $\mathbf{0 . 0 1 2}$ & 0.095 & 0.109 \\
\hline $\begin{array}{c}\text { chronic vs } \\
\text { acute }\end{array}$ & 0.693 & 0.48 & 0.867 \\
\hline
\end{tabular}

Table 1. p-values of the results of the intergroups analysis of variance of the coefficients mean diffusion and mean kurtosis, fractional anisotropy

Discussion: It was suggested the use of the diffusion and kurtosis characteristics of the white matter as the indicator of the brain state and it's may be prognostic factors of the stroke risk. The mean kurtosis coefficient probably indicates the severity of the loss of cellular structures, for example, in degenerative disease or neuronal/axonal atrophy (4). The results can be potentially useful both for early diagnosis and determination of the stroke risk, and for prognosis clinical outcome (5).

\section{References:}

1. https://doi.org/10.1016/j.neurobiolaging.2014.03.011;

2. https://doi.org/10.3389/fneur.2020.00252;

3. https://doi.org/10.2478/raon-2019-0004;

4. https://doi.org/10.2214/AJR.13.11365;

5. https://doi.org/10.3174/ajnr.A5180.

\section{S2.P2.}

\section{Altered brain activation associated with working memory during spontaneous migraine attacks}

\author{
*A. Ruiz-Tagle ${ }^{1}$, P. Figueiredo ${ }^{1}$, J. Pinto ${ }^{2}$, P. Vilela ${ }^{3}$, I. Pavão ${ }^{4}$, \\ R. Gil-Gouveia ${ }^{3}$ \\ ${ }^{1}$ University of Lisbon, ISR Lisbon / Department of Bioengineering, \\ Lisbon, $P T$; \\ ${ }^{2}$ University of Oxford, Institute of Biomedical Engineering/ \\ Department of Engineering Science, Oxford, GB, \\ ${ }^{3}$ Hospital da Luz, Lisbon, PT; \\ ${ }^{4}$ University of Lisbon, Department of Clinical Neurosciences, Lisbon, \\ $P T$
}

Introduction: Migraine is the second most common neurological cause for disability in young and middle-aged adults ${ }^{1}$. It is a cyclic disorder that consists of recurrent migraine attacks (ictal phase), and symptom-free periods in between (interictal phase) ${ }^{2}$. There is evidence showing cognitive disturbance in patients while undergoing the ictal phase, but controversy exists regarding other phases of the cycle $^{3}$. We investigated working memory in a clinical sample of migraineurs using fMRI.

Subjects/methods: 12 adult women with episodic migraine without aura underwent 2 sessions of fMRI (during (1) ictal and (2) interictal phases). fMRI was acquired on a $3 \mathrm{~T}$ Siemens Verio System, with a 12-channel RF coil using T2 weighted gradient-echo EPI sequence $\mathrm{TR}=2000 / 30 \mathrm{~ms}$ and voxel size of $4 \times 4 \times 3.6 \mathrm{~mm}$. N-back paradigm consisted of a block design with two conditions (0-back and 2-back) (NordicNeuroLab fMRI). The fMRI data was analyzed using FSL. Preprocessing included fieldmap distortion correction, spatialsmoothing $(\mathrm{FWHM}=5 \mathrm{~mm}$ ) and non linear registration to $\mathrm{MNI}$ space. For statistical analysis a general linear model was built including standard motion parameters as covariates. For second-level analyses we used flame1 with all sessions and per group of sessions; Session1 (S1) and Session2 (S2). For ROI analysis, a mask was 
extracted subtracting S1-S2 in order to obtain the percentage of BOLD signal change on the working memory contrast for all subjects on each session. SPSS was used for statistical analysis using non parametric tests and correlations.

Results/discussion: Group mean maps of activation during the 2-back task, and significant differences between ictal and interictal sessions, are shown in Figs. 1 and 2. Percentage of BOLD signal change in a left prefrontal ROI showing significantly higher activation during the ictal phase, and its correlation with task performance, are shown in Fig. 3.
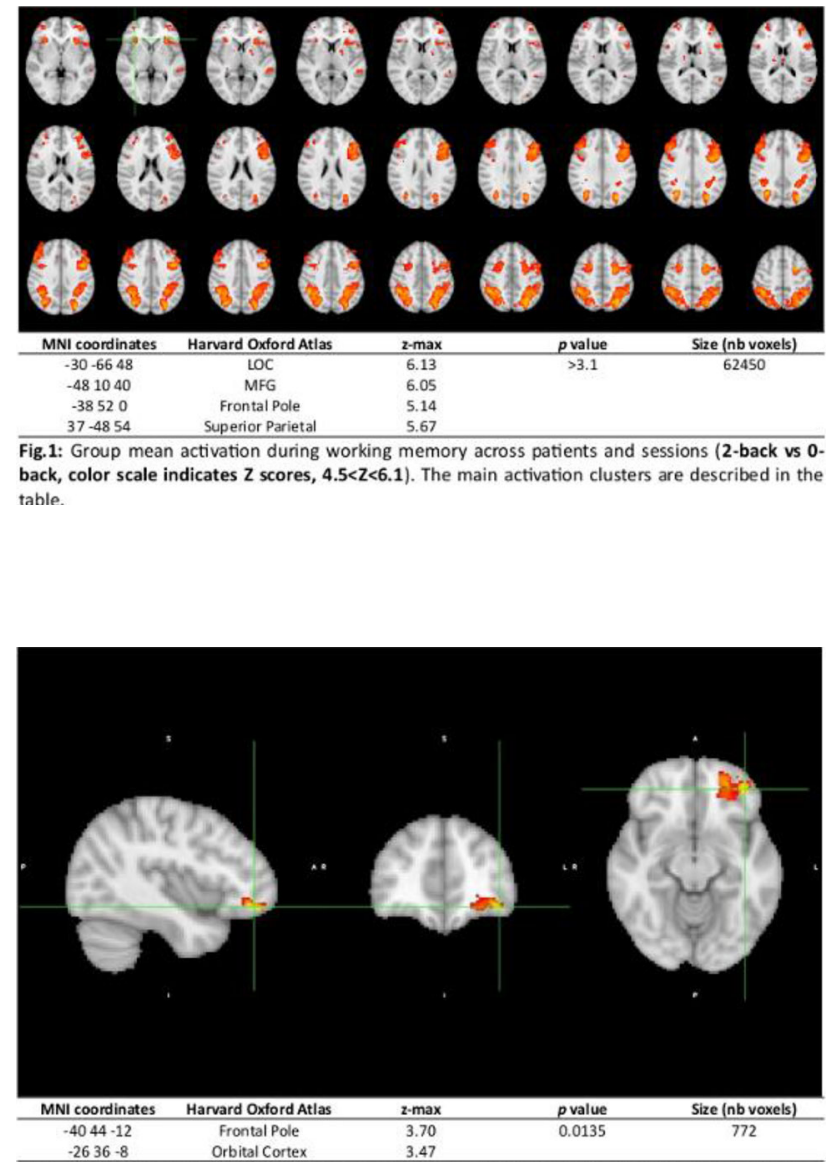

Fig.2. Group mean difference between session of activation during working memory across patients: ictal > interictal, for $\mathbf{2}$-back vs $\mathbf{0}$-back contrast, colour scale indicates $\mathbf{Z}$ scores, $3.2<2<6.1$ ). The main activation clusters are described in the table.
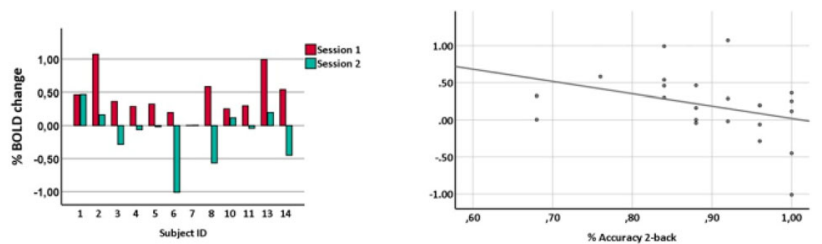

Fig.3. Brain activation in the prefrontal ROI vs. performance during 2-back working memory task. Left: \% BOLD signal change for each patient and session. Right: Spearman correlation between $\%$ BOLD signal change and performance accuracy, across patients and sessions (mean correlation= $-.45, p=0.030$ )

Prefrontal cortex regions on the left hemisphere were significantly more activated while performing a verbal working memory task under pain condition. Learning effect of this task can be observed as a decline in activation as well as improvement in performance, as described in previous literature ${ }^{4}$. This is the first time that working memory is investigated using fMRI to observe test-retest effects during spontaneous migraine attacks.

\section{References:}

1. V. Feigin et al., The Lancet Neurology. 18, 459-480, 2019.

2. M. Ashina et al., The Lancet, 2021.

3. D. Vuralli, C. Ayata \& H. Bolay, The journal of headache and pain. $19,1-14,2018$.

4. M. Plichta et al., Neuroimage. 60, 1746-1758, 2012.

\section{S2.P3.}

Structural and functional covariance in network nodes of victims of violence

\author{
*A. Shymanskaya ${ }^{1}$, U. Habel ${ }^{1,2}$, N. Kohn ${ }^{3}$, L. Wagels ${ }^{1,2}$ \\ ${ }^{1}$ University Hospital RWTH Aachen, Department of Psychiatry, \\ Psychotherapy and Psychosomatics (Faculty of Medicine), Aachen, \\ $D E$; \\ ${ }^{2}$ Jülich Aachen Research Alliance (JARA), Department \\ of Translational Brain Medicine, Aachen, DE; \\ ${ }^{3}$ Radboud University Medical Centre, Donders Institute for Brain, \\ Cognition and Behaviour, Nijmegen, NL
}

Introduction: The experience of violence is a stressor and is often associated with long-term effects on mental health. These effects might lead to changes in the structure and function of brain networks. The aim of the current study was to elucidate this stress-violencebrain relationship by testing structural and functional covariances among four stress-related networks: the default mode (DMN), salience (SN), dorsal attention (DAN), and frontoparietal (FPN) network. The four networks were parcellated into spatially distinct nodes, and brain data were averaged across all voxels of a node. We contrasted covariance in victims of violence $(\mathrm{VoVs}, \mathrm{n}=34)$ and matched controls without experience of violence $(n=32)$.

Methods: Participants underwent an extended scanning protocol, with a pre (R1) and a post-task (R2) resting-state fMRI scans and an anatomical MPRAGE scan. The task consisted of stress/emotion induction paradigms. Structural connectivity was determined from covariance in gray matter mass $\left(\mathrm{GMM}^{1}\right)$ using VBM. Sex, age, psychiatric diagnosis, antidepressants, the number of other psychotropic drugs, and total intracranial volume (for VBM) were controlled. We used sparse inverse covariance with threefold GraphicalLassoCV $\mathrm{CV}^{2}$ to estimate the partial correlation between two regions while conditioning for all other correlations in one step for each group and each modality.

Results: From VBM, we identified 7 pairs of nodes that differed significantly between VoVs and controls, 6 of them showing less GMM covariance in VoVs. Within-network disturbances emerged only in the SN (3), while the FPN showed the highest proportion of inter-network differences between groups (Fig. 1). In RS1 (Fig. 2A, B) 5 node-to-node connections differed significantly between groups: SN-DMN (2 positive, 1 negative), DAN-DMN, and DAN-FPN (2 negative), and 1 increased intra-network connection within FPN. Proportionally, differences were largest in DMN (67\%) and SN (50\%). For RS2 (Fig. 2C, D), 5 connections differed between groups. Less covariance in VoVs was found between SN Insula and DMN LP (R), while more covariance was observed for all other inter- and intranetwork connections. Differences were more frequent in SN and FPN $(60 \%)$. 
Fig. 1

A

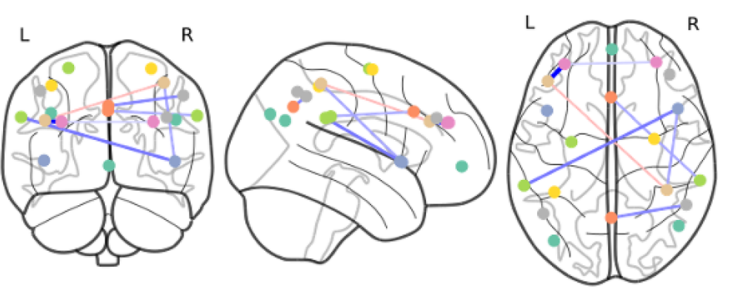

B
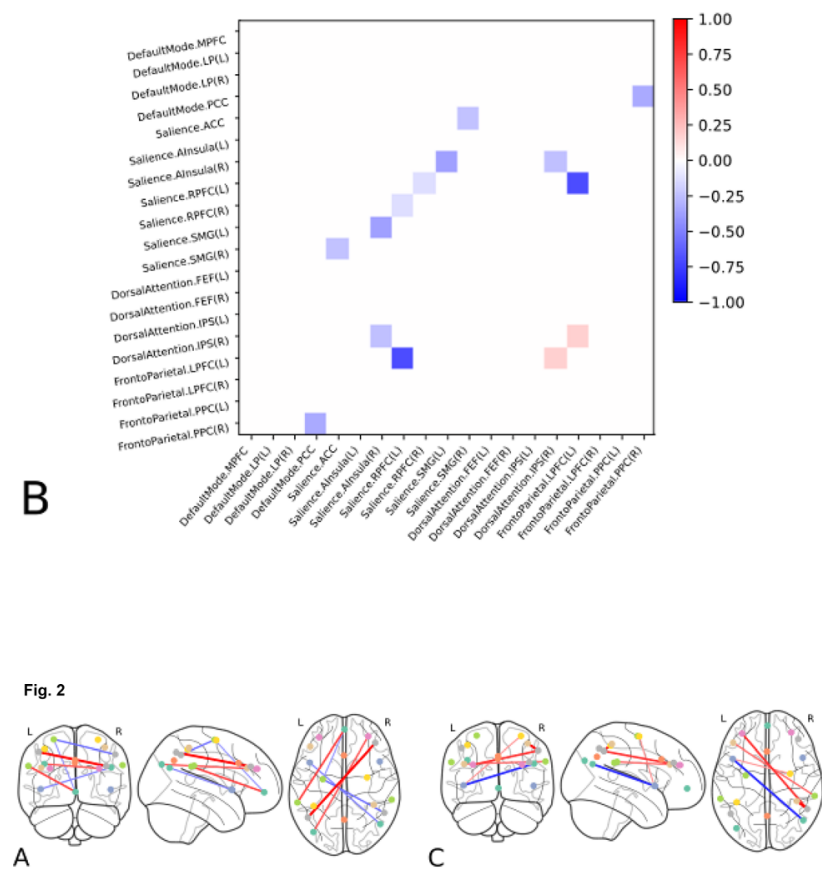

C
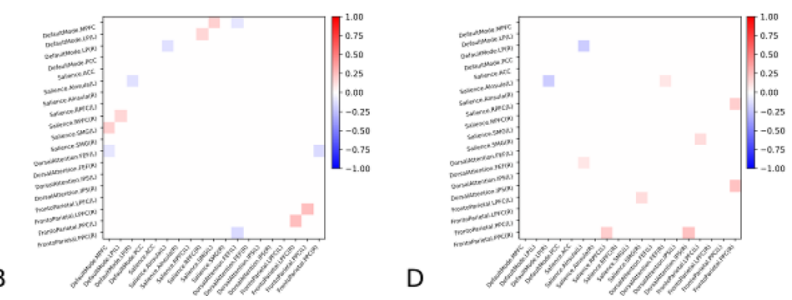

Discussion: Results demonstrate structural and functional connectivity differences prior to and after the two mild experimental stressors in a transdiagnostic and heterogeneous sample with a history of violence compared to unaffected controls. In line with previous findings ${ }^{3,4}$ in normal healthy and traumatized populations, the important role of the $\mathrm{SN}$, and SN-centered network interactions, may reflect dysfunctional mechanisms in $\mathrm{VoVs}$ that depict a risk factor for diverse mental health problems.

\section{References:}

${ }^{1}$ Gennatas et al. Journal of Neuroscience 20 (2017): 5065-5073.

${ }^{2}$ Varoquaux et al. International Conference on Medical Image Computing Springer, Berlin, Heidelberg, 2010.

${ }^{3}$ Akiki et al. Current Psychiatry Reports 11 (2017): 1-10.

${ }^{4}$ Clemens, et al. Scientific reports 1 (2017):1-9.

\section{S2.P4.} Memory functional connectivity study in patients
with temporal lobe epilepsy

\author{
*N. Pereira ${ }^{1,2}$, V. Tavares ${ }^{1,2}$, C. Capela ${ }^{3}$, L. Cerqueira ${ }^{3}$, H. Ferreira ${ }^{1,2}$ \\ ${ }^{1}$ Instituto de Engenharia Biomédica e Biofísica, Lisbon, PT, \\ ${ }^{2}$ University of Lisbon, Faculty of Sciences, Lisbon, PT; \\ ${ }^{3}$ Centro Hospitalar Lisboa Central, Department of Neurology, \\ Lisbon, $P T$
}

Introduction: Temporal Lobe Epilepsy (TLE) is one of the most common epilepsies and is drug-resistant in $76.8 \%$ of cases [1, 2], therefore needing surgery. TLE is often associated with sclerosis of the mesial temporal structures: the hippocampus, entorhinal cortex, and amygdala, which are involved in memory encoding, storage, and retrieval [1]. So, mapping of the memory network is critical for preventing surgical sequelae. Common memory paradigms require complex and costly instrumentation, preventing its more widespread use. Herein, a memory paradigm was adapted with hand grasping to collect patient feedback seamlessly.

Methods: Twelve TLE patients (8 males) aged 25 to 62 were studied. As part of a pre-surgical planning protocol, all participants performed a hand grasping and a face-name memory paradigm adapted from [3]. A block design of $100 \mathrm{~s}$ duration was used for the hand grasping paradigm in which the subjects were asked to alternately move each hand for $10 \mathrm{~s}$.

The memory paradigm consisted of viewing faces unfamiliar to the patient paired with fictional first names, in a modified Novel vs. Repeated design, with a total duration of $10 \mathrm{~min}$. Each face-name pair was presented for $5 \mathrm{~s}$. A total of 4 runs were done, in which each run was composed of Novel face-name pairs followed by a fixation cross for rest, followed by Repeated face-name pairs. The Repeated facename pairs could have wrong names associated, to check whether the patient remembered the correct pair. The patients' answer was provided through hand grasping (left hand to wrong/ right hand to correct).

Brain scans were done using a 1.5 T MRI scanner with a 12-channels head matrix coil. A volumetric T1-weighted scan was done, followed by BOLD sequences for the functional paradigms.

A general linear model (GLM) was used in FSL to analyse the functional images to map hand grasping regions and regions related to memory encoding and retrieval.

The higher-level group analysis included the following contrasts: EncodeRegion, regions active for encoding; and RetrieveFalse and RetrieveTrue, regions related to wrong and correct face-name correspondence, respectively.

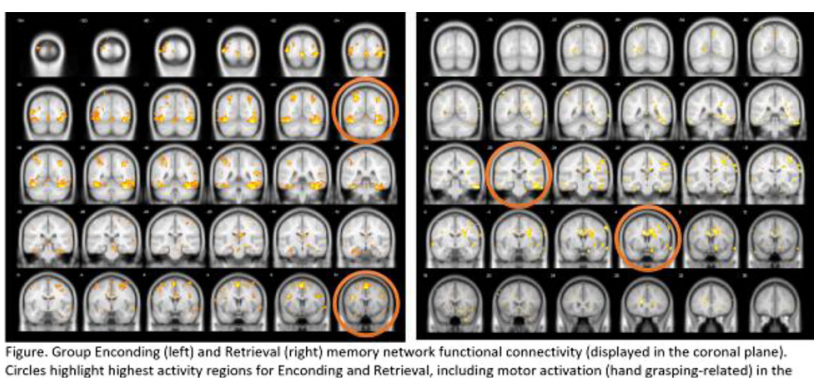

Circles highlight highest activity regions for Enconding and Retrieval, including motor activation (hand grasping-related) in the atter paradigm

Results: The regions observed to be related to encoding and retrieval of memory information were (see Figure):

- Encoding: bilateral temporal lobe, fusiform gyrus, and right inferior orbitofrontal cortex. 
- RetrieveFalse: sensorimotor cortex, procedural memory regions including basal ganglia and the cerebellum, and the culmen, fusiform gyrus, anterior cingulate, and limbic lobe.

- RetrieveTrue: similar activation patterns as those of RetrieveFalse, with additional activation in the occipital lobe and lingual gyrus.

Discussion: This study has demonstrated that memory network mapping can be fairly easily implemented in a clinical setting using a face-name paradigm with hand grasping as a response.

\section{References:}

[1] Bernhardt et al., 2013. https://doi.org/10.3389/fnhum.2013.00624

[2] Sisodiya et al., 2002. https://doi.org/10.1093/brain/awf002

[3] Sperling et al., 2001. https://doi.org/10.1002/hbm.1047

\section{S2.P5.}

\section{Investigating cerebrovascular reactivity of moyamoya disease patients using PET/MRI}

\author{
${ }^{*}$ M. Zhao ${ }^{1}$, M. Moseley ${ }^{1}$, G. Steinberg ${ }^{2}$, G. Zaharchuk ${ }^{1}$ \\ ${ }^{1}$ Stanford University, Department of Radiology, Stanford, CA, US; \\ ${ }^{2}$ Stanford University, Department of Neurosurgery, Stanford, CA, DE
}

Introduction: Cerebrovascular reactivity (CVR) reflects the change in cerebral blood flow (CBF) in response to a vasoactive stimulus often performed in a brain stress test. Studies have demonstrated that impaired CVR was associated with a higher risk of acute stroke ${ }^{1}$. PET imaging with ${ }^{15} \mathrm{O}$-water as the radiotracer has been the standard modality to measure CBF and CVR. However, it is impractical in most hospitals due to the requirement of an on-site cyclotron. Arterial spin labeling is a quantitative MRI technique that enables non-invasive $\mathrm{CBF}$ and CVR measurement and has demonstrated effectiveness in measuring CVR of normal subjects ${ }^{2}$. In this work, we compare the CVR of Moyamoya patients, whose vessel occlusion is a risk factor to acute stroke, measured by ASL MRI with the reference-standard 15Owater PET.

Methods: Imaging data were acquired from 26 Moyamoya patients (18-64 years, 16 females) using a simultaneous 3 T PET/MRI system (GE SIGNA, Waukesha, WI, USA). All patients had unilateral or bilateral vessel occlusion at the anterior cerebral artery (ACA), middle cerebral artery (MCA), and/or posterior cerebral artery (PCA). The scanning parameters of the single post-labeling delay pseudocontinuous ASL (single-PLD PCASL), delay pseudo-continuous ASL (multi-PLD PCASL, 3 PLDs), and PET were described in our previous work ${ }^{2}$. PET and ASL data were acquired simultaneously at baseline and $15 \mathrm{~min}$ after the injection of the vasodilator acetazolamide $(15 \mathrm{mg} / \mathrm{kg}$ with a maximum of $1 \mathrm{~g})$. Each patient received $891 \pm 71.8 \mathrm{MBq}$ of $15 \mathrm{O}$-water. CBF of PET was computed using the single-compartment pharmacokinetic model; CBF of ASL was computed using the general kinetic model ${ }^{3,4}$. CVR was computed as the percentage of $\mathrm{CBF}$ change compared with baseline CBF. Flow territories (right and left ACA, MCA, and PCA) were defined based on the Harvard-Oxford cortical and subcortical structural atlases ${ }^{5}$. Mean CVR within the territories affected by occlusion and normal territories was computed for each subject. Paired t-tests were performed to compare the mean CVR between the affected and unaffected territories. Concordance correlation coefficient values were computed to examine the agreement between CVR measured by ASL and PET. Results: Figure 1 shows the hemodynamic maps of an example patient. Figure 2 shows that the CVR of the affected regions was significantly lower than the normal regions. Figure 3 shows that
multi-PLD PCASL achieved the higher agreement with PET based on higher concordance correlation coefficients.
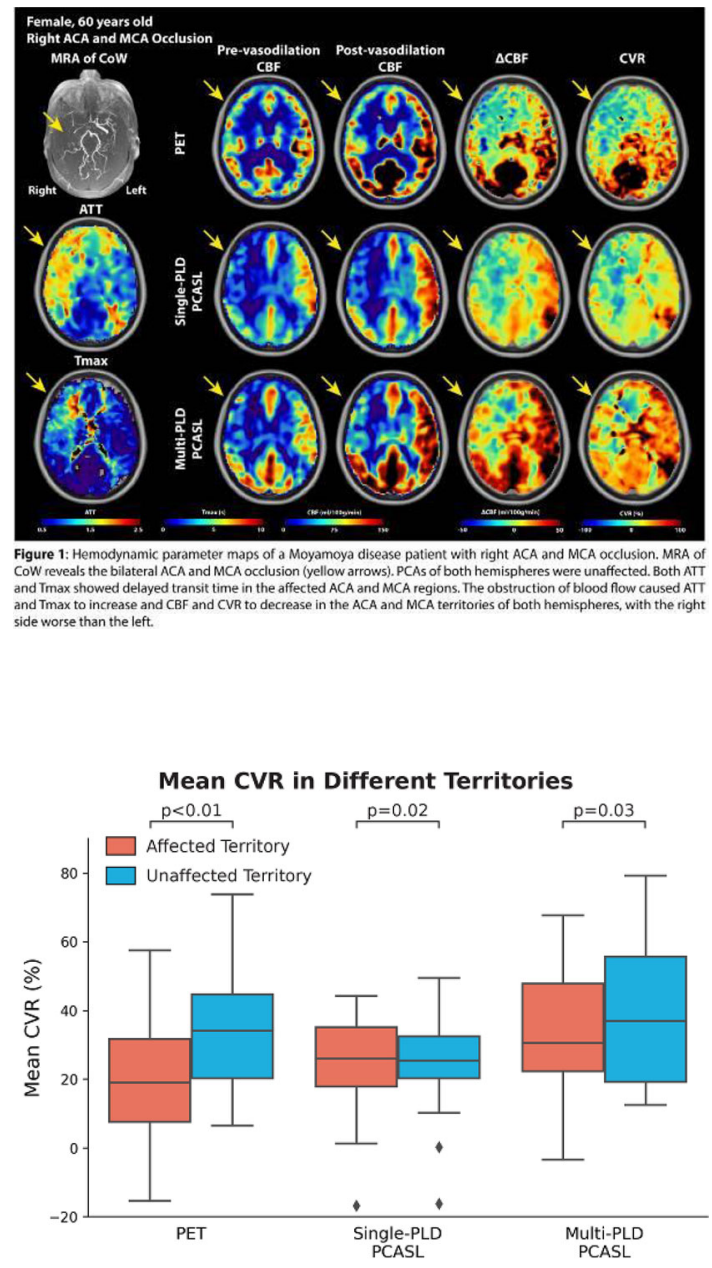

Figure 2: Group mean CVR measured by ASL and PET in affected and normal territories. The mean CVR measured by all modalities was significantly lower in affected regions. Each box plot indicates from top to bottom, the maximum, 75 th, 50th, 25th percentiles, and minimum not considering outliers, and the outliers represented by diamonds.

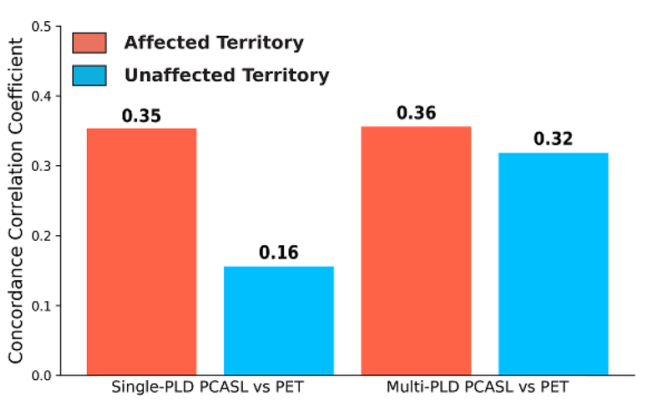

Figure 3: Concordance correlation coefficients between ASL and PET. Both ASL techniques had a similar PCASL showed higher agreement with PET in normal (unaffected) territories! 
Discussion: Multi-PLD PCASL had the highest agreement with the reference PET modality in CVR measurements. Both single and multi-PLD ASL were effective in detecting impaired CVR in Moyamoya patients.

\section{References:}

1 Yonus et al., J. Neurosug. 1993.

2 Zhao et al., NeuroImage, 2021.

3 Zhou et al., IEEE Transactions on Nuclear Science 2001.

4 Buxton et al., MRM, 1998.

5 Smith et al., NeuroImage, 2004.

\section{S2.P6.}

\section{Sensitivity of quantitative MRI for post mortem evaluation of brain edema}

\author{
*M. Bauer ${ }^{1}$, C. Berger ${ }^{1}$, E. Scheurer ${ }^{1}$, C. Lenz ${ }^{1}$ \\ ${ }^{1}$ University of Basel, Institute of Forensic Medicine, Basel, $\mathrm{CH}$
}

Introduction: The post mortem determination of brain edema is routinely performed by forensic pathologists during autopsy by rating macroscopically visible signs [1]. An objective and equally reliable method uses the normalized cerebral weight, which is calculated by dividing the brain weight measured at autopsy by the intracranial volume segmented in computed tomography (CT) images [2]. In this work, we evaluated the sensitivity of quantitative magnetic resonance imaging (MRI) for the assessment of brain edema by correlating the values of post mortem $\mathrm{T} 1, \mathrm{~T} 2, \mathrm{~T} 2 *$, fractional anisotropy (FA) and mean diffusivity (MD) with the normalized cerebral weight.

Methods: In this study, 18 deceased were included. A head CT scan was performed and the MRI protocol, shown in Table 1, was applied to the brains in-situ using a $3 \mathrm{~T}$ scanner (both Siemens, Siemens Healthineers, Erlangen, Germany). For every examined MRI parameter, the values in the automatically segmented cortex, white and deep gray matter were determined separately. To evaluate the agreement of the MRI parameters with the normalized cerebral weight, the correlation coefficient $r$ and the corresponding $p$ value $p$ were calculated. Image and statistical analysis were performed using FSL (FMRIB Software Library, Analysis Group, [3]) and MATLAB (The MathWorks, Inc., Natick, MA, United States).

\begin{tabular}{|c|c|c|}
\hline $\begin{array}{l}\text { MRI } \\
\text { parameter }\end{array}$ & MRI sequence & Properties \\
\hline$T_{1}$ & Inversion recovery spin echo & $6 \mathrm{TI}=30-1200 \mathrm{~ms}, \mathrm{TE}=12 \mathrm{~ms}, \mathrm{TR}=7060 \mathrm{~ms}$ \\
\hline $\mathrm{T}_{2}$ & Multi-contrast spin echo & $12 \mathrm{TEs}=9.8-117.6 \mathrm{~ms}, \mathrm{TR}=5720 \mathrm{~ms}$ \\
\hline $\mathrm{T}_{2}{ }^{*}$ & Multi-echo gradient echo & $12 \mathrm{TEs}=5.79-50.94 \mathrm{~ms}, \mathrm{TR}=68 \mathrm{~ms}$ \\
\hline $\mathrm{FA}, \mathrm{MD}$ & $\begin{array}{l}\text { Diffusion weighted single shot } \\
\text { spin echo }\end{array}$ & $\begin{array}{l}\mathrm{TE}=109 \mathrm{~ms}, \mathrm{TR}=18700 \mathrm{~ms}, \mathrm{~b}=2000 \mathrm{~s} / \mathrm{mm}^{2}, \\
64 \text { directions }\end{array}$ \\
\hline
\end{tabular}

Results: The obtained correlations and p values are given in Table 2. The correlations between the normalized cerebral weight and T1, T2 and $\mathrm{MD}$, respectively, are significant in the cortex. FA exhibits a significant correlation with the normalized cerebral weight not only in the cortex, but also in the white and deep gray matter.

\section{fig. 2}

Table 2: Correlation coefficients $\mathrm{r}$ and corresponding p-values $\mathrm{p}$ of correlations between MRI parameters $T_{1}, T_{2}, T_{2}^{*}, F A$ and $M D$ and the normalized cerebral weight for cortex, white and deep gray parameters $\mathrm{T}_{\mathrm{l}}, \mathrm{T}_{2}, \mathrm{~T}_{2}, \mathrm{FA}$ and $\mathrm{MD}$ and the normalized cerebral weight for cortex, white and deep gray
matter. High correlations $|\mathrm{r}|>0.7$ and significant $\mathrm{p}$-values $\mathrm{p}<0.05$ are highlighted in bold.

\begin{tabular}{c|cccccc}
\hline Region & \multicolumn{2}{|c}{ Cortex } & \multicolumn{2}{c}{ White matter } & \multicolumn{2}{c}{ Deep gray matter } \\
Parameter & $\mathbf{r}$ & $\mathbf{p}$ & $\mathbf{r}$ & $\mathbf{p}$ & $\mathbf{r}$ & $\mathbf{p}$ \\
\hline $\mathbf{T}_{\mathbf{1}}$ & -0.59 & $\mathbf{0 . 0 1 0}$ & -0.28 & 0.253 & -0.47 & 0.051 \\
$\mathbf{T}_{2}$ & $-\mathbf{0 . 7 8}$ & $\mathbf{0 . 0 0 0}$ & -0.19 & 0.453 & 0.04 & 0.889 \\
$\mathbf{T}_{2}{ }^{*}$ & -0.24 & 0.342 & -0.09 & 0.727 & 0.21 & 0.410 \\
$\mathbf{F A}$ & $\mathbf{0 . 7 8}$ & $\mathbf{0 . 0 0 0}$ & 0.63 & $\mathbf{0 . 0 0 5}$ & 0.49 & $\mathbf{0 . 0 4 0}$ \\
$\mathbf{M D}$ & -0.62 & $\mathbf{0 . 0 0 6}$ & 0.11 & 0.677 & -0.02 & 0.933 \\
\hline
\end{tabular}

Discussion: Especially FA values and the cortex are suitable to detect brain edema post mortem as significant correlations were detected in all regions for FA as well as in the cortex for almost all MRI parameters. However, potential influencing factors like the post mortem interval, the age at death and the brain temperature of the deceased during MRI scanning were not evaluated in this study and need to be carefully investigated in future work.

\section{References:}

[1] Hausmann et al., IJLM, 2006, 120.

[2] Bauer et al., FSI, 2020, 308. [3] Jenkinson et al., NeuroImage, 2012,62 .

S2.P7.

Multiparametric MRI to evaluate the antiinflammatory effect of a non-steroideal drug in the tumoral development of a glioma rat model

\author{
*I. J. Veríssimo Cabete ${ }^{1}$, *N. Arias-Ramos ${ }^{1}$, P. López-Larrubia ${ }^{1}$, \\ M. J. Guillén Gómez ${ }^{1}$ \\ ${ }^{1}$ Instituto de Investigaciones Biomédicas, CSIC/UAM, Madrid, ES
}

Introduction: Glioblastoma multiforme (GBM) is the most aggressive manifestation of brain tumors [1]. The prognosis and survival remain poor and tumors generally recur after standard surgery and coadjuvant radio-chemotherapy [2]. Many studies have been done with GBM models, identifying magnetic resonance imaging (MRI) parameters to be used as non-invasive surrogate biomarkers of this pathology [3], which are able to determine the therapeutic response during the initial stages of treatment or even before. Anti-inflammatory (AI) agents have been studied as anti-tumoral drugs [4] and tested in GBM models with promising results [5]. Furthermore, the modulation of inflammation in GBM by AIs may improve responses to current treatments $[6,7]$. In this study we aimed to assess the MRI tumor features evolution in GBM-bearing rats treated with a nonsteroidal AI to investigate its impact on the evolution and treatment of the disease.

Methods: GBM was induced in Sprague-Dawley rats by intracranial injection of C6 cells [8]. Animals were subcutaneously (s.c). injected with the AI drug Meloxicam $(2 \mathrm{mg} / \mathrm{kg}$ ) or saline (control) during 15 days, starting the treatment 5 days after tumor cell injection. MRI evaluations were carried out in a $7 \mathrm{~T}$ system until day 27 post-cell injection (or until end-point criteria). Tumor volume was followed-up with Gd-T1W images at different time points. Multiparametric MRI studies with magnetization transfer, diffusion-weighted, T2W, T2*W and dynamic contrast enhancement images were acquired at an early and an advanced tumor stage. Parametric maps were generated with home-made software developed with Matlab.

Results: So far, we have observed lower MTR\% and axial diffusivity (AD) values in the tumor area of treated rats compared with control rats at the advanced tumor stage (Fig. 1), while fractional anisotropy (FA) values were higher in the peritumoral region. 


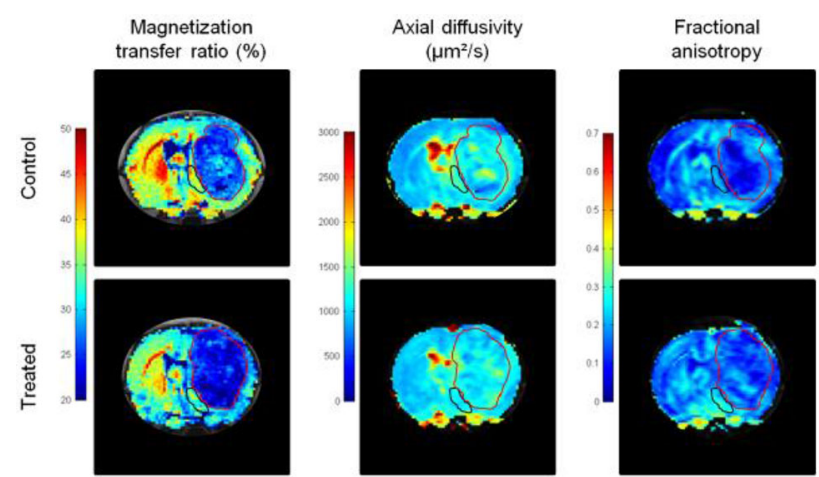

Figure 1. Parametric maps of magnetization transfer ratio (MT\%), axial diffusivity (AD) and fractional anisotropy (FA) of control and treated rats at the advanced tumor stage. The selected ROIs are tumor area (red) and peritumoral region (black).

Discussion: These results could suggest that treated animals presented a greater structural integrity of the tumor tissue, which means the absence of characteristic necrosis of a developing cancer, while in the peritumoral region there was not so much accumulation of cells that would occur in an proliferation process, pointing Meloxicam as potential candidate therapy against GBM.

\section{References:}

[1] Hanif et al., Asian Pac J Cancer Prev, 18 (2017), 3-9.

[2] Davis et al., Clin J Onc Nurs, 20 (2016), S2-S8.

[3] Shaver et al., Cancers, 11 (2019), 829.

[4] Rayburn et al., Mol Cell Pharmacol, 1 (2009), 29-43.

[5] De Souza et al., J Magn Reson, 42 (2015), 1582-1591.

[6] Nifterik et al., Int J Radiat Biol, 87 (2011), 192-201.

[7] Jin et al., Mater Express, 10 (2020), 551-555.

[8] Pérez-Carro et al., EJNMMI Res, 4 (2014) 44.

\section{S2.P8.}

\section{Longitudinal hybrid PET/MRI in juvenile Huntington disease: a multimodal structural, functional and metabolic pilot study}

\author{
*M. E. Caligiuri ${ }^{1}$, ${ }^{*}$. Vizza $^{2}$, P. Veltri ${ }^{3}$, F. Squitieri ${ }^{4}$, G. L. Cascini ${ }^{5}$, \\ U. Sabatini ${ }^{6}$ \\ ${ }^{1}$ University Magna Graecia of Catanzaro, Neuroscience Research \\ Center, Catanzaro, IT; \\ ${ }^{2}$ Mater Domini University Hospital, Dpeartment of Neuroradiology, \\ Catanzaro, IT; \\ ${ }^{3}$ University Magna Graecia of Catanzaro, Department of Surgical \\ and Clinical Science, Catanzaro, IT; \\ ${ }^{4}$ CSS-Mendel Institute of Human Genetics, IRCCS Casa Sollievo della \\ Sofferenza Hospital, Rome, IT; \\ ${ }^{5}$ University Magna Graecia of Catanzaro, Department \\ of Experimental and Clinical Medicine, Catanzaro, IT; \\ ${ }^{6}$ University Magna Graecia of Catanzaro, Department \\ of Neuroradiology, Catanzaro, IT
}

Introduction: Huntington disease is a rare and autosomal dominant inherited disorder caused by an expanded CAG repeat mutation in the Huntingtin gene [1]. Juvenile Huntington"s disease (JHD, onset $\leq 20$ years) is even rarer and it is associated with large CAG repeats $(>60)$. The clinical features of JHD are heterogeneous, and differ from those characterizing the adult form (e.g., rigidity and seizures are more common than chorea). For this reason, it is crucial to monitor and characterize patterns of disease progression. In this work, we propose an hybrid-imaging approach for the longitudinal assessment of brain changes in JHD, using multimodal PET-MRI data fusion (i.e., merging information regarding structural, functional and metabolic alterations).

Methods: A 20-year-old female with JHD underwent 3 T PET-MRI (Siemens Biograph mMR) at two timepoints (18 months follow-up). The protocol included FDG-PET as well as the simultaneous acquisition of T1-weighted and resting state functional MRI (rs-fMRI) data. Figure 1 shows the proposed analysis workflow, carried out using FSL and AFNI, applied over single-subject longitudinal data. Briefly, voxel-wise percentage changes at follow-up relative to baseline status were calculated on (i) grey matter (GM) density, (ii) FDG-PET uptake, (iii) rs-fMRI regional homogeneity (ReHo), and (iv) rs-fMRI amplitude of low frequency fluctuation (fALFF).

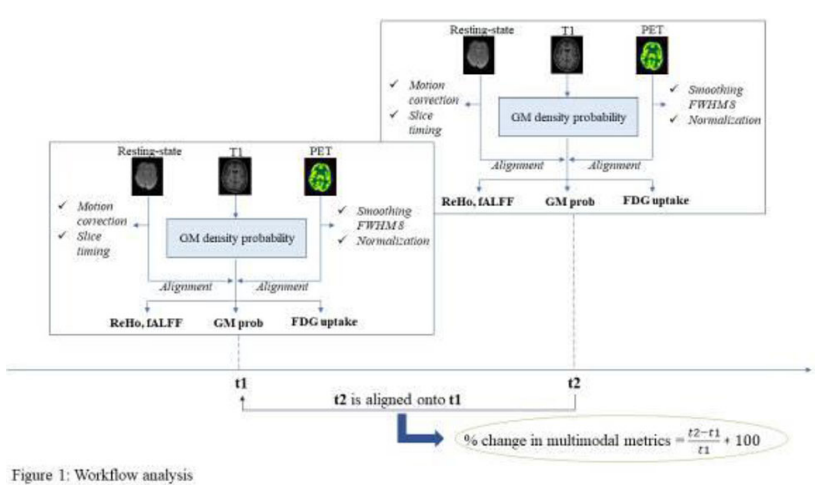

Results: Figure 2 shows voxel-wise maps of percentage changes at follow-up in the single JHD patient. GM density decreased on average over the whole brain of $31.9 \pm 21.1 \%$ (median [interquartile range $]=28.7[14.2-47.5]$ ). Percentage change in FDG uptake was increased in the frontoparietal network, while widespread reduction was observed in the remaining regions (including thalamus). ReHo and fALFF followed the pattern of PET changes, also highlighting increased functional connectivity in the frontal cortex opposed to decreased connectivity in posterior regions.

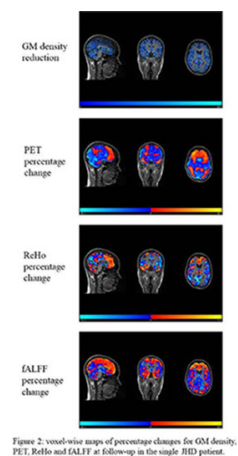

Discussion: Results provide a comprehensive evaluation of complementary changes that affect a single JHD patient over 18 months. These changes were widespread and the percentage change was relevant, compared to known trajectories of healthy aging over the same time period. This pilot study suggests that hybrid imaging is a promising technique for monitoring of JHD changes over time, especially in the context of future clinical trials to test disease-modifying drugs. 


\section{Reference:}

Fusilli C. et al. Biological and clinical manifestations of juvenile Huntington's disease: a retrospective analysis. Lancet Neuro, 2018, 17.11:986-993.

\section{S2.P9.}

\section{The functional and structural consequences} of microglia in major depressive disorder

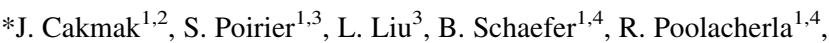
A. Burhan ${ }^{1,3}$, P. Sabesan ${ }^{1,4}$, K. St. Lawrence ${ }^{1,3}$, J. Theberge ${ }^{1,3}$,

J. Hicks $^{1,3}$, E. Finger ${ }^{1,3}$, L. Palaniyappan ${ }^{1,2,4}$, U. Anazodo ${ }^{1,3}$

${ }^{1}$ Western University, London, CA;

${ }^{2}$ Robarts Research Institute, London, CA;

${ }^{3}$ Lawson Health Research Institute, Lawson Imaging Department,

London, $C A$;

${ }^{4}$ London Health Sciences Centre, London, CA

Introduction: Major depressive disorder (MDD) is a highly debilitating neuropsychiatric illness linked to increases in both peripheral and central inflammation, as well as to changes in both functional and structural connectivity. Although countless studies have investigated these topics, the relationship between neuroinflammation and functional/structural connectivity in MDD has not been explored.

Methods: Here we explore this relationship in 12 treatment-resistant late-life MDD patients and 23 healthy controls using simultaneous acquisition of [18F]-FEPPA PET and MRI. Relative standardized uptake values of microglia activity measured with PET and Pearson"s r-to-z scores representing functional connectivity were extracted from the insula and the subgenual subregion of the anterior cingulate cortex ( $\operatorname{sgACC}$ - two key areas implicated in MDD pathogenesis $(1,2)$. Diffusion tensor imaging metrics were extracted from the cingulum bundle, intersecting these key regions. Regression analyses were performed to assess the associations between sgACC microglial activity and functional connectivity, structural connectivity, and HAM-D scores.

Results: We found significantly increased [18F]-FEPPA uptake in the depressed patients compared to the healthy controls in the left sgACC. Controls compared to patients showed an overall increase in connectivity between the sgACC and the insula, and functional connectivity was a significant non-linear factor in explaining the microglial activity in the left sgACC. This left sgACC microglial activity was also linearly associated with structural connectivity of the cingulum bundle measured by diffusion tensor imaging. Furthermore, [18F]-FEPPA uptake predicted severity scores of HAM-D.

Discussion: In this pilot observational study, we show that there are distinct differences in microglial activity between MDD patients and controls that are associated with altered functional connectivity between the sgACC and the insula and which can influence the structural connectivity of the cingulum bundle. The non-linear relationship between microglial activity and functional connectivity may indicate peaks of microglial activity that correspond with specific functional outcomes. This study, albeit preliminary, suggests that neuroinflammation relates to both functional and structural brain network function in MDD.
A.

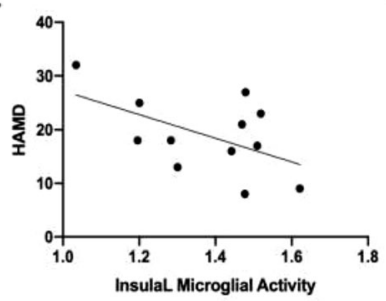

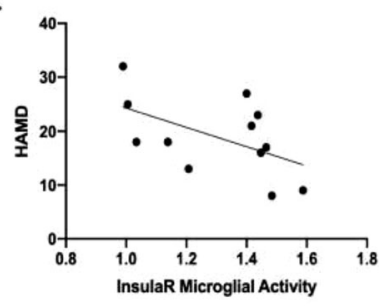

Figure 1. The relationship between insula microglial activity and HAM-D scores.

A.

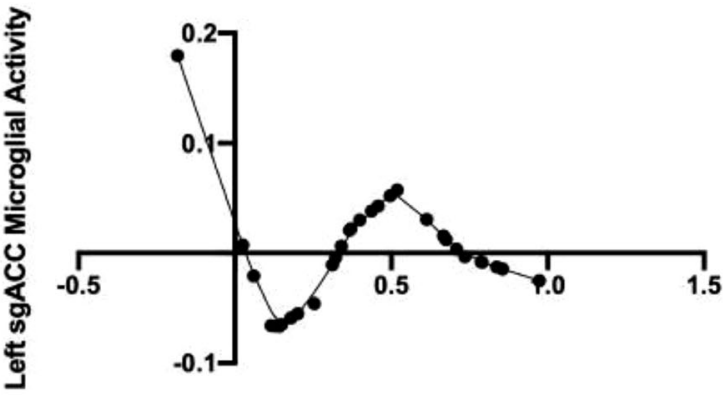

Functional Connectivity

B.

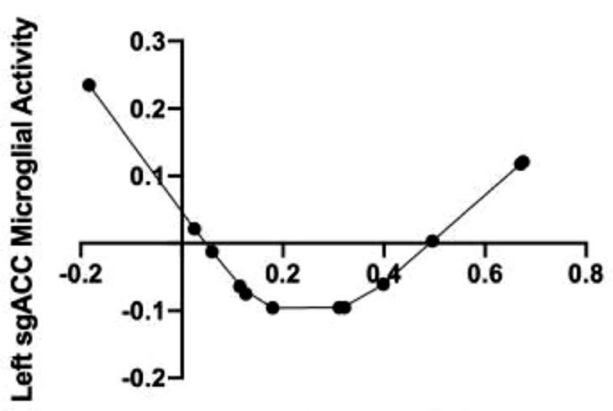

Functional Connectivity

C.

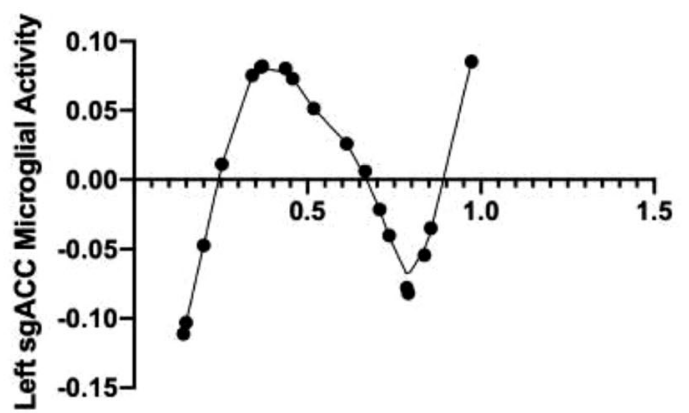

Functional Connectivity

Figure 2. The relationship between microglial activity and functional connectivity. 

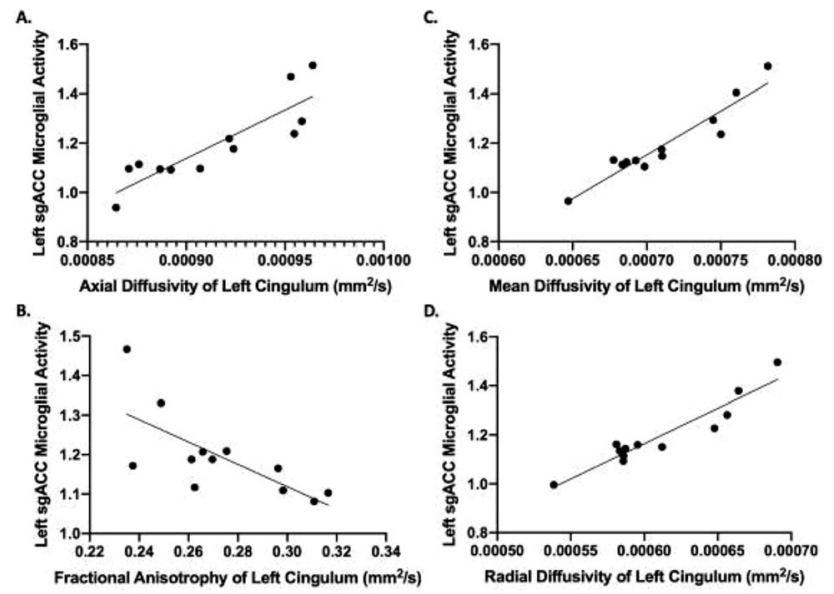

Figure 3 . The relationship between microglial activity and structural connectivity.

\section{References:}

Wiebking C, Bauer A, De Greck M, Duncan NW, Tempelmann C, Northoff G (2010): Abnormal body perception and neural activity in the insula in depression: An fMRI study of the depressed "material me.” World J Biol Psychiatry 11. 10.3109/15622970903563794 Stevens FL, Hurley RA, Taber KH (2011): Anterior cingulate cortex: Unique role in cognition and emotion. $J$ Neuropsychiatry Clin Neurosci 23: 121-125.

\section{S2.P10.}

\section{Assessment of cerebral perfusion symmetry in retired Canadian Football League players}

*E. Danielli ${ }^{1,2}$, B. Padrela ${ }^{3}$, M. Doughty ${ }^{1,2}$, J. Petr ${ }^{3,4}$, H. J. Mutsaerts ${ }^{3}$, M. D. Noseworthy ${ }^{1,2,5}$

${ }^{I}$ McMaster University, School of Biomedical Engineering, Hamilton, $C A$;

${ }^{2}$ St. Joseph's Healthcare, Imaging Research Centre, Hamilton, CA; ${ }^{3}$ Amsterdam University Medical Center, Department of Radiology and Nuclear Medicine, Amsterdam, NL;

${ }^{4}$ Helmholtz Center Dresden-Rossendorf, Institute

of Radiopharmaceutical Cancer Research, Dresden, DE;

${ }^{5}$ McMaster University, Department of Electrical and Computer Engineering, Hamilton, CA

Introduction: High contact sports such as American football can result in athletes sustaining concussions and countless sub-concussive blows, leading to cumulative neurological damage [1]. This study aimed to use cerebral blood flow (CBF) measured with ASL and a Z-scoring approach to quantify focal brain damage and left/right symmetry in retired Canadian Football League (rCFL) players. It was hypothesized that subjects would have asymmetric CBF and regional hypoperfusion due to their history of repetitive head trauma.

Methods: Seventeen rCFL players (100\% male, aged $58 \pm 6.15 \mathrm{y})$ were scanned using a $3 \mathrm{~T}$ GE Discovery MR750 MRI and a 32-channel head coil. 3D T1-weighted fSPGR and 3D pseudo-continuous ASL (pCASL) scans acquired. The pCASL scans were processed using ExploreASL to perform brain segmentation, motion correction, spatial normalization to MNI152 T1 $1.5 \mathrm{~mm}$ space, partial volume correction, and spatial covariance $(\mathrm{CoV}) \mathrm{CBF}$ quantification (Fig. 1) [2]. CoV was expected to be more robust with compromised cerebrovasculature and physiological differences [3]. Twelve concussion-related regions-of-interest (ROIs) were selected from the Harvard-Oxford (HO) and Hammers (HS) Atlases (Table 1) [4]. ROI $\mathrm{CBF}$ was analyzed for the left and right hemispheres separately and bilaterally. Prior to Z-scoring, ROIs that failed Shapiro-Wilk normality testing or had insufficient data were excluded. A Z-scoring approach was applied in MATLAB with the bilateral $\mathrm{CoV}$ value as the comparable metric. Z-score outliers that fell 2, 3 or 4 standard deviations from the bilateral means were classified as mild (1), moderate (2), or severe (3) injury burden (IB). A subject-specific IB symmetry index was calculated as: $(\mathrm{L}-\mathrm{R}) /(0.5(\mathrm{~L}+\mathrm{R}))$. Normality, multiple linear regression, and correlation tests were performed with RStudio. Age, type of positional head impact, and career length were used as covariates.

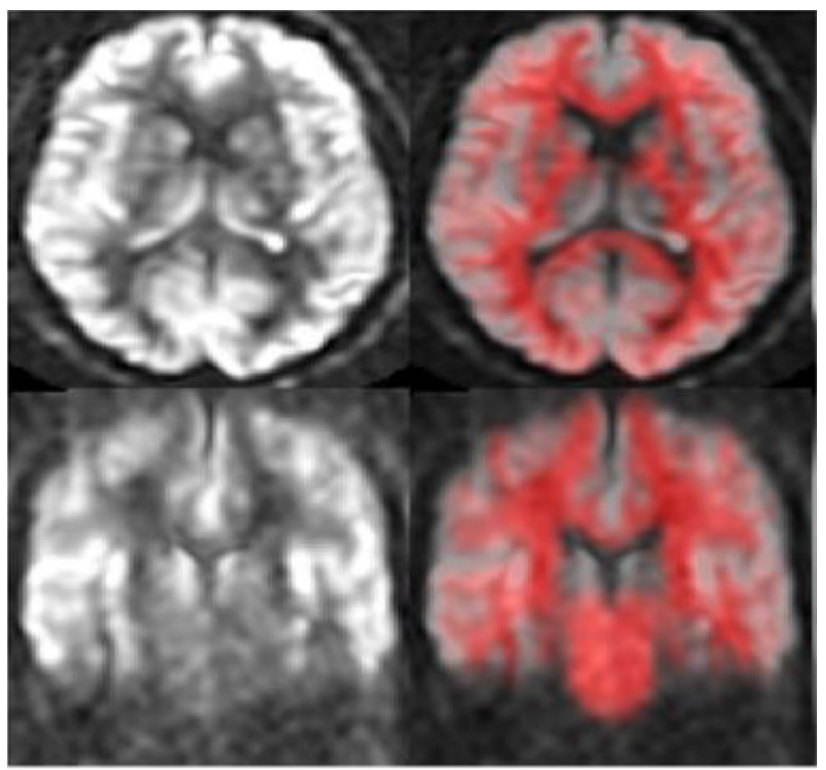

Figure 1. CBF perfusion map for a subject that, with the Hammers Atlas (HS) and spatial covariance (CoV) combination applied, sustained bilateral, positive Z-score outliers in the anterior aspect of the cingulate gyrus. The anterior aspect of their left cingulate gyrus was mildly atypical $(\mathrm{Z}$-score $=+2.01, \mathrm{IB}=1)$, while the right was severely atypical $(\mathrm{Z}$-score $=+4.23, \mathrm{IB}=3)$. High-resolution $3 \mathrm{D}$ T1-weighted fSPGR (TR/TE/flip $=11.34 / 4.25 \mathrm{~ms} / 12^{\circ}, 256 \times 256$ matrix with $1 \mathrm{~mm}$ slice thickness, $1 \mathrm{~mm}$ isotropic) and 3D pseudocontinuous ASL ( $\mathrm{pCASL}$ ) ( $\mathrm{PLD}=1525 \mathrm{~ms}$, labeling duration $=1450 \mathrm{~ms}, \mathrm{TR} / \mathrm{TE}=4886 / 10.528 \mathrm{~ms}, \mathrm{NSA}=4$, background-suppressed, separate M0 scan) scans were acquired and used for the analysis of this study.

Results: The cingulate gyrus (anterior) ( $\mathrm{IB}=6)$, parahippocampal gyrus (anterior) (IB =5), and inferior temporal gyrus (posterior) $(\mathrm{IB}=4)$ had the greatest IBs. IB was symmetrical $(-0.02 \pm 1.31)$ with equivalent mean IBs (left $=0.94 \pm 1.20$; right $=1 \pm 1.41$ ), but almost twice as many positive Z-scores (positive $=18$; negative $=10)$. Left IB and negative $Z$-scores had a significant variance $(\mathrm{p}=0.0102)$ and correlation $(\mathrm{r}=+0.695)$, and right IB and positive Z-scores had a significant variance $(\mathrm{p}=0.00377)$ and correlation $(\mathrm{r}=+0.773)$ (Fig. 2). Also, age was significantly correlated with total IB $(\mathrm{r}=+0.526)$. 


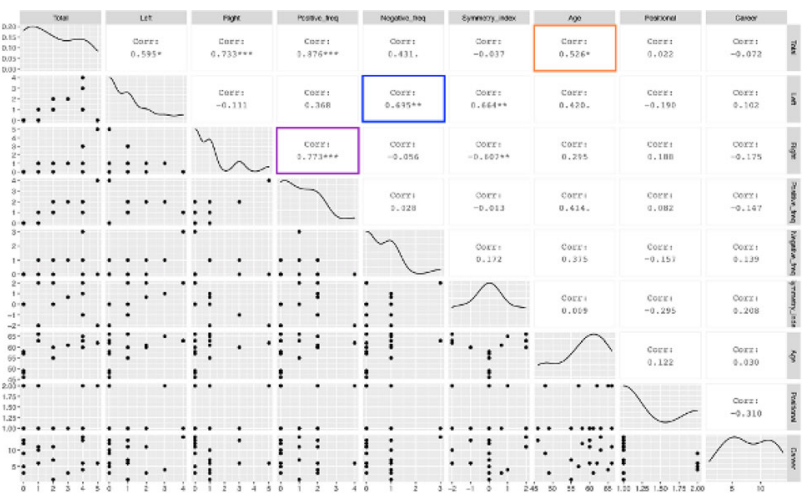

Figure 2. Correlation matrix to examine the strength and significance of correlations between the total, left and right injury burden (IB), the frequency of positive (hyperperfusion) and negative (hypoperfusion) Z-score outliers, the IB symmetry index, and the demographic information of age, type of positional contact, and career length. Noteworthy significant, positive correlations were found between positive $Z$-scores and right IBs (in purple, $\mathrm{r}=0.773, \mathrm{p}<0.001$ ), negative $Z$-scores and left IB (in blue, $\mathrm{r}=0.695, \mathrm{p}<0.01$ ), and age and total IB (in orange, $\mathrm{r}=0.526, \mathrm{p}<0.05$ ). More simply,

hyperperfusion correlated with right IB and hypoperfusion correlated with left IB.

Fig. 3

Table 1. Outlining the total, left and right injury burden (IB) calculated for the 12 concussion and neuropathological-related ROIs used in this study and each rCFL subject. The Z-scores and subsequent IBs were calculated based on the left, right and bilateral spatial covariance (CoV) CBF values. The Hammers (HS) Atlas was preferred if a ROI existed in both the Harvard-Oxford (HO) Atlas and the HS Atlas.

\begin{tabular}{|c|c|c|c|}
\hline ROI & $\begin{array}{c}\text { Total injury } \\
\text { burden }\end{array}$ & $\begin{array}{c}\text { Left injury } \\
\text { burden }\end{array}$ & $\begin{array}{l}\text { Right injury } \\
\text { burden }\end{array}$ \\
\hline Superior parietal cortex (HS) & 1 & 1 & 0 \\
\hline Anterior aspect of cingulate gyrus (HS) & 6 & 2 & 4 \\
\hline Posterior aspect of cingulate gyrus (HS) & 1 & 1 & 0 \\
\hline Inferior temporal gyrus posterior division $(\mathrm{HO})$ & 4 & 1 & 3 \\
\hline Parahippocampal gyrus anterior division (HO) & 5 & 3 & 2 \\
\hline Parahippocampal gyrus posterior division $(\mathrm{HO})$ & 2 & 0 & 2 \\
\hline Medial orbital gyrus (HS) & 2 & 2 & 0 \\
\hline Cerebellum (HS) & 2 & 0 & 2 \\
\hline Inferior frontal gyrus pars opercularis (HO) & 2 & 2 & 0 \\
\hline Hippocampus (HS) & 3 & 1 & 2 \\
\hline Amygdala(HS) & 3 & 1 & 2 \\
\hline Superior frontal gyrus (HS) & 2 & 2 & 0 \\
\hline Subject & $\begin{array}{c}\text { Total injury } \\
\text { burden }\end{array}$ & $\begin{array}{c}\text { Left injury } \\
\text { burden }\end{array}$ & $\begin{array}{l}\text { Right injury } \\
\text { burden }\end{array}$ \\
\hline 1 & 2 & 1 & 1 \\
\hline 2 & 4 & 1 & 3 \\
\hline 3 & 2 & 2 & 0 \\
\hline 4 & 0 & 0 & 0 \\
\hline 5 & 4 & 3 & 1 \\
\hline 6 & 5 & 0 & 5 \\
\hline 7 & 0 & 0 & 0 \\
\hline 8 & 0 & 0 & 0 \\
\hline 9 & 4 & 1 & 3 \\
\hline 10 & 0 & 0 & 0 \\
\hline 11 & 0 & 0 & 0 \\
\hline 12 & 1 & 1 & 0 \\
\hline 13 & 1 & 0 & 1 \\
\hline 14 & 4 & 4 & 0 \\
\hline 15 & 3 & 2 & 1 \\
\hline 16 & 2 & 1 & 1 \\
\hline 17 & 1 & 0 & 1 \\
\hline
\end{tabular}

Discussion: IB was overall symmetrical, but subject-wise IB was often unilateral. In agreement with literature, the cingulate cortex may be especially vulnerable to injury [4]. We also found indications of left ROI hypoperfusion and right ROI hyperperfusion. Further studies may analyze concussion CBF in relation with DTI and fMRI.

\section{References:}

[1] Hart et al. (2013) JAMA Neurol.

[2] Mutsaerts et al. (2020) NeuroImage.

[3] Mutsaerts et al. (2017) J Cereb Blood Flow Metab.

[4] Churchill et al. (2017) J Neurotrauma.

\section{S2.P11.}

Evaluation of changes perfusion and white matter volume of the brain in clinically isolated syndrome

\author{
${ }^{*}$ L. Vasilkiv ${ }^{1}$, Y. Stankevich ${ }^{1}$, O. Bogomyakova ${ }^{1}$, A. Tulupov ${ }^{1}$ \\ ${ }^{I}$ Russian Academy of Sciences, International Tomography Centre, \\ Novosibirsk, $R U$
}

Introduction: Clinically isolated syndrome (CIS) is the initial stage of the formation of multiple sclerosis, has a symptom complex identical to multiple sclerosis, but does not meet all the criteria for dissemination in time and space. According to the literature, $30 \%$ of patients had a conversion of a clinically isolated syndrome to multiple sclerosis within 1 year and about $50 \%$ after 5 years 1 ; however, Bates et al. it has been found that early treatment of patients with CIS accelerates recovery and may delay the development of multiple sclerosis2. The early initiation of effective therapy with disease modifying drugs (DMDs) is a hot topic, which raises the question of when to start it.

Purpose: To assess changes in perfusion and volume of the brain substance in clinically isolated syndrome (CIS).

Materials and methods: The MR study was carried out on a MRscanner "Ingenia" ("Philips") 3 Tesla. The study included 12 healthy volunteers and 6 patients with demyelinating disease of the central nervous system-with clinically isolated syndrome (CIS) and 13 patients with multiple sclerosis (MS). To assess perfusion using the method of dynamic susceptibility contrast (DSC). Quantitative and qualitative assessment of $\mathrm{CBF}$ and $\mathrm{CBV}$ in white and gray matter of different lobes of the brain. To assess morphometry, the obtained T1WI and FLAIR images were loaded into an automated system for calculating the volume of brain structures based on the segmentation method. White matter brain (relWMV) and gray matter (relGMV) volumes were calculated relative to total intracranial volume as a percentage.

Results: A moderate correlation was found between a decrease in the volume of the white matter of the brain and the severity of focal changes (coefficient of $\mathrm{r}$-Spearman correlation 0.4, $\mathrm{p} \leq 0.05$ ), compared with CIS in patients with relapsing-remitting multiple sclerosis (RRMS) in remission and patients with secondary-progressive multiple sclerosis (SPMS) significantly reduced by $32 \%$ and $40 \%$ relCBF ( $\mathrm{p} \leq 0.05$ ), compared with the CIS group in patients with RRMS in the stage of exacerbation and remission, the volumes were significantly reduced by $8 \%$ and $11 \%(\mathrm{p} \leq 0.05)$.

Discussion/conclusion: Based on the data obtained, it follows that the pathogenesis of CIS and MS is based on identical processeschanges in perfusion and neurodegeneration, starting at the earliest stages of the disease.

Source of financing: We thank Ministry of Science and Higher Education of the RF for access to MRI equipment (AAAA-A16116121510090-5). The work was supported by Russian Science Foundation (the project \#19-75-00052).

\section{S2.P12.}

Comparison of cerebrovascular reserve measurements from arterial spin labelling and 4D flow MRI with acetazolamide challenge in healthy volunteers and patients with sickle cell disease

*L. Vaclavu ${ }^{1}$, B. Biemond ${ }^{2}$, P. van Ooij ${ }^{3}$, M. J. P. van Osch ${ }^{1}$, A. Nederveen ${ }^{3}$ 
${ }^{1}$ Leiden University Medical Center, Department of Radiology, Leiden, $N L$;

${ }^{2}$ Amsterdam University Medical Center, Department of Hematology, Amsterdam, NL;

${ }^{3}$ Amsterdam University Medical Center, Department of Radiology and Nuclear Medicine, Amsterdam, NL

Introduction: Patients with sickle cell disease (SCD) exhibit both small and large-vessel vasculopathy ${ }^{1}$. Both ischaemic and haemorrhagic strokes are prevalent but risk varies with age $^{2}$. Little is known about differences between small and large-vessel health across age in $\mathrm{SCD}$. Cerebrovascular reserve (CVR) is a measure of hemodynamic compensation and vascular health. Our aim was to investigate the agreement between CVR from 4D flow MRI (large vessels) and arterial spin labelling (ASL) MRI (small vessels).

Methods: 10 adults with SCD and 8 healthy controls were included. Images were acquired at $3 \mathrm{~T}$ (Philips Healthcare, Best, NL) and included 3D anatomical, 2D multi-slice single-delay pseudo-continuous ASL, and 3D time-resolved phase-contrast MRI (4D flow MRI) ( $0.5 \mathrm{~mm}$ isotropic resolution, 4 cardiac frames) sequences. Acetazolamide (ACZ) was administered intravenously after baseline images. ASL CVR was calculated as \%change in grey matter cerebral blood flow $(\mathrm{mL} / 100 \mathrm{~g} / \mathrm{min})$ from baseline and post-ACZ ASL. Time-averaged velocity $(\mathrm{m} / \mathrm{s})$, peak time-frame flow $(\mathrm{mL} / \mathrm{s})$ and peak timeframe lumen area $\left(\mathrm{mm}^{2}\right)$ perpendicular to each vessel were calculated from 4D flow MRI. 4D flow CVR (\%) was calculated as \%change in flow over both internal carotid arteries. Wilcoxon rank tests were used to compare groups $(\mathrm{p}<0.05$ statistically significant). Agreement between ASL CVR and 4D flow CVR was assessed using BlandAltman and regression analysis.

Results: While velocity did not differ between groups, flow was higher in most vessels in SCD compared to controls as well as lumen area (Fig. 1). ACZ induced an increase in flow and lumen area with smaller changes noted in SCD patients. Results from orthogonal regression and Bland-Altman in Fig. 2. shows that there was poor agreement between ASL-CVR and 4D flow CVR particularly for higher CVR. Interestingly, there was a positive correlation with age for 4D flow CVR but not for ASL CVR (Fig. 3).
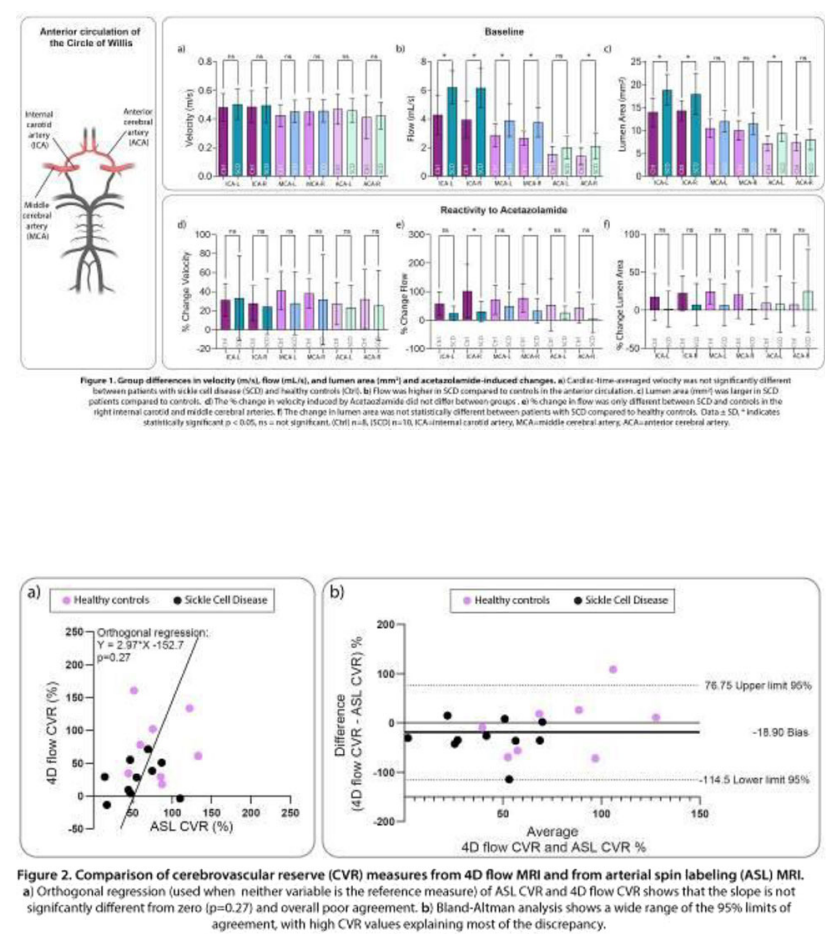
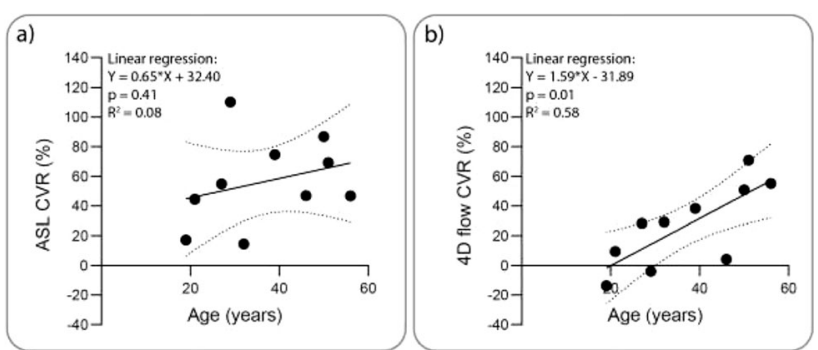

Figure 3. Association between age and CVR in SCD patients $(n=10)$.

a) ASL CVR was not significantly associated with age in SCD patients. b) $4 D$ flow CVR was significanly associated with age in SCD such that CVR was higher in older patients.

Discussion: The discrepancy between ASL and 4D flow CVR could be related to technical errors: (i) noise propagates when calculating CVR as \% change; (ii) ASL covered the whole grey matter but 4D flow was only assessed in anterior vessels; (iii) flow changes and downstream perfusion changes should have similar physiological responses but flow territory mapping would be better suited to determine the exact correspondence between flow changes in a large vessel and its specific perfusion territory. The correlation with age was only significant for $4 \mathrm{D}$ flow CVR. Although interesting, this could be inclusion bias since relatively healthier SCD patients are more likely to participate in research. Recent studies show dilation of the MCA to $\mathrm{CO}_{2}$ on high resolution $7 \mathrm{~T}^{3}$, and 4D flow MRI ${ }^{4}$. Here we also found small increases in area $\left(\sim 2 \mathrm{~mm}^{2}\right)$, and conclude that the large conduit arteries of the circle of Willis are capable of dilation in response to $\mathrm{ACZ}$.

\section{References:}

${ }^{1}$ Kassim, Blood (2016).

${ }^{2}$ Ohene-Frempong, Blood (1998).

${ }^{3}$ Verbree, JAP (2014).

${ }^{4}$ Kellawan, JCBFM (2017).

\section{S2.P13.}

CSF space volumetry using 3D-CISS in Aqp4-deficient mice - quantitative analysis and technical advances

\author{
*R. Gomolka ${ }^{1}$, M. Nedergaard ${ }^{1}$, Y. Mori ${ }^{1}$ \\ ${ }^{1}$ University of Copenhagen, Center for Translational Neuromedicine, \\ Copenhagen, DK
}

Introduction: Crucial for brain functions, the glymphatic system encompasses brain metabolite and waste transport involving an exchange of CSF with interstitial fluid [1]. CSF flows into the brain via periarterial spaces facilitated by astrocytic aquaporin-4 (Aqp4) channels at the astrocyte end-feet and disperses into the neuropil [2]. MRI is the most suitable for in-vivo imaging of the whole brain, but CSF space imaging often requires tracer injection altering CSF dynamics. Herein, we present fully noninvasive high-resolution livemice CSF volumetry using 3D constructive interference steady-state (3D-CISS) [3] at 9.4 T. We investigated if 3D-CISS can disclose subtle differences in CSF volumes between Aqp4-null (KO) vs. wildtype (WT) mice, and compared imaging with $50 \mathrm{vs} .33 \mu \mathrm{m}^{3}$ isometric voxels.

Methods: Aqp4-KO and WT littermates $(\mathrm{n}=30 ; 40 \% \mathrm{~F}$, mean \pm SD $13 \pm 3$ (range 9-20) weeks age, $25.8 \pm 3.6 \mathrm{~g}$; Danish Animal Experiments Inspectorate approval) underwent $9.4 \mathrm{~T}{ }^{1} \mathrm{H}-\mathrm{MRI}$ (BioSpec 94/30USR, Bruker) using: 1) $1500 \mathrm{mT} / \mathrm{m}$ gradient coil 
(BFG6S, Bruker) with room-temperature volumetric Tx/Rx resonator (in. diam. $=40 \mathrm{~mm}$ ); 2) $240 \mathrm{mT} / \mathrm{m}$ gradient coil $($ BGA-12S) with cryogenically-cooled surface $\mathrm{Tx} / \mathrm{Rx}$ quadrature-resonator (CryoProbe). All animals were under anesthesia (Ketamine/Xylazine, $100 / 10 \mathrm{mg} / \mathrm{kg}, 11.5 \mathrm{ml} / \mathrm{kg} / 45 \mathrm{~min}$ ) with monitored temperature and respiration. Every 3D-CISS image was calculated as a maximum intensity projection from at least 2 realigned 3D-TrueFISP volumes, acquired in the opposite phase encoding direction (Table 1). For every 3D-CISS volume (Fig. 1), CSF space was segmented and the CSF/ brain volume ratio was calculated in few steps:

- 1) Semiautomatic brain volume extraction in ITK-SNAP

- 2) Automatic:

- Brain volume box-bounding for removal of remaining residual regions

- In-house CSF space separation in Matlab R2019a:

- Adaptive CSF separation based on cumulative distribution of the brain intensities

- Slice-wise 2D region-growing for CSF boundaries correction based on contrast calculation [4], in all 3 planes.

Obtained CSF/brain volume ratios from $\mathrm{KO}$ and WT animals were compared with Kruskal-Wallis ANOVA (Dunn's correction).

Fig. 1

\begin{tabular}{|c|c|c|c|c|c|c|c|c|c|c|}
\hline 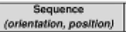 & $\begin{array}{l}T x \text { TxiR } \\
\text { coill }\end{array}$ & $\begin{array}{c}\text { TR } \\
{[\text { [ms] }}\end{array}$ & $\begin{array}{c}\mathrm{TE} \\
{[\mathrm{ms}]}\end{array}$ & $\begin{array}{l}\text { FA } \\
\text { [dog] }\end{array}$ & Fov $\left[\mathrm{mm}^{3}\right]$ & NEX & $\begin{array}{l}\text { Vorel size [mm'] } \\
\text { (interpolation) }\end{array}$ & $\begin{array}{l}\text { Bandwidtit } \\
\text { [Hz pix] }\end{array}$ & $\begin{array}{c}\text { PE } \\
\text { directions }\end{array}$ & TA \\
\hline $\begin{array}{c}\text { 3D-TruefiSP } \\
\text { (8syitlat, prone) }\end{array}$ & $\begin{array}{l}\text { Aasmim } \\
\text { semperatura }\end{array}$ & 4 & 2 & 50 & 19.2.216 $6 \times 16$ & 3 & $\begin{array}{l}0.0650 .0550 .01 \\
\{20 \times 1.6 \times 1.09\end{array}$ & 387 & 2 & $19 \min$ \\
\hline $\begin{array}{l}\text { 3D-TruefiSP } \\
\text { (sagittal, prone) }\end{array}$ & Cryofirabe & 5.2 & 2.6 & 50 & $19.2 \times 12.8 \times 12.9$ & 2 & $\begin{array}{l}0.03 \times 0.03 \times 0.03 \\
\{2.0 \times 1.6 \times 1.0\}\end{array}$ & 250 & 2.4 & $\begin{array}{l}13 \text { min } \\
30000\end{array}$ \\
\hline
\end{tabular}

Results: The brain volumes were significantly different between combined from 2 MRI setups KO and WT groups (Mann-Whitney: $\mathrm{U}=64, \mathrm{p}<0.05$, med. KO-WT $=17.8 \mathrm{~mm}^{3}$; Fig. 2C). Automatically segmented $\mathrm{CSF} / \mathrm{brain}$ volume ratios were different between KO's and WT's (Kruskal-Wallis: KS $(4,30)=8.4, \mathrm{p}<0.05$ ), but the difference was visible only between the results from CryoProbe $(\mathrm{p}=0.036$; Fig. 2D)

Fig. 2

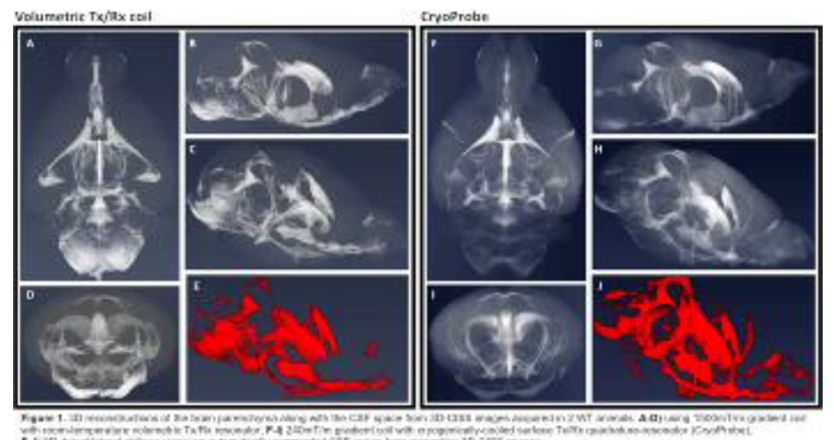

Fig. 3

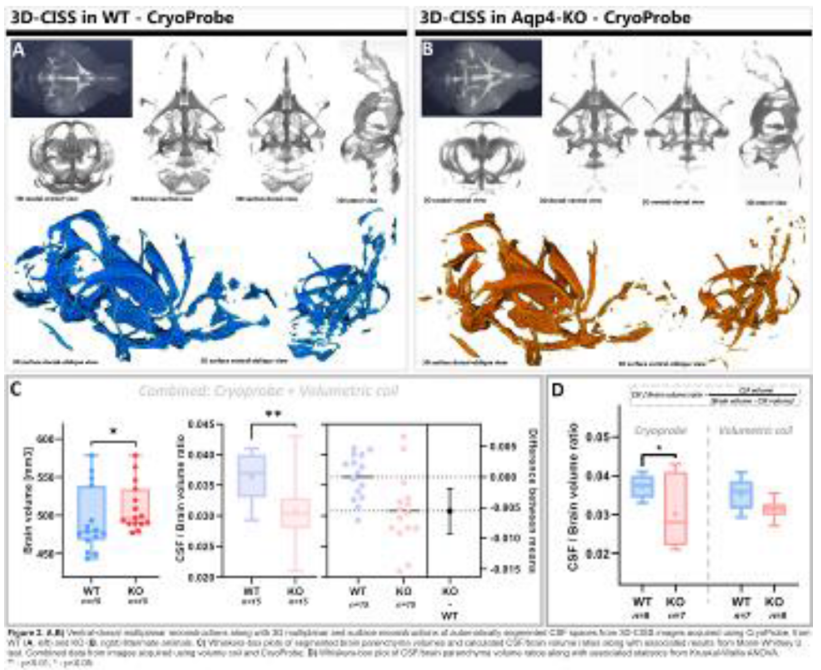

Discussion: 3D-CISS MRI using CryoProbe is feasible to depict subtle differences in CSF volumes between Aqp4-KO and WT mice. Despite smaller voxels, reduced averaging and longer TEs, the setup provided good differentiation without invasive tracer injection.

\section{References}

1. Iliff et al., Sci Transl Med 2012;4(147):147ra111.

2. Mestre et al., Nature Communications 2018;9.

3. Tanioka et al., Radiology 1991;178(1):141-4.

4. Gomolka et al., Neuroradiol J 2017;30(1):15-22.

\section{S2.P14.}

\section{Assessment of aquaporin- 4 role in brain activity detected by diffusion magnetic resonance imaging}

*B. Yagüe Jiménez ${ }^{1}$, I. Guadilla Gómez ${ }^{1}$, N. Arias-Ramos ${ }^{1}$, B. Lizarbe ${ }^{1}$, S. Cerdán ${ }^{1}$, P. López-Larrubia ${ }^{1}$

${ }^{1}$ Instituto de Investigaciones Biomédicas Alberto Sols (UAM), Madrid, ES

Introduction: AQP4 is a transmembrane water channel highly expressed in central nervous system, regulating fluid exchange [1] by water transport between two sides of plasmatic membrane, and depending on concentration gradients of solutes [2]. Previously, we have shown that functional diffusion MRI can detect cellular swelling associated to glucose uptake [3]. On these grounds, role of APQ4 in this process was studied.

Methods: Sodium salt of AQP4 inhibitor TGN-020 [4] was used to assess cerebral changes after glucose bolus administration in C57BL6/J adult male mice with TGN administration plus glucose, saline administration plus glucose, and saline administration only. Diffusion tensor imaging studies were acquired in a $7 \mathrm{~T}$ equipment and mean diffusivity (MD) and fractional anisotropy (FA) were measured in hypothalamus, hippocampus, and cortex regions. Temporal distribution of MRI studies and administration of treatments: basal diffusion study was acquired without any dispensed treatment. Then, inhibitor $(100 \mu \mathrm{L} / 25 \mathrm{~g}$ per animal of $0.24 \mathrm{M}$ TGN-020) or saline $(300 \mu \mathrm{L} / 25 \mathrm{~g}$ body weight (b.w.) of saline) were administered intraperitoneally through a catheter. After $20 \mathrm{~min}$, glucose $(200 \mu \mathrm{L} /$ $25 \mathrm{~g}$ b.w. of $2.08 \mathrm{M}$ glucose in saline) or saline were injected and subsequently diffusion study was performed. $30 \mathrm{~min}$ after, last 
diffusion study was acquired. In a parallel cohort, we acquired $1 \mathrm{H}$ High Resolution Magic Angle Spinning (HRMAS) spectra in a $11.7 \mathrm{~T}$ system. Statistical analysis: for MRI data, parametric maps are still acquaring. For HRMAS; a linear mixed model was done.

Fig. 1
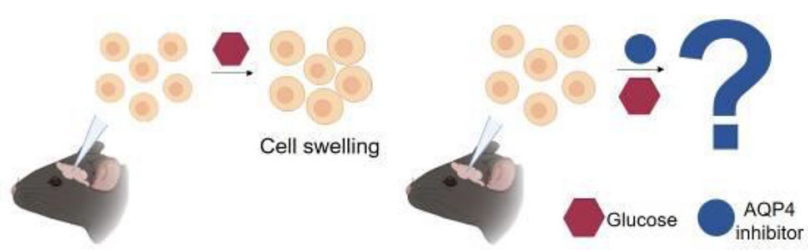

Results: Glucose group experienced a decreased in MD 30 min after glucose injection. However, this response seems to be partially inhibited in TGN group. Metabolic studies show a decreased glutamate concentration in cortex and hippocampus of TGN group, as compared to glucose and control groups, while GABA concentration is higher.

Fig. 2

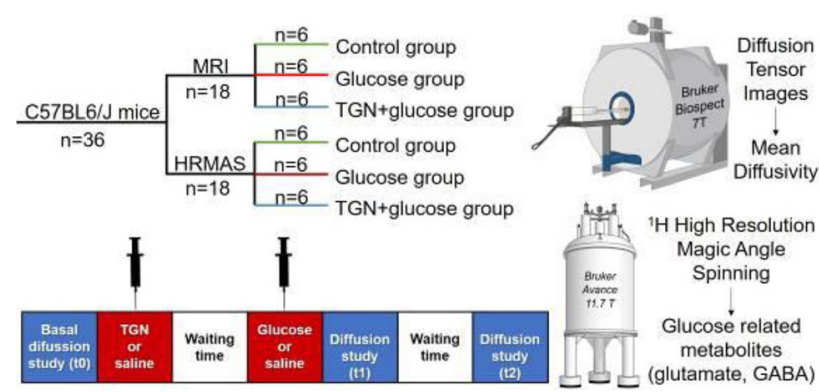

Fig. 3

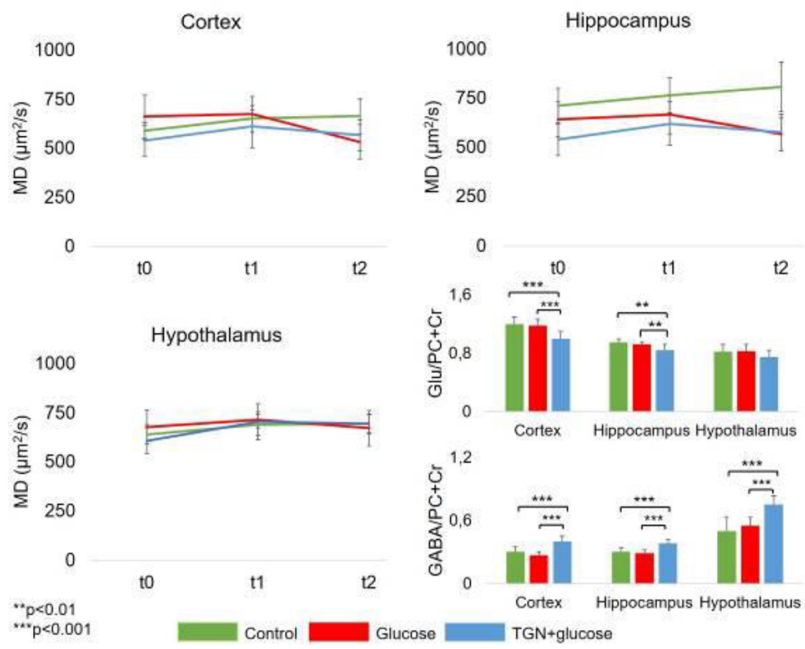

Discussion: Glucose administration induces a cerebral response detectable with DTI. Use of TGN inhibits AQP4 and partially avoids its function, noting that AQP4 is involved in cellular volume regulation. Metabolic results correspond with an alteration of the glutamate cycle due to AQP4 obstruction, that yield a net increase of this metabolite concentration.

\section{References}

[1] Mader S et al. Aquaporin-4 Water Channel in the Brain and Its Implication for Health and Disease. Cells. 2019;8(2).

[2] Venero et al. Aquaporins in the central nervous system. Progress in Neurobiology 2001, 63:321-336.

[3] Lizarbe, B et al. Systemic Glucose Administration Alters Water Diffusion and Microvascular Blood Flow in Mouse Hypothalamic Nuclei-An fMRI Study. Front Neurosci. 2019. 13: 921.

[4] Igarashi et al. Pretreatment with a novel aquaporin 4 inhibitor, TGN-020, significantly reduces ischemic cerebral edema. Neurol Sci 2011, 32:113-116.

\section{S2.P15.}

Tumor burden of heterotopic glioblastoma in mice: tumor growth evaluation by visual assessment and texture analysis using clinical 3 T MRI

${ }^{*}$ S. Wagner ${ }^{1}$, D. Freitag ${ }^{2}$, K. H. Herrmann ${ }^{3}$, M. Skalej ${ }^{1}$, W. Wohlgemuth ${ }^{4}$, H. Gufler ${ }^{4}$

${ }^{I}$ Martin Luther University Halle-Wittenberg, Clinic and Polyclinic of Neuroradiology, Halle (Saale), DE;

${ }^{2}$ Jena University Hospital, Department of Neurosurgery, Jena, DE;

${ }^{3}$ Jena University Hospital, IDIR Medical Physics Group, Jena, DE;

${ }^{4}$ Martin Luther University Halle-Wittenberg, Clinic and Polyclinic

of Radiology, Halle (Saale), DE

Introduction: Using MRI to non-invasively study animals over a prolonged course of disease development and treatment would eliminate the need to sacrifice similarly sized groups of animals at each given time point during a study (1). However, how to quantify tumor growth and treatment response non-invasively poses a challenge to all tumor models. In this animal study, we examined the value of automated texture analysis and visual structure assessment of images derived from a clinical $3 \mathrm{~T}$ scanner to estimate the tumor burden of heterotopically implanted glioblastoma multiforme in mice. Methods: This study received ethics committee (Thüringer Landesamt für Verbraucherschutz) approval. Xenografts were established in 18 immunodeficient mice by subcutaneous injection of human glioblastoma cells into both flanks. The animals were followed up on a clinical 3 T-MR using a $25 \mathrm{~mm}$ Litz volume resonator and were finally sacrificed for histological work up. 3D TSE SPACE and 3D T1-weighted (T1w) FL3D-VIBE sequences before and after contrast agent injection were obtained. The tumors were divided into three groups based on histology and immunocytochemistry (Ki67) findings. Volumes and signal intensities (SI) were calculated. Qualitative assessment of each MR measurement was adapted from the VASARI (Visually AcceSAble Rembrandt Images) MRI feature set (2) and by texture analysis (using MaZda software) (3).

Results: 85 MR-measurements in 21 tumors of 14 animals were analyzed: transplants with densely-packed tumor cells $(10 / 21)$, isolated tumor cells $(5 / 21)$ and no tumor cells $(6 / 21)$. Increasing volumes $(\mathrm{p}<0.05)$, heterogeneity, neovascular proliferation, necrosis in the tumor matrix, and progressive solid tumor components in the margin $(\mathrm{p}<0.05)$ represent the hallmarks of tumor growth. MaZda software extracted 314 features from the images. Haar wavelet transform parameters were the best for distinguishing between transplants with densely-packed tumor cells and transplants with isolated tumor cells $(\mathrm{p}<0.05)$.

Discussion: The visual and texture analysis-based assessment of the internal structure of heterotopically implanted glioblastomas enables the tumor burden to be estimated by 3 T MRI. This approach provides reproducible and quantifiable results with regard to tumor burden, 
which is the basic parameter of any therapeutic study aimed at inhibiting tumor growth.

\section{References:}

1 Herrmann KH, Pfeiffer N, Krumbein I, Herrmann L, Reichenbach JR. MRI compatible small animal monitoring and trigger system for whole body scanners. Z Med Phys. 2014 Mar;24(1):55-64.

2 VASARI project-wiki.cancerimagingarchive.net. Read relevant article. Accessed on 09/06/2016.

3 P. Szczypinski, M. Strzelecki, A Materka, A. Klepaczko, MaZda-A software package for image texture analysis. Computer Methods and Programs in Biomedicine. 2009; 94(1):66-76.

\section{S2.P16.}

\section{Lactate as a neuroprotective treatment after a neonatal hypoxic-ischemic event}

\author{
${ }^{*}$ H. Roumes ${ }^{1}$, S. Sanchez ${ }^{1}$, L. Pellerin ${ }^{2}$, A. K. Bouzier-Sore ${ }^{1}$ \\ ${ }^{1}$ University of Bordeaux, Centre de Résonance Magnétique des \\ Systèmes Biologiques, Bordeaux, FR; \\ ${ }^{2}$ University of Poitiers, French National Institute of Health \\ and Medical Research (Inserm), Poitiers, FR
}

Introduction: Glucose is the main energy substrate for the brain but lactate is also important since it can be used as an efficient neuronal substrate (1) especially during brain activity (2). Interestingly, there is a shift in lactate perception, that start to be no longer considered as a waste metabolic product but rather as an important molecule for the brain. Indeed, lactate has also been shown to be important in physiological processes such as memory consolidation and long-term memory formation (3). So, if lactate is an important molecule for the brain, could it be neuroprotective? We tested this hypothesis on a neonatal model of brain hypoxia.

Methods: Hypoxic-ischemic (HI) damage was produced in neonate Wistar rat (P7) (carotid artery ligation +2 h hypoxia, $n=70$ ). Pups received an lactate injection before (HILb) or after $\mathrm{HI}$ (HILa), or glucose (HIG) or pyruvate (HIP), or $\mathrm{NaCl}$ (HIC), or lactate + oxamate (LDH inhibitor, HILO). In the last group, pups received 3 injection of lactate ( $3 \mathrm{~h}, 24 \mathrm{~h}$ and $48 \mathrm{~h}$ after HI, HI3L). In the HIL group, some pups received [3-13C]lactate and MRS was performed on brain biopsies. Brain lesions were assessed by DWI at $4.7 \mathrm{~T}$ (Bruker Biospec) $3+48 \mathrm{~h}$ after $\mathrm{HI}$. ADC and FA were measured. Behavioral tests were also performed (righting reflex, mNSS and NOR tests).

Results: Results showed that lactate was neuroprotective when administered after the insult, rather than before. Brain lesion volumes (BLV) (3 h after the HI event) were $40 \%$ of the total brain volume (TBV) in HIC compared to $30 \%$ in HILa. BLV $48 \mathrm{~h}$ after the HI were $19 \%$ of the TBV in HIC compared to $8 \%$ in HILa. When oxamate was co-injected (inhibition of the metabolic use of lactate), the neuroprotection was lost. BLV were even bigger in HILO than in HIC, suggesting that endogenous lactate itself could be neuroprotective. Moreover, glucose or pyruvate, two other brain substrates, were not neuroprotective. 13C-MRS of brain biopsies $3 \mathrm{~h}$ after [3-13C]lactate injection indicated that lactate was metabolized by the brain $(13 \mathrm{C}$ incorporation into amino acids). Finally, when lactate was injected 3 times, neuroprotection was the most effective: BLV were around $1 \%$ $48 \mathrm{~h}$ after the insult. ADC values (measured at P7) were also better after lactate injection, indicating a lower edema severity.

Conclusion: A single lactate injection induced a $25 \%$ and a $58 \%$ reduction in BLV compared to HIC $3 \mathrm{~h}$ and $48 \mathrm{~h}$ after HI, respectively, indicating a rapid and efficient neuroprotection. When oxamate was co-injected, the neuroprotection was completely abolished, highlighting the role of lactate metabolism in this protection. After 3 lactate injections, pups presented the smallest BLV and a complete recovery of neurological reflexes, sensorimotor capacities and longterm memory, demonstrating that lactate administration is a promising therapy for neonatal $\mathrm{HI}$ insult.

\section{References}

1 Bouzier-Sore et al. JCBFM 2003.

2 Sampol et al. Front Neuroenergetics 2013.

3 Suzuki et al. Cell 2011.

\section{S2.P17.}

\section{Impact of cognitive training in somatosensorial resting state network in the Tg-F344AD rat model}

\author{
*R. Tudela ${ }^{1}$, E. Muñoz-Moreno ${ }^{2}$, X. López-Gil ${ }^{2}$, G. Soria ${ }^{1,3}$ \\ ${ }^{I}$ Consorcio Centro de Investigación Biomédica en Red de \\ Bioingeniería, Biomateriales y Nanomedicina (CIBER-BBN), Group \\ of Biomedical Imaging / University of Barcelona, Barcelona, ES; \\ ${ }^{2}$ Institut d'Investigacions Biomèdiques August Pi i Sunyer (IDIBAPS), \\ Magnetic Resonance Imaging Core Facility, Barcelona, ES; \\ ${ }^{3}$ University of Barcelona, Institute of Neurosciences, Barcelona, ES
}

Introduction: We have recently demonstrated that the TgF344-AD rat model (TG) shows time-dependent alterations revealing dysfunction in several resting state networks such as default mode, sensorimotor and somatosensory networks [1]. Moreover, amplitude of the somatosensory network of TG rats at 18 months of age was strongly correlated with the behavioral performance in a working memory task, the delayed non-matched to sample test (DNMS), revealing the importance of this network for a proper cognitive outcome.

In this study we present preliminary results regarding the effect of early behavioral training during 8 months on somatosensory networks in non-aged wild type (WT) and TG rats.

Methods: The experimental groups were as follows: Non-trained: WT $\mathrm{n}=11$; $\mathrm{TG} \mathrm{n}=11$; Trained: $\mathrm{WT} \mathrm{n}=9$; $\mathrm{TG} \mathrm{n}=12$. Training and DNMS test were performed as previously described [1] starting at 3 months of age until animals were 11 months old, when MRI was acquired.

rs-fMRI was acquired in a $7 \mathrm{~T}$ scanner by using a single-shot gradient-echo EPI sequence. Images had 600 volumes of $64 \times 64 \times 34$ voxels and $0.4 \times 0.4 \times 0.6 \mathrm{~mm}^{3} /$ voxel with $\mathrm{TR}=2 \mathrm{~s}$ and $\mathrm{TE}=28 \mathrm{~ms}$.

Image preprocessing included: slice-timing, motion correction, skullstripping, spatial normalization, spatial smoothing, detrending and regression by motion parameters, and temporal filtering $(0.01-0.1 \mathrm{~Hz}) .30$ independent components were obtained using FSL MELODIC [2] considering the whole cohort. Based on our previous studies we selected the somatosensorial network as it was the focus of our analysis (Fig. 1A). 
Fig. 1

(A)

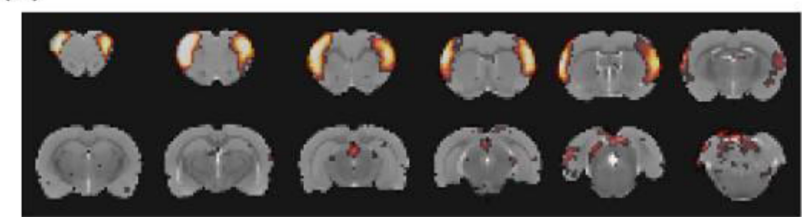

(B)

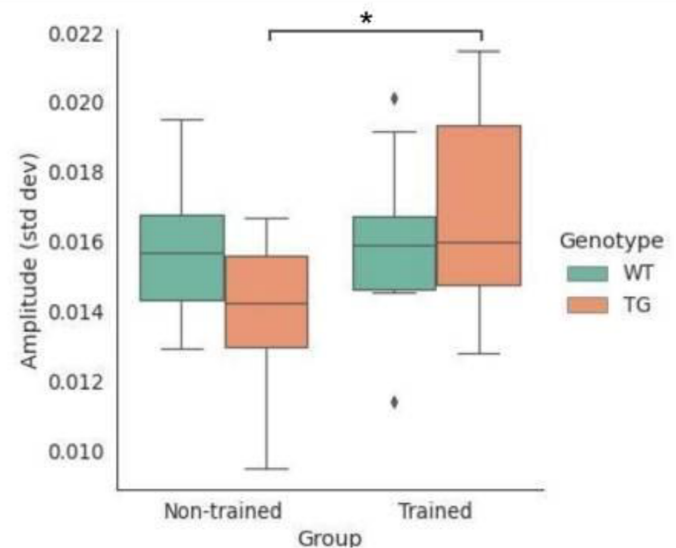

(A) Representative coronal sections of the somatosensorial network $(Z>2.3)$. (B) Boxplot of the Amplitude for wild-type (WT) and TgF344-AD (TG), non-trained and trained experimental groups. The box extends from the lower to upper quartile values of the data, with a black line at the median. The whiskers extend from the box to show the range of the data. Flier points are those past the end of the whiskers. Asterisk indicates significant difference (Kruskal-Wallis $p<0.05$ ) between groups connected by the line.

The standard deviation of the time-series (Amplitude) of the component was computed for each subject and the differences between groups were evaluated using Kruskall-Wallis test.

Results: The amplitude, as a measure of the magnitude of the BOLD activity, in the somatosensorial network was significantly increased in the trained TG group compared to the non-trained TG group $(\mathrm{p}<0.05)$. This was not observed between the respective WT groups (Fig. 1B). Despite the trend to lower amplitude values in non-trained TG versus non-trained WT, no significant differences between genotypes were observed in any of the groups.

Discussion: In our previous work [1] significant differences between cognitive-trained TG and WT rats were observed in the amplitude of somatosensory network only at 18 months of age, while in a similar non-trained cohort, functional alterations in somatosensory cortex connectivity were observed from 6 months on [3].

Although no significant differences between genotypes were observed, there was a significant difference between non-trained and trained TG rats, pointing to an impact of cognitive training in the somatosensorial network of TgF344-AD rats, that could compensate for the initial pathology-related alterations in these animals.

\section{References}

[1] Tudela et al. Front. Aging Neurosci. 2019. 11:213.

[2] Beckman and Smith. NeuroImage 2005. 25(1):294-311.

[3] Anckaerts et al. Neurobiol. Disease 2019. 124:93-107.

\section{S2.P18.}

\section{Characterization of the brain phenotype of $T s h z 3$ $+/$ lac Z mice using in vivo MRI and MRS}

\author{
*A. Comino García-Muñoz ${ }^{1}$, J. Sourdon ${ }^{1}$, I. Varlet ${ }^{1}$, A. Lokossou ${ }^{1}$, \\ M. Bernard ${ }^{1}$, L. Fasano ${ }^{1}$, T. A. Perles-Barbacaru ${ }^{1}$, L. Had $^{1}$, A. Viola ${ }^{1}$ \\ ${ }^{1}$ Aix-Marseille University, Center for Magnetic Resonance in Biology \\ and Medicine, Marseille, FR
}

Introduction: The teashirt 3 gene ( $T s h z 3$ in mice, TSHZ3 in human) codes for a transcription factor expressed in brain which has been linked to autism in humans and in mice with heterozygous deletion ${ }^{1}$. $T s h z 3$ is mainly expressed in the cortex, the amygdala and the hippocampus. The aim of this study was to use in vivo MRI and MRS to characterize the microstructural and metabolic brain phenotype of heterozygous mutant $T s h z 3+$ lacZ mice.

Subjects: Heterozygous mutant $T s h z 3+/ l a c Z$ and control CD1 mice were explored on a vertical spectrometer (Bruker AVANCE $500 \mathrm{WB}$ @ $11.75 \mathrm{~T}$ ) with a transmit/receive birdcage coil under medetomidine sedation and isoflurane anaesthesia. 2D-DTI was performed with 12 directions, $\delta=1.65 \mathrm{~ms}$ and $\Delta=10 \mathrm{~ms}, 2 \mathrm{~b}$ values $(0$ and $1200 \mathrm{~s} /$ $\left.\mathrm{mm}^{2}\right)$. An EPI technique $(\mathrm{TE}=15.7 \mathrm{~ms}, 12$ interleaved segments, $\mathrm{NA}=4$, FOV $15 * 15 \mathrm{~mm}^{2}$, matrix $100 \times 100,5$ non-contiguous slices of $0.5 \mathrm{~mm}$ ) was used. DTI images were processed under ParaVision 5.1 software generating DTI maps including FA, MD, $\lambda 1$, $\lambda 2, \lambda 3$ and radial diffusivity. Post-processing was performed using FSL and ImageJ software (ROI position: corpus callosum and external capsule, cortex, lateral ventricles, thalamus, hypothalamus, hippocampus and amygdala). Localized $1 \mathrm{H}-\mathrm{MRS}$ of the thalamus: PRESS sequence, $\mathrm{TR}=1700 \mathrm{~ms}, \mathrm{TE}=20 \mathrm{~ms}$, voxel of $8 \mathrm{~mm} 3,512$ averages, with and without VAPOR (2 averages). MRS data were processed with CSIAPY using a time domain quantitation algorithm based on QUEST. The metabolite basis set included 17 metabolites and 12 macromolecules. Water signal was used as an internal reference. Statistical analysis was performed using Mann-Whitney test.

Results: the DTI maps of 19 control mice and 19 Tshz3 + /lacZ mice were analysed. Significantly higher mean diffusivity values (MD) were measured in the corpus callosum and external capsule $(\mathrm{P}=0.012)$, the cortex $(\mathrm{P}=0.0185)$ and the thalamus $(\mathrm{P}=0.0403)$ of heterozygous mice. Longitudinal diffusivity $(\lambda 1)$ was increased in the cortex $(\mathrm{P}=0.0236)$ and the amygdala $(\mathrm{P}=0.0157)$ and radial diffusivity in the cortex $(\mathrm{P}=0.0374)$ and the thalamus $(\mathrm{P}=0.0347)$. The analysis of proton spectra from 19 control mice and 16 Tshz $3+1$ lac $\mathrm{Z}$ mice showed an increase in the Glycine/ $\mathrm{H} 20$ and $\mathrm{N}$-acetylaspartate $(\mathrm{NAA}) / \mathrm{H}_{2} \mathrm{O}$ ratios $(\mathrm{P}=0.0161$ and $\mathrm{P}=0.0288$ respectively). Discussion: DTI results show a general increase in diffusivity in brain structures with reduced expression of $T s h z 3$ (cortex and amygdala) as well as in the thalamus. The increase in $\lambda 1$ suggests that water diffusivity along or within the axons is increased, whereas the increase in radial diffusivity could indicate a modification in the myelin sheath in mutant animals. The higher levels of glycine, an excitatory neurotransmitter and of the neuronal marker NAA point to neuronal dysfunction. The unexpected accumulation of NAA could be linked to an altered axonal transport of this compound which is hydrolysed in oligodendrocytes.

\section{Reference:}

1-Caubit et al. Nat Genet. 2016;48(11):1359-1369. 


\section{S2.P19.}

Magnetic resonance imaging of the cerebral appetite regulation centres during high-fat diet consumption in female mice

\author{
D. Galguera ${ }^{1}, *$ N. Arias-Ramos ${ }^{1}$, S. Cerdán ${ }^{1}$, P. López-Larrubia ${ }^{1}$, \\ B. Lizarbe ${ }^{1,2}$
}

${ }^{1}$ Instituto de Investigaciones Biomédicas Alberto Sols (UAM), Madrid, ES;

${ }^{2}$ Autonomous University of Madrid, Department of Biochemistry, Madrid, ES

Introduction: Obese patients and male murine models fed on a high fat diet (HFD) are known to experience an inflammatory response of the hypothalamus, the main brain regulator of energy homeostasis [1]. Likewise, this inflammation has been detected in the reward and mesocorticolimbic centres [2]. Preliminary magnetic resonance imaging (MRI) studies have supported evidence of inflammation in male mice brain, produced within only 15 days of HFD [3].

Methods: We conducted a study with 18 female mice randomly divided into HFD and control (CTRL) groups. We followed their respective food intake, body weight changes and blood glucose levels during 10 weeks, and performed T2-weighted and magnetization transfer (MT) images (7 T Bruker BioSpec) of the brain, 1, 2, 4 and 10 weeks after diet diversification. Mean values of T2 and MT ratios (MTR) were calculated in the hypothalamus, nucleus accumbens, infralimbic area (ILA) and hippocampus. Statistical analysis was performed by a 2-way ANOVA (diet, area) and post-hoc T tests.

Results: Our results show that weight gain and calorie intake were significantly higher on HFD mice, and no differences were detected in blood glucose levels (Fig. 1 left). MTR and T2 showed a significant diet*area interaction effect at day 7, with lower T2 values and higher MTR in ILA ( $\mathrm{p}<0.05)$ (Fig. 1 right).

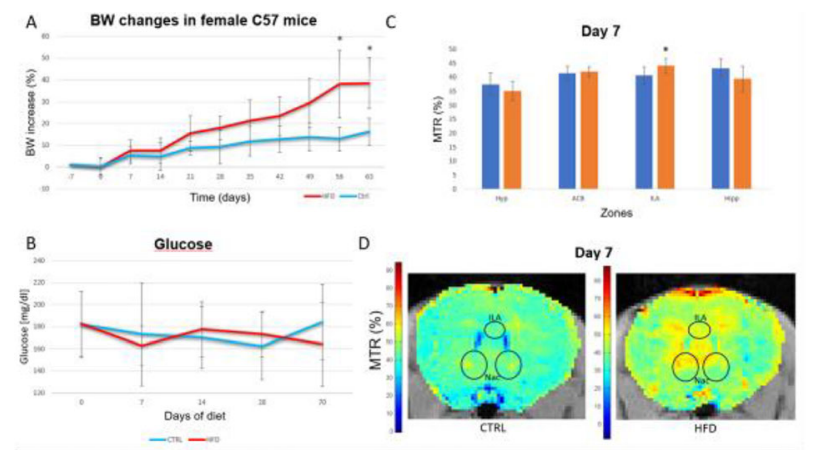

Figure 1: A. Evolution of body weight gain in Ctrl vs HFD animals. B. Evolution of glucose in Ctrl vs HFD animals. C. Comparison of \%MTR mean values between Ctrl and HFD animals in the studied areas at D (Hyp, Hypothalamus; Nac, Nucleus Accumbens; Hipp, Hippocampus). D. Parametric MTR maps of the axial section of the brain of a HFD mouse and a control mouse at D7 in the Nac and ILA.

Discussion: In summary, our work shows that both physiological and MRI biomarkers are altered by HFD, but to a lesser extent than in male mice [3]. However, one week of high-fat diet was sufficient to trigger an increase of MTR and reduction of T2 values in the mouse ILA, on agreement with inflammation.

\section{References}

[1] Thaler JP, Yi C, Schur EA, Guyenet SJ, Hwang BH, Dietrich MO, et al. Obesity is associated with hypothalamic injury in rodents and humans. J Clin Invest 2012 Jan;122(1):153-162.

[ 2 Cazettes F, Cohen JI, Yau PL, Talbot H, Convit A. Obesity-mediated inflammation may damage the brain circuit that regulates food intake. Brain Res [Internet]. 2011;1373:101-9. Available from: 10.1016/j.brainres.2010.12.008
[3] Campillo BW, Cedan S, Lopez-larrubia P and Lizarbe, B. et al. One week high-fat diet alters MTR, T2 and ADC values in the mouse hypothalamus and reward centers. Magn Reson Mater Phy 32, 1-105 (2019). 10.1007/s10334-019-00753-3.

\section{S2.P20.}

\section{Macromolecule-suppressed GABA level increases in posterior cingulate cortex in acute pediatric mild traumatic brain injury}

\author{
*A. Manzhurtsev ${ }^{1,2,3}$, P. Bulanov ${ }^{3}$, M. Ublinskiy ${ }^{1,2}$, \\ P. Menshchikov ${ }^{2,4}$, I. Melnikov ${ }^{1}$, O. Bozhko ${ }^{1}$, A. Yakovlev ${ }^{1,2,5}$, \\ T. Akhadov ${ }^{1,3}$, N. Semenova ${ }^{1,2,3,5}$
}

${ }^{1}$ Clinical and Research Institute of Emergency Pediatric Surgey and Traumatology (CRIEPST), Moscow, $R U$;

${ }^{2}$ Russian Academy of Sciences, Emanuel Institute of Bichemical Physics, Moscow, RU;

${ }^{3}$ Lomonosow Moscow State University, Moscow, $R U$;

${ }^{4}$ Philips Healthcare, Moscow, $R U$;

${ }^{5}$ Russian Academy of Sciences, Federal Research Centre of Chemical Physics, Moscow, RU

Introduction: Concussion or mild traumatic brain injury (mTBI) is one of the most widespread types of trauma. To date, cerebral metabolic alterations after mTBI are studied poorly, especially when it comes to the levels of neurotransmitters. Spectral-edited magnetic resonance spectroscopy allows noninvasive measurement of the gamma-aminobutyric acid (GABA) concentration as well as the concentration of glutamate and glutamine (Glx). The literature data on the $[\mathrm{GABA}]$ change after acute $\mathrm{mTBI}$ is based on the measurement of GABA + levels, where GABA signal is contaminated with macromolecular (MM) compound. The aim of this study is to measure GABA- in the PCC cerebral region in the acute phase of mTBI in children.

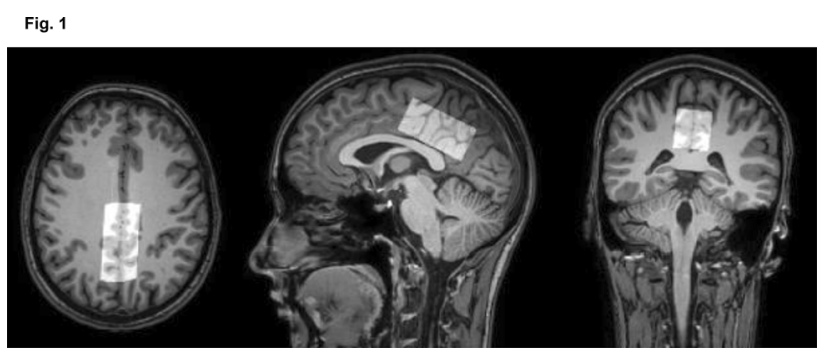

Materials and methods: Nineteen patients with acute mTBI (12-70 $\mathrm{h}$ since the injury, $16.2 \pm 1.4$ y.o.) and twenty-one healthy control $(18.5 \pm 2.3$ y.o. $)$ participated in the study. Philips Achieva 3.0 T was used, standard MRI protocol for TBI patients (T2-, T1wighted images, FLAIR, SWI, DTI) revealed no pathological lesions in brain tissue of any subject. MRS voxel $(50 \times 25 \times 25 \mathrm{~mm})$ was located in posterior cingulate cortex (see Fig. 1). MEGA-PRESS pulse sequence without MM contamination was used [1]: TR = $2000 \mathrm{~ms}$, TE $=80 \mathrm{~ms}, 180$-editing pulses applied on $1.9 \mathrm{ppm}$ and $1.5 \mathrm{ppm}, \mathrm{NSA}=288$ (acq.time $\sim 10 \mathrm{~min}$ ). Spectra were processed in Gannet 3.1 [2], GABA/Cr, Glx/Cr, and GABA/Glx values were obtained.

Results: The data of two controls and three mTBI patients did not match the inclusion criteria (GABA fit error $<12 \%$ ).

GABA-/Cr $(\mathrm{p}<0.01)$ and GABA-/Glx $(\mathrm{p}<0.05)$ were statistically significantly higher in mTBI group than in the normal group (see Fig. 2, 3), while Glx/Cr was unchanged. 

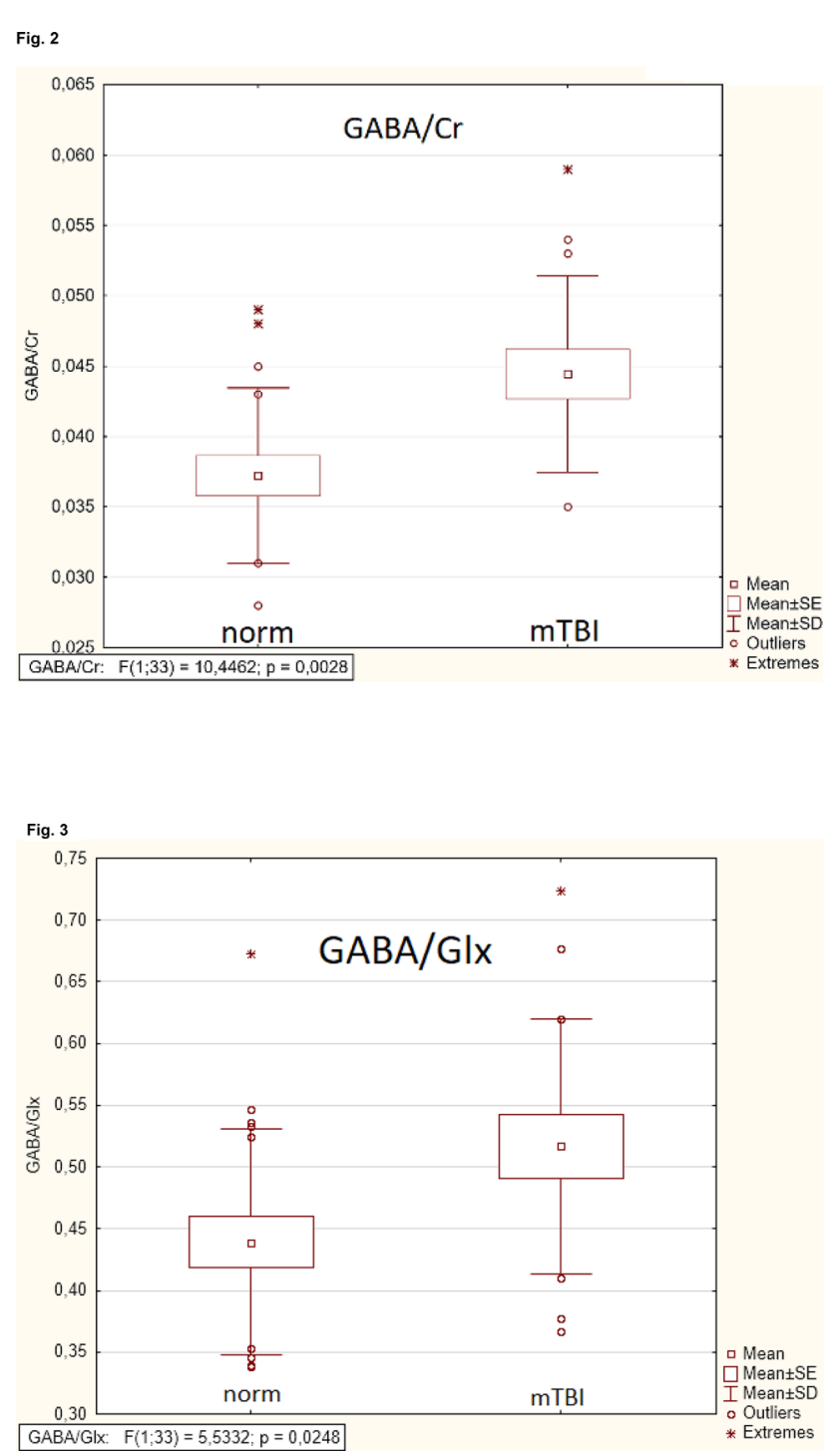

No correlation between the metabolite levels and age was revealed in both groups.

Discussion: It was demonstrated [3] that the measurements of macromolecule-suppressed GABA- may be more effective in search for the metabolic changes than GABA + measurements. In our previous study, we have demonstrated that GABA-/Cr increases in anterior cingulate cortex of children with mTBI [3]. To our knowledge, the mTBI-caused increase in GABA- in PCC, revealed in current study, is reported for the first time and agrees with the previous results. The lack of correlations between age and metabolite levels agrees with the literature data [4] and eliminates possible bias of the results caused by group age difference.

This study provides insight into metabolic alterations caused by mTBI and may facilitate better understanding of the long-term mTBI consequences.

\section{References:}

[1] $10.1002 / \mathrm{mrm} .24391$.

[2] $10.1002 /$ jmri.24478.

[3] 10.1134/S0006350917060161.

[4] 10.1038/s41598-020-80530-8.

\section{S2.P21.}

A correlation between the main excitatory neurotransmitter (Glu) and myo-inositol (mI), as well as a decrease in $\mathrm{pH}$ were found in acute $\mathrm{mTBI}$

*P. Bulanov ${ }^{1}$, A. Manzhurtsev ${ }^{2,1,3}$, A. Yakovlev ${ }^{2,3,4}$, P. Menshchikov ${ }^{5}$, M. Ublinskiy ${ }^{2}$, T. Akhadov ${ }^{2}$, N. Semenova ${ }^{2,1,3,4}$

${ }^{1}$ Lomonosow Moscow State University, Moscow, RU;

${ }^{2}$ Clinical and Research Institute of Emergency Pediatric Surgery and Traumatology (CRIEPST), Moscow, RU;

${ }^{3}$ Russian Academy of Sciences, Emanuel Institute of Biochemical Physics, Moscow, RU;

${ }^{4}$ Russian Academy of Sciences, Semenov Institute of Chemical Physics, Moscow, RU;

${ }^{5}$ Philips Healthcare, Moscow, $R U$

Introduction: Concussion is a mild form of traumatic brain injury (mTBI) with short-term loss of consciousness. Long-term consequences of mTBI can include headaches, attention deficit disorder, sleep problems, etc. [1]. However, with standard MR - tomography or computed tomography (CT), anatomical changes and abnormalities are not observed, which indicates the need to search for biochemical changes in the brain using magnetic resonance spectroscopy (MRS). The aim of this work is to study the effect of mTBI in the acute phase on the $\mathrm{pH}$ of the brain and the concentration of cerebral metabolites available in the methods: PRESS and TE-averaged PRESS.

Materials and methods: The study involved 16 patients with mTBI (scanning was carried out in the acute phase-up to 3 days after injury), the mean age was $15 \pm 3$ years, and 17 healthy volunteers of the same age constituting the control group. Philips Achieva dStream 3.0 $\mathrm{T}$ and 32-channel SENSE quadrature coil were used. The study protocol included a sequence of PRESS pulses with the following parameters: $\mathrm{TR}=2 \mathrm{~s}, \mathrm{TE}=80 \mathrm{~ms}, \mathrm{~N}$ points $=2048, \mathrm{BW}=2000$ $\mathrm{Hz}, \mathrm{NSA}=288$; and TE-averaged PRESS: TR $=2000 \mathrm{~ms}$, TE from 35 to $185 \mathrm{~ms}$ in $2.5 \mathrm{~ms}$ steps. The voxel $(50 \times 25 \times 25 \mathrm{~mm})$ was located in the posterior cingulate cortex (Fig. 1). The values of the central frequency of the signal in the region of $7 \mathrm{ppm}$ were determined, and the $\mathrm{pH}$ values were calculated using the expression from [2].

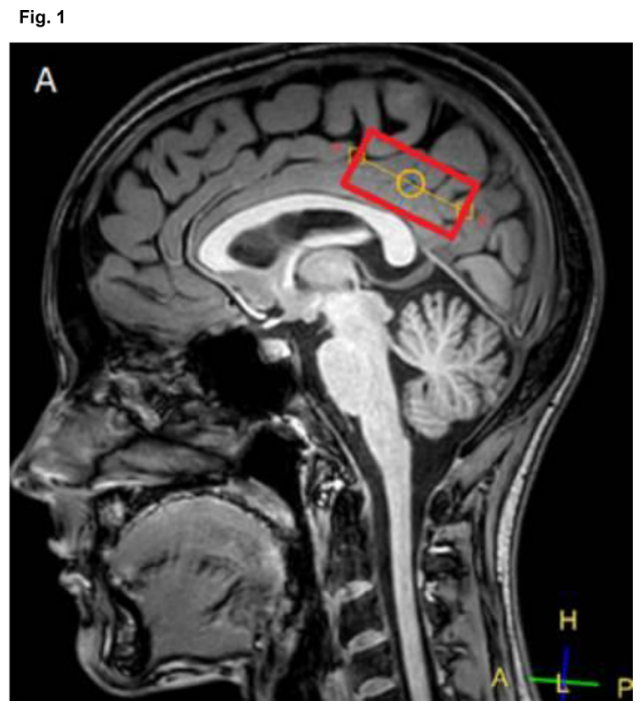


Results: In mTBI a shift in the resonance of the protons in the region of $7 \mathrm{ppm}$ is $0.01 \mathrm{ppm}$. This corresponds to a $1.2 \%$ decrease in $\mathrm{pH}$. At the same time, the change in the chemical shift of NAA and Cr is less by 2 and 3 orders of magnitude, respectively, which excludes an erroneous shift along the frequency axis (Fig. 3). Changes in metabolite concentrations according to PRESS and TE-averaged PRESS data not found. A significant correlation was also found between myo-inositol (mI) and glutamate (Glu) (Fig. 2).

Fig. 2
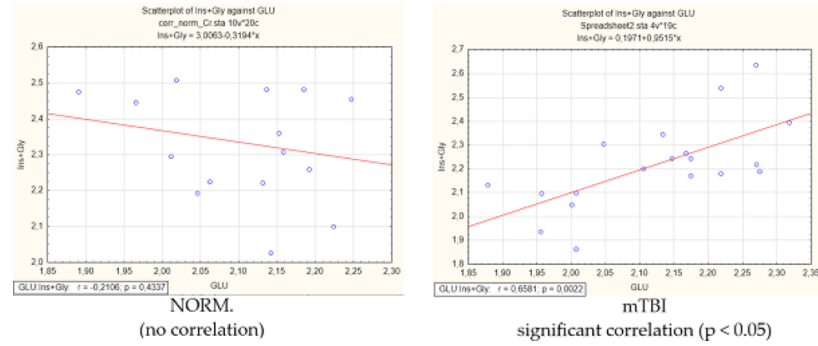

fig. 3

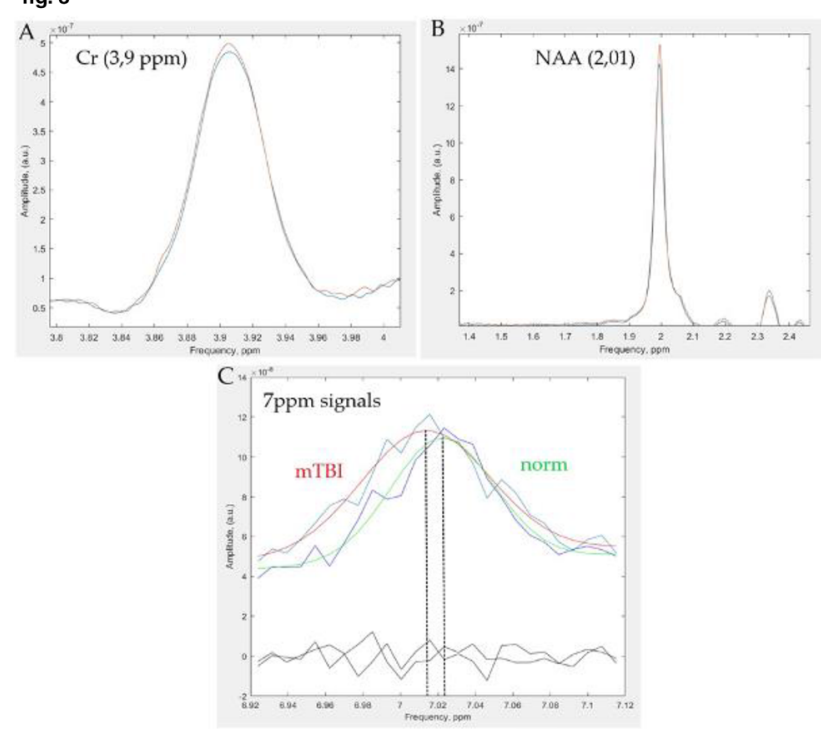

Discussion: The revealed correlation can be associated with the correlation obtained in [3] between NAAG and $\mathrm{mI}$ in hCI. It is due to the fact that NAAG can act as an agonist of Glu receptors [4]. NAAG binding to mGluR 3 receptors and initiates $\mathrm{Ca}^{2+}$ signaling, in which inositol trisphosphate (IP3) is involved, indirectly associated with mI. The lack of changes in the concentration of metabolites in the PRESS and TE-averaged PRESS spectra is also often found in the literature [5]. A decrease in PN may indicate a violation of blood microcirculation and the accumulation of lactate in the area of interest. However, due to the insignificance of the effect $(1 \%)$, additional studies are needed, which are difficult for MRS methods.

\section{References:}

[1] $10.1007 / \mathrm{s} 12178-019-09536-8$.

[2] $10.1002 / \mathrm{mrm} .1910380611$.

[3] $10.1002 / \mathrm{mrm} .28332$.

[4] 10.1111/j.1471-4159.2011.07380.x.

[5] 10.22462/13.15.2019.7.

\section{S2.P22.}

\section{IDH mutation status in brain gliomas evaluated with MEGA-PRESS edited MR spectroscopy}

\section{*D. Juskanic ${ }^{1}$, J. Polakova Mistinova ${ }^{1}$, L. Patrovic ${ }^{2}$, S. Holly ${ }^{2}$,} M. Marjanska ${ }^{3}$

${ }^{1}$ Comenius University in Bratislava, Medical Faculty, Bratislava, SK, ${ }^{2}$ JESSENIUS diagnostické centrum a.s., Nitra, SK;

${ }^{3}$ Center for Magnetic Resonance Research, Department of Radiology, Minneapolis, MN, US

Introduction: The presence of isocitrate dehydrogenase (IDH) mutation is a major diagnostic and prognostic marker in gliomas. Proteomic footprint of IDH mutation is intracellular presence of oncometabolite 2-hydroxy-glutarate $(2 \mathrm{HG})$, which is detectable by MR spectroscopy (MRS). Primary aim of the study was to evaluate the diagnostic performance of in-vivo MRS to determine IDH status. Spectral quality subanalysis was also performed.

Methods: 39 spectra were acquired and reported prospectively in 36 consecutive patients with MR imaging appearence of CNS glioma between February 2018 and June 2019 at tertiary referral hospital with neurosurgery department.Single voxel MRS data were acquired at $3 \mathrm{~T}$ system (Magnetom Skyra, Siemens), with Mescher-Garwood point- resolved spectroscopy (MEGA-PRESS) sequence. Standard voxel size was $8 \mathrm{ml}$, adjustment to the tumor volume was an option. Postprocessing and evaluation of spectra was performed with LCModel. Positive finding was defined as detectability of $2 \mathrm{HG}$ peak with CRLB value less than $60 \%$. Immunohistochemistry or genetic test results were available to compare to 18 spectra.
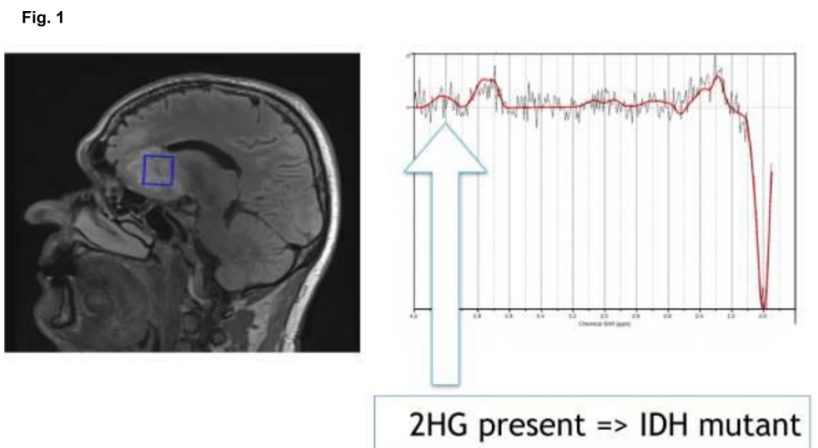

Results: Single voxel MEGA-PRESS MR spectroscopy provided $80 \%$ sensitivity (CI 95\% $0.44-0.96$ ) and $75 \%$ specificity (CI $95 \%$ $0.36-0.95)$ in the detection of 2HG. Spectral quality subanalysis revealed, that the greatest limitation of themethod is lower SNR, which resulted in intermediate quality in $44 \%$ and to non-diagnostic test in $13 \%$ of all acquired spectra.

Fig. 2
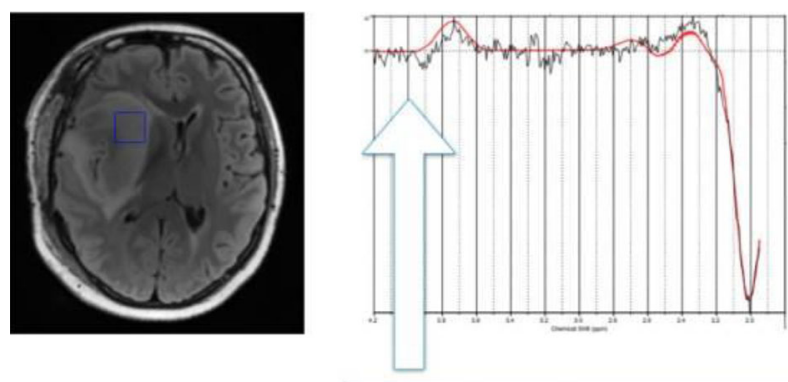

2HG absent $=>$ IDH wilde type 
Conclusion: 2HG MR spectroscopy has a competing diagnostic accuracy, considering its non-invasive, non-contrast and radiation free nature. It may be an integral part of advanced MR tumor protocol, aiding prognostic assessment and multidisciplinary treatment planning for glioma patients.

\section{References:}

1. Tietze, A. et al. Noninvasive assessment of isocitrate dehydrogenase mutation status in cerebral gliomas by magnetic resonance spectroscopy in a clinical setting, Journal of Neurosurgery JNS, 128(2), 391-398. Retrieved Dec 17, 2020

2. Branzoli $\mathrm{F}$ et al. Highly specific determination of IDH status using edited in vivo magnetic resonance spectroscopy, Neuro-Oncology, 20(7), 907-916 (2018).

\section{S2.P23.}

\section{Absolute concentrations of cerebral metabolites in mild cognitive impairment}

*A. Kiryanova ${ }^{1}$, O. Bozhko ${ }^{2,3}$, N. Semenova ${ }^{1,2,3,4,5}$ A. Manzhurtsev ${ }^{1,2,4}$, Y. Fedorova ${ }^{3}$

${ }^{1}$ Lomonosow Moscow State University, Moscow, $R U$;

${ }^{2}$ Clinical and Research Institute of Emergency Pediatric Surgery and Trauma (CRIEPST), Moscow, $R U$;

${ }^{3}$ Mental Health Research Center, Moscow, RU;

${ }^{4}$ Russian Academy of Sciences, Emanuel Institute of Biochemical Physics, Moscow, RU;

${ }^{5}$ Russian Academy of Sciences, Semenov Institute of Chemical Physics, Moscow, $R U$

Introduction: Mild cognitive impairment (MCI) represents a transitional stage between normal aging and dementia. This stage is characterized by a high rate of progress to various types of dementia [1]. In this study, the search for possible metabolic markers is based on proton MR spectroscopy in the posterior cingulate cortex (PCC), a part of the brain with high metabolic activity, playing central role in the default mode network [2]. Consequently, the study of changes in the concentration of metabolites will help to understand the metabolic changes in PCC during MCI. To date, we found no literature data on the change in absolute concentrations of metabolites.

Methods: The measurements were carried out on a Philips Ingenia $3.0 \mathrm{~T}$ scanner, 27 subjects participated in the study:

1)1) Normal (clinically and according to MRI scan)-14 subjects $(12 \mathrm{f}+2 \mathrm{~m})$, aged $47-79$

2)2) Patients with clinically diagnosed $\mathrm{MCI}-13$ subjects (10 $\mathrm{f}+3 \mathrm{~m})$, aged $46-83$

Spectroscopic voxel sized $20 \times 20 \times 30 \mathrm{~mm}$ was placed in the PCC, a typical location is shown in Fig. 1.

\section{Fig. 1}

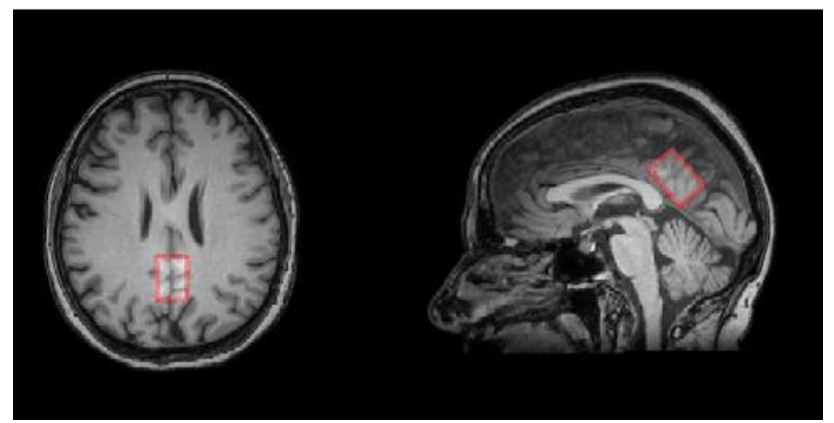

To acquire spectra, PRESS sequence was used with the following parameters: $\mathrm{TE}=35 \mathrm{~ms}$, TR $=2000 \mathrm{~ms}$, NSA $=80$, the number of spectral points: $2048, \mathrm{BW}=2000 \mathrm{~Hz}$. The Reference spectrum of the unsuppressed water signal was also acquired.

Results: Average SNR of spectra of the normal subjects and of patients with MCI were 19.4 and, 19.2 respectively. The values of absolute and relative concentrations of metabolites are presented in the Fig. 2 and 3. Statistically significant increase in the $\mathrm{Cho} / \mathrm{Cr}$, as well as the increase in the absolute concentration of Cho and a decrease in NAA/mI ratio were revealed during MCI.

Fig. 2
\begin{tabular}{|c|c|c|c|c|c|c|}
\hline & tCr & NAA & Glx & mI & Cho* $^{*}$ & NAA/mI* \\
\hline норма & $6,34 \pm 0,15$ & $8,19 \pm 0,20$ & $11,6 \pm 0,5$ & $4,76 \pm 0,15$ & $1,22 \pm 0,04$ & $1,73 \pm 0,04$ \\
\hline MKC & $6,56 \pm 0,19$ & $8,28 \pm 0,32$ & $12,4 \pm 0,7$ & $5,3 \pm 0,3$ & $1,38 \pm 0,05$ & $1,59 \pm 0,07$ \\
\hline
\end{tabular}

Fig. 3

\begin{tabular}{|c|c|c|c|c|}
\hline \multicolumn{1}{|c|}{} & NAA & Glx & mI & Cho* \\
\hline норма & $1,295 \pm 0,022$ & $1,82 \pm 0,06$ & $0,752 \pm 0,014$ & $0,193 \pm 0,004$ \\
\hline MKC & $1,254 \pm 0,021$ & $1,88 \pm 0,06$ & $0,81 \pm 0,03$ & $0,209 \pm 0,004$ \\
\hline
\end{tabular}

Discussions: The increase in [Cho] indicates a violation of phospholipid metabolism in this cerebral zone. This finding may witness for a shift in the balance between synthesis and degradation of cell membranes towards their degradation [3] in MCI. The decrease in $\mathrm{NAA} / \mathrm{mI}$ may signify the change in the neuron-glia ratio towards the gliolysis of the brain [4]. The results of the study are promising as a basis to create the non-invasive markers of dementia progression.

\section{References:}

1. https://doi.org/10.1017/S1092852918001347.

2. https://doi.org/10.1093/brain/awt162.

3. https://doi.org/10.1007/s007020070051.

4. https://doi.org/10.3233/JAD-143225.

\section{S2.P24.}

Identification of metabolic changes of cognitive decline continuum in parkinson's disease using proton magnetic resonance spectroscopic imaging at $3 \mathrm{~T}$

*S. Azamat ${ }^{1,2}$, S. Cengiz ${ }^{1}$, E. Erdoğdu ${ }^{3,4}$, D. B. $\operatorname{Arslan}^{1}$, A. Kicik ${ }^{4,5}$, Z. Tufekcioglu ${ }^{6}$, B. Bilgic ${ }^{6}$, H. Hanagasi $^{6}$, H. Gürvit ${ }^{6}$, T. Demiralp ${ }^{4,7}$, E. Ozturk-Isik ${ }^{1}$

${ }^{1}$ Boğaziçi University, Institute of Biomedical Engineering, Istanbul, $T R$;

${ }^{2}$ Basaksehir Cam and Sakura City Hospital, Department

of Radiology, Istanbul, TR;

${ }^{3}$ Isik University, Faculty of Arts and Sciences/Department

of Psychology, Istanbul, TR;

${ }^{4}$ Istanbul University, Hulusi Behcet Life Sciences Research Center, Istanbul, TR;

${ }^{5}$ Demircioglu Bilim University, Faculty of Medicine, Department

of Psychology, Istanbul, DE;

${ }^{6}$ Istanbul University, Faculty of Medicine, Istanbul, TR;

${ }^{7}$ Istanbul University, Faculty of Medicine / Department of Physiology, Istanbul, TR

Introduction: Proton magnetic resonance spectroscopic imaging (1H-MRSI) is a noninvasive technique that provides information 
about the metabolism of the brain. The aim of this study is to define metabolic changes at different cognitive stages in Parkinson"s disease (PD) using 1H-MRSI at $3 \mathrm{~T}$.

Methods: Twenty-one patients with Parkinson"s disease dementia (PDD), 37 mild cognitively impaired PD (PD-MCI), and 30 cognitively normal PD (PD-CN) were included in this prospective study. The Institutional Review Board approved our research protocol, and all subjects provided written informed consent. Multi-slice 1H-MRSI data was acquired using point resolved spectroscopy (PRESS) sequence at $3 \mathrm{~T}(\mathrm{TR} / \mathrm{TE}=1000 / 52 \mathrm{~ms})$. LCModel software was used for quantification of total $\mathrm{N}$-acetyl-aspartate (tNAA), total creatine (tCr), choline (Cho), glutamate/glutamine complex (Glx), and inositol (Ins). Oryx-MRSI [1] was used to create metabolite maps and overlay them onto the reference anatomical MRI after chemical shift correction. Then, the metabolite intensities were corrected for the cerebrospinal fluid (CSF) fraction at each voxel. Metabolite maps were then registered onto the MNI152 brain atlas using FMRIB Software Library (FSL), and the mean metabolite intensities and ratios were calculated at 400 different functional parcellations of the cerebral cortex [2]. A Kruskal-Wallis test followed by post-hoc Dunn's tests were performed to define metabolic differences among the three patient subgroups.

Results: The mean age of PD-CN, PD-MCI and PDD patients were $60 \pm 9,63 \pm 8$, and $71 \pm 6$, respectively. PDD patients had statistically significantly lower $\mathrm{tNAA} / \mathrm{tCr}$ at the right visual network ( $\mathrm{P}=0.0008$ for $\mathrm{PDD}$ vs. $\mathrm{PD}-\mathrm{CN}$, and $\mathrm{P}=0.022$ for PDD vs. PD$\mathrm{MCI})$ than the non-demented $\mathrm{PD}$, and at the right frontoparietal control network than PD-CN $(\mathrm{P}=0.0006)$ after $\mathrm{CSF}$ correction (Table 1). Additionally, PDD patients had lower $\mathrm{tNAA} / \mathrm{tCr}$ at right somatomotor network $(\mathrm{P}=0.0007)$ and lower tNAA/Ins at left default mode network $(\mathrm{P}=0.003)$ than PD-CN (Fig. 1).

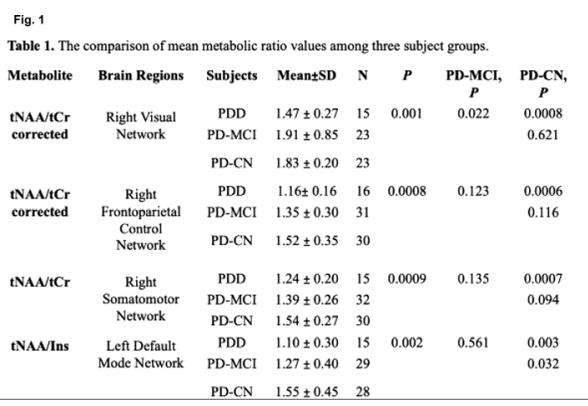

Fig. 2

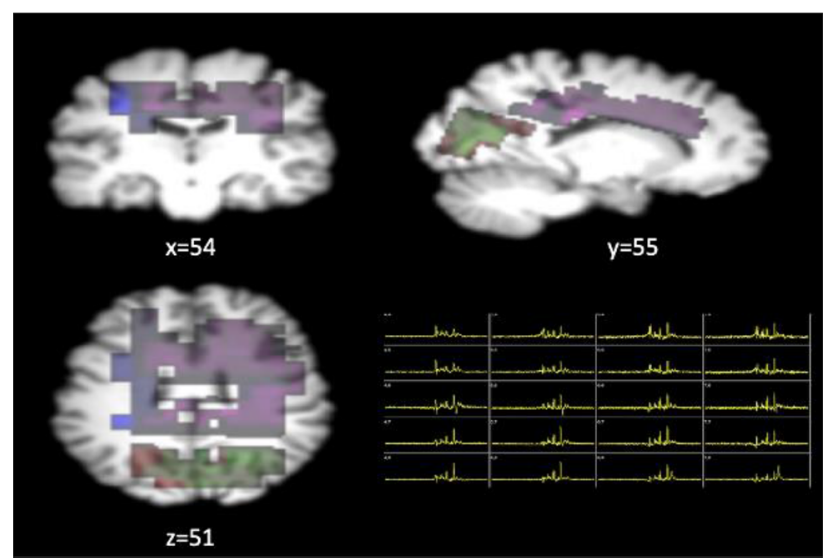

Figure 1. Metabolite maps registered onto MNI brain atlas, red and blue press box represent NAA/tCr metabolite maps, violet and green press box represent NAA/Ins metabolite maps
Discussion: Lower NAA/Cr and NAA/Ins at the regions associated with the visual, frontoparietal control, somatomotor and default mode functional networks may be associated with reduced neuronal viability during the cognitive decline continuum in Parkinson's disease.This study was supported by TUBITAK 1001 project \#115S219, Istanbul University Scientific Research Projects Unit project \#1567/42362 and Bogazici University Scientific Research Projects Unit project \#15222.

\section{References:}

1. Cengiz S, Yildirim M, Bas A, Ozturk-Isik E. ORYX-MRSI: A data analysis software for multi-slice 1H-MRSI. International Society for Magnetic Resonance in Medicine. Virtual Meeting, May 15-20, 2021. (Digital Poster)

2. Schaefer, A., et al., Local-Global Parcellation of the Human Cerebral Cortex from Intrinsic Functional Connectivity MRI. Cereb Cortex, 2018. 28(9): p. 3095-3114.

\section{S2.P25.}

Metabolic profile alterations at ventral attention network detected using proton MR spectroscopic imaging are associated with parkin gene mutation

*S. Azamat ${ }^{1,2}$, S. Cengiz ${ }^{1}$, M. Cebi ${ }^{3}$, D. B. $\operatorname{Arslan}^{1}$, A. Kicik ${ }^{4,5}$, E. Erdoğdu ${ }^{6,4}$, B. Bilgic ${ }^{7}$, T. Demiralp ${ }^{7,8}$, H. Gürvit $^{7}$, H. Hanagasi $^{7}$, E. Ozturk-Isik ${ }^{1}$

${ }^{1}$ Bogaziçi University, Institute of Biomedical Engineering, Istanbul, $T R$;

${ }^{2}$ Basaksehir Cam and Sakura City Hospital, Department of Radiology, Istanbul, TR;

${ }^{3}$ Uskudar University, Faculty of Arts and Sciences,Department of Psychology, Istanbul, TR;

${ }^{4}$ Istanbul University, Hulusi Behcet Life Sciences Research Center, Neuroimaging Unit, Istanbul, TR;

${ }^{5}$ Demircioglu Bilim University, Faculty of Medicine, Department of Psychology, Istanbul, DE;

${ }^{6}$ Isik University, Faculty of Arts and Sciences / Department of Psychology, Istanbul, TR;

${ }^{7}$ Istanbul University, Faculty of Medicine, Istanbul, TR;

${ }^{8}$ Istanbul University, Faculty of Medicine / Department of Physiology, Istanbul, TR

Introduction: Parkin gene (PRKN) mutation is the most common form of autosomal recessive parkinsonism, which causes the familial form classified as PARK2. Proton magnetic resonance spectroscopic imaging (1H-MRSI) provides information about brain metabolism in Parkinson's disease (PD). This study compares the metabolic profiles of PD patients with PRKN mutation with that of the idiopathic PD patients and healthy control (HC) subjects using 1H-MRSI at $3 \mathrm{~T}$.

Material-methods: Sixty patients were enrolled in this prospective study (13 PARK2 patients, 30 idiopathic PD patients with normal cognition (PD-CN), and 17 healthy control subjects (HC)). Our research protocol was approved by the Institutional Review Board, and all subjects provided written informed consent. Multi-slice $1 \mathrm{H}-$ MRSI data was obtained using the point resolved spectroscopy (PRESS) sequence $(\mathrm{TR} / \mathrm{TE}=1000 / 52 \mathrm{~ms})$. LCModel software was utilized for quantification of total $N$-acetyl-aspartate (NAA), total creatine (tCr), choline (Cho), glutamate/glutamine complex (Glx), and myoinositol (Ins). Metabolite maps were created using OryxMRSI [1], followed by overlaying them onto the reference anatomical MRI after chemical shift correction. Afterwards, metabolite intensities were corrected for the cerebrospinal fluid (CSF) fraction at each voxel. FMRIB Software Library (FSL) was used to register 
metabolite maps onto the MNI152 brain atlas and mean metabolite ratios were obtained at 100 distinct functional parcellations of the cerebral cortex [2]. A Kruskal-Wallis test followed by post-hoc Dunn's tests were performed to define metabolic differences among the three patient subgroups.

Results: The mean age of PD-CN, PARK2, and HC patients were $60 \pm 9,44 \pm 4$, and $60 \pm 6$, respectively. PARK2 patients had significantly higher $\mathrm{Glx} / \mathrm{tCr}$ in the left salient/ventral attention network (SN/VAN) $(\mathrm{P}=0.0012$ for PARK2 vs HC) than the healthy control subjects after CSF correction (Table 1).

$$
\begin{aligned}
& \text { Fig. } 1 \\
& \text { Table 1. The comparison of mean metabolic ratio values among three subject groups. } \\
& \text { Metabolite } \\
& \text { Brain Regions } \\
& \text { Subjects } \\
& \text { Mean } \pm \text { SD } \\
& P \quad \text { HC, } P \text { PD-CN, } P \\
& \text { GIx/tCr } \\
& \text { Len } \\
& \text { PARK } \\
& 1.42 \pm 0.17 \\
& \begin{array}{l}
\text { Salient/Ventral } \\
\text { Attention }
\end{array} \\
& \text { Network } \\
& \mathrm{PD}-\mathrm{CN} \\
& 1.28 \pm 0.14 \\
& \begin{array}{lll}
0.0017 & 0.0012 & 0.126 \\
& & 0.081
\end{array}
\end{aligned}
$$

Discussion: Increased $\mathrm{Glx} / \mathrm{tCr}$ might be associated with glutamate/ glutamine toxicity within the SN/VAN, which would lead to eventual cell death. Primary function of this network is to direct attention to unexpected stimuli. VAN activity is modulated by the noradrenergic inputs from the locus coeruleus. PRKN neuropathology involves loss of noradrenergic neurons in the locus coeruleus alongside the hallmark degeneration of dopaminergic neurons in the substantia nigra pars compacta. Our finding may serve as a metabolic imaging marker of PRKN.

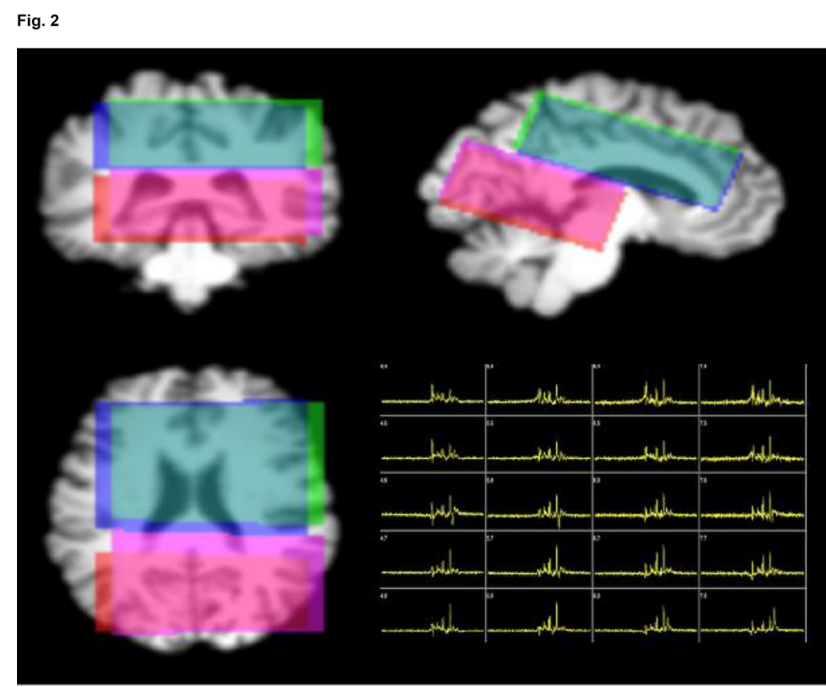

Figure 1. After evaluating the chemical shift misregistration, axial, sagittal, and coronal views of Glx (red and blue), $\mathrm{tCr}$ (violet and green) metabolite image locations are shown within the press box.

\section{Acknowledgements}

TUBITAK 1001 \#115S219, Istanbul University Scientific Research Projects Unit (SRPU) \#1567/42362, and Bogazici University SRPU \#15222.

\section{References}

1. Cengiz S., et al., ORYX-MRSI: A data analysis software for multislice 1H-MRSI.International Society for Magnetic Resonance in Medicine. (Digital Poster) May 15-20, 2021.
2. Schaefer, A., et al., Local-Global Parcellation of the Human Cerebral Cortex from Intrinsic Functional Connectivity MRI. Cereb Cortex. 2018;28(9), 3095-3114

\section{S2.P26.}

\section{GlucoCEST MRI: from phantom to patient}

*S. Derks ${ }^{1,2,3}$, Y. Wu ${ }^{2}$, E. Warnert ${ }^{2}$, L. Knutsson ${ }^{4}$, A. van der Veldt ${ }^{3}$, M. Smits ${ }^{2}$

${ }^{1}$ Erasmus Medical Center, Department of Neurooncology, Rotterdam, $N L$;

${ }^{2}$ Erasmus Medical Center, Department of Radiology and Nuclear Medicine, Rotterdam, NL;

${ }^{3}$ Erasmus Medical Center, Department of Oncology, Rotterdam, NL; ${ }^{4}$ Lund University, Department of Medical Radiation Physics, Lund, $S E$

Introduction: The introduction of new treatments has improved the perspectives of patients with brain tumours. To determine the optimal treatment, there is a need for imaging techniques that not only provide information on anatomy but also on physiology of brain tumours. GlucoCEST, with its potential to indirectly measure D-glucose in tissue and blood, is a promising new MR technique to measure changes in uptake after treatment in brain tumours. The uptake curve reflects delivery, transport and metabolism of D-glucose. Although performing glucoCEST is challenging at clinical scanners (with relatively low B0 field strength), several centres have demonstrated this possibility [1-3]. Here, we describe the development of glucoCEST from phantom to healthy subjects on a 3 Tesla MR scanner.

Methods: We first conducted a phantom study to identify optimal parameter settings in the glucoCEST sequence, at the 3 Tesla MR scanner of our PET/MR modality, with a 24 channel head coil (General Electric, Chicago, USA). We used 3D snapshot CEST[4]. The phantom consisted of 9 plastic $50 \mathrm{ml}$ tubes with a $\mathrm{pH}$ of 7.4 and D-glucose concentrations of $2.5 \mathrm{mM}, 10 \mathrm{mM}$ and $40 \mathrm{mM}$. Four tubes were combined with $8 \%$ cross-linked bovine serum albumin (BSA) to simulate human physiology. Several sequence parameters were explored, including $\mathrm{B} 1$ power $(1 \mu \mathrm{T}, 1,5 \mu \mathrm{T}, 2 \mu \mathrm{T}$ and $3 \mu \mathrm{T})$, radio frequency (RF) pulse (80 and 160 pulses), saturation time (ST) and interval $(40 \mathrm{~ms}+20 \mathrm{~ms}$ and $20 \mathrm{~ms}+20 \mathrm{~ms})$ and frequency offset (between $-100 \mathrm{ppm}$ and $100 \mathrm{ppm}$ with 53 steps). Eight healthy subjects (age range 19-24 years, 2 males and 6 females) were enrolled. They received an intravenous cannula in each arm; one for glucose bolus injection (injection duration of $3.2 \mathrm{~min}, 25 \mathrm{~g}$ dextrose in a $50 \mathrm{ml}$ solution) and the other for regular blood draws to monitor venous blood glucose levels during and after injection. Baseline and post-injection glucoCEST images, as well as dynamic glucose enhanced (DGE) images, were obtained from each subject. Z-spectra and MTR-asymmetry curves for white matter (WM), grey matter (GM) and superior sagittal sinus (SS) were created for MR signal analysis.

Results: In the phantom, the strongest glucoCEST signal was obtained in the tube with $40 \mathrm{mM}$ glucose with a B1 power of $1.5 \mu \mathrm{T}$, $80 \mathrm{RF}$ pulses, saturation module with $20 \mathrm{~ms}$ and $50 \%$ duty cycle, and frequency offset at $1.2 \mathrm{ppm}$ (Fig. 1). Figure 2 shows the difference in $\mathrm{Z}$-spectra from DGE images before and after glucose injection in a healthy subject. 


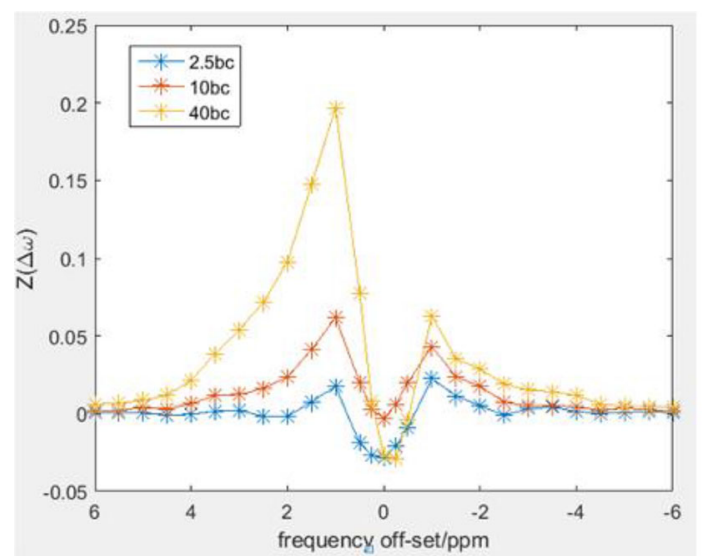

Figure 1. Difference in Z-spectra in tubes containing $8 \%$ cross-linked BSA and different glucose concentrations $(2.5 \mathrm{mM}, 10 \mathrm{mM}$ and $40 \mathrm{mM})$ compared with $8 \%$ cross-linked BSA in the phantom.

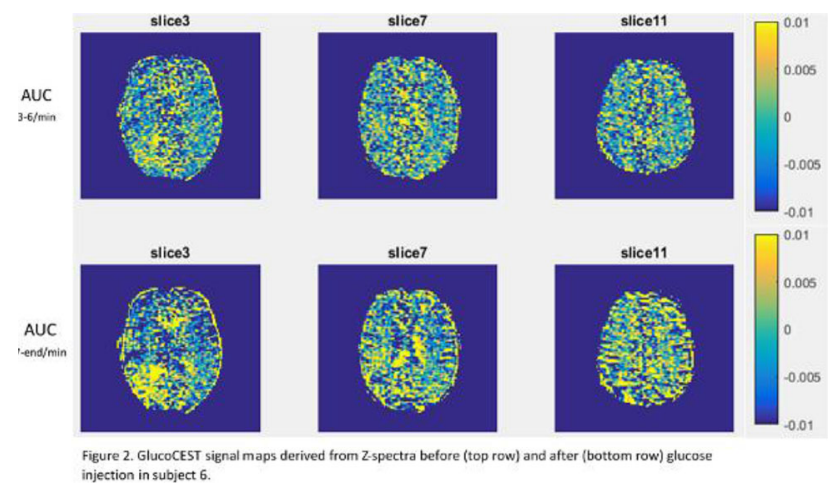

Discussion: In healthy subjects, glucoCEST was optimized for 3 Tesla MR using a PET/MR modality. Although the detected signal was still limited in healthy subjects, glucoCEST is a promising new MR technique to measure D-glucose uptake in brain tumours. Therefore, we will further evaluate and optimize glucoCEST in patients with brain tumours.

\section{References:}

1. Kim et al. PMID31728307.

2. Xu et al. PMID31872916.

3. Zaiss et al. PMID31313865.

4. Deshmane et al. PMID30431179.

\section{S2.P27.}

\section{Study of homocarnosine level and $\mathrm{pH}-\mathrm{value}$ in response to visual activation}

\footnotetext{
*A. Gritskova ${ }^{1}$, A. Yakovlev ${ }^{2,3,4}$, A. Manzhurtsev ${ }^{2,3,4,5}$, M. Ublinskiy ${ }^{2,3,4}$, T. Akhadov ${ }^{2,3,4,5}$, N. Semenova ${ }^{2,3,4,5}$

${ }^{1}$ Lomonosow Moscow State University, Department of Medical Physics, Moscow, RU;

${ }^{2}$ Russian Academy of Sciences, Semenov Institute of Chemical Physics, Moscow, RU;

${ }^{3}$ Russian Academy of Sciences, Emanuel Institute of Biochemical Physics, Moscow, RU;

${ }^{4}$ Clinical and Research Institute of Emergency Pediatric Surgery
}

and Traumatology (CRIEPST), Moscow, $R U$;

${ }^{5}$ Lomonosow Moscow State University, Moscow, $R U$

Homocarnosine $(\mathrm{Hc})$ is a dipeptide of Gamma-Aminbutyric Acid (GABA) and histidine. Hc acts as neurotransmitter in human brain. NMR signals of Hc and GABA are quite similar and differ only with the presence in $\mathrm{Hc}$ spectrum of imidazole protons resonance line. We can receive Hc signals by using pulse sequence MEGA-PRESS (M-P PS) [1]. It is possible to measure $\mathrm{pH}$-value due to $\mathrm{Hc}$ signals [2]. $\mathrm{Hc}$ levels and $\mathrm{pH}$-value in response to visual stimulation (VS) are estimated in this study.

Methods: The study involved 17 healthy people. Scanning was performed on Philips $3 \mathrm{~T}$ scanner. Spectra in rest and during continuous $8 \mathrm{~Hz}$ flashing checkerboard VS were accumulated (10 min each), by using M-P PS (TE $=80 \mathrm{~ms}$, TR $=2000 \mathrm{~ms}$, NSA $=288$ ).

Data from scanner were processed in Matlab using packages FID-A and Gannet. Phase and frequency correction was performed, as a result, resonant lines of creatine protons $(\mathrm{Cr})(\delta=3.027)$ in all spectra were reduced to $3 \mathrm{ppm}$. Then spectra were averaged among participants.

The intensity was determined as one of the approximation parameters for $\mathrm{Hc}(\delta=7.0)$ and $\mathrm{Cr}(\delta=3.027)$. Maximum values of $\mathrm{Hc}$ peaks and their chemical shifts have been found, $\mathrm{pH}$-values were calculated using the equation:

$\mathrm{pH}=\mathrm{pK}_{\mathrm{A}}+\log \left(\left(\delta-\delta_{\mathrm{AH}}\right) /\left(\delta_{\mathrm{A}}-\delta\right)\right)$

$\mathrm{pKA}=6,86$ is the logarithm of the acid dissociation constant, $\delta \mathrm{AH}=7,27 \mathrm{ppm}$ is the chemical shift of $\mathrm{Hc}$ acid, $\delta \mathrm{A}=6,92 \mathrm{ppm}-$ of Hc base [2].

$\mathrm{Hc}$ contribution to the peak $\delta=3 \mathrm{ppm}$ in M-P spectrum at rest was estimated using a model spectrum, as it was done in the work [3]. Hc peaks in absolute values during VS and at rest are shown in Fig. 1.

Fig. 1

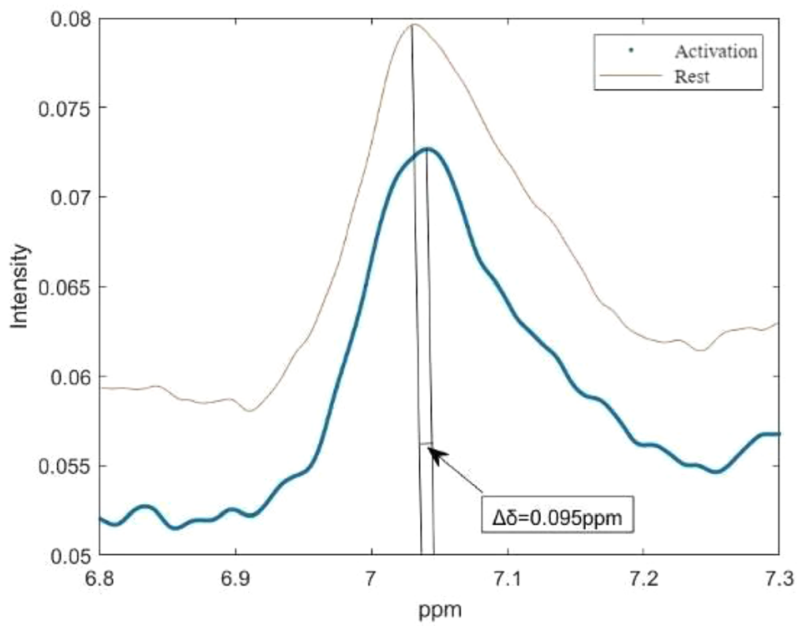

Table 1 Results

\begin{tabular}{llll}
\hline & Peak, ppm & $\mathrm{pH}$ & $\mathrm{IHc} / \mathrm{ICr}$ \\
\hline Act & 7.041 & $7.136 \pm 0.011$ & $0.130 \pm 0.021$ \\
Rest & 7.032 & $7.189 \pm 0.011$ & $0.133 \pm 0.071$ \\
\hline
\end{tabular}




\section{Results:}

For $\mathrm{pH}$-values and $\mathrm{Hc}$ intensities, 2-parameter t-test with different variances was performed $(\alpha<0.05)$, for $\mathrm{pH} \mathrm{p}<0.001$, for $\mathrm{Hc}$ $\mathrm{p}>0.5$. Statistically significant difference is present for $\mathrm{pH}$-values and absent for $\mathrm{Hc}$ intensities during VS and at rest. Hc signal at rest is $30 \pm 8 \%$ of the peak $(\delta=3 \mathrm{ppm})$ intensity in M-P spectrum.

Discussion: The decrease in $\mathrm{pH}$ during VS can be explained by an increase in lactate concentration. The existence of an inverse correlation between these values is proved by various studies [4]. During prolonged VS an increase in lactate concentration in human visual cortex is observed, which confirms our results [5].

\section{References:}

[1] Mescher et al. Simultaneous in vivo spectral editing and water suppression, NMR in Biomedicine (1998)

[2] Rothman et al. Hc and the measurement of neuronal $\mathrm{pH}$ in patients with epilepsy, Magnetic resonance in medicine (1997)

[3] Landheer et al. Elevated Hc and GABA in subject on isoniazid as assessed through $1 \mathrm{H}$ MRS at $7 \mathrm{~T}$, Analytical biochemistry (2020)

[4] Hagihara et al. Decreased brain $\mathrm{pH}$ as a shared endophenotype of psychiatric disorders, Neuropsychopharmacology (2018)

[5] Mangia et al. The in vivo neuron-to-astrocyte lactate shuttle in human brain, Journal of neurochemistry (2009) 
S3.P1.

\section{Comparison of the profile asymmetry of balanced steady-state free precession at $3 \mathrm{~T}$ and $0.55 \mathrm{~T}$}

\author{
*J. Schäper ${ }^{1,2}$, G. Bauman ${ }^{1,2}$, O. Bieri ${ }^{1,2}$ \\ ${ }^{1}$ University of Basel, Department of Biomedical Engineering, Basel, \\ $\mathrm{CH}$; \\ ${ }^{2}$ University Hospital of Basel, Department of Radiology / Division \\ of Radiological Physics, Basel, $\mathrm{CH}$
}

Introduction: The concept of balanced steady-state free precession (bSSFP) has been first introduced by Carr (1) in the late 1950s featuring a frequency response profile that is always symmetric around the on-resonance (e.g. see Freeman and Hill (2)). However, it was reported by Miller (3) that various tissues intrinsically exhibit a strong and rather unexpected asymmetry in their frequency response profile, which was attributed to local asymmetric intra-voxel frequency distributions $(3,4)$. It has been shown that this asymmetry is especially strong in brain white matter where myelination is the probable cause (4).

In a previous work (5), we showed that the bSSFP profile becomes symmetric in the limit of TR $\rightarrow 0$, where voxels "forget" about the underlying spectral composition and become apparently "pure". Whereas this limit can hardly be reached at high-field scanners, lowfields might offer symmetric bSSFP frequency response functions. Here, the profile asymmetry at $3 \mathrm{~T}$ and $0.55 \mathrm{~T}$ is compared.

Methods: Imaging was performed on the head of a healthy volunteer at $3 \mathrm{~T}$ and $0.55 \mathrm{~T}$ (MAGNETOM Prisma and MAGNETOM Free.Max, Siemens Healthineers, Erlangen, Germany), using a Cartesian bSSFP product sequence, with a flip angle $\alpha=10^{\circ}$, TR $=3 \mathrm{~ms}$, FOV $=256 \times 192 \times 160 \mathrm{~mm}^{3}$, imaging matrix: $128 \times 96 \times 80$, yielding $2.0 \mathrm{~mm}$ isotropic resolution. At $3 \mathrm{~T}$ a 20 -channel and at $0.55 \mathrm{~T}$ a 12 -channel head coil were used.

The frequency response was sampled by $\mathrm{N}=36(3 \mathrm{~T})$ and $\mathrm{N}=32$ $(0.55 \mathrm{~T})$ scans with equally distributed linear RF phase increments in the interval $[0,2 \pi)$. Scanning took $20 \mathrm{~min}$ at $3 \mathrm{~T}$ and $17 \mathrm{~min}$ at $0.55 \mathrm{~T}$ to complete.

Results: Representative bSSFP profiles of a white matter ROI are shown in Fig. 1. Using a TR of $3 \mathrm{~ms}$, the asymmetry is obvious at $3 \mathrm{~T}$, whereas the profile at $0.55 \mathrm{~T}$ exhibits almost no asymmetry. As a result, for brain at $0.55 \mathrm{~T}$ the symmetric regime of bSSFP is already reached for common TR settings.
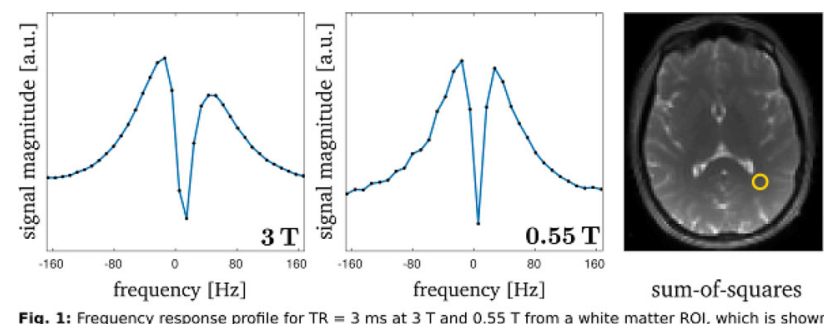
Fig. 1: Frequency response profile for $T R=3 \mathrm{~ms}$ at $3 \mathrm{~T}$ and $0.55 \mathrm{~T}$ from a white matter ROI, which is show on the right. The asymmetry is clearly visible for $3 \mathrm{~T}$, whereas the profile at $0.55 \mathrm{~T}$ is almost completely

Discussion and conclusion: We have shown that at low-fields a symmetric frequency response function can be expected for bSSFP brain imaging even for common TR values. The limit of "pure" bSSFP imaging, as proffered by low-field scanners, is of fundamental interest especially within the context of quantification where any mismatch between theory and experiment can lead to a systematic bias in the estimated parameters.

\section{References:}

1. Carr H. Phys Rev. 1958;112:1693.
2. Freeman, R. \& Hill, H.D.W. JMR 1971;4:366-383.

3. Miller K. L. MRM 2010;63:385-395.

4. Miller K. L., et al. MRM 2010;63:396-406.

5. Schäper J., et al. Proceedings of 29th ISMRM, no. 4195.

\section{S3.P2.}

\section{Free-breathing 3D alpha-mapping for ventilation quantification at $0.55 \mathrm{~T}$}

\author{
*O. Pusterla ${ }^{1,2}$, O. Bieri ${ }^{1,2}$, ${ }^{\mathrm{G}}$. Bauman ${ }^{1,2}$ \\ ${ }^{1}$ University Hospital Basel, Department of Radiology / Division \\ of Radiological Physics, Basel, $\mathrm{CH}$; \\ ${ }^{2}$ University of Basel, Department of Biomedical Engineering, Basel, \\ $\mathrm{CH}$
}

Introduction: Quantification of regional lung ventilation is of great interest for the assessment of respiratory diseases ${ }^{1-4}$. Recently, we proposed $\alpha$-mapping for calculation of lung ventilation maps ${ }^{5}$ from ${ }^{1} \mathrm{H}$ multi-volumetric imaging. In children with cystic fibrosis, $\alpha-$ mapping with breath-holding imaging strongly correlated with lung function markers of ventilation impairment ${ }^{4}$. Transition to freebreathing $\alpha$-mapping may increase its feasibility. In this work, we propose to derive $\alpha$-mapping from data acquired on a low-field MR with a respiratory self-gated 3D balanced steady-state free precession half-radial dual-echo pulse sequence $\left(\right.$ bSTAR $\left.^{6}\right)$.

Methods: The index $\alpha$ reflects a ventilation measure derived from parenchyma signal intensity modulations arising during breathing 5 . The value of $\alpha$ expresses the change in volume of a voxel as a function of the whole lung volume change; e.g. $\alpha=1$ indicates the ventilation in the voxel is the same as the whole lung mean ventilation, $\alpha=2$ that the voxel ventilation is double.

$\alpha$-mapping was evaluated in healthy volunteers and in accordance with the local ethics. Imaging was performed in free breathing on a $0.55 \mathrm{~T}$ clinical MR-scanner (MAGNETOM Free.Max, Siemens Healthineers). The $\mathrm{bSTAR}^{6}$ parameters were: TE1/TE2/TR $=0.08 /$ 2.1/2.2 ms, hard RF pulse $=100$ us, 150,000 radial half-spokes, $\mathrm{FOV}=34 \times 34 \times 34 \mathrm{~cm}^{3}, 300$ interleaves, $\mathrm{FA}=25^{\circ}, \mathrm{BW}=1002$ $\mathrm{Hz} /$ pixel, TA $=5: 30 \mathrm{~min}$.

Before image reconstruction, a trajectory correction of the acquired data was performed. Six volumetric datasets were reconstructed with an isotropic resolution of $2 \mathrm{~mm}$ via retrospective respiratory signal gating. The lung volume of each dataset was determined from 3D deep learning lung segmentations ${ }^{7}$. Image registration was done with Elastix, and the data median filtered ${ }^{5}$ (kernel $5 \times 5 \times 5$ ). Finally, $\alpha$ was computed ${ }^{5}$.

Results: Representative bSTAR images and segmentations are shown in Fig. 1. The sequence shows potential for diagnostic imaging. Exemplary 3D $\alpha$-maps are presented in Fig. 2, revealing ventilation homogeneity on iso gravitational planes (coronal view) and a ventilation gradient from anterior to posterior lung. 


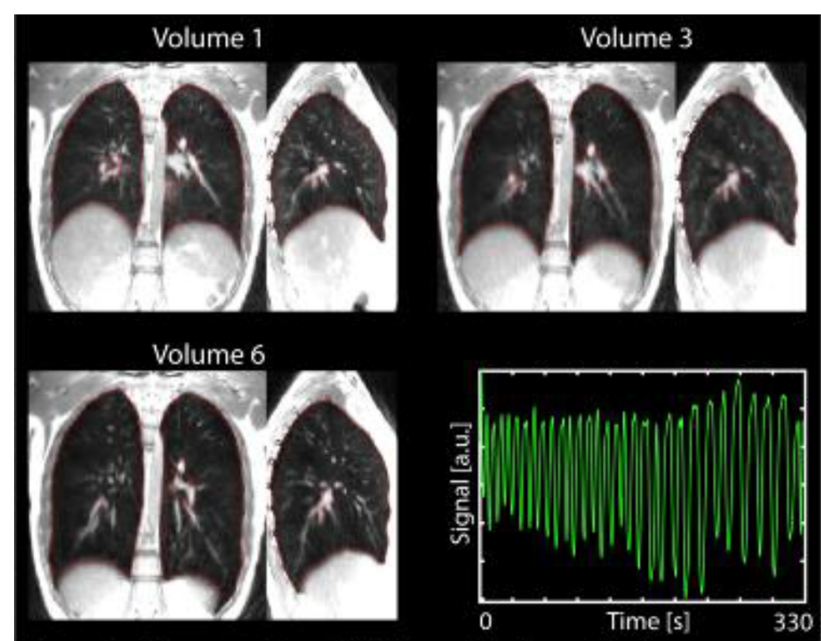

Figure 1. Representative bSTAR multi-volumetric images reconstructed at $2 \mathrm{~mm}$ isotropic resolution. The recurrent neural network lung segmentations are delineated in red. On the bottom-right, the signal modulation of the k-space center used to bin the k-space radial spokes and reconstruct the multi-volumetric images.

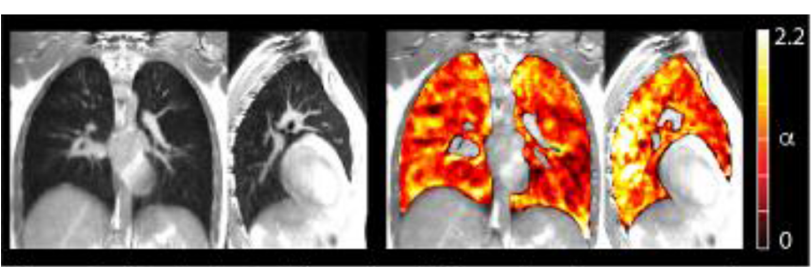

Figure 2. Morphological imaging (left) and pulmonary respiratory $\alpha$-maps (right). The lung ventilation is homogeneous on iso gravitational planes. The posterior lung (lower lobes) has higher ventilation (grater expansion) as compared to the anterior lung. In this subject, the mean $\alpha$ value in the whole lung is 0.95 , indicating excellent agreement with the adapted sponge model. The subject whole lung volumes at expiration and inspiration were 2.2 and 2.8 liters, respectively, and the mean fractional ventilation $27 \mathrm{ml} \mathrm{Air} \mathrm{/} 100 \mathrm{ml}$ Tissue.

Discussion: The derived 3D ventilation maps at $0.55 \mathrm{~T}$ show image features and quality comparable to breath-holding imaging ${ }^{4-5}$ at $1.5 \mathrm{~T}$ and other ventilation techniques (e.g. increased ventilation in dorsal lung as quantified with SPECT).The proposed free-breathing scheme offers good prospects for 3D morphological imaging and ventilation mapping.

In conclusion, we demonstrated initial feasibility to derive $\alpha$-mapping from multi-volumetric data acquired during free breathing using bSTAR on a commercial $0.55 \mathrm{~T}$ low-field MR scanner. In future studies, the clinical value of the method will be further investigated.

\section{S3.P3.}

Functional pulmonary imaging with matrix pencil decomposition at $0.55 \mathrm{~T}$

\author{
*O. Pusterla ${ }^{1,2}$, O. Bieri ${ }^{1,2}, *$ G. Bauman ${ }^{1,2}$ \\ ${ }^{1}$ University Hospital Basel, Department of Radiology/Division \\ of Radiological Physics, Basel, $\mathrm{CH}$; \\ ${ }^{2}$ University of Basel, Department of Biomedical Engineering, Basel, \\ $\mathrm{CH}$
}

Introduction: Thanks to advances in technology, modern low-field MRI systems $(<1 \mathrm{~T})$ allow for clinical imaging and are gaining renewed interest ${ }^{1}$. In addition to having cost advantages and reduced requirements for scanner siting, low-field MRI offers new imaging opportunities.

Balanced steady-state free precession (bSSFP) is an example of a clinical sequence limited by $\mathrm{B}_{0}$ dependent susceptibility and specific absorption rate (SAR), especially at high field strength. Typically, for bSSFP the repetition time (TR) is minimized to mitigate off-resonance-related banding artifacts. Due to the reduced $B_{0}$ inhomogeneities at low field, large field-of-view can be imaged with less stringent restrictions to TR. Furthermore, at low field, bSSFP benefits from increased $T_{2} / T_{1}$ (i.e. higher signal) and the possibility to use optimal flip angle settings, due to reduced SAR limits.

Functional lung imaging with matrix pencil (MP) decomposition ${ }^{2}$ relies on dynamic free-breathing acquisitions with a dedicated ultrafast bSSFP (ufSSFP) imaging sequence in which the TR is kept ultrashort (i.e. $\sim 1.5 \mathrm{~ms}$ at $1.5 \mathrm{~T}$ ). MP-MRI is based on naturally occurring respiratory and cardiac signal modulations in the pulmonary tissue, and from the acquired time-resolved data, maps of fractional ventilation and perfusion are computed. MP-MRI and similar techniques have recently shown compelling results ${ }^{3}$ and are highly desirable for longitudinal chronic pulmonary disease management since non-invasive. As previously mentioned, lung imaging at low field might be beneficial. Therefore, this work aims to demonstrate the feasibility of MP-MRI on a clinical $0.55 \mathrm{~T}$ low-field MRI scanner.

Methods: Healthy volunteers were scanned at 0.55 T (MAGNETOM Free.Max, Siemens Healthineers) and 1.5 T (MAGNETOM AvantoFit, Siemens Healthineers) in accordance with local ethics. Imaging was performed with ufSSFP at $1.5 \mathrm{~T}$, and with bSSFP and gradient echo (GRE) at $0.55 \mathrm{~T}$. Ultra-fast bSSFP sequence parameters and MP postprocessing details are given in Bauman et $\mathrm{al}^{4}$. At $0.55 \mathrm{~T}$, identical parameters were used, but for bSSFP TR was $3.0 \mathrm{~ms}$ (the shortest achievable) and the flip angle to $90^{\circ}$. For GRE, a TR of $4.0 \mathrm{~ms}$ was used and a flip angle of $15^{\circ}$.

Results: Exemplary images acquired with ufSSFP, bSSFP, and GRE, as well as functional maps are presented in Fig. 1. The quality of baseline ufSSFP images acquired at $1.5 \mathrm{~T}$ appears superior to bSSFP at $0.55 \mathrm{~T}$, in which artifacts are present (see the liver). In terms of visual image quality, bSSFP at both field strengths outperform GRE. Overall, functional imaging with bSSFP is feasible at both field strengths.

\section{References:}

1. Nyilas S, Eur Respir J 2017.

2. Heidenreich JF, Radiology 2020.

3. Klimeš F, Magn Reson Med 2021.

4. Pusterla O, ISMRM 2017.

5. Pusterla O, Magn Reson Med 2017.

6. Bauman G, Magn Reson Med 2021.

7. Pusterla O, ISMRM 2018. 


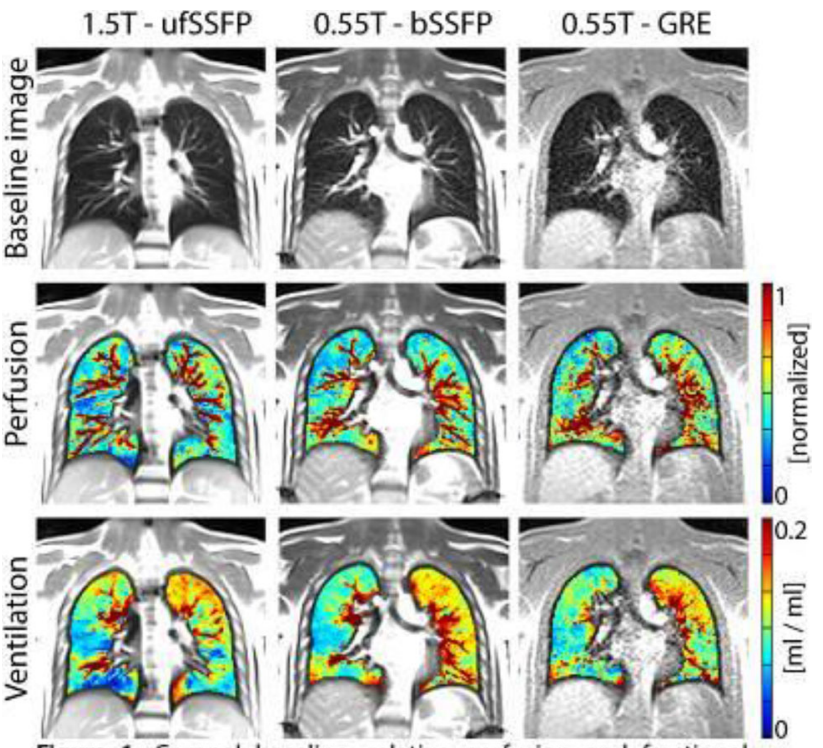

Figure 1. Coronal baseline, relative perfusion and fractional ventilation maps generated using matrix pencil decomposition in a healthy volunteer from the data acquired with ufSSFP at $1.5 \mathrm{~T}$, and bSSFP and GRE at $0.55 \mathrm{~T}$.

Discussion: We demonstrated perfusion and ventilation mapping with bSSFP on a low-cost commercial $0.55 \mathrm{~T}$ MR scanner. Further sequence optimization is required to improve MP-MRI at low field.

\section{References:}

1. Marques, J Magn Reson Ima. 2019.

2. Bauman, Magn Reson Med. 2017.

3. Nyilas S, Eur Respir J. 2017.

4. Bauman, Magn Reson Med. 2016.

\section{S3.P4.}

\section{Diffusion tensor imaging and sodium $\left({ }^{23} \mathrm{Na}\right) \mathrm{MRI}$ :} a correlation study

\author{
*P. Polak ${ }^{1,2}$, M. D. Noseworthy ${ }^{1,2,3}$ \\ ${ }^{1}$ McMaster University, School of Biomedical Engineering, Hamilton, \\ $C A$; \\ ${ }^{2}$ St. Joseph's Healthcare, Imaging Research Centre, Hamilton, CA; \\ ${ }^{3}$ McMaster University, Department of Electrical and Computer \\ Engineering, Hamilton, $C A$
}

Introduction: Due to the essential role of sodium $\left({ }^{23} \mathrm{Na}\right)$ in human metabolism [refs] in vivo ${ }^{23} \mathrm{Na}$ MRI is an enticing prospect, despite numerous technical challenges (1). Part of this attraction lies in the role sodium plays in various neurological disorders, among these multiple sclerosis and cancer $(2,3)$. Comparisons to other MRI techniques, such as diffusion tensor imaging (DTI), are hindered by the low signal and generally large voxel sizes common to ${ }^{23} \mathrm{Na}$ MRI. This study examines DTI measures and ${ }^{23} \mathrm{Na}$ MRI in a healthy population. Methods: Subjects: 8 male, 6 female, mean age: 34.1 scanned in a study approved by research ethics.

Hardware: GE 3 T 750 (Milwaukee, WI) using a quadrature birdcage ${ }^{23} \mathrm{Na}$ coil and a 32-channel ${ }^{1} \mathrm{H}$ head coil.

Acquisition: Two ${ }^{23} \mathrm{Na}$ sequences (flip angles 30/70) acquired using density-adapted 3D radial projections (4) to obtain total sodium content (TSC). Protocol details in Fig. 1.

\begin{tabular}{|c|c|c|c|c|c|c|c|c|}
\hline Sequence & $\mathrm{TR}$ (ms) & TE (ms) & $\mathrm{TI}(\mathrm{ms})$ & $\begin{array}{l}\text { Acquisition } \\
\text { Time (nmm:ss) }\end{array}$ & Flip Angle & $\begin{array}{l}\text { Field of View } \\
(\mathrm{mm})\end{array}$ & $\begin{array}{l}\text { Acquisition Voxels } \\
\left(\mathrm{mm}^{2}\right)\end{array}$ & Notes \\
\hline \multicolumn{9}{|l|}{${ }^{\bar{n}} \mathrm{Na}$} \\
\hline DA-3DRp & 24 & 0.2 & - & $10: 48$ & 30 & 240 & $3.2 \times 3.2 \times 3.2$ & $\begin{array}{c}13500 \\
\text { projections, } \\
2 \text { averages }\end{array}$ \\
\hline DA-3DRp & 24 & 0.2 & - & $10: 48$ & 70 & 240 & $3.2 \times 3.2 \times 3.2$ & $\begin{array}{c}13500 \\
\text { projections, } \\
2 \text { averages }\end{array}$ \\
\hline B1+ Map & 160 & 0.2 & - & $03: 50$ & 90 & 240 & $6.2 \times 6.2 \times 6.2$ & $\begin{array}{l}\text { FLORET, } 3 \\
\text { hubs, } 120 \\
\text { interleaves, } 4 \\
\text { averages }\end{array}$ \\
\hline \multicolumn{9}{|l|}{${ }^{\top} \mathrm{H}$} \\
\hline 2D Axial DTI & 12000 & 78 & - & $13: 30$ & - & 240 & $2.5 \times 2.5 \times 2.5$ & $\begin{array}{l}60 \text { directions, } \\
6 \mathrm{~b}=0 \text { images }\end{array}$ \\
\hline $\begin{array}{c}\text { 2D Axial DTI- } \\
\text { (reverse EPI polarity) }\end{array}$ & 12000 & 78 & - & $02: 30$ & - & 240 & $2.5 \times 2.5 \times 2.5$ & $\begin{array}{l}6 \text { directions, } \\
6 \mathrm{~b}=0 \text { images }\end{array}$ \\
\hline 3D Sagittal T1 & 1200 & 4.25 & 450 & $05: 30$ & 12 & 240 & $0.47 \times 0.94 \times 2.0$ & $\begin{array}{l}- \\
-\end{array}$ \\
\hline 3D Sagittal T2 & 3000 & 149 & - & $07: 30$ & - & 240 & $0.47 \times 0.94 \times 2.0$ & - \\
\hline
\end{tabular}

Reconstruction: ${ }^{23} \mathrm{Na}$ images reconstructed with BART (5) using gridding and a thresholded $l_{1}$ wavelet for noise reduction. ${ }^{23} \mathrm{Na}$ images were reconstructed to $1 \mathrm{~mm}^{3}$ before calculating TSC and T1 Map images using the methods outlined by (6). DTI images were eddy corrected.

Registration: Performed using FSL and gradient unwarping (7). Details in Fig. 2.

a
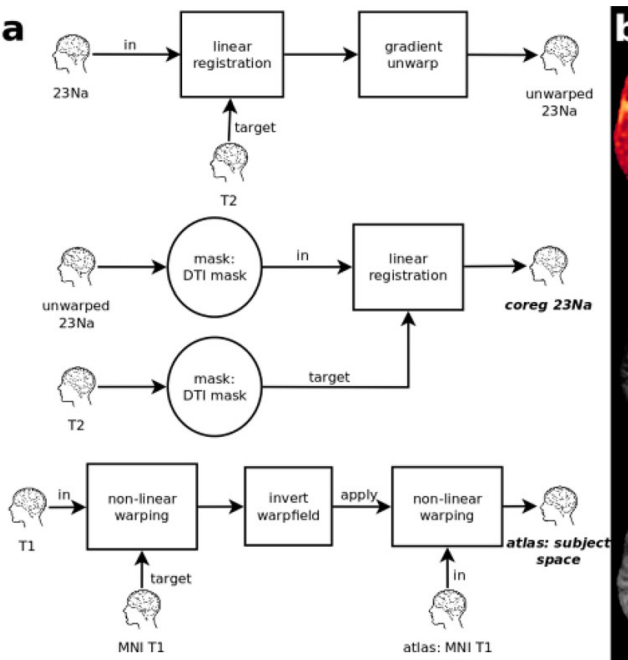

Figure 2: (a) Registration pipeline for ${ }^{23} \mathrm{Na}$ images and T1 atlas. T2, DTI, and mask images are first registered to subject $T 1$ space (not shown). Registration steps then proceed in order top to bottom, using FSL tools and gradient unwarping software. (b) Sample registered images $-{ }^{23}$ Na TSC image (top) in $\mathrm{mM}$; FA image (middle), and T1 weighted anatomical image (bottom).

Analysis: TSC calibrated linearly based on mean signal in regions in eyes $(140 \mathrm{mM})$ and the corona radiata $(38 \mathrm{mM})(6,8)$. An atlas of white (WM) and grey matter (GM) regions was constructed and warped into subject space with FSL. Whole brain mask "bins" were created based on the range of FA values appropriate for either WM or GM regions (i.e. $0.4<\mathrm{FA}<0.5$ ). Mean and standard deviation TSC was measured for each atlas region and separated into the FA bins. Results: Figure 3: (a) representative TSC, T1 and FA images (b) plots for mean TSC and relative volume in GM and (c) WM versus FA. 


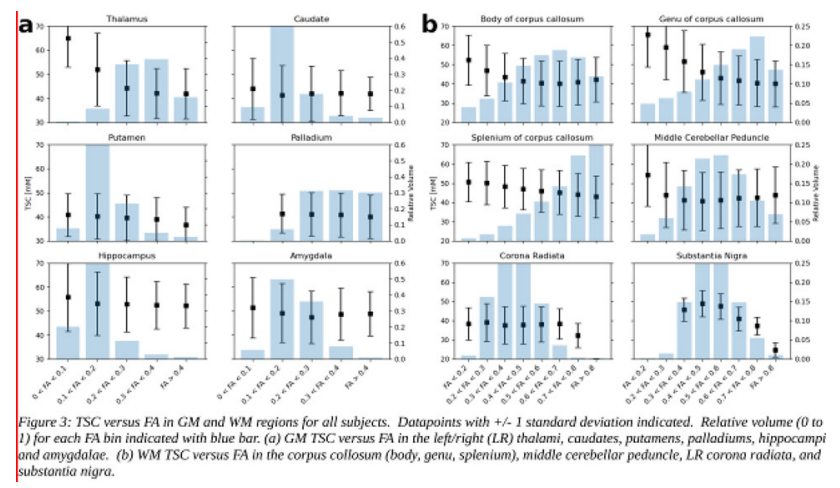

Discussion: This study examined correlative measurements of ${ }^{23} \mathrm{Na}$ MRI and DTI in a healthy population. Other groups have investigated ${ }^{23} \mathrm{Na}$ and diffusion measurements in cancer and traumatic brain injury $(9,10)$, but this work combines TSC, a registration pipeline with a fine-grained analysis of FA in specific WM and GM regions.

In WM near the lateral ventricles (i.e. corpus collosum) there was an inverse correlation between FA and TSC. One possible explanation of this is point spread function of ${ }^{23} \mathrm{Na}$ MRI perturbing WM regions by high ${ }^{23} \mathrm{Na}$ signal from the lateral ventricles. This is a pilot study and further examinations of these effects are required.

A TR of $24 \mathrm{~ms}$ results in a ${ }^{23} \mathrm{Na}$ sequence which is T1-weighted. A sequence without T1-weighting would require a TR of 120-160 ms: a significant increase in acquisition time. The multi-flip angle approach used here allows a high resolution TSC map in a clinically feasible time.

\section{References:}

1. Madelin JMRI 2013.

2. Zaaraoui Rad 2012.

3. Nagel Irad 2011

4. Nagel MRM 2009.

5. Uecker ProcMRM 2015.

6. Coste MRI 2019.

7. Polak ProcMRM 2015.

8. Ouwerkerk Rad 2003.

9. Jacobs JMRI 2009.

10. Chen ProcMRM 2021.

\section{S3.P5.}

\section{Artificial k-space based simulations and rotating phantom measurements to assess detection limits of single cell time-lapse MRI}

\section{${ }^{*}$ E. Wilken ${ }^{1}$, F. Freppon ${ }^{1}$, M. Masthoff ${ }^{1}$, C. Faber $^{1}$ \\ ${ }^{1}$ University Hospital Münster, Clinic of Radiology / Translational Research Imaging Center (TRIC), Münster, DE}

Introduction: By collating repetitive $\mathrm{T} 2 *$-weighted images into movies, time-lapse MRI allows non-invasive detection and tracking of intravascular moving single cells to study the immune response under inflammatory stimulus. ${ }^{1,2}$ Since the generated contrast of ironlabeled cells is motion-dependent, we address the detectable velocity range by simulating movement-induced blurring. To validate the findings, a rotating phantom system was constructed, and time-lapse MRI of iron labeled monocytes in agarose was performed.

Methods: Time-lapse contrast was simulated using MATLAB. A signal voids position was stepwise increased in a synthetic phantom and artificial k-space was filled by fractions of the Fourier transforms

of the individual images. The final image was acquired through inverse Fourier transform (Fig. 1a) and then multiplied with an in vivo time-lapse MR image of mice brain for an overlay. To imitate moving cells, a system was built to rotate agar gel phantoms with embedded ION-labeled monocytes inside an MRI-scanner (9.4 T Biospec (Bruker Biospin) with cryogenic probe) (Fig. 2). A stepper motor (Nanotec) with a mounted planetary transmission is used as drive to achieve cell velocities around $1 \mu \mathrm{m} / \mathrm{s}$. Repetitive T2*weighted images were then acquired with a gradient echo sequence (in-plane resolution: $67 \times 55 \mu^{2}$, scan time: $8 \mathrm{~min} 12 \mathrm{~s}$ ) for a static phantom and a rotation of $4.4 \times 10^{-3} \mathrm{rpm}$.

(a)

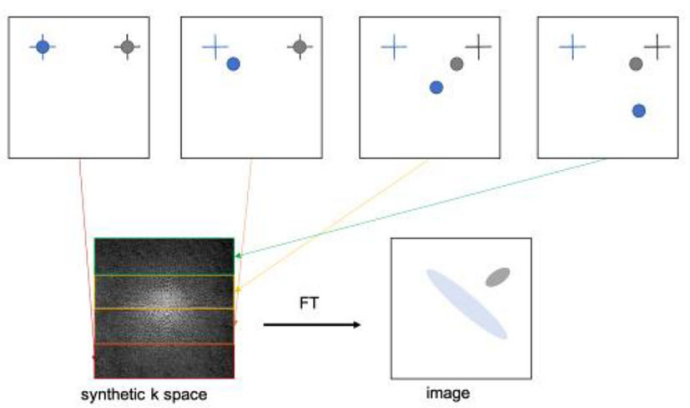

(b)

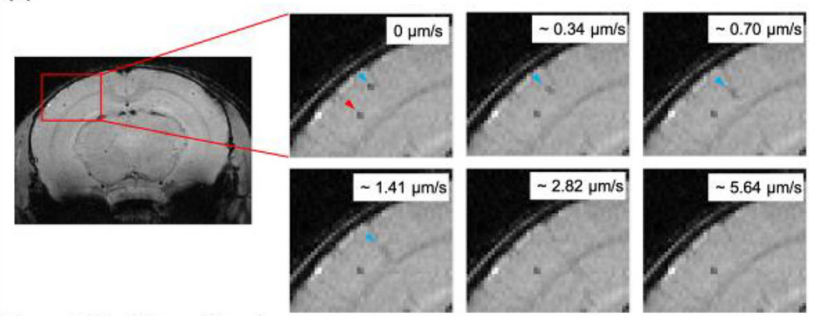

Figure 1: Simulations of time-lapse contrast.

(a) Basic concept of how synthetic k-space is created to simulate blurring of image contrast of moving cells.

(b) Image details, indicated by the red rectangle, of an overlay of a $\mathrm{T} 2 *$-weighted image acquired with the time-lapse MRI protocol and the contrast simulation for different cell motion velocities, showing a real (red arrowhead) and a simulated (blue arrowhead) cell in the mouse brain cortex.

(a)

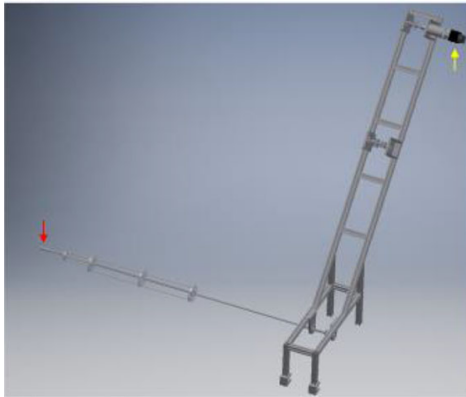

Figure 2: System to rotate an agarose phantom with iron-labeled cells.

(a) Design sketch of the whole system. The phantom (red arrow) is attached to a glass fiber stick mounted with ball bearings inside the MRI scanner assuring a centered position. To reduce disturbance and damage due to the stray field of the MR system, the stepper motor (yellow arrow) is placed away from the bore center and mounted on an aluminum structure. The rotation of the phantom is then driven through toothed belts.

(b) Photo of the system installed at the scanner. 
Results: The superposition of simulated moving cells by assembled artificial k-space and real MRI data accounts for noise, anatomical structures, and imaging artifacts. A direct comparison of real and simulated cells is possible showing that the artificial cells represent the experimental signal voids well (Fig. 1b). The increasing blurring of time-lapse contrast becomes evident supposedly resulting in a maximum detectable speed of $1 \mu \mathrm{m} / \mathrm{s}$. Moreover, the MR images of iron-labeled monocytes inside a rotating agarose phantom (Fig. 3b) are in good agreement with the simulations: In general, slowly moving signal voids close to the axis of rotation remain visible. On the other hand, the contrast of monocytes linked to higher velocities becomes increasingly blurred resembling the simulated time-lapse contrast in shape and size.

(a)

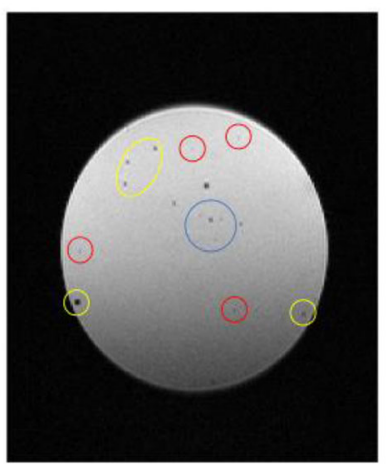

(b)

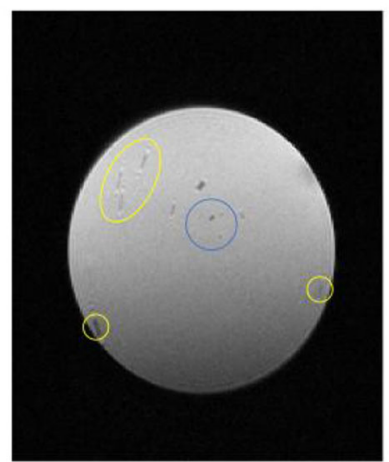

Figure 3: Phantom time-lapse measurements of iron-labeled monocytes embedded in agarose gel in a static position (a) and with a rotation of $4.4 \times 10^{-3}$ $\mathrm{rpm}$ (b). The blue circle indicates cells close to the axis of rotation which are visible in both images regardless of the contrast they generate in the static case. On other hand, for faster moving cells (up to $2.3 \mu \mathrm{m} / \mathrm{s}$ ), the visibility depends strongly on the contrast they have in the non-rotating phantom as displayed by the yellow (visible in both cases) and red (only visible if motionless) circles.

Discussion: Overall, the performed simulations are in line with in vivo data and can be validated by phantom measurements. However, the detectable velocity range of migrating cells is not only dependent on the speed, but also on the contrast in a static position (Fig. 3a). Hence, some cells approaching the edge of the phantom generate enough contrast, while others are not detectable anymore. We understand all three approaches as valid methods to further analyze the detection boundaries of time-lapse MRI regarding size and velocity of iron-labeled cells, which we aim to specify in the future for our standard time-lapse sequence and different acquisition accelerating methods.

\section{References:}

1. PMID: 29934611.

2. PMID: 25385430.

\section{S3.P6.}

\section{Accelerating high field acquisitions with Wave-CAIPI on a single axis insert gradient}

\author{
*T. Roos ${ }^{1}$, E. Versteeg ${ }^{1}$, J. Siero ${ }^{1}$ \\ ${ }^{1}$ University Medical Centre Utrecht, Department of Radiology, \\ Utrecht, NL
}

Introduction: The increased SNR available at higher field strengths, like $7 \mathrm{~T}$, allows for higher undersampling factors $(\mathrm{R})$ and thus faster acquisitions. SNR is however not only reduced by $\sqrt{ }$, but also by the g-factor and that limits the obtainable acceleration of conventional techniques like SENSE. Wave-CAIPI [1] can minimize this penalty by spreading the aliasing and thus fully utilising the $3 \mathrm{D}$ coil sensitivity profiles.

The amount of spreading is limited by the PSF of the sine waves and thus by their strength and amount of cycles. The usage of a highperformance insert gradient [2] allows for better sine waveforms and thus more spreading, even during short readouts. Previous simulation work has shown that this increases the efficiency of Wave-CAIPI for a single axis insert [3].

This work demonstrates highly accelerated Wave-CAIPI acquisitions using a gradient insert while avoiding PSF calibration pre-scans and compares their performance against SENSE.

Methods: To compare the effects of the accelerations techniques, phantom and in vivo $0.8 \mathrm{~mm}$ whole-brain MP-RAGE scans (matrix $320 / 236 / 320, \mathrm{Te} / \mathrm{TR} / \mathrm{TI}=3.0 / 6.0 / 1000 \mathrm{~ms}, \alpha=6 \mathrm{deg}$, TFE factor $300, \mathrm{Bw}=380 \mathrm{~Hz}$ ) were made using a Philips Achieva $7 \mathrm{~T}$ (Best, Netherlands) scanner. Both SENSE and Wave-CAIPI were $8 \times$ oversampled in the readout direction and undersampled by $3.3 \times$ in both phase-encoding directions for a total acceleration of 9.9. 10 sinus cycles were played during, with the $\mathrm{Z}$-wave shifted by $90^{\circ}$.

To study the effect of wave amplitudes on the Z-axis, the highest requestable strength of $8 \mathrm{mT} / \mathrm{m}$ was used for the body gradients and both matched and doubled by the insert.

Offline reconstruction in MATLAB recreates the gradient waveforms and convolves them with the impulse response measured using a Skope field camera (Zurich, Switzerland) for each channel. BART was used for CG-SENSE reconstruction and RMSE was iteratively minimised for auto-calibration of the exact trajectory [4].

Results: Figure 1 shows the resulting predicted gradient waveforms and how they are obtained from the different gradient axes, without the need for additional pre-scans.

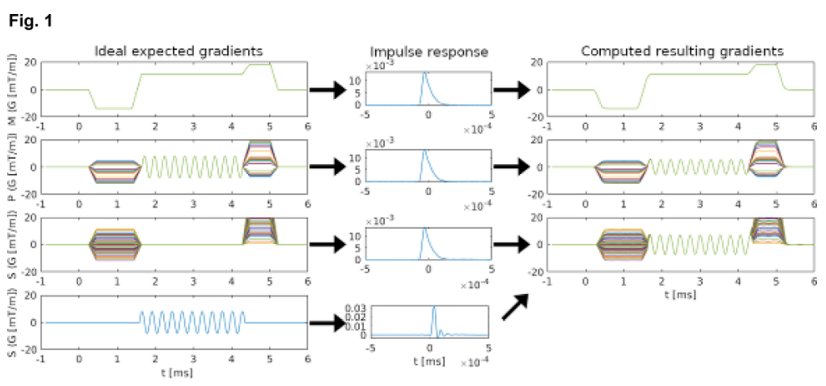

The phantom results depicted in Fig. 2 show sharp artefacts produced by SENSE, that are less visible in the Wave-CAIPI \#2 acquisition, but failed correct wave unfolding in the lower and higher amplitudes. 


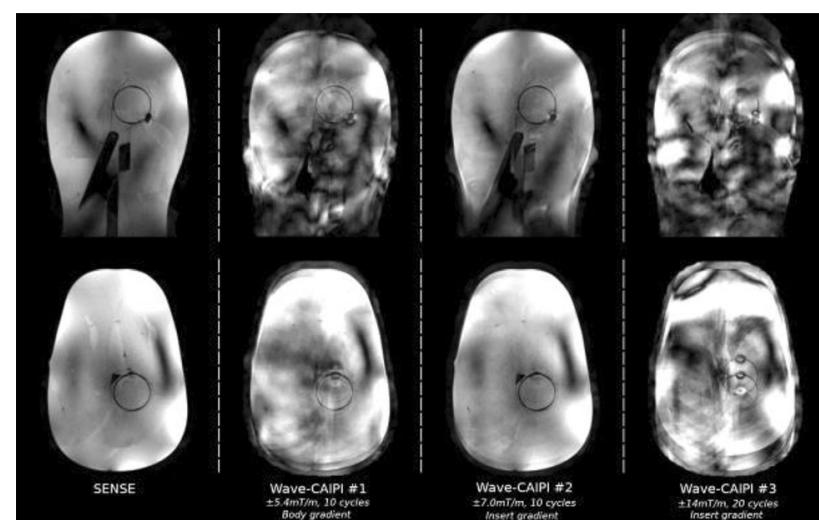

The third Figure shows that the optimal in vivo Wave-CAIPI acquisition improves on SENSE in certain areas (green arrow) and adds artefacts on others (red arrows).

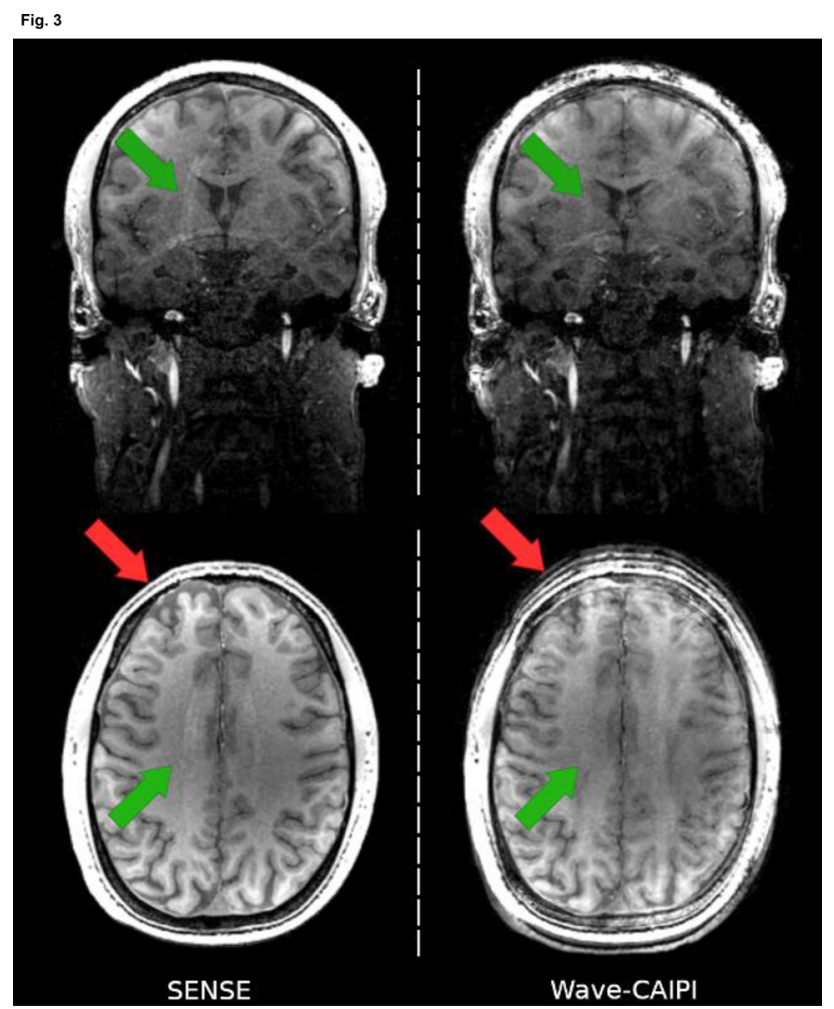

Discussion: Wave-CAIPI is a promising acceleration technique that performs better with higher-performance gradients.

The utilised calibration-less reconstruction produces artefacts when the gradients are pushed to their limits, which PSF pre-scans might mitigate. When then utilising the increased performance of a gradient insert, Wave-CAIPI might be attractive in even the shortest of readouts such as EPI.

\section{References:}

[1] B.Bilgic, 2015.

[2] E.Versteeg, 2021.

[3] A.Madrigal, 2020.

[4] S.F.Cauley, 2017.

\section{S3.P7.}

\section{Implicit ZTE dead-time gap filling with iterative reconstruction}

*T. Wood ${ }^{1}$, Y. Brackenier ${ }^{2}$

${ }^{1}$ King's College London, Department of Neuroimaging, London, GB; ${ }^{2}$ King's College London, Department of Biomedical Engineering, London, $G B$

Introduction: The short, but non-zero, time taken to switch between transmit and receive leads to the dead-time gap in Zero Echo-Time (ZTE) imaging. This gap causes reconstruction artefacts and so methods have been developed for filling the gap ${ }^{1}$. We previously showed that 1D GRAPPA can fill small dead-time gaps ${ }^{2}$. We show here that gap filling is implicit during iterative reconstruction, and that sensitivity maps can be extracted from either a separate lowresolution scan or from the ZTE data using ESPIRiT ${ }^{3}$. This may be beneficial in dynamic acquisitions where acquiring extra data to fill the gap with the correct contrast may be problematic.

\section{Methods}

We simulated a 3D Shepp-Logan phantom, matrix $64 \times 64 \times 64$, radial oversampling 2 , sampled first with a complete trajectory (no dead-time gap), and then with a gap of three samples. We reconstructed the complete dataset using sensitivities directly extracted from the data ${ }^{4}$, followed by 8 iterations of $\operatorname{cgSENSE}^{5}$. Dead-time gap data was reconstructed using sensitivities extracted using ESPIRiT with a kernel size of $7 \times 7 \times 7$, calibration region $23 \times 23 \times 23$. The dead-time gap was excluded from the calibration region.

Volunteer data was acquired on a 3 Tesla scanner (GE MR750) equipped with a 32-channel head coil (Nova Medical) with a ZTE sequence, $3 \mathrm{~mm}$ voxel size, matrix $72 \times 72 \times 42, \mathrm{RBW} \pm 32.5$ $\mathrm{kHz}, 2 \times$ read oversampling, angular undersampling 6 , flip-angle $1^{\circ}$, dead-time gap four samples, and included a low-resolution k-space. Data was compressed from 32 to 8 channels ${ }^{6}$. Sensitivity maps were extracted four ways: merging low- and high-res k-spaces + direct extraction, direct extraction from low-res data, direct extraction from high-res data, and by ESPIRiT from the high-res data. The latter three were then compared to the first as the ground truth.

Results: Figure 1 shows the phantom reconstructed with the different methods. The iterative reconstruction shows no dead-time gap artefacts.

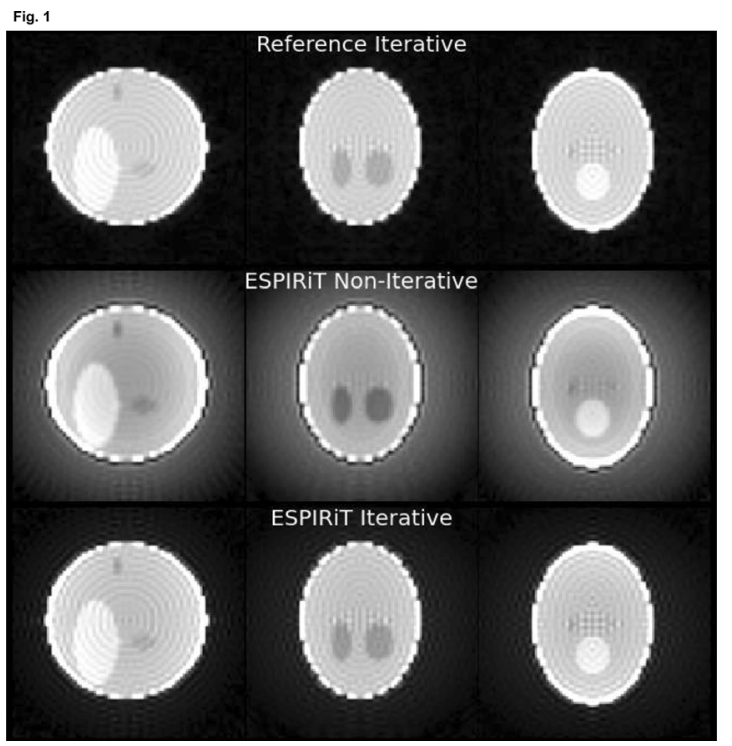


Figure 2 shows the sensitivity maps for the volunteer data. The merged and low-res only maps are similar, ESPIRiT shows different channel phase (as expected), while the high-res only maps are clearly corrupted. Fig. 3 shows the ESPIRiT and low-res maps lead to correct reconstructions, while the high-res maps result in artefacts.
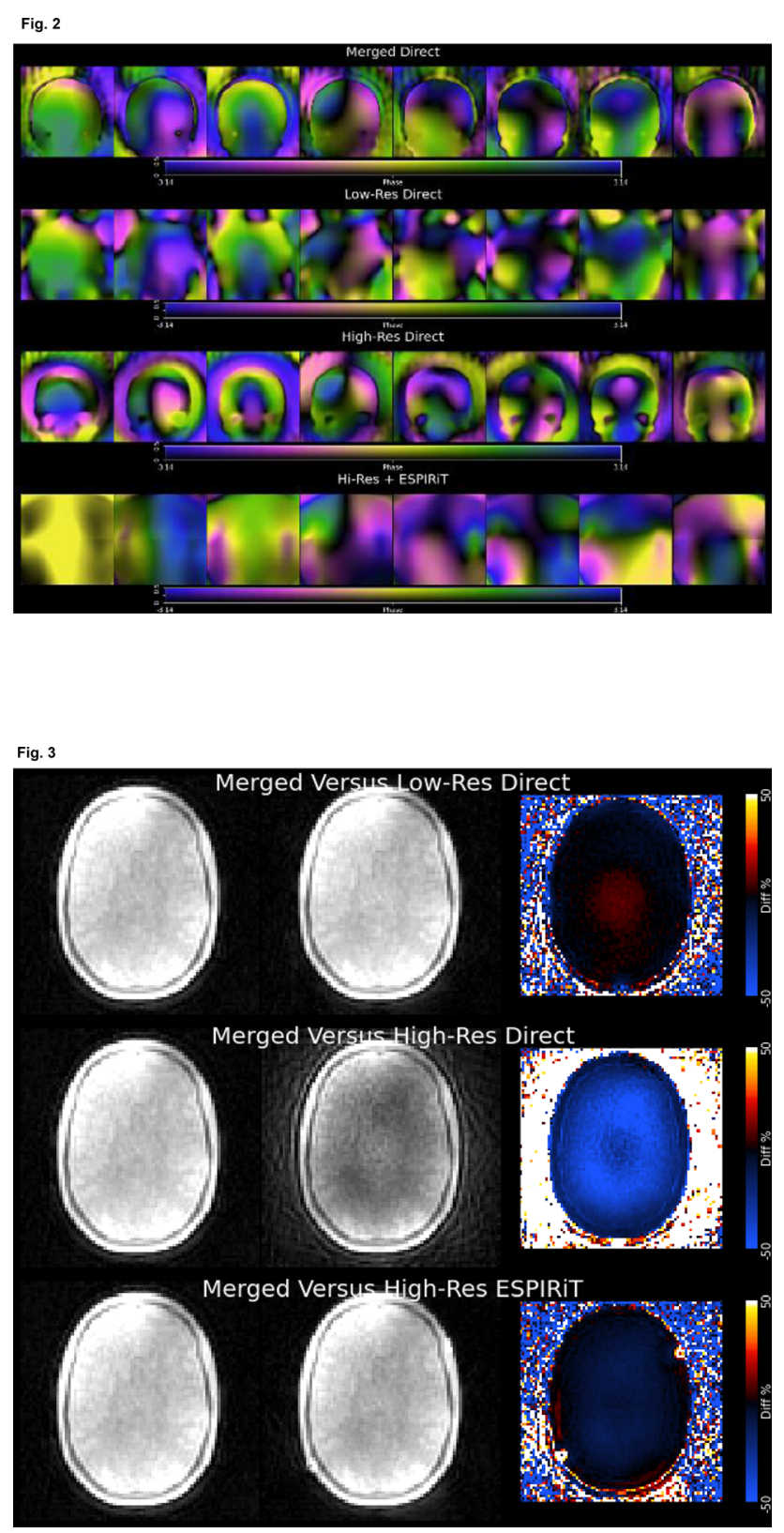

Conclusion: Small dead-time gaps can be implicitly filled during an iterative recon, similar to missing lines of $\mathrm{k}$-space in traditional parallel imaging. High fidelity sensitivity maps are required, but these can be taken either from a separate low-resolution scan or extracted from the data itself using ESPIRiT. The exact size of the gap that can be filled will depend on the coil geometry.

\section{References:}

1 Froidevaux R, NMR in Biomed 2021 DOI:10.1002/nbm.4493

2 Wood TC, ESMRMB 2020 DOI:10.1007/s10334-020-00874-0

3 Uecker M, MRM 2014 DOI:10.1002/mrm.24751

4 Sodickson D, MRM 2002 DOI:10.1002/mrm.10087
5 Pruessmann KP, MRM 2001 DOI:10.1002/mrm.1241

6 Huang F, MRI 2008 DOI:10.1016/j.mri.2007.04.010

\section{S3.P8.}

Quality-aware cine cardiac MRI acquisition and reconstruction from undersampled $k$-space data

*I. Machado ${ }^{1}$, E. Puyol-Antón ${ }^{1}$, K. Hammernik ${ }^{2,3}$, G. Cruz $^{1}$, I. Oksuz ${ }^{4}$, D. Ugurlu ${ }^{1}$, B. Ruijsink ${ }^{1,5}$, A. Young ${ }^{1}$, C. Pietro ${ }^{1}$, J. A. Schnabel ${ }^{1,2,6}$, A. P. King ${ }^{1}$

${ }^{1}$ King's College London, London, GB;

${ }^{2}$ Technical University of Munich, Munich, GB;

${ }^{3}$ Imperial College London, London, DE;

${ }^{4}$ Istanbul Technical University, Istanbul, TR;

${ }^{5}$ Guy's and St. Thomas' NHS Foundation Trust, London, GB;

${ }^{6}$ Helmholtz Center Munich, Munich, GB

Cine cardiac MRI is an important diagnostic tool in cardiovascular medicine. However, cardiac MRI is associated with an inherently slow acquisition process. Reducing the number of $\mathrm{k}$-space profiles and recovering the image via undersampled reconstruction is a common approach to speed up the scan. Several deep learning (DL) undersampled reconstruction methods have been proposed to accelerate image reconstruction. However, the undersampling factor is commonly fixed to conservative values before acquisition to ensure diagnostic image quality, potentially leading to unnecessarily long scan times. Here we aim to address this limitation by assessing image quality from undersampled k-space during acquisition, creating an "active" acquisition process in which only enough $\mathrm{k}$-space data are acquired to enable the reconstruction of an image that can pass automated quality control (QC) checks.

The image analysis pipeline consists of a DL reconstruction algorithm and a DL image QC step to detect poor quality reconstructions. We simulated an active acquisition process by first creating $\mathrm{k}$-space data from cine short-axis cardiac MR images from 270 UK Biobank subjects. We utilised a similar strategy to [1] to generate synthetic phase and use a radial golden angle sampling pattern to simulate undersampled k-t-space data containing increasing numbers of profiles. These were then reconstructed using a deep cascade of convolutional neural networks (DCCNN) [2] (F.1) and subsequently automatically checked for quality. The QC was framed as a binary classification problem and addressed using a ResNet classification network. Binary image quality labels from 225 images of different levels of undersampling were generated by visual inspection and validated by an expert cardiologist. The ResNet network was trained for 200 epochs with a binary cross entropy loss function. The simulated acquisition terminated when the reconstructed images passed the QC check.

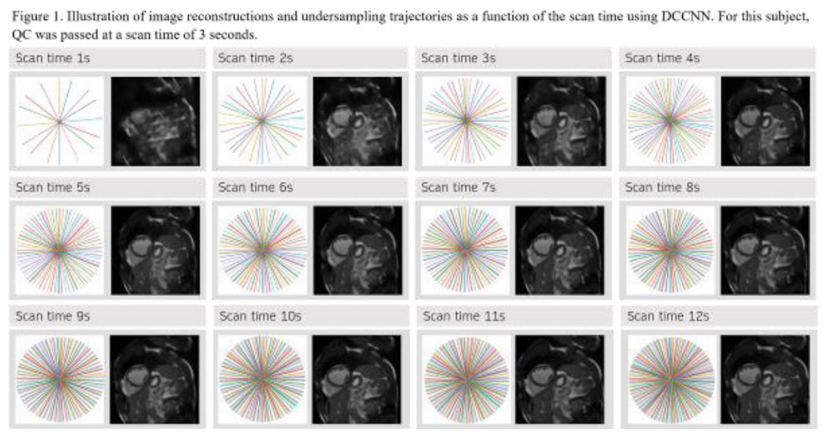


Our results show that by using DCCNN for cine cardiac MRI reconstruction, we can pass image QC checks after approximately $4 \mathrm{~s}$ of simulated acquisition (i.e. undersampling factor of 4.5). This would result in a reduced scan time for $2 \mathrm{D}$ cardiac cine MRI, which takes $\sim 12 \mathrm{~s}$ in our clinical protocol (spatial resol. $=1.8 \times 1.8 \times$ $8.0 \mathrm{~mm}^{3}$, temporal resol. $=31.56 \mathrm{~ms}$, undersampling factor $=2$ ). For the QC step, the average balanced accuracy, sensitivity and specificity were $0.93,0.86$ and 0.99 respectively. Image quality results are shown in T.1.

Fig. 2

Table 1. Image quality was evaluated with the Mean Absolute Error (MAE), Peak Signal to Noise Ratio (PSNR) and Structural image reconstrueted with the minimum amount of $k$-space data to pass the QC echecks. Results were comparable with those reported in $[3]$.

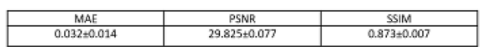

This work demonstrates the feasibility of a DL-based framework for automated quality-controlled "active" acquisition of undersampled cine cardiac MRI without previously defined undersampling factor. Future investigations will consist of linking image acquisition and reconstruction to further downstream tasks such as segmentation, volume curve analysis and estimation of cardiac functional parameters.

\section{References:}

[1] Haldar, J. P. (2013).

[2] Hammernik, K. et al., (2018).

[3] Oksuz, I. et. al (2020).

This work was funded by EPSRC (EP/P001009/1) and HDR UK Health Data Research UK

\section{S3.P9.}

\section{Effect of wave-CAIPI parameters and reconstraction frameworks: LORAKS and SENSE}

*A. Monreal Madrigal ${ }^{1}$, F. Mauconduit ${ }^{2}$, E. Versteeg $^{3}$, A. Vignaud $^{2}$, N. Boulant ${ }^{2}$, B. A. Poser ${ }^{1}$

${ }^{1}$ Maastricht University, Maastricht, NL;

${ }^{2}$ NeuroSpin Centre CEA Saclay, Gif-sur-Yvette, FR;

${ }^{3}$ University Medical Center Utrecht, Utrecht, $N L$

Introduction: LORAKS (low rank modeling of local k-space) a calibration-less constrained low-rank modeling framework estimates the linear dependence structure from the under-sampled acquisition and allows for more flexible sampling schemes [1], it imposes support and phase constraints and is expected to provide more accurate reconstruction than classical parallel imaging approaches. This flexibility of LORAKS makes it applicable to Wave-CAIPI [2], and it has been shown in [3] that LORAKS outperforms SENSE [4] in the reconstruction of this type of acquisitions.

For a more general scope, we simulate different Wave-CAIPI parameters to further investigate their impact on image quality, here we present the preliminary results.

Methods: A fully sampled GRE of a healthy volunteer was acquired on a 7 T SIEMENS scanner, using the following parameters: matrix size $=240 \times 240 \times 60$, voxel size $=1 \times 1 \times 2 \mathrm{~mm}^{3}, \mathrm{BW} / \mathrm{px}=80$ $\mathrm{Hz}, \mathrm{TE} / \mathrm{TR}=11 / 29 \mathrm{~ms}$. Coil compression from 32 to 16 channels and Coil Sensitivity maps were obtaining using ESPIRiT [5,6]. Wave acquisitions were simulated from a fully sampled Cartesian data using the wave equation [2] (Fig. 1a) and different wave parameters (Fig. 3). The k-space data was then under-sampled with a factor of 16 $\left(\mathrm{R}_{\mathrm{y}}=4, \mathrm{R}_{\mathrm{z}}=4\right)$ using a CAIPIRINHA mask [7] (Fig. 1b) and reconstructed using the direct SENSE reconstruction. The same data was also under-sampled using three different masking strategies (Fig. 1b) and reconstructed using a modified version of the opensource LORAKS implementation [1,8] to allow for 3D reconstructions and incorporating the Point Spread Function formulation. The rank 1,000 and regularization $\lambda=1$ were selected as suggested in [3], neighborhood radius was $r=3$. To assess the quality of the reconstructions the NRMSE and High Frequency Error Norm were computed.

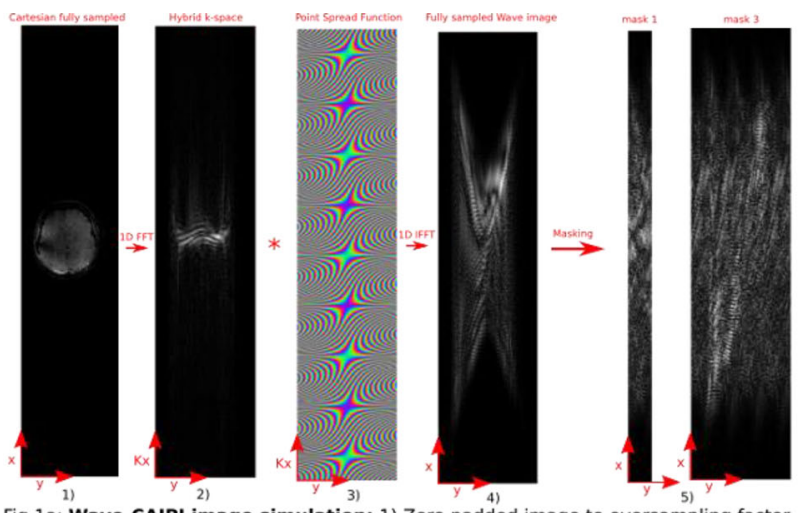

Fig 1a: Wave-CAIPI image simulation: 1) Zero padded image to oversampling factor 2) FFT in readout direction, 3) Multiplication with PSF in the hybird k-space, 4) IFFT to arrive to fully sampled wave image 5) 3D FFT, masking and 3D IFFT to get under-sampled wave-image to be reconstructed
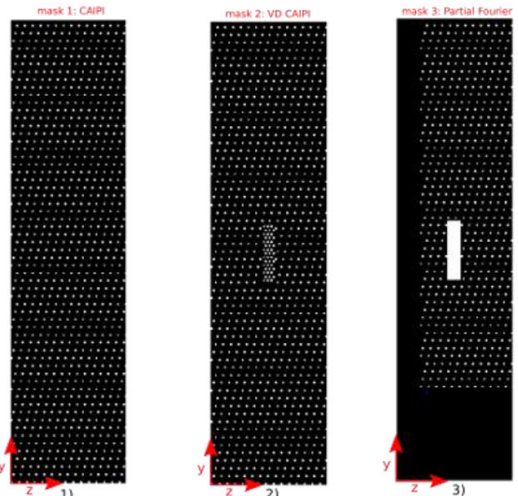

Fig1b: Masks used to under-sample data: 1) CAIPI (SENSE and LORAKS), 2) Variability Density CAIPI (LORAKS), 3) Partial Fourier (LORAKS)

Results:The image quality improves with higher wave gradient amplitudes and number of cycles (Fig. 2), as expected from the larger spreading effect and as previously reported in [9]. Nevertheless, we did not observe a marked improvement in image quality when reconstructing with LORAKS compared to SENSE approaches while the reconstruction time of LORAKS is $>10$ times larger (Fig. 3). 


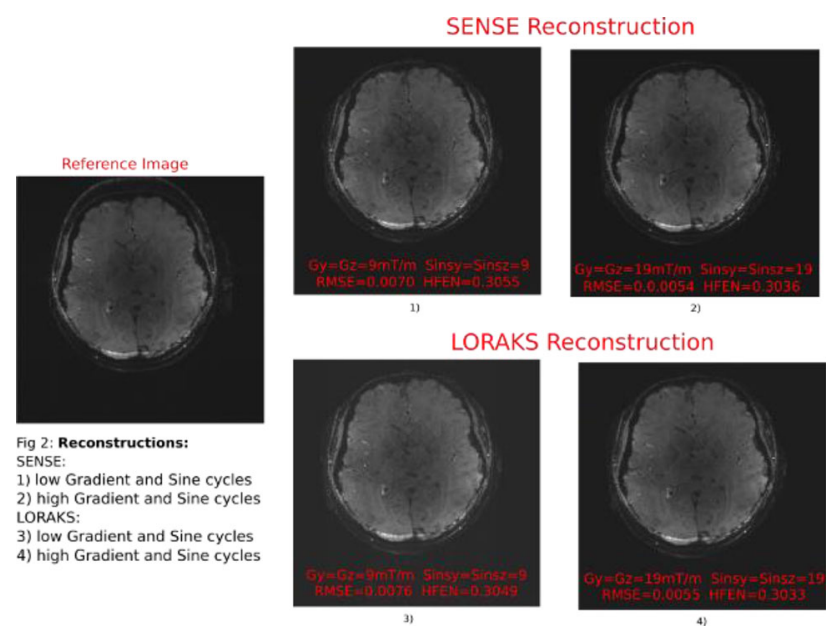

\begin{tabular}{|c|c|c|c|c|c|c|c|c|c|c|}
\hline \multirow[b]{2}{*}{ Gy } & \multirow[b]{2}{*}{ Gz } & \multirow[b]{2}{*}{$\sin _{r}$} & \multirow[b]{2}{*}{$\sin _{z}$} & \multirow[b]{2}{*}{$\begin{array}{c}\text { Max Slew Rate } \\
(\mathrm{mT} / \mathrm{m} / \mathrm{ms})\end{array}$} & \multicolumn{3}{|c|}{ SENSE } & \multicolumn{3}{|c|}{ LORAKS Mask 2} \\
\hline & & & & & NRMSE & HFEN & Recon time (s) & NRMSE & HFEN & Recon time (s) \\
\hline 9 & 9 & 9 & 9 & 43 & 0.0070 & 0.3055 & 109 & 0.0076 & 0.3049 & 5248 \\
\hline 14 & 14 & 14 & 14 & 70 & 0.0056 & 0.3020 & 92 & 0.0057 & 0.3016 & 4385 \\
\hline 19 & 19 & 19 & 19 & 96 & 0.0054 & 0.3036 & 95 & 0.0055 & 0.3033 & 5346 \\
\hline
\end{tabular}

Discussion: In this work the Wave-CAIPI and LORAKS parameters were selected arbitrarily. Further work needs to validate the results shown here with more combinations of LORAKS parameters selected in a more informed manner. Since we used simulated data, a comparison with prospectively under-sampled data for the most promising parameter sets will be performed.

\section{References:}

[1] Haldar, JP, IEEE T-MI 33(3):668-681, 2013.

[2] Bilgic B, et al.MRM 73(6):2152-2162, 2015.

[3] Kim TH, et al. MRM 81(3):1620-1633, 2019.

[4] Pruessmann, KP, et al. MRM 42(5):952-962,1999.

[5] Bahri D, et al. Proceedings of the 21st Annual Meeting of ISMRM, 2013.

[6] Uecker, M, et al. MRM 71(3):990-1001, 2014.

[7] Breuer, FA et al. MRM 53(3):684-691, 2005.

[8] Kim, TH, et al. USC, Tech. Rep. USC-SIPI-443, 2018.

[9] Polak, D, et al. JMRI 50(3):961-974, 2019.

\section{S3.P10.}

\section{Efficient 2D cardiac MR cine reconstruction through a self-supervised motion compensated (SSMoComp) architecture}

\section{E. Martín-González ${ }^{1}$, *J. R. Rodríguez-Galván ${ }^{1}$, C. Alberola-López ${ }^{1}$ \\ ${ }^{1}$ University of Valladolid, Department of Imaging, Valladolid, $S U$}

Introduction: Reducing acquisition time in MRI is a common goal within the MR community. To achieve this, a usual approach is to undersample the k-space and reconstruct the images with the support of involved methods. Both numerical optimization methods and, more recently, Deep Learning (DL) based methods have been reported. Specifically, MoDL [1] is a framework that satisfactorily combines both approaches. However, fully-sampled data are needed for training due to its supervised learning character and the best solution reported (a 10-stage network unroll with weight sharing), it is high computationally expensive.

Materials and methods: The aim of this abstract is to design and test a self-supervised DL architecture for the implementation of the solution reported in [2]. The function there optimised included a data fidelity term and a regularization term with a motion compensation operator $T(\cdot)$. These terms are weighted by a parameter $\lambda$. T(.) is a groupwise elastic registration, implemented by means of a pre-trained network [3]. The architecture is fed with 30 -frame $320 \times 320$ pixel multislice $2 \mathrm{D}$ cine images. which are resampled to $1 \mathrm{~mm}^{2}$ and cropped to $160 \times 160$ pixels. The database is composed by $9-14$ slices from 88 patients, divided into 4 subsets, namely, $60 \%$ for training, $10 \%$ for early stopping, $10 \%$ for validation (i.e. selection of $\lambda$ ) and $10 \%$ for testing. Provision was made so that slices from the same patient belong only to one subset.

An Acceleration Factor (AF) of 10 has been used and we have let $\lambda$ range within the interval $(0.1,0.8)$. Operator $T(\cdot)$ consists in three executions of the network in network [3]. Training has a maximum of 300 epochs and early stopping has a patience parameter of 40 epochs Validation selected $\lambda=0.3$.

Results and discussion: Table 1 presents the results on the test set for SSMoComp vs. MoDL with 5 layers and 10 iterations, trained for 50 epochs. Figure 1 shows a reconstruction example.

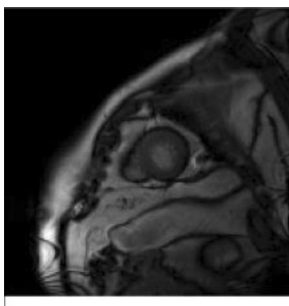

(a)

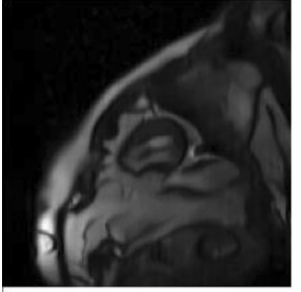

(c)

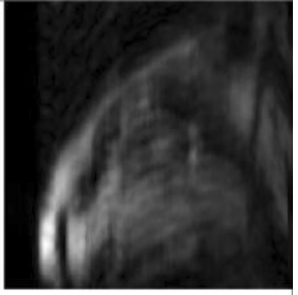

(b)

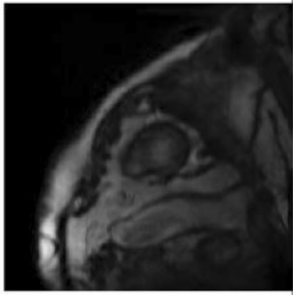

(d)
Figure 1: Comparison between original (a) subssampled (b) reconstrusted using MoDL (c) and reconstructed using SSMoComp (d).

Fig. 2

\begin{tabular}{cccc}
\hline Model & PSNR $(\mathrm{dB})$ & SSIM & Training time $(\min )$ \\
\hline SSMoComp & 25,91 & 0,83 & 70 \\
MoDL & 25,75 & 0,82 & 601 \\
\hline
\end{tabular}

Table 1: Comparison of results between SSMoComp and MoDL.

Results show that SSMoComp is more efficient $(\times 86)$ than MoDL, with comparable quality results. MoDL has less trainable parameters and no free parameters, but very large GPU memory requirements. 


\section{References}

[1] Aggarwal, H. K., Mani, M. P., \& Jacob, M. (2018). MoDL: Model-based deep learning architecture for inverse problems. IEEE transactions on medical imaging, 38(2), 394-405.

[2] Martín-González, E., Moya-Sáez, E., Menchón-Lara, R. M., Royuela-del-Val, J., Palencia-de-Lara, C., Rodríguez-Cayetano, M., ... \& Alberola-López, C. (2021). A Clinically Viable Vendor-Independent and Device-Agnostic Solution for Accelerated Cardiac MRI Reconstruction. Computer Methods and Programs in Biomedicine, 106,143 .

[3] Martín-González, E. et al. (2020). Groupwise Non-Rigid Registration with Deep Learning: An Affordable Solution Applied to 2D Cardiac Cine MRI Reconstruction. Entropy, 22(6), 687.

\section{S3.P11.}

\section{Improving spatial resolution of myocardial T1-mapping} using a model based super-resolution reconstruction

\author{
*F. Cachado ${ }^{1}$, A. Gaspar ${ }^{1}$, R. G. Nunes ${ }^{1}$ \\ ${ }^{1}$ University of Lisbon, Institute for Systems and Robotics / Department \\ of Bioengineering, Lisbon, PT
}

Introduction: Over the last years, T1 mapping has become an important tool for myocardial tissue characterization, including detection of fibrosis1. For a detailed tissue evaluation, a high in-plain resolution and sufficient signal-to-noise ratio (SNR) are required, thus, thick slices are often used, sacrificing the through-plane resolution. To increase it, we propose to incorporate a T1 signal recovery exponential model into a super-resolution (SR) reconstruction.

Methods: SR was implemented by considering Low Resolution (LR) acquisitions with a relative offset (half of the slice thickness) for each inversion time TI. Ground truth (GT) T1 weighted (T1w) images were simulated by applying the MOLLI2 exponential model to the MRXCAT3 phantom (matrix size $=180 \times 140$, and slice thickness $=1 \mathrm{~mm}$ ). To build LR images (y) with a slice thickness of $2 \mathrm{~mm}$, a convolution matrix A (accounting for the slice profile) was applied to the phantom. Random Gaussian noise was added (Signalto-noise ratio of 40).

To reconstruct High Resolution (HR) images (x), we considered both data and model consistency terms (Eq. 1). The exponential signal recovery model in Eq. (2) featuring the parameters: M0, equilibrium magnetization, $\mathrm{k}$, a constant to account for inversion efficiency and $\mathrm{T} 1$, relaxation time was used:

$\min \{\mathrm{x}, \mathrm{u}\} \operatorname{norm}(\mathrm{Ax}-\mathrm{y}, 2)^{2}+\operatorname{lambda} * \operatorname{norm}(\mathrm{x}-\mathrm{u}, 2)$

where

$\mathrm{u}=\mathrm{M} 0\left(1-\mathrm{k} * \mathrm{e}^{(-\mathrm{TI} / \mathrm{T} 1)}\right)$

Using the ADMM4 approach to minimize Eq. (1), we first fixed the model parameters and predicted the HR images $x$, using the closed form solution derived from the generalized Tikhonov regularization applied to the data term, with a regularization weight of 1.0. The next step consists of applying soft-thresholding, using lambda $=\operatorname{norm}(<\mathrm{AT}, \mathrm{y}\rangle, \infty)$, to the model term to minimize the difference between the $x$ and $u$ images, where $u$ is the T1w image predicted from Eq. (2) after fitting the relaxation model to the previous $\mathrm{x}$ estimate.

To evaluate the quality of the reconstruction, the mean absolute error (MAE) was calculated for the input LR and reconstructed HR images compared to the GT.

Results: The example T1w reconstructed images (longest inversion time), as seen in Fig. 1, show a MAE of $0.86 \%$ whereas for the LR images the MAE is $3.95 \%$. The estimated HR T1 maps were correctly estimated compared to the GT map, as can be seen in Fig. 2, showing a global MAE of $2.34 \%$.
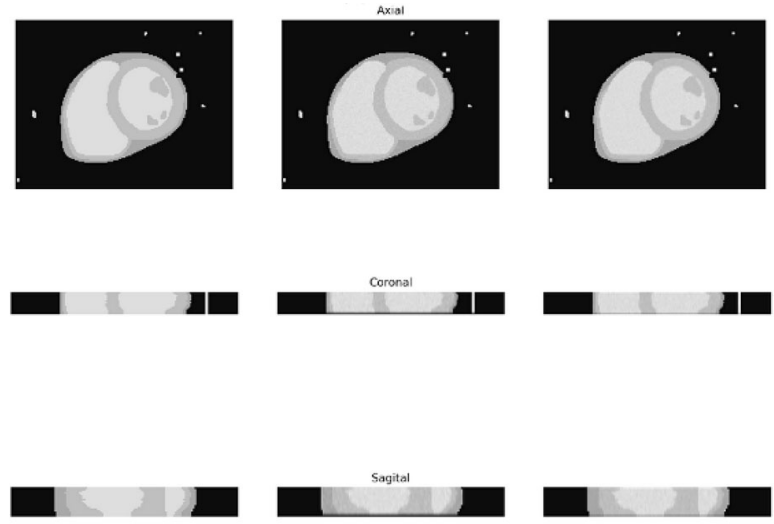

Figure 1 - Example Tl weighted (Tlw) image showing three orthogonal orientations of the MRXCAT phantom. The first column represents the Ground Truth images, the second column the Low Resolution images, and the third the High Resolution images reconstructed using the proposed method.
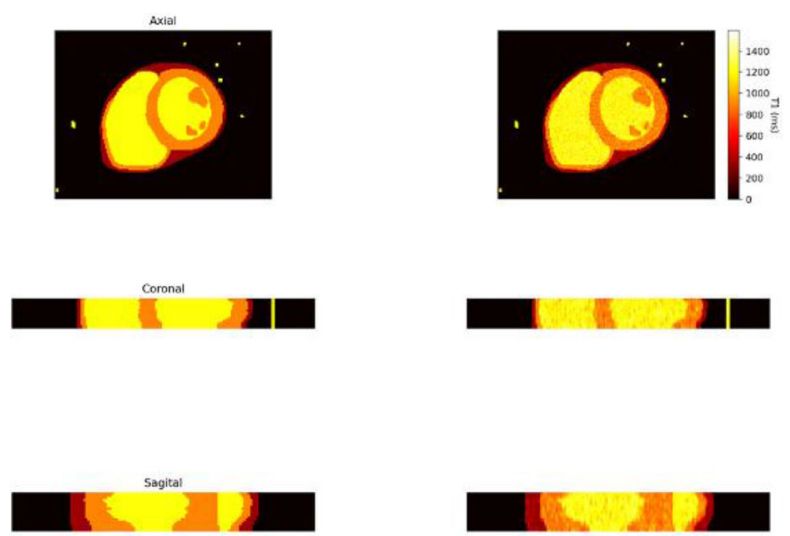

Figure 2 - T1 maps showing three orthogonal orientations of the MRXCAT phantom. The first column represents the Ground Truth T1 maps and the second column the High Resolution T1 maps reconstructed using the proposed method.

Discussion: The results obtained from the proposed model-based SR method show that accurate HR T1 maps can be reconstructed. Future work will focus on phantom and in vivo validation.

\section{References:}

1. C. R. Hamilton-Craig et al., Front. Cardiovasc. Med. 2017, 3.

2 D. R. Messroghli et al., JMRI, 2007;26:1081.

3. L. Wissmann et al. JCMR.2014;16:63.

4. S. Boyd, Foundations and Trends ${ }^{\circledR}$ in Machine Learning 2010, 3, 1.

Acknowledgements: FCT (SFRH/BD/120006/2016, PTDC/EMDEMD/29686/2017); Lisboa 2020 (LISBOA-01-0145-FEDER029686). 


\section{S3.P12.}

Accurate $T_{1}$ mapping of the myocardium with a goldenangle radial MOLLI and a model-based regularized reconstruction SALSA

*A. C. Freitas ${ }^{1}$, A. Gaspar ${ }^{1}$, F. Cachado ${ }^{1}$, N. A. Silva ${ }^{2}$, J. M. Dias ${ }^{3}$, R. G. Nunes ${ }^{1}$

${ }^{1}$ University of Lisbon, Lisbon, PT;

${ }^{2}$ Hospital da Luz, Department of Learning Health, Lisbon, PT;

${ }^{3}$ University of Lisbon, Lisbon, PT

Introduction: Quantitative MRI provides valuable information regarding cardiovascular pathologies as myocardial $\mathrm{T}_{1}$ changes may reflect infarction, diffuse fibrosis or inflammation [1]. The clinical approach consists of acquiring multiple $T_{1}$-weighted images with a MOLLI (MOdified Look-Locker) sequence and fitting a 3-parameter model: $\mathrm{S}(\mathrm{TI})=\mathrm{A}-\mathrm{B} \cdot \exp \left(-\mathrm{TI} / \mathrm{T}_{1} *\right)$; where $\mathrm{T}_{1} \sim \mathrm{T}_{1} *(\mathrm{~B} / \mathrm{A}-1)$ [1]. However, many other factors affect the magnetization state (e.g. effective flip angle and heart rate variability) which lead to $T_{1}$ bias. Another issue of conventional cartesian MOLLI is the lengthy breathhold required. To address this, we propose coupling an accelerated golden-angle (GA) radial MOLLI with a model-based regularized reconstruction SALSA.

Methods: MOLLI acquisition was simulated using Extended Phase Graphs (EPG) [2]: 5(3)3 scheme with a GA radial trajectory and bSSFP readout $\left(\mathrm{FA}=35^{\circ}, 2\right.$ inversions with $\mathrm{TI}=231$ and $331 \mathrm{~ms}$, $\mathrm{TR}=517 \mathrm{~ms}$, heart rate $=70 \mathrm{bpm}$ ). MRXCAT[3] heart phantom was considered with 3 tissue regions: healthy myocardium, fibrotic lesion and blood pool $\left(\mathrm{T}_{1}=1000 / 1300 / 1400 \mathrm{~ms}\right.$ and $\mathrm{T}_{2}=50 / 70 / 250 \mathrm{~ms}$ at 1.5 T-Fig. 1). Gaussian noise was added $(\mathrm{SNR}=40)$. EPG dictionary computed with $\mathrm{T}_{1}=[150: 1: 2300] \mathrm{ms}$ and $\mathrm{T}_{2}=[25: 25: 350] \mathrm{ms}$. Accelerated GA radial data $(\mathrm{R}=4)$ was reconstructed with NUFFT[4] and SALSA[5]. Considering that the noisy $\mathrm{k}$-space (y) of an image ( $\mathrm{x}$ ) can be described as $\mathrm{y}=\mathrm{Bx}+\mathrm{n}$ (B is the direct operator and $\mathrm{n}$ the added noise); SALSA solves the constrained optimization problem $\min 1 / 2\|\mathrm{Ax}-\mathrm{y}\|_{2}+\phi(\mathrm{v}), \mathrm{A}=\mathrm{BW}$ and $\mathrm{W}$ contains the wavelet elements, using an alternating direction method of multipliers (ADMM). The first term describes the $\mathrm{L}_{2}$ data-fidelity term and $\phi(\mathrm{v})$ (if $\mathrm{x}=\mathrm{v}$ due to variable splitting) is the wavelet-based regularizer term. $\mathrm{T}_{1}$ maps were obtained by 3-param fit to the accelerated MOLLI images, and by matching the NUFFT and SALSA reconstructed MOLLI images to the dictionary. Different SALSA weights $(\lambda)$ were investigated (Fig. 2 ).

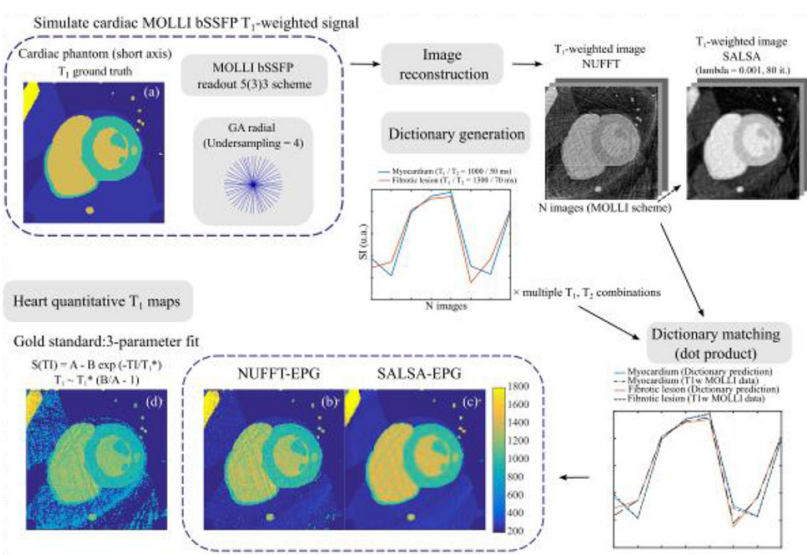

Figure 1 - Schematic representation of the $T_{1}$ mapping simulation environment. (a) Numerical MRXCAT phanton representing the heart on a short axis view with 3 tissue regions and corresponding $T_{1}$ values: healthy myocardium, fibrotic Iesion and blood pool. A MOLLT bSSFP acquisition scheme with a GA radial sampling was used (number of radial spoke corresponding to an undersampling of 4). After this, noise was added considering an SNR of 40. Images were the reconstructed with NUFFT and SALSA with adequate density compensation. An EPG-based dictionary was pre-computed

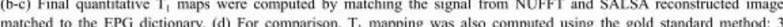
parameter fil.
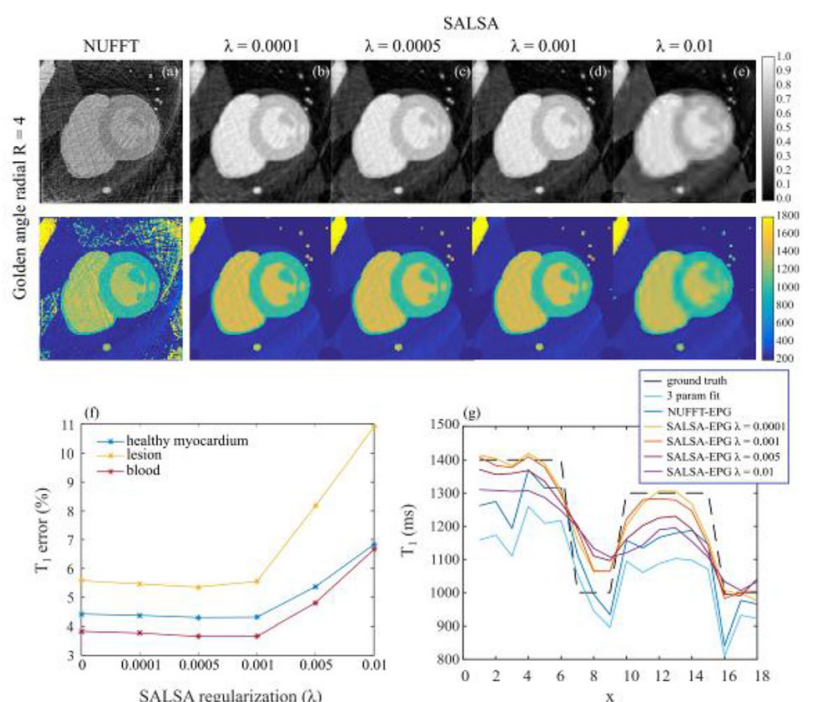

Figure 2 - Optimization of SALSA regularization weights $(\lambda)$. $T_{1}$-weighted images acquired with a $G A$ radial $(R=4)$ and reconstructed with a basic NUFFT (a) and SALSA (b-e). Effect of the SALSA regularization weight being varied from 0.001 to 0.01 is shown in $T_{1}$-weighted images and resulting quantitative $T_{1}$ maps. Use of even a reduce of an optimal SALSA weight $Q=0001$ also weatly inproves ryocadial wall sharpess comped to EPG or the 3-param fit T, maps (Fig 2 g - oramge vs dark and light blue lines). However, an excessive inerene of

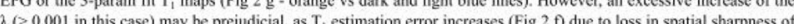
the myocardial wall and fibrotic lesion boundaries (Fig 2 a - purple line).

Results: A reduction of aliasing artifacts present in the MOLLI SALSA reconstructed images is noticeable until an optimal minimum $\mathrm{T}_{1}$ error point at $\lambda=0.001$. Spatial sharpness of the myocardial wall and fibrotic lesion boundaries was also improved at $\lambda=0.001 \mathrm{com}$ pared to 3-param fit and NUFFT $\mathrm{T}_{1}$ maps (Fig. 2 g). However, increasing $\lambda$ past this will lead to excessive smoothing of the $T_{1}$ map and loss of sharpness. Proposed SALSA-EPG resulted in improved $\mathrm{T}_{1}$ accuracy and precision (Fig. 3).
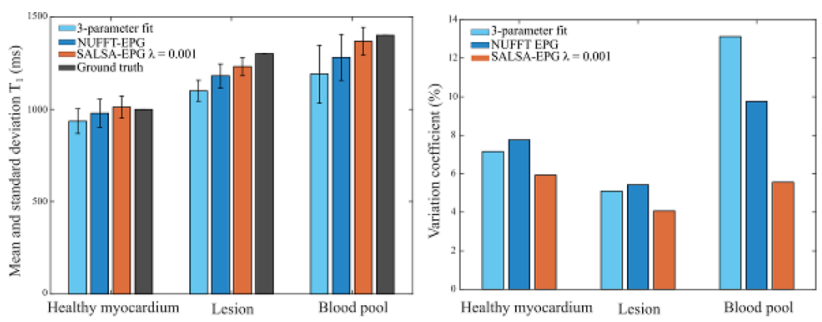

Figure 3 - Comparison of $\mathrm{T}_{1}$ mapping methods, (a) Mean and standard deviation $\mathrm{T}_{1}$ (ms) for 3 tissue regions obtained with 3 methods: 3-parameter fit, matching NUFFT reconstructed images to the dictionary and SALSA reconstructed images matching to the dictionary (with optimal $\lambda=0.001$ ). Grey bar plots represent ground truth value. (b) Bar plots representing $\mathrm{T}_{1}$ precision given as the variation coefficient: standard deviation within the region of interest divided by the mean. Proposed SALSA-EPG method ensures improved $T_{1}$ accuracy (mean values closer to ground truth) and precision (lower variation

Discussion: SALSA-EPG appears to be a promising method to obtain robust and fast $T_{1}$ mapping of the heart. Future work includes adding coil sensitivity information to increase acceleration and validating simulation data with phantom and in vivo acquisitions.

\section{References:}

[1] Taylor, JACC: Card Img (2016) 9:67-81.

[2] Weigel, JMRI (2015) 41:266-295.

[3] Wissmann, JCMR (2014) 16:63.

[4] Fessler, JMR (2007) 188:191-195.

[5] Afonso, IEEE Trans Img Process (2010)19:2345-56.

Acknowledgements: $\quad$ FCT(SFRH/BD/120006/2016,PTDC/EMDEMD/29686/2017); LISBOA-01-0145-FEDER-029686 


\section{S3.P13.}

\section{Under-sampled diffusion-weighted MR image reconstruction through convolution neural network}

*F. Aamir ${ }^{1}$, I. Aslam ${ }^{1}$, H. Omer ${ }^{1}$

${ }^{1}$ COMSATS University Islamabad, Electrical and Computer Engineering, Islamabad, PK

Introduction: Undersampling speed up DWI that helps to reduce the off-resonance, chemical shift, susceptibility and geometric artifacts ${ }^{1}$; however, undersampling leads to aliasing artifacts ${ }^{2}$. This paper proposes a new method 2D Dense U-Net for the reconstruction of artifact free DW image from the acquired 1D variable density undersampled $k$-space data. The proposed 2D Dense U-Net is tested on ten patients" entire brain volume data. The results show better image quality, both visually and in terms of evaluation criteria than contemporary Compressed Sensing (CS) reconstruction ${ }^{2}$.

Method: Figure-1 illustrates a schematic diagram of the proposed method. 2D Dense U-Net has 10 convolution layers with a learning rate of $3 \times 10^{-5}$; weight decay factor of 0.1 ; ReLU as an activation function and RMSProp optimizer. These parameters are chosen after an extensive experimentation.

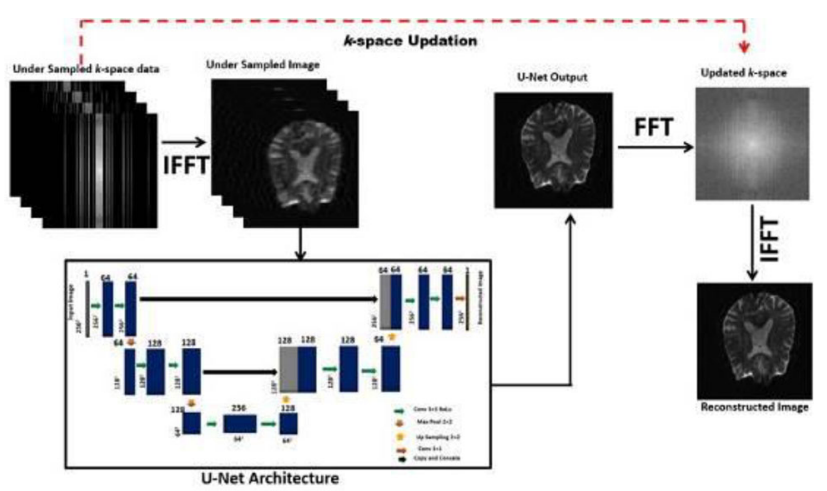

Figure-1: A schematic diagram of the proposed technique (2D Dense U-Net)

Firstly, the variable density undersampled (input) and fullysampled (labels) images are used to train the 2D Dense U-Net. The 2D Dense U-Net (after training) is expected to remove the artifacts from the undersampled image, however, it also updates the originally acquired $k$-space samples. To retain the initially acquired $k$-space data, an additional step of $k$-space updation ${ }^{3}$ is applied. Finally, IFFT of the updated $k$-space provides the solution image.

The proposed method is trained and tested on the Oasis-3 DWI datasets ${ }^{4}$ acquired from a $3 \mathrm{~T}$ Siemens scanner with the following parameters: Scanning sequence $=$ ss-EPI, TR $=14.5 \mathrm{~ms}, \mathrm{TE}=0.11$ $\mathrm{ms}$, and Flip angle $=90^{\circ} .2 \mathrm{D}$ Dense U-Net training is performed with a training set of 5433 images and a validation set of 605 images while the trained 2D Dense U-Net is tested on 20,160 image ${ }^{4}$. The proposed method was trained on Intel(R) Xeon(R) CPU,128 GB RAM, and GPU NVIDIA GeForce GTX 1080Ti with Python 3.8. The network requires approximately $18 \mathrm{~h}$ for training in our experiments. The reconstruction is performed on the whole brain volume with different $\mathrm{AF} 4$ and 6 and $(0 \leq \mathrm{b}$ values $\leq 800) \mathrm{s} / \mathrm{mm}^{2}$.

Results: Figure- 2 shows reconstruction results of our experiments at $\mathrm{AF}=6$ for a single patient. Comparison of different evaluation parameters for the CS and 2D Dense U-Net at $\mathrm{AF}=4$ and 6 is given in Table-1. The results demonstrate that the 2D Dense U-Net provides $57.0 \%$ lower mean AP, $52.0 \%$ lower mean RMSE, and $24.5 \%$ higher mean SSIM values than the contemporary CS for human brain DWI data in our experiments.

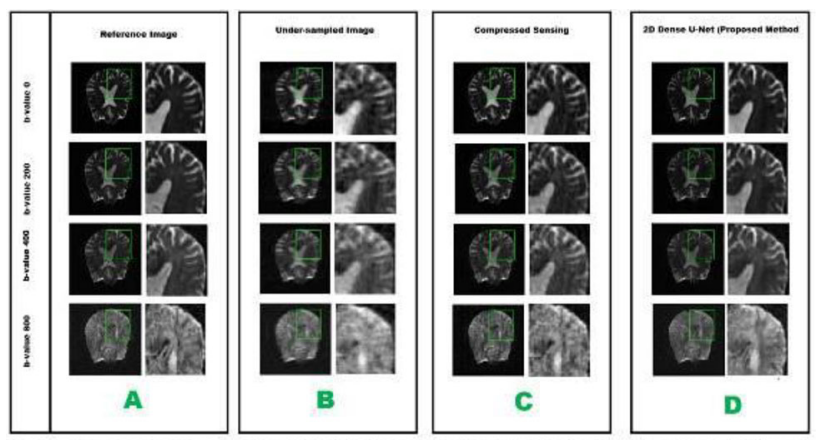

Figure-2: Reconstruction results of the $3 T$ human brain DWI data (central slice) at AF 6 having different b-values i.e. 0,200,400 and $800 \mathrm{~s} / \mathrm{mm}^{2}$ : reference image (Column A), under-sampled image (Column B), Compressed Sensing reconstruction (Column C), the proposed method reconstruction (Column D)

Fig. 3

Table-1: For 3T DWI human brain data (central slice), comparison of the reconstruction results of the proposed method (2D Dense U-Net) and conventional Compressed Sensing with reference to AP, RMSE, and SSIM values at acceleration factors 4 and 6

\begin{tabular}{|c|c|c|c|c|c|c|c|c|c|c|}
\hline \multirow[t]{2}{*}{$\begin{array}{c}\text { Acceleration } \\
\text { Factor }\end{array}$} & \multirow[t]{2}{*}{ b-values } & \multicolumn{3}{|c|}{ Compressed Sensing } & \multicolumn{3}{|c|}{$\begin{array}{l}\text { Proposed Method } \\
\text { (20 Dense U-Net) }\end{array}$} & \multicolumn{3}{|c|}{$\begin{array}{l}\text { \% improvement w.r.t to } \\
\text { Compressed Sensing) }\end{array}$} \\
\hline & & AP & RMSE & sSIM & AP & RMSE & SSIM & AP & RMSE & $\operatorname{ssim}$ \\
\hline 4 & $\begin{array}{l}0 \\
200 \\
400 \\
800\end{array}$ & $\begin{array}{l}0.0055 \\
0.0075 \\
0.0100 \\
0.0180 \\
0\end{array}$ & $\begin{array}{l}0.0103 \\
0.0119 \\
0.0136 \\
0.0196\end{array}$ & $\begin{array}{l}095646 \\
09332 \\
0.9303 \\
0.9998\end{array}$ & $\begin{array}{l}0.0025 \\
0.0032 \\
0.0040 \\
0.0065\end{array}$ & $\begin{array}{l}0.00060 \\
0.0075 \\
0.0008 \\
0.0090\end{array}$ & $\begin{array}{l}.97950 \\
0.9780 \\
0.9754 \\
0.9650\end{array}$ & $\begin{array}{l}3555 \\
573 \% \\
60.0 \% \\
63.8 \%\end{array}$ & $\begin{array}{l}11 \% \% \\
365 \% \\
33.2 \% \\
54.0 \%\end{array}$ & $\begin{array}{l}1.495 \\
4956 \\
4856 \\
7.246\end{array}$ \\
\hline 6 & $\begin{array}{l}200 \\
400 \\
500\end{array}$ & $\begin{array}{l}0.0330 \\
0.0351 \\
0.0380 \\
0.0425\end{array}$ & $\begin{array}{l}0.0325 \\
0.0390 \\
0.0410 \\
0.0480\end{array}$ & $\begin{array}{l}0.5015 \\
0.7986 \\
0.7425 \\
0.7158\end{array}$ & $\begin{array}{l}0.0120 \\
0.0145 \\
0.0150 \\
0.0165\end{array}$ & $\begin{array}{l}0.001 \% 0 \\
0.0198 \\
0.0192 \\
0.0220\end{array}$ & $\begin{array}{l}0.95860 \\
0.5540 \\
0.9450 \\
0.9420\end{array}$ & $\begin{array}{l}4.38 \% \\
58.46 \\
60.5 \% \\
61.1 \%\end{array}$ & $\begin{array}{l}47.6 \% \\
5517 \% \\
53.1 \% 6 \\
54.1 \%\end{array}$ & $\begin{array}{l}196 \% \\
19.46 \\
27.5 \% \\
31.5 \%\end{array}$ \\
\hline
\end{tabular}

Discussion/conclusion: A new CNN based method for DWI reconstruction is proposed and the results compared with CS reconstruction. The proposed method works well on all AFs and $b$ values in our experiments while CS does not perform well at higher $b$ values. This is because the high $b$ values contain more diffusion information and strong background signal suppression.

\section{References:}

1. D. Le Bihan et.al, doi: jmri.20683.

2. C. Zhang et.al, doi: j.neuroimage.2020.116584.

3. C. M. Hyun et.al, doi: 10.1088/1361-6560/aac71a.

4. https://central.xnat.org (accessed Aug. 27, 2020).

\section{S3.P14.}

Improved image quality for static BLADE MRI by using the $L 1$ total-variation regularized least absolute deviation solver

H. C. Chen ${ }^{1}$, H. C. Yang ${ }^{1}$, C. C. Chen ${ }^{2}$, W. H. Yu ${ }^{3}$, F. N. Hwang ${ }^{3}$, S. Harrevelt ${ }^{4}$, Y. C. Chao ${ }^{1}$, H. C. Chang ${ }^{5}$, C. K. Chang ${ }^{2,6}$, *J. M. $\operatorname{Lin}^{7,8}$

${ }^{I}$ Madou Sin-Lau Hospital, Tainan, TW;

${ }^{2}$ National Taiwan University, Taipei, TW;

${ }^{3}$ National Central University, Taoyuan City, TW;

${ }^{4}$ Eindhoven University of Technology, Eindhoven, $N L$;

${ }^{5}$ The Chinese University of Hong Kong, Hong Kong, HK;

${ }^{6}$ King's College London, London, TW;

${ }^{7}$ University of Cambridge, Cambridge, GB;

${ }^{8}$ Trusted Medical Computing Laboratory, Taipei, GB

Introduction: BLADE sequence (also known as the PROPELLER MRI [1] or MultiVane) acquires multiple overlapping rectangular 
$\mathrm{k}$-space patches, which cover the circular region in $\mathrm{k}$-space while sharing k-space center. The motion insensitivity of BLADE MRI is achieved by retrospective correction for translational and rotational motions. It has been accepted that BLADE MRI reduces the motion artifacts and benefits the scanning process for uncollaborative patients. For collaborative patients, however, the current BLADE protocols for collaborative patients yield suboptimal image quality, mostly due to the trade-offs between scanning time, matrix size, and the sensitivity encoding (SENSE) factor. To improve the image quality of BLADE MRI using the index tensor solvers and to evaluate the MRI image quality in a clinical setting. In this study, we investigated the index tensor notation for common Cartesian and nonCartesian MRI encoding methods. We generalized the index notation to recent inverse reconstruction methods in MRI, using simulation and in vivo MRI data.

Methods: BLADE MRI reconstructions using two tensor solvers (the least-square solver and the L1TV-LAD solver) were implemented on the graphics processing unit (GPU). The BLADE rawdata were prospectively acquired and anonymized before the assessments by two independent radiologists with random orders of showing the images. Evaluation scores were examined for consistency and then by repeated-measures analysis of variance (ANOVA) to identify the potential superior algorithm(s).

Results: As shown in Fig. 1, the complex imaginary is influenced by different coil estimation methods. The simulation showed the structural similarity index (SSIM) of various tensor solvers range between 0.995 and 0.999. Evaluation of images (Fig. 2) showed substantial inter-reader agreement (Cohen's Kappa $=0.63$, 95\% confidence interval: $0.57,0.68$ ). The image score of L1TV-LAD was significantly higher than vendor-provided image and the least-square method. The image score of the least-square method was significantly lower than the vendor-provided image. No significance was identified in L1TVLAD with the regularization strength of $\lambda=0.4-1.0$.

Fig. 1

(A)
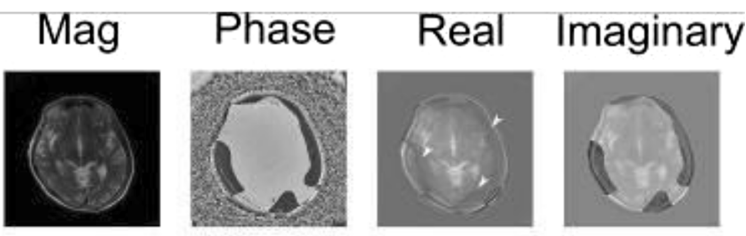

(B)
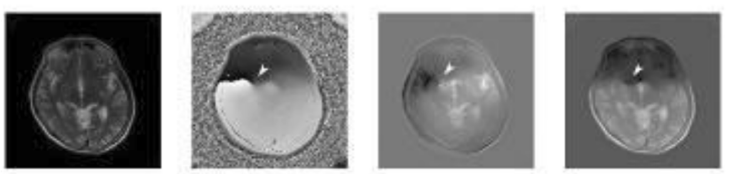

(C)
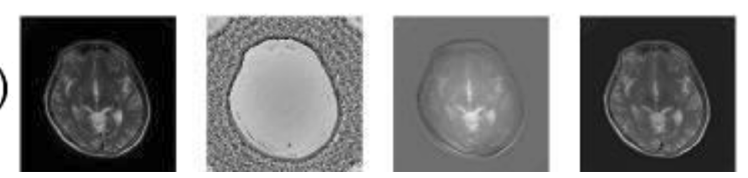

Fig. 2

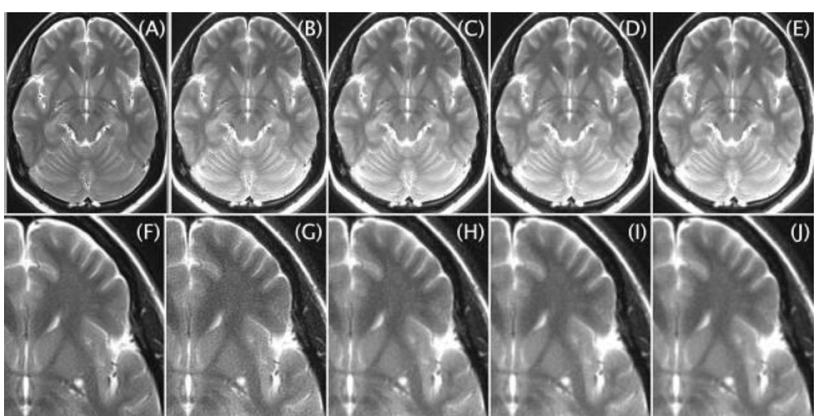

Discussion: Index tensor solvers were implemented and evaluated on the GPU. The L1TV-LAD with regularization strength of $\lambda=0.4-1.0$ was found consistently better than least-square and vendor-provided reconstruction in BLADE MRI with a SENSE factor of 2. Further development of the integrated computing system with the scanner is warranted.

\section{Reference:}

[1] James G Pipe. Motion correction with PROPELLER MRI: application to head motion and free-breathing cardiac imaging. Magnetic Resonance in Medicine: An Official Journal of the International Society for Magnetic Resonance in Medicine, 42(5):963-969, 1999.

\section{S3.P15.}

\section{Data enforcement in MRI kspace reconstruction using deep learning}

\author{
${ }^{*}$ G. Touquet ${ }^{1}$, L. Boussel ${ }^{1}$, P. Douek ${ }^{1}$, M. Sigovan ${ }^{1}$ \\ ${ }^{1}$ CREATIS, Villeurbanne, FR
}

Introduction: Undersampling can be used to accelerate time-consuming MRI acquisitions, but leads to artefacts in the image domain. Non deep learning based reconstruction processes mitigating this effect can provide diagnostic image quality, but can also require significant reconstruction time and computational resources. Deep learning techniques, on the other hand, have shown significant potential in the MRI reconstruction problems with great results and fast evaluations [1]. While deep learning application have already been proposed in the k-space, we intend to offer in this work a novel approach to simplify the training task and ensure data consistency within the network both at training and evaluation.

Methods: All following methods were trained and tested on 4860 knee images from the NYU fastMRI database [3]. The chosen deep learning architecture is a Unet [4], as it provides an easily trainable convolutional approach while having proven its capacities. To accomodate the coil-separated and complex information, the same neural network weights are applied parallelly to each of these dimensions. The consistency enforcement consists in setting the kspace values that were measured to their original values after the correction, by use of a inverse mask and the addition of the original measurement, as described in the joined figure. In order to evaluate the gain from the consistency enforcement, two Unet are trained with kspace data, one with the data enforcement approach, and one without. The network loss is a combination of an L1-norm in the kspace domain and a modified SSIM [5] score in the image domain. 
Fig. 1

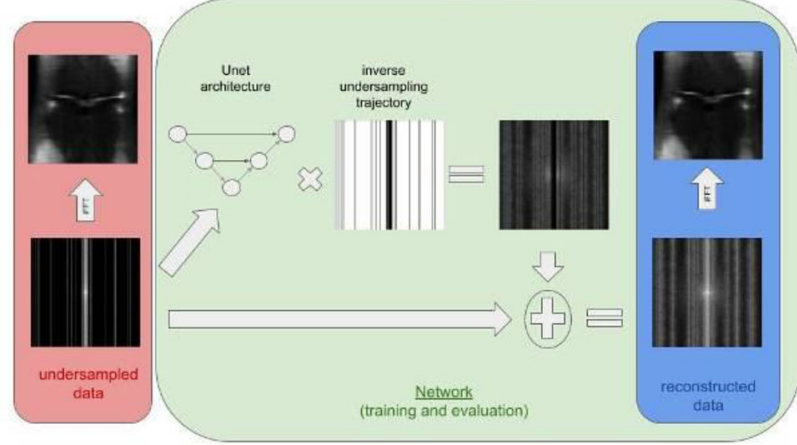

Results: The network trained with the data enforcement method outperforms the other method with a optimum loss of 0.39 compared to 0.45 and the data enforced version converged at least 5 times faster to its final value.

Discussion: The convolutional approach, although fairly close to the GRAPPA kernel approach, might be improved upon by designing a network architecture with intrinsic kspace properties and chosen trajectory in mind. Nevertheless the trajectory masking technique can be applied to any such approach, and will help shortening training phases. Furthermore the use of complex numbers in both kspace and image space, will be ultimately fit for phase contrast applications.

\section{References:}

1. Jin Liu et al. Applications of deep learning to MRI images: A survey. Big Data Mining and Analytics ( Volume: 1, Issue: 1, March 2018) 10.26599/BDMA.2018.9020001.

2. Griswold MA, et al. Generalized autocalibrating partially parallel acquisitions (GRAPPA). MRM 2002;47:1202-10.

3. Knoll et al. Radiol Artif Intell. 2020 Jan 29;2(1):e190007. 10.1148/ ryai. 2020190007

4. O. Ronneberger et al. U-net: Convolutional Networks for Biomedical Image Segmentation. O.Ronneberger, et al. arXiv: 1505.04597.

5. Z. Wang et al. Image quality assessment: From error visibility to structural similarity. IEEE Transactions on Image Processing 13(4): 600-612, avril 2004.

\section{S3.P16.}

\section{Improved and undersampled open-source prototyping myocardial T1 mapping sequence (ProMyoT1)}

\author{
*A. $\operatorname{Gaspar}^{1}$, N. A. Silva ${ }^{2}$, R. G. Nunes ${ }^{1}$ \\ ${ }^{1}$ University of Lisbon, Lisbon, PT; \\ ${ }^{2}$ Hospital da Luz, Department of Learning Health, Lisbon, PT
}

Introduction: Methods such as MOLLI ${ }^{1}$ have had a major impact in the clinic for myocardial tissue characterization, but warrant further improvements (e.g. increased slice coverage, sampling flexibility). These can be hindered by the need for access/knowledge of the vendor specific programming environments. To overcome this limitation, we introduced the open-source Prototyping Myocardial T1 mapping sequence (ProMyoT1) ${ }^{2}$ using Pulseq. Here, we report recent improvements to ProMyoT1, reducing artifacts on the T1 weighted (T1w) images, and implementing an undersampled (US)ProMyoT1 version for faster acquisition.

Methods: Two modifications were performed to the linear filling scheme of ProMyoT1 (github.com/ANG13/ProMyoT1) $-(\mathrm{TR} / \mathrm{TE}=$
$3.04 / 1.52 \mathrm{~ms}$, linear ramp-up of 11 pulses, flip angle $=35^{\circ}$, slice thickness $=6 \mathrm{~mm}$, FOV $=200 \times 200 \mathrm{~mm}^{2}$, and matrix size $=128$ $\times 128) .{ }^{2}$ First, a gradient crusher was added before each T1w image acquisition to remove echo-pathways responsible for undesirable image artefacts. Second, a US-ProMyoT1 version was developed to: 1) reduce the acquisition window (390 ms for fully-sampled FSProMyoT1); 2) shorten the first inversion time (TI) $\left(\mathrm{TI}_{1}=218 \mathrm{~ms}\right.$ for FS). The current US-ProMyoT1 enables an acceleration factor of 2 with 24 auto-calibration lines, acquisition window of $231 \mathrm{~ms}$, and $\mathrm{TI}_{1}=150 \mathrm{~ms}$.

FS-ProMyoT1 and US-ProMyoT1 were tested with and without the additional gradient crusher in a Siemens Aera $1.5 \mathrm{~T}$ scanner in the ISMRM/NIST phantom ${ }^{4}$. A clinical MOLLI scan was acquired and reference tabulated phantom $\mathrm{T} 1$ values used for comparison.

Image reconstruction (inverse FFT for FS and GRAPPA for US) and $\mathrm{T} 1$ estimation were performed offline using Matlab; for T1 mapping the function lsqcurve was employed (3-parameter model).

Results: T1w images show a reduction of artefacts when adding crusher gradients for both FS and US ProMyoT1-see $\mathrm{TI}_{2}$ images in Fig. 1, estimated T1 maps in Fig. 2. Crusher application impacted T1 values of FS-ProMyoT1 for vial \#3 $\left(\mathrm{T} 1^{\text {no }}{ }^{\text {Crusher }}=984 \pm 3 \mathrm{~ms}\right.$ vs $\mathrm{T} 1^{\text {crusher }}=1023 \pm 3 \mathrm{~ms}$ ) (Fig. 2 and 3 ), while $\mathrm{T} 1$ values of FSProMyoT1 differ mainly for vial \#6 $\left(\mathrm{T} 1^{\text {no }}{ }^{\text {Crusher }}=413 \pm 6 \mathrm{~ms}\right.$ vs $\mathrm{T}^{\text {crusher }}=340 \pm 6 \mathrm{~ms}$ ). FS-ProMyoT1 did not provide correct $\mathrm{T} 1$ estimates for reference values $<100 \mathrm{~ms}$.

\section{T1w ProMyoT1}

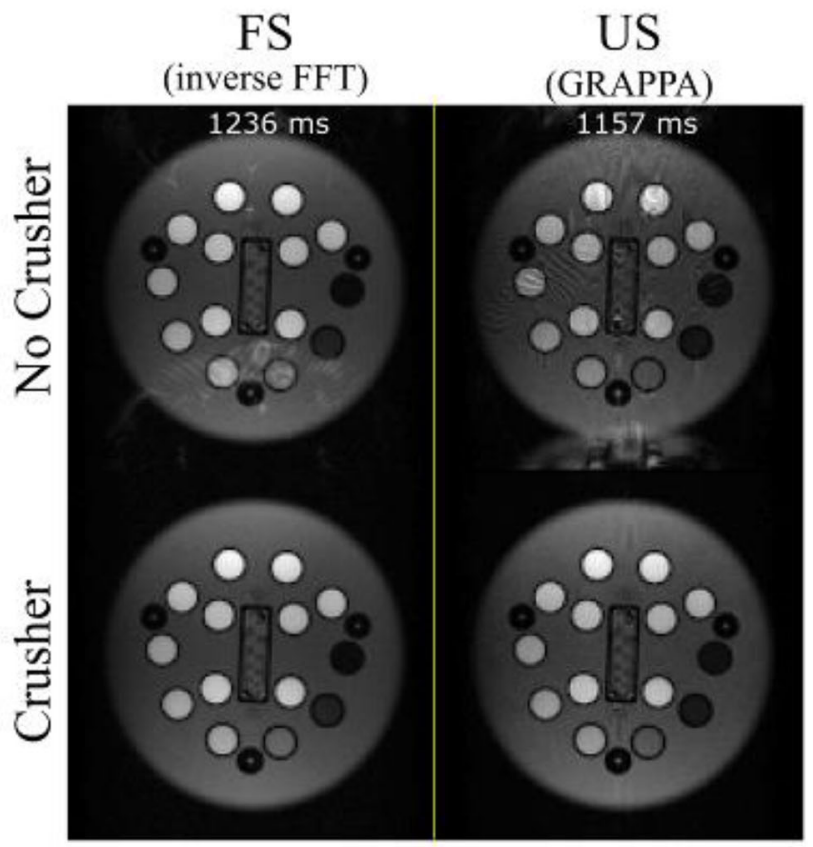

Figure 1 - T1 weighted (T1w) images for second inversion time (in white) obtained with ProMyoT1 with fully sampled (FS), and undersampled (US) acquisitions of NIST/ ISMRM phantom. T1w images for both methods are presented with no crusher (top row) and with the crusher (bottom row) application. Simulated heart rate of $60 \mathrm{bpm}$. 


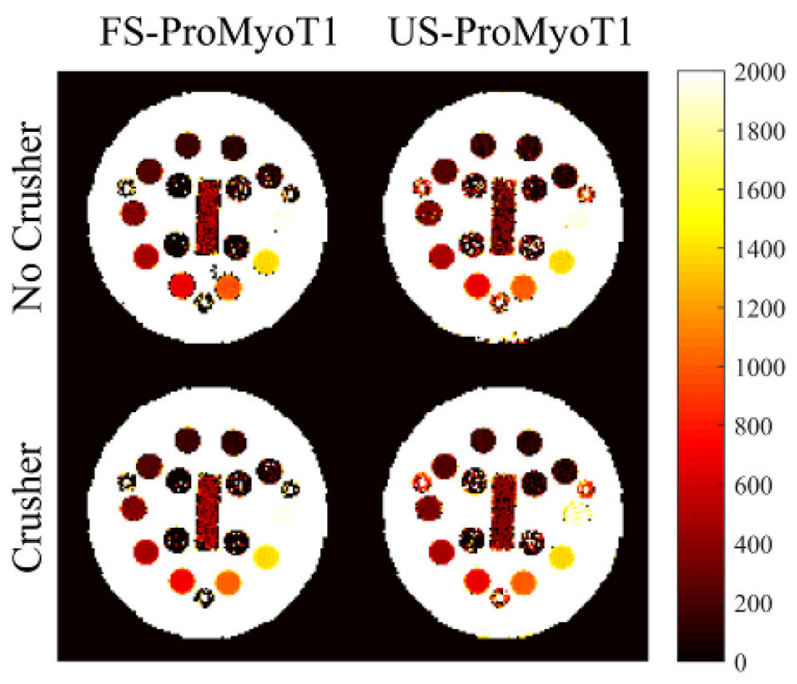

Vial Number location

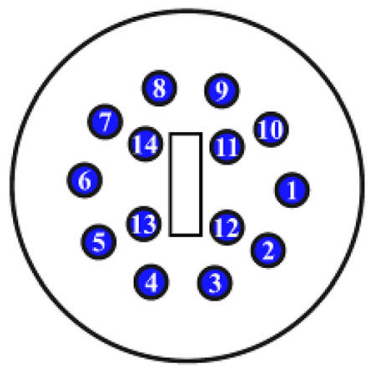

Figure 2 - T1 maps (in ms) estimated from undersampled (US) and fully-sampled (FS) ProMyoTl with crusher (top row) and no crusher (bottom row) application. A scheme of vial number location is presented.

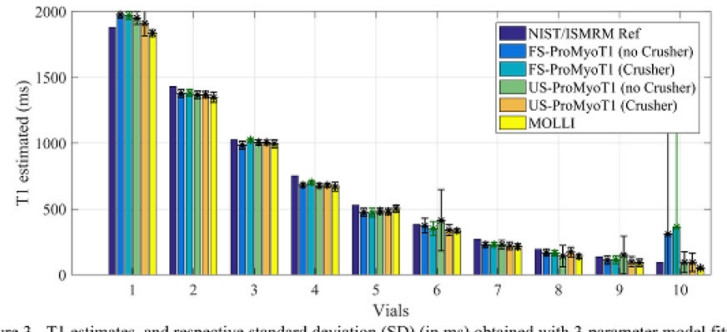

Figure 3 - Tl estimates, and respective standard deviation (SD) (in ms) obtained with 3-parameter model fitting for: fully-sampled (FS-ProMyoTI) and undersampled (US-ProMyoTI) acquisitions with linear filling order, with and phantom are presented in dark blue.

Discussion: This work shows that ProMyoT1 can easily be adapted and improved. T1w artifacts which locally hindered T1 estimation were removed with the addition of gradient crushers. This allowed improving T1 estimates for the affected vials especially for US-ProMyoT1. The US-ProMyoT1 enabled T1 estimates similar to FSProMyoT1 with the advantages of increased flexibility in vivo for different heart rates. Future work will include testing US-ProMyoT1 in different scanners and in vivo for reproducibility.

\section{References}

1. Messroghli D et al. MRM 200;26:1081-6

2. Gaspar A et al. Proc ISMRM 2021;3599

3. Layton KJ et al. MRM 2017;77:1544-52

4. Jiang Y et al. MRM 2017;78:1452-7

Acknowledgements: FCT (SFRH/BD/120006/2016,PTDC/EMDEMD/29686/2017); Lisboa 2020 (LISBOA-01-0145-FEDER029686).

\section{S3.P17.}

Neuro-imaging DW-MR data Reconstruction through cascaded neural network

*F. Aamir ${ }^{1}$, I. Aslam ${ }^{1}$, H. Omer ${ }^{1}$

${ }^{1}$ COMSATS University Islamabad, Electrical and Computer Engineering, Islamabad, $P K$

Introduction: Reconstruction of the under-sampled DW-MR data is challenging because the higher b value images usually have low SNR; however high-quality reconstruction is often needed for clinical use. We propose a DWI Cascaded-Net to recover the artifact free DW-MR image using accelerated 1D Cartesian variable density $k$-space data. Our experiments show improved performance of the proposed DWI Cascaded-Net both visually and in terms of assessment parameters than contemporary U-Net and ResNet reconstruction results.

Subjects and method: Figure-1 depicts block diagram of the proposed scheme. In DWI Cascaded-Net (U-Net + ResNet), initially IFFT is applied to the 1D variable density under-sampled $k$-space data which provides aliased images. The aliased images (input) and the artifact free reference images (label) are fed to train the cascaded-Net i.e. U-Net $+\operatorname{ResNet}^{1,2}$. Once the network has been trained, the under-sampled unseen data is fed to the network to get the output, which recovers the zero-filled spaces of the input under-sampled $k$ space data. To retain the originally acquired $k$-space points, an additional step of data consistency ${ }^{3}$ is applied. Finally, IFFT of the updated $k$-space provides the solution image.

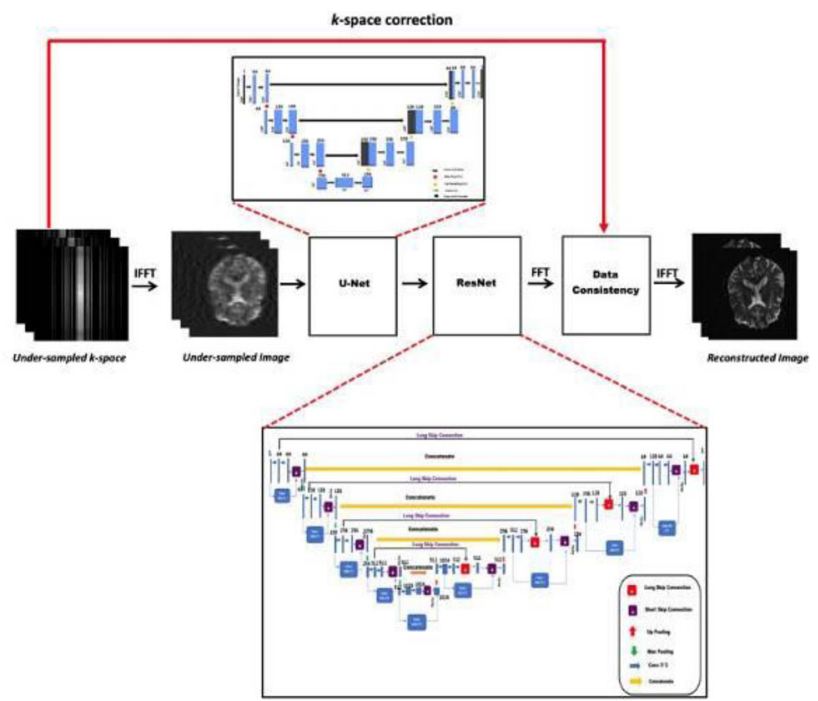

Figure 1: A schematic workflow of the proposed method (i.e. DWI Cascaded-Net)

The proposed DWI Cascaded-Net is trained by using a training set of 5433 images and a validation set of 605 images. In the proposed method, RMSProp optimizer with a learning rate of $5 \times 10^{-4}$ is used 
for optimizing the weights of the network. The network training was implemented on Python 3.8 using TensorFlow, batch size $=5$, epochs $=300$ with early stopping criteria and $12 \mathrm{~h}$ training.

Results: The proposed DWI Cascaded-Net is trained and tested on publicly available OASIS-3 (https://central.xnat.org/)3 DWI neuroimaging data having matrix size $=256 \times 256$ with $\mathrm{TE}=0.11 \mathrm{~ms}$, $\mathrm{TR}=14.5 \mathrm{~ms}$ and Flip angle $=90^{\circ}$. In our experiments, the $\mathrm{b}$ values vary between 0 and $800 \mathrm{~s} / \mathrm{mm}^{2}$.

Reconstruction results of DWI Cascaded-Net, conventional U-Net and ResNet at $\mathrm{AF}=4$ are presented in Figure-2. Table-1 shows the results of DWI Cascaded-Net, conventional U-Net and ResNet in terms of evaluation parameters at $\mathrm{AF}=2,4$ and 6 .

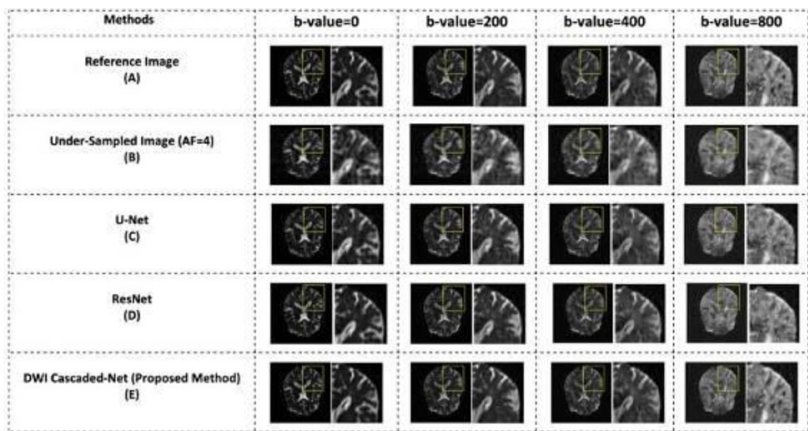

Figure 2: Reconstructed images of 3 T DWI neuro-imaging data with U-Net, ResNet, and DWI Cascaded-Net (Proposed method) at AF=4 having different b-values i.e. 0,200,400 and $800 \mathrm{~s} / \mathrm{mm}^{2}$, respectively
Fig. 3

Table 1: Comparison of the reconstruction quality regarding 'meant std' for PSNR, AP, RMSE, and SSIM values of the neuro-imaging data for different AF i.e. $2 \leq$ AF $\leq 6$ between U-Net, ResNet and DWI Cascaded-Net (Proposec method)

\begin{tabular}{|c|c|c|c|c|}
\hline \multicolumn{2}{|c|}{$\begin{array}{c}\text { Mean Evaluation } \\
\text { Parameters }\end{array}$} & \multicolumn{2}{|c|}{ Conventional Methods } & \multirow{2}{*}{$\begin{array}{l}\text { Proposed Method } \\
\text { DWI Cascaded-Net }\end{array}$} \\
\hline & & U-Net & ResNet & \\
\hline \multirow{4}{*}{$\frac{\pi}{\frac{\pi}{\alpha}}$} & PSNR & $85.290 \pm 0.0141$ & $87.462 \pm 0.0161$ & $90.021 \pm 0.0190$ \\
\hline & AP & $0.0027 \pm 0.0017$ & $0.0014 \pm 0.0010$ & $0.9964 \pm 0.0007$ \\
\hline & RMSE & $0.0030 \pm 0.0020$ & $0.0023 \pm 0.0013$ & $0.0015 \pm 0.0009$ \\
\hline & SSIM & $0.9964 \pm 0.0060$ & $0.9983 \pm 0.0089$ & $0.9990 \pm 0.0096$ \\
\hline \multirow{4}{*}{$\frac{\pi}{\frac{\pi}{4}}$} & PSNR & $76.100 \pm 0.1640$ & $78.367 \pm 0.1831$ & $86.438 \pm 0.206$ \\
\hline & AP & $0.0093 \pm 0.0023$ & $0.0074 \pm 0.0017$ & $0.0026 \pm 0.0012$ \\
\hline & RMSE & $0.0176 \pm 0.0023$ & $0.0165 \pm 0.0023$ & $0.0045 \pm 0.0016$ \\
\hline & SSIM & $0.9679 \pm 0.0160$ & $0.9783 \pm 0.0187$ & $0.9984 \pm 0.0220$ \\
\hline \multirow{4}{*}{$\frac{\mathscr{I}}{\frac{1}{\alpha}}$} & PSNR & $68.312 \pm 0.2053$ & $72.526 \pm 0.2100$ & $80.290 \pm 0.2679$ \\
\hline & AP & $0.0200 \pm 0.0080$ & $0.0226 \pm 0.0021$ & $0.0100 \pm 0.0028$ \\
\hline & RMSE & $0.0267 \pm 0.0234$ & $0.0290 \pm 0.0220$ & $0.0126 \pm 0.0100$ \\
\hline & SSIM & $0.9413 \pm 0.0204$ & $0.9500 \pm 0.0237$ & $0.9843 \pm 0.0298$ \\
\hline
\end{tabular}

Discussion/conclusion: Proposed DWI Cascaded-Net shows improved results as compared to conventional U-Net and ResNet. The proposed method utilizes all the advantages of both the networks together without losing the spatial information during training and reconstructs the high-quality DW-MR images.

\section{References:}

1. C. M. Hyun et.al, doi: 10.1088/1361-6560/aac71a.

2. https://medium.com (accessed May 04, 2021).

3. https://central.xnat.org (accessed Aug. 27, 2020).

\section{S3.P18.}

Slice selection with zero echo time to 2D MRI of short$\mathbf{T}_{2}$ tissues

*J. Borreguero ${ }^{1}$, F. Galve ${ }^{2}$, J. M. Algarín ${ }^{2}$, J. Alonso ${ }^{2}$

${ }^{1}$ Tesoro Imaging S.L., Valencia, ES;

${ }^{2}$ Technical University of Valencia, Institute for Molecular Imaging and Instrumentation (i3M), Valencia, ES

Introduction: MRI of hard biological tissues remains technically challenging due to the fleeting lifetime of their MRN signals [1]. Special-purpose protocols, such as the Zero Echo Time (ZTE) sequences, are arguably most suitable for ultra-short $T_{2}$ samples [2]. However, current approaches to ZTE make it incompatible with slice selection. Here we present a new protocol for slice selection with ZTE (SS-ZTE, [3]).

Methods: SS-ZTE sequence is composed by four main building blocks (Fig. 1): a) sample excitation, where a hard $\mathrm{RF} \pi / 2$ pulse is applied after the onset of the slice selection gradient $\left(\mathrm{G}_{\mathrm{ss}}\right)$; b) slice selection, where a spin-locking ( $\mathrm{SL}$ ) RF pulse $\left(\mathrm{B}_{1 \mathrm{SL}}\right)$ detuned as $\gamma\left(B_{0}+G_{s s} z_{0}\right)$ selectively locks the sample magnetization at slice $z_{0}$, spoiling the rest due to $\mathrm{T}_{2}$ decay during gradient aplication (slice thickness $\Delta=2 \mathrm{~B}_{1 \mathrm{SL}} / \mathrm{G}_{\mathrm{ss}}$ ); c) preservation, to protect the magnetization and coherence of the selected slice from being affected by gradient reconFigurations; and d) acquisition, after the onset of the readout gradient and termination of $\mathrm{G}_{\mathrm{ss}}$ gradient.

Results: As a first demonstration of SS-ZTE performance, we have run tests on our $0.26 \mathrm{~T}$ DentMRI scanner [1]. The sample is a cylindrical tube filled with $3 \% \mathrm{CuSO}_{4}$ doped water $\left(\mathrm{T}_{2}=3 \mathrm{~ms}\right)$. For this $\mathrm{T}_{2}$, we can use a simplified version of the preservation block in Fig. 1 (top) which does not require the storage/spoiling pulses.

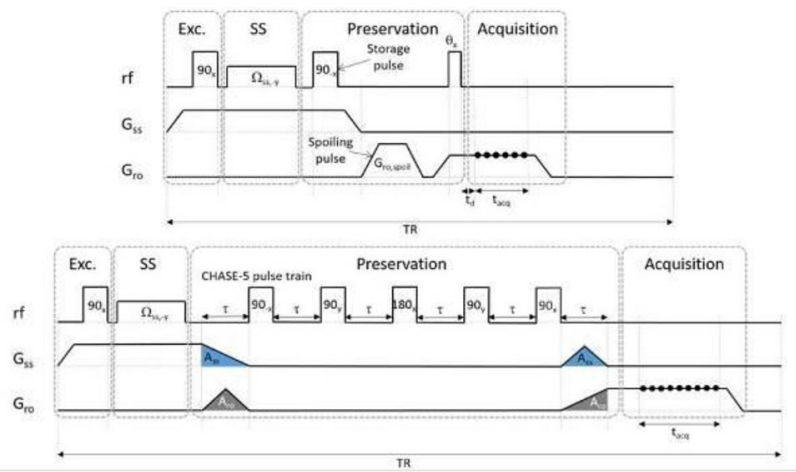

Figure 1. Up) Embodiment of SS-ZTE where the Preservation Block includes a magnetization storage pulse, a gradient spoiling pulse and an excitation pulse. Down) Embodiment of SS-ZTE where the Preservation Block includes a CHASE-5 pulse train length, allowing for zero dead time before data Preservation
acquisition.

Figure 2 (left) shows 1D projections of slices for $\mathrm{G}_{\mathrm{ss}}=30 \mathrm{mT} / \mathrm{m}$ and several SL pulse durations. SL times above $1 \mathrm{~ms}$ result in rather clean Lorentzian slice profiles (square profiles would result from a sinc SL pulse). Figure 2 (middle) presents the dependence of $\Delta$ as a function of $\mathrm{B}_{1 \mathrm{SL}}$ strength for $\mathrm{G}_{\mathrm{ss}}=30 \mathrm{mT} / \mathrm{m}$ and $\mathrm{t}_{\mathrm{SL}}=1 \mathrm{~ms}$, with $\Delta$ ranging from 2 to $10 \mathrm{~mm}$. Figure 2 (right) shows three (superimposed) $4 \mathrm{~mm}$ slices selected with $\mathrm{B}_{1 \mathrm{SL}}=160 \mu \mathrm{T}$ during, $\mathrm{t}_{\mathrm{SL}}=900 \mu \mathrm{s}$ and $\mathrm{G}_{\mathrm{ss}}=80$ $\mathrm{mT} / \mathrm{m}$. For comparison, we have also included $4 \mathrm{~mm}$ slices obtained with $450 \mu$ s and $100 \mu \mathrm{T}$ sinc RF pulses for standard slice selection based on gradient echo (GRE). 


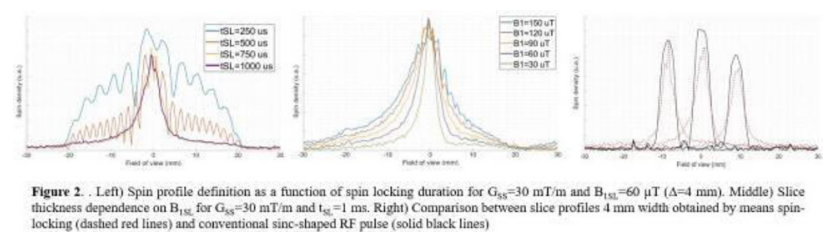

In Fig. 3 we compare 2D images with slice selection $(6 \mathrm{~mm})$ based on standard GRE (left) and SS-ZTE (right). The GRE parameters are the same as in Fig. 2 (right), with the shortest possible echo time (3.1 ms). For SS-ZTE, we chose $B_{1 \mathrm{SL}}=120 \mu \mathrm{T}, \mathrm{t}_{\mathrm{SL}}=1 \mathrm{~ms}$ and $\mathrm{G}_{\mathrm{ss}}=40 \mathrm{mT} / \mathrm{m}$. Acquisition starts $400 \mu \mathrm{s}$ after SL pulse. SS-ZTE enables a SNR improvement by a factor 2.8 .
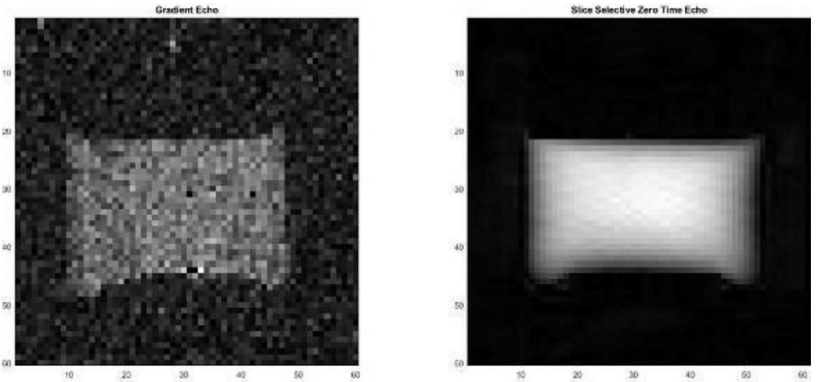

Figure 3. Left) 2D Gradient Echo image obtained with slice selection through sinc-shaped RF pulse in presence of a lineal gradient. Right) 2D SS-ZTE image whose slice selection has been performed by means spin-locking plus a calibrated triangular waveform. To most reliable comparison between they, both images have been obtained with same slice thickness $6 \mathrm{~mm}$, number of averages, spatial resolution of $1 \mathrm{~mm}^{2}$ in a square FOV of $60 \mathrm{~mm}$, acquisition bandwidth in $37.5 \mathrm{kHz}$ and rise fall gradient times fixed to 100 us for all transitions. These images ar presented in raw previously reconstructed by direct FF

Discussion: SS-ZTE removes one of the main constraints associated to previously existing ZTE sequences: their inability to produce $2 \mathrm{D}$ images. A second limitation is the need for a wait time between the excitation and acquisition windows, due to dead times in RF electronics $[1,2]$. In SS-ZTE, the preservation block can also be implemented with dynamical decoupling schemes (e.g. CHASE [4]), instead of SL, making the wait time unnecessary.

\section{References}

[1] Algarín et al., Sci. Rep. (2020).

[2] Weiger et al., Prog. Nucl. Magn. Reson. Spectrosc. (2019).

[3] Galve et al., SS-ZTE patent P202030504.

[4] Waeber et al., Nature (2019).

\section{S3.P19.}

\section{Implementation of stack-of-spiral arterial spin labeling at $7 \mathrm{~T}$ using Pulseq and MR field monitoring}

*R. E. Ma ${ }^{1}$, D. Kurban ${ }^{1}$, D. Ivanov ${ }^{1}$, S. Kashyap ${ }^{1}$, L. Huber ${ }^{1}$, B. A. Poser

${ }^{1}$ Maastricht University, Faculty of Psychology and Neuroscience, Maastricht, NL

Introduction: Pulseq [1] is an open-source MR pulse sequence development package for implementing sequences with novel acquisition and sampling strategies, without going through the timeconsuming vendor-specific sequence programming for initial investigation of sequence behavior. Field monitoring with NMR probes [2] enables high-fidelity measurement of the gradient performance and therefore field correction during image reconstruction. In this study, a 3D stack-of-spiral (SOSP) Arterial Spin Labeling (ASL) sequence was developed and tested at $7 \mathrm{~T}$ combining these two techniques.
Methods: FAIR-QUPISS II labeling module using a tr-FOCI inversion pulse [3] was implemented for the SOSP ASL sequence using Pulseq in Matlab. Acquisition parameters include: TR/TI2/TI1 = $3000 \mathrm{~ms} / 1800 \mathrm{~ms} / 700 \mathrm{~ms}, \mathrm{FOV}=200 \times 200 \mathrm{~mm}^{2}$, in-plane resolution $=(2.1 \mathrm{~mm})^{2}$, slice thickness $=2 \mathrm{~mm}, 36$ slices per slab, undersamping factor $=1.6, \mathrm{FA}=20^{\circ}, \mathrm{BWTP}=25$. FAIR-QUIPSS II labeling blocks and imaging blocks of the sequence are shown in Fig. 1. MR images were acquired on a water phantom and a subject. To examine reconstruction performance, we acquired additionally Cartesian-sampled GRE reference images with identical resolution to their spiral counterpart on the phantom. The in vivo scan contained 50 control/label pairs.

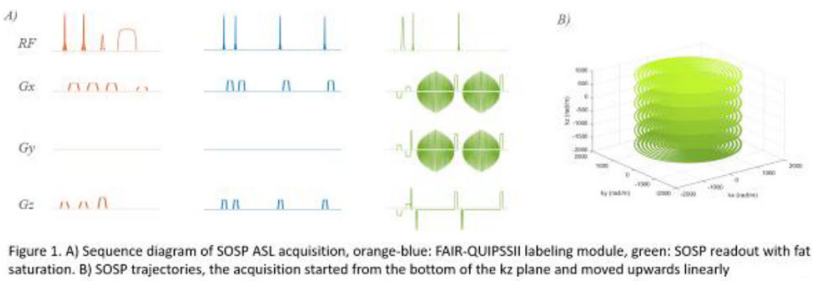

Field monitoring was performed after image acquisition, using $16 \mathrm{~F}^{19}$ NMR probes (Skope, $\mathrm{CH}$ ) placed at the iso-center. GPU-accelerated algebraic reconstruction with up-to- $2^{\text {nd }}$-order field correction based on 3D SENSE model [4] was programmed in Matlab. Off-resonance maps were calculated using the topup function in FSL by acquiring a pair of echo-planar images with the opposite phase encoding and readout direction.

Results: As shown in Fig. 2 on reconstructed phantom images, reconstruction with off-resonance correction effectively reduced geometric distortion and blurring from spiral acquisition, yielding comparable image quality with Cartesian-sampled GRE images. The subtraction images from in vivo acquisition, as in Fig. 3, showed perfusion signal primarily in the cortical area. The central brain showed darker signal most likely due to over-flipping as can be also seen from the control images.

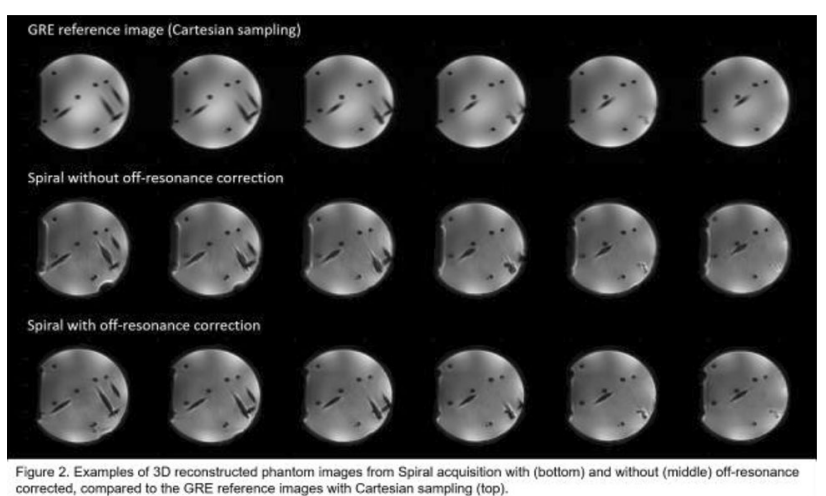

Figure 2. Examples of $3 \mathrm{D}$ reconstructed phantom images from Spiral acquisition wit
corrected, compared to the GRE reference images with Cartesian sampling (top).

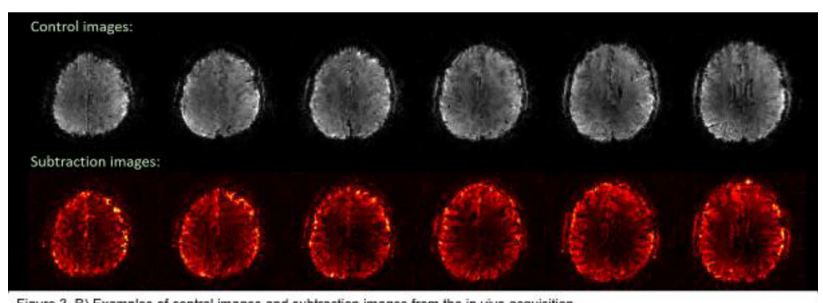

Figure 3. B) Examples of control images and subtraction images from the in vivo acquisition. 
Discussions: The combination of Pulseq and field monitoring makes it feasible to quickly implement and examine the performance of novel MR acquisitions, as shown by our pilot study on SOSP ASL acquisition. The 3D-SENSE based reconstruction with dynamic field and off-resonance correction was successfully implemented, which will be even more beneficial when concurrent field monitoring is performed to correct for physiological fluctuations. The in vivo ASL acquisition indicates non-ideal RF implementation. Next steps include optimizing RF design and improving k-space sampling efficiency.

\section{References:}

1, Layton et al. 2017 MRM 77:1544-1552.

2, Barmet et al. 2008 MRM 60:187-197.

3, Ivanov et al., 2017 MRM 78:121-129.

4, Wilm et al., 2011 MRM 65:1690-1701.

\section{S3.P20.}

Skull lipid signal suppression by means of an outer volume crusher coil for diffusion-weighted half fourier acquisition single shot turbo spin echo with smooth transition between pseudo steady states at $3 \mathrm{~T}$

\author{
*A. Arbabi ${ }^{1}$, D. G. Norris ${ }^{1}$ \\ ${ }^{1}$ Radboud University Medical Centre, Nijmegen, $N L$
}

Introduction: The most widely used method to suppress the bright fat signal in turbo spin echo (TSE) images is the Fat-Sat technique, where a low-bandwidth RF pulse tuned to the fat resonance frequency and an extra gradient pulse to crush the fat signal are used immediately before the imaging scan. All these lead to higher specific absorption rate (SAR), increased scan time, and sequence design complexity.

We demonstrate the application of a crusher coil for the skull lipid signal suppression, which works based on the principle of surface spoiling gradients ${ }^{1}$. We also introduce a variation of diffusionweighted half Fourier acquisition single shot TSE with smooth transition between pseudo-steady states ${ }^{2}$ (TRAPS-DW-HASTE). This method provides a significantly lower SAR level, the same level of sensitivity, and similar image quality as compared to the standard manufacturer"s sequence at the same field strength.

Theory: DW-HASTE is characterized by long echo times (TE) due to a long time block reserved for the inserted diffusion preparation part. To achieve the shortest attainable TE, a centre-out phase encoding scheme is followed, which preserves the full sensitivity for the central k-space region. In TRAPS-DW-HASTE, $180^{\circ}$ refocusing RF pulses are used for the early echoes. Afterwards, the nutation angle is gradually ramped down to a fixed $90^{\circ}$ flip angle (Fig. 1). If the magnetization is continuously in a pseudo-steady state, the greatest signal can be obtained.
For crusher coil application, a trigger signal with a variable duration was implemented at the start of the diffusion preparation event block. Methods: MRI scans were performed on a whole body 3 T Siemens Prisma scanner $(80 \mathrm{mT} / \mathrm{m}$ strength and $200 \mathrm{mT} / \mathrm{m} / \mathrm{ms}$ slew rate) with a 32-channel receive-only head coil in 2 healthy volunteers. Data were collected with DW-HASTE and TRAPS-DW-HASTE for $b=0$ (4 reps and $112 \mathrm{~s}$ scan time) and $1000 \mathrm{~s} / \mathrm{mm}^{2}$ (10 reps and $280 \mathrm{~s} \mathrm{scan}$ time): TE/TR $=109 / 2000 \mathrm{~ms}$, FOV $=200 * 200 \mathrm{~mm}^{2}, \sim 1 \mathrm{~mm}$ isotropic in-plane resolution, 14 axial slices $(3 \mathrm{~mm})$, and GRAPPA $=2$. A 3D diagonal diffusion weighting scheme was used. Fat saturation was performed by either standard Fat-Sat technique or the crusher coil. SAR value measured on the scanner was $78 \%$ and $42 \%$ for DW-HASTE and TRAPS-DW-HASTE, respectively.

Results: Figure 2 shows DW-HASTE and TRAPS-DW-HASTE example images. Figure 3 compares the fat saturation methods.
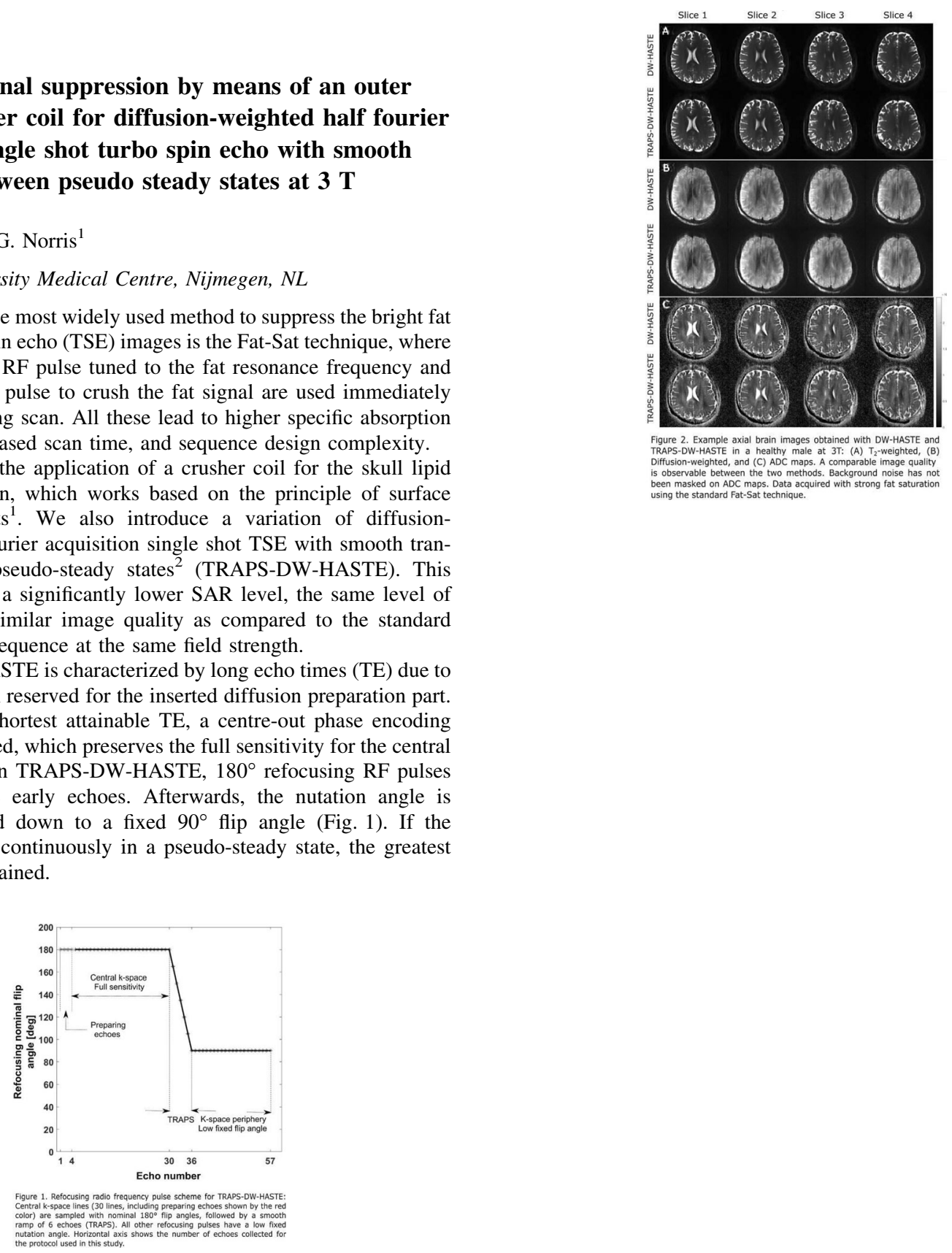


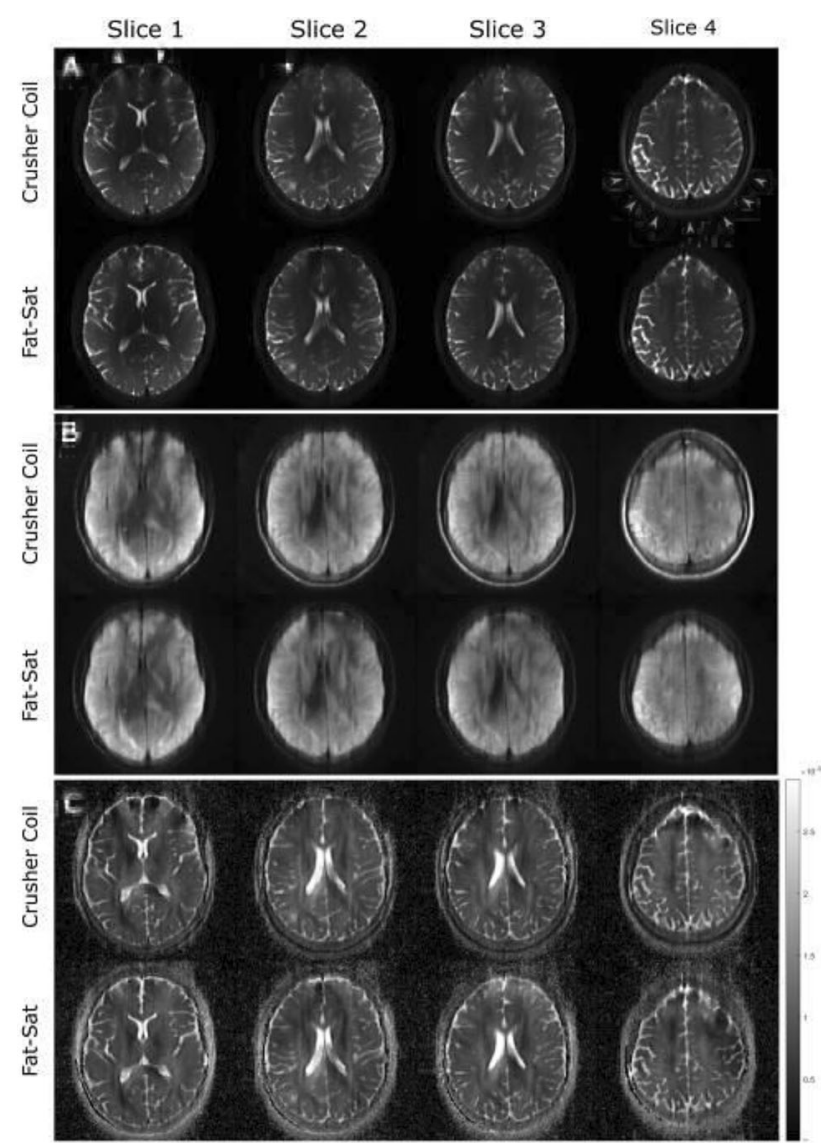

Figure 3. Example fat saturated axial brain images obtained with TRAPS-DW-HASTE in a healthy female at $3 \mathrm{~T}$ : (A) $\mathrm{T}_{2}$-weighted, (B) Diffusion-weighted, and (C) ADC maps. In each panel, application of the crusher coil (top row) and the standard Fat-Sat technique (bottom row) are shown for the same sample slices. Due to the crusher coil's rigid design and varying head sizes and shapes, it is possible that skull fat is partially spoiled on the dorsal region (green arrowheads), or parts of the brain tissue are unwantedly crushed on the frontal lobe (red arrowheads) where the separation distance between the head and coil is small. Here, a $3 \mathrm{~ms}$ trigger signal was played out with the amplifier driven at its $\sim 20 \%$ capacity. Driving higher current would result in unwanted signal crushing over a larger part of the brain frontal lobe for this specific case. Background noise has not been masked on ADC maps.

Discussion: TRAPS-DW-HASTE deposits significantly lower RF power at $3 \mathrm{~T}$ while maintaining image quality. The crusher coil can be used in conjunction with any pulse sequence due to its easy implementation. Due to its rigid design in combination with varying head sizes and shapes, it is possible that skull fat is partially spoiled, or parts of the brain tissue are unwantedly crushed. This problem could be resolved by introducing the capability to locally adjust the strength of the crusher field.

\section{References:}

1. Boer et. al., MRM 73:2062-2068 (2015).

2. Hennig et. al., MRM 49:527-535 (2003).

\section{S3.P21.} A driven equilibrium approach to in-vivo diffusion-
weighted imaging of the liver using spatial encoding

\author{
*S. Gonçalves ${ }^{1}$, Q. Bao ${ }^{2}$, M. Yon ${ }^{2}$, L. Frydman ${ }^{2}$, N. Shemesh ${ }^{1}$ \\ ${ }^{I}$ Champalimaud Centre for the Unknown, Lisbon, PT; \\ ${ }^{2}$ Weizmann Institute, Department of Chemical and Biological \\ Physics, Rehovot, DE
}

Introduction: Spatiotemporal encoding (SPEN) is an alternative to Fourier imaging that is robust against distortions and motion [1]. Recent studies have shown the potential of SPEN for diffusionweighted imaging (DWI-SPEN) of challenging body regions like the pregnant mouse abdomen [2]. DWI-SPEN attains TEs $>35 \mathrm{~ms}$, which hinders its application to the liver at $\mathrm{B} 0^{3} 7 \mathrm{~T}$, where $\mathrm{T} 2 £ 23 \mathrm{~ms}$ [3]. We propose a driven equilibrium approach [4] to DWI-SPEN by placing a diffusion preparation module in front of the SPEN sequence, here named as DP-SPEN, which obtains in-vivo images of the liver with TEs as short as $26 \mathrm{~ms}$.

Methods: The DP module (Fig. 1) consists of 3 non-selective block pulses- $\mathrm{p} / 2 \mathrm{x}-\mathrm{py}-(\mathrm{p} / 2-\mathrm{x})$ — and one pair of monopolar diffusion gradients. Spoiler gradients are placed around and after the py and $(\mathrm{p} / 2-$ $\mathrm{x})$ respectively. The tip-up pulse is phase-cycled between $(\mathrm{p} / 2-\mathrm{x})$ and (p/2-y) to correct for Eddy current artifacts [5]. DP is followed by the SPEN sequence [2].

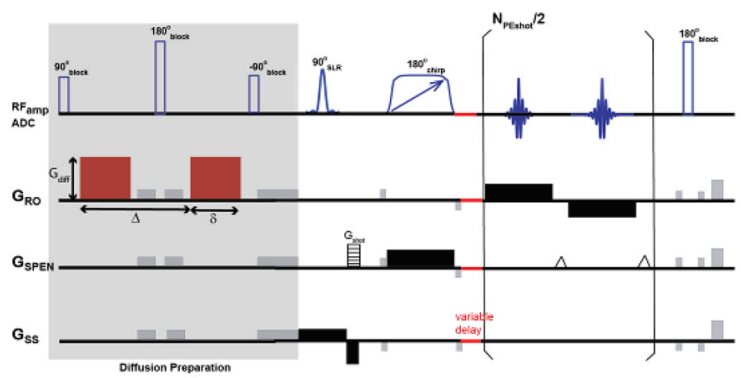

Experiments on a $9.4 \mathrm{~T}$ horizontal bore scanner (Bruker-Biospin, Karlsruhe, Germany) using a 4-element array cryocoil (Bruker BioSpin, Fallanden, Switzerland) for signal reception. Animal experiments were preapproved by the institutional and national authorities. DWI images acquired from water (WP) and brain (BP) phantoms. The WP consisted of a 30/70\% mixture of H20/D2O. The BP consisted of a fixed rat brain immersed in Fluorinert (Sigma Aldrich, Lisbon, Portugal). Images acquired with DWI-EPI and DPSPEN (Fig. 2). In-vivo DP-SPEN images of the liver (Fig. 3) acquired in $\mathrm{N}=2$ mice (wild type, females, 8 weeks)

SPEN images reconstructed as described in [2]. For DP-SPEN, images of the two phase-cycles were combined, using the sum of squares, to obtain the final Eddy current artifact free images.

Results: Fig. 2 shows that DP-SPEN provides mean diffusivity (MD) and fraction of anisotropy (FA) estimates that agree well with those of DWI-EPI. For WP, the MD/FA estimate by DP-SPEN was slightly higher in the upper region of the phantom due to an incomplete correction of Eddy current artifacts. In-vivo results in Fig. 3 show that DP-SPEN accurately images the targeted FOV and successfully maps diffusion in the liver and surrounding organs, in both axial and coronal views. 
A) Water Phantom
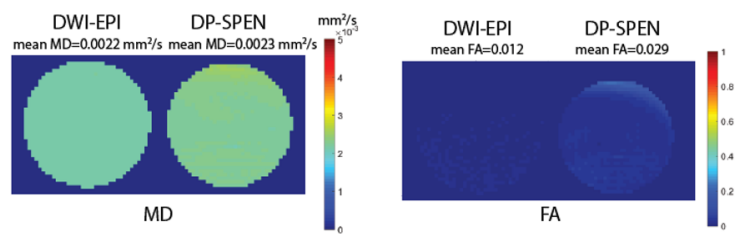

B) Rat Brain Phantom

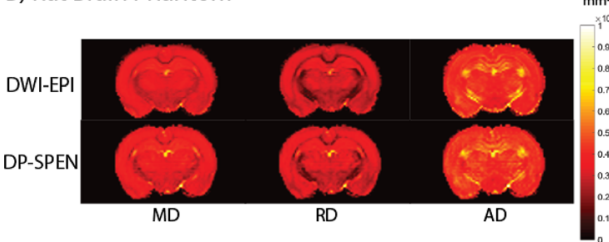

$\mathrm{mm}^{2 / \mathrm{s}}$

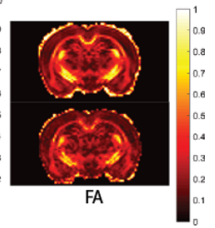

Figure 2-Results for the water and rat phantom. A) Water phantom. TR=8000 ms, TE=44.4 ms (DWI-EPI), $63.3 \mathrm{~ms}$ (DP.SPEN), FOV $=35 \times 35 \mathrm{~mm}$ 12 xilas sice $1 \mathrm{~mm}$ thick, acquistition matti $x=90 \times 90,2$ repettitions, 8 averages, 3 b-values $(800,1200,1800$, 15 directions. B) Rat braln phanton.

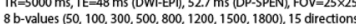

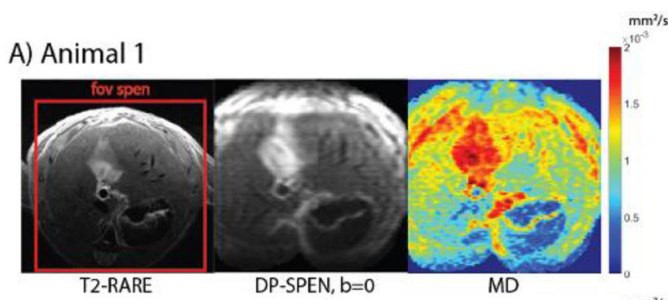

B) Animal 2

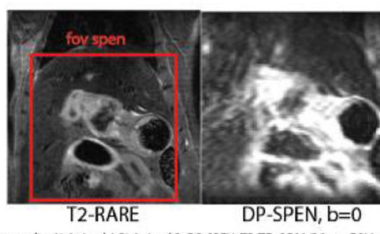
DP-SPEN, b=

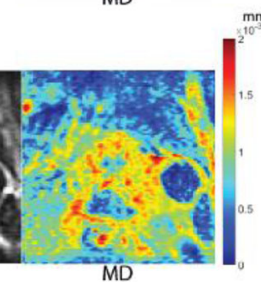

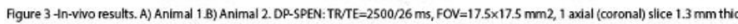

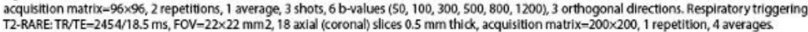
Respiratory triggering.

Discussion: DP-SPEN can map liver diffusion at TEs that are $10 \mathrm{~ms}$ shorter than the minimum TEs of DWI-SPEN, endowing DPSPEN images with higher SNR, which is relevant when mapping diffusion in the liver. B-matrix calculation is greatly simplified when compared to DWI-SPEN because shorter TEs minimize diffusion effects from SPEN imaging gradients. Finally, the decoupled diffusion preparation module offers DP-SPEN larger flexibility in the type of diffusion preparation to be implemented.

\section{References:}

[1] NMR Biomed. 2011 Dec;24(10):1191-201.

[2] NMR Biomed. 2020 Mar;33(3):e4208.

[3] J Magn Reson Imaging. 2012 Jul;36(1):152-8.

[4] Magn Reson Med. 1984 Dec;1(4):502-7.

[5] Magn Reson Med. 1998 Jun;39(6):950-60.

\section{S3.P22.}

\section{Glucose mutarotation kinetics determined by ultrafast CEST line scanning}

\author{
*D. Schache ${ }^{1}$, S. Bardin ${ }^{2}$, L. Ciobanu ${ }^{2}$, C. Faber ${ }^{1}$, V. Hoerr ${ }^{1}$ \\ ${ }^{1}$ University Hospital Münster, Clinic for Radiology, Münster, DE; \\ ${ }^{2}$ Paris-Saclay University, Gif-sur-Yvette, FR
}

Introduction: By Chemical Exchange Saturation Transfer (CEST) Line Scanning (LS) localized Z-spectra can be acquired in less than a minute [1]. This allows to detect kinetic reactions of enzymes and stereoisomeric changes e.g. mutarotation [2].

Conventionally mutarotation is measured by optical methods, although large sample volumes are required. Further, measurements by NMR often require deuterated environments. In contrast, CEST LS can be performed in small sample volumes under physiological conditions and is therefore a promising alternative.

The aim of this study is to measure mutarotation of glucose by CEST LS and to determine the kinetics of its stereoisomers over time.

Methods: For the measurements of glucose mutarotation kinetics, a $75 \mathrm{mM}$ solution of $\alpha$-glucose was prepared in aqua injectabilia $(n=3)$ and was measured by CEST LS for a total time duration of $4 \mathrm{~h}$. Reference datasets were acquired by a CEST-EPI and -RARE sequence. All datasets were corrected by WASSR [3].

Measurements were performed at $20{ }^{\circ} \mathrm{C}$ on a $9.4 \mathrm{~T}$ small animal MR Biospec system (Bruker) equipped with a $72 \mathrm{~mm}$ quadrature coil. To quantify the ratio of the two stereoisomers, the asymmetry for $\alpha$ at $2.1 \mathrm{ppm}$ and $\beta$ at $2.8 \mathrm{ppm}$ was calculated. Data were fitted by using a modified model of 2-side chemical exchange (Fig. 2a) including a correction factor for the sensitivity of detection $\gamma$ and for the uncertainty of the starting point $\mathrm{t}_{\mathrm{d}}$.

Based on CEST measurements of a concentration series (15-75 mM) at the equilibrium, the ratio of isomers in the glucose solution of $75 \mathrm{mM}$ was determined at the endpoint of mutarotation.

Results: Z-spectra acquired by LS agree very well with data measured conventionally by CEST-EPI or -RARE (Fig. 1), and lead to a significant reduction of the measurement time (EPI: 1280 s., RARE: 32,640 s., LS: 40 s.).
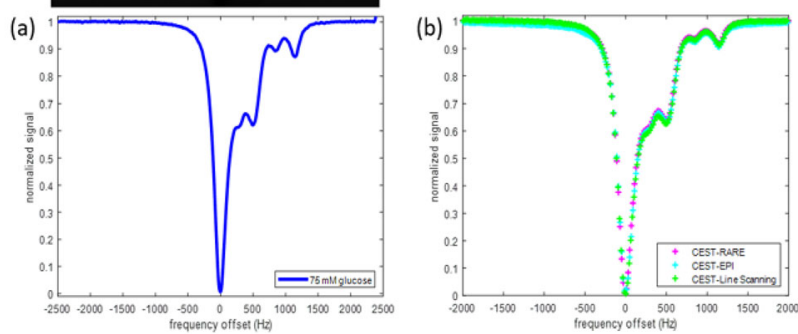

Figure 1: (a) Profile obtained by CEST LS and corresponding (WASSR-) corrected Z-spectrum measured on a $75 \mathrm{mM}$ glucose phantom. (b) CEST spectrum of a $75 \mathrm{mM}$ glucose phantom acquired by CEST-RARE, -EPI, and -LS.

In the LS spectra, a decrease in the peak of the $\alpha$ isomer and an increase in the $\beta$ isomer is detected over time and the corresponding asymmetry values approach an equilibrium state (Fig. 2). 

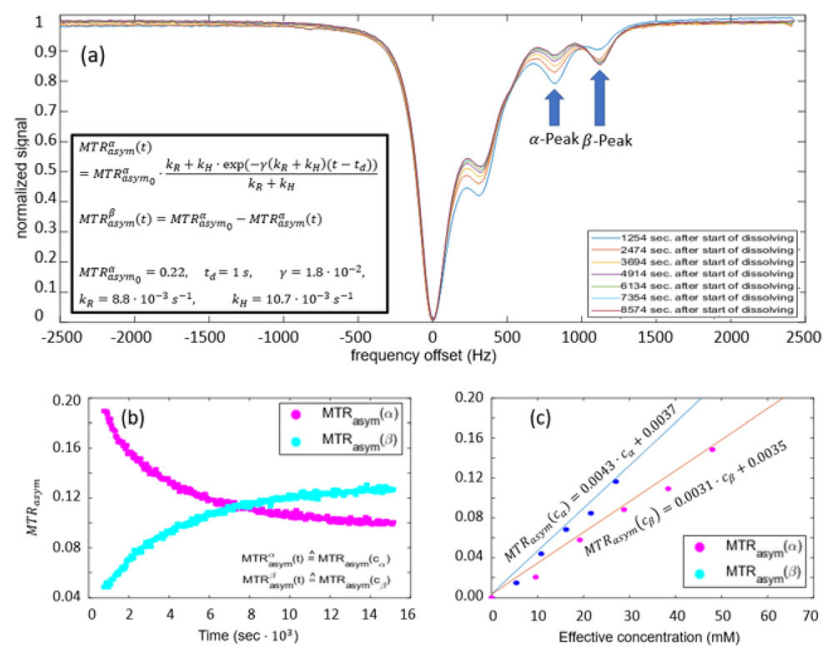

Figure 2: (a) Representative selection of CEST spectra measured every 20 minutes. The asymmetry analysis shows a decrease of the signal of the $\alpha$-isomer and an increase of the $\beta$-isomer over time, which was fitted by the modified model $\left(k_{R}, k_{H}\right.$ exchange rates) (b). The output was related to the results of the concentration series measurements, indicating a linear dependency between the effective concentration (used concentration weighted by isomer ratio at steady state) and the corresponding asymmetry value $\left(R^{2}=0.99\right)$ (c).

From the ratio of the two fits, including the correlation between concentration series and asymmetry, the isomer ratio $\mathrm{R}$ in the steady state can be calculated (Fig. 3): $R_{\text {calc }}=1.61 \pm 0.02$ (expected value $\left.\mathrm{R}_{\text {theo }}=1.78\right)$.

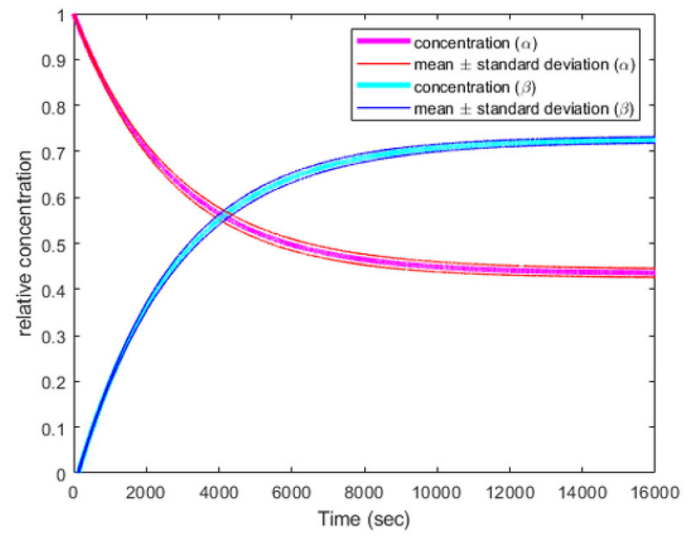

Figure 3: Mean and standard deviation of the relative concentration of the two glucose stereoisomers plotted over measurement time.

Discussion: Currently, the quantification of mutarotation kinetics by CEST LS shows an accuracy of approximately 90\%. To further improve the analysis, it is necessary to enhance the model by optimizing the sensitivity factor in dependency of Larmor frequency, concentration, $\mathrm{pH}$ and ionic environment.

Nevertheless, even in a simplified model assuming the sensitivity factor to be linear and equal for both exchanging resonance frequencies, glucose mutarotation can be measured by CEST LS and the isomer ratio can be verified in good agreement to conventionally measured values.

\section{References:}

[1] Xu X et al., Angew. Chem. 2013; 52(32).

[2] Chou C et al., J. Phys. Chem. A 2010, 114.

[3] Van Zijl P et al., Magn Reson Med. 2009, 61(6).

\section{S3.P23.}

\section{Open-source gradient response function and BO eddy current characterization}

\author{
*T. T. Fernandes ${ }^{1}$, A. Gaspar ${ }^{1}$, A. C. Freitas ${ }^{1}$, K. S. Ravi ${ }^{2}$,
} N. A. Silva ${ }^{3}$, S. Geethanath ${ }^{2}$, R. G. Nunes ${ }^{1}$

${ }^{1}$ University of Lisbon, Institute Superior Técnico, Lisbon, PT; ${ }^{2}$ Columbia University, Magnetic Resonance Research Center, New York, $N Y, I N$;

${ }^{3}$ Hospital da Luz, Department of Learning Health, Lisbon, PT

Introduction: The impact of eddy-currents (EC) [1] can be corrected by determining the gradient impulse response function (GIRF). Here we test recently introduced open source (OS) calibration pulse sequences [2] on $1.5 \mathrm{~T}$ and 3.0 $\mathrm{T}$ scanners, to which we added the ability to measure B0 EC with the method proposed in [3].

Methods: We implemented two pulse sequences to measure the GIRF: H1 (M1) [1, 4] and (M2) [3] using the OS tool PyPulseq [5]Fig. 1. To speed up the acquisition, we decreased the repetition time while spoiling undesirable echo pathways.The GIRF was measured using each method in two different systems in doped water phantoms, through autonomous MRI on a 3.0 T Siemens Prisma Fit [6] and in a Siemens Aera 1.5 T. The data were processed in Matlab ${ }^{\circledR}$ with an inhouse script making use of the GIRFOS_tool [6]. Finally, we explored the ability to measure the $\mathrm{B} 0 \mathrm{EC}$ ( $\mathrm{H} 0$ response function) with [5] on both scanners. As this estimation was found to be more sensitive to noise, a Savitzky-Golay filter was applied to the measurements and a regularization factor included during the deconvolution process. To test whether a plausible $\mathrm{H} 0$ function had been estimated, its convolution with the input gradient to produce a predicted B0 EC phase was compared with the measured phase. Code available at github.com/ ZemaTimoteo/GIRFOS_tool.
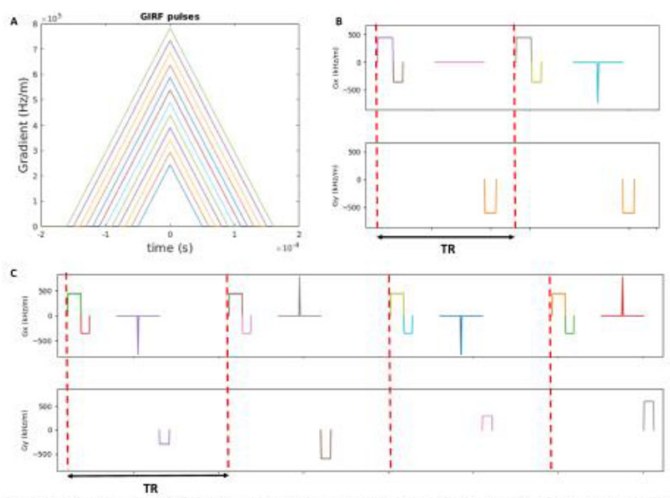

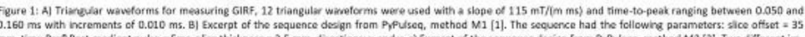

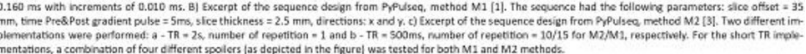

Results: The magnitude GIRF (H1) functions for both the $\mathrm{x}$ and $\mathrm{y}$ axes are depicted in Fig. 2 for both scanners and methods; in all cases the low-pass characteristics were observed as expected. As predicted, the $\mathrm{H} 1$ is a scanner specific function, and in most cases it was possible to obtain comparable curves using different methods and number of repeats; an exception is the shape of $\mathrm{H} 1$ for the $\mathrm{x}$ axis at $1.5 \mathrm{~T}$ as the estimated shapes differed between methods. Figure 2 also demonstrates that the profile of $\mathrm{H} 1$ was similar for long and short TR acquisitions, confirming that undesirable signal contributions could be avoided. 

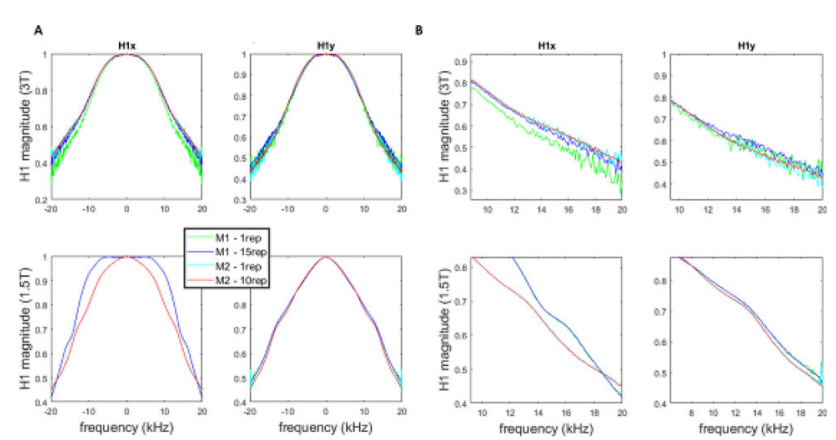

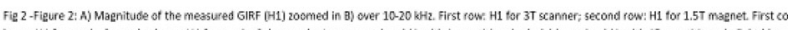

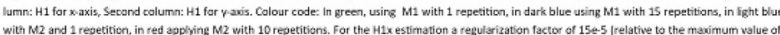
with $M 2$ and 1 repection, in red applying

The H0 functions depicted in Fig. 3 also display low-pass filter properties for both scanners and for the two measured axes. Comparison of the measured and predicted phases suggest that the implemented scheme is not yet suitable to capture fast phase polarity changes. The best post-processing approach for limiting the impact of noise was found to be scanner specific.
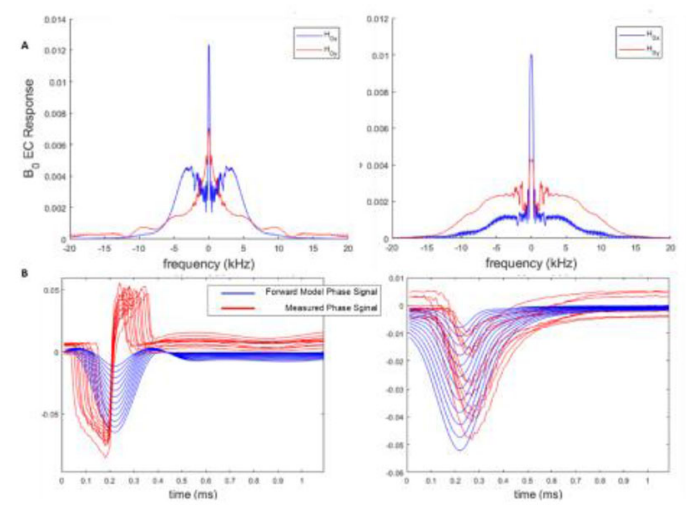

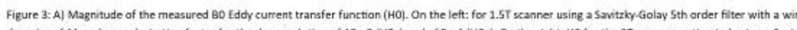

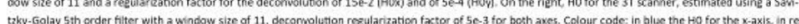

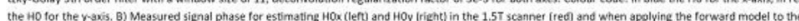
the Ho tor the rasks. B)

Discussion and conclusions: We demonstrated the ability to perform fast and reproducible GIRF and $\mathrm{HO}$ calibration measurements. At present, the measured $\mathrm{H} 0$ does not yet capture fast changes in phase polarity contrarily to [3]; a possible explanation is the limitation to use a longer dwell time of $10 \mu$ s (4 times larger) compared to the original implementation; the differences between methods also requires further investigations.

Acknowledgments: FCT grants UIDB/50009/2020,SFRH/BD/ 120006/2016 and LISBOA-01-0145-FEDER-029686

\section{References:}

1 Vannesjo MRM 2013,69.

2 Fernandes ISMRM 2021.

3 Robinson MRM 2019,81.

4 Duyn JMR 1998,153.

5 Ravi Op Sourc Softw 2019,4.

6 Ravi MRM 2020,73.

\section{S3.P24.}

Blurring artefact correction on MR images with spiral $k$-space by modifying $k$-space trajectory

\section{*O. B. YURDAKOS ${ }^{1}$, Y. GOKPEK ${ }^{1}$, O. Doganay ${ }^{1}$ \\ ${ }^{1}$ Health Sciences Institute, Department of Basic Oncology, Izmir, TR}

Introduction: The magnetic field inhomogeneities results in blurred MR image artefact due to the frequency off set with spiral k-space sampling [1]. The blurring artefact may be introduced by deviations in gradient imperfections that are normally corrected by the use of modified $\mathrm{k}$-space trajectories rather than the original $\mathrm{k}$-space trajectories for improving image resolution in the reconstruction process [2]. However, there is no general methodology to guide the correction of k-space necessary to achieve a robust offline blurring correction. The purpose of this study is to calculate and correct the blurring artefact due to the frequency off-set and/or gradient imperfections by modifying the original k-space trajectory.

Methods: The original spiral k-space[3], was modified multiplying original k-space by the correction functions including Stairs and Dirac Delta functions in k-space without changing the number of point as shown in Fig. 1. Then, Spearman"s correlation was used to measure the improvement of blurring artefact in comparison to a Reference image that does not include any of resonance blurring. Two sets of phantom images were used with $\Delta \mathrm{f}=100 \mathrm{~Hz}$ representing the blurred image and $\Delta \mathrm{f}=0 \mathrm{~Hz}$ representing the reference image. The blurred image was corrected with kernel step size of 0.01 from 1 to 10 . A line profile was selected from corrected images and compared to the reference image.

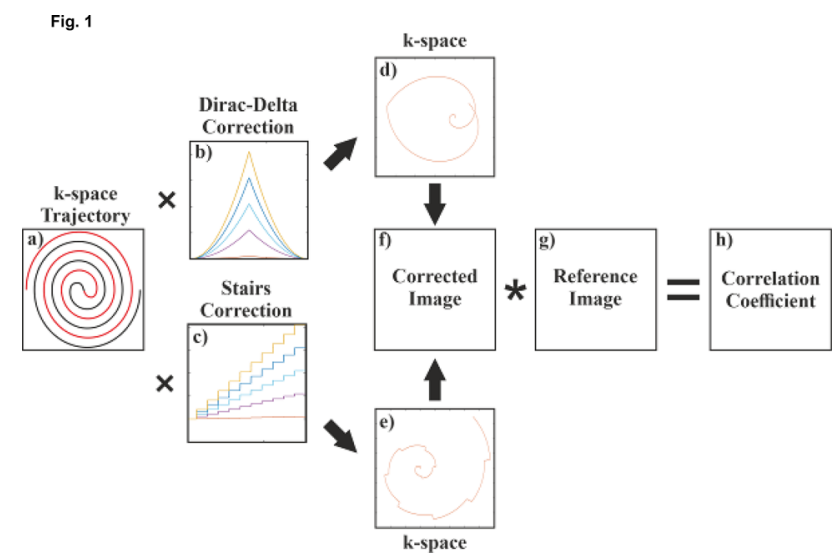

Results: Figure 2 shows the blurred image in (a) the improvement the image resolution as a function of Stairs in (b) and Dirac Delta in (c) with respect to the Reference image in (d). with the line profiles from point A to B (e). The best correlation in line profiles between the reference image and the corrected were summarized in Table 1, was 0.983 showing that the best kernel was 1.421 achieved by Stairs correction with having 4 steps providing an improvement of image resolution of approximately $80 \%$ with a small ringing artefact as shown in Fig. 2c. 


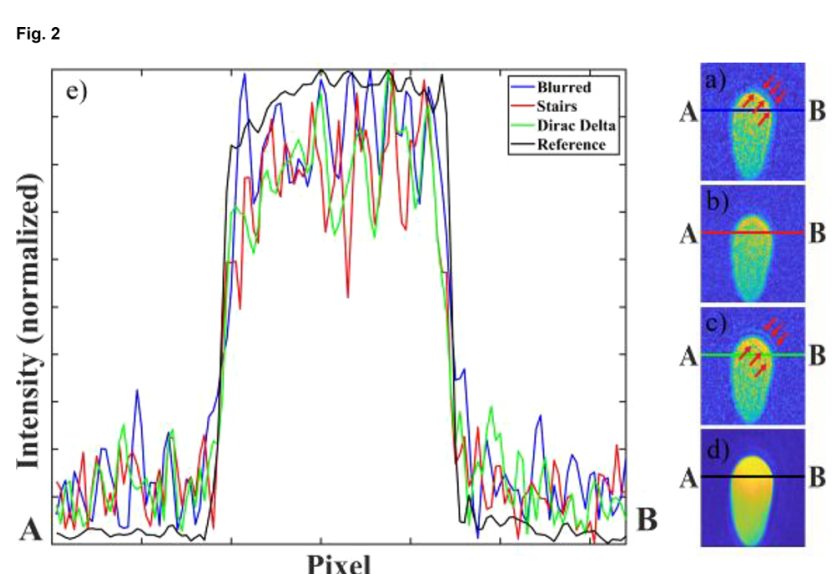

Discussion: Both Stairs and Dirac Delta functions were successful at correction of blurring artefact with an improvement of $80 \%$ and $60 \%$. Stairs function corrected image created a ringing artefact along the external and internal side of the phantom edges, seen in Fig. 3c. Dirac Delta function has created the best image in terms of increasing foreground intensity while having slightly less correlation coefficient compared to the Stairs function. Nevertheless, both corrected images have shown better spatial resolution than the blurred image.

Fig. 3

\begin{tabular}{|c|c|}
\hline $\begin{array}{c}\text { Relation } \\
\text { between images }\end{array}$ & $\begin{array}{c}\text { Correlation Coefficient } \\
\text { from A to B }\end{array}$ \\
\hline Blurred and Reference & $0.967(\mathrm{P}<0.005)$ \\
\hline Stairs and Reference & $0.983(\mathrm{P}<0.005)$ \\
\hline Dirac Delta and Reference & $0.979(\mathrm{P}<0.005)$ \\
\hline
\end{tabular}

Figure 1: Trajectory segmented deblurring. Deblurred images are reconstructed by the manipulation of $\mathrm{k}$-space trajectory multiplicated of chosen two functions (a) k-space trajectory, (b) Dirac Delta and (c) Stairs function from kernel $=1$ to kernel $=10$ (d) and (e) multiplied $\mathrm{k}$-space representation of one-interleave respectively (f) reconstructed images (g) blurrless Reference image and (h) calculated correlation coefficients.

Figure 2: (a) Blurred phantom image having additional frewuency off-set resulting in Blurring artefact at the contrast zone as shown with red arrows. Improved image modified by (b) Dirac Delta with a peak value at 5.10 (c) by Stairs at 1.42, red arrows shows the ringing. (d) Reference image (e) Normalized linear line profiles of blurred, Stairs, Dirac Delta and reference image for each one from the point A to $\mathrm{B}$.

Table 1. Correlation coefficients of blurred, Stairs, Dirac Delta corrected images compared to reference image.

\section{References}

Nolte, T., et al., Spiral blurring correction with water-fat separation for magnetic resonance fingerprinting in the breast. Magn Reson Med, 2020. 83(4): p. 1192-1207.

Pedersen, J.O., et al., Inductive measurement and encoding of k-space trajectories in MR raw data. MAGMA, 2019. 32(6): p. 655-667.

Doganay, O., et al., Fast dynamic ventilation MRI of hyperpolarized (129) Xe using spiral imaging. Magn Reson Med, 2018. 79(5): p. $2597-2606$.

\section{S3.P25.}

Quantitative and qualitative assessment of retrospective motion correction with residual learning for clinical 3D magnetic resonance fingerprinting

\author{
G. Donatelli ${ }^{1,2}$, P. Bosco ${ }^{3}$, C. Pirkl ${ }^{4,5}$, M. Cencini ${ }^{3,2}$, C. Ticci ${ }^{3}$, \\ G. Migaleddu ${ }^{1}$, C. D’Amelio ${ }^{6}$, P. Cecchi ${ }^{1,2}$, L. Biagi $^{3,2}$, \\ J. Kurzawski, ${ }^{3,2}$, R. Pasquariello ${ }^{3}$, R. Battini ${ }^{3}$, G. Buonincontri ${ }^{3}$, \\ B. Menze $e^{4,7}$, M. Menzel ${ }^{4,5}$, M. Cosottini ${ }^{6}$, M. Tosetti ${ }^{3,2}, * M$. \\ Costagli $^{8,3}$ \\ ${ }^{1}$ Azienda Ospedaliero Universitaria Pisana, Pisa, IT; \\ ${ }^{2}$ Imago 7 Research Foundation, Pisa, IT; \\ ${ }^{3}$ IRCCS Stella Maris, Pisa, IT; \\ ${ }^{4}$ Technical University of Munich, Garching, DE; \\ ${ }^{5}$ GE Healthcare, Munich, DE; \\ ${ }^{6}$ University of Pisa, Pisa, IT; \\ ${ }^{7}$ University of Zurich, Zurich, DE; \\ ${ }^{8}$ University of Genoa, Genoa, IT
}

Introduction: A 3D Magnetic Resonance Fingerprinting (MRF) acquisition scheme has recently been proposed to enable retrospective motion correction for improved quantitative T1, T2 and proton density (PD) mapping [1]. In this study we evaluate the further improvement provided by the use of a convolutional neural network $(\mathrm{CNN})$ to learn and correct for residual motion artifacts [2].

Methods: 3D MRF acquisitions [3,1] of 26 patients with negative radiological report were included in this study. 13 were paediatric patients (age: 4-10, acquired on an $1.5 \mathrm{~T}$ HDxT scanner) while 13 were adults (age: 19-74, acquired on a 3 T MR750 scanner). Motioncorrected k-space data [1] were transformed to image space and matched with a pre-computed dictionary of MR signal evolutions to obtain quantitative maps of T1, T2 and PD [4]. Maps went through a multi-scale 3D patch-based $\mathrm{CNN}$ to correct for residual motion artifacts [2]: using artificially motion-corrupted data of healthy adult volunteers, the $\mathrm{CNN}$ was trained to output residual maps, i.e. difference between motion-corrupted and motion-free maps, which were then used to produce corrected maps. Synthetic images mimicking conventional MRI contrasts were generated from the T1, T2 and PD maps, both before and after CNN-based motion correction, by using the equations describing signal intensities of clinical T1w FSPGR, MP2RAGE, T2-FLAIR, T2-FSE sequences.

One neuroradiologist evaluated and compared the synthetic contrasts before and after the CNN-based motion correction. A 6-point scale (0: not assessable; 5: very good) was used to assess image quality and anatomical detail of each sequence in each lobe, limbic system, basal ganglia and cerebellum. The Wilcoxon signed rank test was used to assess differences in assigned scores before and after correction. WM-GM contrast and SNR were computed [5] on maps of T1, T2 and PD, as well as on synthetic FSPGR, MP2RAGE, T2-FLAIR, T2-FSE images before and after the use of the $\mathrm{CNN}$, by using automated tissue segmentations of conventional $\mathrm{T} 1 \mathrm{w}$ images coregistered to the MRF data.

Funding: Grant GR-2016-02361693 (Italian Ministry of Health).

Results: A significant improvement in image quality was observed by radiological evaluation in images that underwent $\mathrm{CNN}$-based motion correction $(\mathrm{p}<0.005$ uncorrected in all comparisons with the exception of the basal ganglia in synthetic MP2RAGE images; Fig. 1 and 2). 


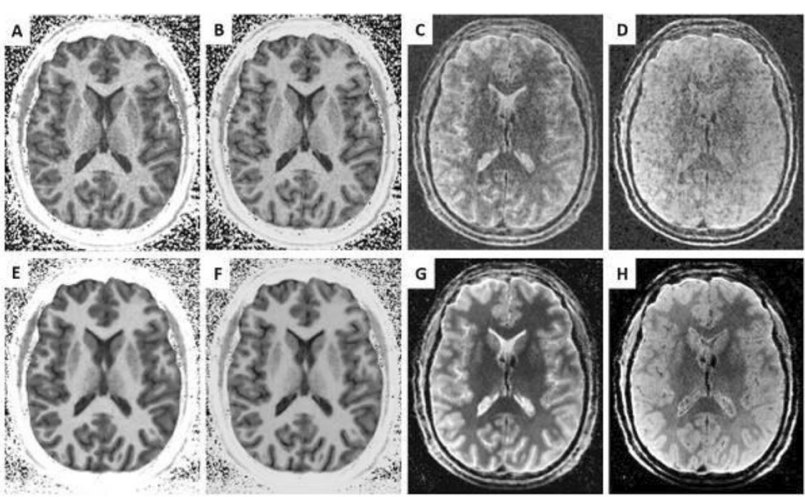

Figure 1. Images of a representative adult patient. Synthetic T1-FSPGR (A, E), T1-MP2RAGE (B, F), T2 FSE (C, G) and T2-FLAIR (D, H) images were obtained from quantitative maps both before (upper row) and after (bottom row) the use of the CNN.

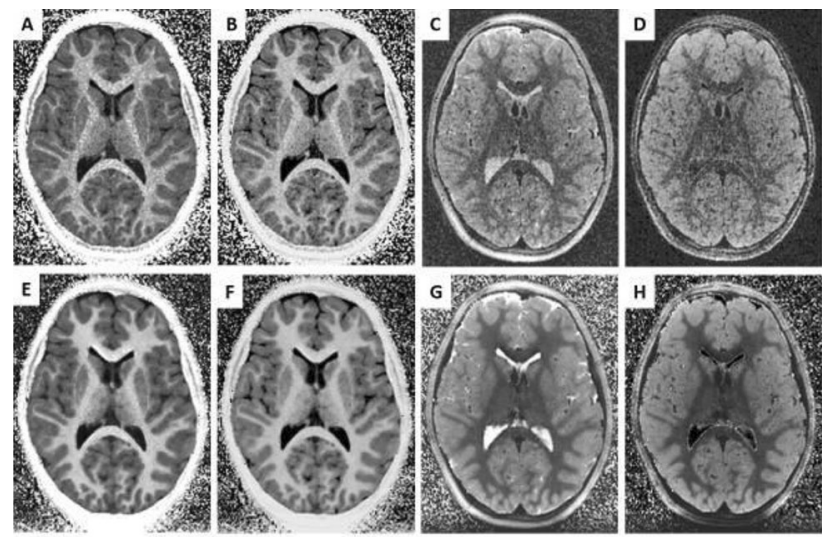

Figure 2. Images of a representative paediatric patient. Synthetic T1-FSPGR (A, E), T1-MP2RAGE (B, F), T2-FSE (C, G) and T2-FLAIR (D, H) images were obtained from quantitative maps both
before (upper row) and after (bottom row) the use of the CNN.

The use of the CNN determined a significant increase in WM-GM contrast and SNR in most cases (Fig. 3).

\begin{tabular}{|c|c|c|c|c|c|c|}
\hline \multirow[t]{2}{*}{$1.5 \mathrm{~T}$} & \multicolumn{3}{|c|}{ WM-GM tissue contrast } & \multicolumn{3}{|c|}{$\overline{S N R}$} \\
\hline & cerebrum & cerebellum & deep nuclei & cerebrum & cerebellum & brainstem \\
\hline T1-map & 8.1 & 24.7 & -10.2 & 3.3 & $\overline{14.4}$ & -12.5 \\
\hline T2-map & 49.1 & 74.1 & 81.1 & 24.3 & 67.8 & 103.7 \\
\hline PD-map & 25.6 & 40.7 & 135.3 & 48.8 & 76.7 & 92.2 \\
\hline T1-FSPGR & 14.2 & 39.4 & -4.4 & 10.9 & 31.9 & 10.5 \\
\hline T1-MP2RAGE & 9.9 & 28.8 & -8.7 & 6.2 & 21.6 & -6.8 \\
\hline T2-FLAIR & 32.0 & 28.4 & 25.1 & 4.3 & 12.5 & 8.2 \\
\hline T2-FSE & 23.4 & 23.4 & 32.5 & 2.4 & 11.8 & 5.4 \\
\hline
\end{tabular}

\begin{tabular}{|l|ccc|ccc|}
\cline { 2 - 7 } \multicolumn{1}{c|}{3} & \multicolumn{3}{c|}{ WM-GM tissue contrast } & \multicolumn{3}{c|}{ SNR } \\
\cline { 2 - 7 } \multicolumn{1}{c|}{} & cerebrum & cerebellum & deep nuclei & cerebrum & cerebellum & brainstem \\
\hline T1-map & 4.6 & 9.2 & 2.7 & 2.0 & -1.7 & -0.6 \\
T2-map & 25.4 & 24.7 & 38.8 & 45.6 & 76.0 & 93.4 \\
PD-map & 74.0 & 214.8 & 32.8 & 38.3 & 54.8 & 33.8 \\
T1-FSPGR & 4.3 & 4.1 & 2.1 & 4.5 & 0.4 & 0.2 \\
T1-MP2RAGE & 4.6 & 9.5 & 2.2 & 6.2 & 1.9 & 3.4 \\
T2-FLAIR & 19.3 & 13.8 & 14.4 & 16.9 & 22.9 & 37.7 \\
T2-FSE & 9.4 & 13.0 & 11.2 & 14.5 & 21.0 & 29.1 \\
\hline
\end{tabular}

Figure 3: percent improvement in WM-GM contrast and SNR after the application of CNN-based motion correction. Colored values indicate statistically significant differences in paired t-test (p<0.005, uncorrected)

Discussion: Residual motion learning and correction with a CNN agnostic to the anatomy of evaluated patients enables a significant further improvement of quantitative maps and synthetic radiological contrasts obtained with MRF.

\section{References}

[1] Kurzawski, doi:10.1002/mrm.28301

[2] Pirkl, MIDL 2021, https://openreview.net/forum?id=hxgQM71 AuRA

[3] Gómez, doi:10.1038/s41598-020-70789-2

[4] Ma, doi:10.1038/nature11971

[5] Esteban, doi:10.1371/journal.pone.0184661

\section{S3.P26. \\ Respiratory motion-corrected T1 mapping of the abdomen}

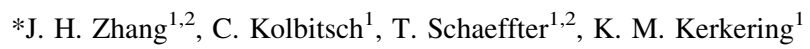 \\ ${ }^{1}$ Physikalisch-Technische Bundesanstalt, Berlin, DE; \\ ${ }^{2}$ Technische Universität Berlin, Department of Biomedical Engineering, Berlin, DE}

Introduction: Quantitative T1 mapping of the abdomen is a noninvasive technique that can help to characterize pathologies and monitor therapy effects in liver and kidney ${ }^{1}$. However, respiratory motion due to breathing of the patient can lead to inaccurate T1 quantification $^{2}$. For an accurate diagnosis, it is important to minimize such motion artifacts. Commonly, T1 maps are acquired during a breath hold, which is often strenuous for patients. Here, we present a respiratory motion correction approach that uses an image-based navigator for improved correction of respiratory motion. The resulting motion-corrected T1 maps were evaluated in two healthy volunteers. Methods: Data was acquired in two healthy volunteers $(\mathrm{m}-29 \mathrm{y}$, $\mathrm{f}-26 \mathrm{y}$ ) at a $3 \mathrm{~T}$ MRI Scanner (Siemens Healthineers). We used a multi-step approach (Fig. 1) for motion-corrected T1 mapping similar as proposed for cardiac T1 mapping ${ }^{3}$. We used a continuous 2D golden-radial trajectory ${ }^{4}$ and applied 7 equally spaced inversion pulses during a scan time of $16 \mathrm{~s}(\mathrm{TR} / \mathrm{TE}=4.9 / 2.2 \mathrm{~ms}$, flip angle $=$ $5^{\circ}$, resolution $\left.=1.3 \times 1.3 \times 8 \mathrm{~mm}^{3}\right)$. In a first step, we reconstructed real-time images ( $100 \mathrm{~ms}$ per image) using iterative SENSE ${ }^{5}$. The position of the diaphragm was tracked and spline-interpolated to obtain an image-based respiratory navigator. Next, we used this image-based navigator to bin the original k-space data into 10 respiratory motion states for the reconstruction of respiratory-resolved images at higher spatial resolution. Respiratory motion fields were estimated using non-rigid spline-based image registration ${ }^{6}$. In the last step, we carried out a motion-corrected image reconstruction of images at different inversion times (TI images). Motion-corrected T1 maps were calculated as $\mathrm{T} 1$ values for each pixel using the inversion recovery Look-Locker concept ${ }^{7}$.

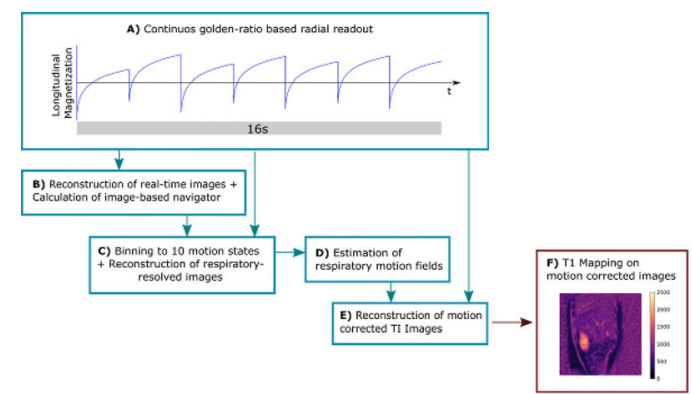

Figure 1: Workflow for motion-corrected T1 mapping of the abdomen. A) Data is continuously acquired while applying seven inversion rf-pulses. B) Image-based navigator is calculated from reconstructed real-time images. C) The navigator signal is used to bin the data into 10 motion states and to reconstruct the respiratory-resolved images accordingly. D) Respiratory motion fields are estimated from the respiratory-resolved images. E) Motion correction is applied during the reconstruction of Tl images. F) Final motion-corrected T1 map of the abdomen 
Results: The calculation of the image-based navigator was successful in both volunteers. The image-based navigator (Fig. 2) shows 4 breathing cycles during $16 \mathrm{~s}$. Fig. 2 further illustrates the reconstruction of respiratory-resolved images using the image-based navigator. The registered respiratory-resolved images show that motion correction was successful. T1 maps for two volunteers are displayed in Fig. 3. Compared to the uncorrected T1 maps, the corrected $\mathrm{T} 1$ maps show less artifacts at the diaphragm and sharper blood vessels and kidney structures. T1 maps with breath hold are given as reference.

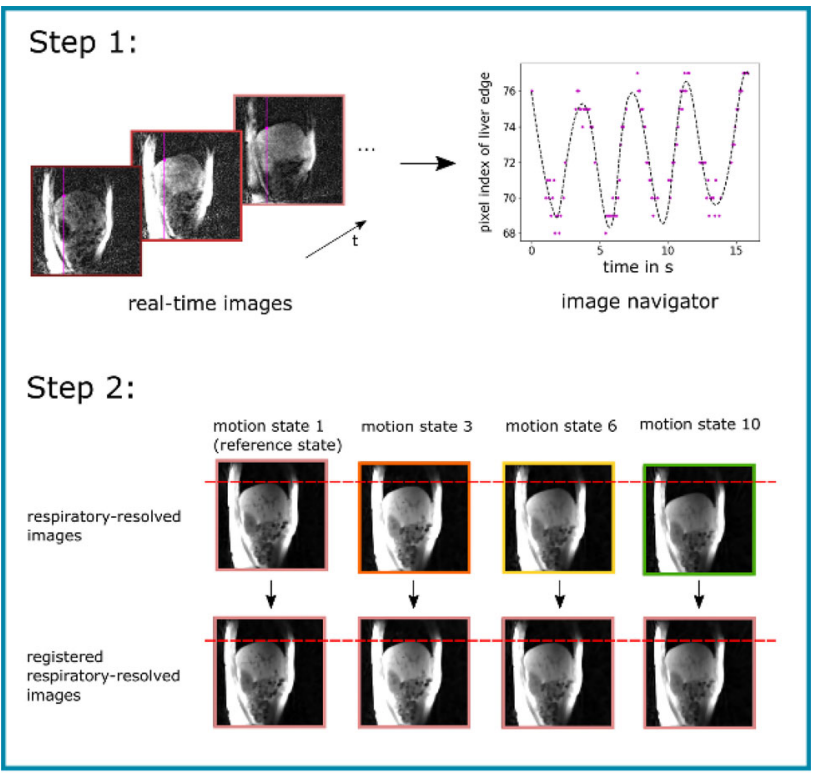

Figure 2: Step 1 shows the reconstructed real-time images on the left side. Along a vertical line, the diaphragm (marked with magenta colored point) is determined over time. For the image navigator, the position of the diaphragm is then plotted over time. The spline interpolation of these points is depicted by the black dashed line. The image navigator is used in step 2 to bin the data into 10 motion states. At the top, the respiratory-resolved images are shown for motion state 1,3,6 and 10. At the bottom, the images were registered using the estimated motion fields for mation correction. The dashed red line indicates the position of the diaphragm, which changes for each motion state of the respiratory resolved images, but stays constant for the registered ones.
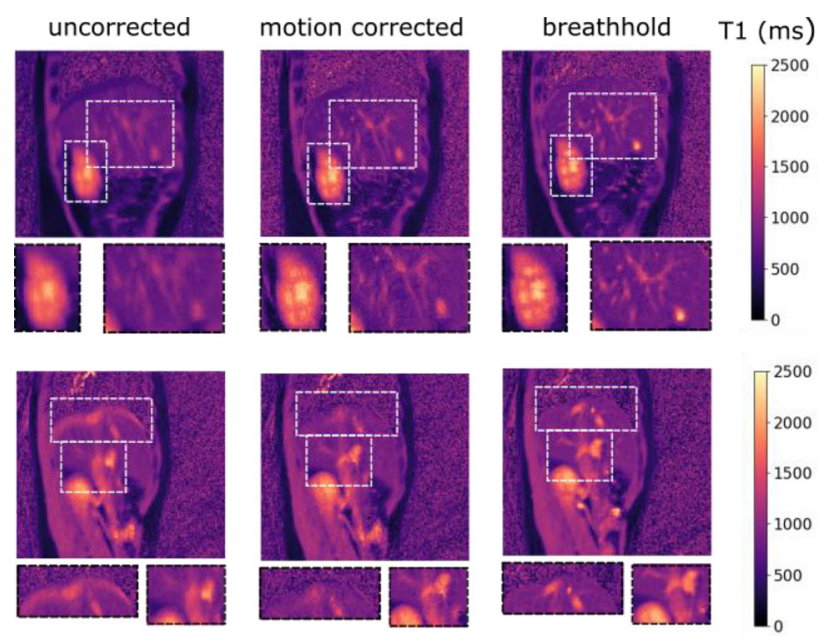

Figure 3: In-vivo T1 maps without motion correction (left), with motion correction (middle) and with breath hold (right). The white dotted squares are a zoomed view of blood vessels, kidney and diaphragm. For both volunteers, the corrected T1 maps show less artifacts at the diaphragm and sharper blood vessels and kidney structure than the uncorrected ones. Breath hold T1 maps are given as reference.
Discussion/conclusion: In this study, feasibility for respiratory motion-corrected T1 mapping in $16 \mathrm{~s}$ with a voxel resolution of $1.3 \times 1.3 \times 8 \mathrm{~mm}^{3}$ at $3 \mathrm{~T}$ was shown, using continuous data acquisition and an image-based navigator for motion correction.

\section{References:}

1 Shah et al., Radiographics, 2011:31:867-880.

2 McClelland et al., Med Image Anal. 2013:17:19-42.

3 Becker et al., MRM, 2019:81:1080-1091.

4 Winkelmann et al., IEEE TMI. 2007:26:68-76.

5 Pruessmann et al., MRM 2001:46:638-651.

6 Rueckert et al., IEEE TMI, 1999:18:712-721.

7 Messroghli et al., MRM, 2004:52:141-146.

\section{S3.P27. \\ Image registration shortcut for prospective motion correction using unseparated multiband slices}

\author{
*S. Winata ${ }^{1}$, D. C. Hoinkiss ${ }^{2}$, G. A. Keith ${ }^{1}$, D. A. Porter ${ }^{1}$, \\ M. Günther ${ }^{2}$ \\ ${ }^{1}$ University of Glasgow, Imaging Centre of Excellence, Glasgow, GB; \\ ${ }^{2}$ Fraunhofer Institute of Digital Medicine (MEVIS), Bremen, DE
}

Introduction: When multiband (MB) excitation is used, image-registration based prospective motion correction (PMC) is typically performed after the MB-acquired slices are separated into individual slices. This study explores the possibility of programming a pulse sequence to register the unseparated/collapsed $\mathrm{MB}$ slices in a Prospective Acquisition CorrEction (PACE) scheme and explore its effects. In PACE, translational and rotational motion estimates are calculated by registering the current imaging volume to a Reference volume. These estimates are then used to adjust the slice position and orientation [1]. This also results in a time delay, in addition to the mutlislice separation-related delay [2]. Registering the volumes without a prior separation of the MB images will allow for a quicker processing and an increase in temporal resolution.

Methods: The study was performed using retrospective reconstruction of scan data acquired from a phantom on a Siemens MAGNETOM Terra $7 \mathrm{~T}$ scanner. A 2D EPI with BOLD (blood oxygen level dependent) contrast time series was acquired (30 volumes, 36 slices, MB factor 3, TR/TE $2210 / 32 \mathrm{~ms}$, matrix size $64 \times 64$, slice thickness $3 \mathrm{~mm}$ ). Using a processing algorithm developed in the Siemens' Image Calculation Environment, volumeto-volume registration (as in PACE [1]) was performed for two different cases: 1) MB slices are unseparated; 2) MB slices are separated using the slice-GRAPPA method. Translational and rotational parameters were then calculated.

Results: Figure 1 shows a sample of the unseparated and separated slices. Figure 2 shows part of the results with the $\mathrm{x}$-axis translation and $y$-axis rotation estimates for the unseparated (blue) and separated (red) image registration cases. 


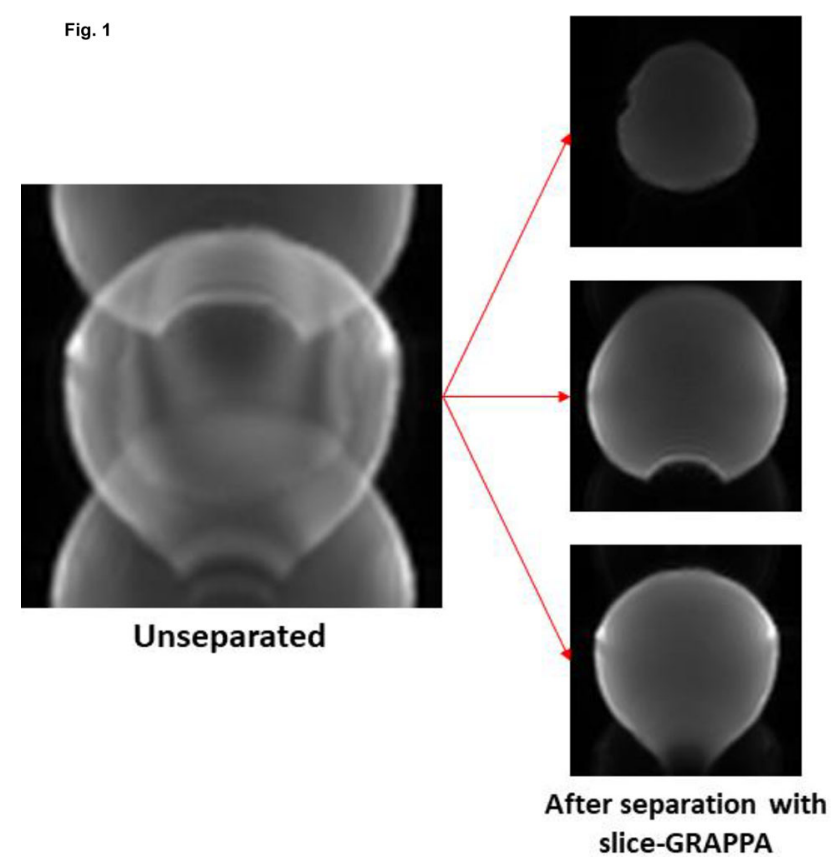

Fig. 2
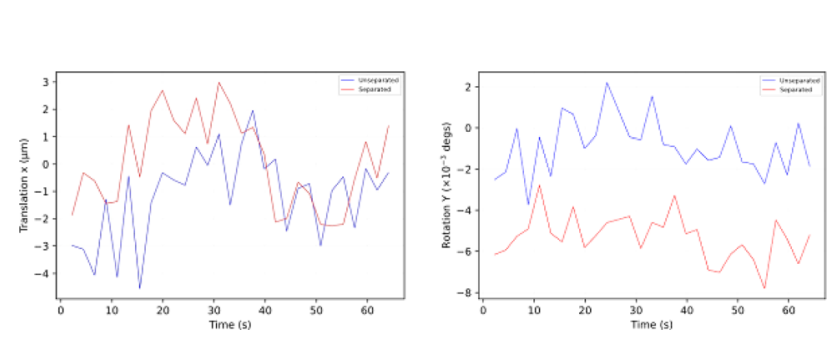

\begin{abstract}
slice-GRAPPA
\end{abstract}
Discussion: It is possible to integrate the technique into the processing pipeline. Figure 2 shows that the unseparated slice registration's shift pattern is in close agreement with the separated slice registration, however the parameter values are small due to the phantom study's inherent limitations. Volunteer studies involving motion protocols were planned to validate the method further in brain imaging, but these have been postponed due to ongoing scanner and recruitment restrictions related to the COVID-19 pandemic. The use of a subset of slices instead of a full volume for registration akin to in MS-PACE[3] will also be evaluated. Overall, pending further validation, it shows a potential in applying direct registration of unseparated images for PMC.

Figure 1. An unseparated MB slice and its corresponding slices after slice-GRAPPA separation.

Figure 2. Image registration results for translation along the $\mathrm{x}$-axis and rotation along the $y$-axis. The red curve shows the separated slice registration, while the blue shows the unseparated slice registration.

\section{References}

[1] Thesen S et al. Magn Reson Med. 2000;44(3):457-65.

[2] Hoinkiss DC et al. Neuroimage. 2019;200:159-73.

[3] Hoinkiss DC, Porter DA. Magn Reson Med. 2017;78(6):2127-35. 


\section{S4.P1.}

Separating tissue transit effects to improve robustness of multi-TE ASL-based blood brain barrier integrity measurements

\author{
*A. Mahroo ${ }^{1}$, M. A. Buck ${ }^{1}$, J. Huber ${ }^{1}$, M. Günther ${ }^{1,2}$ \\ ${ }^{I}$ Fraunhofer Institute of Digital Medicine (MEVIS), Division of MR \\ Physics, Bremen, DE; \\ ${ }^{2}$ University of Bremen, Faculty 01 (Physics, Electrical Engineering), \\ Bremen, $D E$
}

Introduction: Arterial spin labeling (ASL) utilizes water as an endogenous tracer and thus can act as a sensitive method for detecting minute and subtle damage to the blood-brain barrier (BBB). The general kinetic model assumes instantaneous exchange of water molecules with surrounding tissue. But even restricted exchange does not fully describe reality, as labeled blood has to first reach the capillaries within a voxel, before exchange with tissue takes place. In this work, we have extended the T2-based two-compartment model (2-CM) [1] by incorporating an additional delay called "Intra-voxel Transit Time" (ITT) to account for transit within the voxel. The main goal of incorporating ITT is to separate the two distinct mechanisms of tissue transit and exchange time (Texch). We compare extended model with 2-CM using simulations and in vivo data. Modeling details can be found in a separate abstract (Nr. 085).

Methods: Data was simulated for two multi-TE Hadamard [2] (HAD) ASL protocols: HAD-8; SBD: $400 \mathrm{~ms}$, PLD = $200 \mathrm{~ms} \&$ HAD-4; $\mathrm{SBD}=1000 \mathrm{~ms}, \quad \mathrm{PLD}=600 \mathrm{~ms}$ and both with $8 \times \mathrm{TEs}=$ 13.8-207.6 ms, increment of $27.7 \mathrm{~ms}$. Both datasets were generated with arterial transit time: $500 \leq \mathrm{ATT} \leq 2500 \mathrm{~ms}$, Texch $=10 \leq$ Texch $\leq 1000 \mathrm{~ms} ; \quad$ ITT $=200 \mathrm{~ms}$ and $\mathrm{CBF}=60 \mathrm{ml} / 100 \mathrm{~g} / \mathrm{min}$. Texch error in terms of percentage deviation was calculated relative to the simulated Texch value.

In-vivo data from ten healthy subjects ( 4 females, aged $28-40$ years) was acquired with the same protocol at $3 \mathrm{~T}$ (MAGNETOM Skyra, SIEMENS Healthineers AG). Data was analyzed with FSL fabber [3] and Texch was estimated with both models. Extended model also estimated ITT. Mean gray matter (GM) values were calculated.

Results: Figure 1 shows the Texch error estimates from the two models. As can be seen, Texch is overestimated with the 2-CM. Figure 2(A) shows the Texch and ITT maps from a representative subject. Figure 2(B) shows the mean GM Texch estimated with the two models $(\mathrm{P}<0.002)$. Table 1 shows mean GM Texch and ITT values.
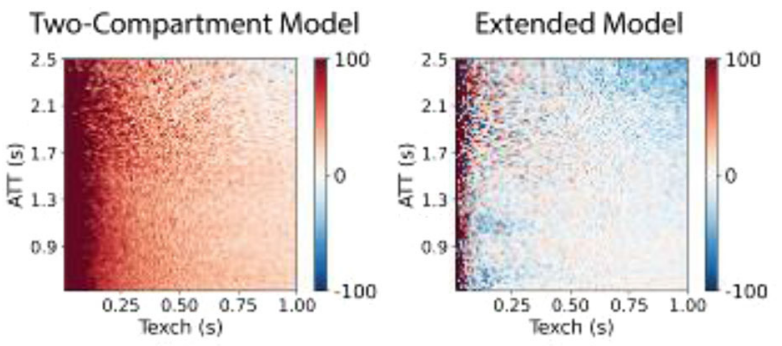

Figure 1: Texch percentage deviation error for data simulated with ITT $=200 \mathrm{~ms}$. Two-compartment model not considering ITT, shows more error for lower Texch values ( $<300 \mathrm{~ms})$.
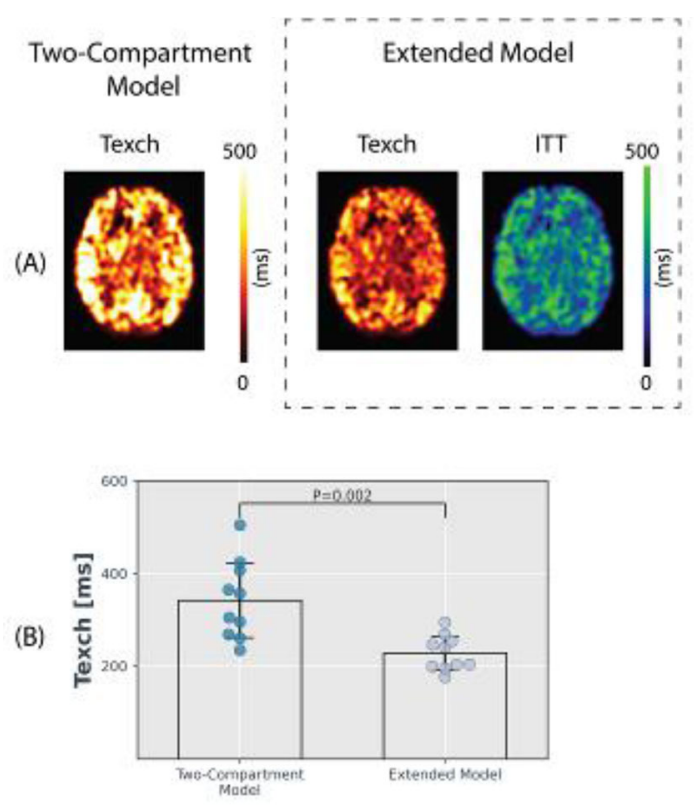

Figure 2: (A) Texch and ITT maps from a representative volunteer. (B) Mean gray matter Texch values and error (standard deviation (SD)) from 10 subjects. Mean Texch and associated SD are smaller for the extended model.

Discussion and conclusion: Data was simulated with ITT $=200 \mathrm{~ms}$ and 2-CM not considering ITT, overestimates Texch especially for lower Texch values $(<300 \mathrm{~ms})$. This shows that fast exchange dynamics - which would be expected in a disrupted BBB-show the highest error and are partially disguised by the ignorance of ITT. This highlights an important issue that if the tissue transit effects are not modelled carefully, this may lead to a low sensitivity of the technique to differentiate a disrupted BBB from a healthy one.

Fig. 3

Table 1: Mean gray matter Texch (ms) and ITT (ms) values.

\begin{tabular}{lll}
\hline Parameter & $\begin{array}{l}\text { Two-Compartment } \\
\text { Model }\end{array}$ & Extended Model \\
\hline Texch & $342 \pm 85.3$ & $227.9 \pm 37.9$ \\
ITT & - & $310.3 \pm 52.9$ \\
\hline
\end{tabular}

The extended model shows stable results for in-vivo data. The mean GM Texch estimated with $2-\mathrm{CM}(342 \pm 85.3)$ was $50.6 \%$ higher relative to the extended model $(227.9 \pm 37.9, P=0.002)$. Mean GM ITT was $310.3 \pm 52.9 \mathrm{~ms}$. Robustness of Texch estimation as a proxy measure of BBB permeability can be improved by separating transit effects from the exchange dynamics.

\section{References}

1. Gregori et al., JMRI 2013, 37(2).

2. Günther et al., ISMRM 2007, 380.

3. Chappell et al., IEEE 2008, 57(1). 


\section{S4.P2.}

\section{An ExploreASL toolbox for DICOM to BIDS conversion}

*M. Stritt ${ }^{1,2}$, B. Padrela ${ }^{2}$, M. Dijsselhof ${ }^{2}$, P. Clement $^{3}$, A. OliverTaylor $^{4}$, F. Barkhof ${ }^{2}$, X. Golay ${ }^{4}$, M. Günther ${ }^{5}$, J. Gregori ${ }^{1}$, J. Petr $^{6}$, H. J. Mutsaerts ${ }^{2}$

${ }^{l}$ mediri GmbH, Heidelberg, DE;

${ }^{2}$ Amsterdam University Medical Center, Department of Radiology and Nuclear Medicine, Amsterdam, NL;

${ }^{3}$ Ghent University, Department of Radiology and Nuclear Medicine, Ghent, BE;

${ }^{4}$ Gold Standard Phantoms Ltd, London, GB;

${ }^{5}$ University of Bremen, Bremen, DE;

${ }^{6}$ Helmholtz, Center Dresden-Rossendorf, Dresden, DE

Introduction: Most data scientists spend about $45 \%$ of their time on tasks like data loading and cleansing [1]. This is especially problematic in ASL-MRI, which is available in a variety of acquisition flavors, export forms, and differs between vendors and laboratories. ASL-BIDS [2] has recently addressed this issue by defining a standardized data structure. Current DICOM to BIDS conversion tools are still working on a complete support of ASL-BIDS [3]. Here, we introduce a tool within ExploreASL [4], that converts DICOMs to ASL-BIDS using DCMTK [5] and dem2niiX [6].

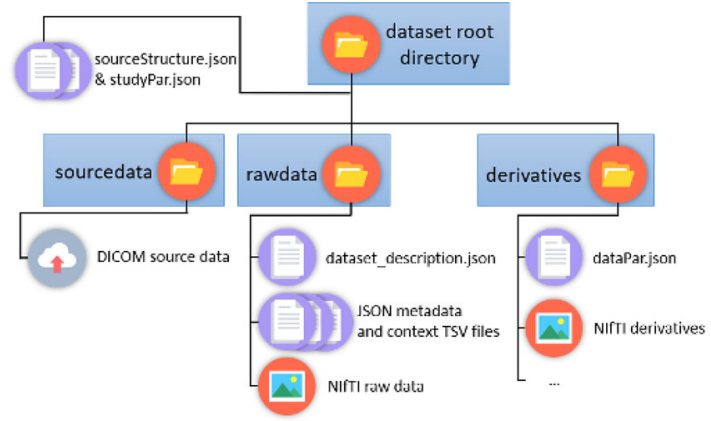

Figure 1: Date structures and descriptive text files within ASL-BIDS. To reduce the complexity, only the most relevant data structures and text files are shown. The sourceStructure JSON file describes the sourcedata and is used for the DCM2NII conversion. The studyPar JSON file describes the scanned study. If not defined and is used for the DCM2NII conversion. The studyPar JSON file describes the scanned study. If not defined manually, both dataset_description and dataPar JSON files will be generated automatically within the third
and fourth import module. The dataPar JSON file is used to configure ExploreALS pipeline settings. Detailed descriptions can be found on the GitHub website and within the corresponding code documentation.

Methods: This tool comprises four modules. The DCM2NII module flexibly converts DICOMs to NIfTI images and descriptive JSONs. In this conversion step, relevant fields (Fig. 2) are extracted. Full ASLBIDS rawdata are generated by the second module. Output of the first module is improved by interpretation of vendor specific fields, such as Philips scale slopes and Siemens Phoenix protocols. The tool validates the metadata files for completeness according to BIDS 1.6.0. Module three allows defacing of structural scans. Module four converts the BIDS rawdata to the ExploreASL format. To reliably support a variety of ASL sequences, anonymized single-subject data from 48 previously-processed studies were converted (Table 1). Integration and unit testing scripts were written to consistently inspect data transfer results.

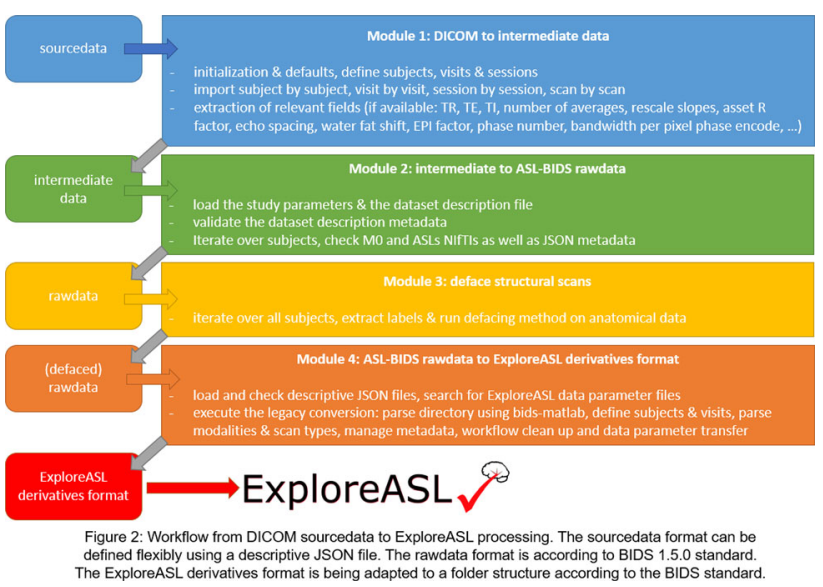

Fig. 3

\begin{tabular}{|c|c|c|c|c|}
\hline Vendor & Model & Labeling type & Sequence & Software version \\
\hline \multirow[t]{4}{*}{ GE } & \multirow[t]{2}{*}{ Signa HDxt } & \multirow[t]{2}{*}{ PCASL } & 2D EPI & 23 LX MR (HD23.0) \\
\hline & & & 3D SPIRAL & 14 LX MR (14.0), 15 LX MR (15.0) \\
\hline & \multirow[t]{2}{*}{ Discovery MR750 } & \multirow[t]{2}{*}{ PCASL } & \multirow[t]{2}{*}{ 3D SPIRAL } & 23 LXMR (DV23.1), 23 LX MR (DV22.0) \\
\hline & & & & 24 LX MR (DV24.0) \\
\hline \multirow[t]{5}{*}{ Philips } & Achieva & PCASL & 2D EPI & 3.2.1.1, 3.2.3.0, 3.2.3.1, 3.2.3.2, 5.1.7.2 \\
\hline & Ingenuity & PCASL & 2D EPI & $3.2 .2 .0,3.2 .2 .1$ \\
\hline & \multirow[t]{2}{*}{ Ingenia } & PCASL & 2D EPI & 4.1.2.1, 5.3.1.1, 5.1.8.2, 5.1.9.1 \\
\hline & & PCASL & 3D GRASE & $5.4 .1 .0,5.4 .0 .2,5.1 .7 .2$ \\
\hline & Intera & PCASL & 2D EPI & 3.2 .1 .1 \\
\hline \multirow[t]{8}{*}{ Siemens } & \multirow[t]{2}{*}{ Skyra } & PCASL & 3D GRASE & VD13A \\
\hline & & PASL & 3D GRASE & VE11C \\
\hline & \multirow[t]{3}{*}{ TrioTim } & PASL & 2D EPI & VB15A, VB17A, VB19A \\
\hline & & PCASL & 2D EPI & VB17A \\
\hline & & PCASL & 3D GRASE & VB17A \\
\hline & Verio & PASL & 2D EPI & VB17A \\
\hline & \multirow[t]{2}{*}{ Prisma } & PASL & 3D GRASE & MR E11, VE11C \\
\hline & & PCASL & 3D GRASE & VD13D, VE11C \\
\hline
\end{tabular}

Table 1: Simplified overview of ASL "flavors" used for consistent unit and integration testing. The table shows various vendor, model, labeling type and software version combinations. Additional differences between some of the "flavors", like differing encoding schemes or minor differences of acquisition settings, were omitted to reduce complexity. Included sequence types are product, work in progress (WIP) and concept for production (C2P) sequences.

Results: Improved processing parallelization, improved reproducibility, and platform-independence were achieved through dockerization of ExploreASL including the ASL-BIDS conversion tool. ExploreASL v1.7.0 fully supported the conversion of the sequences in the ASL consensus paper and ASL-BIDS 1.6.0. A variety of relevant fields were read from DICOM metadata (Fig. 2). Testing was done internally using datasets from 10 different scanners of 3 vendors with 3 different sequences and 25 software versions.

Discussion: This toolbox improves ASL data curation, creating an easy-to-use tool to convert DICOM source data. The described workflow creates a standardized structure ready to be analyzed and shared between researchers. The strength of this tool is the diversity of supported ASL sequences, vendors, and scanners. A limitation of ASL-BIDS in general is that more advanced ASL sequences are not yet supported. We anticipate that this work will increase research time efficiency and allow pooling multi-center ASL datasets for advanced statistical analyses.

Acknowledgments: This work is part of the Eurostars Project ASPIRE 01QE2026A, which is funded by the German Federal Ministry of Education and Research, Innovate UK, and the Netherlands Enterprise Agency. HM and MD are supported by the Dutch Heart Foundation (2020T049). FB and XG are supported by NIHR funding through the UCLH Biomedical Research Centre. 


\section{References:}

1. www.anaconda.com/state-of-data-science-2020.

2. Clement 2019 MAGMA.

3. pypi.org/project/dcm2bids/.

4. Mutsaerts 2020 NeuroImage.

5. dicom.offis.de/dcmtk.php.en.

6. Li 2016 J Neurosci Methods.

\section{S4.P3.}

\section{Quantification of background suppressed arterial spin labeling images without an M0 image}

*Y. Prysiazhniuk ${ }^{1}$, M. Dijsselhof ${ }^{2}$, K. C. C. van de $\operatorname{Ven}^{3}$, K. P. A. Baas $^{4}$, A. Oliver-Taylor ${ }^{5}$, D. L. Thomas ${ }^{6,7}$, H. J. Mutsaerts ${ }^{2}$, J. Petr ${ }^{8}$

${ }^{1}$ Czech Technical University in Prague, Department of Cybernetics, Prague, CZ;

${ }^{2}$ Amsterdam University Medical Center, Department of Radiology and Nuclear Medicine, Amsterdam, NL;

${ }^{3}$ Philips Healthcare, Best, NL;

${ }^{4}$ Amsterdam UMC, Location AMC, Department of Radiology and Nuclear Medicine, Amsterdam, NL;

${ }^{5}$ Gold Standard Phantoms Ltd, London, GB;

${ }^{6}$ UCL Queen Square Institute of Neurology, Department of Brain

Repair and Rehabilitation, London, GB;

${ }^{7}$ UCL Queen Square Institute of Neurology, Wellcome Centre

for Human Neuroimaging, London, GB;

${ }^{8}$ Helmholtz Center Dresden-Rossendorf, Dresden, DE

Introduction: Arterial spin labeling (ASL) is a non-invasive perfusion MRI scan technique used in pathologies such as Alzheimer"s disease, cerebrovascular disease, and brain tumors [3]. To obtain absolute quantification of cerebral blood flow (CBF) in $\mathrm{mL} / 100 \mathrm{~g} /$ min, the blood equilibrium magnetization (M0b) is required [1]. For that, M0-scan acquisition is recommended [1]; however, in practice, clinical studies often lack an M0-scan. A control image can be used as an alternative only when background suppression (BSup) is not used. Here, we investigate the possibility to reconstruct an M0 scan from a control image with BS and compare it with acquired M0 scans from the same patients.

Methods: Five healthy volunteers (mean age $66 \pm 13$ years, 1 female) were scanned in two sessions using a Philips 3 T MRI. Each session consisted of a 3D T1w scan, and two pCASL scans with 2D EPI and 3D GraSE readouts: 4 BSup pulses at 716/1949/2875/ $3391 \mathrm{~ms}$ after labeling start, inter-slice timing $33.8 \mathrm{~ms}$ (2D), and an M0 scan without BSup, details in [2]. ASL and T1w data were processed using ExploreASL [5]. For each slice, the level of BSup in GM/WM was calculated assuming $90^{\circ}$ pre-saturation, $95 \%$ inversion efficiency of BSup and respective tissue T1-time (Fig. 1). The slicewise BSup levels were used to correct the control images, generating a pseudo M0 image. Two models were tested: 1) single-tissue, assuming either $100 \% \mathrm{GM}$ or $100 \% \mathrm{WM}$ voxels, and 2) mixed-tissue, allowing voxels with GM and WM partial volume-there, the signal was separated using partial volume correction [4], BSup-corrected using respective tissue models, and then aggregated. Mean relative error between $\mathrm{CBF}$ quantified with the pseudo- and true-M0 was obtained for the whole brain in the central slices (3-13 in 2D, 3-12 in 3D). A voxel-wise within-subject coefficient of variation (wsCoV) in CBF was calculated between sessions.

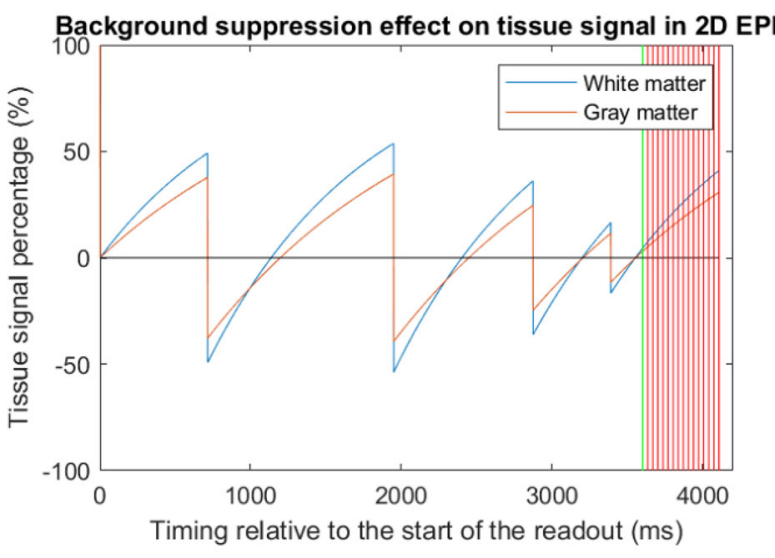

Figure 1: Estimated time-course of longitudinal magnetization in $\mathrm{GM}(\mathrm{T} 1=1500 \mathrm{~ms})$ and $\mathrm{WM}(\mathrm{T} 1=1050 \mathrm{~ms})$. One $90^{\circ}$ pre-saturation and four $180^{\circ}$ BSup pulses were used. Green vertical line indicates the start of the readout. Red vertical lines indicate the readout start of the following slices.

Results: Examples of pseudo-M0 are in Fig. 2. For 2D EPI, the average error in $\mathrm{CBF}$ was $6.1 \%$ for mixed-tissue and $14.8 \%$ for single-tissue model (Fig. 3). The mean wsCoV was $5.74 \%$ for mixedtissue and $6.75 \%$ for single-tissue model. For 3D GraSE, the error in $\mathrm{CBF}$ was $7.4 \%$ for mixed-tissue and $8.6 \%$ for single-tissue. The mean wsCoV was $5.38 \%$ for mixed-tissue and $4.22 \%$ for single-tissue model.
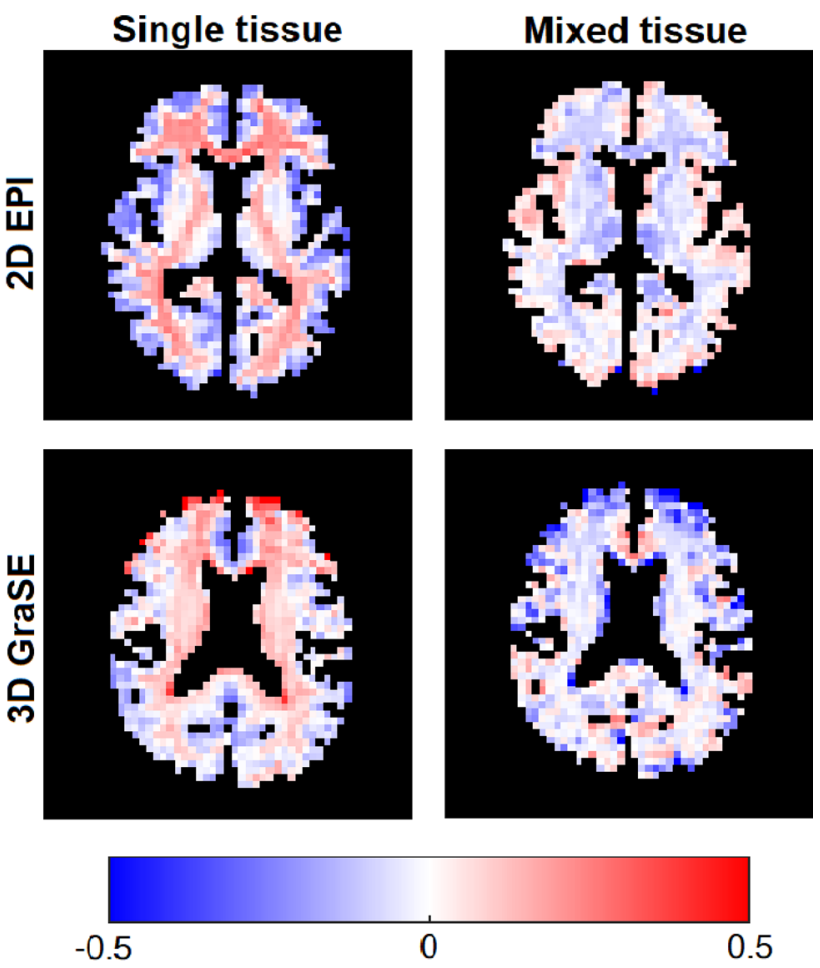

Figure 2: An example axial slice of a single subject showing spatial error distribution in estimated M0-scans. Voxel-wise relative error with respect to the acquired reference $\mathrm{MO}$ scans is calculated for $2 \mathrm{D}$ EPI and 3D GraSE using single-tissue and mixed-tissue methods. 

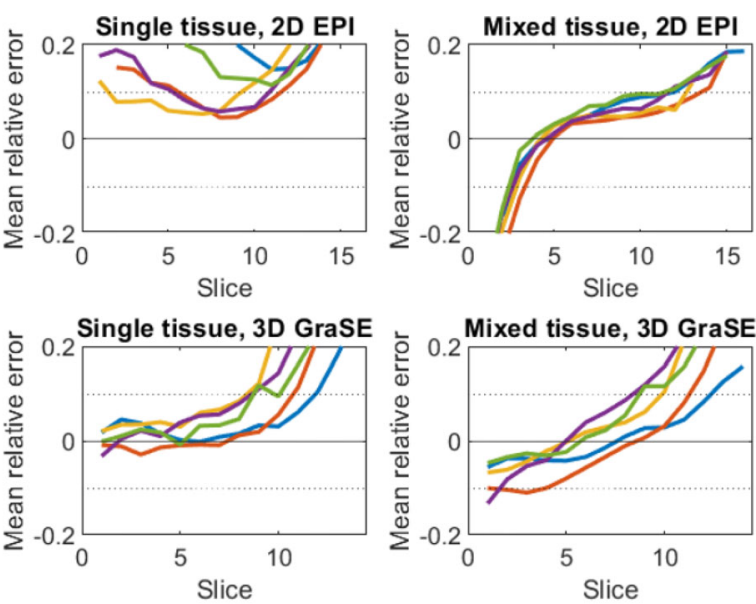

Figure 3: Accuracy of quantified CBF in 2D EPI and 3D GrasE using single-tissue and mixed-tissue methods.

Discussion: The mixed-tissue approach provided the best accuracy. The between-subject variation was comparable to previous results using a real M0 scan [2]. Lower level of BSup in 2D EPI allows a more robust M0 estimation than with 3D GraSE. Using M0-estimation from the control scans with BSup is a feasible option to CBF quantification if M0-scans are missing.

Acknowledgment: This work is part of the COST Action CA18206 Glioma MR Imaging 2.0 (www.glimr.eu), supported by COST (European Cooperation in Science and Technology) www.cost.eu.

\section{References}

[1] Alsop MRM 2015.

[2] Baas AJNR 2021.

[3] Telischak JMRI 2015.

[4] Asllani MRM 2008.

[5] Mutsaerts NI 2020.

\section{S4.P4.}

\section{Individually optimized background suppression} for ASL using a real-time feedback loop on the scanner

\author{
*K. Koolstra ${ }^{1}$, M. Staring ${ }^{1}$, P. de Bruin ${ }^{2}$, M. J. P. van Osch ${ }^{1}$ \\ ${ }^{1}$ Leiden University Medical Center, Department of Radiology, Leiden, \\ $N L$; \\ ${ }^{2}$ Philips Healthcare, Best, $N L$
}

Introduction: Background suppression (BGS) in ASL leads to perfusion images with higher SNR compared to ASL without BGS ${ }^{1}$. BGS is obtained by applying multiple inversion pulses before and during the post-label delay (PLD). The optimal inversion times (TI), and therefore the quality of the BGS, depends on the relaxation times of the underlying tissue and on inhomogeneities of the magnetic fields. Although this results in inter-subject differences, current ASL protocols make use of one set of predefined TI for all subjects, primarily because these inter-scan variations are not known at the moment of scanning. This means that the quality of the perfusion images is not optimal for all subjects. In this work, we develop a feedback loop (FBL) that optimizes the TI of ASL BGS pulses realtime on the scanner, generating individually optimized perfusion images for each subject.

Methods: Data acquisition: Experiments were performed in 2 subjects (informed consent obtained), using a 3 T MR system (Philips,
NL) with a 32-channel head coil. PCASL data were acquired with a single-shot EPI readout: label duration/PLD $=2050 / 1750 \mathrm{~ms}$, TE/ TR $=17 / 4000 \mathrm{~ms}$. Initial TI $(683 / 1948 / 2980 / 3597 \mathrm{~ms})$ were optimized via simulations for suppression of CSF, gray matter, white matter and corpus callosum.

Feedback mechanism: After each dynamic, label/control images were sent to an external computer via the remote connection software XTC (Philips, NL). On this computer, we developed a Python tool that receives and processes the images in real time. Updated TI were sent back to the scanner and imported during scanning (Fig. 1A).

TI optimization: 4 TI ( 2 during labelling, 2 after) were optimized in real-time using Nelder-Mead ${ }^{2}$ (80 dynamics), such that the label signal was minimized while maximizing the perfusion signal to avoid magnitude subtraction errors for near-optimal BGS, i.e.

$\mathbf{T I}^{\prime}=\operatorname{argmin}_{\mathbf{T I}}\left\|\mathbf{m}_{\mathrm{L}}(\mathbf{T I})\right\|_{2}-\lambda\left\|\mathbf{m}_{\mathrm{C}}-\mathbf{m}_{\mathrm{L}}\right\|_{2}$,

with $\mathbf{m}_{\mathrm{L} / \mathrm{C}}$ the label/control image obtained with $\mathbf{T I}=$ $\left[\mathrm{TI}_{1}, \mathrm{TI}_{2}, \mathrm{TI}_{3}, \mathrm{TI}_{4}\right]^{\mathrm{T}}$.

Results: Figure 1 shows the TI, the cost function and the label images during the FBL scan in 1 subject. Figure 2 shows the averaged ASL images over the last 10 dynamics of the FBL scan for $\lambda=5$ (regularized) and $\lambda=0$, compared to a scan with and without standard BGS. Figure 3 shows the results for a stimulus scan at the end of a FBL scan, showing a signal increase in the visual cortex both in perfusion and control images.

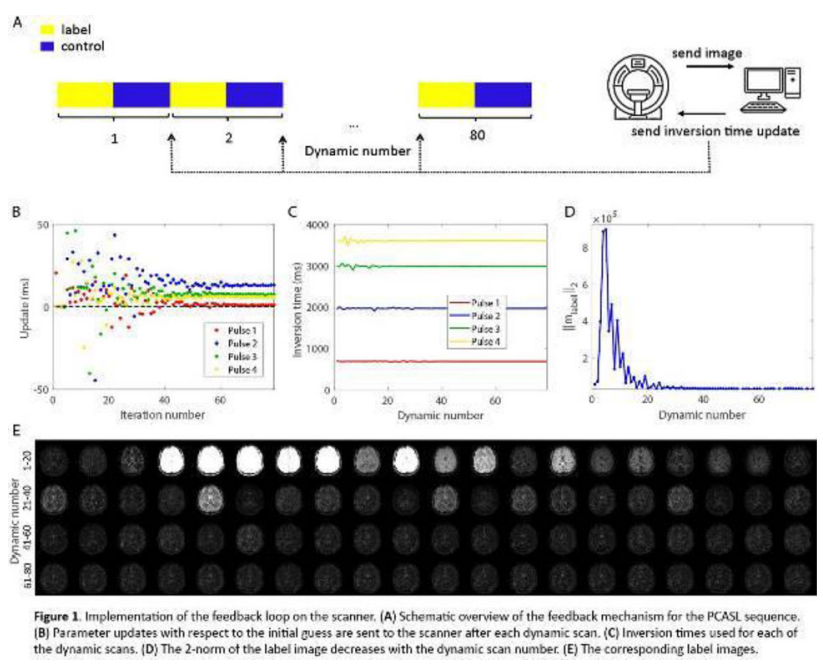

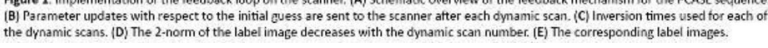




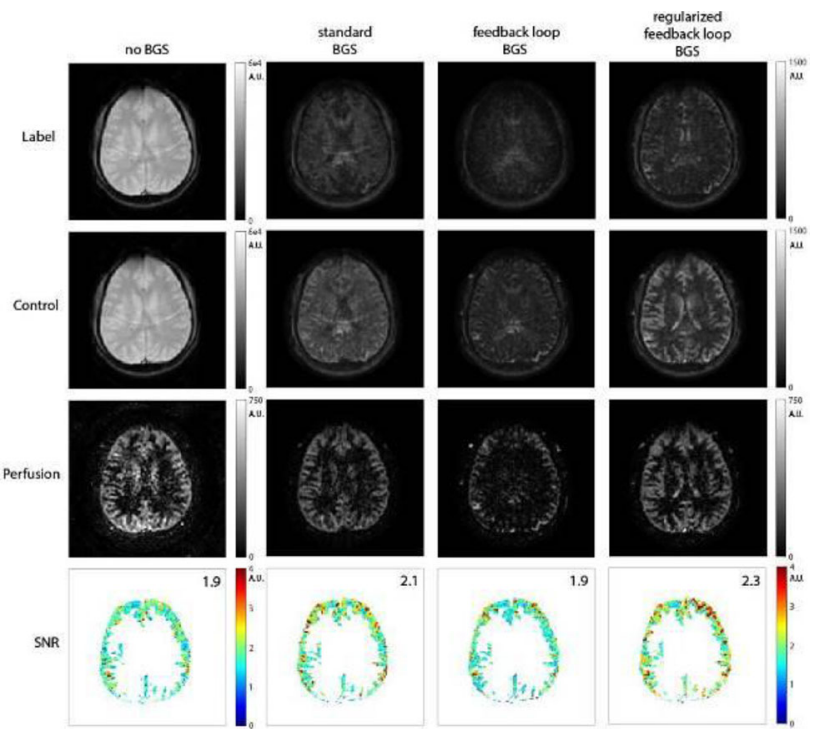

Figure 2. Averaged label, control and perfusion images over 10 dynamics for different amount of BGS. Standard BGS results in a perfusion image with higher temporal SNR compared to without BGS. Optimized BGS $(\lambda=0)$ with the feedback loop results in a lower quality perfusion image compared to standard BGS due to magnitude subtraction errors. Regularized optimization (A-5) ais welts top of a good initial estimate of the inversion times.

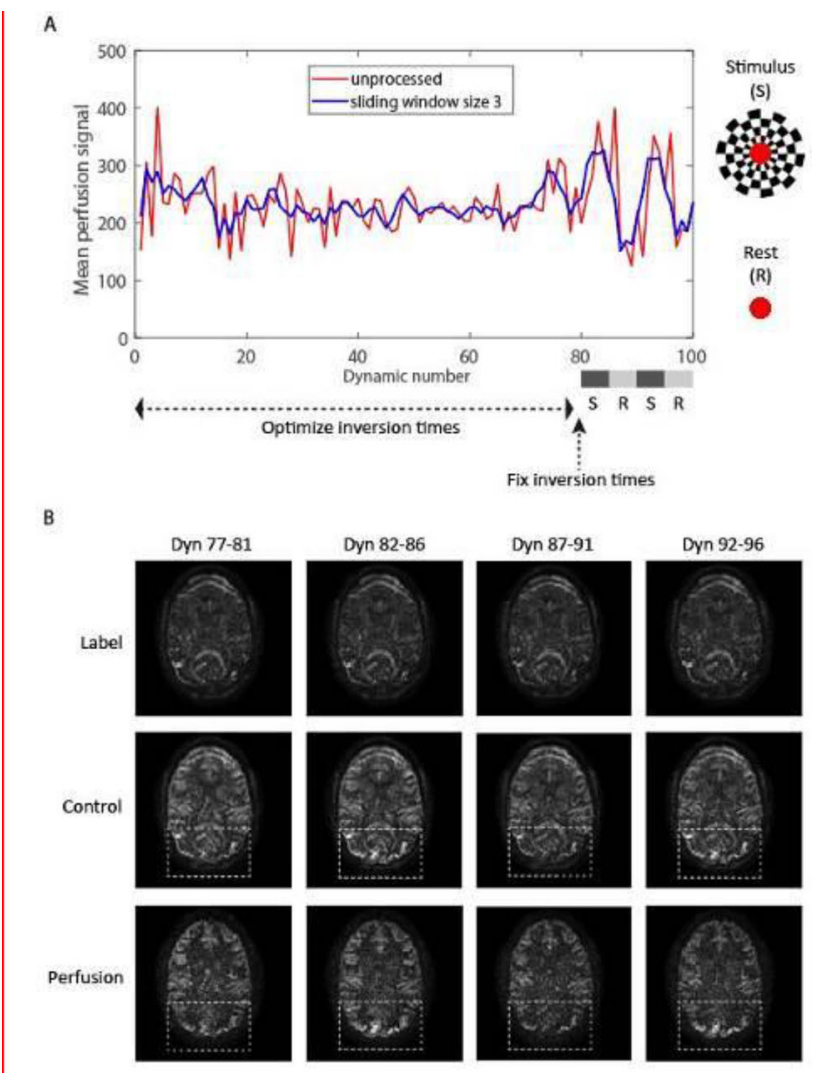

Figure 3. Neuronal activation measured by performing a stimulus at the end of a feedback loop scan. (A) The mean perfusion signal in an ROI in the visual cortex shows activation shortly after presenting the checkerboard (S), followed by a period of rest (R). (B) The visual cortex (dashed rectangle) shows an increase in the perfusion signal, which can directly be observed in the control images (all averaged over 5 neighboring dynamics), allowing to track the neuronal activation at twice the temporal resolution.
Discussion: The developed FBL scan increased the SNR of the perfusion images by $23 \%$ compared to $15 \%$ for standard BGS. Regularization prevents the FBL scan to converge to label and control images with opposite signs, otherwise leading to magnitude subtraction artifacts. The improved BGS leads to a control image in which the perfusion signal can directly be appreciated. This patient-optimized approach could therefore be a first step towards subtractionless ASL to allow twice the temporal resolution when monitoring neuronal activation.

\section{References:}

${ }^{1}$ Maleki (2012).

${ }^{2}$ Nelder (1965).

\section{S4.P5.}

\section{Adaptive adjustment of background suppression in pseudo-continuous arterial spin labeling}

\author{
*J. Huber ${ }^{1}$, D. Hoinkiss ${ }^{1}$, M. Günther ${ }^{1,2}$ \\ ${ }^{I}$ Fraunhofer Institute of Digital Medicine (MEVIS), Bremen, DE; \\ ${ }^{2}$ University of Bremen, Bremen, DE
}

Introduction: Background suppression (BS) of static tissue signal plays a significant role in successful application of arterial spin labeling to avoid subtraction errors due to subject motion or signal fluctuation. However, common BS approaches can be difficult to set up properly and premise prior knowledge about the tissues T1 distribution [1]. Therefore, a novel approach for adaptive BS in pseudocontinuous arterial spin labeling is presented. Instead of assuming the $\mathrm{T} 1$ distribution in an organ of interest, the actual spectrum of encountered $\mathrm{T} 1$ values is calculated from the preceding M0 scan, which is then used to suppress the static tissue to a desired level of residual magnetization.

Methods: In typical ASL experiments, a M0 scan with multiple saturation times is performed for quantification purposes. In this work M0, T1 and the saturation efficiency $M_{\text {sat }}$ are fitted for each voxel from the M0 scan using a monoexponential. A two-dimensional histogram with a bin size of $100 \mathrm{~ms}$ for $\mathrm{T} 1$ values and $2.5 \%$ for the saturation level is calculated from resulting $\mathrm{T} 1$ and $\mathrm{M}_{\mathrm{sat}}$ maps. The resulting $2 \mathrm{D}$ histogram is then used to find optimal timings of $n$ FOCI inversion pulses by minimizing the functional as given in Fig. 1a. In short, the algorithm tries to suppress all $\mathrm{T} 1$ values present in the histogram to a user defined level of residual magnetization. The algorithm is evaluated in a watermelon and in-vivo by adjusting the adaptive BS to levels of $0 \%, 4 \%, 8 \%$ and $12 \%$ with application of one to four inversion pulses. Additional imaging parameters are given in Fig. 1b. 
Fig 1

$$
\begin{aligned}
& \text { a.) } \quad \mathrm{e}(\vec{t})=\sum_{T_{1}}\left[\frac{M_{\mathrm{z}}\left(T_{1}, M_{\text {sat }} T_{\mathrm{i}}, \vec{t}\right)}{M_{0}\left(T_{1}\right)}-M_{\text {res }}\left(T_{1}, M_{\text {sat }}\right)\right] \cdot w_{\mathrm{c}}\left(T_{1}, M_{\text {sat }}\right) \\
& \vec{t}=\text { Optimal timings of inversion pulses } \\
& M_{\mathrm{z}} / M_{0}=\text { Longitudinal magnetization after inversion time } T_{\mathrm{i}} \\
& M_{\text {res }} / M_{\text {sat }}=\text { Desired level of residual magnetization in percent/Magnetization after presaturation }
\end{aligned}
$$
$w_{c}=$ Number of corresonding counts in histogram

b.)
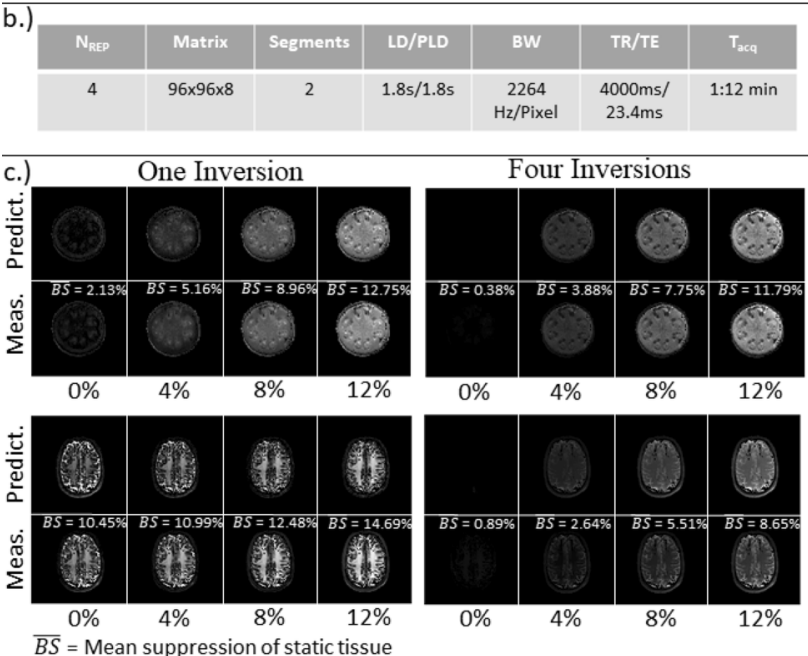

Results: Figure 1c shows a comparison between measured and predicted image contrasts. Figure 2 shows the mean level of suppression for different numbers of inversion pulses. Quality of perfusionweighted images is shown in Fig. 3.
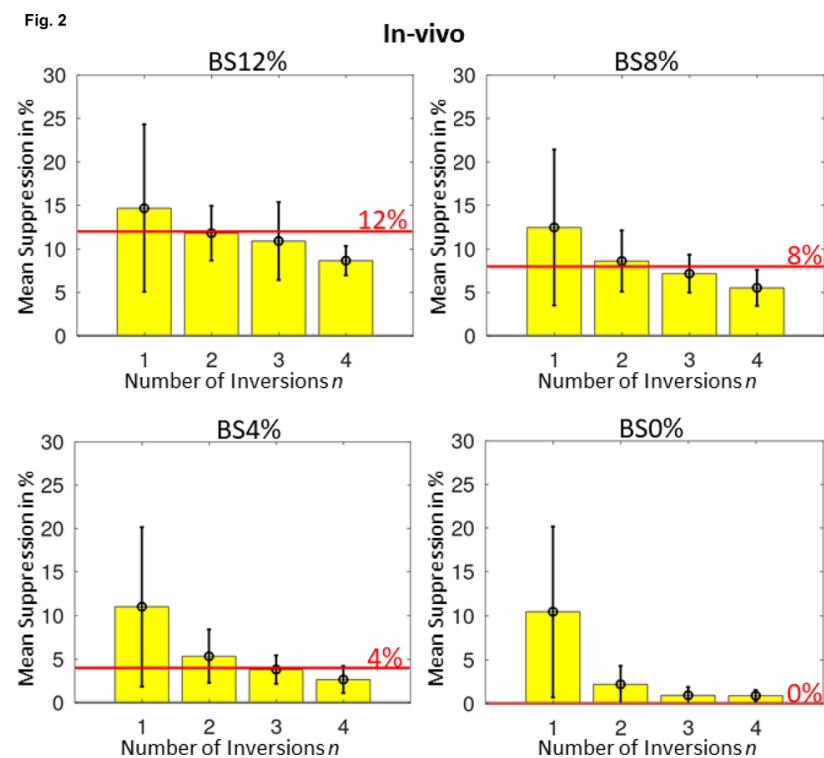

Fig. 3

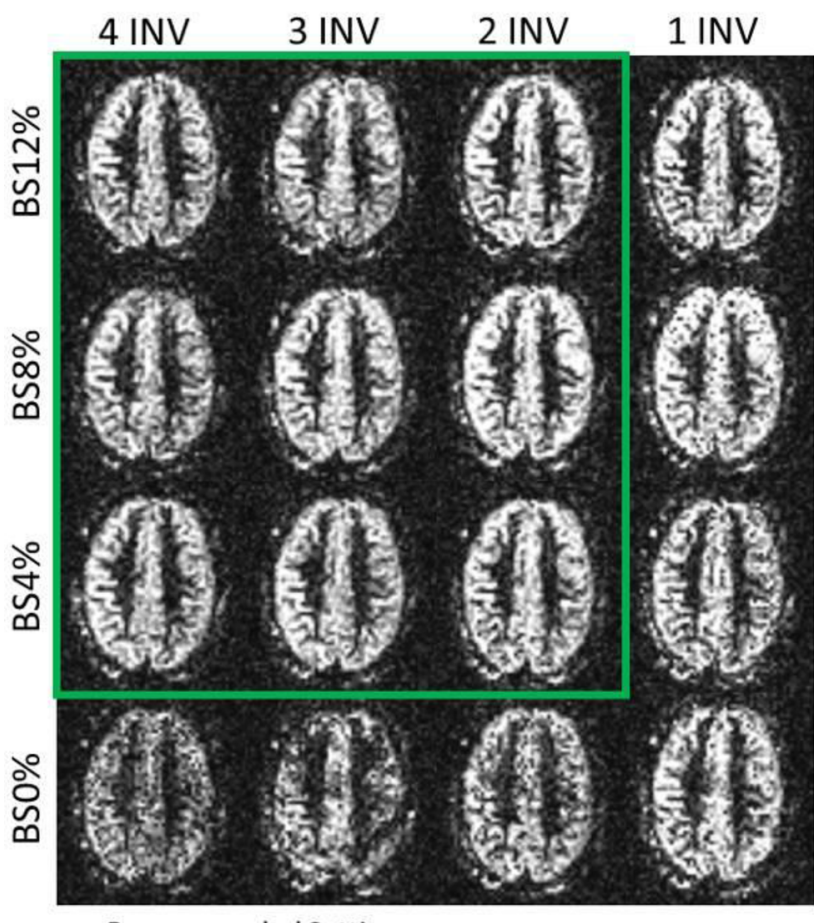

- Recommended Settings

Discussion: The proposed BS technique produces accurate levels of residual magnetization in the watermelon phantom, even for a small number of inversion pulses (cf. Fig. 1c, top). For in-vivo data of the brain, the $\mathrm{T} 1$ distribution is broader such that only one inversion pulse is not able to produce the desired suppression (cf. Fig. 1c, bottom). Using four inversion pulses, predicted and measured BS get closer to the desired levels of magnetization. However, larger deviations are observed in contrast to the phantom data, which might result from restricted inversion efficiency in-vivo. This might also explain why BS levels using two or three inversions are closer to desired levels (cf. Fig. 2) which needs further investigation. Figure 3 shows that high quality perfusion weighted images are obtained using the proposed BS with levels of 4\%-12\% and two-four inversions. We conclude, that the proposed technique is able to produce high quality perfusionweighted images while simplifying the BS adjustment in pCASL experiments.

\section{References:}

[1] Günther et al., Single-Shot 3D Imaging Techniques Improve Arterial Spin Labeling Perfusion Measurements, MRM 54, 2005

\section{S4.P6.}

Arterial spin labeling in carotid artery stenosis: evaluation of partial volume effect and transit delay bias

${ }^{*}$ G. Hoffmann ${ }^{1}$, J. Göttler ${ }^{1}$, C. Zimmer ${ }^{1}$, C. Preibisch ${ }^{1}$, N. Sollmann ${ }^{1}$, S. Kaczmarz

${ }^{1}$ Technical University of Munich, Department of Neuroradiology, Munich, DE

Introduction: Arterial Spin Labeling (ASL) is highly promising for non-invasive perfusion imaging in cerebrovascular diseases. ${ }^{1}$ Pseudo- 
continuous ASL (pCASL) measurements in patients with unilateral internal carotid artery stenosis (ICAS) previously showed ipsilaterally decreased cerebral blood flow $(\mathrm{CBF}){ }^{2,3}$ However, lower CBF may also originate from partial volume effects $(\mathrm{PVE})^{4,5}$ and arterial transit time (ATT) delays. ${ }^{1,6}$ The aim of our study was to evaluate PVE and ATT bias on pCASL-based CBF in grey matter (GM) of ICAS patients and age-matched healthy controls (HC). First, PVE were assessed by a linear regression algorithm. ${ }^{7}$ Second, ATT effects were evaluated by means of spatial coefficients of variation (sCOV). ${ }^{6}$

Methods: 15 asymptomatic ICAS patients (70.2 \pm 4.4 years) and 24 HC $(69.9 \pm 7.3$ years) were scanned on a 3 T Philips Ingenia (Best, $\mathrm{NL}$ ). The imaging protocol and derived parameters are summarized in Fig. 1, including single post label delay pCASL. PVE correction $(\mathrm{PVEc})^{7}$ on GM-CBF was compared globally as well as differentiated between anterior and posterior circulation using a perfusion territory atlas. ${ }^{8}$ Moreover, CBF asymmetry indizes ${ }^{2}$ (AI, Fig. 1) between hemispheres were evaluated and SCOV compared between patients and HCs.

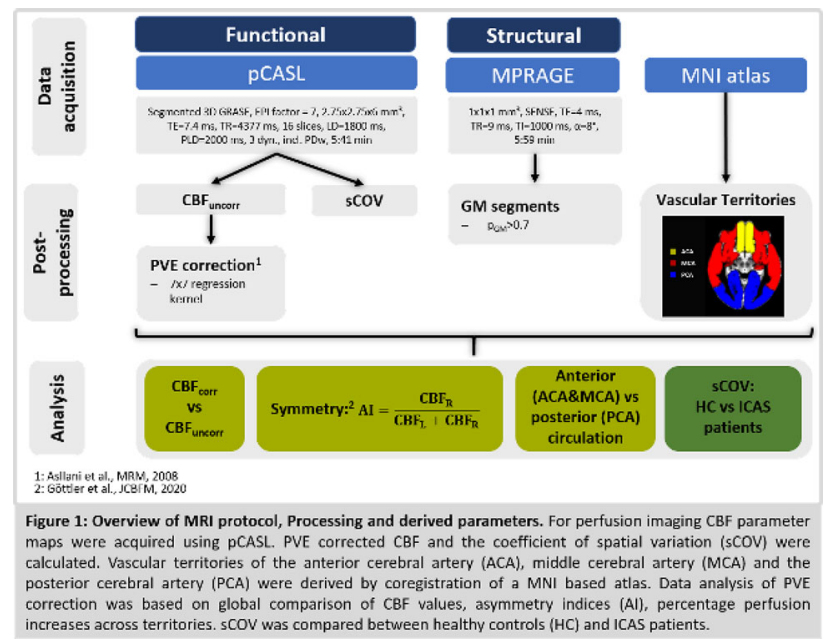

Results: Exemplary data show PVEc induced GM-CBF increases, with strongest effects in frontal regions of ICAS patients (Fig. 2). Group analysis in ICAS showed globally increased GM-CBF $(+10.3 \pm 2.5 \%$, Fig. $3 \mathrm{~A})$ by PVEc, most pronounced in the anterior circulation (Fig. $3 \mathrm{~B}$ ), and preserved $\mathrm{CBF}$ symmetry $(\mathrm{AI} \approx 0,47$, Fig. 3C). HCs" GM-CBF increased by PVEc (+9.1 $\pm 3.1 \%)$, without regional differences. Spatial COV was 0.37 for $\mathrm{HC}$ and 0.33 for patients (Fig. 3D).

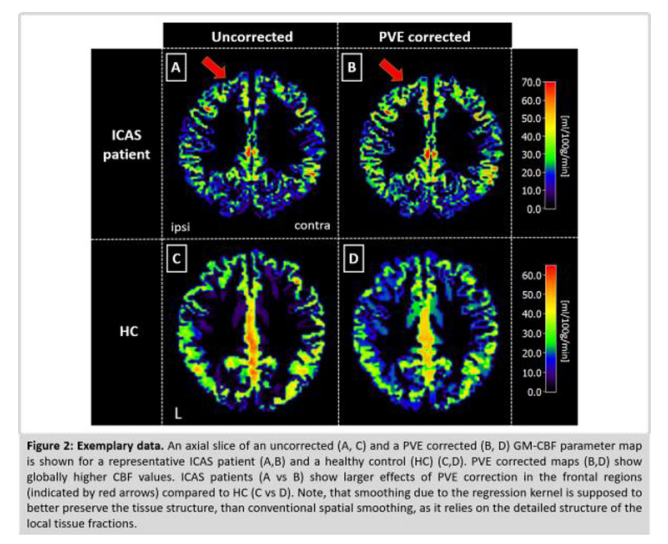

Fig. 3

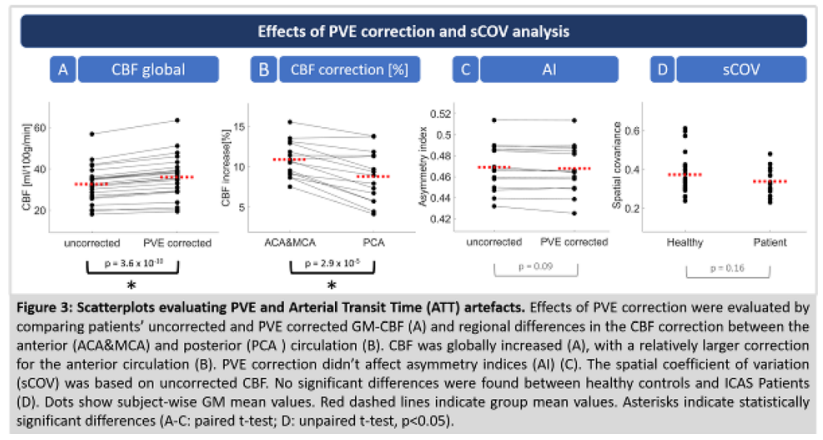

Discussion: Our results indicate systematic underestimation of uncorrected GM-CBF in patients and $\mathrm{HCs}$, in line with literature. ${ }^{4,9}$ Therefore, PVEc is recommended to better differentiate disease driven $\mathrm{CBF}$ changes. Interestingly, PVE of $\mathrm{HCs}$ were spatially homogenous, while patients showed strongest effects in the anterior circulation. This may be due to accelerated parenchymal volume loss in areas supplied by branches of a (stenosed) ICA, as ICAS is known to be related to atrophy. ${ }^{10}$ Unchanged AI indicates similar bilateral effects and absence of severe unilateral atrophy, while analysis of sCOV indicates no severe delay effects. Prospectively, vessel selective $\mathrm{ASL}^{11}$ could further differentiate PVE within perfusion territories and time-encoded ASL ${ }^{12}$ could exclude delay effects.

To conclude, patients' lowered ipsilateral CBF seems to be pathophysiologically driven, as we found neither effects of PVEc on AI nor severe ATT artefacts. Therefore, widely available standard pCASL is applicable for clinical perfusion imaging.

\section{References:}

1: Donahue, JCBFM, 2018.

2: Göttler, JCBFM, 2020.

3: Kaczmarz, JCBFM, 2020.

4: Asllani, HBM, 2009.

5: Mutsaerts, NeuroImClin, 2014.

6: Mutsaerts, JCBFM, 2017.

7: Asllani, MRM, 2008.

8: Tatu, Karger, 2012.

9: Petr, MAGMA, 2018.

10: Muller, Nrad, 2011.

11: Helle, MRM, 2010.

12: vanOsch, JCBFM, 2018.

\section{S4.P7.}

Towards free-breathing liver perfusion imaging (using prospective motion correction)

\author{
${ }^{*}$ J. Huber ${ }^{1}$, M. Channaveerappa ${ }^{1}$, D. Hoinkiss ${ }^{1}$, M. Günther ${ }^{1,2}$ \\ ${ }^{I}$ Fraunhofer MEVIS, Bremen, DE; \\ ${ }^{2}$ University of Bremen, Bremen, DE
}

Introduction: Assessment of liver perfusion can yield valuable functional information about diseases like carcinoma and cirrhosis. However, being a subtractive technique, ASL in abdominal organs is challenging due to breathing motion. Prospective motion correction using additional navigator images, however, is hampered by background suppression used to avoid subtraction errors in ASL. Therefore, a technique for prospective adjustment of saturation and imaging slices of a pCASL sequence is presented, based on motion 
estimates obtained from a preceding reference cycle and a crosscorrelation matching approach during the ASL experiment.

Methods: Prospective tracking of the liver position during an axial pCASL experiment is accomplished by additional 2D EPI navigator readouts as shown in Fig. 1a. First, multiple navigators are acquired over a period of around $10 \mathrm{~s}$, covering the full cycle of breathing motion of the liver (reference cycle) and a look-up table is filled with calculated positional shifts. During the actual ASL experiment, 2D navigator images (ASL navigators) are acquired before the slice-selective presaturation pulse as well as the 3D GRASE imaging module. These ASL navigators are matched into the reference cycle by calculation of the normalized cross-correlation between the navigator and all reference images. After that, translational shifts, corresponding to the position of the image with maximum correlation value, are sent back to the sequence, adjusting the saturation/imaging position. The algorithm is validated for protocol parameters as given in Fig. 1b. A phantom is placed on a movable base (cf. Fig. 2a), which is shifted continuously during the acquisition of the reference cycle. During the experiment, the phantom is moved between two distinct positions. Motion experiments are performed, with/without application of prospective correction.

Fig. 1

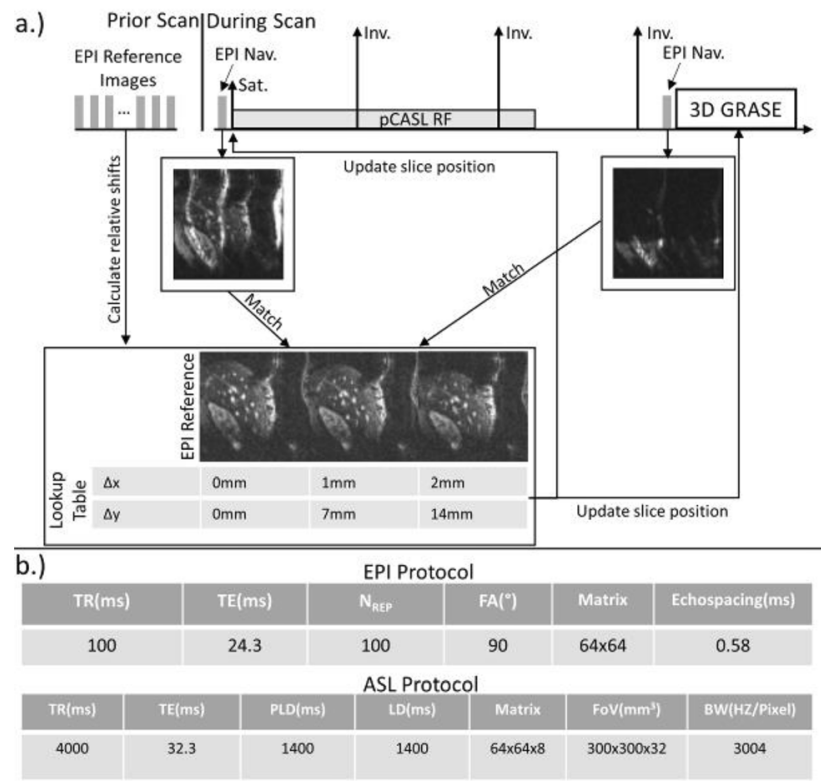

Results/discussion: Figure $2 \mathrm{~b}$ shows the motion trajectory estimated from the reference cycle, corresponding to the applied shifts. Figure $2 \mathrm{c}$ shows an exemplarily plot of the correlation metric between the first navigator of the ASL experiment and all navigators from the reference cycle. Maxima are clearly visible such that correctly identified corresponding shifts from the lookup table were sent back to the sequence during the experiment (cf. Fig. 2d). Figure 3 shows successful prospective adaption of the sequence to the changes in positioning of the object when compared to the scan without adaption of the sequence. Additional retrospective in-plane motion correction using a PROPELLER readout will be further investigated.
Fig. 2
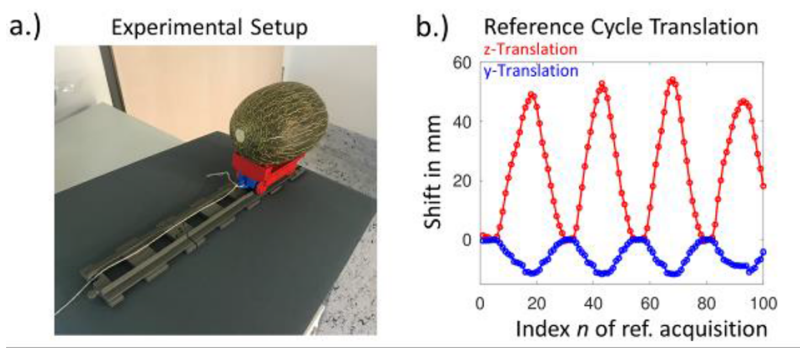

c.) Correlation Matching of Nav. 1

d.) Feedback during ASL Acquisition
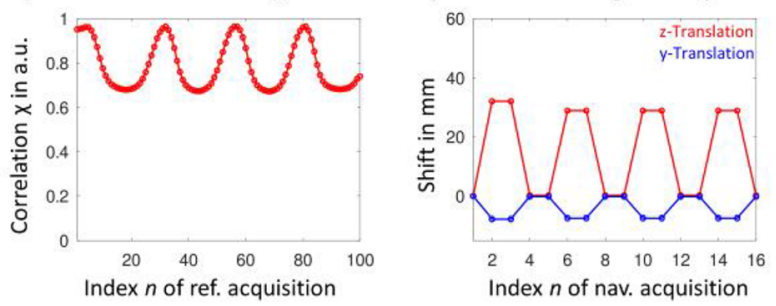

Fig. 3

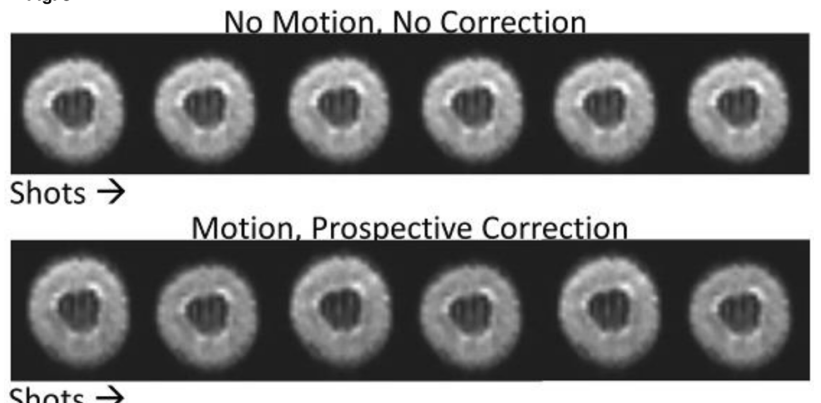

Shots $\rightarrow$

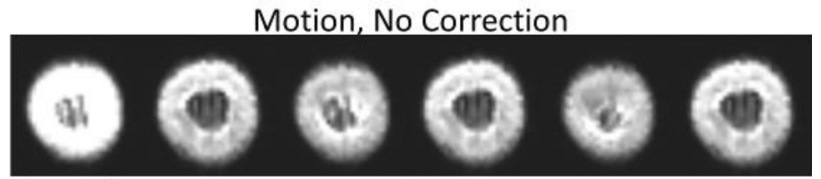

Shots $\rightarrow$

Conclusion: Successful tracking of the objects position during the ASL experiment was achieved using the proposed reference cycle correlation matching technique. Therefore, future work will focus on the application of the proposed technique to free-breathing ASL imaging of the liver.

\section{S4.P8.}

\section{Using a digital phantom for quality control of ExploreASL image processing}

*M. Stritt ${ }^{1,2}$, B. Padrela ${ }^{2}$, M. Dijsselhof ${ }^{2}$, A. Oliver-Taylor ${ }^{3}$,

F. Barkhof ${ }^{2}$, X. Golay ${ }^{3}$, M. Günther ${ }^{4}$, J. Gregori ${ }^{1}$, H. J. Mutsaerts ${ }^{2}$, J. Petr ${ }^{5}$

${ }^{I}$ mediri GmbH, Heidelberg, DE;

${ }^{2}$ Amsterdam University Medical Center, Department of Radiology and Nuclear Medicine, Amsterdam, NL;

${ }^{3}$ Gold Standard Phantoms Ltd, London, GB; 
${ }^{4}$ University of Bremen, Bremen, DE;

${ }^{5}$ Helmholtz Center Dresden-Rossendorf, Dresden, DE

Introduction: In recent years, there has been an increasing focus on open-science and reproducible research, including reliable and robust software. To this end, methods of requirements engineering and quality control were integrated in the ExploreASL pipeline and development workflow. Here, we used the recently developed digital reference object (DRO) "ASL-DRO" to evaluate the reliability of ExploreASL with a main focus on motion correction [1,2].

Methods: Synthetic ASL scans were generated by the ASL-DRO [1] using average $\mathrm{CBF}$ and segmentations from the EPAD study [3] as DRO input (Fig. 1). To study the influence of motion, pseudo-random motion was introduced to the rawdata using the default ASL-DRO. 3D translations and rotations were defined for the ASL time-series starting with zero for the first volume. For the following volumes, a uniformly distributed random number within a specific range was added to the previous volume. The synthetic data were processed with ExploreASL using default settings. Estimated motion and mean CBF were compared with ground truth motion and CBF.

Results: CBF results based on the EPAD ground truth (Fig. 1) were $36-58 \%$ underestimated. ExploreASL estimated motion with minor errors for most motion settings (Fig. 2a-i). Both the euclidean norm as well as absolute displacement (Fig. 2j-k) display a high effect of rotation on overall voxel-wise motion.

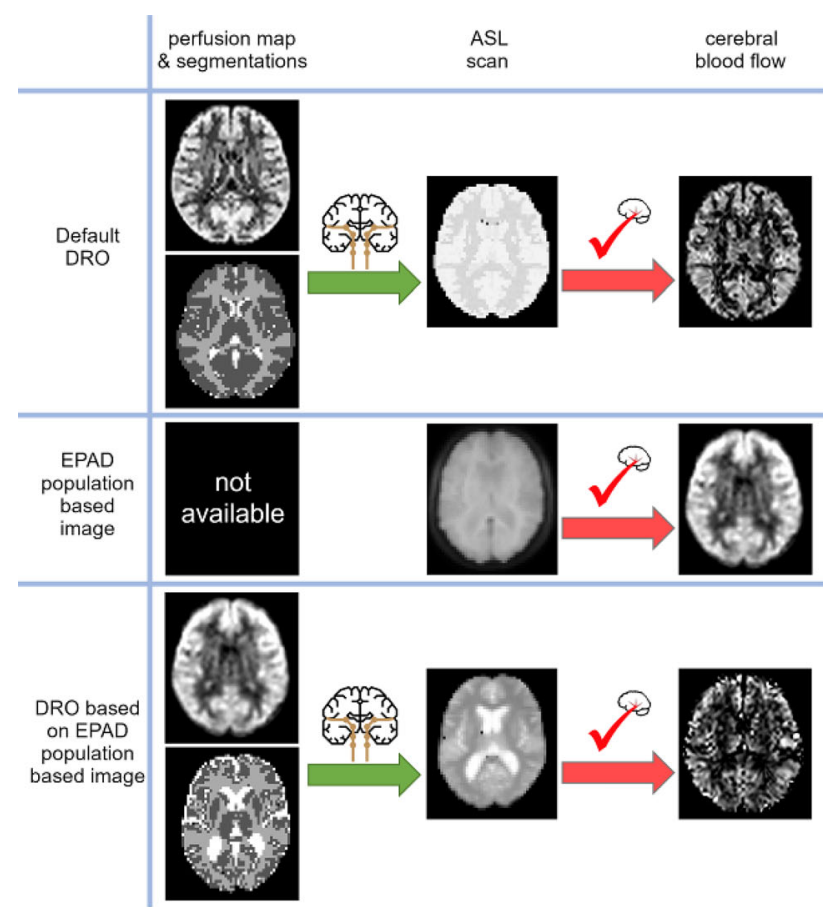

Figure 1: Simplified workflows. Row one shows some of the atlas-based input ground truth maps of the ASL-DRO (perfusion map \& segmentations), the generated ASL scan and the resulting CBF of ExploreASL. Row two displays a population-based average image based on datasets from the EPAD study and the resulting CBF map, which is used as a ground truth perfusion map for the DRO in row three. The additional ground truth maps $\left(T 1, T 2, T 2^{*}\right.$ \& transit times) and the synthetic structural images corresponding to each row were omitted to simplify the overview. In the workflow of row three, DRO settings similar to the EPAD study were used (PLD 2.025s, lab. dur. 1.65s, lab. eff. 0.85, acq. matrix [64,64,20], SNR 100.0 30 acq. pairs, $\mathrm{T} 1, \mathrm{~T} 2, \mathrm{~T} 2 *$ \& ATT according to default DRO, TR/TE $5 \mathrm{~s} / 0.01 \mathrm{~s}$ ).
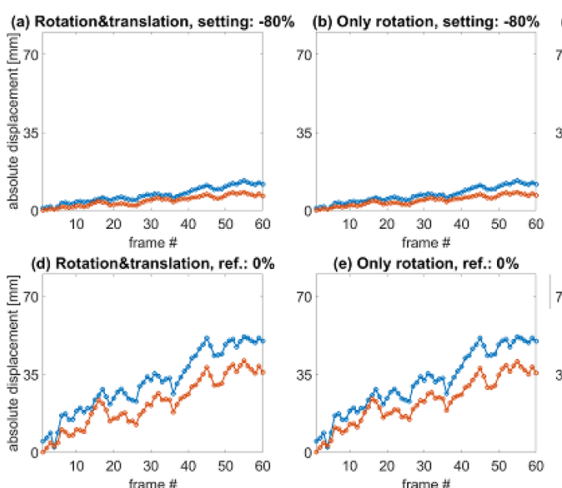

(c) Only translation, setting: $-80 \%$
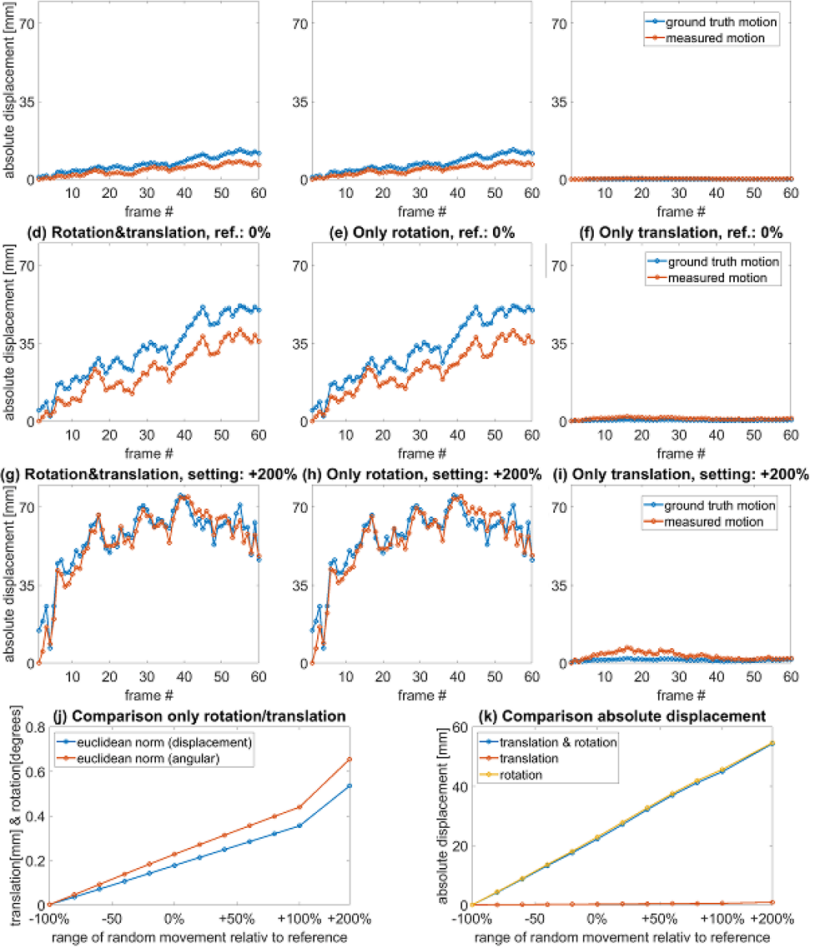

Figure 2: Motion related results. The reference setting $(0 \%)$ contains random frame-by-frame rotation between $[-0,3,+0.3]$ degrees as well as random frame-by-frame translation between $[-0.125,+0.125] \mathrm{mm}$. Three exemplary settings are shown in graphs (a) to (i). Graph (j) shows the euclidean norms for both translational and rotational values for each setting. Graph ( $k$ ) displays the absolute displacement

comparing all settings for both translation \& rotation as well as translation \& rotation individually.

The voxel-wise error in CBF increased by 3.8 between $-100 \%$ and $0 \%$ and $19.4 \mathrm{ml} / 100 \mathrm{~g} / \mathrm{min}$ between $-100 \%$ and $+200 \%$ (Fig. 3a). For peripheral brain regions, the error increases with motion exacerbated by the sharp contrast between brain and background in the DRO (Fig. 3b). CBF underestimation increased with motion based on increased blurring [4] (Fig. 3c). The increased effect of motion on individual smaller regions (Fig. 3d) led to higher variation in CBF.

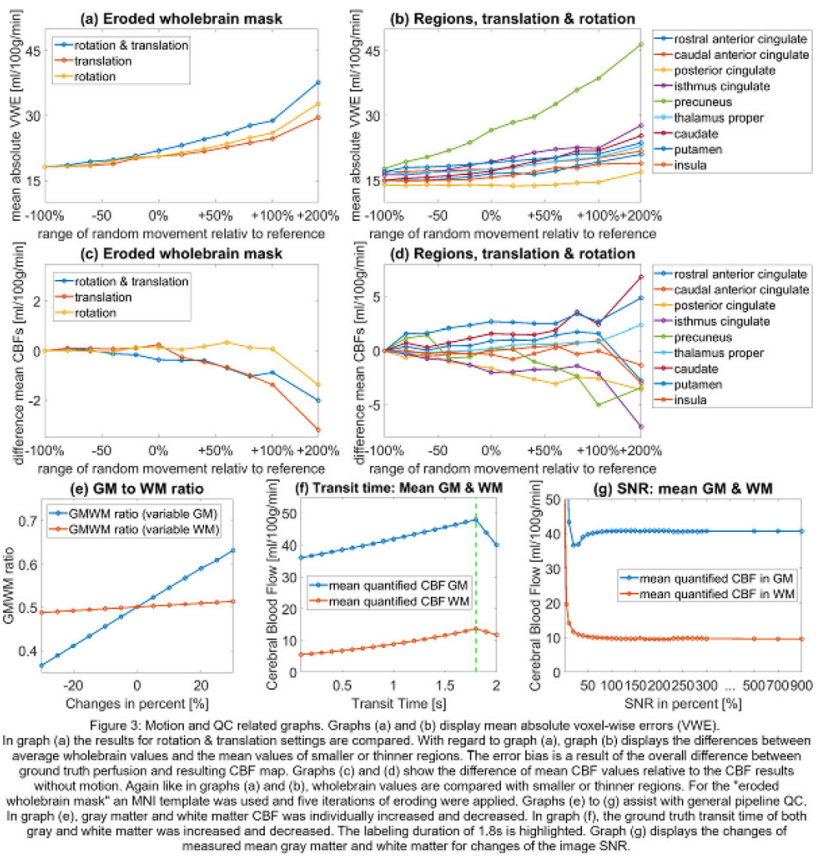


Discussion: The ASL-DRO proved to be useful for software QC and evaluation. It was found that a motion measure based on displacement of a single-voxel is not representative and mean-voxel motion needs to be used. CBF underestimation was partly caused by input data undersampling, partly by partial volume artifacts. Both the high impact of rotational movement and the increased effect of motion on thinner or smaller regions is visible in the results (Fig. 3a, b), and the DRO can be utilized in the future to help correct for these artifacts. Integration of DRO based QC workflows is a promising way to improve methods stability and pipeline results.

Acknowledgments: This work is part of the Eurostars Project ASPIRE 01QE2026A, funded by the German Federal Ministry of Education and Research, Innovate UK, and the Netherlands Enterprise Agency. HM and MD are supported by the Dutch Heart Foundation (2020T049). FB and XG are supported by NIHR through the UCLH Biomedical Research Centre.

\section{References}

1. Oliver-Taylor, ISMRM 2021.

2. Mutsaerts, NeuroImage 2020

3. Ritchie, EPAD Study 2020.

4. Petr, ESMRMB 2016.

\section{S4.P9.}

\section{Impact of susceptibility distortion correction on perfusion imaging by pCASL with 3D GRASE readout}

${ }^{*}$ C. Domingos ${ }^{1}$, A. Fouto ${ }^{1}$, A. Ruiz-Tagle ${ }^{1}$, I. Esteves ${ }^{1}$, R. Gouveia Nunes $^{1,2}$, N. A. Silva ${ }^{3}$, P. Vilela ${ }^{2}$, P. Figueiredo ${ }^{1}$

${ }^{1}$ University of Lisbon, Lisbon, PT;

${ }^{2}$ Hospital da Luz, Neurology Department, Lisbon, PT;

${ }^{3}$ Hospital da Luz, Department of Learning Health, Lisbon, PT

Introduction: Arterial spin labeling (ASL) is a noninvasive perfusion imaging technique with great clinical potential, and a consensus paper has been published with recommendations for its clinical implementation $^{1}$. In particular, a segmented 3D GRASE readout is indicated for optimal SNR. Because this is known to be affected by susceptibility artefacts, appropriate distortion correction techniques are often used ${ }^{2}$. One study showed that distortion correction increased diagnostic precision of ASL perfusion images ${ }^{3}$, but the impact on ASL data quality and ensuing perfusion measurements has not been systematically assessed. Here, we investigate the effects of susceptibility distortion correction on perfusion imaging by pCASL with 3D GRASE in terms of temporal SNR (tSNR) as well as perfusion.

Methods: A group of 15 women (20-48 years, 7 controls and 8 migraine patients) was studied on a $3 \mathrm{~T}$ Siemens Vida MRI System using a 64-channel head RF coil. Perfusion imaging was performed using pCASL with 3D GRASE $(\mathrm{TR}=5.6 \mathrm{~s}$, TE $=18.4 \mathrm{~ms}$, labeling duration $=1.8 \mathrm{~s}$, post-labeling delay $=1.8 \mathrm{~s}$, background suppression, 4 repetitions $)^{1}$. A fieldmap (TE1/TE2 $\left.=4.92 / 7.38 \mathrm{~ms}\right)$ and a T1weighted structural image (1 $\mathrm{mm}$ isotropic resolution) were also acquired.

Image analysis was performed using FSL (fsl.fmrib.ox.ac.uk). After motion correction, two preprocessing options were considered: without or with distortion correction (FSL's PRELUDE\&FUGUE). Relative perfusion maps were obtained by averaging pairwise controllabel subtraction images with spatial regularisation (FSL's BASIL). The tSNR was calculated voxelwise on the pairwise control-label subtraction images as the temporal mean value divided by the temporal standard deviation (tStd). The structural image was segmented and 8 anatomical ROIs were defined. Registration was performed between the relatively distortion-free structural images and each of the two preprocessed ASL datasets (without and with distortion correction) (FSL's FLIRT).

Results: An example of the effects of distortion correction on the tSNR and perfusion maps is shown in Fig. 1. The tStd significantly decreased and tSNR significantly increased in all ROIs (Fig. 2). For perfusion, we found significant changes in the occipital lobe, cerebellum and brainstem (Fig. 3).
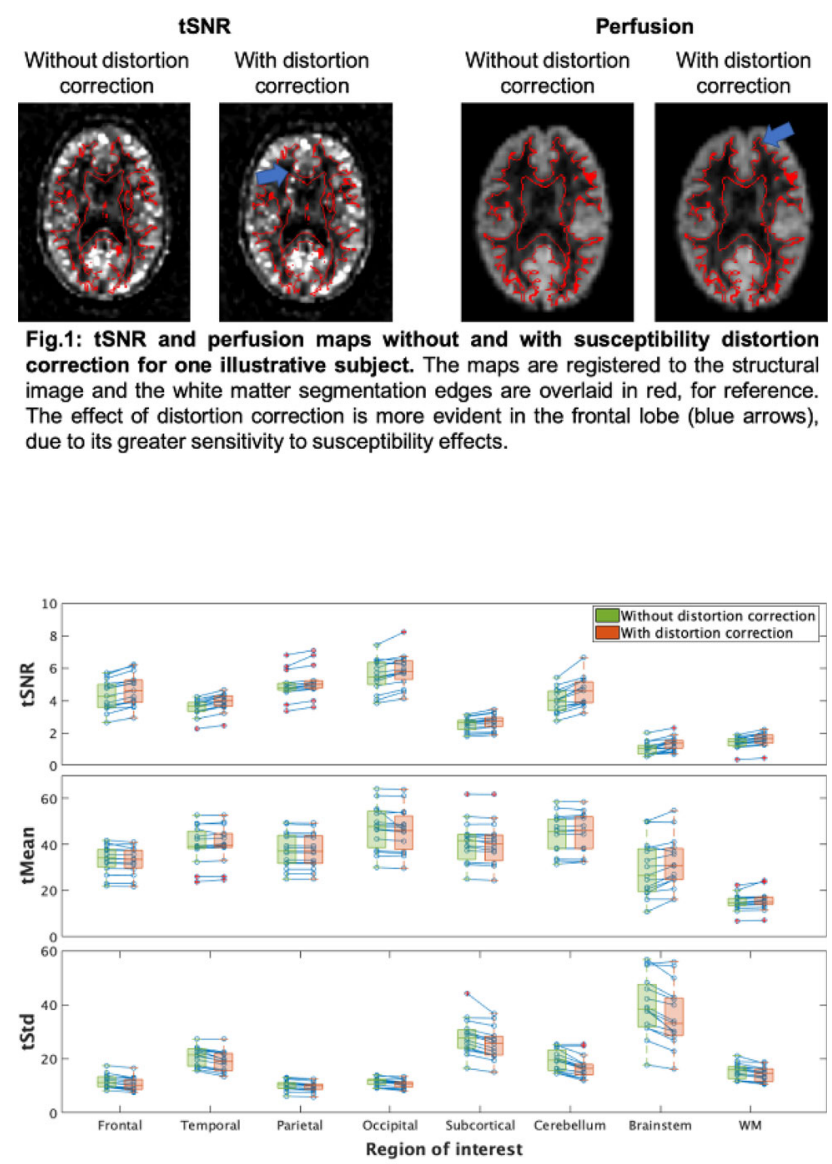

Fig.2: tSNR Results. tSNR, tMean and tStd values obtained for the eight ROls. Boxplots represent distributions across subjects, for ASL data without distortion correction (green), and with distortion correction (orange). The blue lines connect the values obtained for each subject. Two-way repeated measures ANOVA showed significant main effects of correction and $\mathrm{ROI}$ as well as a significant interaction between them, with all ROls exhibiting significant increases in ISNR. For tMean no significant main effects were observed for correction. Finally, tSTd showed significant main effects for correction, ROI and their interaction, with significant decreases for temporal, subcortical, cerebellum and brainstem ROIs. 


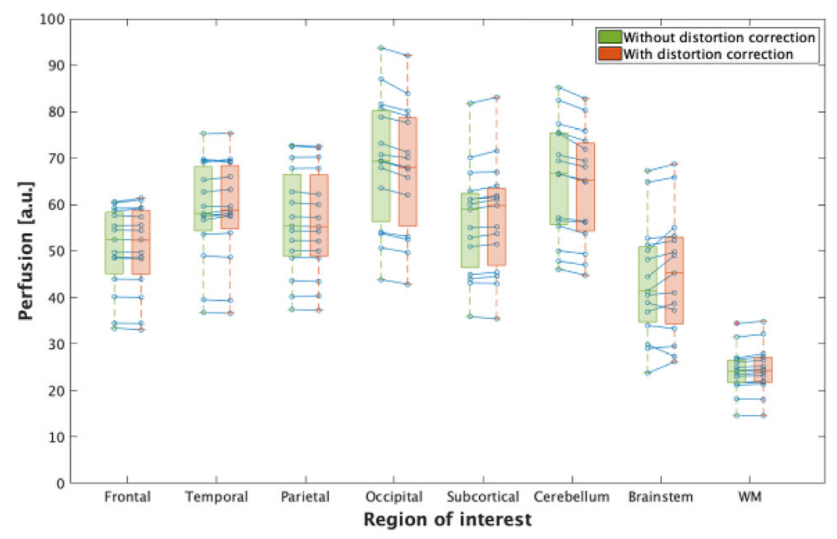

Fig.3: Perfusion Results. Perfusion values obtained for eight ROIs. Boxplots represent distributions across subjects, for ASL data without distortion correction (green), and with distortion correction (orange). The blue lines connect the perfusion values obtained for each subject. Two-way repeated measures ANOVA showed a significant main effect of $\mathrm{ROI}$ and a significant interaction between $\mathrm{ROI}$ and correction. Posthoc tests revealed significant changes with correction for the occipital, cerebellum and brainstem ROIs.

Conclusion: We showed that susceptibility distortion correction significantly improved the tSNR of pCASL-3D GRASE data across the whole brain, with impact on perfusion in some regions. Improvements in tSNR have been previously reported for BOLD signals, even with static correction ${ }^{4}$, but not for ASL. This step should hence be integrated in the ASL preprocessing pipeline, especially due to its intrinsically low SNR.

\section{References:}

1. Alsop et al., MagResonMed 73(1):102-116, 2015.

2. Andersson et al., Neuroimage 20(2), 870-888, 2003.

3. Madai et al., JNeuroimaging 26:436-444, 2016.

4. Dymerska et al., Neuroimage 168:321-331, 2018.

\section{S4.P10.}

\section{Impact of ICA denoising on ASL perfusion imaging across different groups of participants}

*S. P. Monteiro ${ }^{1}$, J. Pinto ${ }^{2}$, A. Fouto ${ }^{1}$, R. Gouveia Nunes ${ }^{1}$, L. Alves ${ }^{3}$,

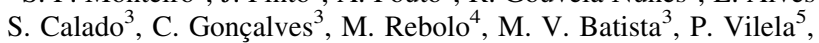
P. Figueiredo ${ }^{1}$

${ }^{1}$ University of Lisbon, Lisbon, PT;

${ }^{2}$ University of Oxford, Institute of Biomedical Engineering, Oxford, $G B$;

${ }^{3}$ University of Lisbon, Department of Neurology, Lisbon, PT;

${ }^{4}$ University of Lisbon, Lisbon, PT;

${ }^{5}$ Hospital da Luz, Imaging Department, Lisbon, PT

Introduction: Arterial spin labeling (ASL) has great clinical potential for the noninvasive evaluation of cerebral blood flow (CBF), as well as other haemodynamics parameters such as the arterial transit time (ATT) if sampling multiple post-labeling delays (PLD) [1]. The intrinsically low signal to noise ratio (SNR) of ASL has motivated the development of various acquisition and post-processing strategies. In particular, Independent Component Analysis (ICA) has been shown to be valuable in differentiating the ASL signal of interest from structured noise sources [2]. Here, we further investigate the impact of ICA-based denoising on ASL perfusion imaging comparing two groups of participants: Small Vessel Disease (SVD) patients and agematched controls.

Methods: Multi-PLD pulsed ASL data was acquired from 17 SVD patients and 12 age-matched controls on a Siemens Verio scanner using a PICORE-Q2TIPS sequence with a 2D multi-slice GE-EPI readout (TR/TE $=2500 / 11 \mathrm{~ms}$; 11 TIs: $400-2400 \mathrm{~ms})$. Data analysis was performed using FSL (fsl.fmrib.ox.ac.uk). ICA was performed on the motion-corrected control-label subtraction time series. Noise-related Independent Components (ICs) were manually identified and removed following 2 approaches: conservative ICA (ICAc)—removal of ICs clearly related to motion, susceptibility and multiband artifacts; and aggressive ICA (ICAa) - removal of all ICs not consistent with the expected ASL signal location/dynamics (Fig. 1). These two approaches were compared to the non-denoised data (noICA) in terms of the \% change in the temporal SD of the subtraction time series as well as the gray matter mean values of CBF and ATT and respective variances estimated by fitting an extended kinetic model to the data using BASIL [1].

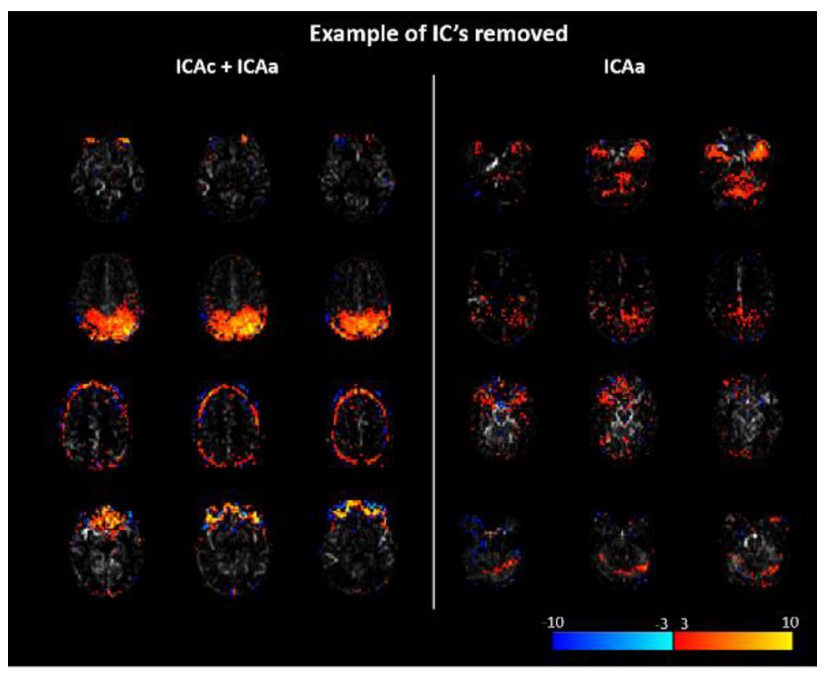

Figure 1. Explicative examples of ICS removed on ICA aggressive (ICAa) and ICA conservative (ICAC) (left) and only on ICAa (right).

Results: The voxelwise temporal SD changes produced by the 2 ICA denoising approaches (Fig. 2) were up to $\pm 10 \%$ greater in patients relative to controls. The $\mathrm{CBF}$ and ATT estimation variances were significantly reduced with ICA denoising, indicating an improvement in estimation precision, with a trend for greater effects in patients (Fig. 3). Although global gray matter CBF and ATT values did not change, ICA denoising did impact the voxelwise statistical analysis between patients and controls: after denoising, significant CBF differences found in apparently artifactual locations were eliminated (results not shown). 


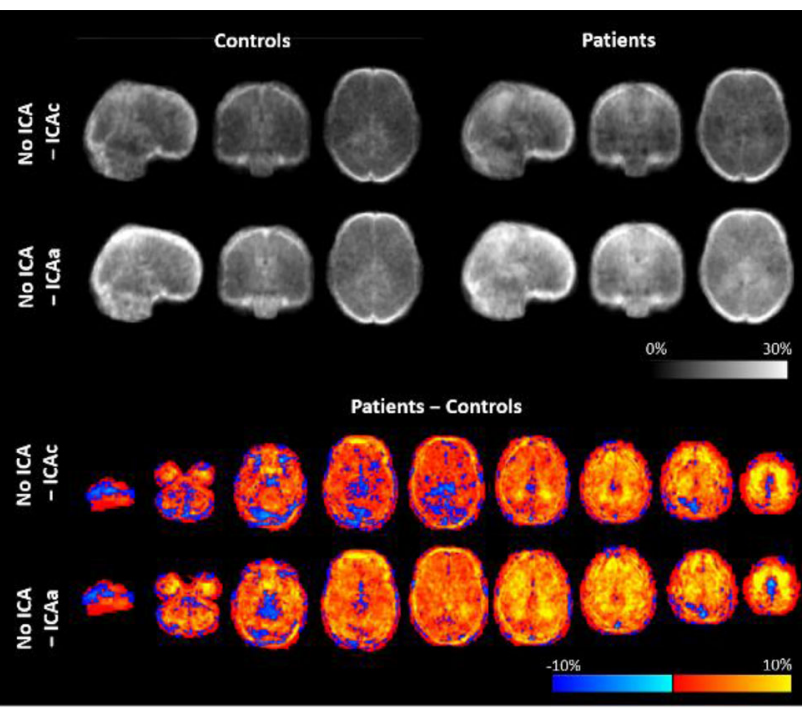

Figure 2. Impact of ICA denoising on the temporal SD of the ASL control-label subtraction timeseries: \% change between non-denoised data (no ICA) and denoised data with ICA conservative (ICAC) and ICA aggressive (ICAa), for Controls and Patients (top) and respective difference (bottom). The top maps show reductions of up to $30 \%$ in temporal SD with both denoising approaches on the edges and the central part of the brain, larger for ICAa vs ICAC and for patients vs controls. The bottom maps show that these changes are up to $\pm 10 \%$ greater in patients relative to controls.
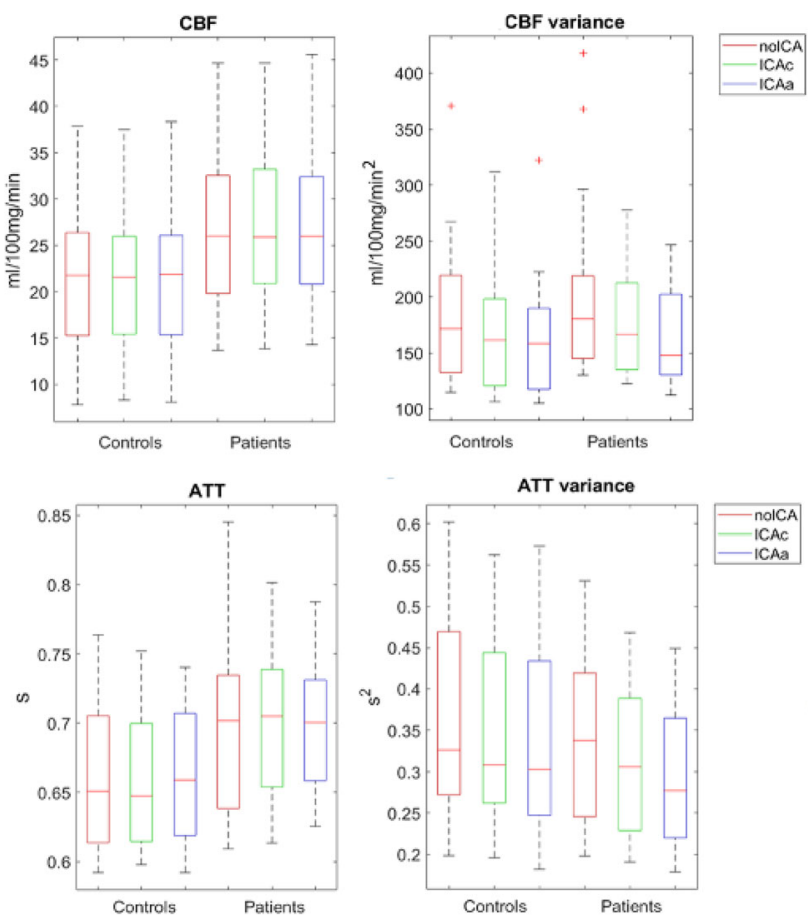

Figure 3. Gray matter mean values of $\mathrm{CBF}$ and $\mathrm{ATT}$, as well as their respective estimation variance, using the three methodologies: No ICA, ICA conservative (ICAC) and ICA aggressive (ICAa), for both controls and patients. Estimation variance is reduced with ICA denoising, with a trend for a greater effect in patients relative to controls.

Discussion: Our results support the use of ICA denoising of multiPLD pulsed ASL data to improve the quality of CBF and ATT estimates, and further suggest that it may differentially affect data collected from patients and controls, significantly influencing the statistical analysis of group differences in perfusion parameters. ICA denoising appears to have more impact in the patients" group, most likely because these are more prone to artifacts such as head motion.

\section{References}

1. Chappell et al., MRM 63, 1357-1365, 2010.

2. Carone et al., Neuroimage 200, 363-372, 2019.

\section{S4.P11.}

Evaluation of physiological contributions to restingstate BOLD fluctuations based on ECG-derived signals in simultaneous EEG-fMRI studies

*I. Esteves ${ }^{1}$, M. Xavier ${ }^{1}$, A. Fouto ${ }^{1}$, A. Ruiz-Tagle ${ }^{1}$,

A. Vourvopoulos ${ }^{1}$, N. A. Silva ${ }^{2}$, R. G. Nunes ${ }^{1}$, R. Gil-Gouveia ${ }^{3}$,

A. Rosa ${ }^{1}$, P. Figueiredo ${ }^{1}$

${ }^{1}$ University of Lisbon, Lisbon, PT;

${ }^{2}$ Hospital da Luz, Learning Health, Lisbon, PT;

${ }^{3}$ Hospital da Luz, Neurology Department, Lisbon, PT

Introduction: Non-neuronal physiological sources, such as heartbeat and respiration, may compromise resting-state BOLD-fMRI analysis. Physiological noise correction usually demands additional experimental setup to record cardiac and respiratory signals. In EEG-fMRI acquisitions, the ECG is typically recorded anyway; since it is modulated by respiration, an ECG-derived respiration (EDR) signal may also be derived without extra equipment ${ }^{1}$. We evaluate the performance of EDR signals for physiological noise correction in restingstate EEG-fMRI, by comparison with measured respiratory signals. Methods: Resting-state EEG-fMRI data was acquired from 6 female migraine patients on a $3 \mathrm{~T}$ Siemens Vida system with a 64-channel $\mathrm{RF}$ coil using 2D-EPI (TR/TE $=1260 / 30 \mathrm{~ms}$, in-plane GRAPPA-2, SMS-3, 60 slices, $2.2 \mathrm{~mm}$ iso resolution). Respiratory signal (Resp) was acquired with the integrated BioMatrix Sensors at $400 \mathrm{~Hz}$ and ECG as part of the MR-compatible EEG system (Brain Products) at $1000 \mathrm{~Hz}$. Gradient artifact correction ${ }^{2}$, downsampling $(250 \mathrm{~Hz})$ and bandpass filtering $(0.05-40 \mathrm{~Hz})$ were applied to ECG. EDRs were extracted with 7 different methods: ECG envelope (ENV), heart-rate variability (HRV), amplitude modulation (AM), QRS area modulation (QRS-AM), principal component analysis (PCA), kernel PCA (kPCA), and empirical mode decomposition (EMD) ${ }^{1}$. The similarity between each EDR and Resp was assessed by correlation and coherence $^{3}$. Three general linear models (GLM) of physiological noise were fitted to the average BOLD signal in grey matter (GM), white matter (WM) and cerebrospinal fluid (CSF): Respiratory only (RETROICOR respiratory terms; respiratory volume per time, RVT), cardiac only (RETROICOR cardiac terms; cardiac rate, CR) and full model with all regressors ${ }^{4,5,6}$. The variance explained (VE) was computed based on the adjusted $\mathrm{R}^{2}\left(\mathrm{R}^{2}{ }_{\text {adj }}\right)$.

Results: Among the EDR methods yielding the highest correlation coefficients for each category, EMD, PCA and kPCA achieved the highest correlation values, but they were surpassed by HRV in terms of coherence (Fig. 1). Regarding the physiological noise models, all EDRs yielded significant VEs and no significant differences were found relative to Resp (Figs. 2, 3). 

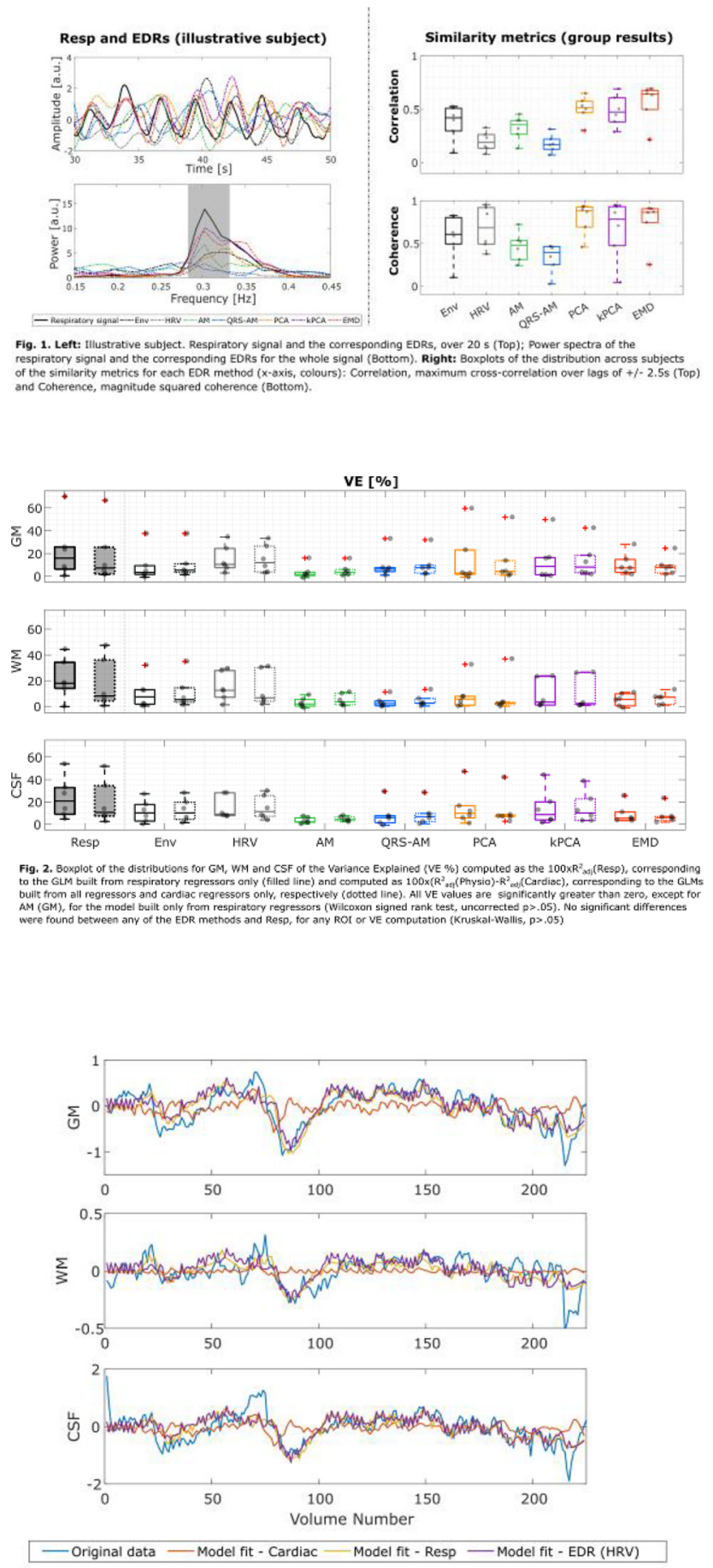

Fig. 3. Illustrative subject. Percent signal change (\%) for the original preprocessed functional data (corrected for distortion and motion, and high-pass filtered) and the model fit using only cardiac regressors, only respiratory regressors obtained
from Resp and only respiratory regressors obtained from EDRs, in this case for HRV method.

Discussion: The EMD, PCA and kPCA methods showed the highest similarity with Resp, in line with previous work outside the MR environment. The VE obtained from the EDRs is equivalent to the one obtained from Resp, indicating the feasibility of using EDRs for physiological noise correction in resting-state EEG-fMRI. Although derived from ECG, EDR-based regressors explained more BOLD signal variance than using only cardiac regressors.

\section{References}

1. Abreu et al., NeuroImage, 2017.

2. Niazy et al., NeuroImage, 2005.

3. Widjaja et al., IEEE Trans. Biomed. Eng., 2012.

4. Birn et al., Hum. Brain Mapp., 2008.

5. Chang et al., NeuroImage., 2009.

6. Glover et al. Magn Reson Med., 2000.

\section{S4.P12.}

How independent is the community structure of static and dynamic functional networks from the brain's underlying structure?

\author{
*F. Ayres-Ribeiro ${ }^{1,2}$, M. Xavier ${ }^{2}$, I. Esteves ${ }^{2}$, R. Abreu ${ }^{3,4}$, J. Jorge ${ }^{5,6}$, \\ P. Figueiredo ${ }^{2}$, A. Francisco ${ }^{1}$ \\ ${ }^{1}$ University of Lisbon, Department of Computer Science \\ and Engineering/Instituto Superior Técnico, Lisbon, PT; \\ ${ }^{2}$ University of Lisbon, Department of Bioengineering/ISR Lisbon, \\ Lisbon, PT; \\ ${ }^{3}$ University of Coimbra, Institute of Nuclear Sciences Applied \\ to Health (ICNAS), Coimbra, PT; \\ ${ }^{4}$ University of Coimbra, Coimbra Institute for Biomedical Imaging \\ and Translational Research (CIBIT), Coimbra, PT; \\ ${ }^{5}$ École polytechnique fédérale de Lausanne (EPFL), Laboratory \\ for Functional and Metabolic Imaging, Lausanne, $\mathrm{CH}$; \\ ${ }^{6}$ Swiss Center for Electronics and Microtechnology (CSEM), Systems \\ Division, Neuchâtel, $\mathrm{CH}$
}

Functional connectivity (FC) measured by resting-state fMRI (rsfMRI) can be analysed using graph theory to characterize brain networks' topology over time. Since these are spatially embedded, the question arises whether the structure captured can be explained exclusively by proximity constraints determined by the brain's underlying structure, or whether there is some degree of functional specialization responsible for the patterns found. This question has been recently addressed in terms of the static functional connectome (sFC) [1]. Here, we further investigate the community structure captured over time by analysing dynamic $\mathrm{FC}(\mathrm{dFC})$ against a spatially informed null model.

A rs-fMRI dataset collected from 9 healthy subjects at $7 \mathrm{~T}$ was used [2]. The data was parcellated into 68 regions using the Desikan atlas, and $\mathrm{dFC}$ was computed (for each $\mathrm{TR}=1 \mathrm{~s}$ ) using phase coherence [3]; this was averaged over all time points to obtain sFC. The networks were thresholded by keeping the giant component structure for most time points. Community analysis was performed using the Louvain algorithm, before and after thresholding both $\mathrm{sFC}$ and $\mathrm{dFC}$ networks, for all time points deviating from a rewiring null model. To investigate the impact of spatial embedding, we used a degree-constrained spatial null model and applied a modified Louvain algorithm regressing out this influence [4]. Finally, the community structure captured with and without the proximity constraints was compared using Normalized Mutual Information (NMI).

We found an overall increase in the modulary values when thresholding both sFC and dFC (Fig. 1), as expected [5]. However, this increase was substantially higher for $\mathrm{dFC}$, pointing to a more modular structure captured over time than for the temporal average. Comparing against the rewiring null model, dFC modularity was statistically significant for all time points, while about $88 \%$ were selected when using the spatial null model, resulting in higher z-score values than for the $\mathrm{sFC}$ (Fig. 2). This suggests that dFC community structure is less influenced by spatial constraints, whereas sFC favors short-range connections expected by the spatial embedding. This observation was further confirmed by comparing the community 
structure with and without regressing out this influence, since we found higher similarity values for dFC (Fig. 3).

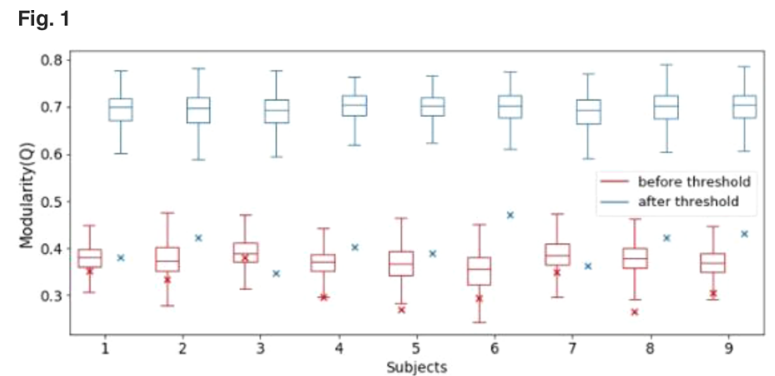

Comparison of the modularity values obtained for both SFC (crosses, $\mathrm{x}$ ) and dFC (boxplots) before (in red) and after (in blue) applying a density threshold (11\%), for each subject, using the Louvain algorithm (resolution parameter set to 1 )

Fig. 2

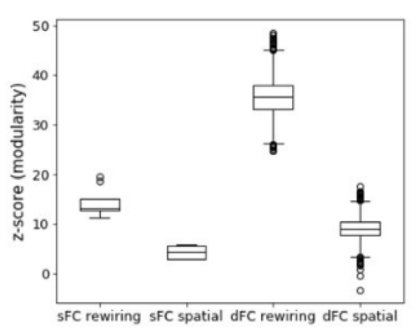

$\mathrm{Z}$-scores resulting from the comparison of $\mathrm{SFC}$ and $\mathrm{dFC}$ modularity values (concatenating all subjects) with the ones from 100 surrogates of the rewiring and spatial null models.

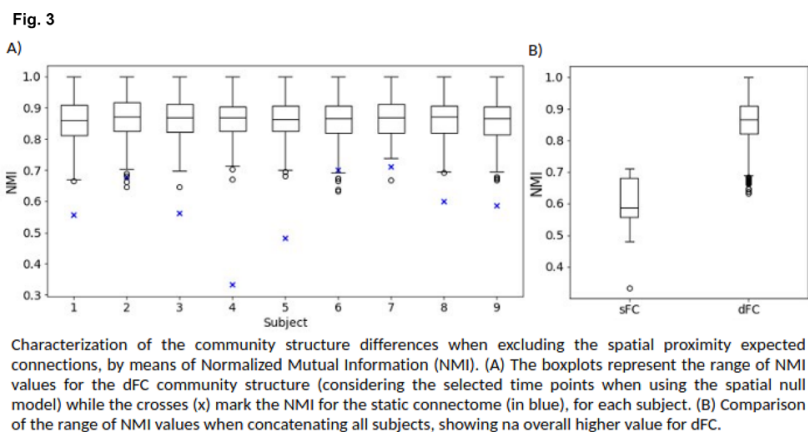

We found that, although the topology and community structure of the rs-fMRI functional networks is mainly explained by the spatial embedding, a significant degree of functional specialization can still be detected which is space-independent, particularly for the dFC.

\section{References:}

[1] Esfahlani et al., NeuroImage 2020.

[2] Wirsich et al., Neuroimage 2021.

[3] Cabral et al., Sci Rep. 2017.

[4] Cazabet et al., 8 th Conference on Complex Networks, pp. 26118-55.

[5] Joudaki et al., PLoS One. 2012.

\section{S4.P13. \\ BOLD signal data trimming results in increased functional connectivity}

\author{
$*$ D. Saraiva ${ }^{1}$, H. Ferreira ${ }^{1}$ \\ ${ }^{1}$ University of Lisbon, Faculty of Sciences, Lisbon, PT
}

Introduction: For performing robust and generalizable machine learning functional neuroimaging studies, combining multi-site data has been essential. Considering the impact of scan parameters on fMRI images, researchers usually trim multi-site data to the same number of time points. However, the effects of trimming BOLD signal data in terms of functional connectivity (FC) are still poorly understood.

Methods: Resting-state functional MRI data from thirty healthy subjects were pre-processed for five different numbers of time points. Individual FC matrices were generated by performing dual regression with the brain template from Shirer et al. [1]. The correlation matrices were binarized for several thresholds, which excluded weak correlations and included both positive and negative correlations for the analysis. To study FC differences between different numbers of time points, network degrees were computed for each brain region, and the degree distributions were compared. In addition, the median degree numbers per subject and per brain region were subtracted between different trimming conditions. Wilcoxon tests were then performed to evaluate which brain region degrees were significantly different between different trimming conditions.

Results: Increasing degree numbers for the global network were observed, together with a non-significant right-shifting trend of the degree distributions, associated with fewer time points (Fig. 1 left). Additionally, when comparing the degree distribution per brain region, it was observed that the majority of resting-state networks show increased degree numbers, whilst only the auditory network showed a decreased degree for fewer time points (Fig. 1 right).

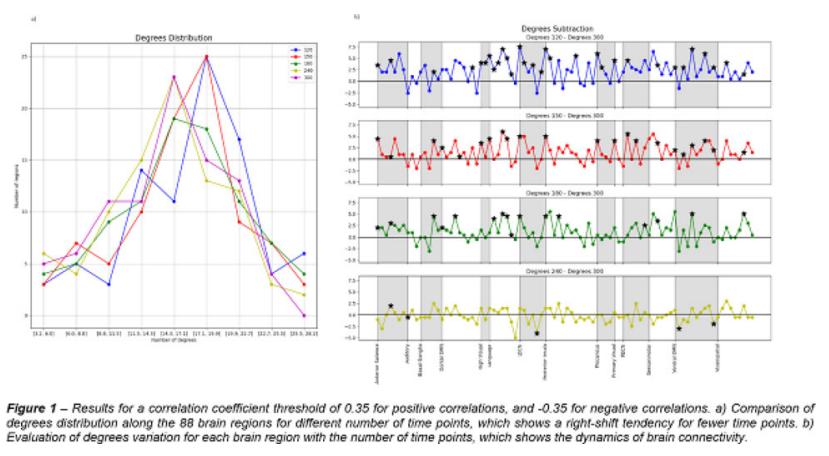

Discussion: The overall increase in the degree numbers for fewer time points, suggests that there are fewer stronger correlations between brain regions with longer scanning times. In fact, some brain networks seem to present a more dynamic pattern than others, namely the higher visual, language, and left executive control networks. In contrast, the auditory, the visuospatial, the basal ganglia, and the dorsal default mode networks present a more stable and less dynamic pattern. This may be related to environmental demands (e.g. at rest during MRI scanning, visual stimuli) that engage some networks to change communication patterns more than others. Finally, although the overall characteristics of the global networks seem to be maintained, care should be taken to account for the trimming effects on FC at the regional level. 


\section{Reference:}

[1] Shirer WR, Ryali S, Rykhlevskaia E, Menon V, Greicius MD: Decoding subject-driven cognitive states with whole-brain connectivity patterns. Cereb Cortex (2012)

\section{S4.P14.}

\section{Functional magnetic resonance imaging analysis revisited: a model free approach}

\author{
*J. E. McGillivray ${ }^{1,2}$, H. Debruin ${ }^{1,3}$, M. D. Noseworthy ${ }^{1,2,4,5}$ \\ ${ }^{1}$ McMaster University, School of Biomedical Engineering, Hamilton, \\ $C A$; \\ ${ }^{2}$ St. Joseph's Healthcare, Imaging Research Centre, Hamilton, CA; \\ ${ }^{3}$ McMaster University, Electrical and Computer Engineering, \\ Hamilton, $C A$; \\ ${ }^{4}$ McMaster University, Department of Radiology, Hamilton, CA; \\ ${ }^{5}$ University of Toronto, Department of Medicine and Biomedical \\ Engineering, Toronto, $C A$
}

Introduction: Functional magnetic resonance imaging (fMRI) employs the blood oxygen dependent level (BOLD) effect to determine stimulus induced brain physiological state changes [1]. The BOLD effect leverages hemoglobin"s oxygenation dependant magnetic properties to describe changes in blood-flow and metabolic need [1]. The hemodynamic response function (HRF) mathematically characterizes the BOLD response, to describe neural activity via amplitude, latency, and duration measures [2]. Although BOLD signal temporal correlation with the HRF is the accepted norm to determine neural activation, it is non-ideal because of its assumed canonical form [3]. An assumed HRF model also masks information to be gained from its variability. As such, a model independent method is proposed.

Methods: Six participants (5 male, 3 right-handed) performed finger tapping during two fMRI runs. Each run was divided into six repeated sets of two 30 s blocks: (i) right finger tapping (RT) then rest (ii) left finger tapping (LT) then rest. Data was collected using a 32-channel head coil and GE 3 T MR750 scanner. 3D fSPGR $(0.5 \times 0.5 \times 1$ $\mathrm{mm}, \mathrm{TE} / \mathrm{TR} / \mathrm{flip}=2.36 / 7.86 \mathrm{~ms} / 120)$ images provided anatomic reference. Functional images $(3.44 \times 3.44 \times 4 \mathrm{~mm}, \mathrm{TE} / \mathrm{TR} / \mathrm{flip}=35 /$ $2000 \mathrm{~ms} / 900$ ) were preprocessed (eddy current and motion correction), prior to co-registration using FSL [4]. Co-registered images were temporally $(0-0.05 \mathrm{~Hz})$, and spatially ( $5 \mathrm{~mm}$ FWHM Gaussian) filtered. Voxel-wise analysis was performed based off the BOLD time series phase space. Phase space separability into tap versus rest states was assessed with a linear discriminant classifier. Voxel-wise HRF correlation analysis served as an activation reference. Phase space metric and the correlation maps were Fischer transform $\mathrm{z}$ score normalized and spatially warped to the MNI-152 atlas for group analysis. Statistical maps were generated with AFNI [5].

Results: Bonferonni corrected T-tests showed significantly active regions for correlation and separation metrics for RT and LT trials. A larger activation using the separation metric, as noted for RT (Fig. 1), also held for LT. ANOVAs comparing HRF correlation to boundary separation activity, with participants separated by handedness, were performed for RT and LT. The dominant tappers showed less similarity between the two metrics (Fig. 2). Regardless of handedness, the metrics show a greater similarity during left hand tapping.

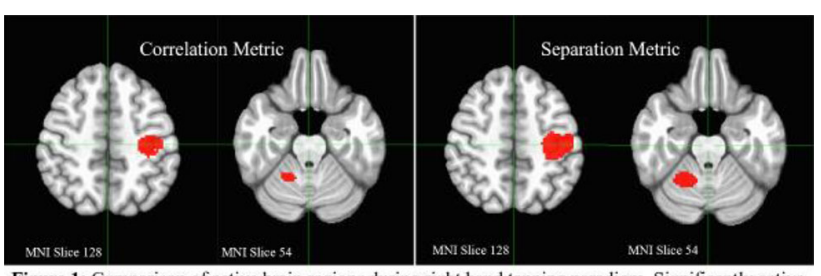

Figure 1: Comparison of active brain regions during right hand tapping paradigm. Significantly active regions were required to have, $p$ value of less than 0.05 and boundary separation accuracy or HRF correlation of greater than $75 \%$. Phase space separability shows larger activation regions in the motor cortex and cerebellum than convention HRF correlation metric. Image left is anatomical right.

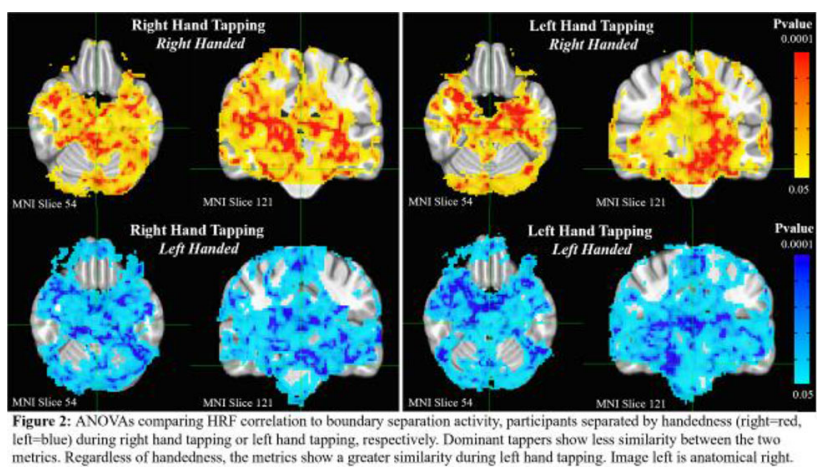

Discussion: The results indicate that the separability metric agrees with HRF correlation activation patterns yet is model free. It is sensitive to hand-dominance and thus could show activation as a gradient due to brain state organization and not, subject specific, amplitude. The results suggest that handedness affects motor activation, with left-handed patterns tending to show additional and bilateral recruitment. A feasible extension would compare paretic and non-paretic tapping activation for stroke patients to characterize activation and assess rehab induced changes.

\section{References}

[1] Ogawa S, et al. (1990) Proc, Natl. Acad. Sci. 87(24);9868-9872.

[2] Ogawa S. et al. (1992) Proc. Natl. Acad. Sci. 89(13):595 I-5955.

[3] Lindquist MA, et al. (2009) Neurolmage, 45(1 Suppl):S187-198.

[4] Smith SM, et al. (2004) Neurolmage, 23(Suppl 1):S208-219.

[5] Cox RW, et al. (1996) Compu. Biomed. Res. 29(3):162-173.

\section{S4.P15.}

Using mouse hemodynamic response functions to detect BOLD fMRI with different mechanical stimulations

\author{
*H. F. Chen ${ }^{1}$, *B. Pradier $^{1}$, H. Lambers ${ }^{1}$, A. Allelein ${ }^{1}$, \\ N. Nagelmann ${ }^{1}$, C. Faber ${ }^{1}$ \\ ${ }^{1}$ University Hospital Münster, Translational Research Imaging \\ Center (TRIC), Münster, DE
}

Introduction: Task functional MRI (fMRI) has been well-established combining the measurement of hemodynamic response function (HRF) with the general linear model (GLM) to detect blood-oxygenlevel-dependent (BOLD) changes in rat brain structures 1 . However, it is unclear whether species (rat vs. mouse) or sex differences in mice 
exist regarding HRF. Further, a strong bias exists towards male study subjects in the literature 2. Therefore, we investigated HRF in male and female mice using 2 natural stimulation modalities (pinprick (pp) and von Frey $(\mathrm{vF})$ ) and compared these findings to our previously published rat HRF1.

Methods: 8 to 10-week-old C57BL/6 J mice were stimulated on their right hind paw and scanned using a 9.4 T MRI scanner with a Cryoprobe. After shimming, task fMRI measurements were performed using a single-shot gradient EPI sequence (TR/TE 1000/18 ms, Matrix $76 \times 66$, Resolution $200 \times 200 \mu \mathrm{m}^{2}$ ) under medetomidine sedation combined with $0.2 \%$ isoflurane. Experiments started $40 \mathrm{~min}$ after bolus injection. For mechanical $\mathrm{pp}$ and $\mathrm{vF}$ stimulation, we used an inhouse developed rotating stimulator and a block paradigm with $10 \mathrm{~s}$ stimulation $/ 20 \mathrm{~s}$ rest and a frequency of $1 \mathrm{~Hz}$ and a pulse duration of $0.5 \mathrm{~s}$ (Fig. 1). SPM12 was used for MR data preprocessing and to perform GLM-based analysis (using the FIR basis set). Time courses of BOLD responses of S1HL were extracted with MarsBaR. Time courses were fitted (Fig. 2A), HRFs were calculated (Fig. 2B) and compared with a functional t-test using custom written Matlab scripts as previously published 1 .

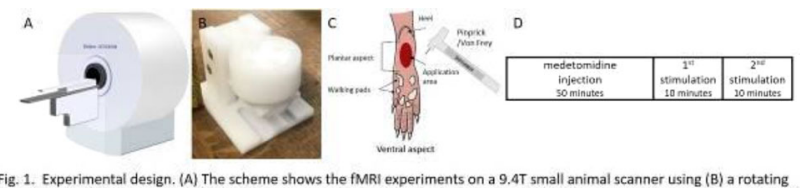
Fig. 1. Experimental design. (A) The scheme shows the fMRI experiments on a $9.4 \mathrm{~T}$ small animal scanner using (B) a rotating
stimulator. (C) Mice were stimulated at the same position of right hind paw using calibrated von Frey flilaments or pin prick (D).

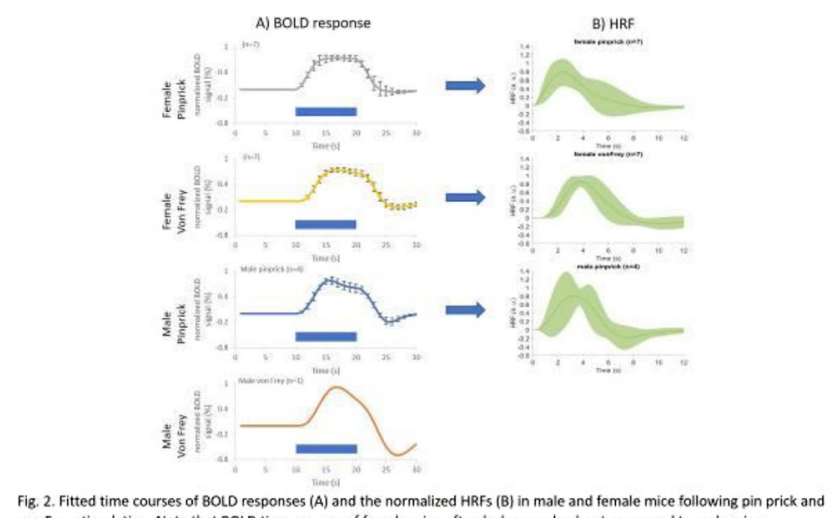

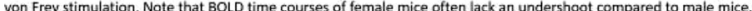

Results: We found significant differences in the HRFs between both sexes during pp stimulation $(\mathrm{p}=0.029)$, which was accompanied by $0.5 \mathrm{~s}$ delayed onset in male mice (Fig. 3A). Further, we detected a different HRF during $\mathrm{pp}$ and $\mathrm{vF}$ stimulation in female mice $(p=0.034)$ (Fig. 3B). Again, the female pp-HRF showed a significantly faster onset. Next, we tested GLM performance with different model orders. Preliminary data show comparable performance of mouse-derived and rat-specific HRFs at the first order, while human HRF performed poorly. Using a 3rd order model all HRFs showed similar performance.

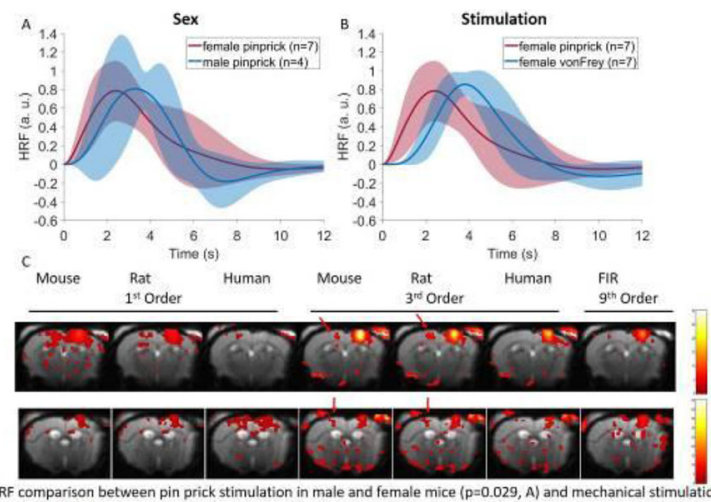

g. 3. HRF comparison between pin prick stimulation in male and female mice $(p=0.029, A)$ and mechanical stimulation odalitites in female mice $(\mathrm{p}=0.034, \mathrm{~B})$. Data were analyzed using a functional $\mathrm{t}$-test. (C) GLM performance with different basis inctions (canonical $1^{\text {th }}$ order, canonical $3^{\text {at }}$ order) and the finite impulse response (FIR). Performance was compared using 3 REs from SPM. Shown are exempland datasets for pinprick (top row) and von Frey stimulation (bottom row). Color bar dicates t-values for models of $1^{\prime \prime}$ order and f-values for $\mathrm{FIR}$ and $3^{\text {rd }}$ models of $3^{\text {rd }}$ order.

Discussion: In conclusion, our study shows sex- and stimulation modality-specific differences in HRFs. These differences might account for the increased sensory sensitivity of female compared to male mice. Further, the difference in modality might arise from increased recruitment of peripheral nociceptors when stimulating with pin prick. Since most preclinical studies are performed with male animals, we therefore recommend to use sex-specific HRFs or to use models of higher order to form regressors for the GLM.

\section{References}

1. Lambers, H. et al. A cortical rat hemodynamic response function for improved detection of BOLD activation under common experimental conditions. Neuroimage 208, 116446 (2020).

2. Mogil, J. S. Qualitative sex differences in pain processing: emerging evidence of a biased literature. Nat. Rev. Neurosci. 21, 353-365 (2020).

\section{S4.P16.}

\section{A framework for optimal planning during ultrahigh spatiotemporal resolution line-scanning}

\author{
${ }^{*}$ J. Heij ${ }^{1}$, L. Raimondo ${ }^{1}$, J. Siero ${ }^{1,2}$, S. Dumoulin ${ }^{1,3}$, W. van der \\ Zwaag $^{1}$, T. Knapen ${ }^{1,3}$ \\ ${ }^{1}$ Spinoza Centre for Neuroimaging, Amsterdam, NL; \\ ${ }^{2}$ University Medical Centre Utrecht, Department of Radiology, \\ Utrecht, $N L$; \\ ${ }^{3}$ Vrije Universiteit Amsterdam, Department of Experimental \\ and Applied Psychology, Amsterdam, NL
}

Introduction: To achieve sufficient spatial and temporal resolution to distinguish features of laminar BOLD responses, we recently implemented line-scanning $\mathrm{fMRI}^{1}$. Here, a single line is acquired with $\sim 250 \mu \mathrm{m}$ resolution and $\sim 100 \mathrm{~ms}$ TR, requiring very precise positioning. To negate the convoluted folding of the cortex and maximize the resolution in laminar direction, we developed a framework for planning of the line based on functional (population receptive field $[\mathrm{pRF}]$ parameters) and structural (minimal curvature) information. Our planning strategy allows us to direct the line through a patch of cortex with distinct functional and structural properties, thereby greatly enhancing the utility of line-scanning data.

Methods: The pipeline includes two different scan sessions at different days (Fig. 1). In the first session, we acquired a high-resolution MP2RAGE and performed pRF-mapping at $7 \mathrm{~T}$. The line-scanning acquisition in session 2 used a modified $2 \mathrm{D}$ gradient-echo sequence where the phase-encode gradients are removed and outer volume 
suppression (OVS) is used to suppress signal outside the line. Rigidbody transformation mapping of session 1 to session 2 was applied to the coordinate and normal vector of the selected best vertex to plan the line (Fig. 1). Four 5-min line-scanning runs were acquired with flashing checkerboard stimuli presented on the left and right side of fixation in a randomized event-related design to elicit lateralized BOLD responses (Fig. 2BC) ) $^{2,3}$.
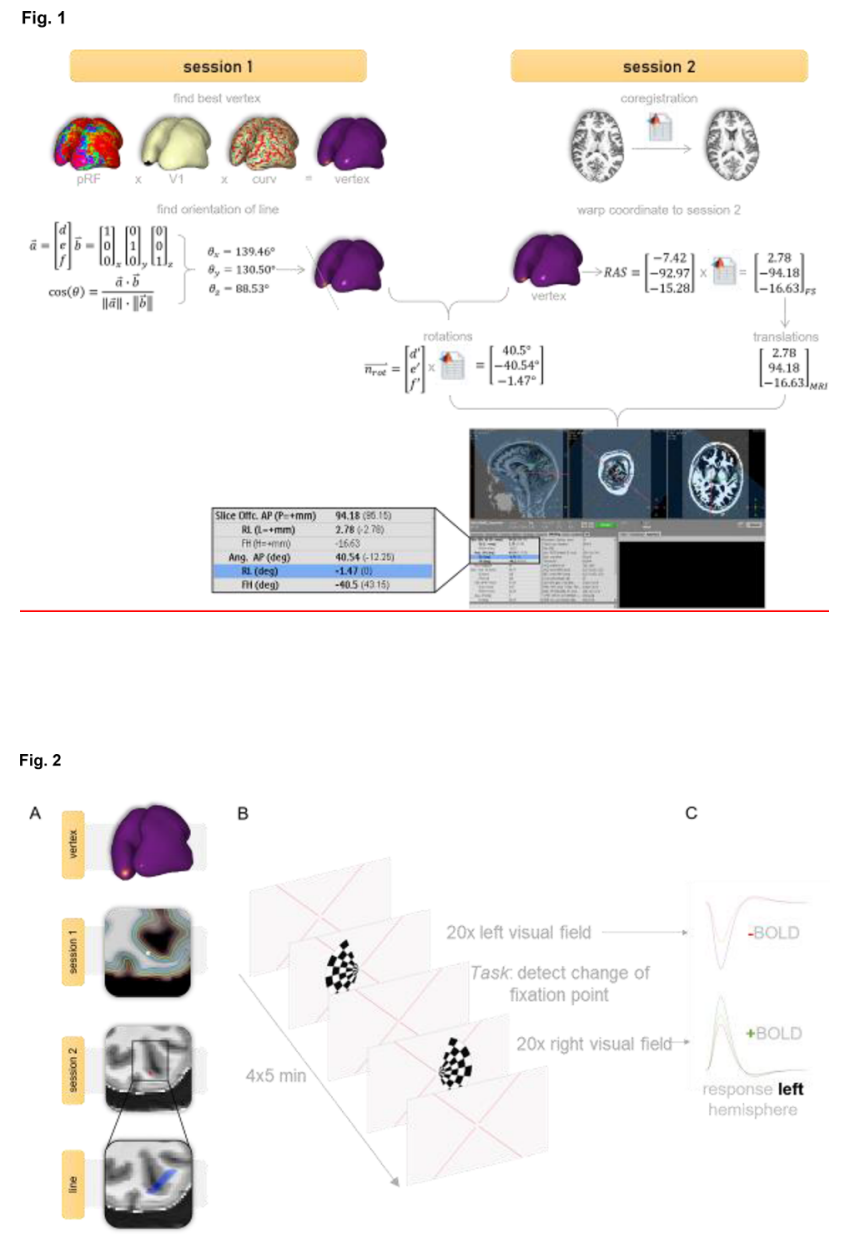

Results: The line was perfectly perpendicular to the cortical band covering our patch of interest (Fig. 2A), indicating successful navigation of the best vertex from surface to scanner. To determine which voxels of the line belonged to grey matter, we used the tissue segmentation obtained in session 1 (Fig. 3A). Finite impulse response (FIR) time-courses showed a strong BOLD response in the left hemisphere following stimulation to the right, contralateral, visual hemifield (Fig. 3B). This response was absent or inverted for ipsilateral stimulation, which caused negative BOLD-response in a few voxels per subject (Fig. 3C).

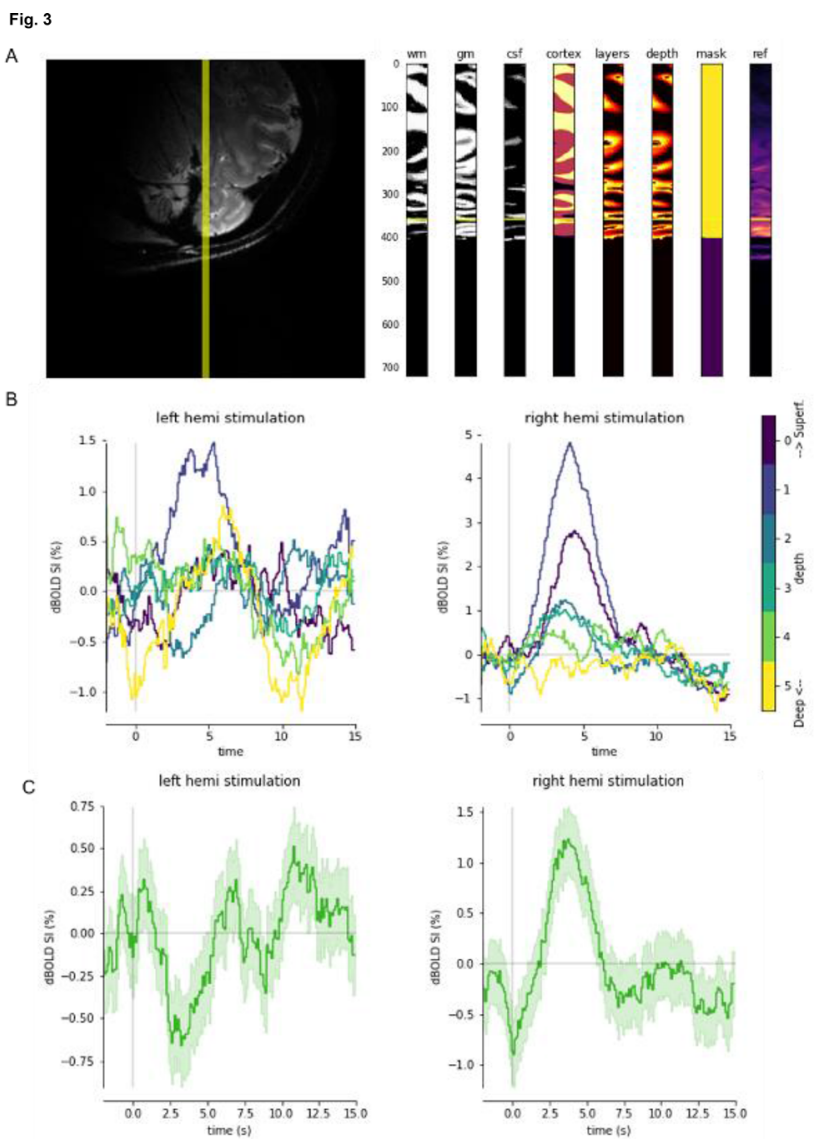

Discussion: We have presented a method that combines functional and structural data to increase the accuracy of planning and enrich the information obtained with line-scanning fMRI. A hemifield experiment evoked a strong positive BOLD-effect in response to stimulation of the right visual field, while stimulation of the left visual field evoked more negative responses. We aim to build upon these promising results by optimizing the pipeline further in a variety of ways; eye tracking (effect of eye blinks on laminar processing), motion correction and denoising strategies, and an attention task to elicit stronger negative BOLD responses ${ }^{4}$.

\section{References}

1. Raimondo, L. et al. ISMRM (2020).

2. Goense, J. et al. Neuron 76, 629-639 (2012).

3. Fracasso, A. et al. Neuroimage 164, 100-111 (2018).

4. Gouws, A. D. et al. J. Neurosci. 34, 10347-10360 (2014).

S4.P17.

Neurovascular coupling: fMRI of human brain during visual stimulation, effect of acetylsalicylic acid

*M. Ublinskiy ${ }^{1,2}$, A. Manzhurtsev ${ }^{1,2}$, A. Yakovlev ${ }^{1,2}$,

N. Semenova ${ }^{1,2}$, T. Akhadov ${ }^{1}$

${ }^{I}$ Clinical And Research Institute Of Emergency Pediatric Surgery And Trauma (CRIEPST), Department of Radiology, Moscow, RU;

${ }^{2}$ Russian Academy of Sciences, Institute of Biochemical Physics, Moscow, $R U$ 
Purpose: The study is aimed at explanation of the mechanism of neurovascular coupling a pulse increase in the supply of oxygen and energy metabolites to the excitation area of central nervous system in response to neurostimulation.

Methods and materials/background: Study group consisted of 15 healthy participants in age from 18 to 28 years (mean age 24 years). All MRI studies were performed on a Philips Achieva dStream 3.0 T scanner equipped with a 32-channel Philips dStream head coil. A 5 min fMRI echo planar imaging (EPI) sequence was acquired $(\mathrm{TR}=3000 \mathrm{~ms}$, echo time $[\mathrm{TE}]=30 \mathrm{~ms}, 100$ dynamics with dynamic scan time $=3 \mathrm{~s}$ ). A flashing chessboard with a frequency of $8 \mathrm{~Hz}$ will be used as a visual stimuli for fMRI study (Fig. 1).

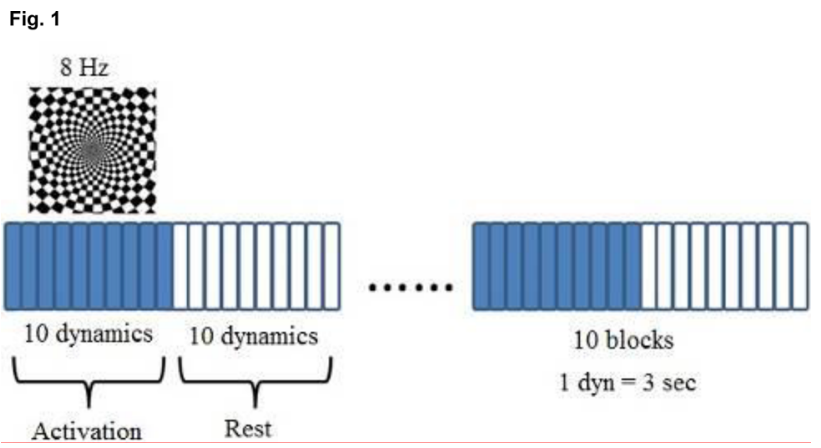

All fMRI studies for each participant were repeated twice: after the first fMRI scan all participants took a pill of acetylsalicylic acid (aspirin) to inhibit the prostaglandin synthesis-one of the main ways of vasodilation.

An intergroup analysis (before/after pill) was estimated using paired t-test.

Results: In all subjects, the visual cortex was activated statistically significantly ( $p<0.05$, FWE). Rest of brain structures do not show a reliable response to neurostimulation (Fig. 2A). The study made it possible to discover and study effect of habituation-desentization of induced vasodilation upon repeated video stimulation. The resistance of BOLD signal level in visual cortex to repeated video stimulation and effect of acetylsalicylic acid (aspirin) was revealed. BOLD was found to be sensitive to aspirin in thalamus ( $p<0.09$, FWE), a structure involved in the transmission of visual information (Fig. 2B).

Fig. 2
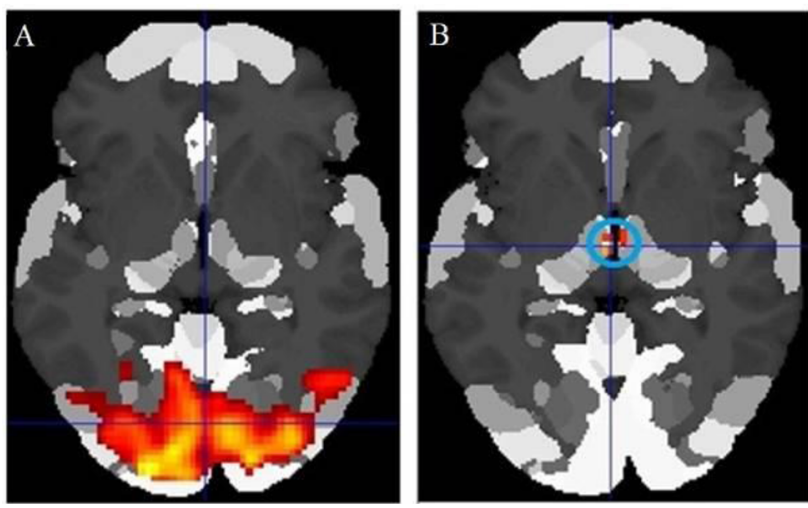

Conclusion: The observed decrease in hemodynamic response to visual stimulation in thalamus under the action of aspirin, absence of the effect of aspirin in the visual cortex and a strong correlation between changes in BOLD in both structures indicate a different ratio of the activities of drug-initiated vasodilation and vasoconstriction processes. Analysis of the distribution of prostaglandin E synthetase in the human brain showed that the expression of the corresponding gene in occipital cortex is lower than in the thalamus. The lower content of the enzyme, the precursor of PGH synthetase, may be the reason for the lesser effect of its inhibition by aspirin in the cortex as compared to the thalamus. This creates a statistically significant vasoconstrictor effect of the drug in thalamus during video stimulation.

\section{S4.P18.}

Does the field strength matter? Comparing layer-fMRI VASO across $3 \mathrm{~T}, 7 \mathrm{~T}$, and $9.4 \mathrm{~T}$

${ }^{*}$ R. Huber ${ }^{1}$, M. Kronbichler ${ }^{2,3}$, R. Stirnberg ${ }^{4}$, L. Kronbichler ${ }^{5}$, S. Fernández-Cabello ${ }^{6}$, T. Stöcker ${ }^{4}$, B. A. Poser ${ }^{1}$

${ }^{1}$ Maastricht University, Faculty of Psychology and Neuroscience, Maastricht, NL;

${ }^{2}$ Christian Doppler Medical Centre (PMU), Neuroscience Institute, Salzburg, AT:

${ }^{3}$ University of Salzburg, Centre for Cognitive Neuroscience / Department of Psychology, Salzburg, AT;

${ }^{4}$ German Center for Neurodegenerative Diseases (DZNE), Bonn, DE;

${ }^{5}$ Christian-Doppler Medical Centre (PMU), Department

of Psychiatry, Psychotherapy and Psychosomatics, Salzburg, AT;

${ }^{6}$ University of Oslo, Norwegian Centre for Mental Disorders Research (NORMENT), Oslo, NO

Introduction: Recent methodological advances in fMRI contrast and readout strategies allow researchers to approach the mesoscopic regime of cortical layers. Specifically, high-resolution blood-volume sensitive vascular space occupancy (VASO) (Lu 2003) enables functional mapping of cortical information processing within and across brain systems (Bollmann 2020). While layer-fMRI VASO has commonly been performed at $7 \mathrm{~T}$, the goal of this work is to investigate how well it can be extended to other field strengths, $3 \mathrm{~T}$ and $9.4 \mathrm{~T}$.

Methods: We used an SS-SI VASO implementation at $3 \mathrm{~T}, 7 \mathrm{~T}$, and 9.4 $\mathrm{T}$ based on the 3D-EPI sequences by (Poser 2010) and (Stirnberg 2020), respectively. All experiments were performed on MagnetomSIEMENS scanners with 32-channel (3 T, $7 \mathrm{~T})$ and 31ch $(9.4 \mathrm{~T})$ receive coils, using a $12-15$ min finger tapping task. Layer analysis and within-layer smoothing $(\mathrm{FWHM}=0.8 \mathrm{~mm}$ ) was done in LayNii (Huber 2021). Here, data from two traveling heads were augmented with separate cohorts across field strengths $(\mathrm{N}=12) .9 .4 \mathrm{~T}$ experiments used a modified adiabatic TR-FOCI inversion pulse and toggling between two separate $\mathrm{pTx}$ shims for inversion and excitation (Huber 2018).

Results: We find that a single run for BOLD and VASO can provide reliable task-induced signal changes at sub-millimeter resolutions across all tested field strengths (Fig. 1). The GE-BOLD activation map shows more significantly activated voxels than VASO. VASO's higher spatial specificity is also visible in profile plots as two separate peaks (blue arrows). The blurry nature of the GE-BOLD signal is expected due to the locally unspecific signal of pial and diving veins. 
Fig. 1

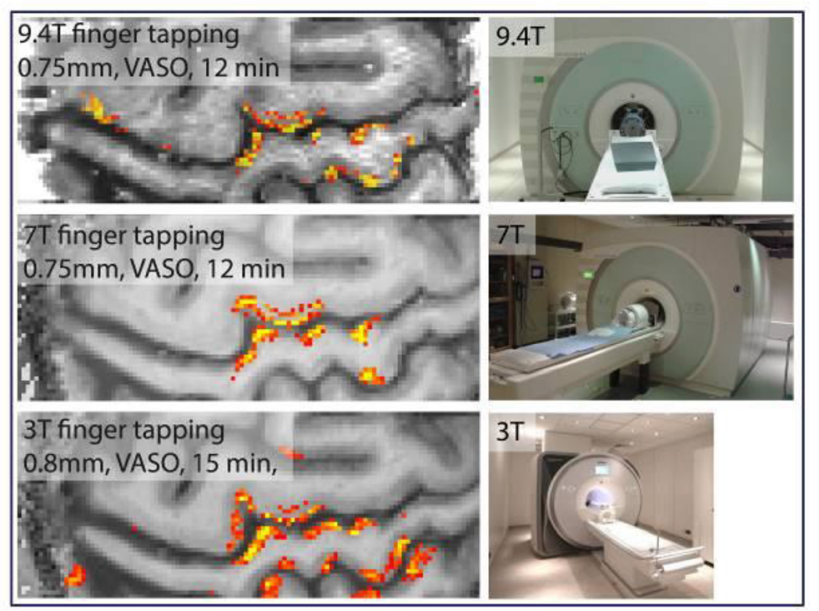

3 T vs. 7 T: For VASO at 3 T, we find that the relatively low SNR can be partially accounted for by means of the divergence of blood and tissue $\mathrm{T} 1$ at lower field strengths. Furthermore, layer-specific smoothing, longer runs, and NORDIC-based (Vizioli 2021) component selection can result in additional sensitivity gains.

9.4 $T$ vs. $7 \mathrm{~T}$ : At $9.4 \mathrm{~T}$, we find that the practical constraints of $\mathrm{B} 1+$-challenges can be largely accounted for by advanced pulse specific pTx-shimming with 9.4 T-optimised excitation and inversion pulses.

Fig. 2

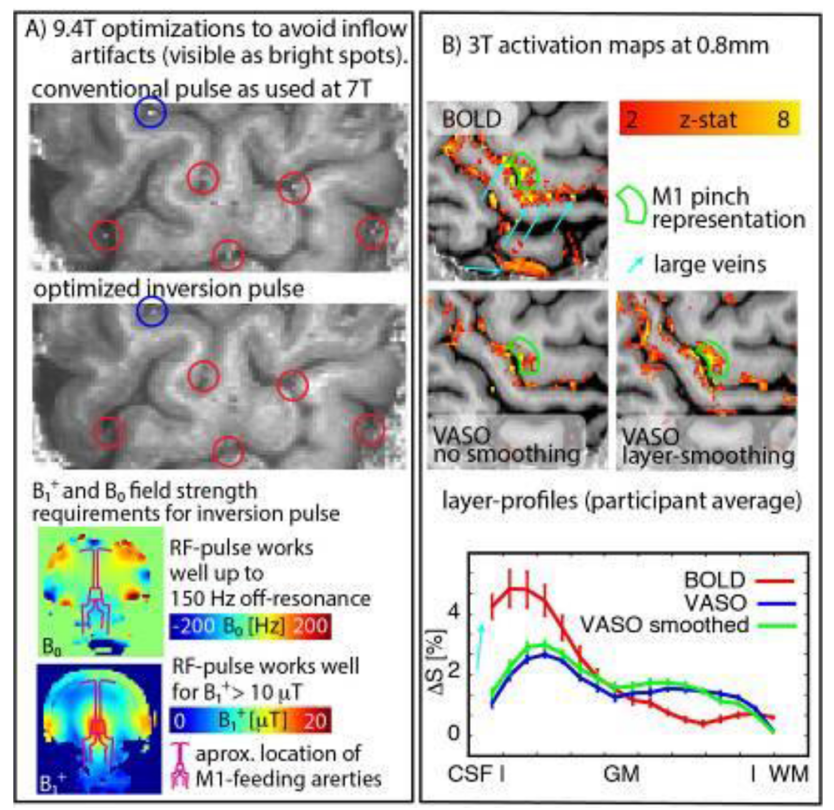

Discussion and conclusion: In this work we find that high quality layer-specific CBV-fMRI data can be acquired across a wide range of field strengths, as suggested previously (Markuerkiaga 2021). Each field strength has specific challenges that can be largely accounted for. We believe that the usability of sub-millimeter VASO sequences across field strengths will contribute to an increased accessibility of laminar-fMRI across a wider community.

\section{References:}

Bollmann \& Barth 2020, PNB,

Huber et al., 2021, NI,

Huber et al. 2018. NI,

Lu et al. 2003, MRM,

Markuerkiaga et al., 2021, NM,

Strinberg \& Stöcker, 2020, MRM,

Vizioli et al., 2021, BioRvix.

\section{S4.P19.}

Total glutamate and glutamine concentration dynamic after short visual stimulus

*A. Yakovlev ${ }^{1,2,3}$, A. Manzhurtsev ${ }^{1,2,4}$, M. Ublinskiy ${ }^{1,2}$, O. Bozhko ${ }^{1}$, T. Akhadov ${ }^{1,4}$, N. Semenova ${ }^{1,2,3,4}$

${ }^{1}$ Clinical and Research Institute of Urgent Pediatric Surgery and Trauma (CRIEPST), Moscow, $R U$;

${ }^{2}$ Institute of Biochemical Physics RAS, Moscow, RU;

${ }^{3}$ Russian Academy of Sciences, Moscow, RU;

${ }^{4}$ Lomonosow Moscow State University, Moscow, $R U$

Introduction: Currently, it is important to measure the concentration of the main excitatory neurotransmitter glutamate (Glu) after a single stimulus using proton magnetic resonance spectroscopy (1H-MRS) ${ }^{1,2}$. The peculiarity of the kinetics of changes in [Glu], measured using MRS, in a short period is that the resulting change in [Glu] can be caused not only by slow metabolic mechanisms ${ }^{3}$ but also by the rapid movement of the neurotransmitter from the vesicles ${ }^{4}$. Hence, it becomes necessary to determine the kinetic characteristics of Glu dynamics during neuroactivation. For this purpose, in this work, the total concentration of Glu and glutamine (Gln) was measured in response to a short visual stimulus in $24 \mathrm{~s}$.

Methods: MRI and single voxel 1H-MRS were performed using the Philips Achieva dStream 3 T MRI System (Eindhoven, Netherlands) using the 8 coil SENSE Head coil. Eighteen healthy subjects (11 males; mean age $=23.6 \pm 2.2$ years) took part in the study. The $4 \mathrm{~Hz}$ flashing checkerboard was used for stimulation in similar blocks $(3 \mathrm{~s}$ stimulus, 21 s-black screen). FMRI images were acquired using gradient-echo echo-planar imaging (GE EPI) sequence with TR/ $\mathrm{TE}=3000 / 40 \mathrm{~ms}, \quad$ flip angle $=90^{\circ}, \quad$ in-plane resolution $=2.4 \times 2.4 \mathrm{~mm}$, slice thickness $=4 \mathrm{~mm}$, number of slices $=30$ and 120 measurements. An activation map was obtained using SPM12 in response to visual stimulation. The spectra were obtained using the PRESS sequence $(\mathrm{TR}=2000 \mathrm{~ms}, \mathrm{TE}=35 \mathrm{~ms}, \mathrm{NSA}=444$ $(12 * 37)$, voxel size $-20 \times 30 \times 20 \mathrm{~mm})$ and were localized in the activated region of the visual cortex (Fig. 1). Spectra were preprocessed. We identified the impact of BOLD effects in metabolite spectra by estimating the differences in total creatine (tCr) singlet (3.03 ppm) linewidth between each time point and the value at $-1 \mathrm{~s}$ ( $1 \mathrm{~s}$ before the start presentation of the stimulus. Metabolites were quantified using LCModel with a basis set of 17 simulated metabolites. The difference between the obtained values $(\mathrm{Glx} / \mathrm{Cr}, \mathrm{NAA} / \mathrm{Cr}$, and linewidth of $\mathrm{tCr}$ ) corresponding to individual time points was statistically determined by repeated measures of ANOVA statistical test. Post hoc Dunnett's test was then run. 
Fig. 1
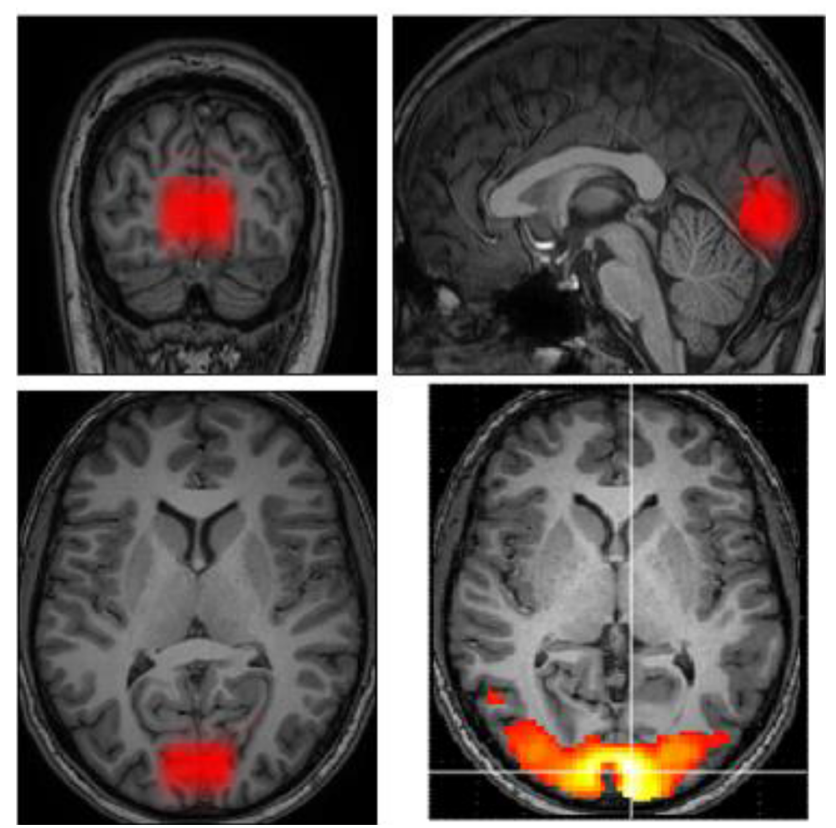

Results: Dunnett's post hoc test showed a statistically significant increase in $\mathrm{Glx} / \mathrm{Cr}$ between $-1 \mathrm{~s}$ and $1 \mathrm{~s}$, $19 \mathrm{~s}$ (Fig. 2, $\mathrm{p}<0.05, \sim+7 \%$ at each point; rmANOVA: $\mathrm{p}=0.06)$. There is no correlation between the change in $\mathrm{Glx} / \mathrm{Cr}$ concentration and the amplitude of the fMRI response $(r=0.24, \mathrm{P}>0.05)$.

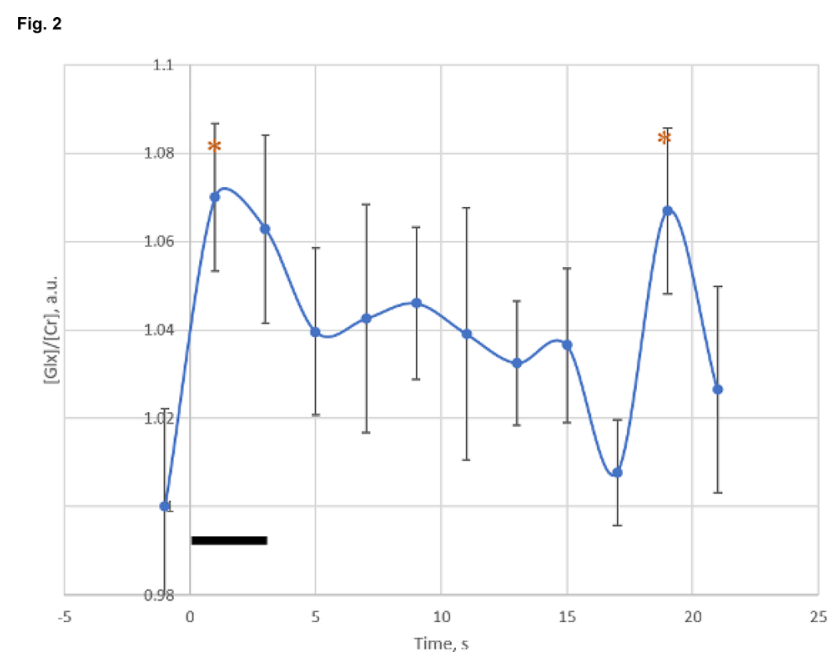

Discussion: Analysis of the main rates of reactions for the synthesis of Glu and Gln showed that there is no reaction with the rate obtained in the present study $(\sim 0.8 \mathrm{mM} / \mathrm{s})$. It follows from this that the result obtained is most likely a consequence of the transition of Glu from the "invisible" (vesicular) pool to the "visible" one $\mathrm{e}^{5}$. The peak at $19 \mathrm{~s}$ is considered as the end of the Glu reuptake process.

\section{References:}

1. Bednařík, P. et al., 2015.

2. Betina Ip et al., 2019.

3. Rothman, D. L. et al., 2011.

4. Hori, T. et al., 2012.

5. Kauppinen R. A. et al., 1994.

\section{S4.P20.}

The iron-complex of Deferasirox: the molecular and functional characteristics that can make it a viable alternative to Gd-based agents

\author{
*E. Gianolio ${ }^{1}$, L. Palagi ${ }^{1}$, E. Di Gregorio ${ }^{1}$, R. Stefania ${ }^{1}$, \\ C. Cavallotti ${ }^{2}$, S. Aime ${ }^{1}$ \\ ${ }^{1}$ University of Turin, Department of Molecular Biotechnology \\ and Health Science, Turin, IT; \\ ${ }^{2}$ Cage Chemicals, Novara, IT
}

Introduction: Currently, the search for alternatives to gadoliniumcontaining MRI contrast agents addresses the field of $\mathrm{Fe}$ (III) bearing species with the view that the use of an essential paramagnetic metal ion may avoid the issues raised by the exogenous $\mathrm{Gd}$. In this context attention is specifically devoted to highly stable, hexacoordinated $\mathrm{Fe}(\mathrm{III})$ complexes [1-3]. Particularly interesting are Fe(III) complexes with ligands that are represented by clinically approved iron sequestering agents, as their established biocompatibility properties may provide a good support for facilitating their clinical translation as MRI CAs. Herein, the in vitro and in vivo studies on $\mathrm{Fe}$ (deferasirox)2 (Fe(DFX)2-Fig. 1), a Fe(III) complex with a sequestering ligand largely used for thalassemic patients [4], are reported.

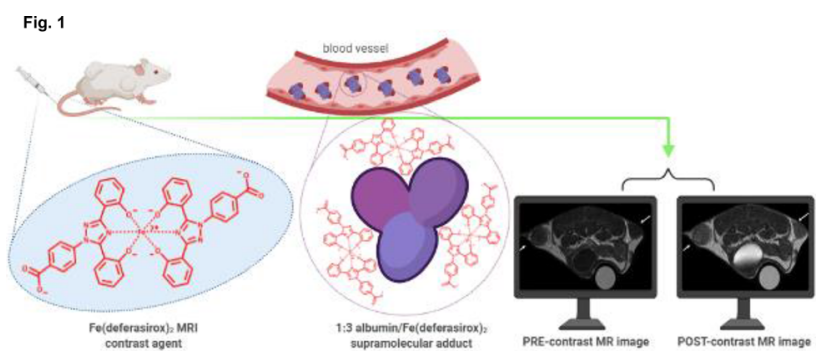

Methods: $T 1$ and $T 2$ relaxivities were investigated in water and in human serum at $25^{\circ} \mathrm{C}$ and $37^{\circ} \mathrm{C}$ in the range of magnetic field strengths $0.01-80 \mathrm{MHz}$ on a Stelar SpinMaster FFC-NMR relaxometer. The interaction of $\mathrm{Fe}(\mathrm{DFX}) 2$ with human serum albumin was studied using the proton relaxation enhancement method and the involved binding sites on the protein have been identified by relaxometric competitive assays. The absence of any inner sphere water molecule coordinated to $\mathrm{Fe}$ (III) ion was assessed through a variabletemperature 17O-R2-NMR experiment at $14.1 \mathrm{~T}$. Fe(DFX)2 efficiency as MRI contrast enhancer was investigated in vivo on a Bruker BioSpec $3 \mathrm{~T}$ scanner in a TS/a tumor bearing mouse model and compared with the contrast enhancement obtained by Gd(DTPA).

Results: Fe(DFX)2 owns an outstanding thermodynamic stability and a good relaxivity, comparable to those of clinically used Gd-based CAs, even if lacking any inner sphere water molecule. Its high affinity for HSA enables, in serum, the formation of a supramolecular adduct with three units of complex bound to the protein. The binding sites were identified to belong to IB, IIA and IIIA subdomains. Preliminary in vivo imaging studies on a tumor mouse model indicate that, on a 3 T MRI scanner, the contrast ability of $\mathrm{Fe}(\mathrm{DFX}) 2$ is well comparable to the one shown by the commercial Gd(DTPA) agent.

Discussion: The relaxation enhancement capability, the very high stability, the overall biodistribution and excretion properties and the expected good biocompatibility of $\mathrm{Fe}(\mathrm{DFX}) 2$, make this system a promising candidate as an alternative to the currently used Gd-based MRI CAs.

\section{References:}

[1] Boehm-Sturm P, Haeckel A, Hauptmann R, Mueller S, Kuhl CK, Schellenberger EA. Radiology 2017; 286; 537-546. 
[2] Bales BC, Grimmond B, Johnson BF, Luttrell MT, Meyer DE, Polyanskaya T, Rishel MJ, Roberts J. CMMI 2019; 1-10.

[3] Snyder EM, Asik D, Abozeid, SM, Burgio A, Bateman G, Turowski SG, Spernyak JA, Morrow JR. Angewandte Chemie 2020; 132; 2435-2440.

[4] Moukalled, N. M.; Bou-Fakhredin, R.; Taher, A. T. Mediterr J Hematol Infect Dis 2018, 10 (1). 10.4084/MJHID.2018.066.

\section{S4.P21.}

\section{Hybrid PET/MRI validation of arterial input functions after dual injection of MR contrast agents and their radioactive analogs in mice using an extracorporeal circulation approach at $9.4 \mathrm{~T}$ BioSpec high-field MRI}

\author{
*F. Gierse ${ }^{1,2}$, F. Büther ${ }^{1,3}$, B. Maus ${ }^{2}$, S. Hermann ${ }^{1}$, M. Claesener ${ }^{3}$, \\ M. Schäfers ${ }^{1,3}$, C. Faber $^{2}$, P. Backhaus ${ }^{1,2,3}$ \\ ${ }^{1}$ University of Münster, European Institute for Molecular Imaging \\ (EIMI), Münster, DE; \\ ${ }^{2}$ University of Münster, Translational Research Imaging Center \\ (TRIC), Münster, DE; \\ ${ }^{3}$ University Hospital Münster, Department of Nuclear Medicine, \\ Münster, DE
}

Introduction: Dynamic contrast-enhanced (DCE) MRI asks for exact determination of the dynamic arterial blood concentration (arterial input function, AIF). Image derived AIF measurement is heavily biased by partial volume or motion effects which leads to insufficent quantification of perfusion MRI especially in small animals. In a previous study, we could introduce a novel method to measure the AIF DCE-MRI in mice in an extracorporeal circulation [1]. In the current study, we simultaneously recorded rapid MRI extracorporeal AIFs and radioactive contrast agent analogs in a hybrid cross-validation approach.

Methods: 12 intracranial tumor bearing nude mice were measured in a 9.4 T Bruker Biospec small animal MRI. $35 \mathrm{mM}$ Gd-DO3A-butrol (Gadovist, 7 mice) or Gd-DTPA (Magnevist, 5 mice) were co-injected i.v. in $100 \mu \mathrm{l}$ mixed with their radioactive analog; ${ }^{68} \mathrm{Ga}$-DO3Abutrol (mean 7.2 Mbq \pm std 2.4) or ${ }^{99 \mathrm{~m}} \mathrm{Tc}$-DTPA $(23.1 \mathrm{MBq} \pm 6.2)$. An extracorporeal circulation was applied shunting the femoral artery to the tail vein. The circulation featured 2 reservoirs in the MR field of view and a MR compatible unit (Swisstrace, Twilite) for measurements of blood radioactivity (Fig. 1A). A Golden-angle Radial Sparse Parallel (GRASP) sequence was used for DCE covering the whole brain and reservoirs in isotropic $0.2 \mathrm{~mm}$ voxels [2]. Compressed sensing MP2RAGE with identical spatial resolution was employed for T1 mapping [3].

Results/discussion: Integrated simultaneous recordings of PET and DCE-MRI AIFs using the Twilite measuring unit were technically feasible. Shunt flows varied across different animals but were constant in individual measurements as established by laser doppler flow recordings (Fig. 3A/B). Dynamic acquisition employing rapid MRI techniques demonstrated very little noise at $5 \mathrm{~s}$ temporal resolution while allowing 3D isotropic whole brain coverage. Compressed sensing MP2RAGE established quantitatively plausible 3D T1 maps with identical coverage and spatial resolution in less than $6 \mathrm{~min}$. Well quantitative correspondence on the whole range of dynamic contrast agent and radiotracer concentrations was established when applying a fixed correction factor of 1.8 (Figs. 1B and 2). Although our preliminary analysis does not yet feature dispersion correction, the findings point towards a high precision across individual animals with both contrast agent and radioactive analog pairs. The robust quantitative relationship of simultaneous recordings of different contrast agent/radiotracer analog pairs establishes a basis for quantitative comparison of AIF recordings in hybrid small animal PET/MRI. Moreover, it enables to perform integrated PET/MRI modeling to explore quantitative relationships of contrast agent and radiotracer kinetics.
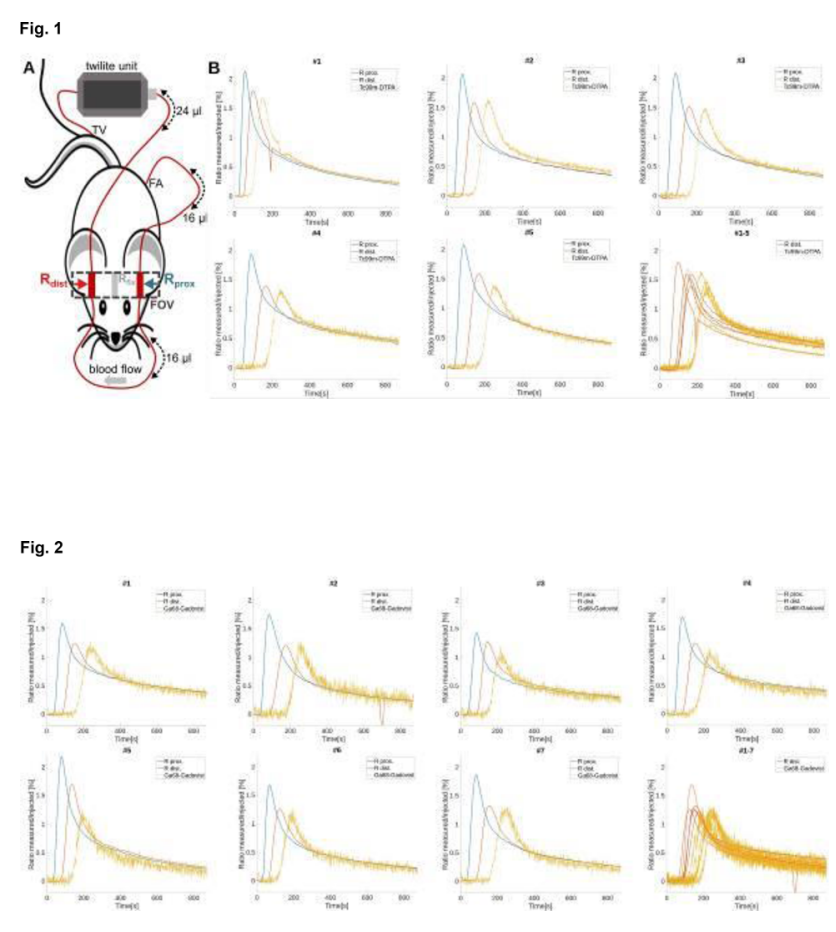

Fig. 3
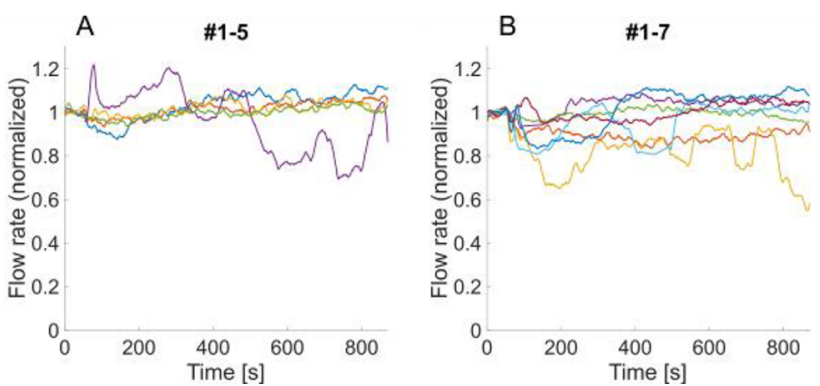

References:

[1] Backhaus P, Büther F et al. 2020, MRM, 2019, 1-12.

[2] Zhang J, Feng L, Otazo R, Kim SG. 2018, MRM, 2018, 140-152.

[3] Trotier AJ, Rapacchi S, Faller TL, Miraux S, Ribot EJ, MRM, 2019, 551-559. 


\section{S4.P22.}

Evaluation of the cerebrovascular reactivity in patients with moyamoya angiopathy by use of resting-state fMRI: comparison to breath-hold fMRI and $\left[{ }^{15} \mathrm{O}\right]$ water PET

*L. Zerweck ${ }^{1}$, C. Roder ${ }^{2}$, T. K. Hauser ${ }^{1}$, J. Thurow ${ }^{3}$, A. Mengel $^{4}$, M. Tatagiba ${ }^{2}$, N. Khan ${ }^{2,5}$, P. T. Meyer ${ }^{3}$, U. Ernemann ${ }^{1}$, U. Klose ${ }^{1}$

${ }^{I}$ University Hospital Tübingen, Department of Diagnostic and Interventional Neuroradiology, Tübingen, DE;

${ }^{2}$ University Hospital Tübingen, Department of Neurosurgery,

Tübingen, DE;

${ }^{3}$ Albert-Ludwig-University of Freiburg, Department of Nuclear

Medicine, Medical Center-University of Freiburg, Faculty

of Medicine, Freiburg im Breisgau, DE;

${ }^{4}$ University Hospital Tübingen, Department of Neurology and Stroke, Tübingen, DE;

${ }^{5}$ University Children's Hospital Zurich, Moyamoya Center, Zurich, $D E$

Introduction: To assess the risk of stroke hemodynamic evaluation is required in patients with Moyamoya Angiopathy (MMA). Estimation of the cerebral perfusion reserve (CPR) by use of $\left[{ }^{15} \mathrm{O}\right]$ water PET with acetazolamide (ACZ) challenge is considered the diagnostic standard. Evaluation of the cerebrovascular reactivity (CVR) by use of breath-hold-triggered fMRI (bh-fMRI) as an index of the CPR has been proposed as a readily available and reliable alternative [1]. Recent findings suggest the use of resting-state fMRI (rs-fMRI) to estimate the CVR [2] . The aim of this study was to compare the rsfMRI approach to bh-fMRI and $\left[{ }^{15} \mathrm{O}\right]$ water PET in patients with MMA.

Methods: A comparison of 25 rs-fMRI data sets of patients with MMA to the corresponding bh-fMRI data sets and, in a subgroup of 7 patients, to $\left[{ }^{15} \mathrm{O}\right]$ water PET data sets was performed. The rs-fMRI images were realigned, normalized, spatially smoothed $(12 \mathrm{~mm}$ FWHM) and frequency filtered $(0.02-0.04 \mathrm{~Hz})$. The cerebellar signal time-course was determined and a cross-correlation analysis was performed in which the correlation coefficients between the cerebellar Reference time-course and the individual voxels' signal time-courses were calculated. The data sets of all three modalities were segmented into 12 standardized VOIs [3]. A comparative analysis between rsfMRI and bh-fMRI as well as $\left[{ }^{15} \mathrm{O}\right]$ water PET was performed by calculating the correlation between the rs-fMRI CVR values (correlation coefficients) of the 12 VOIs and the corresponding bh-fMRI CVR values (relative signal change after breath-hold stimulation) and $\left[{ }^{15} \mathrm{O}\right]$ water PET CPR values (relative CBF change after ACZ stimulation) [4].

Results: Both, the comparison of the 25 rs-fMRI data sets to the bhfMRI data sets (Pearson's $r=0.71 \pm 0.13$, minimum $=0.35$, maximum $=0.95)$ and the 7 rs-fMRI data sets to the corresponding $\left[{ }^{15} \mathrm{O}\right]$ water PET data sets (Pearson's $r=0.80 \pm 0.19$, minimum $=0.41$, maximum $=0.95)$ revealed high level of agreement [4] . Exemplary maps of one patient with high agreement and the corresponding scatterplots can be seen in Figs. 1 and 2.
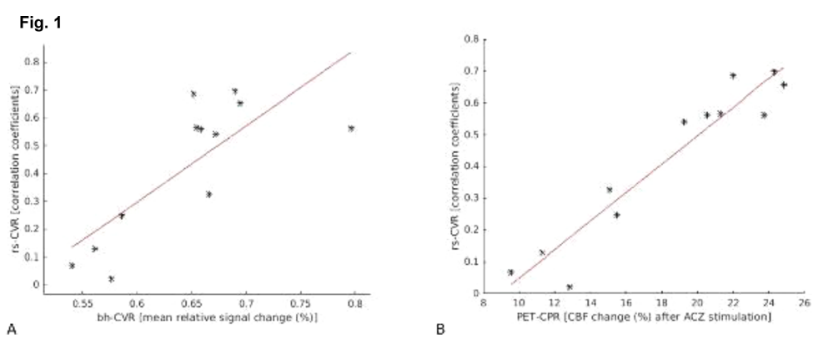

Fig. 2
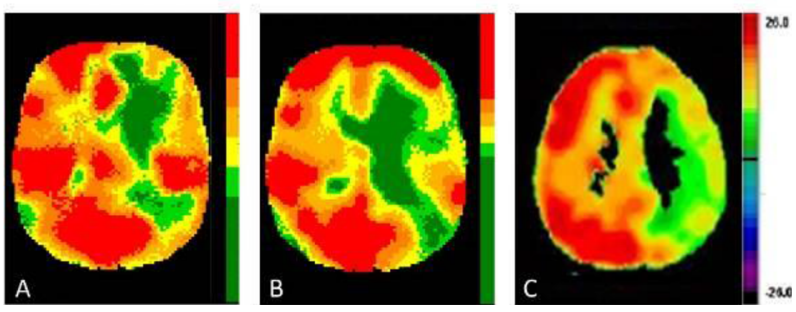

Discussion: The present analysis indicates that rs-fMRI might be a promising readily available method for hemodynamic evaluation with almost no patient cooperation required. Further studies are needed.

\section{References}

1 Hauser TK et al. (2019) Neuroimage Clin 22:101713.

2 Liu P et al. (2017) Neuroimage 146:320-326.

3 Tatu L et al. (1998) Neurology. 50(6):1699-708.

4 Zerweck L et al. (2021) AJNR Submitted.

Figure 1 Scatter plots of one patient showing the correlation between rs-fMRI and bh-fMRI $(r=0.78)$ (A) and between rs-fMRI and $\left[{ }^{15} \mathrm{O}\right]$ water PET $(r=0.95)(\mathbf{B})$

Figure 2 Exemplary rs-fMRI- (A), bh-fMRI- (B) and $\left[{ }^{15} \mathrm{O}\right]$ water PETmaps $(\mathbf{C})$ of one patient

\section{S4.P23.}

Feasibility of glioblastoma tissue response mapping with physiological BOLD using precise oxygen and carbon dioxide modulation

\footnotetext{
*V. Stumpo ${ }^{1,2}$, M. Sebök ${ }^{1,2}$, C. H. B. van Niftrik ${ }^{1,2}$, K. Seystahl ${ }^{3,2}$, M. Weller ${ }^{3,2}$, L. Regli ${ }^{1,2}$, J. Fierstra ${ }^{1,2}$

${ }^{1}$ University Hospital Zurich, Department of Neurosurgery, Zurich, $\mathrm{CH}$;

${ }^{2}$ University Hospital Zurich, Clinical Neuroscience Center, Zurich, $\mathrm{CH}$;

${ }^{3}$ University Hospital Zurich, Department of Neurology, Zurich, $\mathrm{CH}$
}

Introduction: Innovative physiological MRI development focusses on better depiction of the heterogenous vascular and metabolic imaging aspects posed by GBM. In this regard, Blood oxygenationlevel dependent (BOLD) MRI with standardized and precise carbon dioxide $(\mathrm{CO} 2)$ and oxygen $(\mathrm{O} 2)$ modulation may provide novel imaging markers to better detail GBM characteristics.

Materials and methods: Seven newly diagnosed, untreated GBM patients prospectively underwent a BOLD study with precise standardized changes in $\mathrm{CO} 2$ and $\mathrm{O} 2$ provided by a computer controlled gasblender (RespirActTM). Optimal delay on a voxel-wise basis was 
identified by applying an iterative analysis to the data to determine the correct voxel-wise temporal $\mathrm{CO} 2 / \mathrm{O} 2$ shift to match the start of the hypercapnia/hypoxia/hyperoxia induced BOLD signal change. \%BOLD signal change/mmHg during hypercapnic, hypoxic and hyperoxic stimulus was calculated for the whole brain, tumor lesion and segmented regions of interest (contrast-enhancing-CE-tumor, necrosis, edema).

Results: Mean \%BOLD signal change/mmHg during hypercapnic stimulus was 0.017 (SD 0.072) in whole brain, 0.028 (SD 0.069) in the tumor mask, 0.029 (SD 0.07) in CE tumor, 0.029 (SD 0.069) in necrosis and 0.047 (SD 0.052) in edema. Mean \%BOLD signal change $/ \mathrm{mmHg}$ during hypoxic stimulus was -0.014 (SD 0.006) in the whole brain, -0.020 (SD 0.017) in the tumor mask, -0.019 (0.015) in CE tumor, -0.029 (SD 0.025) in necrosis and -0.021 (0.021) in edema. Mean \%BOLD signal change/mmHg during hyperoxic stimulus was 0.004 (SD 0.001) in the whole brain, 0.004 (SD 0.002) in tumor mask, 0.009 (SD 0.013) in CE tumor, 0.004 (SD 0.003 ) in necrosis, 0.003 (SD 0.002) in edema (Table 1).

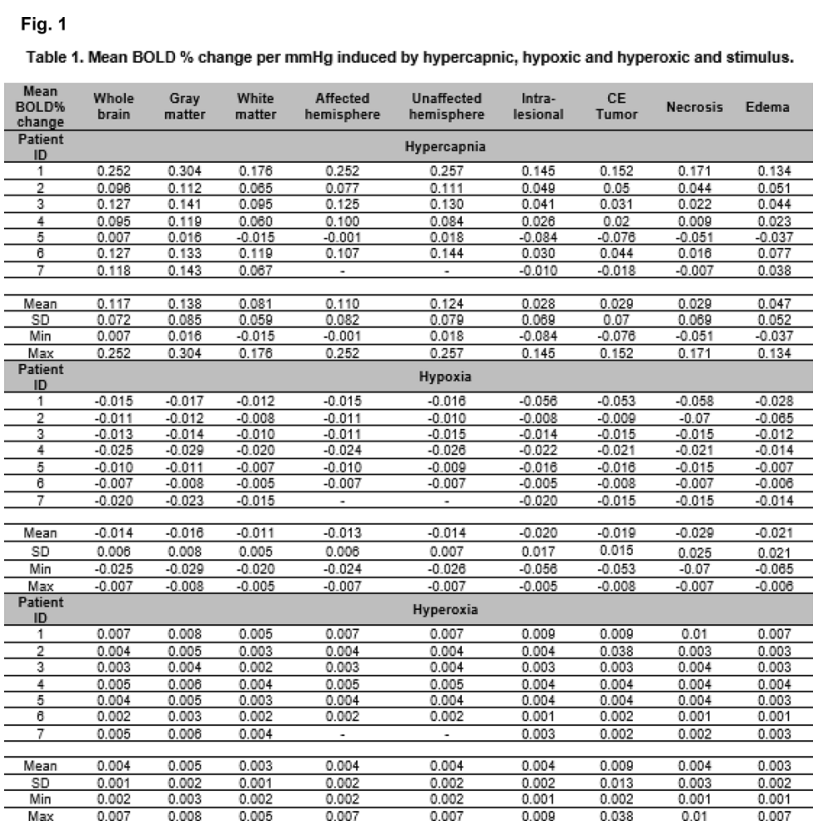

Discussion: Our preliminary study, shows a good feasibility of BOLD with standardized and precise $\mathrm{CO} 2$ and $\mathrm{O} 2$ application as an emerging physiological imaging technique to detail specific GBM characteristics. In particular, specific tissue responses for hypercapnia, hypoxia and hyperoxia were found (Fig. 1), whereas unique whole brain patterns could also be appreciated (Fig. 2). This emerging BOLD technique poses two advantages, since standardized and precise gas application results in a high inter- and intrasubject agreement between follow-up studies, whereas the BOLD MRI contrast provides a high imaging contrast covering the entire brain. The unique tissue response patterns generated from this technique, can therefore be tested as novel imaging markers to better detail GBM lesions and gauge treatment response.
Fig. 2
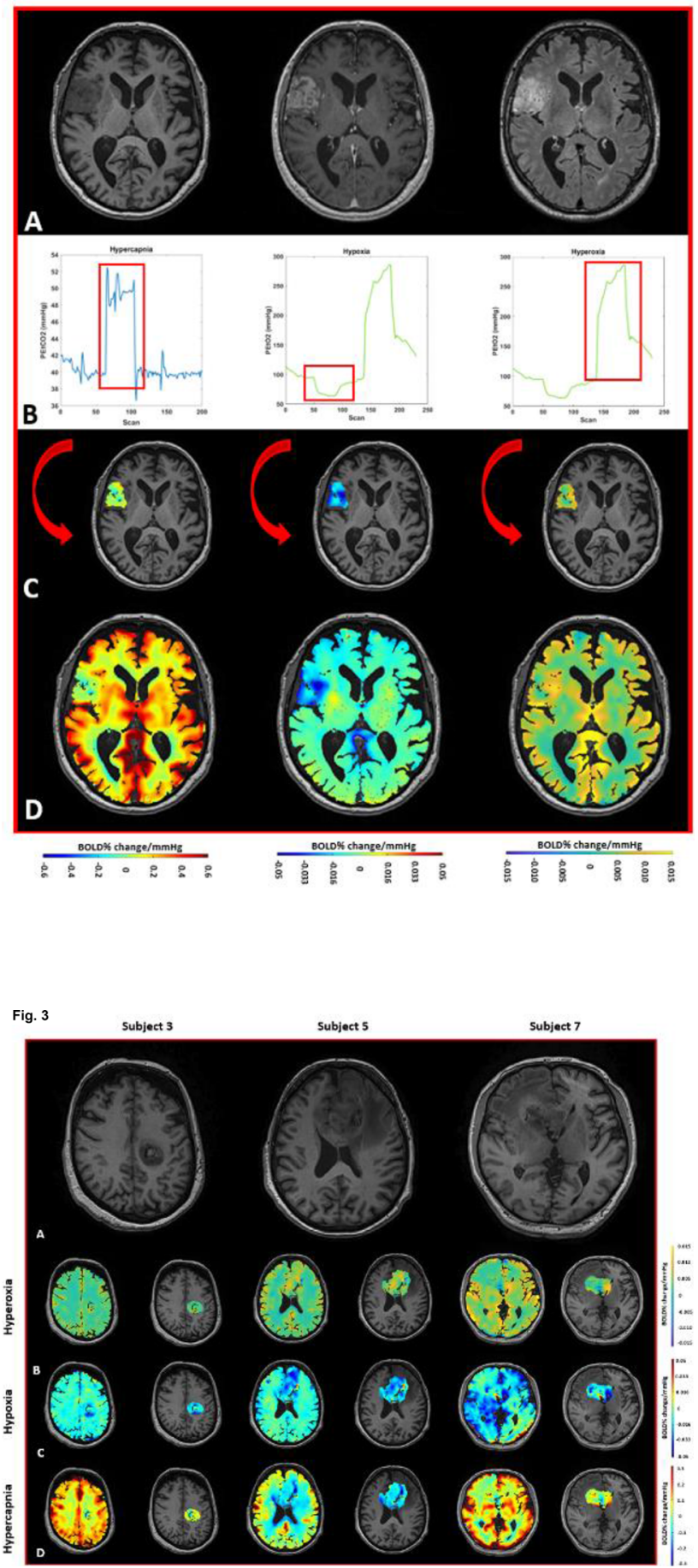
References:

Tofts PS, Kermode AG (1991) Magn. Reson. Med. 17(2):357-367.

'Patel JP, Frey BN (2015) Neural Plast. 2015:708306 doi: 10.1155/2015/708306

${ }^{3} K$ amintsky L. ct al. (2020) Neuroimage Clin. 26:102049 doi: 10.1016/j. nicl.2019.102049

\section{S4.P24.}

\section{Comparing common blood-brain barrier (BBB) permeability models for potential use in neuropsychiatric diseases}

\author{
*A. Gilbank ${ }^{1,2}$, B. N. Frey ${ }^{3}$, N. A. Bock ${ }^{4}$, M. D. Noseworthy ${ }^{1,2,5}$ \\ ${ }^{1}$ St. Joseph's Healthcare, Imaging Research Centre, Hamilton, CA; \\ ${ }^{2}$ McMaster University, School of Biomedical Engineering, Hamilton, \\ $C A$; \\ ${ }^{3}$ McMaster University, Department of Psychiatry and Behavioural \\ Neurosciences, Hamilton, CA; \\ ${ }^{4}$ McMaster University, Department of Psychology, Neuroscience \\ and Behaviour, Hamilton, CA; \\ ${ }^{5}$ McMaster University, Department of Electrical and Computer \\ Engineering, Hamilton, $C A$
}

Introduction: Dynamic contrast enhanced MRI (DCE-MRI) as a tool for quantifying microvasculature permeability has been used to investigate many brain pathologies. In this technique, a series of $\mathrm{T}_{1^{-}}$ weighted images are acquired as a bolus of gadolinium-based contrast agent is injected to record its time-course through the tissue. A mathematical model is then used to fit the data and find physiological parameters, such as mass-transfer rate $\left(\mathrm{K}^{\text {trans }}\right)$ and plasma space. The brain's tissue environment has very low permeability due to the tightly-regulated nature of the blood-brain barrier (BBB). Certain pathologies, such as multiple sclerosis, have been found to strongly disrupt this barrier [1]. However, other disorders like bipolar disorder have been hypothesized [2] and found to cause more subtle disruption [3], which is harder to detect and quantify. In this study, different mathematical models were compared in healthy brains to find if any model had higher precision with the goal of using the optimal model for our neuropsychiatric studies.

Methods: Four models were compared: Tofts, modified Tofts, TwoCompartment Exchange, and the Uptake model. Eight healthy subjects ( $22 \pm 4$ years old, 2 male, 6 female) were scanned, 6 of whom returned for a repeat scan. Subjects were scanned with a 3Tesla MRI and 32 channel head coil (GE Healthcare, Milwaukee WI). A precontrast whole-brain(?) $\mathrm{T}_{1}$ map was calculated from an acquired $\mathrm{B}_{1}{ }^{+}$ map and $\mathrm{T}_{1}$-weighted and proton density weighted images. Subsequently the DCE data was acquired. For DCE Gadovist (Bayer; $0.1 \mathrm{mmol} / \mathrm{kg}$ body weight) was injected with a $3 \mathrm{cc} / \mathrm{s}$ injection velocity into the left antecubital vein. Region-ofinterest (ROI) analysis was performed bilaterally on putamen, thalamus, hippocampus, caudate head and amygdala, and on midline structures: pons and cerebellum. The posterior pituitary was used as a "maximal permeability region", as its vasculature is void of a BBB. Data was fitted with each model in Matlab (MathWorks Inc., Natick MA) and the $\mathrm{R}^{2}$ values were calculated. ANOVA and ANCOVA tests were applied to find significant differences between models $(\mathrm{p}<0.05)$

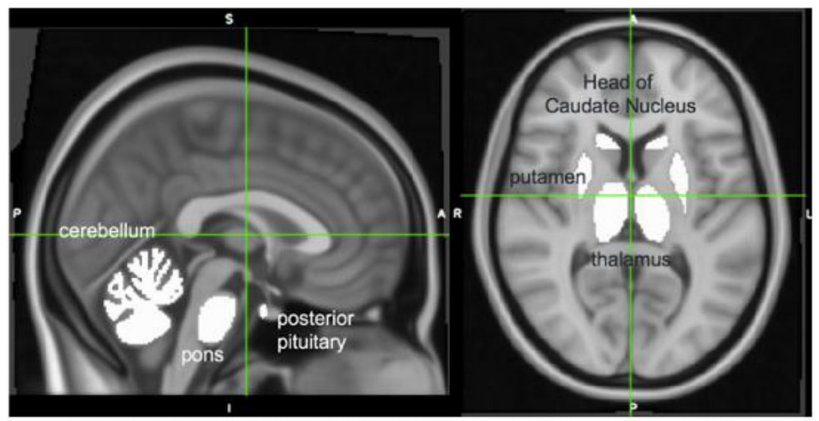

Figure 1: Regions of interest: cerebellum, pons, posterior pituitary, head of caudate nucleus, putamen, and thalamus.

Results: The Tofts model was found to have a significantly lower $\mathrm{R}^{2}$ in ROIs with a BBB, compared to the modified Tofts and Uptake models. Additionally, ROI size (voxel count) was found to be a significant covariate in the ANCOVA comparing the models' $\mathrm{R}^{2}$ values.
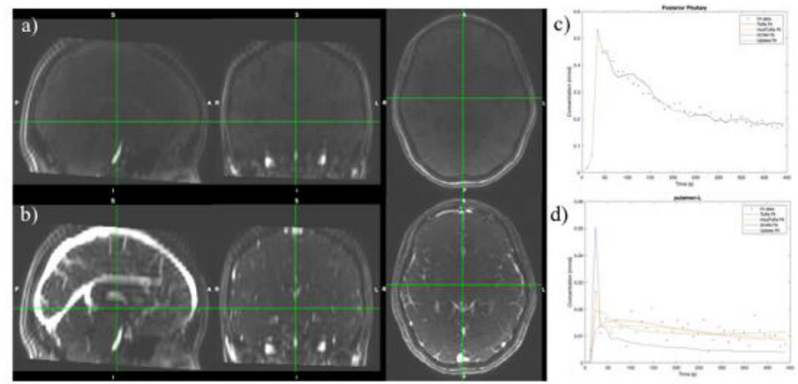

Figure 2: DCE image of registered brain before (a) and after (b) Gadovist bolus injection has reached brain. Concentration curves with fitted models for posterior pituitary (c) and left putamen (d). Scan parameters: GRE scan, flip angle $=30^{\circ}, T R / T E=3.8 / 1.2 \mathrm{~ms}$, field-of-view $=24 \mathrm{~cm}$, matrix $=192 \times 192$, receiver bandwidth $=62.5 \mathrm{kHz}$, ASSET factor $=2,40$ temporal phases, 11 . $3 \mathrm{sec}$ time resolution.

Discussion: As there is no "gold standard" for DCE-MRI modelling, measurement of model accuracy is not possible. Model precision, however, can be estimated through the error in model fit. The Tofts model may have performed worse due to its assumption of negligible tracer concentration in plasma space, or because it had the least parameters. Based on model error there was no difference between modified Tofts, Two-Compartment Exchange, and Uptake models in brain regions having an intact $\mathrm{BBB}$. More data is needed to determine any differences between these three models.

\begin{tabular}{c|c|c|c|c}
\hline ROI & $\mathrm{R}_{\text {Tofts }}^{2}$ & $\mathrm{R}^{2}$ modTofts & $\mathrm{R}^{2}{ }_{\text {2CXM }}$ & $\mathrm{R}^{2}$ Uptake \\
\hline Posterior Pituitary & $0.88 \pm 0.09$ & $0.91 \pm 0.09$ & $0.93 \pm 0.08$ & $0.91 \pm 0.07$ \\
Pons & $0.55 \pm 0.2$ & $0.71 \pm 0.21$ & $0.66 \pm 0.21$ & $0.69 \pm 0.21$ \\
Cerebellum & $0.73 \pm 0.17$ & $0.8 \pm 0.15$ & $0.61 \pm 0.39$ & $0.8 \pm 0.16$ \\
Putamen L & $0.38 \pm 0.31$ & $0.53 \pm 0.24$ & $0.39 \pm 0.63$ & $0.52 \pm 0.26$ \\
Putament R & $0.53 \pm 0.3$ & $0.63 \pm 0.28$ & $0.56 \pm 0.27$ & $0.59 \pm 0.27$ \\
Amygdala L & $0.47 \pm 0.35$ & $0.59 \pm 0.29$ & $0.39 \pm 0.56$ & $0.57 \pm 0.32$ \\
Amygdala R & $0.51 \pm 0.35$ & $0.63 \pm 0.33$ & $0.42 \pm 0.41$ & $0.59 \pm 0.34$ \\
Thalamus L & $0.57 \pm 0.24$ & $0.66 \pm 0.21$ & $0.64 \pm 0.19$ & $0.67 \pm 0.2$ \\
Thalamus R & $0.63 \pm 0.22$ & $0.7 \pm 0.21$ & $0.66 \pm 0.21$ & $0.7 \pm 0.19$ \\
H of C N L & $0.52 \pm 0.25$ & $0.62 \pm 0.2$ & $0.53 \pm 0.3$ & $0.6 \pm 0.21$ \\
H of C N R & $0.57 \pm 0.25$ & $0.66 \pm 0.23$ & $0.56 \pm 0.33$ & $0.64 \pm 0.24$ \\
Hippocampus L & $0.65 \pm 0.22$ & $0.73 \pm 0.19$ & $0.6 \pm 0.44$ & $0.74 \pm 0.17$ \\
Hippocampus R & $0.78 \pm 0.12$ & $0.84 \pm 0.09$ & $0.76 \pm 0.17$ & $0.82 \pm 0.08$ \\
\hline
\end{tabular}

Figure 3: Mean $\mathrm{R}^{2}$ values of each model in each ROI.

References

'Tofts PS, Kermode AG (1991) Magn. Reson. Med. 17(2):357-367.

'Patel JP. Frey BN (2015) Neural Plast. 2015:708306. doi: 10.1155/2015/708306

${ }^{3}$ Kamintsky L. ct al. (2020) Neuroimage Clin. 26:102049. doi: 10.1016/j.nicl.2019.102049 


\section{S4.P25.}

Comparison of vessel size imaging and cerebral blood volume derived from DSC MRI on nonenhancing glioma subtypes

\section{*F. Arzanforoosh ${ }^{1}$, S. van der Voort ${ }^{1}$, M. Smits ${ }^{1}$, E. Warnert ${ }^{1}$ \\ ${ }^{1}$ Erasmus Medical Center, Department of Radiology and Nuclear Medicine, Rotterdam, $N L$}

Introduction: Relative cerebral blood volume (rCBV) is a clinically well-known parameter and reflects information of both microvasculature density and diameter in brain tumors. Studies prove that rCBV can be used for predicting tumor aggressiveness in glioma (1). However, in lower grade oligodendroglioma, due to "chicken-wire" nature of its vasculature, rCBV could also be elevated (2). Vessel size imaging (VSI) is an emerging MRI technique enabling us to calculate the mean diameter of vessels within an image voxel (3). In this study we aimed to investigate the added value of VSI in two nonenhancing glioma subtypes of oligodendrogliomas and astrocytoma.

Subjects and Methods: A retrospective dataset consisting of 33 patients with confirmed non-enhancing glioma was used in this study (4). All patients underwent 3 T MRI scanning (GE, Milwaukee, WI, USA) including hybrid EPI (HEPI); a 2D, simultaneous acquisition of GRE- and SE-EPI DSC MRI with acquisition parameters of $122 \mathrm{TRs}$, TR: $1500 \mathrm{~m}, 15$ slices, voxel size: $1.88 \times 1.88 \times 4.00 \mathrm{~mm}^{3}$, TE GE/ SE: $18.6 / 69 \mathrm{~ms}$. DSC MRI was performed with administration of $7.5 \mathrm{ml}$ of gadolinium-based contrast agent with a pre-load bolus of equal size. rCBV maps were calculated using DSC data from the gradient-echo. Estimates of mean vessel diameter were made according to previously described methods (5). The average of vessel size measurements and normalized rCBV within a tumor ROI (hyperintense on T2/FLAIR) were calculated for each group of patients. Results: The histological classification includes oligodendroglioma (grade II/III) and astrocytoma (grade II/III) (Table 1). As seen in Fig. 1, no significant differences between VSI and rCBV values between grade II and grade III oligodendroglioma and astrocytoma were found within this study. In the oligodendroglioma subgroup both average rCBV and VSI are higher in grade II compared to grade III (T Test, $\mathrm{p}=0.21$ and $\mathrm{p}=0.08$ respectively). Whereas, in astrocytoma subgroup both rCBV and VSI are higher in grade III compare to grade II ( $p=0.51$ and $p=0.41$ respectively). See Fig. 2 . for visual inspection.

Fig. 1

Table 1: Summary of clinical description of patients in the dataset.

\begin{tabular}{|c|c|c|c|}
\hline & & & umber of patients \\
\hline Diagnosis & Tumor Grade & Gender & IDH status \\
\hline \multirow[t]{2}{*}{ Oligodendrogliomas } & II & $\begin{array}{c}14 \\
(5 \mathrm{~F} / 9 \mathrm{M})\end{array}$ & $\begin{array}{c}14 \\
\text { (14 IDH mutant) }\end{array}$ \\
\hline & III & $\begin{array}{c}4 \\
(4 \mathrm{M})\end{array}$ & $\stackrel{4}{4}$ \\
\hline \multirow[t]{2}{*}{ Astrocytoma } & II & $\begin{array}{c}10 \\
(6 \mathrm{~F} / 4 \mathrm{M})\end{array}$ & (9 $\mathrm{DH}$ mutant / $1 \mathrm{IDH}$ wild type) \\
\hline & III & $\begin{array}{c}\mathbf{5} \\
(2 \mathbf{F} / 3 \mathbf{M})\end{array}$ & $\begin{array}{c}5 \\
\text { (3 } \mathrm{IH} \text { mutant / } 2 \mathrm{WH} \text { wild type) }\end{array}$ \\
\hline
\end{tabular}

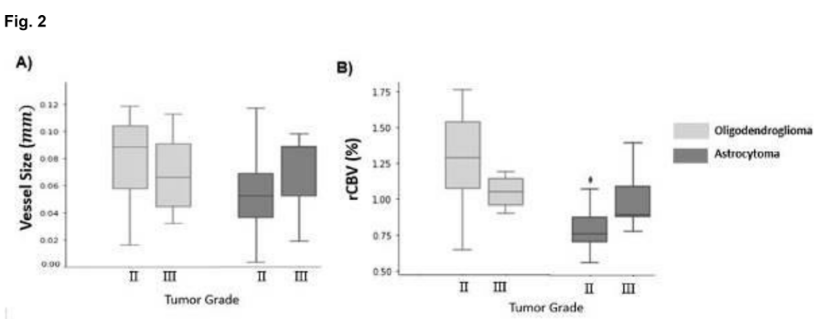

Figure 1: Group average of vessel size (A) and relative cerebral blood volume (B) within tumor ROI, for both Olizodendroglioma and astrocytoma subtype glioma.

Discussion: The results of this study suggests that rCBV and VSI are not able to predict the grade of oligodendrogliomas. Moreover, rCBV and VSI measurements in oligodendrogliomas grade II inclines to be even higher than astrocytoma grade III, while the clinical prognoses shows that the latter is more aggressive than the former. Note that the IDH mutation status of both groups (Table 1) is a likely reason for not finding any significant differences. Future investigations will include increasing the sample size as well as investigating correlations of molecular status with rCBV and VSI.

Fig. 3

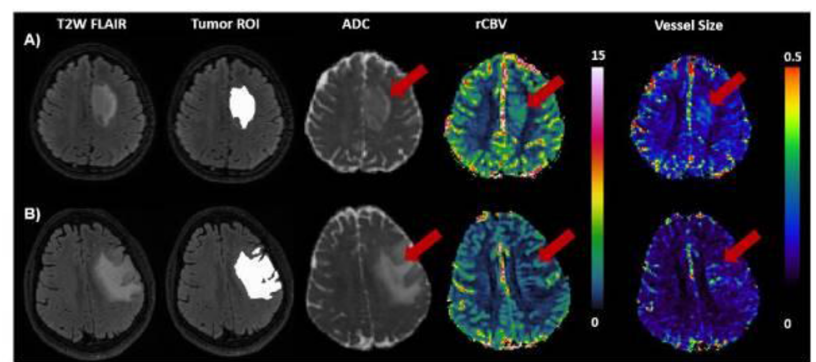

Figure 2: Single slice of exemplary MiRI images of two patients of oligodendroglioma (grade I) (A) and astrocytorna ( $\mathrm{grade}$ I) (B). The images from left to right respectively are: T2W FLAIR; T2W FLAIR overlaid with tumor mask, apparent diffusion coefficient, relative cerebral blood volume, vessel size map.

\section{References:}

1. Kremer S. et al., J Neuroradiol (2002).

2. Fellah S. et al., Am J Neuroradiol (2013).

3. Kiselev VG. et al., Magn Reson Med (2005).

4. van der Voort SR. et al., Clin Cancer Res (2019).

5. Kellner E. et al., J Magn Reson Imaging (2015).

\section{S4.P26.}

Magnitude vs. real data for mapping apparent soma and neurite density using diffusion MRI

\author{
*A. Ianus ${ }^{1}$, J. Carvalho ${ }^{1}$, F. Fernandes ${ }^{1}$, C. Chavarrias ${ }^{1}$, M. Palombo ${ }^{2}$, \\ N. Shemesh ${ }^{1}$ \\ ${ }^{1}$ Champalimaud Centre for the Unknown, Department \\ of Champalimaud Research, Lisbon, PT; \\ ${ }^{2}$ University College London, Centre for Medical Image Computing, \\ London, PT
}

Introduction: Diffusion MRI (dMRI) is a prominent MRI modality to provide contrast to the tissue structure at the microscopic scale, and many techniques have been developed so $\mathrm{far}^{1-4}$. Among them, the SANDI approach ${ }^{5}$ has been recently proposed to map the apparent 
soma size and density and neurite density based on dMRI data. SANDI employs a 3-compartment model and powder averaged diffusion measurements up to very high diffusion weightings, thus the estimates might show bias due to the Rician noise floor ${ }^{6}$. Here we investigate the effects of using either magnitude or real data on the SANDI parameters in the mouse brain, in-vivo.

Methods: Acquisition: dMRI data from $\mathrm{N}=6 \mathrm{C} 57 \mathrm{BL} / 6 \mathrm{~J}$ mice were acquired on a $9.4 \mathrm{~T}$ Bruker scanner with a 4 channel cryprobe using a PGSE-EPI sequence $(\Delta / \delta=20 / 5.5 \mathrm{~ms})$ with 8 shells with $\mathrm{b}=\{1,2.5$, $4,5.5,7,8.5,10,12.5\} \mathrm{ms} / \mu \mathrm{m}^{2}$ and 40 directions each, TE $=36.8$ $\mathrm{ms}, \mathrm{TR}=4 \mathrm{~s}, 4$ averages, slice thickness $=0.4 \mathrm{~mm}, 35$ slices, in plane resolution $=0.12 \times 0.12 \mathrm{~mm}$, matrix $=118 \times 100$, Partial Fourier $=1.35$, per-slice triggering and fat suppression, total time $\sim 3 \mathrm{~h}$. All experiments were performed after approval from the ethics committee following European Directive 2010/63.

Data analysis: To compare the effects of using either magnitude or real data, pre-processing was performed as illustrated in Fig. 1, with calculation of real data ${ }^{7}$ after denoising ${ }^{8}$, ghost correction ${ }^{9}$ and coil combination $^{10}$. After pre-processing, the powder averaged data ${ }^{11}$ was fit using the 3-compartment SANDI model (spheres, sticks and Gaussian diffusion) and a Random Forest regression algorithm in Matlab. Then, parameters estimated from magnitude and real data were compared.

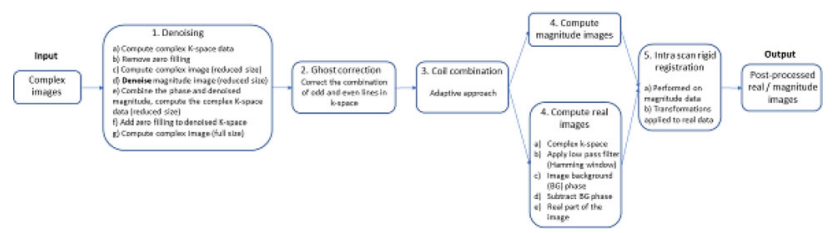

Results: Figure 2 shows representative SANDI parameter maps obtained from magnitude and real data. Overall, parameters have similar contrasts for magnitude and real data, although real data yields more homogeneous maps across the brain. The scatterplots presented in Fig. 3 for both white matter (WM) and gray matter (GM) ROIs show that magnitude data, which includes the Rician noise floor, results in slightly higher apparent neurite fraction $\left(f_{\text {neurite }}\right)$. This is also observed in the maps in Fig. 2, especially at the bottom of the brain where the SNR is lower, as the data was acquired with a surface coil.

Fig. 2

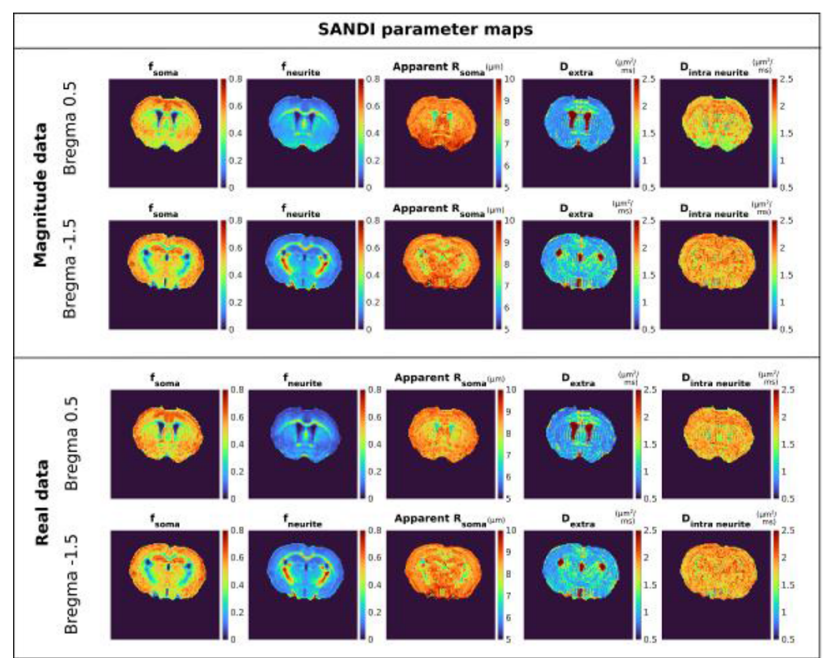

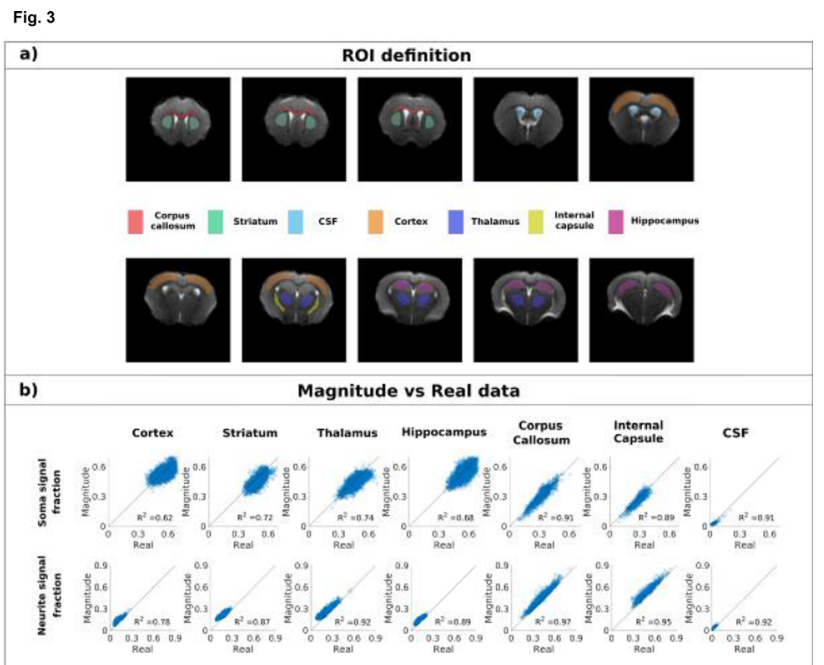

Discussion and conclusion: SANDI maps obtained from both magnitude and real data follow expected patterns with high $\mathrm{f}_{\text {soma }}$ in GM and high $\mathrm{f}_{\text {neurite }}$ in WM. Rician noise affects mostly $\mathrm{f}_{\text {neu- }}$ rite which has slightly higher values when estimated from magnitude data, especially at lower SNR.

\section{References}

[1] Alexander et al., NMRBiomed (2018).

[2] Novikov et al., NMRBiomed (2018).

[3] Jelescu et al., J Neurosci Methods (2020).

[4] Veraart et al., eLife (2020).

[5] Palombo et al., NI 2020.

[6] Afzali et al., bioRxiv, (2020).

[7] Eichener et al. NI (2015).

[8] Veraart et al. MRM NI 2016.

[9] Buonocore and Gao, MRM 1997.

[10] Walsh et al., MRM 2000.

[11] Callaghan et al. Biophys J. (1979)

\section{S4.P27.}

Free water DTI at high and standard spatial resolution - optimal parameters and fitting procedures

\author{
${ }^{*}$ M. Levardon ${ }^{1},{ }^{*}$ G. Arribarat ${ }^{1}$, Y. Fave ${ }^{1}$, H. Gros-Dagnac ${ }^{1}$, \\ J. A. Lotterie ${ }^{1,2}$, P. Péran ${ }^{1}$ \\ ${ }^{1}$ University of Toulouse, Toulouse Neuro-Imaging Center (ToNIC), \\ Toulouse, FR; \\ ${ }^{2}$ University of Toulouse, Toulouse, FR
}

Introduction: Diffusion tensor imaging (DTI) is a classic approach to extract parameters related to tissue microstructure. Free water elimination DTI model aims to evaluate fraction of free water inside a voxel. For FW estimation, two shell acquisitions and fitting procedure with weighted linear and non-linear least squared (NLS) are recommended [1]. Another procedure has been used in clinical studies with regularized gradient descent (RGD), which was initially proposed to manage single-shell acquisitions. Our work aims to investigate differences between the two fitting procedures as well as the influence of the partial volume and the angular resolution to estimate accurately FW maps.

Methods: We assessed a 3 T MRI exam (Achieva, Philips) on 30 healthy subjects (see parameters in Fig. 1). The gradient directions 
were calculated to be angularly equidistributed in single shell acquisitions and complementary between several sets.

\section{Fig. 1}

\begin{tabular}{|c|c|c|c|}
\hline $\begin{array}{c}\text { Spatial } \\
\text { reoluniona (and) }\end{array}$ & $\begin{array}{l}\text { bavilues } \\
\text { p.ent? }\end{array}$ & $\begin{array}{c}\text { Nb. of } \\
\text { dinstibas }\end{array}$ & $\begin{array}{l}\text { Nb. of } \\
\text { weti }\end{array}$ \\
\hline High & 300 & 35 & 2 \\
\hline$(0.9573 \times 0.9975 \times 2)$ & 100 & 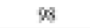 & 4 \\
\hline Standand & 300 & 16 & $\mathrm{~L}$ \\
\hline$(1,579 \times 1,975 \times 2)$ & 1000 & 32 & i \\
\hline
\end{tabular}

All DWIs were processed following: denoising with LPCA [2] filter using DIPY [3], correcting for susceptibility and eddy current artefacts using SPM [4] and FSL [5] respectively. FW maps were calculated using RGD [6] and NLS [7] from DIPY. At high resolution, to evaluate the number of gradients needed for free water estimation, parametric maps were calculated with combinations of sets.

Results: The Fig. 2 shows correlations between FW calculated with NLS and FW calculated with RGD in the corpus callosum (CC) and cerebrospinal fluid (CSF). The correlation is higher in the $\mathrm{CC}$ $\left(\mathrm{R}^{2}=0.9415\right)$ than in the CSF $\left(\mathrm{R}^{2}=0.719\right)$ and it is better for high resolution than the standard one $\left(C C: R^{2}=0.5966 ; C S F: R^{2}=0.409\right)$. Other white and gray matter structures were investigated and results are the same as for the CC.
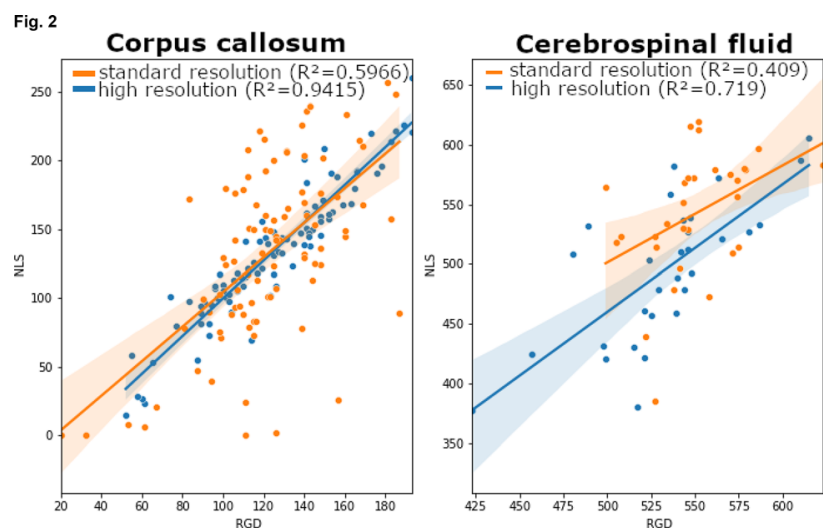

Considering high resolution, we found that the estimation of FW did not differ using only one set or using all sets.

Discussion/conclusion: Our results showed that at high resolution, only one set is enough to estimate FW with accuracy. It allows us to reduce acquisition time or to increase the resolution with the same duration as with more directions. We also demonstrate the fact that RGD results should be interpreted carefully especially for standard resolution. With high resolution the two fitting algorithms corelates more because of the reduction of partial volume effect. This result supports the interest of acquiring DWIs with the highest possible resolution.

\section{References:}

[1] Hoy \& al. (2014) NI.

[2] Manjón \& al. (2013) PO.

[3] Garyfallidis \& al. (2014) $F N$.

[4] Jezzard \& al. (1995) MRM.

[5] Jenkinson (2012) NI.

[6] Golub \& al. (2021) MRM.

[7] Henriques \& al. (2017) $R S$ 


\section{S5.P1.}

\section{Theoretical validation of simulated B1 for a cavity resonator at $300 \mathrm{MHz}$}

F. Vazquez ${ }^{1}$, S. Solis-Najera ${ }^{1}$, R. Martin ${ }^{1}$, O. Marrufo ${ }^{2}, *$ A. Rodriguez ${ }^{3}$

${ }^{I}$ National Autonomous University of Mexico (UNAM), Faculty of Sciences, Mexico City, $M X$;

${ }^{2}$ INNN-MVS, Department of Neuroimage, Mexico City, MX;

${ }^{3}$ UAM Iztapalapa, Department of Electrical Engineering, Mexico City, $M X$

Introduction: A transceiver volume coil based on Mansfield 's cavity resonator design was previously developed and showed a better uniformity and performance compared to the standard birdcage [1]. The $\mathrm{B}_{1}$ improvement of this resonator design was also demonstrated theoretically [2]. However, the study of RF coils importantly rely on numerical simulations which usually facilitate the computation of $\mathrm{B}_{1}$. These results motivated us to theoretically validate its numerical magnetic field.

Method: $B_{1}$ numerical simulations of a cavity resonator with 6 rungs and 6 circular cavities were conducted at $7 \mathrm{~T}$. This coil design is intended to be used in preclinical MRI. All numerical computations were performed with the commercial software tool COMSOL Multiphysics (COMSOL 3.2, Burlington, MA, USA). Coil was simulated in the quadrature-drive mode and tuned at $300 \mathrm{MHz}$. Figure 1.a) shows an illustration of the coil design used in all simulations. We used the following analytical expression for the magnetic field of the cavity resonator, $\mathrm{B}_{1}[3]$ :

$\mathrm{B}_{\text {icav }}=2.2 i_{\text {cav }}\left(l^{2}+d^{2}\right) \mathrm{d}^{-1}\left(1^{2}+\mathrm{d}^{2}\right)^{3 / 2}$

where $l=9 \mathrm{~cm}$ (length) and $d=9 \mathrm{~cm}$ (diameter) and $i_{\text {cav }}$ is the current.

Results and discussion: $B_{1}$ numerical simulations were computed and and show in Fig. 1.b). A really good uniformity and intensity of the magnetic field can be observed for this particular design. Then, a comparison plot was computed using the numerical data and theoretical calculations obtained with the equation above. Figure 2 shows the comparison plots. The simulated profile was taken along the white line in the $B_{1}$ simulation, as shown in Fig. 2. A very good correspondence can be observed between numerical and theoretical computations. This supports the electromagnetic simulations of our transceiver resonator design. These results will allow us to reliably study the coil performance using different layouts.

\section{Fig. 1}

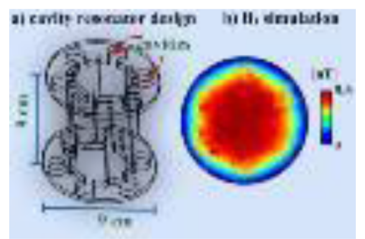

Fig. 2

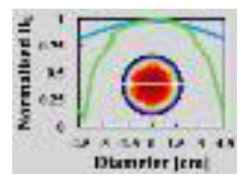

\section{References:}

1. Vazquez, JF, Marrufo O, Solis-Najera SE, Hidalgo SS, Rodriguez AO. (2009). Magnetron volume coil for Magnetic Resonance Imaging of rodents. In Proc. ISMRM.17, p. 4750.

2. Solis-Nájera, S, D. Tomasi, A. O. Rodriguez. (2020) Slotted end ring coil sensitivity experimentally validated at $4 \mathrm{~T}$. Magn Reson Mater Phy 33, 28.

3. Solis-Najera S, Martin R, Vazquez F, Marrufo O, Rodriguez AO. (2020). Experimental development and evaluation of a volume coil with slotted end-rings coil for rat MRI at $7 \mathrm{~T}$. arXiv preprint arXiv:2008.05957.

\section{S5.P2.}

\section{Analytical model and experimental validation} of birdcage coil $B_{1}$ at high frequency

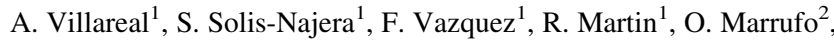 \\ *A. Rodriguez ${ }^{3}$ \\ ${ }^{1}$ National Autonomous University of Mexico (UNAM), Faculty \\ of Sciences, Mexico City, MX; \\ ${ }^{2}$ INNN-MVS, Department of Neuroimage, Mexico City, MX; \\ ${ }^{3}$ UAM Iztapalapa, Department of Electrical Engineering, Mexico \\ City, $M X$
}

Introduction: In MRI is vital to accurately map B1 with suitable phantoms and, ultimately, in vivo [1]. A straightforward theoretical frame of $B_{1}$ is most desirable to actually validate experimental results obtained with the popular birdcage coil. We derived an expression to compute $\mathrm{B}_{1}$ of a birdcage coil at high frequency.

Method: We derived an expression for $\mathrm{B}_{1}$ based on the theoretical work reported in $[1,2]$, together with the expression obtained by De Zanche et al. for a 4 rung birdcage coil [3]:

$$
\begin{aligned}
\mathbf{B}= & 3 / 2\left(i\left(l^{2}+2 d^{2}\right) \mathrm{d}^{-1}\left(l^{2}+d^{2}\right)^{-3 / 2}\right)\left(j_{0}(k r)-j_{1}(k r)(\mathrm{kr})^{-1}\right) \\
& \cdot \mathbf{v}+\left(3 j_{1}(k r)(\mathrm{kr})^{-1}+j_{0}(k r)\right) \cdot(\mathbf{n} \cdot \mathbf{v}) \mathbf{n}
\end{aligned}
$$

where $\mathbf{v}=0.5(1, i, 0)$ is a unit vector in the direction of the circularly polarized RF field generated by the volume coil, and $\mathbf{n}=r^{-1}(x, y, z)$ is the unit normal vector to the radial direction. Functions $j(k r)$ represent the Bessel polynomials. $l$ and $d$ are the length and the diameter of the birdcage coil, respectively. Phantom images were acquired with a standard spin echo sequence. The acquisition parameters were used: $\mathrm{TE} / \mathrm{TR}=25 \mathrm{~ms} / 900 \mathrm{~ms}, \quad \mathrm{FOV}=40 \mathrm{~mm} \times 40 \mathrm{~mm}, \quad$ matrix size $=256 \times 256$, slice thickness $=2 \mathrm{~mm}, \quad \mathrm{NEX}=1$, with a transceiver quadrature birdcage coil (4 rungs, length $012 \mathrm{~cm}$ and diameter $=7.7 \mathrm{~cm}$ ) at $7 \mathrm{~T}$ [4]. Figure 1.a) shows a photograph of the coil prototype.

Results and discussion: Unlike other difficult formulas for $B_{1}$, reported in the literature, our theoretical model is fairly easy to follow for the further developments of this type of volume coils. A comparison plot of the theoretical and experimental results is shown in 
Fig. 1.b). A remarkable concordance can be observed, validating the experimental results obtained with the birdcage coil prototype. The profile patterns also show an excellent agreement with those reported for a $7 \mathrm{~T}$ birdcage coil [4]. An analytical expression of the magnetic field for the popular birdcage coil was derived, and experimentally validated for high field applications.

Fig. 1

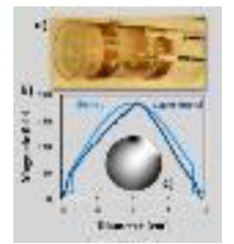

\section{References:}

1. Bidinosti, C. P., Chapple, E. M., \& Hayden, M. E. (2007). Concp Magn Reson Part B: Magn Reson Eng. 31(3), 191-202. 10.1002/ cmr.b.20090.

2. Cline, H., Mallozzi, R., Li, Z., McKinnon, G., \& Barber, W. (2004). Magn Reson Med. 51(6):1129-37. 10.1002/mrm.20064.

3. De Zanche, N., \& Allen, P. S. (2002). Magn Reson Med, 47(2), 364-371. 10.1002/mrm.10039.

4. Solis-Najera, S., Martin, R., Vazquez, F., Marrufo, O., \& Rodriguez, A. O. (2020). arXiv preprint arXiv:2008.05957.

\section{S5.P3.}

\section{Numerical study of magnetic field improvement for a volume resonator at 7 Tesla}

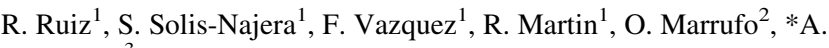 \\ Rodriguez $^{3}$ \\ ${ }^{I}$ National Autonomous University of Mexico (UNAM), Faculty \\ of Sciences, Mexico City, MX; \\ ${ }^{2}$ INNN-MVS, Department of Neuroimage, Mexico City, MX; \\ ${ }^{3}$ UAM Iztapalapa, Department of Electrical Engineering, Mexico \\ City, $M X$
}

Introduction: The development of an RF coil with improved performance is still an important quest in MRI. We proposed to modify the standard birdcage coil by adding circular petals to the rungs to improve its magnetic field, $\mathrm{B}_{1}$ and uniformity.

Method: We numerically simulated the magnetic field of the coil prototype as shown in Fig. 1, using 4 rungs with 4 petals on each rung. The separation between the circles is 3 times the petal radius, to diminish the unwanted effect of the mutual inductance. The commercial software CST Microwave Studio (CST MICROWAVE STUDIO, CST GmbH, Darmstadt, Germany) was used to calculate the electromagnetic fields. A saline-solution spherical phantom was used $\left(\sigma=0.55 \mathrm{~S} / \mathrm{m}, \varepsilon=78.4, \rho=998 \mathrm{~kg} / \mathrm{m}^{3}\right.$, and $\left.\mu=0.999991\right) . \mathrm{B}_{1}$ simulations of a birdcage coil with similar dimensions and 8 rungs were also conducted for comparison purposes.
Fig. 1

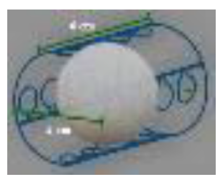

Results and Discussion: Two-dimensional maps were numerically computed and shown in Fig. 2.a) and b). To investigate the uniformity and sensibility of this resonators design, a comparison plots was computed with the simulation data, as shown in Fig. 2. c). Profiles were calculated with data taken along the black line in Fig. 2.d). Finally, a comparison histogram was also done and shown in Fig. 2.e). A clear improvement of sensibility can be observed from Fig. 2, considering that the comparison was done against a birdcage coil with 8 rungs. Numerical results show very concordance with data reported previously [1]. These results showed that it is feasible to build a volume resonator with a better sensibility and similar uniformity that a birdcage coil a larger number of rungs.

Fig. 2

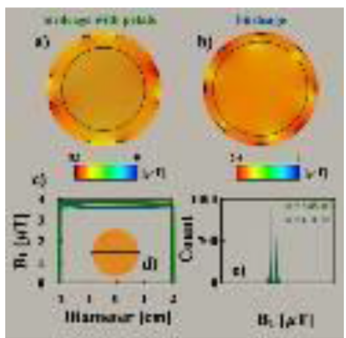

\section{Reference:}

[1] Martin, R., et al. Measurement 82 (2016): 482-489. 10.1016/ j.measurement.2016.01.013.

\section{S5.P4.}

High-performance quadrature biplanar volume $R F$ coil for flexible and fast in vivo imaging at low field $(0.1 \mathrm{~T})$

\author{
*M. Yushchenko ${ }^{1}$, N. Salameh ${ }^{1}$, M. Sarracanie ${ }^{1}$ \\ ${ }^{1}$ University of Basel, Department of Biomedical Engineering, \\ Allschwil, $\mathrm{CH}$
}

Many low-field MRI systems employ open, biplanar magnets to combine the advantages of low field with enhanced access to the patient. The biplanar volume coil ${ }^{1}$ follows the same philosophy, enabling simple positioning and comfortable patient scanning from its open access while maintaining good B1 homogeneity and filling factor. Such a geometry appears particularly handy for MSK imaging in various positions, as opposed to conventional closed volume coil geometries $^{2}$ (e.g. solenoids or Helmholtz). Here, we demonstrate an optimized coil composed of two biplanar coils operating in quadrature mode with good intrinsic decoupling at $4.33 \mathrm{MHz}(0.1 \mathrm{~T})$.

The two coils A and B of the quadrature coil are based on 4 and 5-strip $150 \times 100 \mathrm{~mm} \mathrm{Cu}$ planes connected in series and tuned by variable capacitors at $4.33 \mathrm{MHz}$ (Fig. 1A). Inductive coupling was used to interface both coils ${ }^{3}$ with an optimized coupler design. Good 
geometric decoupling between the two coils is achieved $\left(\mathrm{S}_{21}-\right.$ $\left.36 \mathrm{~dB}, \mathrm{~S}_{11, \mathrm{~A}}-5.4 \mathrm{~dB}, \mathrm{~S}_{22, \mathrm{~B}}-4.8 \mathrm{~dB}, \mathrm{Q}_{\mathrm{A}} 222, \mathrm{Q}_{\mathrm{B}} 267\right)$. MRI was performed on a small footprint resistive biplanar $0.1 \mathrm{~T}$ magnet (Bouhnik SAS, France). A noise equalization scaling was applied to the 2nd channel by considering the noise standard deviations in a corner region of the magnitude images. 3D sequences were run on a Cameleon 3 spectrometer (RS2D, France) with a variable Gaussian density k-space sampling pattern of $50 \%$ for phase-encoding. For in vivo data, the $\mathrm{k}$-space was filtered with a Tukey window $(\mathrm{r}=0.2)$ using MATLAB (Mathworks, USA), and zero-filled to double the matrix size in $3 \mathrm{D}$.

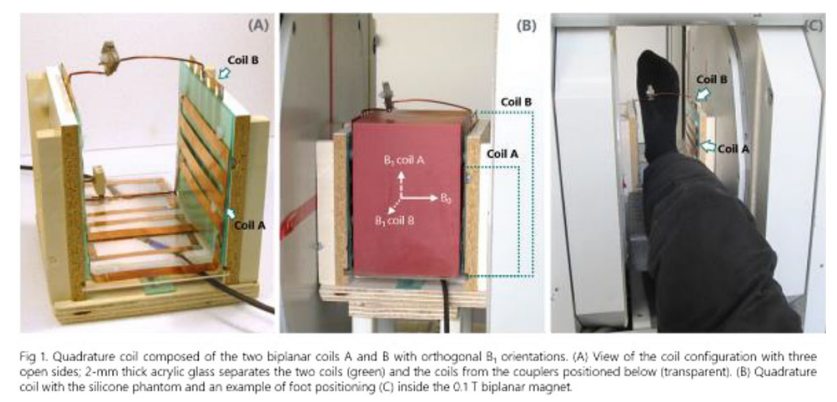

GRE images on a silicone phantom (Fig. 2) show a homogeneous sensitivity of both coils at their center (ROI size: $30 \times 30 \times 12$ voxels). The SNR of the combined image in the central ROI was on average $\sim 36 \%$ greater than that of the separate channels. The distorted peripheral regions of the phantom, due to B0 inhomogeneity and gradient nonlinearities, are located far from the volume of interest. Figure 3 shows examples of 3D views obtained with the quadrature biplanar coil in vivo (ankle and elbow of two healthy volunteers). bSSFP images exhibit good contrast between bone, skin, muscles and ligaments. Typical bSSFP banding artifacts are visible at the edges of the FOV. A similar contrast is obtained from the first echo of the DESS sequence in the flexed elbow (Fig. 3).
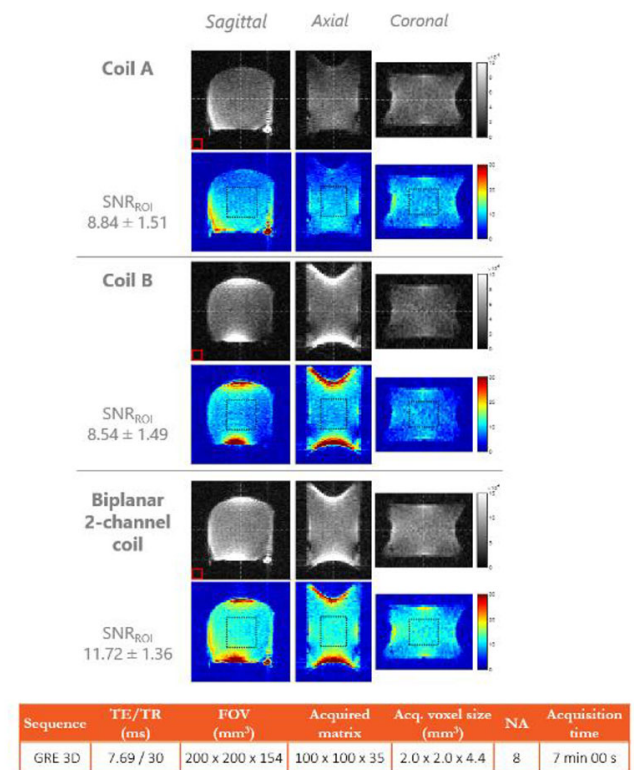

ig 2. Phantom data: magnitude images (grayscale) at 3 orthogonal planes (white dashed lines) extracted from the 3D datasets and the corresponding SNR maps (color). 1st and 2nd rows: data acquired on the silicone phantom with the separate channels $A$ and $B$ of the quadrature coil; $3 r d$
row: the resulting two-channel combination after noise equalization. 1st column: sagittal view, 2 nd

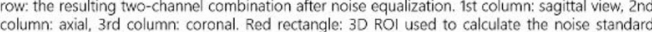
deviation for the SNR maos, black dotted rectangle: 3D SNR and standard deviation values. The bottom of the biplanar quadrature coil is the lower side on the sagittal and axial images. The details of the MR sequences are shown in the table. GRE: spoiled gradient-echo, TR: repetition time, TE: echo time, FOV: field-of-view, NA: number of averages.

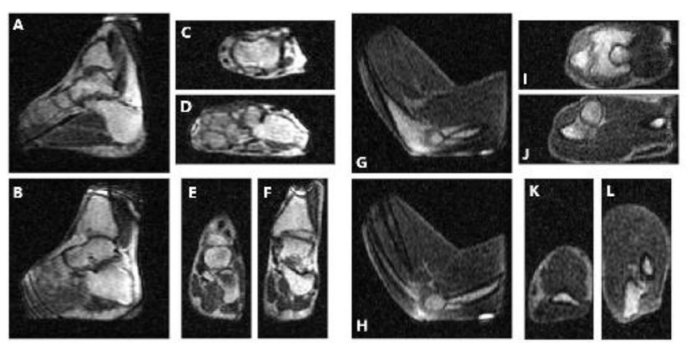

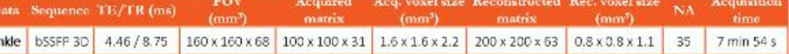
\begin{tabular}{|l|l|l|l|l|l|l|l|l|l|}
\hline Elbow & DESS 30 & $5.16 / 30.00$ & $160 \times 160 \times 68$ & $103 \times 100 \times 31$ & $1.6 \times 1.0 \times 2.2$ & $200 \times 200 \times 63$ & $0.8 \times 0.8 \times 1.1$ & 12 & 2 min 185 \\
\hline
\end{tabular}

F 3. Example vews of the 3D datasets acquired in wo with the quadrature biplanar coli: dorsiflexed ankle of a healthy female

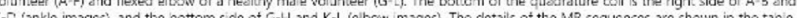

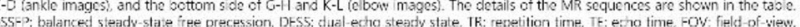
NAS- number of averages.

Overall, the quadrature coil enables good sensitivity and enhanced SNR over its entire volume. This optimized design makes in vivo imaging at low frequency with high $3 \mathrm{D}$ resolution within acceptable acquisition times possible. In addition, different extremities can be easily scanned in various positions thanks to its three open sides. Complementing the advantages of low-field with open access detectors shows great potential for a variety of MRI applications, such as weight-bearing and intra-operative MRI without artifacts from surgical instruments and prosthetics.

\section{References:}

1 Roberts 1993 J Magn Res

2 Mispelter 2008 Comp Rend Chim

3 Hoult 2002 Conc Magn Res

\section{S5.P5.}

\section{Ultra-fast transmit receive switch for high performance} short T2 imaging

\section{${ }^{*}$ C. M. Schildknecht ${ }^{1}$, M. Weiger ${ }^{1}$, K. P. Pruessmann ${ }^{1}$ \\ ${ }^{I}$ ETH Zürich, Zurich, $\mathrm{CH}$}

Introduction: Short T2 MRI methods open up pathways to image compounds with very short coherence times [1]. An object of interest is for example the myelin bilayer in the brain, which has signal components with T2s down to 8 us [2]. However, to perform such measurements high performance gradient systems and RF chains are required. One important part of such RF chain is the transmit-receive switch. Not only does such switch has to change state very fast, ideally in less than one microsecond, but they need to withstand high RF power as well. In all of this the induced transient signals in the RF chain have to be low enough not the saturate the LNA, which is challenging for such switch speeds and power levels. There were only a few TR switches fulfilling the base requirements [3] witch were based on PIN diodes. Recently a novel switch was introduced based on GaN MOSFET capable of switching in less than $100 \mathrm{~ns}$ and allowing more than $1 \mathrm{~kW}$ of peak RF power [4]. In this work we demonstrate that such switch introduces no image artifacts when challenging state of the art sequence is performed.

Methods: Figure 1 shows TR switch topology, the GaN mosfet are turned on during transmission insulating the receive port. During reception the mosfets are turned off insulating the transmit port and connecting the TR port to the receive port. Figure 2 shows the implementation of such switch topology, additionally a LDO, mosfet driver and LNA are on the PCB. 

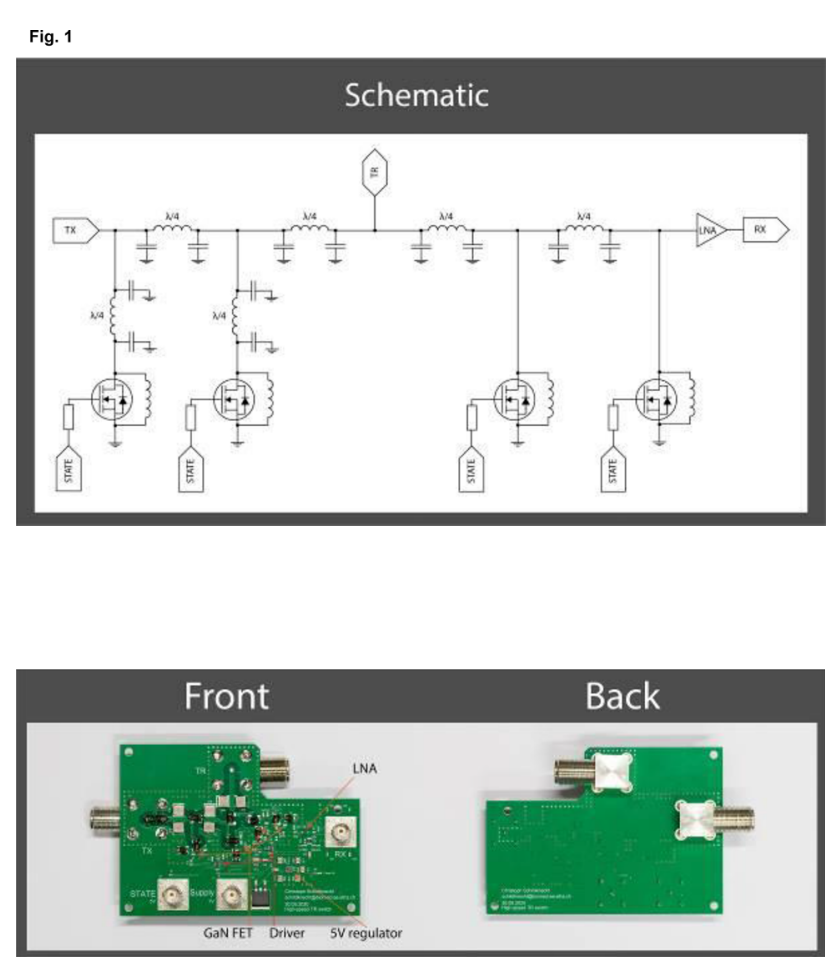

For testing the TR switch a 3D ZTE sequence with SPI gap filling was performed. Relevant scan parameter were $1 \mathrm{~kW}$ peak RF power, $1 \mathrm{MHz}$ bandwidth with a gradient of $200 \mathrm{mT} / \mathrm{m}$. The TR switch was changed state as soon as the coil was sufficiently rung down. Which was in this experiment was approximately $400 \mathrm{~ns}$.

For imaging, a proton free loop coil was used. As phantom, a cylindrical water phantom doped with $\mathrm{MnCl} 2$ to shorted $\mathrm{T} 2$ and $\mathrm{NaCl}$ to increase the coil loading and thereby shortening the ring down was used.

Results: Figure 3 shows image of the water phantom. For better visibility, the image was besides linear also plotted with logarithmic magnitude.

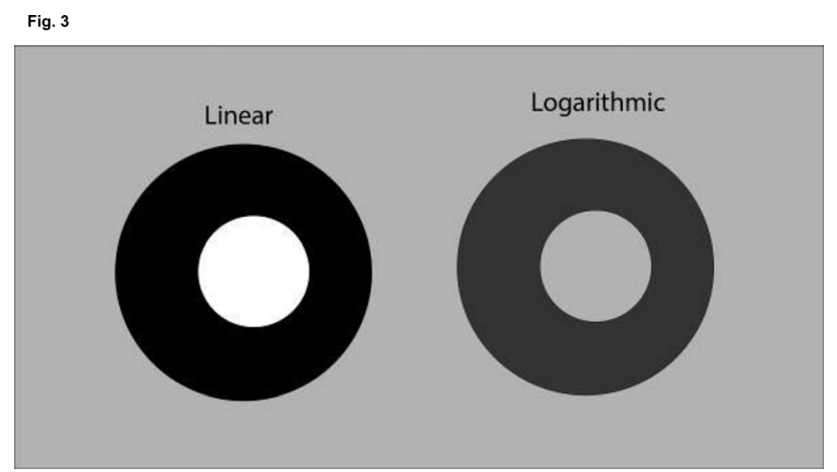

Discussion: As can be seen in Fig. 3 neither the foreground or the background have any artifacts that could be attributed to the transmit receive switch. This shows that the switch is able to support such short $\mathrm{T} 2$ measurements without issues.

With a switch time of less than 100 ns this switch topology is not limiting short $\mathrm{T} 2$ imaging in the foreseeable future and could be one part to enable imaging of ever shorter $\mathrm{T} 2$ compounds.

\section{References:}

1. Weiger Progress in Nuclear Magnetic Resonance Spectroscopy 2019.

2. Weiger NeuroImage 2020.

3. Brunner JMR 2016.

4. Schildknecht ISMRM 2021.

S5.P6.

Robust validation of a parallel-transmit dipole array simulation setup for infant MRI at 7 Tesla

\author{
*J. Clement ${ }^{1}$, O. Ipek ${ }^{1}$ \\ ${ }^{1}$ King's College London, Department of Biomedical Engineering, \\ London, $G B$
}

Introduction: Using parallel-transmit $(\mathrm{pTx}) 7$ Tesla $(\mathrm{T})$ promises to improve image quality and safety margins. Up to date, pTx is not used extensively due to its very restrictive safety margins $(\sim 5 \times$ more restrictive compared to single-Tx for commercial coils). These margins are the main ones responsible for the limited use of acquisition parameters. In this work, robust validation of electromagnetic simulation with the experiment will be explored for $7 \mathrm{~T}$ pTx infant MRI. Methods: A realistic infant size in-house designed phantom was 3Dprinted and filled with a saline solution $([\mathrm{NaCl}]=5.8 \mathrm{~g} / \mathrm{L}$, volume $=4.8 \mathrm{~L}, \varepsilon \mathrm{r}=79, \sigma=0.95 \mathrm{~S} / \mathrm{m})($ Fig. $1 \mathrm{~A})$. The $8 \mathrm{ch}$ dipole array ${ }^{1}$ (23 cm long, $1.5 \mathrm{~cm}$ width on FR-4) was tuned/matched at 297.2 MHz and its S-matrix was measured with VNA (Keysight E5080A-ENA, USA) on the bench using the phantom. In simulation, the 8ch dipole array was modeled (Sim4Life 6.2, ZMT, Switzerland), including the FR-4 substrate $(\varepsilon \mathrm{r}=4, \sigma=0 \mathrm{~S} / \mathrm{m})$, and conductive parts defined as lossy metal $(\sigma=5.8 \mathrm{e} 7 \mathrm{~S} / \mathrm{m})$. The tuning/matching circuit layout was modeled with an exact design of copper trace. A similar range of values to realistic array components was used with losses for inductors $(50-70 \mathrm{nH}$ with series resistors (1-80hms)) and capacitors $(18-25 \mathrm{pF}$ for series/2-15 $\mathrm{pF}$ for parallel,with Q-factor $1500^{2}$ ) to adjust the lumped elements for tuning/matching with the measured S-matrix as a target using a co-simulation method (Optenni Ltd,Finland). MR acquisitions were performed using the phantom on a $7 \mathrm{~T}$ MR scanner (MAGNETOM Terra, Siemens Healthcare, Erlangen, Germany). Individual $\mathrm{B}_{1}{ }^{+}$-field maps (magnitude/phase) were acquired $^{3}$, normalized to $1 \mathrm{~kW}$ total output power at RF amplifier, and compared to simulated $\mathrm{B}_{1}{ }^{+}$-field maps. The $\mathrm{B}_{1}{ }^{+}$-field distributions in $\mathrm{CP}$ mode and for one RF shimmed case were measured with the actual-flip-angle method ${ }^{4}$ and compared to simulations.
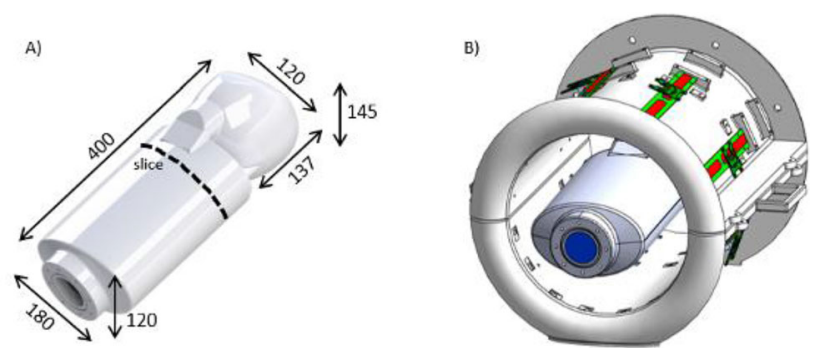

Figure 1-A) CAD model of the infant phantom with general dimensions (in $\mathrm{mm}$ ) and B) View of
the simulated model including the coil array frame and the infant phantom. The frame was included in the model for accurate placement of the dipoles but not simulated.

Results: The mean difference for the simulated and experimental reflection coefficients (Sii) is lower than 1\%, while it is about $12 \%$ for the nearest neighbor couplings (Sij) (Fig. 2). A good correlation is observed between experimental and simulated $\mathrm{B}_{1}{ }^{+}$-field maps for 
magnitude and phase (Fig. 3B). Dipoles 1 to 7 present an individual averaged $\mathrm{B}_{1}{ }^{+}$-field value within $\pm 10 \%$ of the measured value (Fig. 3C) while for dipole 8 it is $23 \%$ higher in simulations. The simulated $\mathrm{CP}$ and shimmed $\mathrm{B}_{1}{ }^{+}$maps were correlated better than $90 \%$ with measured maps (Fig. 3D).
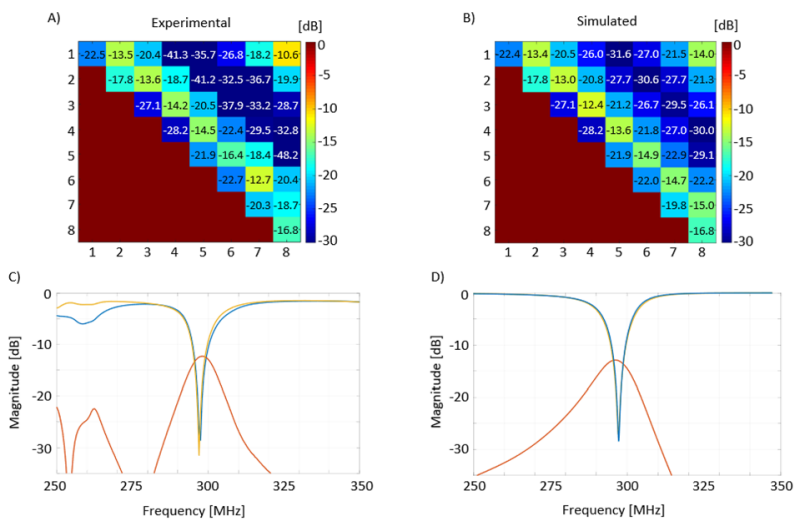

Figure 2-A) Experimentally measured and B) simulated S-matrices for the infant phantom at the isocentre of the 8Tx dipol coil array (Figure 18). Mean differences between experimental and simulated values were less than $1 \%$ for self-reflection around $12 \%$ for nearest-neighbour couplings. C) Experimentally measured and D) simulated S-curves
dipoles in the array. The reflection coefficients and couplings are shown over the same bandwidth.

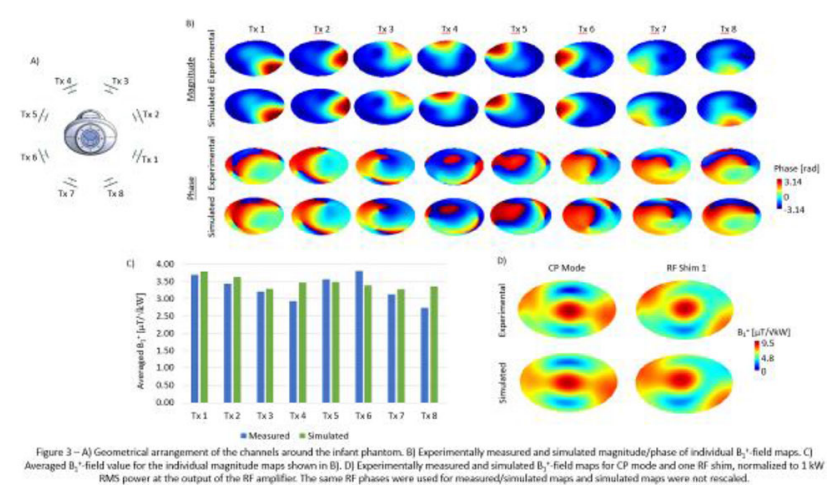

Discussion and conclusion: Robust correlations were found between experiments and simulations for $\mathrm{S}$-matrices, individual $\mathrm{B}_{1}{ }^{+}$-field amplitude/phase maps and RF shimmed $\mathrm{B}_{1}^{+}$-maps. The absence of coaxial cables in simulations or slight differences in coupling values, notably for dipole 8 with 1 may explain the remaining mismatches. We conclude that a robust validation of electromagnetic simulation with the experimental setup was shown for safe infant MRI at 7 Tesla.

\section{References:}

${ }^{1}$ Clement et al. ISMRM 2020, 4618.

${ }^{2}$ Chen et al. MRM 79(3), 2018.

${ }^{3}$ Fautz et al. ISMRM 2008, 1247.

${ }^{4}$ Yarnykh et al. MRM 57(1), 2007.

\section{S5.P7.}

\section{Polymer nanosystems as dual-imaging theranostic agents in a glioblastoma mouse model}

*N. Arias-Ramos ${ }^{1}$, M. Serrano-Torres ${ }^{1}$, L. E. Ibarra ${ }^{2}$, M. J. Guillén Gómez $^{1}$, M. D. Caverzán ${ }^{2,3}$, R. E. Palacios ${ }^{3}$, C. A. Chesta ${ }^{3}$, P. LópezLarrubia $^{1}$
${ }^{1}$ Instituto de Investigaciones Biomédicas Alberto Sols (UAM), Department of Endocrine and Nervous System Pathophysiology, Madrid, ES;

${ }^{2}$ University of Río Cuarto, Departamento de Biología Molecular, Facultad de Ciencias Exactas Físico-Químicas y Naturales, Rio Cuarto, AR;

${ }^{3}$ CONICET, Instituto de Investigaciones en Tecnologiás Energéticas y Materiales Avanzados, Rio Cuarto, AR

Introduction: Glioblastoma multiforme (GBM) is considered the most lethal of the malignant primary brain tumors ${ }^{1}$. In fact, even after aggressive treatment, prognosis and survival remain poor ${ }^{2}$. Recently, nanosystems have become promising candidates for GBM diagnosis and treatment, due to their exceptional magnetic properties, biocompatibility and blood brain barrier (BBB) penetrability ${ }^{3}$. In our previous investigations, metallated doped conjugated polymer nanoparticles (CPNs) (conjugated with fluorescent polymer F8BT) were visualized in tumors by $\mathrm{T} 2$ weighted (T2W) $\mathrm{MRI}^{4}$. In this sense, this project aims to evaluate the biodistribution of two CPNs with different types of cores, $\mathrm{Fe}_{3} \mathrm{O}_{4}$ or $\mathrm{NiFe}_{2} \mathrm{O}_{4}$, in mice bearing GBM flank-tumors and control mice.

Methods: In vitro validation of both CPNs was performed in a phantom study with different CPNs dilutions (Fig. 1A). Afterwards, NOD-SCID mice were injected intravenously with CPNs with a $\mathrm{Fe}_{3} \mathrm{O}_{4}$ or $\mathrm{NiFe}_{2} \mathrm{O}_{4}$ core. CPN's biodistribution was studied by T2W magnetic resonance imaging (MRI) pharmacodynamics (Fig. 1C \& D) and T2 maps (Fig. 1B) were obtained before and after the CPNs injection in a $7 \mathrm{~T}$ system. An hour after injection, mice were sacrificed, organs were resected and studied by fluorescent imaging. Additionally, mice bearing C6-GBM flank tumors were studied by T2W MRI before and 15 min after intertumoral injection of $\mathrm{Fe}_{3} \mathrm{O}_{4}$ or $\mathrm{NiFe}_{2} \mathrm{O}_{4}$ CPNs. Then, mice were sacrificed and flanks removed for fluorescence studies using a IVIS Lumina II system.

Results: We observed a higher CPNs uptake in the liver and a moderate accumulation in the renal cortex and the renal medulla as seen in $\mathrm{T} 2 \mathrm{~W}$ pharmacodynamics (Fig. $1 \mathrm{C} \& \mathrm{D}$ ). The CNPs liver accumulation seem to be higher with $\mathrm{Fe}_{3} \mathrm{O}_{4}$ than $\mathrm{NiFe}_{2} \mathrm{O}_{4}$ core nanoparticles (Fig. 1C). Intratumor injection studies revealed that both CPNs can be visualized in flank tumors by T2W images, showing signal decreasing in the location where the CPNs were injected (Fig. 2A). Both CPNs were also detected in the xenograft tumors by fluorescence imaging and not observed in not injected (control) tumors (Fig. 2B).

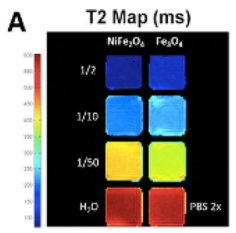

C
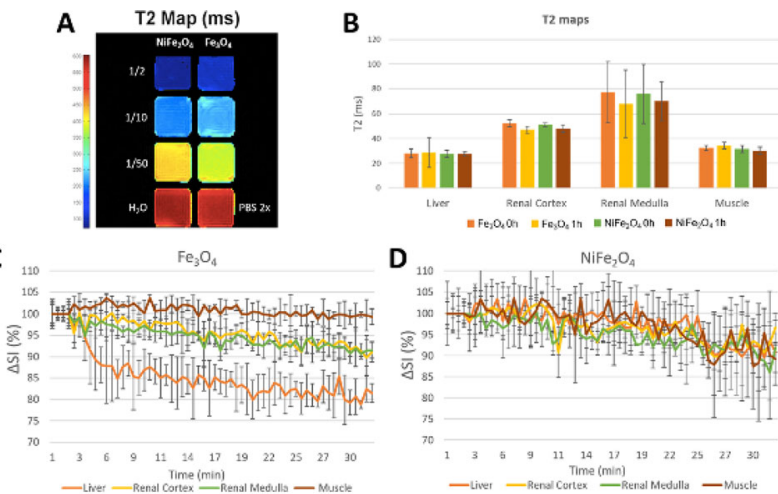

Figure 1: A) T2 map in vitro study of the CPNs with $\mathrm{NiFe}_{2} \mathrm{O}_{4}$ or $\mathrm{Fe}_{3} \mathrm{O}_{4}$ core in different dilutions and using water and $\mathrm{PBS} 2 \mathrm{x}$ as controls. B) $\mathrm{T2}$ ( $\mathrm{ms}$ ) values in different organs, obtained from $\mathrm{T} 2$ maps before and one hour after CPNs injection. C, D) Examples of T2W 


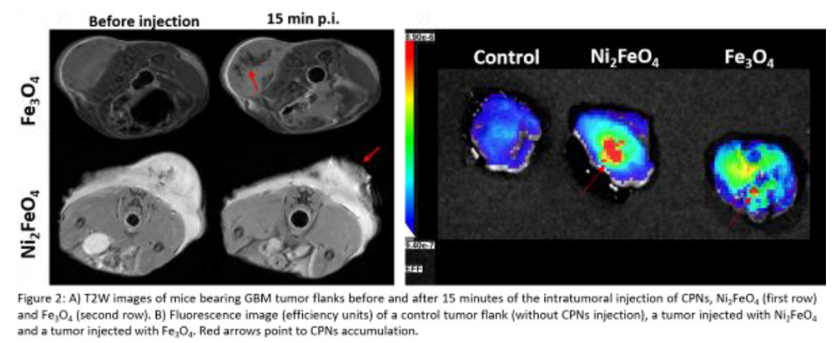

Discussion: Overall, results suggest that both CPNs are good candidates to study whether they reach and accumulate in the tumor of an orthotopic and xenograft GBM model.

\section{References:}

1. Louis, D. N. et al. Acta Neuropathol. 131, 803-820 (2016).

2. Davis, M. E. et al. Clin. J. Oncol. Nurs. 20, 1-8 (2016).

3. Hsu, J. F. et al. Cancers vol. 13 1-22 (2021).

4. Ibarra, L. et al. Magn. Reson. Mater. Physics, Biol. Med 32(1): 107-233 (2019).

\section{S5.P8.}

\section{The impact of $\mathrm{B}_{1}{ }^{+}$optimisation on high-resolution perfusion and diffusion acquisitions at $7 \mathrm{~T}$}

*S. Kashyap ${ }^{1}$, R. A. M. Haast ${ }^{2}$, F. J. Fritz ${ }^{3}$, D. Kurban ${ }^{1}$, D. Ivanov ${ }^{1}$, A. Roebroeck ${ }^{1}$, B. A. Poser ${ }^{1}$

${ }^{1}$ Maastricht University, Department of Cognitive Neuroscience, Maastricht, NL;

${ }^{2}$ Aix-Marseille University, Center for Magnetic Resonance in Biology and Medicine, Marseille, FR;

${ }^{3}$ Universitätklinikum Hamburg-Eppendorf (UKE), Institute of Systems Neuroscience, Hamburg, DE

Introduction: We previously ${ }^{1}$ addressed the cause of apparently absent perfusion in the right inferior temporal lobe by optimising the spatial positioning of dielectric pads to achieve a more symmetric $\mathrm{B}_{1}{ }^{+}$field. Our earlier work ${ }^{2,3}$ has shown that optimisations to improve labelling efficiency such as tr-FOCI inversion pulses ${ }^{4}$ and dielectric pads $^{5,6}$ can yield robust perfusion imaging using ASL at $7 \mathrm{~T}$. Dielectric pads provide a means to locally improve the $\mathrm{B}_{1}{ }^{+}$field, and Pads of smaller size $\left(13 \times 13 \mathrm{~cm}^{2}\right)$ have proven to be a preferable compromise for participant comfort owing to differences in head shapes and sizes. We observed that optimised pad positioning allows a symmetric $\mathrm{B}_{1}{ }^{+}$in near whole-brain acquisitions. Thereby the adiabatic condition for the inversion pulse is fulfilled, resulting in robust measurement of perfusion in the right inferior temporal lobes. Dielectric pads have been previously shown to yield improvements for spin-echo based diffusion imaging that is also $\mathrm{B}_{1}^{+}$sensitive ${ }^{7}$. Here, we extend our investigation to also include diffusion-weighted imaging using a PGSE sequence to assess the impact of our setup. Methods: Data were acquired on 6 participants $(30 \pm 3$ years, 2 female) on a Siemens Magnetom $7 \mathrm{~T}$ using the 1Tx/32Rx NOVA head coil. Up to three $13 \times 13 \mathrm{~cm}^{2} \mathrm{CaTiO}_{3}$ dielectric pads were employed using (a) 1-1 and (b) 2-1 conFiguration (Fig. 1,2 schematic). $\mathrm{B}_{1}^{+}$maps were acquired using an Sa2RAGE sequence ${ }^{8}$ with $2 \mathrm{~mm}$ isotropic voxels. Perfusion-weighted $($ GRAPPA $=3$, TIs $=$ $700 / 1700 \mathrm{~ms}, \mathrm{TR} / \mathrm{TE}=2760 / 12 \mathrm{~ms}, \mathrm{BW}=2164 \mathrm{~Hz} / \mathrm{px})$ and $\mathrm{M} 0$ $(\mathrm{TR}=20 \mathrm{~s})$ data were acquired with a pulsed ASL sequence with a FAIR QUIPSS II labelling and 2D EPI readout with $1.8 \mathrm{~mm}$ isotropic voxels. Diffusion-weighted data $(\mathrm{GRAPPA}=3, \mathrm{~b}$ val $(\#$ dir $)=$ $1000(35) / 2000(53) \mathrm{s} / \mathrm{mm}^{2}$ including 5 interleaved b0 images, TR/
$\mathrm{TE}=6500 / 62 \mathrm{~ms}, \mathrm{BW}=1776 \mathrm{~Hz} / \mathrm{px})$ were acquired using a 2D-EPI spin-echo sequence with $1.5 \mathrm{~mm}$ isotropic voxels. Data processing was carried out using FSL"s oxford_asl, eddy_cuda9.1 and dtifit 9 .

Results: Please see figure captions.

Discussion: In this $7 \mathrm{~T}$ study, we show that whole brain $\mathrm{B}_{1}{ }^{+}$distribution can be improved by optimising the placement of dielectric pads given a priori knowledge of the coil "s inherent $\mathrm{B}_{1}{ }^{+}$asymmetry. We demonstrate that $\mathrm{B} 1+$ has a direct impact on perfusion and diffusion measurements in the right temporal lobes and therefore, on their potential clinical utility at $7 \mathrm{~T}$. We urge caution for translating $3 \mathrm{~T}$ protocols to $7 \mathrm{~T}$ because the skewed $\mathrm{B}_{1}{ }^{+}$can be limiting on the measured perfusion and diffusion data. Next steps include to study the impact of the B1 + asymmetry on regional perfusion and diffusion signals. We will use MESMERISED ${ }^{10}$ to study the impact of $\mathrm{B}_{1}{ }^{+}$ optimisation on high $\mathrm{b}$ value acquisitions as well as more advanced diffusion models.
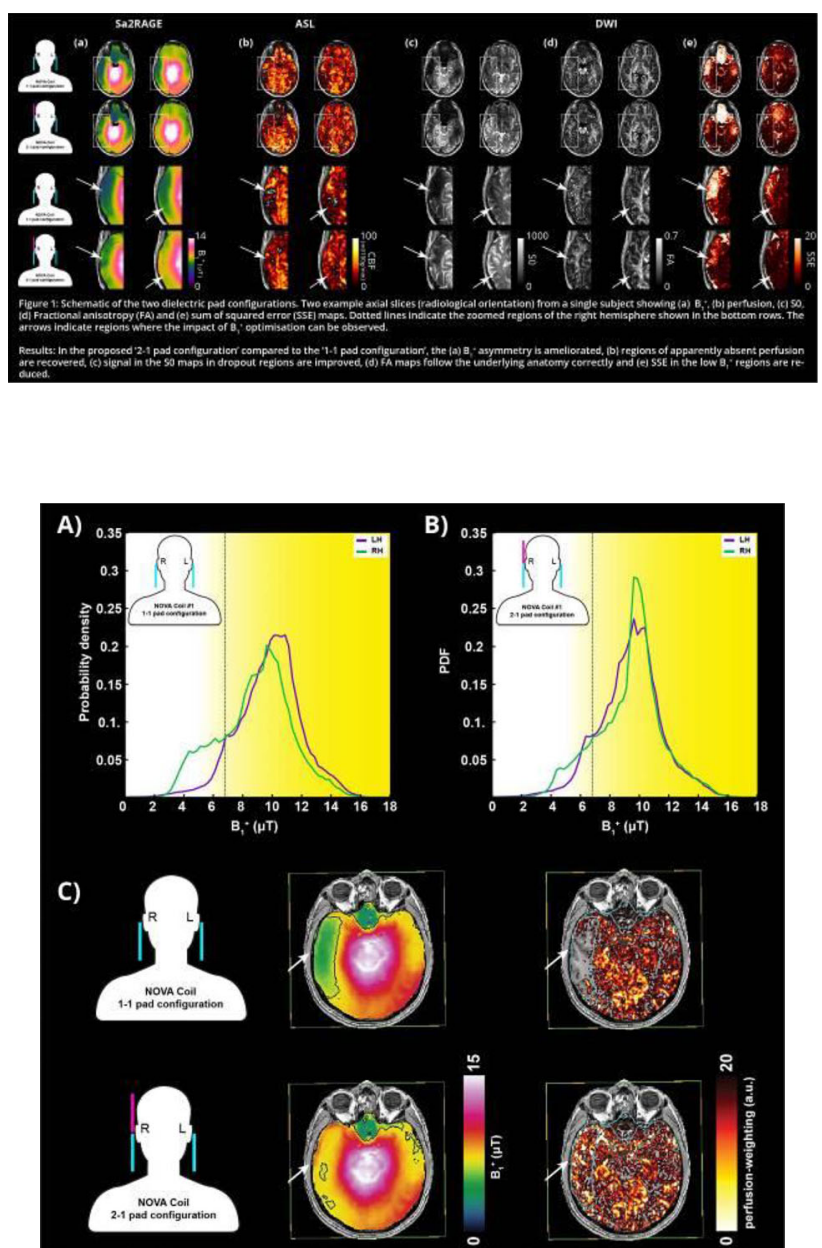

Figure 2: Histograms of $\mathrm{B}_{1}^{+}$in left and right hemispheres of another participant for 1-1 (A) and 2-1 (B) dielectric pad configuration (schematic inset). (C) Impact of improved $\mathrm{B}_{+}^{+}$symmetry on high-resolution $1.5 \mathrm{~mm}$ isotropic perfusion acquisitions. Outlines on the brain indicate low $\mathrm{B}_{1}^{+}$regions.

Results: The improvement achieved by the '2-1 pad configuration' (B) compared to the '1-1 pad configuration' $(A)$ is clearly seen by the increase in the number of voxels which meet the adiabatic condition of the inversion pulse indicated by yellow coloured background and dotted vertical black line. Particularly the green line which represents the right hemisphere wherein the $32 \mathrm{ch}$ coil has inherently a lower $\mathrm{B}_{1}^{+}$. The consequent impact on perfusion measurements at $1.5 \mathrm{~mm}$ in the right hemisphere is shown in (C). Compared to the $1.8 \mathrm{~mm}$ perfusion data in Fig $1 \mathrm{~b}$, the impact of the inherently asymmetric $\mathrm{B}^{+}$seems to affect $1.5 \mathrm{~mm}$ resolution more than the $1.8 \mathrm{~mm}$ resolution acquisitions. 


\section{References}

[1] Kashyap 2021, ISMRM.

[2] Ivanov 2016, MRM.

[3] Ivanov 2017 NI.

[4] Hurley 2010 MRM.

[5] Webb 2011 MRM.

[6] Teeuwisse 2010, IMA.

[7] Vu 2015 NI.

[8] Eggenschwiler 2012, MRM.

[9] Glasser 2013 NI.

[10] Fritz 2020, biorXiv.

\section{S5.P9.}

\section{Deuterium brain imaging at $7 \mathrm{~T}$ during $\mathrm{D}_{2} \mathrm{O}$ loading}

*D. Cocking ${ }^{1,2}$, *R. Damion ${ }^{1,3}$, D. Auer ${ }^{3}$, H. Franks ${ }^{3}$, D. Wilkinson ${ }^{3}$, M. Brook ${ }^{4}, *$ R. Bowtell ${ }^{1,2}$

${ }^{1}$ University of Nottingham, Sir Peter Mansfield Imaging Centre, Nottingham, GB;

${ }^{2}$ University of Nottingham, School of Physics and Astronomy, Nottingham, GB;

${ }^{3}$ University of Nottingham, School of Medicine, Nottingham, GB;

${ }^{4}$ University of Nottingham, School of Life Sciences, Nottingham, GB

Introduction: Deuterium magnetic resonance measurements following injection or ingestion of ${ }^{2} \mathrm{H}$-labelled compounds are of increasing interest for metabolic studies [1, 2]. The quadrupole moment of ${ }^{2} \mathrm{H}$ offers sensitivity to molecular orientation [3] and different relaxation time behaviour compared to ${ }^{1} \mathrm{H}$. Analysis of body fluid samples during heavy water loading is used for studies of protein synthesis [4]. Here we report ${ }^{2} \mathrm{H}$ MR measurements made at $7 \mathrm{~T}$ on human subjects loading with $\mathrm{D}_{2} \mathrm{O}$ to $\sim 1.5 \%$ concentration over a 6 -week period for a protein synthesis study.

Methods: Measurements were made on a $7 \mathrm{~T}$ Philips Achieva scanner using a dual-tuned ${ }^{2} \mathrm{H} /{ }^{1} \mathrm{H}$ birdcage coil (Rapid). Two subjects were scanned weekly during the loading period. In each session, we acquired 3D multi-echo, gradient echo (MEGE) ${ }^{2} \mathrm{H}$ images with a range of TR-values to allow calculation of $\mathrm{T}_{1}$ and $\mathrm{T}_{2}{ }^{*}$ maps, using simple fitting to the saturation recovery and TE variation. Low resolution MEGE ${ }^{1} \mathrm{H}$ images were also acquired for comparison. In an additional scanning session, we used the 32-channel Nova receiver coil to acquire high-resolution (0.7 mm isotropic) ${ }^{1} \mathrm{H}$ MPRAGE and $\mathrm{T}_{2}{ }^{*}$-weighted images for tissue segmentation, along with a low resolution MEGE ${ }^{1} \mathrm{H}$ image for co-registration. Relaxation times were measured in CSF, grey matter and white matter regions defined on the segmented images.

Results: Fig. 1 shows exemplar $3 \mathrm{D}{ }^{2} \mathrm{H}$ GE image data $(5 \mathrm{~mm}$ isotropic resolution, $\mathrm{TR}=50 \mathrm{~ms}, \mathrm{FA}=30^{\circ}$, 4-echoes $\mathrm{TE}_{1}=6 \mathrm{~ms}$, $\Delta \mathrm{TE}=8.5 \mathrm{~ms}$, acquisition time $6.5 \mathrm{~min}$ ) acquired after 8 days of $\mathrm{D}_{2} \mathrm{O}$ loading. The $\mathrm{T}_{2}{ }^{*}$ contrast in the image, which is formed from the average of the 4 echoes, mainly differentiates CSF from the other tissues. Fig. 2 shows example ${ }^{2} \mathrm{H} \mathrm{R}_{1}=1 / \mathrm{T}_{1}$ and $\mathrm{R}_{2}{ }^{*}=1 / \mathrm{T}_{2}{ }^{*}$ maps $\left(6 \times 6 \times 10 \mathrm{~mm}^{3}\right.$ voxels $)$ from two axial slices. These were formed by fitting to images with $\mathrm{FA}=60^{\circ}$ and TR $=80,120,240$ and $480 \mathrm{~ms}$ and to 5 echoes with $\mathrm{TE}_{1}=\Delta \mathrm{TE}=9 \mathrm{~ms}$, respectively. Corresponding ${ }_{1} \mathrm{H} \mathrm{R}_{2}{ }^{*}$ maps formed by fitting to MEGE data with 5 echoes $(\mathrm{TE} 1=8.9 \mathrm{~ms}$ and $\Delta \mathrm{TE}=5 \mathrm{~ms})$ are also shown. Fig. 3 details the average ${ }^{2} \mathrm{H}, \mathrm{T}_{1}$ and $\mathrm{T}_{2}{ }^{*}$ values, found by averaging over pixels classified as only containing GM, WM or CSF in the segmentation.

Fig. 1

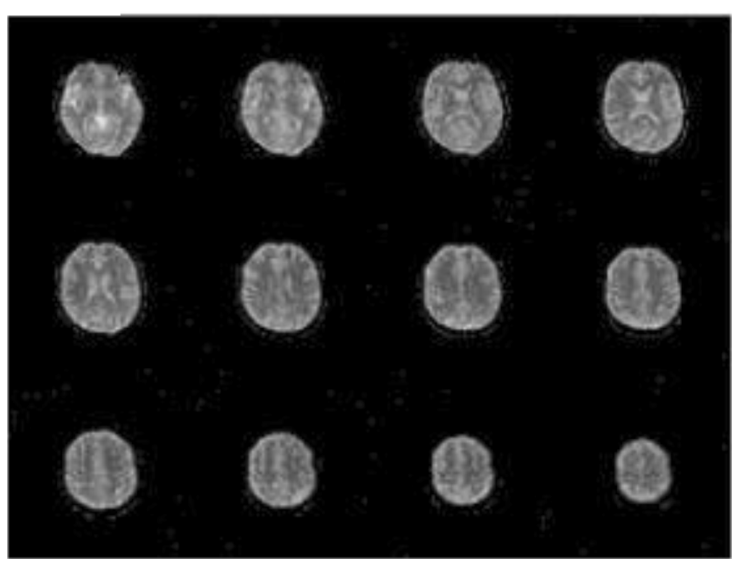

Fig. 2

Deuterium $\mathrm{R}_{1}\left[\mathrm{~ms}^{-1}\right]$

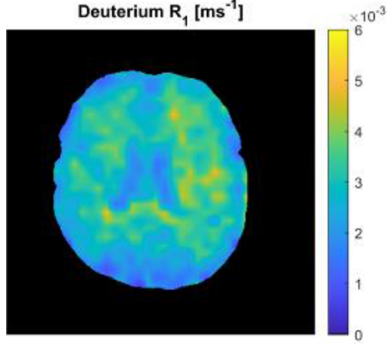

Deuterium $\mathbf{R}_{2}{ }^{*}\left[\mathrm{~ms}^{-1}\right]$

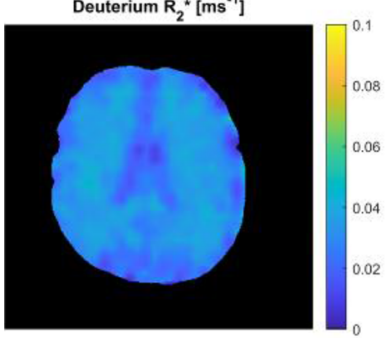

Proton $R_{2}^{*}\left[\mathrm{~ms}^{-1}\right]$

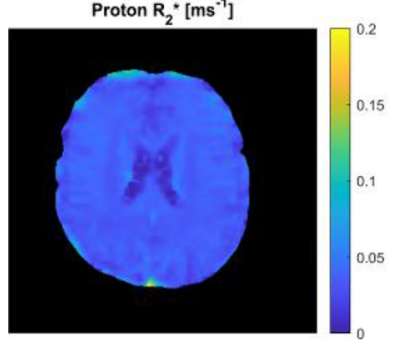

Deuterium $R_{1}\left[\mathrm{~ms}^{-1}\right]$
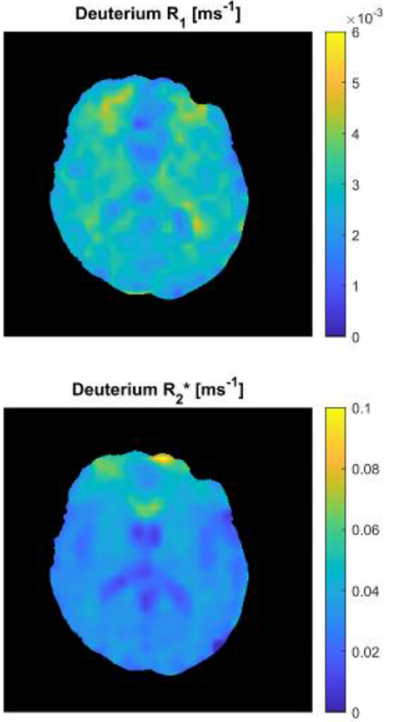

Proton $R_{2}{ }^{*}\left[\mathrm{~ms}^{-1}\right]$

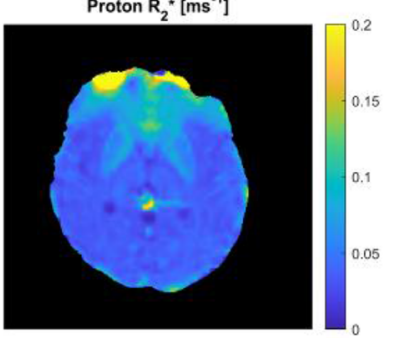


Fig. 3

\begin{tabular}{|c|c|c|c|c|}
\hline \multirow[b]{2}{*}{ Brain Tissue } & \multicolumn{2}{|c|}{ Case 1} & \multicolumn{2}{|c|}{ Case 2} \\
\hline & $\mathrm{T}_{1}[\mathrm{~ms}]$ & $\mathrm{T}_{2}^{*}[\mathrm{~ms}]$ & $\mathrm{T}_{1}[\mathrm{~ms}]$ & $\mathrm{T}_{2}{ }^{*}[\mathrm{~ms}]$ \\
\hline CSF & $428 \pm 159$ & $66 \pm 64$ & $456 \pm 153$ & $54 \pm 47$ \\
\hline GM & $367 \pm 125$ & $37 \pm 22$ & $384 \pm 111$ & $38 \pm 18$ \\
\hline WM & $352 \pm 107$ & $34 \pm 22$ & $332 \pm 88$ & $31 \pm 11$ \\
\hline
\end{tabular}

Discussion: Images with good SNR could be obtained in times of a few minutes at $7 \mathrm{~T}$ with $5 \mathrm{~mm}$ resolution in subjects loading with $\mathrm{D}_{2} \mathrm{O}$ to $\sim 1.5 \%$ concentration $(\sim 100$ times natural abundance). Similar levels of signal enhancement were found over the 6-week loading period. $\mathrm{T}_{1}$-values for deuterium in HOD were significantly shorter than corresponding values for ${ }^{1} \mathrm{H}$ in $\mathrm{H}_{2} \mathrm{O}$ [5], as expected due to the effect of quadrupolar ${ }^{2} \mathrm{H}$ relaxation. ${ }^{2} \mathrm{H}$ and ${ }^{1} \mathrm{H}, \mathrm{T}_{2}{ }^{*}$ values were similar, but the quadrupolar ${ }^{2} \mathrm{H}$ relaxation did not show strong sensitivity to iron content in deep grey matter (Fig. 2).

\section{References:}

1. De Feyter et al., Science Advances, 4, 7314, 2018.

2. De Feyter et al., JMR, 326, 106932, 2021.

3. Gursan et al. Proc. ISMRM 2021 \#1784.

4. Miller et al., J Appl. Physiol 128: 1163, 2020.

5. Wright et al., MAGMA, 21, 121, 2008.

\section{S5.P10.}

\section{MR safety of electrical muscle stimulation at 1.5 and 3 Tesla}

\author{
*X. Deligianni ${ }^{1,2}$, P. Madörin ${ }^{1}$, O. Bieri ${ }^{1,2}$, F. Santini ${ }^{1,2}$ \\ ${ }^{1}$ University Hospital of Basel, Department of Radiology / Division \\ of Radiological Physics, Basel, $\mathrm{CH}$; \\ ${ }^{2}$ University of Basel, Department of Biomedical Engineering, Basel, \\ $\mathrm{CH}$
}

Introduction: Electrical muscle stimulation (EMS) has been successfully used during MRI imaging ${ }^{1-3}$, but reports on the safety aspects are scarce ${ }^{4,5}$. Quantitative data on heating in realistic usage situations help to ensure the safety of the subjects and optimize the acquisition parameters and hardware setup for high-SAR sequences. Methods: Temperature was measured with a fiber optical thermometer. The two electrodes of the EMS device were in contact with a muscle tissue-simulating gel for MRI and temperature sensors were placed below them. A $3^{\text {rd }}$ sensor was inside the medium far from the electrodes and a 4th sensor was inside the bore in the air. An overview of the experiments is presented on Table 1 .

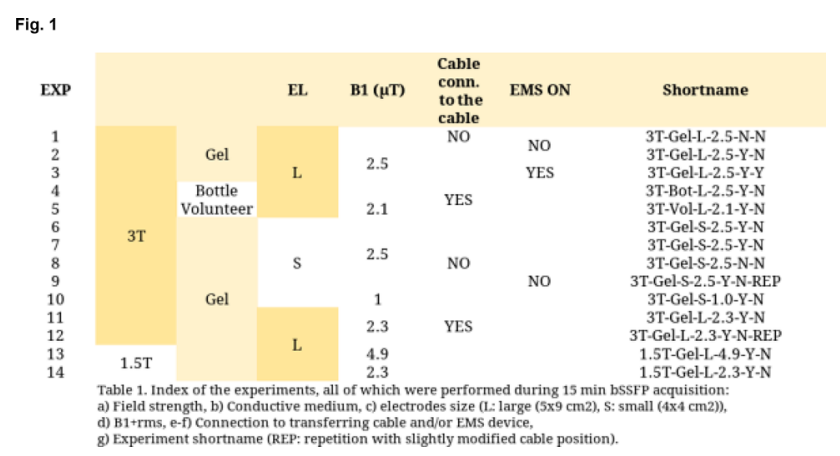

The experiment was repeated with the electrodes attached to a large plastic bottle ${ }^{4}$, and in vivo. The objects were placed off-center to achieve an E-field coupling similar to the one experienced on a limb. For the in vivo measurements, two large electrodes were connected to the EMS device and placed on the thigh ${ }^{2}$ and two on the other leg, in skin contact and under a non-connected electrode.

At $3 \mathrm{~T}$, a balanced SSFP sequence (scan time: $15.6 \mathrm{~min}$, TR $=3.74$ $\mathrm{ms}, \alpha=27-31^{\circ}$ ) was used to maximize SAR.

At $1.5 \mathrm{~T}$ we measured once at maximum SAR and once at half the maximum SAR. In all cases, the calculated B1 + rms field was recorded.

Results: The rise of temperature never reached a safety-threatening level. A rise of temperature was observed on the sensors that were in touch with the electrodes and was different for the two electrodes (Table 2).

\section{Fig. 2}

\begin{tabular}{|c|c|c|c|c|c|}
\hline \multirow{3}{*}{ EXP } & \multicolumn{5}{|c|}{ Max Temp difference } \\
\hline & \multicolumn{3}{|c|}{ Sensor No } & \multicolumn{2}{|c|}{ Shortname } \\
\hline & 1 & 2 & 3 & 4 & \\
\hline 1 & 0.9 & 1.1 & 1 & 0.7 & 3T-Gel-L-2.5-N-N \\
\hline 2 & 3.8 & 0.6 & 0.3 & 0.3 & 3T-Gel-L-2.5-Y-N \\
\hline 3 & 4 & 0.3 & 0.2 & 0.1 & 3T-Gel-L-2.5-Y-Y \\
\hline 4 & 2.7 & 5.2 & 1.2 & 0.2 & 3T-Bot-L-2.5-Y-N \\
\hline 5 & 2.5 & 3.8 & 3.6 & 2.8 & 3T-Vol-L-2.1-Y-N \\
\hline 6 & 9.6 & 4.5 & 0.2 & 0.5 & $3 \mathrm{~T}-\mathrm{Gel}-\mathrm{S}-2.5-\mathrm{Y}-\mathrm{N}$ \\
\hline 7 & 7.3 & 4.1 & 0.4 & 0.6 & 3T-Gel-S-2.5-Y-N \\
\hline 8 & 0.1 & 0 & 0.3 & 0.1 & $3 \mathrm{~T}-\mathrm{Gel}-\mathrm{S}-2.5-\mathrm{N}-\mathrm{N}$ \\
\hline 9 & 1.3 & 0.8 & 0.4 & 0.5 & 3T-Gel-S-2.5-Y-N-REP \\
\hline 10 & 1 & 0.8 & 0.4 & 0.1 & 3T-Gel-S-1.0-Y-N \\
\hline 11 & 2 & 0.8 & 0.4 & 0.3 & 3T-Gel-L-2.3-Y-N \\
\hline 12 & 6.5 & 5.1 & 0.4 & 0.5 & 3T-Gel-L-2.3-Y-N-REP \\
\hline 13 & 12.5 & 2.2 & 0.4 & 0.4 & 1.5T-Gel-L-4.9-Y-N \\
\hline 14 & 3 & 0.4 & 0 & 0.1 & 1.5T-Gel-L-2.3-Y-N \\
\hline
\end{tabular}

Table 2. Temperature rise in optic sensors 1 to 4 for all experiments. Darker colors correspond to higher heating.

The highest temperature rise at $3 \mathrm{~T}$ was $9.6{ }^{\circ} \mathrm{C}$, when smaller electrodes were used. In vivo, the temperature rise did not exceed $4{ }^{\circ} \mathrm{C}$. For larger electrodes, maximum temperature rise was $6.5^{\circ} \mathrm{C}$. At $1.5 \mathrm{~T}$ a higher $\mathrm{B} 1+$ rms was allowed, therefore the resulting temperature rise was higher and for the maximum allowed SAR this was $12{ }^{\circ} \mathrm{C}$.

Temperature curves (Fig. 1) show that placing the electrodes on top of a bottle lead to an overestimation of the heating rate (1A\&B). The rise of temperature in vivo at $3 \mathrm{~T}$ was roughly linear and did not exceed $4{ }^{\circ} \mathrm{C}(1 \mathrm{C})$, and was very similar to the free probe. Finally, at $1.5 \mathrm{~T}$ we observed a fast and large change of temperature (1D). 


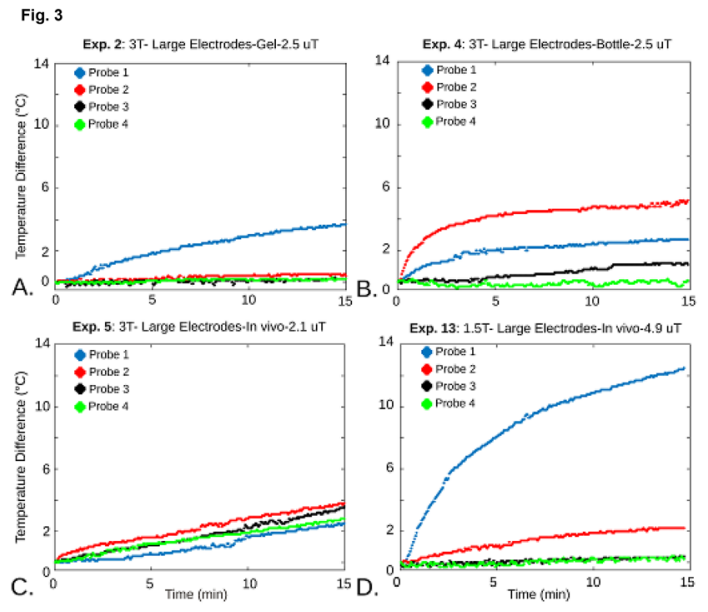

Temperature rise curves during a b-SSFP acquistion: A) At $3 \mathrm{~T}$ with large electrodes in a tissue-mimicking gel and $2.5 \mathrm{TT} \mathrm{B1}+\mathrm{rm}$ electrodes in vivo and $2.5 \mu \mathrm{T}$ B1+rms (Experiment No.5), D) At 1.5T with large electrodes in a tissue-mimicking gel and $4.9 \mu \mathrm{T}$ $1+$ rms (Experiment No.13)

Discussion: The largest temperature rise was observed at $1.5 \mathrm{~T}$, with higher $\mathrm{B} 1+\mathrm{rms}$, and at $3 \mathrm{~T}$ with smaller electrodes connected to the cable. The highest temperature rise at $3 \mathrm{~T}$ was less than $10^{\circ} \mathrm{C}$.

Therefore, it is suggested that during EMS-synchronized MRI, the electrodes can stay attached to the subject during the scan even in case of high SAR sequences. For an additional safety margin, the cable can be detached from the electrodes when possible.

Using electrical muscle stimulation in vivo during MRI scans causes no major risk of excessive RF heating, neither at $1.5 \mathrm{~T}$ nor at $3 \mathrm{~T}$.

\section{References (DOI):}

1. $10.1152 /$ jappl.1993.74.2.532

2. $10.1002 / \mathrm{mrm} .26154$

3. $10.1249 / \mathrm{MSS} .0000000000000491$

4. $10.1002 /$ jmri. 25316

5. $10.1002 /$ jmri. 1088

Acknowledgments: Supported by the SNSF (Nr. 172876).

\section{S5.P11.}

\section{Estimation of RF response at multi-electrode lead hotspots using lead electromagnetic models}

\author{
*M. Hussain ${ }^{1}$, G. Schaefers ${ }^{1,2}$ \\ ${ }^{1}$ Magnetic Resonance Institute for Safety, Technology and Research \\ GmbH (MRI-STaR), Gelsenkirchen, DE; \\ ${ }^{2} M R:$ Comp GmbH, Gelsenkirchen, DE
}

Introduction: This work focus on identifying the optimal clinical relevant pathway from a given set of trajectories and estimating the RF-induced dissipated power at the multi-electrode array of cortical implant (CorTec GmbH, Germany, see Fig. 1) by means of lead electromagnetic models (LEM). To develop the LEM, the transfer functions for each of the electrode are computed along with the tangential electric fields. The unscaled LEM is then used to compute the dissipated power at the electrodes and identify the optimal trajectory.

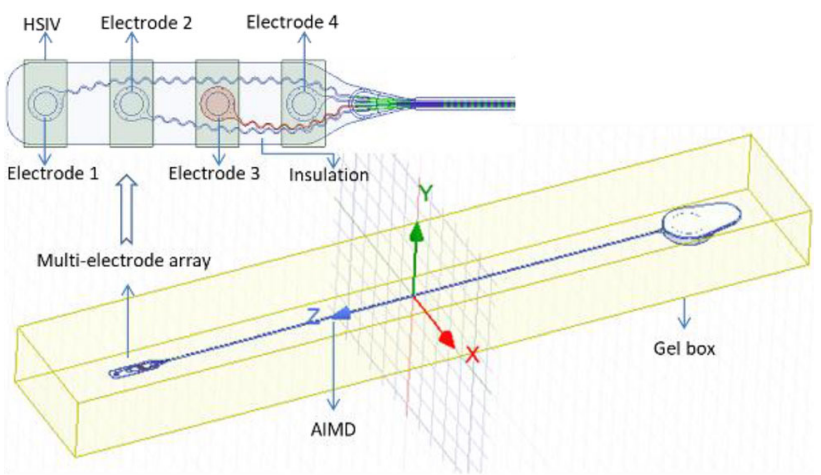

Figure 1. Sketch of the investigated multi-electrode implant.

Methods: The lead electromagnetic models as described in ISO/TS 109741 [1] comprise of a transfer function of the implant, the tangential electric field along the lead pathway and a constant. The LEM is given by

$\mathrm{P}_{\mathrm{lem}}=\mathrm{A}\left|\int_{0}^{1} \mathrm{~S}(1) \mathrm{E}_{\mathrm{tan}}(1) \mathrm{dl}\right|^{2}$. (1)

Here, $S(1)$ is the transfer function, $E_{\tan }(1)$ is the tangential electric field along the lead pathway, $\mathrm{A}$ is the calibration factor, and 1 is the length of the lead. The transfer functions of all four leads of CorTec's implant are computed by reciprocity method [2] using ANSYS HFSS (ANSYS Electromagnetics $2020 \mathrm{R} 1$ ) at resonance frequency of $64 \mathrm{MHz}$ with leads being inserted in gel medium with physical properties defined as per ASTM standard. Further, an MRI birdcage coil at $64 \mathrm{MHz}$ is simulated with a human head model along with 11 clinical relevant trajectories (Fig. 2). The tangential electric fields along these trajectories are computed and used in Eq. 1 to develop the unscaled LEM.

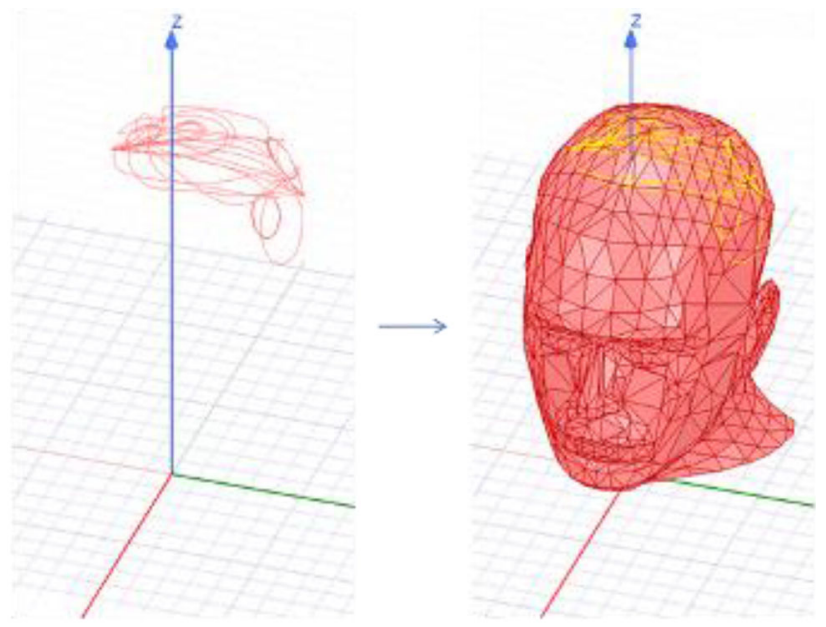

Figure 2. Clinical relevant trajectories in the human head model.

Results and discussion: The unscaled lead electromagnetic model (Eq. 1 without constant A) is then used to compute the power dissipation at the hotspots for all the clinical trajectories in the human head model. Fig. 3 shows the normalized power dissipation for all 11 clinical trajectories computed using unscaled LEM. It can be observed from Fig. 3 that for the given set of trajectories and simulation setup, trajectory 2 dissipates the highest power at the tip of the implant. 

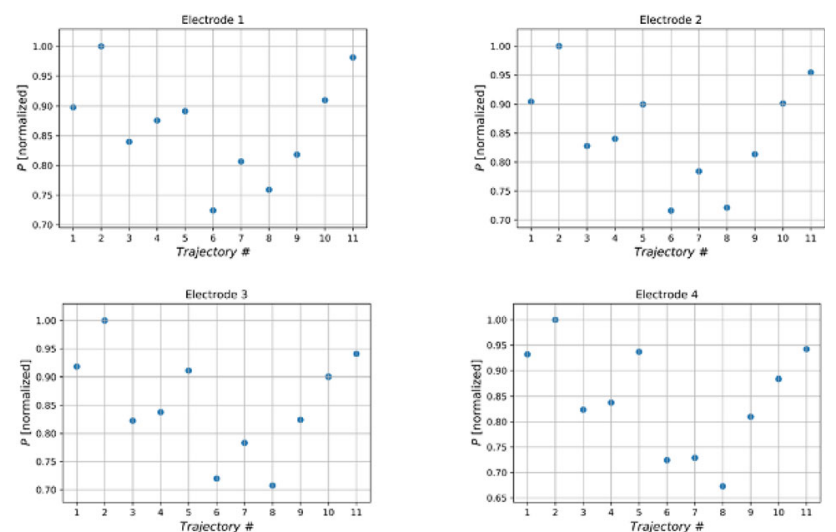

Figure 3. Normalized power dissipation at the electrodes 1-4 of the multi-electrode cortical implant using unscaled LEM.

Conclusion: The developed framework to identify the optimal clinical trajectory from a given set of trajectories and then estimating the power dissipation at the hotspots is quiet robust and generic. The framework is applicable to arbitrary structure with multiple electrode leads and helpful in optimizing the RF response with respect to the lead trajectories.

\section{References}

[1]. ISO/TS 10974: Assessment of the safety of magnetic resonance imaging for patients with an active implantable medical device, International Organization for Standardization, Geneva, Switzerland; 2018.

[2]. Feng, R. Qiang, W. Kainz, and J. Chen. A technique to evaluate mri-induced electric fields at the ends of practical implanted lead. IEEE Transactions on microwave theory and techniques, 63:305-313, 2015.

\section{S5.P12.}

\section{Regulation of the temperature during the vibration measurement according to ISO/TS 10974 to $37^{\circ} \mathrm{C}$ body temperature}

\author{
*M. Scholten ${ }^{1}$, G. Schaefers ${ }^{1,2}$, R. Laser ${ }^{1}$ \\ ${ }^{I} M R:$ comp GmbH, Gelsenkirchen, DE; \\ ${ }^{2}$ Magnetic Resonance Institute for Safety, Technology and Research \\ GmbH (MRI-STaR), Gelsenkirchen, DE
}

Introduction: The vibration behaviour of active implantable medical devices (AIMD) in the MR environment can have a strong temperature dependency. These vibrations can cause malfunction and pose potential risk to patients. A method to evaluate this interaction is defined in ISO/TS 10974 Sect. 10.3.7 and specifies that the measurement must take place at constant around the body temperature $\left(37{ }^{\circ} \mathrm{C} \pm 5{ }^{\circ} \mathrm{C}\right)$, if the material properties are significantly affected by ambient temperature [1]. However, it has been identified that especially implantable pulse generators (IPG) require an advanced test setup as these are more likely to fail. It is important to note that the measurement setup is permanently exposed to the static magnetic field. In order to fulfil these requirements we have developed a temperature controlled measurement setup to be able to test these critical implants within the MR environment.

Setup: The setup shown in Fig. 1 is built to utilize the $\mathrm{dB} / \mathrm{dt}$ at the top of the MR bore. The setup contains six controllable chambers, and each chamber was equipped a heating resistor (HR) to imitate the behaviour of an IPG. Cooling of the HR is achieved by a continuous flow of tempered air. Fibre optical probes are attached to each HR to monitor the temperature. Two more probes are pinned outside of the chamber to monitor Reference temperature. The Reference temperature was monitored all the time and used to control the air flow for an automatically readjusted of the temperature inside the system. Focus of the whole test setup is the MR compatibility which is achieved by the usage of only MR Safe labelled materials.

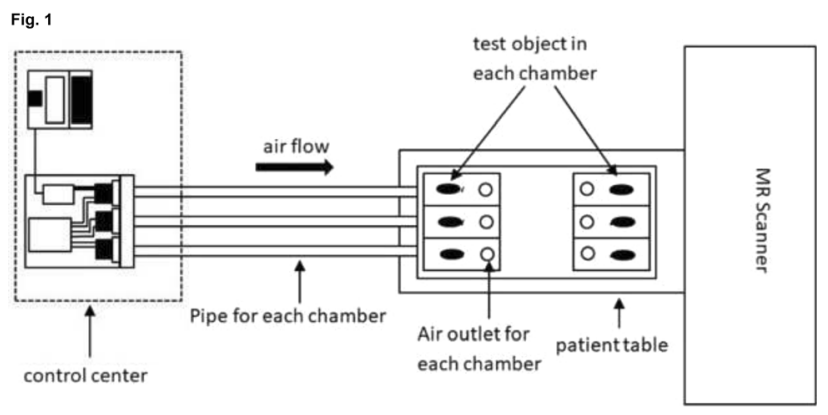

Schematic thermoregulation measurement setup

Results: The green, red, and purple graphs in Fig. 2, shows the heating of $5.5 \mathrm{~W}, 3.3 \mathrm{~W}$ and $1.1 \mathrm{~W}$ at the heating resistor. The first dip in the graph shows the time point where the heating was switched off and the second dip shows where the active cooling was switched on. The yellow graph shows the temperature curve with a maximum cooling for three different heating powers. This was used to determine the maximum power that can be cooled to reach the required temperature range. At a power of $3.3 \mathrm{~W}$ the temperature can be cooled down to a value of $38{ }^{\circ} \mathrm{C}$ which is within the required range. The brown graph shows the constant temperature curve at approximately $37{ }^{\circ} \mathrm{C}$ using the cooling system.

Fig. 2

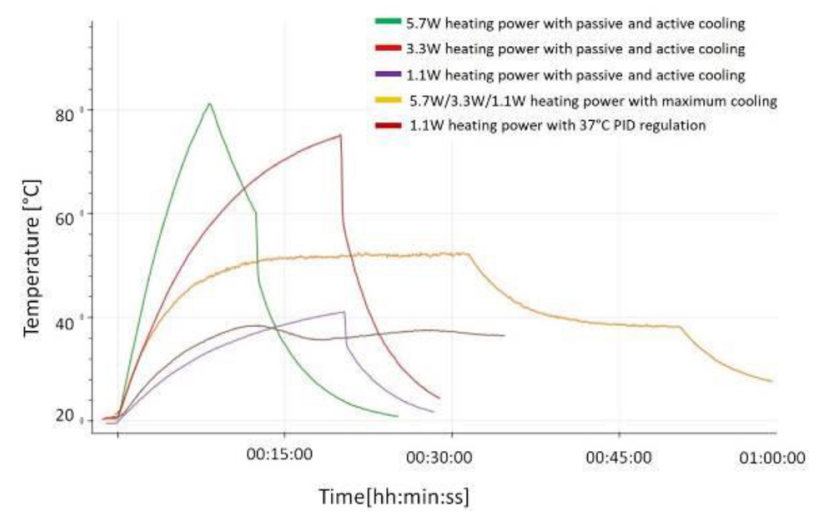

Discussion: This setup shows that it is possible to meet the standard prescribed in the ISO/TS 10974. This approach provides a more realistic way of performing gradient induced vibration measurement, which leads to more reliable measurement results. As a conclusion it is no longer necessary to distinguish between devices that have to be measured at $20{ }^{\circ} \mathrm{C}$ and those that have to be measured at $37{ }^{\circ} \mathrm{C}$, as all AIMD can be measured at body temperature in a simple manner. With the presented setup an advanced temperature control is realised for the vibration measurement of critical implants.

\section{References:}

[1] Technical specification ISO/TS 10974 www.iso.org. 


\section{S5.P13.}

\section{Designing unconventional MRI magnets using a self- trained deep neural network}

\author{
${ }^{*}$ S. Tewari ${ }^{1}$, S. Yousefi ${ }^{1}$, A. G. Webb ${ }^{1}$ \\ ${ }^{1}$ Leiden University Medical Center, Department of Radiology, Leiden, \\ $N L$
}

Introduction: Low-cost, point-of-care magnetic resonance imaging (MRI) setups are mostly built using permanent magnets and commonly use cylindrical Halbach type configurations [1]. More novel magnet design conFigurations need to be explored for designing unconventional custom-built magnets. We present here a deep neural network-based artificial intelligence (AI) model to tackle this.

Method: AI model: The deep neural network model that we propose is a modification of a standard encoder-decoder model. In the model shown in Fig. 1, the neural network in the decoder part has been replaced by an analytic equation solver. This uses an analytic expression derived for the magnetic field generated by a cubic permanent magnet [2]. We call the combination an encoder-analytic hybrid model (EA model). The encoder part is a 3D convolution neural network that takes a 3D target field as an input and generates the magnet design parameters as an output in the form of a $1 \mathrm{D}$ vector. This EA model thus does not require a dedicated training data set. It can simply learn from the analytic part of the model, which acts also as a form of regularization.

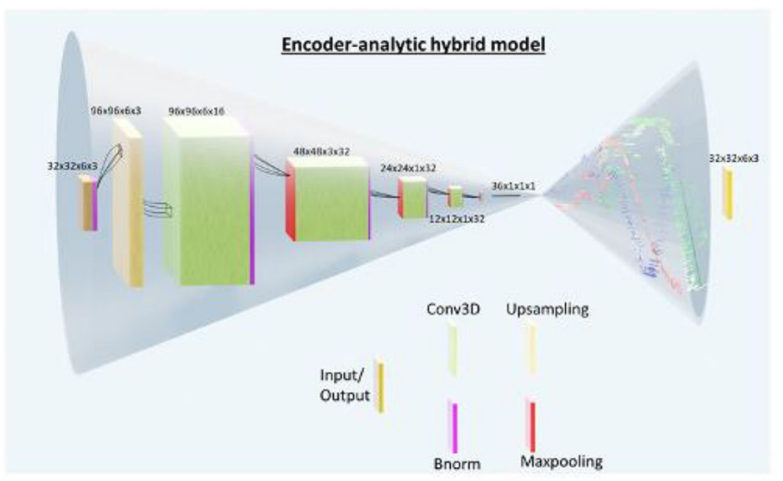

Figure 1: Encoder-analytic hybrid model. The left side of the model is the encoder which takes a 3D target vector field as input and outputs a ID position vector of length 36 for the 36 permanent magnets used here. The right side of the model is the analytic part and doesn't have any neural network inside. It takes the magnet design output of the encoder and generates a 3D vector field. which is compared with the target field and the error is propagated backwards.

Results/discussion: We put our model in a task to generate the best surface magnet design. This should have a small perfectly linear gradient $\left(G_{z}\right)$ perpendicular to the magnet surface which can be used for spatial encoding perpendicular to the surface of the magnet and at the same time the field should be perfectly homogeneous in the $\mathrm{x}-\mathrm{y}$ plane in a region of interest (ROI) defined above the magnet. The Figure of merit that is minimized here is thus inhomogeneity defined over a reduced magnetic field $\left(B_{r}=B-z G_{z}\right)$.

We start with a $6 \times 6$ grid of permanent cubic magnets and the optimization task looks to find the best z-positions for each magnet within a $\pm 5 \mathrm{~mm}$ translation (see Fig. 2). Our AI model would look for the solution by iteratively learning from the forward map of the problem (the analytic part) and fine-tuning the parameters for subsequently building the inverse map (the encoder part). For the current case, these inhomogeneity values are taken as the error which is then propagated backwards to train the encoder. The ROI of dimensions $16 \times 16 \times 6 \mathrm{~mm}^{3}$ was defined at around $30 \mathrm{~mm}$ above the zero position of the magnets. Figure 3 (a) shows the result of the inhomogeneity convergence obtained using the EA model. Figure 3 (b,c,d) provides for the best magnet design, the line plots for the $B_{x}$, $B_{y}$ and $B_{z}$ components in the ROI along the x-, y- and z-directions. We compared this result with a genetic algorithm model and found that the best magnet output of our EA model is about 3-4 times better.

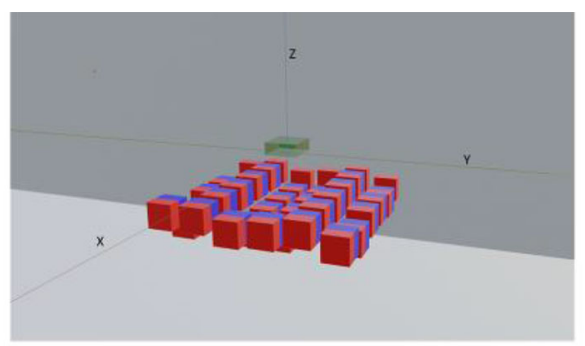

Figure 2: Surface Magnet constructed using 36 permanent cubic magnets. The blue and red color corresponds to the north and south pole respectively. The region of interest of dimension $\left(16 \times 16 \times 6 \mathrm{~mm}^{3}\right)$ is depicted with a green color.
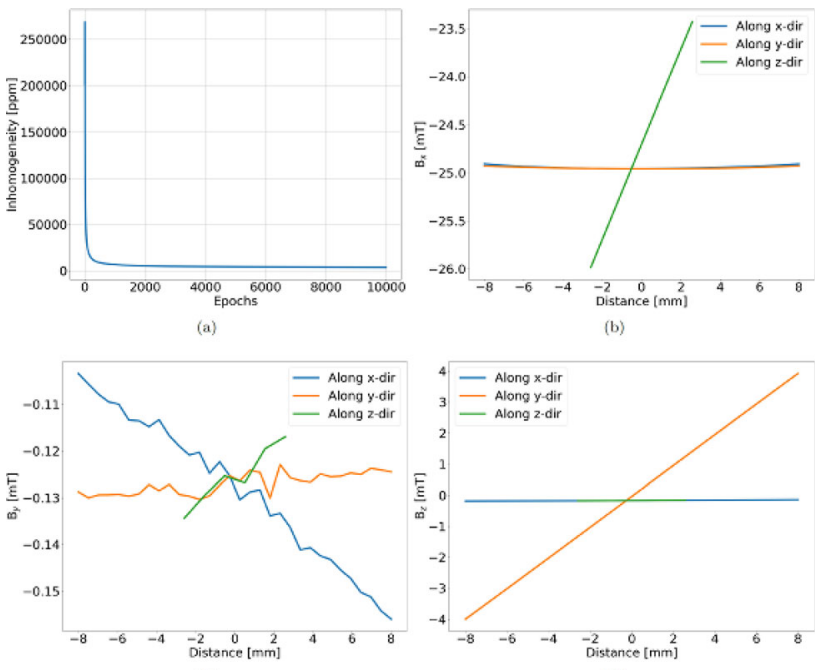

Figure 3: (a) Convergence of the inhomogeneity values in our EA model. (b.c.d) Simulation results for the best magnet: Bx, By, Bz line plots at the center of the ROI along $x$ - $y$ - and $z$-direction.

\section{References:}

[1] Sarracanie, Mathieu, and Najat Salameh. "Low-field MRI: how low can we go? A fresh view on an old debate." Front Phys 8 (2020): 172.

[2] Engel-Herbert, Roman, and Thorsten Hesjedal. "Calculation of the magnetic stray field of a uniaxial magnetic domain. " Journal of Applied Physics 97.7 (2005): 074504. 


\section{S5.P14.}

\section{A fast $0.5 \mathrm{~T}$ prepolarizer module for preclinical magnetic resonance imaging}

*J. P. Rigla ${ }^{1}$, J. Borreguero ${ }^{1}$, C. Gramage ${ }^{2}$, E. Pallás ${ }^{2}$,

J. M. González ${ }^{1}$, R. Bosch ${ }^{1}$, J. M. Algarín ${ }^{2}$, J. V. Sanchez-Andres ${ }^{3}$,

F. Galve ${ }^{2}$, D. Grau-Ruiz ${ }^{1}$, R. Pellicer ${ }^{2}$, A. Ríos ${ }^{1}$, J. M. Benlloch ${ }^{2}$,

J. Alonso ${ }^{2}$

${ }^{1}$ Tesoro Imaging S.L., Valencia, ES;

${ }^{2}$ Technical University of Valencia, MRILab / Institute for Molecular Imaging and Instrumentation (i3M), Valencia, ES;

${ }^{3}$ Jaume I University, Department of Medicine, Castellón, ES

Introduction: We present a description and the experimental test of a home-made PMRI (Prepolarized Magnetic Resonance Imaging), preclinical system designed for simultaneous visualization of hard and soft biological tissues [1] for the HISTO-MRI project [2].

Subjects/methods: The PMRI system is shown in Fig. 1. This setup is composed of a main, $\mathrm{C}$-shaped permanent magnet, a prepolarization magnet (PM), an RF coil, a cooling system for the PM, and highpower electronics. The PM is a water-cooled solenoid capable of generating a dc pulses with an intensity, $\mathrm{Bp}$, of $0.48 \mathrm{~T}$ for a drive current, IP, $255 \mathrm{~A}$. To generate the prepolarization pulses, the system uses high-power electronics based on a high-power switching module (IPM-16P from Eagle Harbor Technologies Inc.). This is driven from a battery bank consisting of 16 commercial batteries connected in pairs, each pair with both batteries connected in series. A more detailed description of this system can be found in [3]

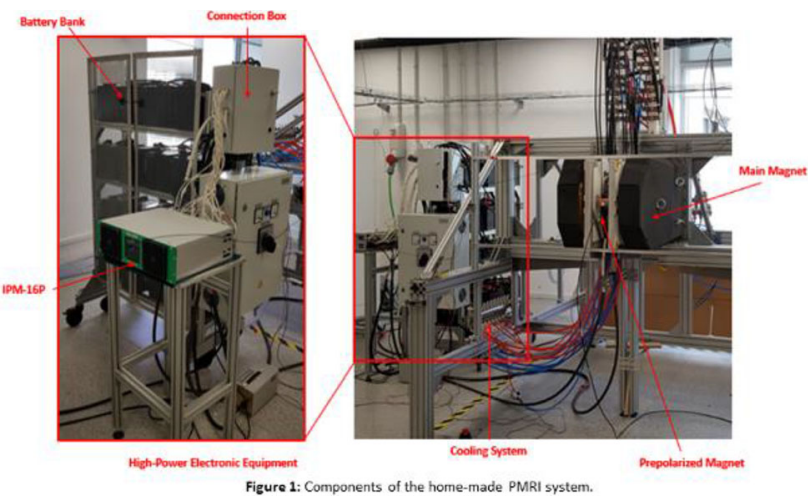

Discussion/results: Two different studies were carried out. Firstly, we demonstrated the SNR enhancement directly in the reconstructed images using the PMRI system. For this we used a mouse brain sample. The images in Fig. 2(a) and (c) are obtained using a RARE pulse sequence which a prepolarization pulse of $700 \mathrm{~ms}$ at $\mathrm{p} \approx$ $0.48 \mathrm{~T}$, applied at the beginning of a sequence repetition, and where ?dead $=40 \mathrm{~ms}$. The color scale is common to both datasets to highlight the gain in SNR when we prepolarize the sample. The SNR enhancement is evident in both sets of images. To quantify the influence of prepolarization, we plot in Fig. 2(d) the signal intensity profile along a horizontal line around the middle portion of the images in Fig. 2(a). The results shown are low pass filtered (in image space) to aid visual estimation of the SNR increase $\approx 1.72$. Secondly, we demonstrated the capacity of our system to obtain images with tissue contrast. In this case, we used a sample with different organic samples, (with different T1, see Fig. 3(b)). The images reconstructed in Fig. 3 (a) are taken for a RARE PMRI sequence with different prepolarization times. The evolution of CNRs as a function of the prepolarization pulse length is also shown in Fig. 3(c).

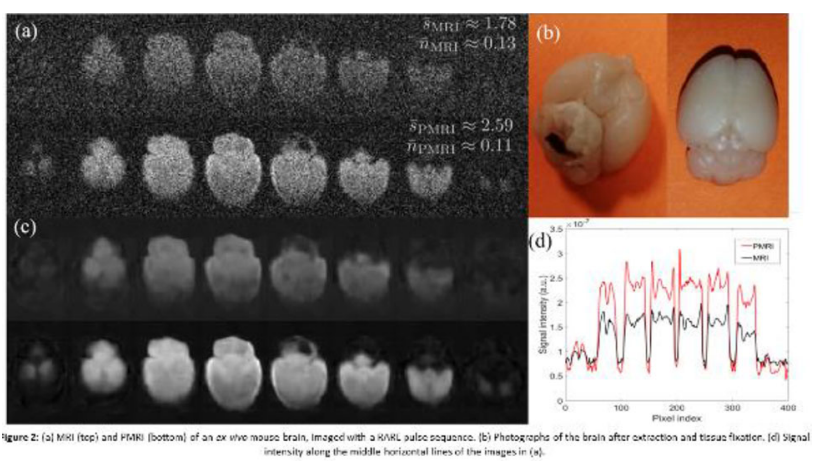

$0 \mathrm{~ms} \quad 100 \mathrm{~ms} \quad 200 \mathrm{~ms} \quad 300 \mathrm{~ms} \quad 400 \mathrm{~ms} \quad 500 \mathrm{~ms} \quad 600 \mathrm{~ms} \quad 700 \mathrm{~ms} \quad 800 \mathrm{~ms} \quad 900 \mathrm{~ms}$

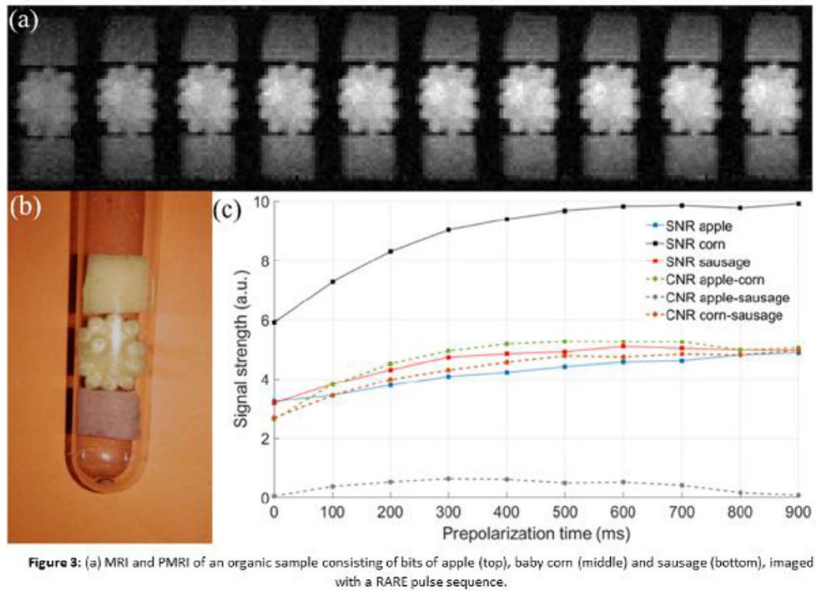

Conclusions/discussion: We have demonstrated the advantages of prepolarization on long T1samples, with a home-made PMRI system, and the observed increase in signal-to-noise ratio agrees with our models for the electronics and time evolution of the magnetization. The prepolarization pulse fall times are $\sim 10 \mathrm{~ms}$ and can be made even shorter $(<300$ us) with high power electronics under development, enabling hard tissue PMRI [3].

\section{References}

[1] J.M. Algarín et al. Doi:10.1038/s41598-020-78456-2

[2] HISTO-MRI Project, http://www.histo-mri.i3m.upv.es/

[3] J. P. Rigla et al., "A Fast 0.5 T Prepolarizer Module for Preclinical Magnetic Resonance Imaging," in IEEE Transactions on Magnetics, doi: 10.1109/TMAG.2021.3080840.

\section{S5.P15.}

\section{MaRCoS: a high-performance open-source control system for affordable MRI}

*V. Negnevitsky ${ }^{1}$, T. O’Reilly ${ }^{2}$, R. Pellicer-Guridi ${ }^{3,4}$, Y. VivesGilabert $^{3,4}$, L. Craven-Brightman ${ }^{5}$, D. Schote ${ }^{6}$, J. M. Algarín ${ }^{4}$, M. Prier ${ }^{6}$, J. Stockmann ${ }^{5}$, T. Witzel ${ }^{7}$, *B. Menküc ${ }^{8}$, J. Alonso ${ }^{4}$, A. G. $\mathrm{Webb}^{2}$

${ }^{I}$ ETH Zurich, Institute for Quantum Electronics, Zurich, $\mathrm{CH}$; ${ }^{2}$ Leiden University Medical Centre, Department of Radiology, Leiden, $N L$; 
${ }^{3}$ PhysioMRI, Valencia, ES;

${ }^{4}$ Spanish National Research Council (CSIC), MRILab/Institute for Molecular Imaging and Instrumentation (i3M), Valencia, ES; ${ }^{5}$ Massachusetts General Hospital, A. A. Martinos Center for Biomedical Imaging, Boston, MA, US;

${ }^{6}$ Otto von Guericke University, Research Campus STIMULATE, Magdeburg, DE;

${ }^{7} Q$ Bio Inc., San Carlos, CA, US;

${ }^{8}$ University of Applied Sciences and Arts Dortmund, Dortmund, DE

Unconventional MRI platforms, including low-field scanners, have seen rapid advancement toward clinical and research applications in the past few years [1]. The creation of open-source platforms has lowered the entry barrier for education, clinical and advanced research [2]. In this work we show the status of MaRCoS (MAgnetic Resonance COntrol System), an open-source MR console and software package with powerful features, that offers higher versatility than many closed-source commercial spectrometers, and show its use in several MR setups.

Figure 1 shows the MaRCoS stack. It is based on the previouslydeveloped OCRA [3], upgraded to improve its features. The core hardware is a Red Pitaya SDRLab ([4], \$600) controlling a custom gradient driver board (either [5] or [6], \$300). The SDRLab FPGA (Field Programmable Gate Array) runs the flocra firmware. Compared to OCRA, flocra uses a 'FLow-based' structure with two RF transmit and two receive channels with independent frequencies, phases, amplitudes, downmixing parameters and sampling data rates. It also provides four gradient outputs and six TTL I/Os, with a timing resolution of $8.13 \mathrm{~ns}$. The shortest time between RF, TTL or receiver events is $33 \mathrm{~ns}$, and $\sim 1$ us for gradients. Sequence length, timing and complexity have fewer restrictions than before, e.g. there are no raster clocks, and sequences with hundreds of coherent TRs, soft pulses, and arbitrary gradient waveforms have been tested. Changes to a parameter (RF properties, receiver rate, etc.) can be precisely timed. As with OCRA, this facility allows, for example, real-time alteration of sequence parameters based on inputs from external sensors.

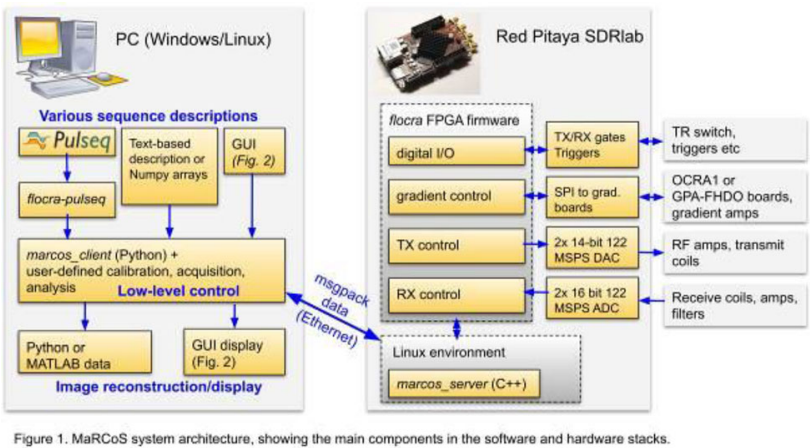

The SDRLab is controlled using Python libraries. Sequences can be written using i) Pulseq [7], a hardware-agnostic pulse sequence prototyping framework, ii) a simple domain-specific language, or iii) sets of time/value Numpy arrays. A hardware simulator and a test suite greatly simplify new feature development. Figure 2 shows a GUI that runs standard sequences, and an example TSE waveform. Figure 3 shows images acquired at three sites using MaRCoS, demonstrating the capabilities of the system for diverse and high-quality imaging.

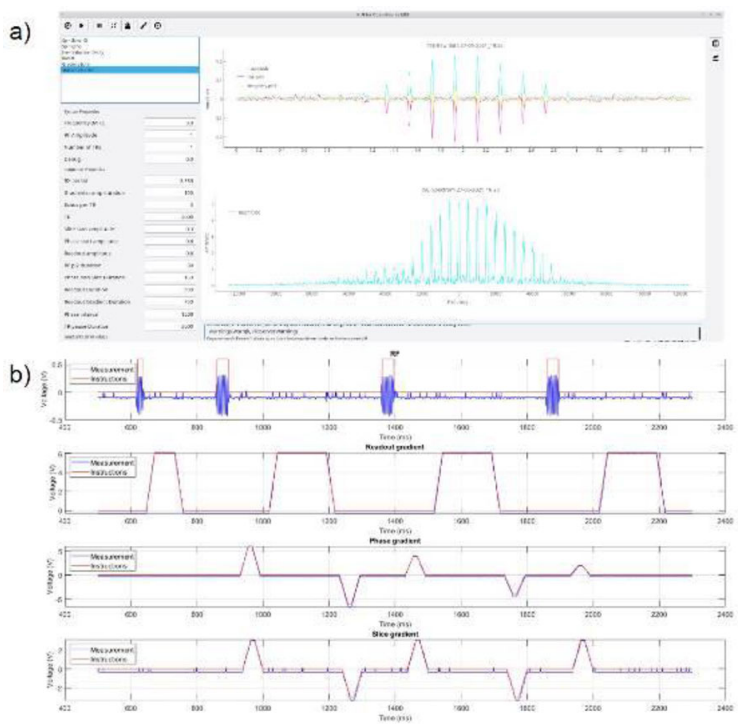

Figure 2. a) PhysioMRI GUI showing raw data for a turbo-spin-echo (TSE) sequence. b) Previewed and measured waveforms for a single TSE TR.

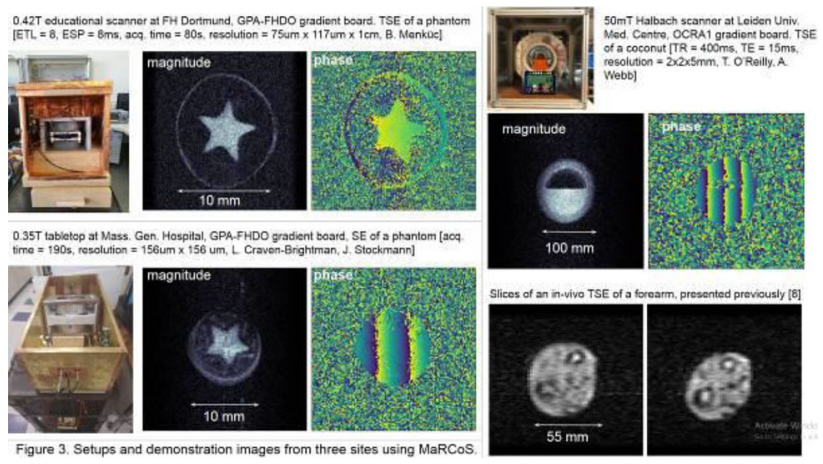

Thanks to its demonstrated performance, wide applicability to lowfield MR, accessible documentation and friendly user community [9], MaRCoS is rapidly growing in scope, with parallel multi-device operation in development. It is already able to provide much of the functionality of a modern MR spectrometer at a fraction of the cost and without the drawbacks of proprietary systems.

We acknowledge the generous contributions of Suma Anand, Hengjie Liu and Ivan Fomin.

\section{References:}

[1] 10.3389/fphy.2020.00172.

[2] 10.1002/mrm.28396.

[3] https://zeugmatographix.org/ocra.

[4] https://bit.ly/3gfOkGC.

[5] https://bit.ly/3w4Myi4.

[6] https://bit.ly/3uYrpoE.

[7] 10.1002/mrm.26235.

[8] ISMRM21, Abstract Nr. 0748.

[9] https://bit.ly/2TPLXmk. 


\section{S5.P16.}

\section{Cardiac MRI with active cardiac devices at a clinical $0.55 \mathrm{~T}$ ultra-wide-bore scanner}

\author{
*F. Santini ${ }^{1,2}$, X. Deligianni ${ }^{1,2}$, O. Bieri ${ }^{1,2}$, O. Pusterla ${ }^{1,2}$ \\ ${ }^{1}$ University Hospital Basel, Department of Radiology / Division \\ of Radiological Physics, Basel, $\mathrm{CH}$; \\ ${ }^{2}$ University of Basel, Department of Biomedical Engineering, \\ Allschwil, $\mathrm{CH}$
}

Introduction: Cardiac Magnetic Resonance Imaging (CMR) is a tool of utmost importance for the diagnosis of cardiovascular diseases. However, MR investigations of the heart are time-consuming and, thus, not feasible at all institutions. Additionally, the presence of active cardiac devices (such as pacemakers and implanted cardiac defibrillators), poses an additional safety risk in MRI, because of the potential of heating and device malfunction. For these reasons, cardiac MRI at lower field strengths has recently gained interest [1, 2]. Lower field strength scanners are potentially cheaper, and pose reduced safety concerns. The aim of this work was to assess feasibility of cardiac imaging on a novel commercial reduced-cost lowfield scanner, with ultra-wide bore size and reduced gradient performance in presence of active cardiac devices. In contrast to existing dedicated research systems, this is a commercial device that can have immediate application in the clinical routine; however, cardiac scanning on this scanner is currently not supported by the manufacturer.

Methods: A prospectively triggered balanced steady-state free precession protocol was implemented on two commercial whole-body MRI systems (MAGNETOM Free.Max, $0.55 \mathrm{~T}$, and MAGNETOM AvantoFit, $1.5 \mathrm{~T}$, Siemens, Germany) with a resolution of $1.7 \times 1.7 \times 6.0 \mathrm{~mm}^{3}$, FOV $320 \times 290 \mathrm{~mm}^{2}$, flip angle $80^{\circ}$, and parallel imaging factor 2 . TR/TE was $4.26 / 1.78$ at $0.55 \mathrm{~T}$ and $2.7 /$ $1.2 \mathrm{~ms}$ at $1.5 \mathrm{~T}$. A healthy volunteer was scanned at both field strengths in three conditions: with no cardiac device, with an MRConditional Pacemaker (PM, Identity 5386, St Jude Medical, CA), and with an MR-Unsafe Implanted Cardiac Defribillator (ICD, Lumax 540, Biotronik, Germany). The devices were placed on top of the right pectoral muscle to simulate a clinically relevant position. Two- and four-chamber views (CV) of the heart were obtained.

Results: In the no-device condition, the images at $0.55 \mathrm{~T}$ exhibited a lower signal-to-noise with respect to the $1.5 \mathrm{~T}$ images. The contrast between blood and myocardium was visible in both the 2- and 4-CV planes (Fig. 1), but not the trabeculations. In the presence of implants, the PM did not impair the evaluation of the left ventricle, whereas the ICD caused major artifacts, especially visible in the $4 \mathrm{CV}$ at both field strengths (Fig. 2).
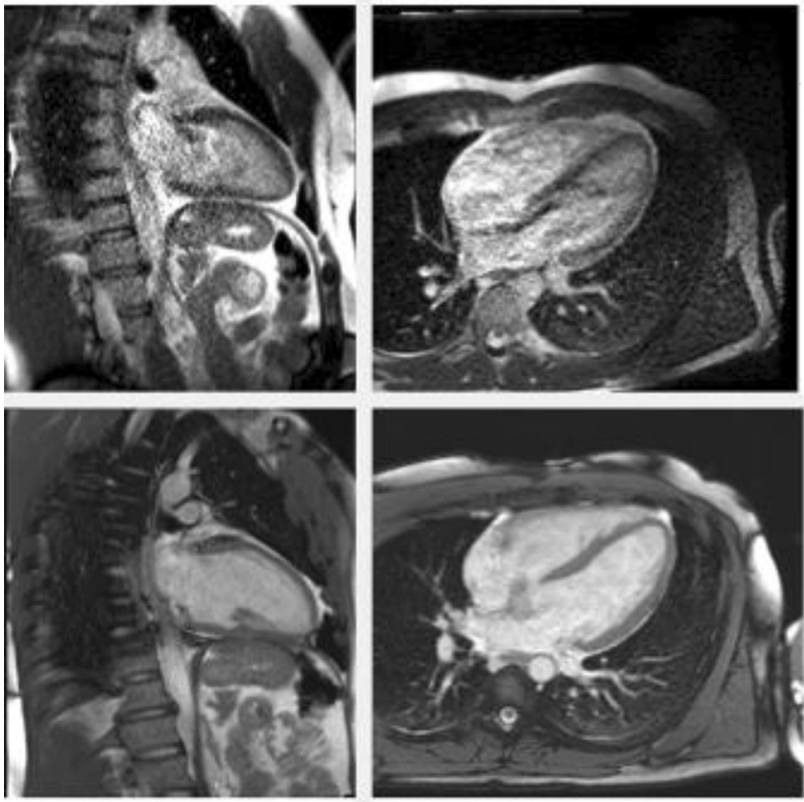

Figure 1: 2- and 4-chamber views of the heart at $0.55 \mathrm{~T}$ (top) and $1.5 \mathrm{~T}$ (bottom).
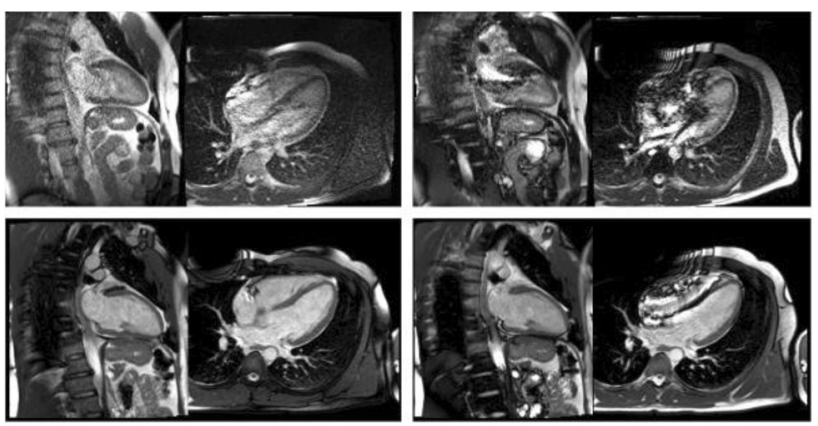

Figure 2: 2-and 4-chamber views of the heart at $0.55 T$ (top) and $1.5 T$ (bottom) with an MR-conditional pacemaker
(leff) and an MR-unsafe ICD (right)

Discussion: Despite the theoretical advantage of a lower field strength with respect to susceptibility, the larger bore size of the $0.55 \mathrm{~T}$ scanner $(80 \mathrm{~cm}$ vs $60 \mathrm{~cm})$ and the increase in TR due to reduced gradient performance made the size of the artifacts comparable at both field strengths. The $0.55 \mathrm{~T}$ system suffered from highly reduced signal-to-noise, however the delineation of the main structure was possible in normal conditions. Alternative k-space sampling strategies able to exploit the gradients more efficiently while keeping TR short (e.g. radial) are necessary for improved diagnostic performance.

\section{References}

1. Bandettini et al., JCMR 2020 2.Vishnevskiy et al., ISMRM 2021. 


\section{S6.P1.}

\section{Frequency and phase shifts correction of MR spectra using deep learning in time domain}

\author{
*A. Shamaei ${ }^{1}$, J. Starčuková ${ }^{1}$, I. Pavlova ${ }^{1}$, Z. Starčuk $\mathrm{Jr}^{1}$ \\ ${ }^{1}$ Czech Academy of Sciences Research Institute, Institute of Scientific \\ Instruments, Brno, $\mathrm{CZ}$
}

Introduction: Processing magnetic resonance spectroscopy (MRS) signals remains challenging due to hardware and physiologic processes, which may lead to frequency and phase shifts (FPS). Thus, frequency-and-phase correction (FPC) is a useful step in MRS signal processing.

Deep learning (DL) has proved to be successful in a wide range of tasks, including the MR field. DL applications in MRS have recently emerged ${ }^{1}$. It has been shown that DL can also be used for $\mathrm{FPC}^{2}$ in the frequency domain with two separated networks.

In this study, we proposed a novel deep autoencoder (DAE) network for FPC. We showed that a single DAE network could learn a nonlinear low-dimensional model to predict frequency and phase shifts. Methods: Single voxel spectroscopy in vivo 1H MRS signal was acquired from a rat's right hippocampus in a $9.4 \mathrm{~T}$ small animal MR system (Bruker BioSpec) using point resolved spectroscopy (PRESS) sequence (spectral width $=4400 \mathrm{~Hz}, 4096$ points, $\mathrm{TE}=16.5 \mathrm{~ms}$, $\mathrm{TR}=2500 \mathrm{~ms}, 256$ averages). The proposed DAE (Fig. 1) was implemented in the Pytorch framework.

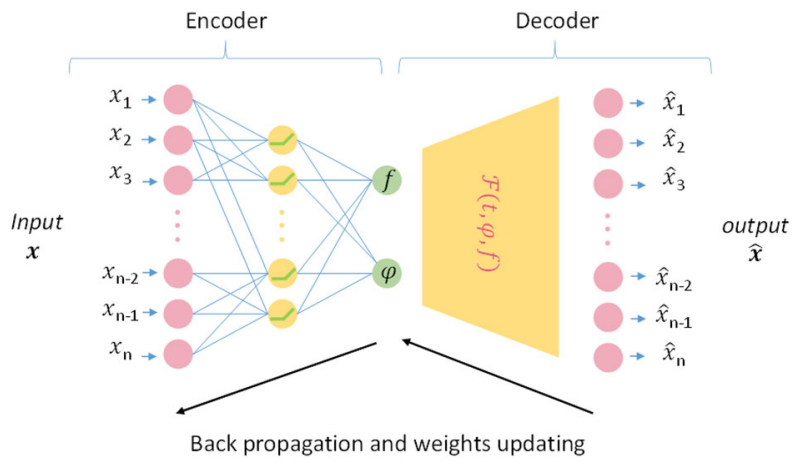

Fig 1. The DAE structure. The encoder constitutes a linear fully connected (FC) layer followed by a Relu layer and a linear FC layer. The decoder is a mathematical model $\left(\mathcal{F}(t, \varphi, \omega)=R(t) \cdot e^{(i(2 \pi f t+\varphi))}\right)$ with the input of estimated frequency $(f)$ and phase $(\varphi)$ and acquired signal $(R(t))$. The DAE was trained to encode the input vector $(\boldsymbol{x} \in \boldsymbol{X})$ into two features ( $f$ and $\varphi$ ) which can be used to reconstruct a copy of $\boldsymbol{x}$ (the output vector $(\hat{\boldsymbol{x}} \in \hat{\boldsymbol{X}})$ ) with the same dimension as $\boldsymbol{x}$. DAE is trained by minimizing the differences between the original input and the consequent reconstruction.

Training and testing set $(\boldsymbol{X})$ with 10,000 samples were created by applying uniformly distributed artificial frequency and phase offsets in the range of -20 to $20 \mathrm{~Hz}$ and $-90^{\circ}$ to $90^{\circ}$, respectively, to the acquired scan. The training was performed using 9000 samples, the mean-squared error loss, and an Adam optimizer ${ }^{3}$ with a batch size of 32 , learning rate $1 \mathrm{e}-5$, and 150 epochs.

In addition, the test dataset is processed (or corrected) using the spectral registration (SR) ${ }^{4}$ method.

Results: Figure 2 shows the error (True-DL-Predicted) against the true offset.

In the test dataset, the mean error of the frequency and phase shift correction achieved with our proposed method was $0.002 \pm 0.042 \mathrm{~Hz}$ and $-0.009^{\circ} \pm 0.254^{\circ}$, respectively, while SR correction errors were $0.048 \pm 5.5 \mathrm{~Hz}$ and $-0.471^{\circ} \pm 5.973^{\circ}$.

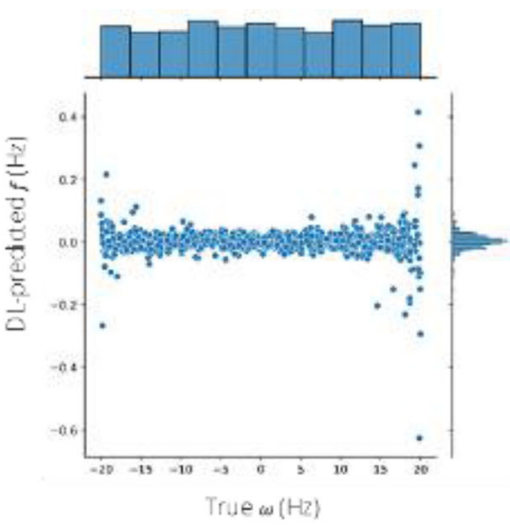

(a)

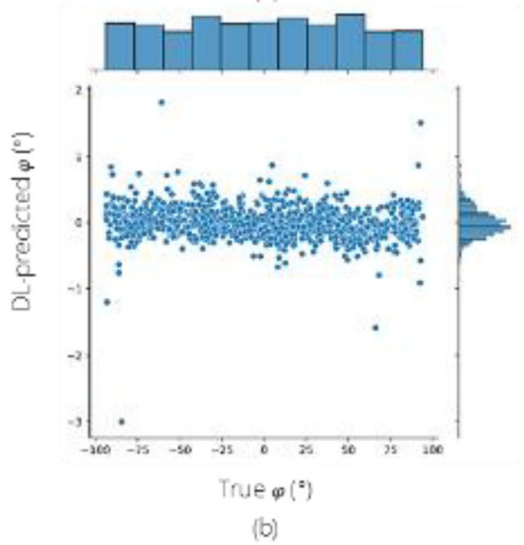

Fig. 2 Testing results. (a, b) Plots of DL-predicted frequency and phase shift against the actual shifts, respectively. Histogram plots show the distribution of random-chosen and predicted offsets.

Discussion: While the previous attempt of using DL for FPC uses two sequential networks, and the first network estimation might bias the other network estimation, our proposed DAE demonstrated a good DL potential for simultaneous FPC.

\section{References}

1. Chen et al., Chem.-A Eur. J., DOI: 10.1002/chem.202000246

2. Tapper et al., Magn. Reson. Med., DOI: $10.1002 / \mathrm{mrm} .28525$

3. Kingma et al., 3rd Int. Conf. Learn. Represent., arXiv:1412.6980

4. Near et al., Magn. Reson. Med. 2015, 10.1002/mrm.25094

Acknowledgment: EU Marie Sklodowska-Curie grant No 813120.

\section{S6.P2.}

\section{Denoising ${ }^{1} \mathrm{H}$ MR spectra in a time-frequency representation by deep learning}

\author{
${ }^{*}$ M. Dziadosz ${ }^{1}$, R. Rizzo ${ }^{1}$, S. P. Kyathanahally ${ }^{2}$, R. Kreis ${ }^{1}$ \\ ${ }^{1}$ University of Bern, Department of Radiology and Biomedical \\ Research, Bern, $\mathrm{CH}$; \\ ${ }^{2}$ Eawag, Department System Analysis, Integrated Assessment \\ and Modelling, Dübendorf, $\mathrm{CH}$
}

Introduction: MRS is limited by SNR. Long signal averaging or large voxels are often needed. Hence, numerous denoising methods were suggested: apodization, signal decomposition, wavelet features analysis or principal component analysis [RK_1]. 
However, it is disputed whether denoising can decrease estimation uncertainties or if it is just a cosmetic tool reducing noise in signalfree areas. We propose a deep learning (DL) approach for noise removal in the time-frequency domain where noise and signal is distributed in a 2D fashion before reconversion to pure 1D spectral or time domain. Here, we judge denoising performance in the relevant spectral regions with a novel score.

Methods: Human brain spectra were synthetized in VESPA (TE = $35 \mathrm{~ms}$ sLASER). Datasets consist of 16 metabolites with concentrations varying from 0 to twice the typical concentrations in healthy brain. For quantification purposes a constant water Reference is added at $0.5 \mathrm{ppm}$. To mimic in vivo conditions, uniformly varying parameters are used: shim 2-5 Hz, water SNR 5-40, macro-molecular background by $\pm 33 \%$.

Time-domain signals (4096 points, 1 s) were transformed into spectrograms using a short-time Fourier transform with window size 128 and overlap interval of 97 samples yielding a $128 \times 128$ matrix.

Figure 1 illustrates the DL model, a U-net with symmetric skip connections (adapted from 2D audio signal processing1), that used real and imaginary channels and mapped spectrograms into apparently noise-free representations.

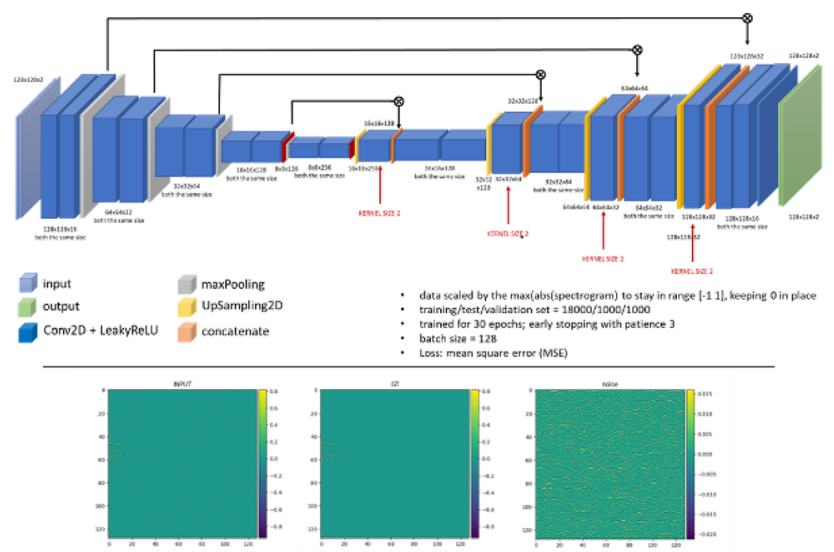

Figure 1. (UPPER) U-net network detailed architecture. (LOWER) Simulated data in the time-frequency representation (spectrograms), that consists of two channels - absorption and dispersion, each represented by a $128 \times 128$ matrix. From the left - noisy INPUT, noiseless GT and the noise (INPUT-GT).

The effect of denoising (DQ, Denoising Quality) is evaluated as a weighted and non-weighted mean absolute deviation from ground truth (GT) relative to the mean absolute deviation of pure noise. Without weighting, DQ is defined like fit quality2. Weighting with the GT spectrum makes this measure (DQw) sensitive to the spectral areas crucial for quantification. (DQ and DQw were calculated according to the equation presented in Fig. 3).

Results and discussion: A novel MRS denoising technique in timefrequency domain was implemented and performed very well as judged visually in Fig. 2.

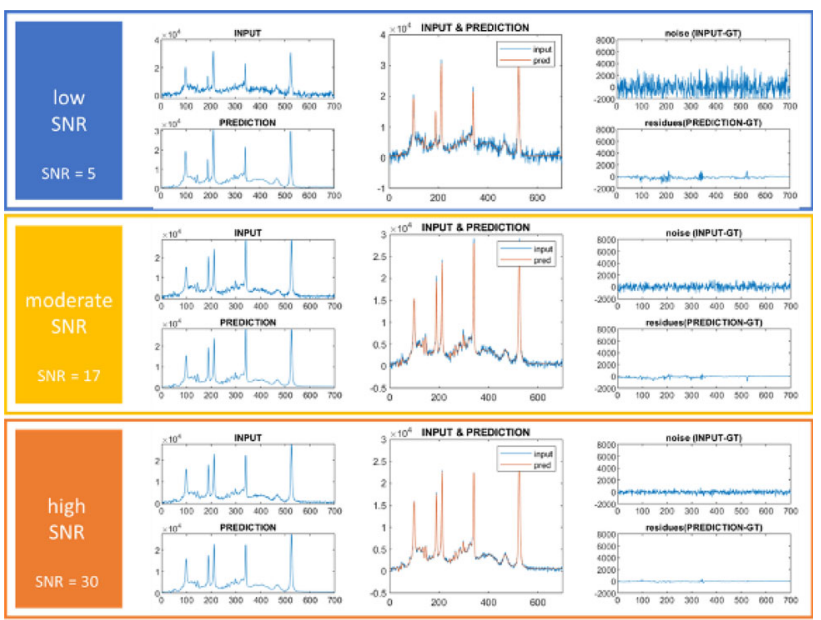

Figure 2. Representative simulated spectra (INPUT) with low (upper row), moderate (middle row) and high (lower row) SNR plotted with a respective the U-net reconstructed spectra (PREDICTION). Noisy, zoomed spectra were plotted along with the prediction and presented in the middle column. The right column presents the noise for each of the case, together with the effectiveness of denoising (residues).

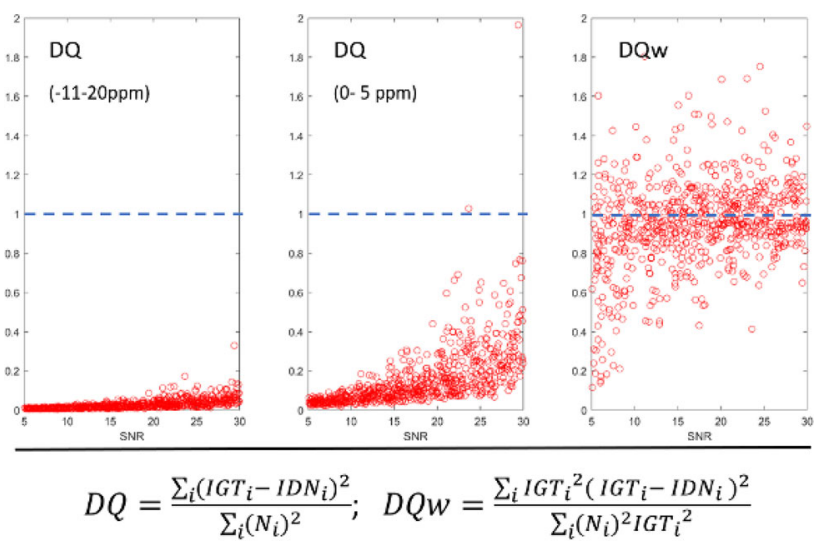

IGT, intensity of GT spectrum as function of frequency, DN: denoised, IDN intensity of DN spectrum.

Figure 3. (top) Results of denoising determined as mean absolute deviation from the GT relative to the mean absolute deviation of pure noise. Outcomes were judged by the non-weighted DQ for the full spectral range (left), spectral range restricted to metabolites area (middle) and weighted DQw (right), that have $0.04,0.24$ and 0.97 mean values, respectively. Based on the traditional error measures there is a huge denoising effect. However, to judge how well the noise was removed in the metabolites area, DQw has to be considered, which on the average is close to 1.

(bottom) Equations to calculate denoising quality as a non-weighted (DQ) and weighted (DQw) mean absolute.

Results of denoising as judged by the traditional and a novel denoising score are presented in Fig. 3 for a test set of $1 \mathrm{k}$ datasets. While DQ is close to 0 for a wide range of SNR, DQw is close to one on average, suggesting that denoising is mostly effective in non-signal containing areas. For some spectra, it is $<1$, for others $>1$.

Conclusions: We show that time-frequency domain denoising by DL is well suited for short TE MR spectra to yield visually pleasing spectra. However, the mean residuals in the spectral areas of the metabolite signals (reflected in DQw) seem to be almost as large as without denoising. Further investigations including model fitting of denoised spectra and investigations of the variance of such results are now needed for final judgment of the practical benefit of denoising in MRS. 


\title{
References:
}

1. https://github.com/vbelz/Speech-enhancement.

2. Near J, NMR Biomed. 2020; e4257.

\section{S6.P3.}

\section{Uncertainties in metabolite quantitation: CNN vs. traditional modeling}

\author{
*R. Rizzo ${ }^{1}$, M. Dziadosz ${ }^{1}$, S. P. Kyathanahally ${ }^{2}$, R. Kreis ${ }^{1}$ \\ ${ }^{1}$ University of Bern, Department of Biomedical Research \\ and Neuroradiology, Bern, $\mathrm{CH}$; \\ ${ }^{2}$ Eawag, Department System Analysis, Integrated Assessment \\ and Modelling, Dübendorf, $\mathrm{CH}$
}

Introduction: Quantification in spectroscopy is traditionally based on model fitting. Prior knowledge, fitting boundaries and choice of algorithms represent crucial steps that introduce dependencies on users and/or fit packages. Deep Learning (DL) has introduced the possibility to speed up quantitation. However, questions arise in regards to how to access uncertainties. Here, a preliminary comparison of accuracy metrics between the two methods is explored using simulated datasets with known ground truth (GT).

Methods: Datasets with 16 metabolites were simulated. Concentrations vary independently between 0 and twice a normal reference concentration from literature. A constant downscaled water reference is added at $0.5 \mathrm{ppm}$ to ease quantitation. Macro-molecular background (MMBG), shim and SNR mimic in vivo acquisitions and were independently and uniformly varied (time domain water referenced SNR 5-40, shim 2-5 Hz, MMBG amplitude 1-2), Fig. 1.

a)

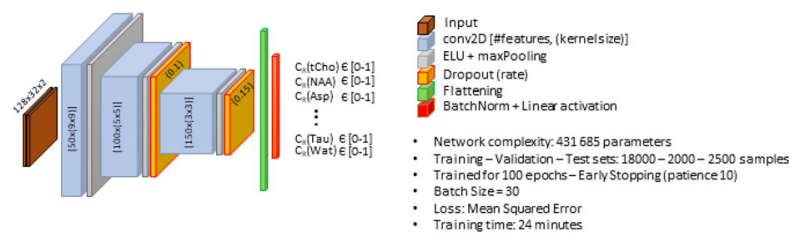

b)

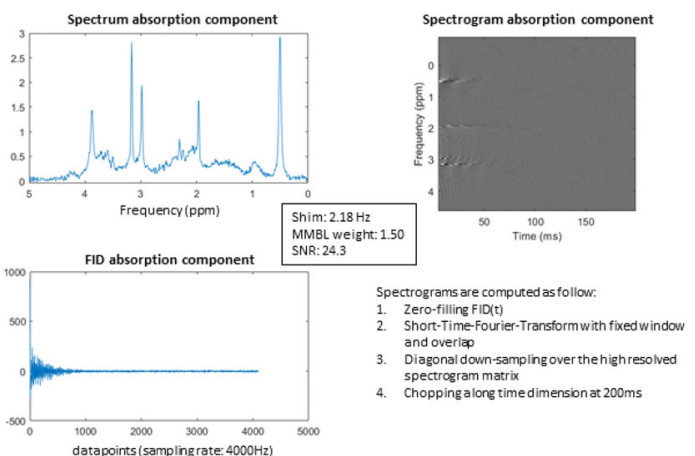

Fig.1: a) Shallow $\mathrm{CNN}$ architecture. b) Absorption components for a sample in training dataset. CNN input consists of a 2 channel $128 \times 32$ matrix. Channels consist of absorption and dispersion perts

A shallow Convolutional Neural Network $(\mathrm{CNN})^{1}$ uses spectrograms ${ }^{2}$ calculated via an in-house script as input, Fig. 1. Relative concentrations are provided as output. Referencing to an estimated water content, absolute concentrations can be evaluated.

A simulated spectrum of a typical median acquisition scenario (SNR, shim) was taken as reference and fitted. Cramer Rao Lower Bounds (CRLBs) were used as uncertainty reference for the comparison.
Results:

- CNN predicts well-represented singlets and characteristic metabolites of human brain spectra well whereas it is less accurate on predicting low-SNR coupled metabolites. However, CNN accuracy resembles for most cases CRLBs of a median spectrum, Fig. 2.

a)
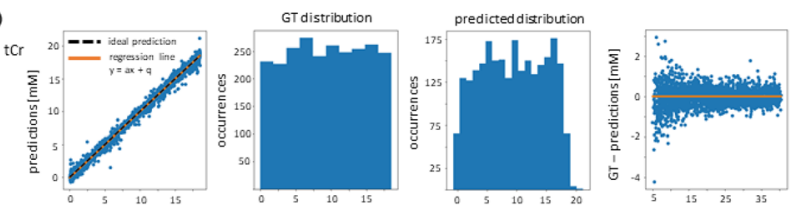

Gln
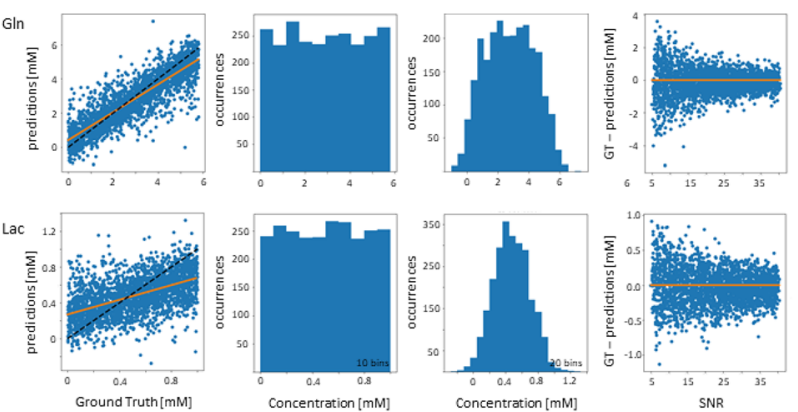

b)

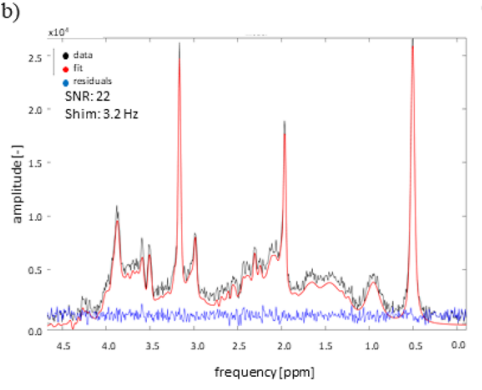

\begin{tabular}{c|ccc|cc} 
& \multicolumn{3}{|c|}{$\mathrm{CNN}$} & \multicolumn{2}{c}{ fitting } \\
\hline & $\mathrm{a}$ & $\mathrm{R}^{2}$ & mse & area & CRLB \\
\hline tCho & 0.98 & 0.99 & 0.11 & 4.30 & 0.08 \\
NA.AG & 0.87 & 0.86 & 0.21 & 2.16 & 0.32 \\
NAA. & 0.99 & 0.99 & 0.55 & 13.10 & 0.34 \\
Asp & 0.51 & 0.41 & 0.61 & 2.87 & 0.44 \\
tCr & 1.00 & 0.99 & 0.35 & 2.57 & 0.14 \\
GABA & 0.25 & 0.13 & 0.41 & 0.77 & 1.05 \\
Glc & 0.88 & 0.87 & 0.15 & 0.81 & 0.20 \\
Glu & 0.96 & 0.96 & 0.86 & 11.53 & 1.08 \\
Gln & 0.81 & 0.77 & 0.60 & 0.80 & 0.94 \\
GSH & 0.80 & 0.74 & 0.22 & 0.14 & 0.40 \\
Gly & 0.56 & 0.45 & 0.35 & 1.36 & 0.76 \\
Lac & 0.41 & 0.29 & 0.20 & 0.91 & 0.52 \\
mI & 0.96 & 0.97 & 0.52 & 9.34 & 0.62 \\
PE & 0.52 & 0.44 & 0.57 & 3.01 & 0.88 \\
sI & 0.67 & 0.61 & 0.08 & 0.20 & 0.18 \\
Tav & 0.49 & 0.39 & 0.36 & 1.53 & 0.72 \\
\hline
\end{tabular}

Fig.2: a) CNN prediction plots for 3 sampled metabolites respectively stressing different goodness of predictions compared to the ground truth (GT). Distributions of prediction skew towards Gaussian output whereas SNR < 15 yields higher errors. b) Fit of a typical spectrum. c) Table with summarized results. a: slope of regression line, $R^{2}$ : coefficient of determination, mse: mean squared error. area: fitted metabolite areas, CRLBs: Cramer-Rao Lower Bounds. CNN shows comparable uncertainties to typical CRLBs.

- Low concentrations tend to be overestimated, high concentrations underestimated.

- CNN uncertainties scale proportionally with $1 / \mathrm{SNR}$, i.e. noise.

Discussion:

- Optimized spectrogram representations are found to be well suited for spectral quantification. Comparison with otherwise used onedimensional $\mathrm{CNNs}^{3}$ is needed.

- DL has recently shown comparable uncertainties to traditional fitting ${ }^{4}$, but is faster and user-friendlier.

- Ideally, CNN or traditional estimations should be unbiased, thus return uniform distributions for uniform test data. However, our CNN predictions tend towards the mean of the training data, in particular for weakly represented metabolites. Intrinsic uncertainties can explain this phenomenon: predictions at the boundaries of the training range are folded back towards the mean value in case of strong uncertainty, given lack of knowledge outside the boundary. Stronger regularization, longer training and deeper network architecture do not reduce this effect substantially.

- Active learning with training data stressing difficult cases may improve predictions. 
- Evaluating and respecting fitting uncertainties is equally crucial for DL and traditional approaches.

\section{References:}

1 Espi, J AUDIO SPEECH MUSIC PROC, 2015, 26.

2 Kyathanahally, MRM 80:851.

3 Lee, MRM 84:1689.

4 Landheer, NMR Biomed 2021:e4521.

\section{S6.P4.}

\section{D-CNN for grading of meningiomas using proton magnetic resonance spectroscopy}

*B. Sacli-Bilmez ${ }^{1}$, A. Bas ${ }^{1}$, A. Ersen Danyeli ${ }^{2,3}$, C. Yakıcıer ${ }^{3,4}$, M. N. Pamir ${ }^{3,5}$, K. Ozduman ${ }^{3,5}$, A. Dincer ${ }^{3,6}$, E. Ozturk-Isik ${ }^{1}$

${ }^{1}$ Boğaziçi University, Biomedical Institute, Istanbul, TR;

${ }^{2}$ Acıbadem Mehmet Ali Aydinlar University, Department of Medical Pathology, Istanbul, TR;

${ }^{3}$ Acibadem Mehmet Ali Aydinlar University, Center

for Neuroradiological Applications and Reseach, Istanbul, TR;

${ }^{4}$ Aclbadem Mehmet Ali Aydinlar University, Department of Molecular

Biology and Genetics, Istanbul, TR;

${ }^{5}$ Aclbadem Mehmet Ali Aydinlar University, Department of Neurosurgery, Istanbul, TR;

${ }^{6}$ Aclbadem Mehmet Ali Aydınlar University, Department

of Radiology, Istanbul, TR

Introduction: Meningiomas are the most common adult brain tumors [1]. The 5-year recurrence rate in grade I meningiomas is $5 \%$ while this rate is $40 \%$ in grade II (atypical) meningiomas [2, 3]. Furthermore, grade III (anaplastic) meningiomas have the worst overall survival rate of up to 2 years [3]. Therefore, tumor grading plays a significant role in the prognosis and treatment planning of meningiomas [4]. This study aims to develop a one-dimensional convolutional neural network (1D-CNN) to determine the grade of meningiomas based on single-voxel proton magnetic resonance spectroscopic (1H-MRS) data.

Methods: Fifty-seven meningioma patients $(15 \mathrm{M} / 42 \mathrm{~F}$, mean age: $52.55 \pm 13.22$ years, range: $18-80$ years, 20 WHO grade I, 37 higher-grade ( 34 WHO grade II and 3 WHO grade III)) were included in this study. The patients were scanned at pre-surgery time points using a $3 \mathrm{~T}$ clinical MR scanner (Siemens Healthcare, Erlangen, Germany) with a 32-channel head coil. 1H-MRS was obtained from a manually placed region of interest with no necrosis, edema, and hemorrhage, using a Point Resolved Spectroscopy (PRESS) sequence $(\mathrm{TR} / \mathrm{TE}=2000 / 30 \mathrm{~ms})$. Fitted spectra obtained by LCModel [5] were used in a 1D-CNN model to classify the grades of meningiomas. Preprocessing steps including L2 normalization, smoothing (Savitzky-Golay filter ( window size $=11$, order $=2$ ), Yeo-Johnson power transformation, and min-max normalization were applied on the input data before classification [6]. Optuna [7] was employed for hyperparameter tuning of the models with 50 trials. ADASYN was used to overcome the imbalanced dataset problem. Table 1 shows the hyperparameters of the proposed model. The model architecture and details are shown in Fig. 2.
Fig. 1
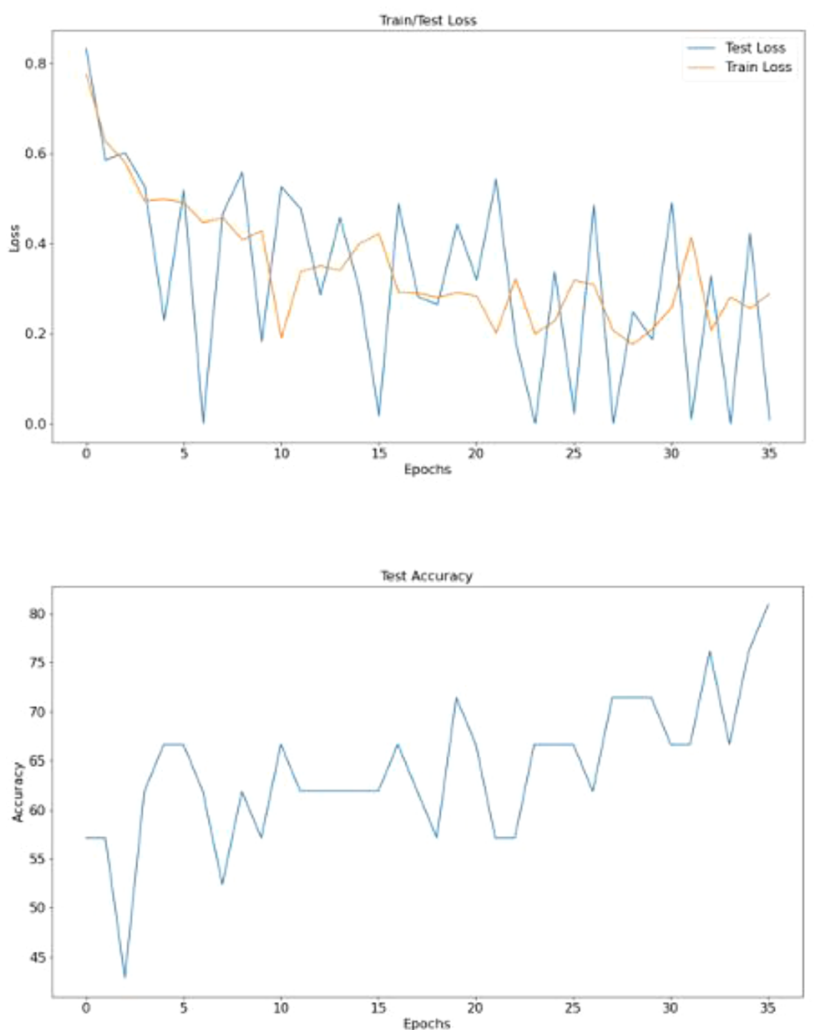

Results: Fig. 1 shows the train and test losses and the test accuracy of the proposed model for 36 epochs. The accuracy of the proposed model was $80.95 \%$ (specificity $=83.33 \%$, sensitivity $=77.78 \%$ ).

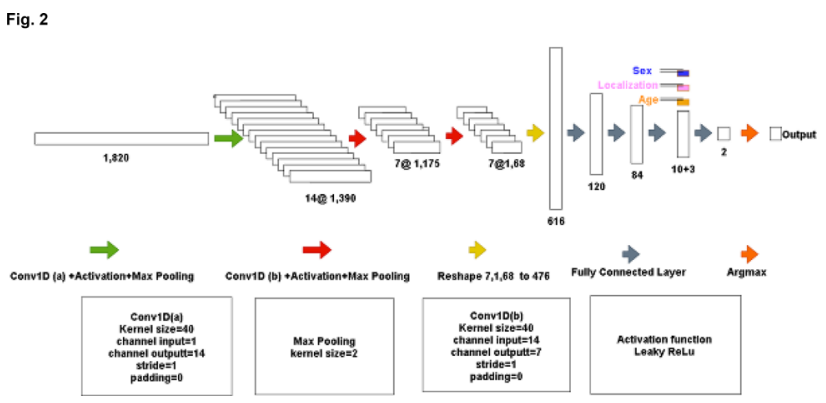




Fig. 3
\begin{tabular}{ll} 
Table 1. The hyperparameters of the proposed $1 \mathrm{D}-\mathrm{CHN}$ model. \\
\hline Hyperparameters & Values \\
\hline Classes & $\begin{array}{l}\text { WHO Grade I (20) vs WHO } \\
\text { Grade II-III(34-3) }\end{array}$ \\
Epochs & 36 \\
Optimizer & RMSProp \\
Activation Function & Leaky ReLu \\
Batch Size (Training/Test) & $10 / 10$ \\
Learning Rate & 0.00436
\end{tabular}

Conclusion and discussion: This study proposed a 1D-CNN model for grading meningiomas based on 1H-MRS. Our results indicated that $1 \mathrm{H}-\mathrm{MRS}$ provides relevant features to the proposed deep learning model for meningioma grading. Future studies will be conducted to verify our results in a larger patient cohort.

Acknowledgments: This study has been supported by TUBITAK 1001 grant 119 S520.

References:

1. 2018 Research Project Summaries-American Brain Tumor Association. (2021). Retrieved 8 June 2021, from https://www.abta. org/2018-research-project-summaries/

2. Perry, A., et al., The American Journal of Surgical Pathology, 1997. 21(12).

3. Perry, A., et al., Cancer, 1999. 85(9): p. 2046-2056.

4. Zhao et al. Neurosurgical Review, 2015.38(1):p.101-107.

5. Provencher. NMR Biomed, 2001.14(4):p.260-4.

6. Engel et al. TrAC Trends in Analytical Chemistry,2013(50):p.96-106.

7. Akiba et al., Proc of the 25th ACM SIGKDD International Conference on Knowledge Discovery\&Data Mining, 2019:p.2623-2631.

\section{S6.P5.}

\section{An alternative non-linear least squares method for fitting 2D T2-T2 relaxation-exchange data}

\section{*E. Caldas de Almeida Araujo ${ }^{1}$, P. Y. Baudin ${ }^{1}$, H. Reyngoudt ${ }^{1}$, B. Marty $^{1}$ \\ ${ }^{1}$ Institute of Myology, Neuromuscular Investigation Center, Paris, FR}

Introduction: In skeletal muscle, the multi-exponential behavior of T2 relaxation seems to reflect histological compartmentation [1]. Nonetheless, in order to assess compartments' intrinsic properties from the 1D T2 spectra, knowledge of the compartmental exchange rates is needed. 2D relaxation-exchange methods could provide such information [2].

Inversion of $2 \mathrm{D}$ relaxation data into $2 \mathrm{D}$ spectra is an ill-posed problem. Although stable solutions can be obtained via regularized Inverse Laplace Transform (rILT) [3], these are penalized by relatively low spectral resolution. In this work we propose a non-linear least squares (NLLS) approach and confront it to the conventional rILT method on simulated data.

Methods: Analytical solutions of the Bloch-McConnell equations were used in order to simulate signals from a CPMG-storage-CPMG sequence (Fig. 1) for 4 bi-compartment systems, characterized with distinct compartment sizes $\left(m_{a}{ }_{a}=90\right.$ or $\left.80 \%\right)$, residence times ( $\tau_{a}=750$ or $500 \mathrm{~ms}$; equilibrium condition: $m^{0}{ }_{a} / \tau_{a}=m^{0}{ }_{b} / \tau_{b}$ ) and fixed intrinsic $\mathrm{T} 1$ and $\mathrm{T} 2$ values $\left(T_{1 a} / T_{1 b}=1250 / 1650, T_{2 a} / T_{2 b}=30 /\right.$ $190 \mathrm{~ms}$ ). The sequence (Fig. 1) was parametrized as follows: IES =
$1 \mathrm{~ms}, \mathrm{n}_{2}=100, \mathrm{n}_{1}=1 \ldots 100 \mathrm{~ms}$ in 16 logarithmically spaced steps, and $\mathrm{TS}=10 \ldots 500 \mathrm{~ms}$ in 15 logarithmically spaced steps. This resulted in a $16 \times 1002 \mathrm{D}$ relaxation data per TS value. Five replicates of each $15 \times 16 \times 100$ data set were built by adding complex white noise $(\mathrm{SNR}=104)$ and taking the absolute value.

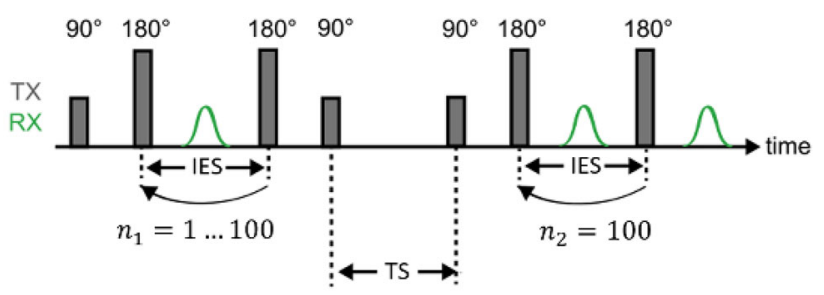

Fig. 1. Pulse sequence for the T2-T2 exchange relaxometry experiment.

T2-T2 spectra were obtained from rILT [4] of each 2D relaxation data, and the relative fractions of each peak were calculated. Each 2D relaxation data was also fitted, via NLLS, to the following model:

$$
\begin{aligned}
\mathrm{S}\left(n_{1}, n_{2}\right)= & P_{11} \exp \left(-\left(\mathrm{n}_{1}+\mathrm{n}_{2}\right) \times \mathrm{IES} / T_{21}\right) \\
& +P_{22} \exp \left(-\left(\mathrm{n}_{1}+\mathrm{n}_{2}\right) \times \mathrm{IES} / T_{22}\right)+P_{12} \\
& \times\left(\exp \left(-\left(\mathrm{n}_{1} / T_{21}+\mathrm{n}_{2} / T_{22}\right) \times \mathrm{IES}\right)\right. \\
& \left.+\exp \left(-\left(\mathrm{n}_{1} / T_{22}+\mathrm{n}_{2} / T_{21}\right) \times \text { IES }\right)\right)(1)
\end{aligned}
$$

Where $P_{11}, P_{22}, P_{12}, \mathrm{~T}_{21}, T_{22}$ are the adjustable variables; $P_{11}$ and $P_{22}$ are the fractions of magnetization that did not exchange during TS (diagonal peaks in the 2D spectra), $P_{12}$ represents the fraction that did exchange (identic off-diagonal peaks in the $2 \mathrm{D}$ spectra), and $T_{21}$ and $T_{22}$ are the apparent $\mathrm{T} 2$ values.

Finally the intrinsic parameters, $m_{a}^{o}, T_{2 a}, T_{2 b}$ and $\tau_{a}$ were estimated by fitting the experimental curves for the peaks" areas as functions of TS, obtained from both methods (rILT and NLLS), to a theoretical model derived from the analytical solutions of the Bloch-McConnell equations (see Fig. 2).
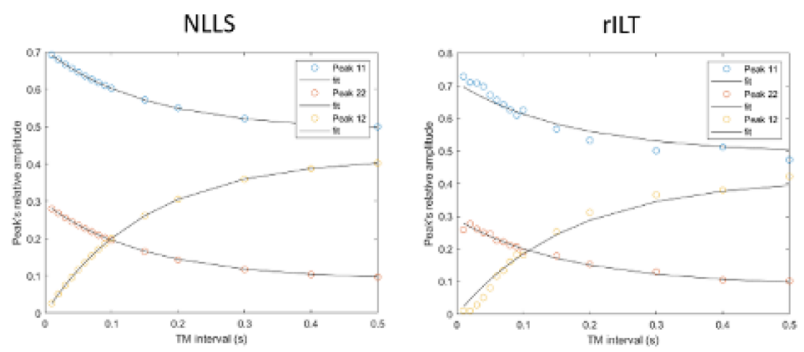

Fig. 2. Example of plots of the T2 components's fractions as functions of TS, as estimated using either the non-linear least squares method (NLLS) or by integration of the 2D T2-T2 spectra obtained from regularized inverse Laplace Transform (rILT). Data was fitted by adjusting the compartments' intrinsic parameters.

Results: The results obtained with the NLLS method showed much higher accuracy and precision then the conventional rILT method (see Table 1). 
Fig 3

Table 1. Estimated compartments' intrinsic parameters for each simuated system, obtained using the conventional rlLT and the proposed NLLS methods

\begin{tabular}{|c|c|c|c|c|c|}
\hline & & $\tau_{a}(\mathrm{~ms})$ & $T_{2 a}(\mathrm{~ms})$ & $T_{2 b}(\mathrm{~ms})$ & $m_{a}^{0}(\%)$ \\
\hline System 1 & & 750 & 30 & 190 & 90 \\
\hline \multirow{2}{*}{ rILT } & mean & 757,6 & 28,4 & 230 & 88,2 \\
\hline & stdDev & 27 & 0,5 & 44,7 & 0,8 \\
\hline \multirow{2}{*}{ NLLS } & mean & 747,8 & 30 & 188,2 & 90 \\
\hline & stdDev & 2 & 0,0 & 0,8 & 0,0 \\
\hline \multicolumn{2}{|l|}{ System 2} & 750 & 30 & 190 & 80 \\
\hline \multirow{2}{*}{ rILT } & mean & 853,2 & 31 & 250 & 82,2 \\
\hline & stdDev & 14 & 0,0 & 0,0 & 0,8 \\
\hline \multirow{2}{*}{ NLLS } & mean & 749,8 & 30 & 189,8 & 80 \\
\hline & stdDev & 0 & 0,0 & 0,4 & 0,0 \\
\hline \multicolumn{2}{|l|}{ System 3} & 500 & 30 & 190 & 90 \\
\hline \multirow{2}{*}{ rILT } & mean & 454 & 30 & 190 & 87,2 \\
\hline & stdDev & 57 & 0,0 & 54,8 & 2,6 \\
\hline \multirow{2}{*}{ NLLS } & mean & 498,6 & 30 & 189 & 90 \\
\hline & stdDev & 3 & 0,0 & 1,7 & 0,0 \\
\hline \multicolumn{2}{|l|}{ System 4} & 500 & 30 & 190 & 80 \\
\hline \multirow{2}{*}{ rILT } & mean & 520,4 & 30 & 230 & 79,2 \\
\hline & stdDev & 14 & 0,0 & 44,7 & 0,4 \\
\hline \multirow{2}{*}{ NLLS } & mean & 499,2 & 30 & 189,6 & 80 \\
\hline & stdDev & 0 & 0,0 & 0,5 & 0,0 \\
\hline
\end{tabular}

Discussion: When one can reasonably assume the number of compartments in the compartmental model, a non-linear fit of $2 \mathrm{D}$ relaxation data to the proposed model (Eq. 1) provides more reliable results than the conventional rILT method.

\section{References}

1. Araujo et al. Biophys J. 106 (2014) 2267-2274.

2. Dortch et al. J Chem Phys. 131 (2009) 164502.

3. Whittall and MacKay. J Magn Reson. 84 (1989) 134-152.

4. Venkataramanan et al. IEEE Trans Signal Process. 50 (2002) 1017.

\section{S6.P6.}

\section{Radiomics analysis of the hippocampus in a transgenic rat model of Alzheimer's disease}

\section{E. Sorribes Torrent ${ }^{1}$, G. Piella ${ }^{1}$, G. Soria ${ }^{2}$, *E. Muñoz-Moreno ${ }^{3}$ \\ ${ }^{1}$ Universitat Pompeu Fabra (UPF), Department of Simulation, Imaging and Modelling for Biomedical Systems, Barcelona, ES, ${ }^{2}$ University of Barcelona, Institute of Neurosciencies, Barcelona, ES; ${ }^{3}$ Institut d'Investigacions Biomèdiques August Pi i Sunyer (IDIBAPS), Magnetic Resonance Imaging Core Facility, Barcelona, ES}

Introduction: Animal models of Alzheimer"s disease (AD) are essential in the research of the pathology and potential treatments. TgF344-AD rats progressively show all AD pathological hallmarks [1]. In this work, radiomics was applied to classify between wild-type (WT) and transgenic (Tg) animals at early and advanced stages of the disease. This can provide a translational approach to identify imaging biomarkers and predict $\mathrm{AD}$ progression.

Methods: 30 Tg344-AD rats and 34 WT littermates underwent MRI on a $7 \mathrm{~T}$ Bruker scan at different ages each from 3 to 18 months, resulting in 194 images. MRI included: T1-weighted images $($ MDEFT, $\mathrm{TE}=2 \mathrm{~ms}, \mathrm{TR}=4 \mathrm{~s}$, voxel size $=0.14 \times 0.14 \times 0.5$ $\mathrm{mm}^{3}$ ) and T2-weighted images (RARE, TE $=35.3 \mathrm{~ms}, \mathrm{TR}=6 \mathrm{~s}$, voxel size $=0.12 \times 0.12 \times 0.8 \mathrm{~mm}^{3}, 40$ slices $)$.
Hippocampus was segmented in $\mathrm{T} 2-\mathrm{w}$ images by automatic registration of a rat brain atlas as described in [2] and translated to T1-w images by affine registration. T1-w images were preprocessed (isotropic voxel interpolation, intensity normalization and discretization) and 107 intensity, shape, and texture radiomics features of the hippocampus were extracted by pyradiomics [3]. To reduce dimensionality, features with extremely high or low variance and highly correlated features were discarded, and the most relevant ones for classification were selected. Machine learning was applied to the selected features to classify into 4 groups: adult $(<1$ year-old) WT, adult $\mathrm{Tg}$, aged ( $>1$ year-old) WT and aged $\mathrm{Tg}$, to differentiate early pathological from healthy brain and from advanced pathology. The dataset was split into training-validation (142 images) and test (52 images) sets. Machine learning was trained on the training-validation set with a $\mathrm{k}$-fold $(\mathrm{k}=10)$ cross-validation scheme and different models to find the most accurate. The accuracy of the selected model was assessed in the test set.

Results: Six features resulted from the selection step (Table 1) and linear Discriminant Analysis was the selected model to classify. Figure 1 shows the confusion matrix in the test dataset. The classification between $\mathrm{Tg}$ and control animals was $69 \%$ accurate. It achieved $78 \%$ if only aged rats were considered, but it was lower $(64 \%)$ in the no-aged animals. In the images correctly identified as $\mathrm{Tg}$, the accuracy to classify between aged and adult $\mathrm{Tg}$ rats was $75 \%$.

Fig. 1

Table 1. Radiomics features selected for classification $\left(\mathrm{P}_{10}: 10^{\text {th }}\right.$ percentile of voxel intensity; $\mathrm{X}$ : voxel intensity; $\mu_{\mathrm{x}}, \mu_{\mathrm{y}}$ : mean gray level intensity of the marginal row and columns probabilities of the gray level co-ocurrence matrix respectively; $p$ : normalized coocurrence matrix; $p_{i}$ : gray level probability in the neighborhood; $s_{i}$ : sum of absolute differences for gray-level $i ; \mathrm{P}$ : dependence matrix)

\begin{tabular}{|l|l|l|l|}
\hline Intensity & First order statistics & 10 th percentil & $\mathbf{P}_{10}$ \\
\cline { 3 - 4 } & & Minimum & $\min (\mathbf{X})$ \\
\cline { 3 - 4 } & Energy & $\sum_{i=1}^{N_{y}} \mathbf{X}(i)^{2}$ \\
\hline Texture & $\begin{array}{l}\text { GLCM: Gray Level } \\
\text { Co-ocurrence Matrix }\end{array}$ & Cluster Shade & $\sum_{i=1}^{N_{y}} \sum_{j=1}^{N_{y}}\left(i+j-\mu_{x}-\mu_{y}\right)^{3} p^{3}(i, j)$ \\
\cline { 2 - 4 } & $\begin{array}{l}\text { NGTDM: } \\
\text { Neighbouring Gray } \\
\text { ToneDifference } \\
\text { Matrix }\end{array}$ & Complexity & $\frac{1}{N_{p, p}} \sum_{i=1}^{N_{9}} \sum_{j=1}^{N_{g}}|i-j| \frac{p_{i} s_{z}+p_{j} s_{j}}{p_{i}+p_{j}}$ \\
\cline { 2 - 4 } & $\begin{array}{l}\text { GLDM: Gray Level } \\
\text { Dependence Matrix }\end{array}$ & $\begin{array}{l}\text { Large } \\
\text { dependence } \\
\text { emphasis }\end{array}$ & $\frac{\sum_{i=1}^{N_{g}} \sum_{j=1}^{N_{d}} \mathrm{P}(i, j) j^{2}}{N_{z}}$ \\
\hline
\end{tabular}

Fig. 2

Figure 1. Confusion matrix of the classifier performance on the test dataset.

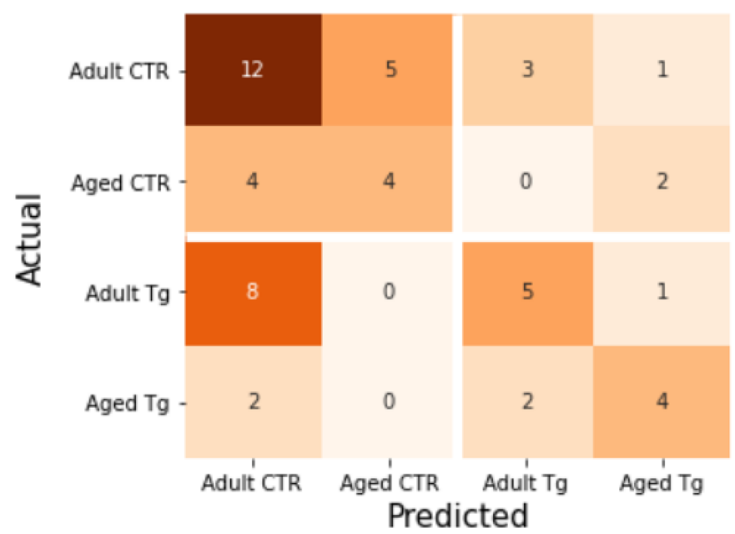


Discussion: Radiomics has shown promising results in AD patients [4] but to the best of our knowledge, this is the first time it is applied to an animal model of $\mathrm{AD}$. Although $\mathrm{Tg}$ aged rats, where frank pathology is expected, were more accurately identified, promising results were also obtained in early ages. Age was considered as indicator of disease stage, but more accurate individual assessment could result in better classification.

\section{References:}

1 Cohen RM, et al. J Neurosci. 2013 33:6245-56.

2 Muñoz-Moreno, E et al. Alz Res Therapy 2018 10:16.

3 Griethuysen JJM et al. Cancer Research, 2017 77:e104-7.

4 Feng Q et al. Curr Alzheimer Res. 2020 17:297-309.

\section{S6.P7.}

\section{Classification of low- and high-grade meningiomas using radiomics features of post-contrast T1-weighted MRI}

\author{
*E. Sümer ${ }^{1}$, M. N. Pamir ${ }^{2}$, A. Dinçer ${ }^{2}$, K. Özduman ${ }^{2}$, E. Ozturk-Isik ${ }^{1}$ \\ ${ }^{I}$ Boğaziçi University, Institute of Biomedical Engineering, Istanbul, \\ $T R$; \\ ${ }^{2}$ Acibadem Mehmet Ali Aydinlar University, School of Medicine, \\ Istanbul, TR
}

Introduction: Meningiomas are the most frequent tumors of the central nervous system that account for $38 \%$ of primary brain tumors. Meningiomas are classified into benign (Grade I), atypical (Grade II), and malignant (Grade III) meningiomas based on their histological features [1]. Grade I, or low-grade meningiomas (LGMs) are associated with a better prognosis than Grade II/III, or high-grade meningiomas (HGMs), which have invasive characteristics and rapid progression [2]. Radiomics extract a large number of quantitative and minable features from medical images, and have potential for noninvasive meningioma grading. The aim of this study is to investigate the potential of radiomics features calculated from contrast-enhanced (CE) T1-weighted (T1-w) for grade prediction in meningiomas.

Methods: Thirty-three low-grade (Grade I) and 43 high-grade (Grade II/III) meningioma patients (mean $( \pm$ std) age $=50.36 \pm 12.9$ years, age range $=[27,80]$, male/female: $30 / 46)$ were included in this study. Post-contrast (gadolinium) T1-w MRI data $(\mathrm{TR} / \mathrm{TE}=415 / 10 \mathrm{~ms}$ ) were acquired using a $3 \mathrm{~T}$ clinical MR scanner (Siemens Healthcare, Erlangen, Germany). The workflow of the study is given in Fig. 1. First, the CE tumor volumes were manually segmented using 3D Slicer. To minimize the effect of inhomogeneity between acquisitions, images were $\mathrm{z}$-score normalized and resampled to the same resolution $(1 \times 1 \times 1 \mathrm{~mm})$. Then, a full set of 1132 features were extracted using PyRadiomics [3]. Feature selection followed by collinear feature elimination was conducted using Gini index and Pearson"s correlations $(r> \pm 0.9)$. The relationships between radiomics features and meningioma grades were assessed using Mann-Whitney U test. Machine learning algorithms were applied to classify LGMs and HGMs using tenfold cross-validation with 50 repetitions.

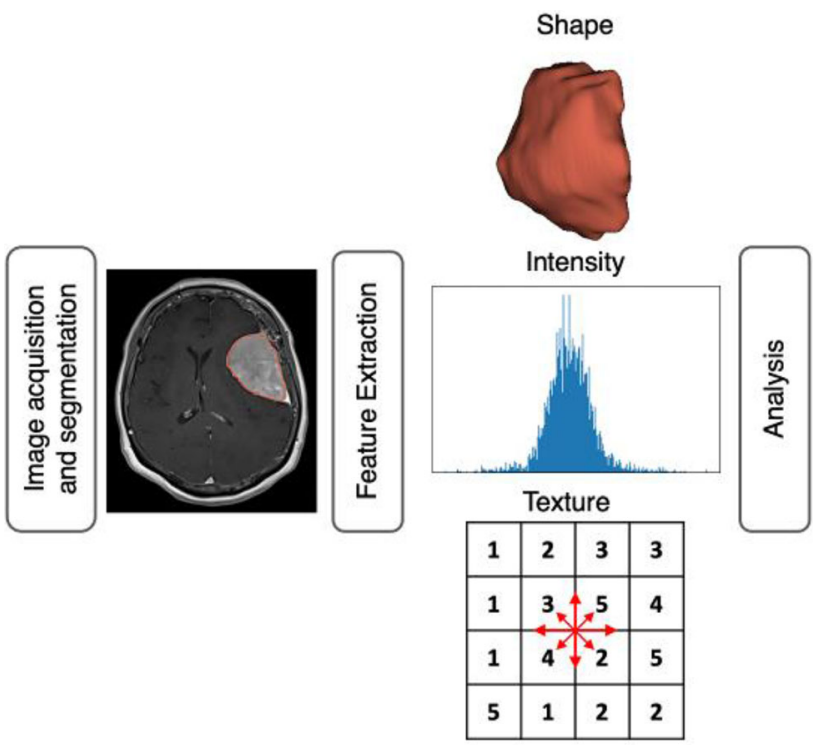

Fig. 1. The workflow of this study.

Results: The feature elimination process resulted in nine predictive features for differentiating LGMs from HGMs $(P<0.005$ for all $)$ (Fig. 2). The best performance for grading meningiomas was obtained with a gradient boost classifier (loss function: deviance, learning rate: $0.1)$ yielding an accuracy of $0.76 \pm 0.043(0.82 \pm 0.06$ sensitivity and $0.69 \pm 0.07$ specificity).

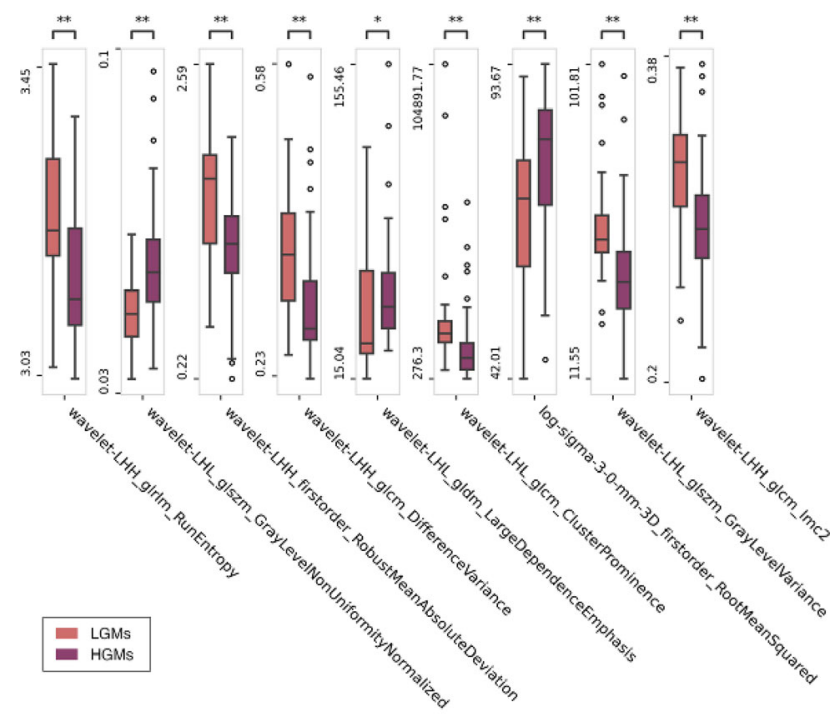

Fig. 2. The most informative radiomic features for differentiating LGMs and HGMS. ( $\left.{ }^{*} P: 0,005, * * *: 0.0005\right)$

Discussion: The results of this study indicated that conventional CE T1-w texture radiomics could predict meningioma grade. The heterogeneous signal enhancement of CE T1-w MRI indicates tumor heterogeneity due to intratumoral hemorrhage, cystic degeneration, and necrosis [4]. Thereby, texture analysis of CE T1-w MRI could provide quantitative information about the tumor signal intensity patterns that are difficult for the human eye to perceive.

Acknowledgments: This study was supported by The Scientific and Technological Research Council of Turkey (TUBITAK) grant \#119S520.

\section{References:}

[1] Louis et al., Acta Neuropathol. 131, 803 (2016). 
[2] Wang et al., Sci. Rep. 6, 1 (2016).

[3] van Griethuysen et al., Cancer Res. 77, e104 (2017).

[4] Hu et al., Eur. J. Radiol. 131, 109251 (2020).

\section{S6.P8.}

\section{Assessment of the stability of radiomic shape features of T2-weighted images of meningiomas}

*E. Sümer ${ }^{1}$, A. Arpak ${ }^{1}$, M. N. Pamir ${ }^{2}$, K. Özduman ${ }^{2}$, A. Dinçer ${ }^{2}$, E. Ozturk-Isik ${ }^{1}$

${ }^{I}$ Boğaziçi University, Institute of Biomedical Engineering, Istanbul, $T R$;

${ }^{2}$ Acibadem Mehmet Ali Aydinlar University, School of Medicine, Istanbul, TR

Introduction: Shape-based radiomic features, like sphericity index, have been associated with overall patient survival [1]. However, 3D representation of tumors might lack natural look and smoothness (staircase artifact) due to the anisotropic voxel sizes in MRI, which might potentially compromise the morphological measurements of segmented tumors. This study aims to assess the effect of staircase reduction methods on shape features of T2-weighted MRI of meningiomas.

Methods: Fourteen radiomic shape-based features were extracted from T2-weighted MRI of 19 patients with meningiomas. All segmentations $(0.21 \times 0.21 \times 3.6 \mathrm{~mm}(N: 13), 0.26 \times 0.26 \times 6.5 \mathrm{~mm}$ $(N: 2), 0.24 \times 0.24 \times 6.5 \mathrm{~mm}(N: 1), 0.43 \times 0.43 \times 3.6 \mathrm{~mm}(N: 3))$ were resampled to the same resolution of $1 \times 1 \times 1 \mathrm{~mm}$. The first method (M1) directly extracted shape-based radiomic features, whereas the second method (M2) applied median smoothing with kernel size set to the original slice thickness (Fig. 1). On the other hand, the third strategy (M3) used morphological contour interpolation [2] followed by median smoothing. Then, several shape features were calculated using PyRadiomics [3]. A Friedman test followed by Wilcoxon post-hoc test was used to determine differences in extracted features. For Friedman test, the cut-off $P$ value was 0.0036 due to Bonferroni multiple comparisons, and for Wilcoxon post-hoc test a $P$ value of 0.05 was considered statistically significant. The influence of smoothing and interpolation strategies was assessed by intraclass correlation coefficients (ICC) and confidence intervals (CI).
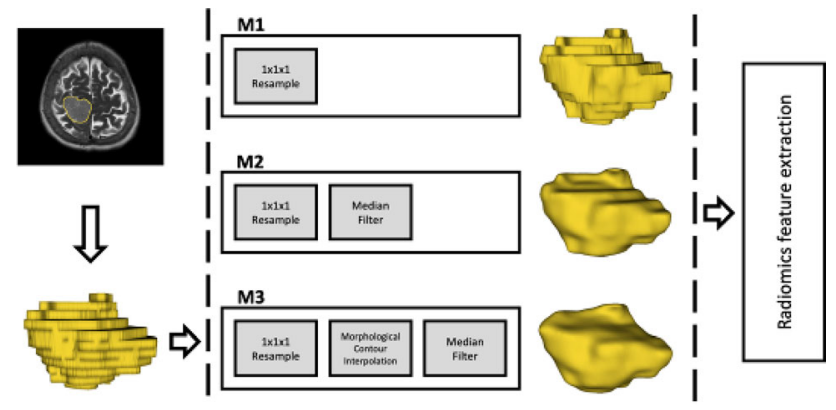

Fig, 1. The three different pre-prosessing apposaches (M1, M2 and Mi3) foe radionics feature extraction.

Results: M1 had the highest surface area and surface to volume ratio $(P<0.01$ for all) (Fig. 2). In contrast, M1 had the lowest sphericity index $(P<0.0001$ for all). M1 resulted in higher maximum 2D diameter column and maximum 3D diameter than both M2 and M3 $(P<0.01$ for all). On the other hand, M2 had a significantly lower maximum 2D diameter slice, mesh volume, and voxel volume than the other approaches $(P<0.05$ for all). M1 showed a significantly higher minor axis length than M2 $(P<0.001)$. The remaining five measures did not significantly differ between the methods $(P>0.004)$. The stability of features was excellent for $13 / 14$ radiomic features (ICC $>0.9$ ), but not for the sphericity index $(\mathrm{ICC}=0.82, \mathrm{CI}=0.17,0.95)($ Fig. 3).
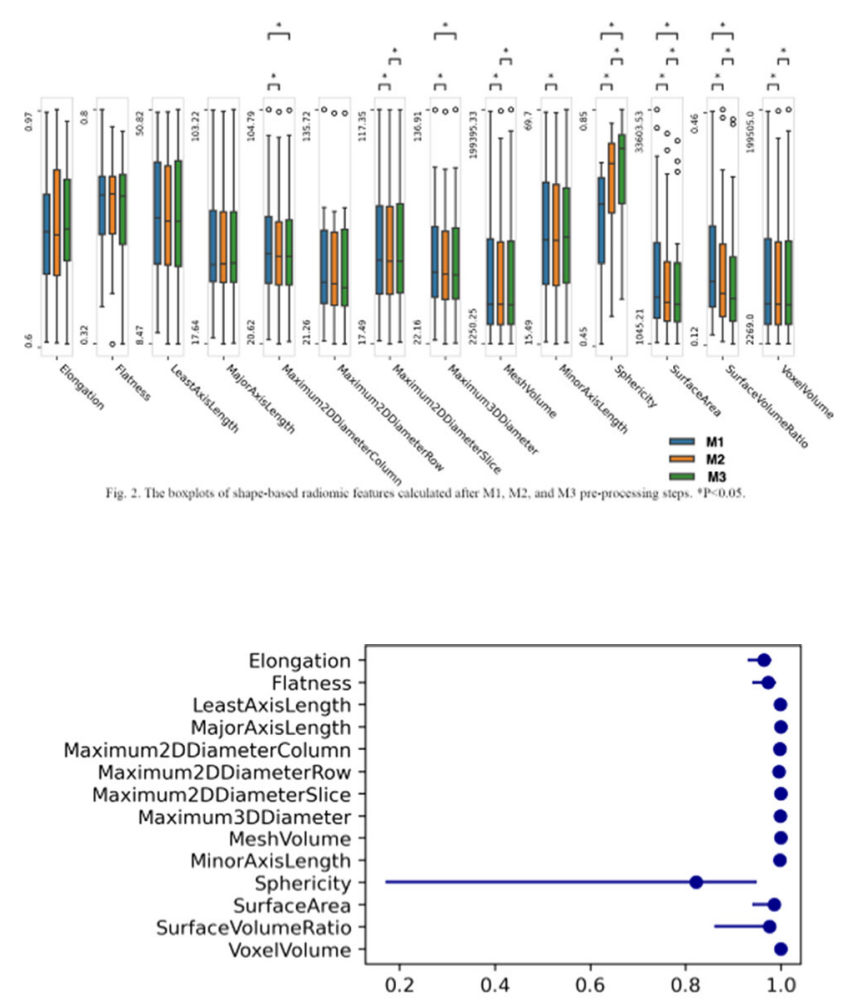

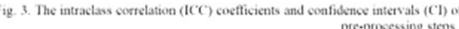

Discussion: The shape features calculated after M1, M2, M3 preprocessing approaches were similar in intensities except for the sphericity index, which was highly affected by the pre-processing approach. Our results indicated that the shape-based radiomic features were generally stable across different smoothing and interpolation strategies.

Acknowledgments: This study was supported by The Scientific and Technological Research Council of Turkey (TUBITAK) grant \#119S520.

\section{References:}

[1] O. Morin et al., Neuro-oncology Adv., 1, 1, 2019.

[2] A. B. Albu et al., IEEE Trans. Biomed. Eng., 55, 8, 2008.

[3] J. J. M. Van Griethuysen et al., Cancer Res., 77, 21, 2017.

\section{S6.P9.}

The effect of MRI signal intensity normalization for radiomics analysis on PCNSL patients

*M. Destito ${ }^{1}$, P. Zaffino ${ }^{1}$, A. Marzullo ${ }^{2}$, R. Leone ${ }^{3}$, E. De Momi ${ }^{4}$, N. Anzalone ${ }^{3}$, F. Calimeri ${ }^{2}$, S. Steffanoni ${ }^{5}$, T. Calimeri ${ }^{5}$, A. J. Ferreri ${ }^{5}$, M. F. Spadea ${ }^{1}$

${ }^{1}$ University Magna Graecia of Catanzaro, Department of Experimental and Clinical Medicine, Catanzaro, IT;

${ }^{2}$ University of Calabria, Department of Mathematics and Computer Science, Cosenza, IT; 
${ }^{3}$ Vita-Salute San Raffaele University, Department of Neuroradiology, Milan, IT;

${ }^{4}$ Polytechnic University of Milan, Department of Electronics, Information and Bioengineering, Milan, IT;

${ }^{5}$ San Raffaele Scientific Institute, Department of OncoHematology, Milan, IT

Introduction: Primary central nervous system lymphoma (PCNSL) is a rare form of highly-aggressive lymphoma. Magnetic Resonance Imaging (MRI) is widely performed for the diagnosis, staging and response assessment. Radiomics extracts quantitative features from medical images to improve diagnosis, prognostic and personalization of therapy in the oncology field. One of the limitations of radiomics in MRI concerns the reproducibility of the extracted features. MRI intensities are not standardized, and they strongly depend on the type of scanner, contrast sequence and acquisition parameters [1]. Herein, we report a study focused on the effect of signal intensity normalization on the reproducibility of radiomics features extracted from MRI scans acquired in different centres and with different scanners. Methods: Twenty-three patients scanned with T1 weighted (T1w) MRI in 3 different time points were selected from the PCNSL Institutional Database of San Raffaele Hospital (Milan). All images were pre-processed as follows: bias field correction, skull stripping, intensity normalization and voxels resampling [2]. Z-score, WhiteStripe and Nyul [1] normalization methods were applied for MRI intensities normalization. In the region of the pons, where no pathological modifications were seen, a sphere of $1 \mathrm{~cm}$ of diameter was created. This choice was made to estimate the sole effect of normalization, thus avoiding any possible disease-specific signal intensity modification. Radiomic features were extracted using the Pyradiomics library; morphological features were not included in the intensity normalization analysis. The Interclass Correlation Coefficient (ICC) was calculated to evaluate the reproducibility of each feature for both original and normalized images [3]. Kruskal-Wallis test and its post hoc were used to compare the ICCs obtained with different normalization methods.

Results: Figure 1 shows median \pm quartiles of ICCs computed on both original and normalized images. The highest ICC was obtained for features extracted from images normalized by Z-score, with a significant improvement $(p<10-9)$ of about $30 \%$ on average if compared to original images.

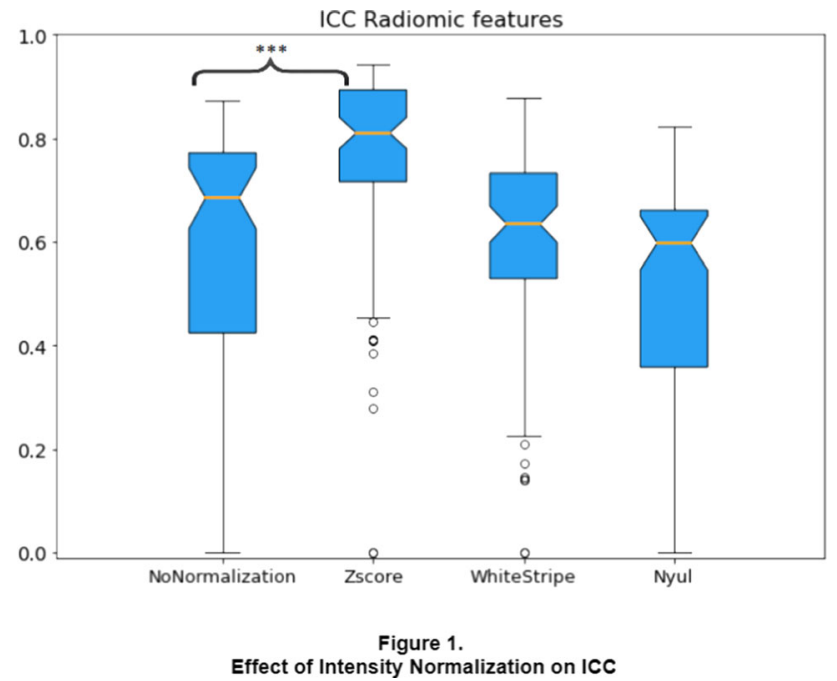

Discussion: Intensity normalization in T1w MRI has a significant impact on the extracted features. Z-score method increases the reproducibility of radiomic features. A larger image study is required to confirm these preliminary results and to better investigate the effect of normalization for prognostic radiomics studies of PCNSL.

\section{References:}

[1] Carré et al. Standardization of brain MR images across machines and protocols: bridging the gap for MRI-based radiomics. Sci Rep 10 (2020).

[2] Moradmand et al. "Impact of image preprocessing methods on reproducibility of radiomic features in multimodal magnetic resonance imaging in glioblastoma." Journal of applied clinical medical physics (2020).

[3] Scalco et al. "T2w-MRI signal normalization affects radiomics features reproducibility". Med Phys (2020).

\section{S6.P10.}

\section{Using transfer learning for IDH mutation prediction in gliomas using whole brain diffusion anisotropy indices}

*H. Halilibrahimoğlu ${ }^{1}$, A. Kaykayoğlu ${ }^{2}$, A. Baş ${ }^{1}$, K. Özduman ${ }^{3,4}$, C. Yakıcıer ${ }^{5}$, A. Ersen Danyeli ${ }^{4,6}$, M. N. Pamir ${ }^{4}$, A. Dinçer ${ }^{4,7}$, E. Öztürk-Iş1k ${ }^{1,4}$, A. Özcan ${ }^{4,8}$

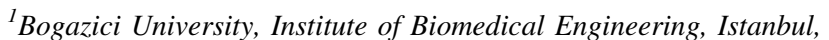
$T R$;

${ }^{2}$ Aclbadem Mehmet Ali Aydinlar University, Department of Medical Engineering, Istanbul, TR;

${ }^{3}$ Aclbadem Mehmet Ali Aydinlar University, Department

of Neurosurgery, Istanbul, TR;

${ }^{4}$ Aclbadem Mehmet Ali Aydinlar University, Center

for Neuroradiological Applications and Research, Istanbul, TR

${ }^{5}$ YoctoSensum Biotechnoogy, Istanbul, TR;

${ }^{6}$ Aclbadem Mehmet Ali Aydinlar University, Department of Medical Pathology, Istanbul, TR;

${ }^{7}$ Acıbadem Mehmet Ali Aydinlar University, Department of Radiology, Istanbul, TR;

${ }^{8}$ Bogaziçi University, Department of Electrical and Electronics Engineering, Istanbul, TR

Introduction: IDH mutation in gliomas is an important genetic biomarker, associated with longer overall survival [1, 2]. Deep learning modalities have been studied to predict IDH with anatomical magnetic resonance imaging (MRI) [3, 4]. In this study, a pre-trained ResNet network was used to predict the IDH mutation status of glioma patients from whole-brain volumetric diffusion anisotropy indices (DAIs) maps.

Methods: 96 patients $(43 \mathrm{~F} / 53 \mathrm{M}$, age: $45.38 \pm 15.28)$ with written informed consent, diffusion tensor imaging (DTI) and IDH mutation (50MUT/46WT) data were included in this IRB approved study. DTI $(\mathrm{TE} / \mathrm{TR}=89.1 / 7733 \mathrm{~ms}, 1.8 \times 1.8 \times 1.8 \mathrm{~mm}, 60$ slices, $\mathrm{b}=1000 \mathrm{~s} /$ $\mathrm{mm}^{2}, 20$ directions) was acquired on a $3 \mathrm{~T}$ TimTrio scanner (Siemens Healthineers, Erlangen, Germany) before surgery. IDH mutation was identified using Sanger sequencing with an Applied Biosystems ${ }^{\mathrm{TM}}$ 3500 Series Genetic Analyzer. DAIs (apparent diffusion coefficient (ADC), fractional anisotropy (FA), relative anisotropy (RA)) were calculated from diffusion tensor eigenvalues[5]. For preprocessing first and last 5 slices of DAI maps were removed. Volumetric ADC, FA, and RA data were assigned to one of the 3 input channels of the deep learning model. No other preprocessing was performed. Data were split into training and test sets retaining $90 \%$ of data in the training set. Then the training set was further split into a training and validation set retaining $90 \%$ of the data in the training set. Pre-trained ResNet networks with varying depths (ResNet34, ResNet50, ResNet100) were used for IDH mutation prediction [6,7]. The last 5 
layers of each network were fine-tuned for 100 epochs to volumetric whole-brain DAI maps, with Adam optimizer (learning rate $=10-4$ ) using TensorFlow [8]. Google Colab GPU environment was used to train the networks.

Results: The training and validation accuracy of each model are shown in Fig. 1, and the performance on the test set is shown in Table 1. In the test set, ResNet-34 trained for 100 epochs predicted IDH status best with $70 \%$ accuracy (AUC: 0.87 ).
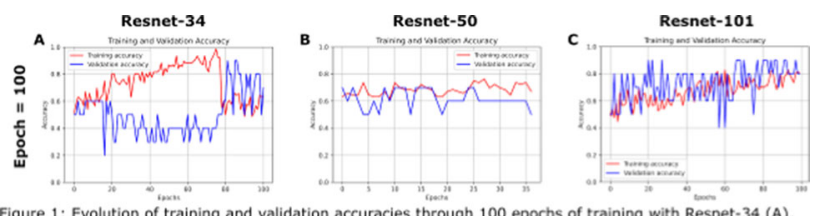
Figure 1: Evolution of training and

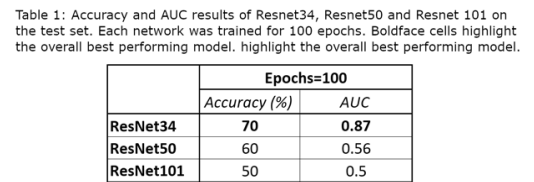

Discussion: This study demonstrates the potential of transfer learning with ResNet in predicting IDH mutation from whole-brain DAI maps. However, the relatively small cohort size led to overfitting. Furthermore, optimizing model depth may decrease the difference between training and validation accuracies.

Acknowledgement: This work was funded by The Scientific and Technological Research Council of Turkey (TUBITAK) 1003 Grant No: $216 \mathrm{~S} 432$.

\section{References:}

[1] Yan et al. N Engl J Med 2009 Feb 19;360(8):765-73.

[2] Louis et al. Acta Neuropathol. 2016 Jun;131(6):803-20.

[3] Chang et al. AJNR Am J Neuroradiol. 2018 Jul;39(7):1201-1207.

[4] Bangalore et al. Neuro Oncol. 2020 Mar 5;22(3):402-411.

[5] Basser et al. J Magn Reson B. 1996 Jun;111(3):209-19

[6] He et al. J. in Proc. of the IEEE CVPR. 2016;770-778.

[7] Solovyev et al. arXiv. 2021; arXiv:2104.01687.

[8]Abadi et al. Proc. of USENIX OSDI. 2016; pp. 265-283.

\section{S6.P11.}

\section{Classification of high- and low-grade meningiomas} using diffusion anisotropy indices with deep learning

\author{
${ }^{*}$ H. Halilibrahimoğlu ${ }^{1}$, B. Buz Yaluğ ${ }^{1}$, A. Kaykayoğlu ${ }^{2}$, A. Ersen \\ Danyeli ${ }^{3,4}$, M. Ş. Ekşi ${ }^{5}$, C. Yakıcıer ${ }^{6}$, M. N. Pamir ${ }^{3}$, A. Dinçer ${ }^{3,7}$, \\ K. Özduman ${ }^{5,3}$, A. Özcan ${ }^{3,8}$, E. Öztürk-Işık ${ }^{1,3}$

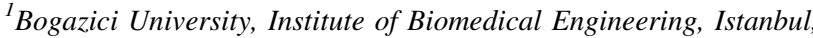 \\ $T R$ \\ ${ }^{2}$ Acıbadem Mehmet Ali Aydinlar University, Department of Medical \\ Engineering, Istanbul, TR; \\ ${ }^{3}$ Aclbadem Mehmet Ali Aydinlar University, Center \\ for Neuroradiological Applications and Research, Istanbul, TR; \\ ${ }^{4}$ Acıbadem Mehmet Ali Aydinlar University, Department of Medical \\ Pathology, Istanbul, TR; \\ ${ }^{5}$ Aclbadem Mehmet Ali Aydinlar University, Department \\ of Neurosurgery, Istanbul, TR; \\ ${ }^{6}$ YoctoSensum Biotechnoogy, Istanbul, TR;
}

${ }^{7}$ Aclbadem Mehmet Ali Aydınlar University, Department of Radiology, Istanbul, TR;

${ }^{8}$ Bogaziçi University, Department of Electrical and Electronics Engineering, Istanbul, TR

Introduction: Meningiomas are the most common intracranial brain tumor and accurate pre-treatment grading is crucial to guide treatment decisions [1]. Various deep learning models were tested to non-invasively predict meningioma grade using apparent diffusion coefficient (ADC) images coupled with other magnetic resonance imaging modalities [2]. In this study, a ResNet-50 model was trained to differentiate low-grade and high-grade meningiomas using diffusion anisotropy indices (DAIs).

Methods: In this IRB approved study, 76 meningioma patients $(46 \mathrm{~F} /$ $30 \mathrm{M}, 50.74 \pm 12.89$ years of age, grade I: 34 , grade II: 39 , grade III: 3) with post-contrast T1-weighted MRI (T1-post, TE/TR $=10 / 589$, $0.69 \times 0.69 \times 3.6 \mathrm{~mm}, 40$ slices) and diffusion tensor imaging (DTI, $\mathrm{TE} / \mathrm{TR}=69 / 4900,1.8 \times 1.8 \times 2.34 \mathrm{~mm}, 42$ slices, $\mathrm{b}=1000 \mathrm{~s} /$ $\mathrm{mm}^{2}, 30$ directions) were included. The patients were stratified into low-grade (Grade I) and high-grade (Grade II and III) groups for classification.

Enhancing tumor lesions were semi-automatically segmented [3] on T1-post MRI and registered to DTI space[4]. DAIs (ADC, fractional anisotropy (FA), relative anisotropy (RA)) were calculated from diffusion eigenvalue images [5], then enhancing tumor regions were masked.

Masked DAI images were cropped to include the tumor slice with the largest cross-section including the former and latter slices. These 3 slices in each DAI map were resized to $(224,224)$ and combined to create an RGB image as the input to the deep learning model. The cohort was stratified into training, validation, and test sets with a $80 / 20$ split ratio between train-test and train-validation sets.

A ResNet-50 network [6] was trained for 50 epochs using Tensorflow [7]. The model was compiled with Adam optimizer, whose learning rate was initialized as 10-4 and reduced when validation accuracy plateaued during training. Three ResNet-50 models were separately trained for ADC, FA, and RA.

Results: Training and validation accuracy plots of the ResNet-50 model are given in Fig. 1, and the model performance on the test set is shown in Table 1. FA images performed best among all DAIs with a test accuracy of $63 \%$ and 0.76 test AUC.
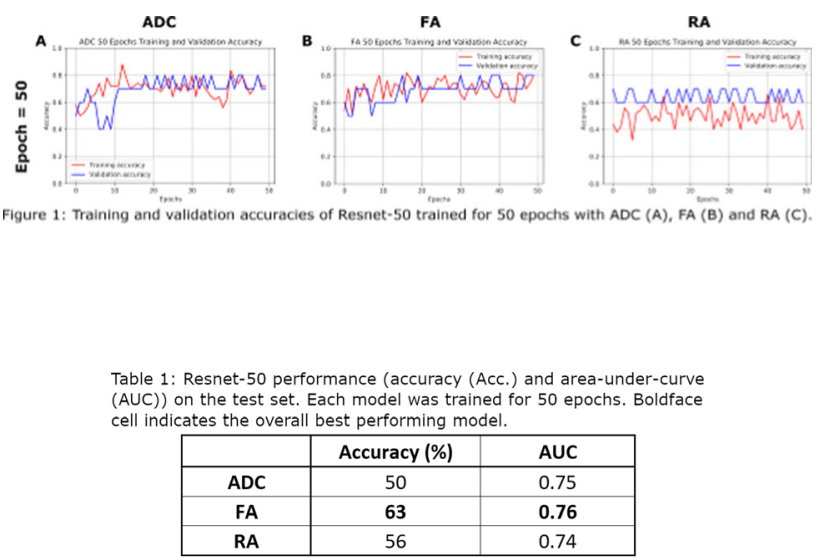

Discussion: This study demonstrates that deep learning can be used to differentiate between low- and high-grade meningiomas. However, the difference between training and test accuracy indicates overfitting and can be improved by increasing the cohort size.

Acknowledgement: This work is funded by The Scientific and Technological Research Council of Turkey (TUBITAK) 1001 Grant No: 119S520. 


\section{References:}

[1] Nassiri et al. Neuro Oncol. 2019 Jan;21(Suppl 1): i2-i3.

[2] Banzato et al. J Magn Reson Imaging. 2019;50,1152-1159.

[3] Fedorov et al. Magn Reson Imaging. 2012 Nov;30(9):1323-41.

[4] Avants et al. Med Image Anal. 2008 Feb;12(1):26-41.

[5] Basser et al. J Magn Reson B. 1996 Jun;111(3):209-19

[6] He et al. J. in Proc. of the IEEE CVPR. 2016;770-778.

[7] Abadi et al. Proc. of USENIX OSDI. 2016;pp. 265-283.

\section{S6.P12.}

\section{Clinical evaluation of the learning-based reconstruction for BLADE MRI}

\author{
H. C. Chen ${ }^{1}$, H. C. Yang ${ }^{1}$, Y. C. Chao ${ }^{1}$, *J. M. $\operatorname{Lin}^{2,3}$ \\ ${ }^{I}$ Madou Sin-Lau Hospital, Tainan, TW; \\ ${ }^{2}$ University of Cambridge, Cambridge, GB; \\ ${ }^{3}$ Trusted Medical Computing Laboratory, Taipei, GB
}

Introduction: Machine learning (ML) has been recognized as an emerging novel technology for building an automatic image reconstruction pipeline from large data sets.

However, most of the current general-purpose ML frameworks are not available to clinical applications, which are limited by the scarcity of data and the lengthy learning times. Recently, the generative interpolation network has been found to learn the feature with fewer data. This can save time and deliver high throughput.

Previously, we demonstrated a feasible model for reconstructing nonCartesian MRI. In this study, we examine the new PyNUFFT based network [1] for static BLADE MRI [2].

Method: The comparative study was approved by the Institutional Review Board (IRB). A total number of 32 in vivo T2 weighted brain MRIs were obtained from the DICOM database for evaluation. The $\mathrm{k}$-space of the BLADE sequence includes multiple overlapping rectangular k-space patches, which cover the circular region in $\mathrm{k}$-space while sharing the central k-space. No patient data were needed to build the network, and the complex-valued back-propagation was performed by quasi-orthogonal complex random bases generated in the image space and k-space. An interpolation sparse matrix in k-space and the image domain were obtained from a Python package.

We used Numpy and Scipy to implement the network. With the coordinate format (COO) and a batch number of 3 , the learning time on a single core CPU is fast and the total learning time for 7500 epochs was $14 \mathrm{~h}$. The reconstruction results of 2500, 5000, and 7500 epochs were compared against conjugate gradient (CG) and density compensation (DC) methods. Two board-certified radiologists evaluated the subjective image quality by means of a five-point score.

Results: The structural similarity metric (SSIM) of the five reconstruction methods (Fig. 1 (A)-(E)) ranged from 0.993 to 0.999 , which were significantly different $(\mathrm{p}<0.001)$.

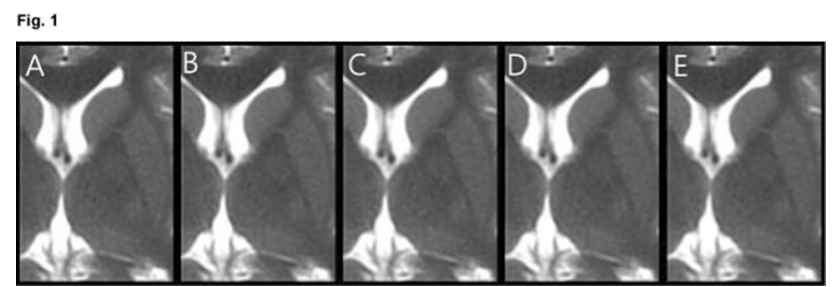

The SSIM of DC was the lowest $(0.993 \pm 0.0038)$, while the four other methods appeared to perform similarly $(0.999 \pm 0.0002)$. The SSIM of the CG method was significantly superior to the learning-based methods $(\mathrm{p}<0.001)$. Two radiologists reported that all five reconstruction methods were

visually identical (see Fig. 1). The subjective image quality scores were: noise level $(4.67 \pm 0.54)$, tissue contrast $(4.59 \pm 0.63)$, sharpness $(4.62 \pm 0.63)$

artifacts $(4.21 \pm 0.89)$ and the overall image quality $(4.53 \pm 0.77)$. Discussion: The proposed machine learning method for BLADE MRI generates identical image quality as the sampling DC and the CG with 2500-7500 epochs.

The proposed learning method is fast without in vivo data needed. The method could be integrated into the clinical scanning process because the

sparse matrix model can be readily ported to contemporary software packages without major difficulties.

\section{References}

[1] H.-C. Chen, J.-M. Lin, J. ESMRMB, 2019

[2] J.Pipe, MRM, 42.5 (1999): 963-969.

\section{S6.P13.}

Identification of most sensitive hemodynamic parameters to predict asymptomatic unilateral internal carotid artery stenosis by random forest ensemble classifier

${ }^{*}$ C. Gleißner ${ }^{1}$, S. Kaczmarz ${ }^{1}$, J. Kufer ${ }^{1}$, L. Schmitzer ${ }^{1}$, M. Kallmayer ${ }^{2}$, C. Zimmer ${ }^{1}$, B. Wiestler ${ }^{1}$, C. Preibisch ${ }^{1}$, J. Göttler ${ }^{1}$

${ }^{I}$ Technical University of Munich, Department of Neuroradiology, Munich, DE;

${ }^{2}$ Technical University of Munich, Department of Vascular and Endovascular Surgery, Munich, DE

Introduction: Asymptomatic internal carotid artery stenosis (ICAS) is a major public health issue ${ }^{1}$ and causes up to $15 \%$ of all strokes. ${ }^{2}$ Often these strokes are located at the edge of vascular territories, i.e. in individual watershed areas (iWSAs), which are most vulnerable to hemodynamic dysfunction. ${ }^{3}$ It is currently unclear, which of several hemodynamic MRI parameters are best suited to predict disease severity and whether hemodynamic changes within iWSA have a higher discriminative ability. ${ }^{4}$ Identifying most sensitive parameters and volumes of interest (VOIs) can increase clinical applicability, provide deeper understanding of the pathology and point out most relevant parameters for further research. To this end, we aim to predict ICAS by applying a random forest classifier (RFC) 5 to an extensive set of eight multi-modal MRI parameters within and outside iWSA in patients and healthy controls (HC). ${ }^{6}$

Methods: Twenty-four asymptomatic unilateral ICAS-patients (70.6 \pm 6.4 years) and $24 \mathrm{HC}(70.4 \pm 4.6$ years $)$ underwent multimodal MRI (Philips $3 \mathrm{~T}$ Ingenia) comprising breathhold-fMRI, mqBOLD, pseudo-continuous ASL and DSC-MRI. ${ }^{6}$ Eight perfusion, oxygenation and microvascular parameters were included (see Fig. 1). Feature vectors were generated from mean parameter values inside and outside iWSA $^{3}$ in grey matter $(\mathrm{GM})$ and white matter (WM) for each hemisphere and for ipsi- vs. contralateral differences. An RFC (MathWorks MATLAB 9.8) was trained for feature ranking using 96 features ( 12 VOIs $\times 8$ parameters). Importance scores and out-of-bag accuracies were calculated. ${ }^{5}$

Results: Bootstrapped importance scores of an RFC trained with 96 features are shown in Fig. 1. After eliminating correlated features, highest ranked features in order of decreasing importance scores are inter-hemispheric differences of TTP in WM, CBF in GM and 
ipsilateral CVR in GM, all inside iWSAs (Fig. 2). Using only the highest ranked feature as input, the accuracy is $73.2 \%$. It rises to $82.3 \pm 2.8 \%$ and $87.7 \pm 2.1 \%$ (area under the curve 0.88 ) for the three and seven most important features, respectively (Fig. 2, 3). Actually, more than four features do not provide significant improvement. Whole hemisphere GM and WM VOIs, neglecting iWSAs, yielded a significantly lower accuracy $(81.7 \pm 2.3 \%$ for seven features, t-test, $\mathrm{p}<0.001$ ).

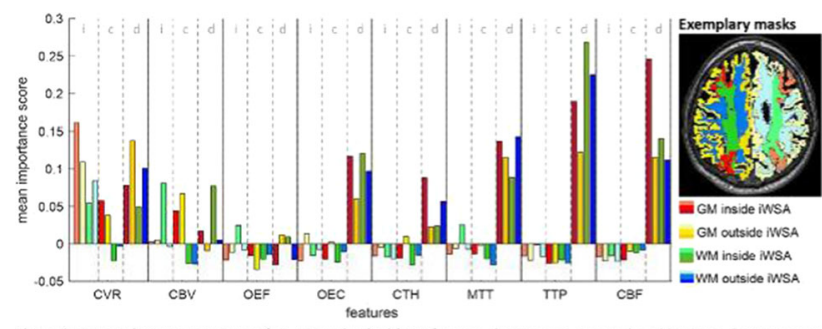

Figure 1: Feature importance scores of an RFC trained with 96 features. Scores were averaged a 1000 times. Features were extracted from eight MRI parameter maps (cerebrovascular reactivity (CVR), cerebral blood flow (CBF), cerebral blood volume (CBV), oxygen extraction fraction (OEF), oxygen extraction capacity (OEC), capillary transit time heterogeneity (CTH), mean trans
time (MTT) and time to peak (TTP), each sampled from 12 different vOls. For each parameter, the first, second and third Eroug of four bars refer to ipsilateral (i) and contralateral ici) mean values, and interhemispheric differences (d) between mean values, respectively, for each group, the colour scheme is similar for GM inside iWSAs (red), GM outside iWSAs (vellow), WM inside iWSAs (green) and WM outside iWSAs (blue). The colour intensity increases from group one to three

\begin{tabular}{|c|c|c|c|c|c|}
\hline rank & parameter & hemisphere & matter & IWSA & mean importance score ( \pm standard deviation) \\
\hline 1 & TTP & difference & WM & inside & $0.60 \longmapsto$ \\
\hline 2 & CBF & difference & GM & inside & $0.53 \longmapsto$ \\
\hline 3 & CVR & ipsilateral & GM & inside & $0.38 \longmapsto$ \\
\hline 4 & CVR & difference & GM & outside & $0.31 \longmapsto$ \\
\hline 5 & MTT & difference & WM & outside & $0.26 \mapsto$ \\
\hline 6 & OEC & difference & WM & inside & $0.22 \longmapsto$ \\
\hline 7 & CTH & difference & GM & inside & $0.18 \longmapsto$ \\
\hline
\end{tabular}

Figure 2: Feature importance scores of an RFC trained with 7 highest ranked features. Scores were averaged 200 times. For each feature, corresponding parameter, VOI characteristics, mean importance score and standard deviation are shown.

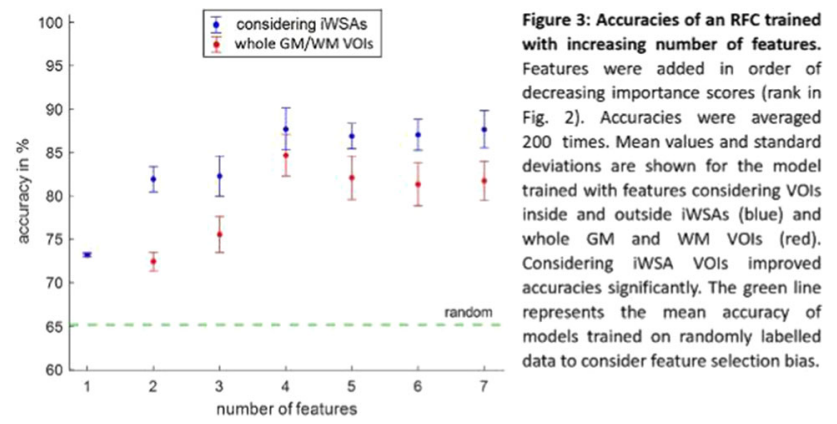

Discussion: We successfully applied an RFC to determine most relevant parameters and VOIs to predict ICAS. Ranking order and increased accuracy of hemodynamic parameters within iWSAs are in line with ${ }^{3,6}$. Few sensitive features, i. e. difference of TTP, CBF and ipsilateral CVR allow to detect ICAS patients. In conclusion, RFCs can identify most sensitive hemodynamic parameters to predict ICAS and may reveal most relevant features for diagnostics and further research in cerebrovascular diseases.

\section{References:}

1: de Weerd, Stroke, 2010.

2: Kamel, Stroke, 2019.

3: Kaczmarz, Neuroradiology, 2018.
4: Chen, NeuroImage, 2019.

5: Breiman, Machine Learning, 2001.

6: Kaczmarz, JCBFM, 2020.

\section{S6.P14. \\ Classification of multiple sclerosis clinical profiles using tensor-based structured data fusion of brain morphological connectivity}

\author{
*B. Barile ${ }^{1}$, A. Marzullo ${ }^{2}$, C. Stamile ${ }^{3}$, F. Durand-Dubief ${ }^{1,4}$, \\ D. Sappey-Marinier ${ }^{1,5}$ \\ ${ }^{1}$ Claude Bernard University Lyon 1, Villeurbanne, FR; \\ ${ }^{2}$ University of Calabria, Rende, IT; \\ ${ }^{3} 3 R \& D$ Department CGnal, Milan, IT; \\ ${ }^{4}$ Hospices Civils de Lyon, Hôpital Neurologique, Bron, FR; \\ ${ }^{5}$ CERMEP-Imagerie du Vivant, Bron, FR
}

Introduction: MS is an autoimmune inflammatory disease characterized by demyelination and neurodegeneration processes, leading to cognitive and physical impairments [1]. Brain morphological connectivity represents a new method for characterizing the brain networks which can be obtained directly from the anatomical T1w image by measuring the Gray Matter (GM) features [2]. The goal of this work is to exploit the multiparametric GM information obtained from three morphological features of the cortical GM such as: thickness, curvature and area. The multi-features connectome data were combined together using tensor-based Structured Data Fusion (SDF) technique [3] for the unsupervised classification of MS clinical profiles.

Methods: A tensor-based multilinear rank-(Lr, Lr, 1) [4] SDF application was used to jointly factorize three GM morphological tensors (Thickness, Curvature and Area) describing characteristics of GM tissue (Fig. 1). 90 patients, distributed in four clinical profiles (12 CIS, 30 RRMS, 28 SPMS, 20 PPMS) underwent an MR examination protocol composed of a 3-dimensional T1-weighted MPRAGE sequence with repetition time/echo time/time for inversion (TR/TE/ TI $=1970 / 3.93 / 1100 \mathrm{~ms}$, flip angle $=15^{\circ}$, voxel size $=1 \times 1 \times 1$. The parcellation task was performed using the FSAverage atlas [5] yielding 68 brain regions in total. Afterward, each voxel was classified into four classes [WM, cortical GM, sub-cortical GM, cerebrospinal fluid (CSF)] and the three morphometric features were calculated. The connectome data were obtained comparing all GM regions yielding a total of three connectomes for each patient which were stacked together obtaining three final tensors (Fig. 2). The multilinear rank-factorization ( $\mathrm{Lr}, \mathrm{Lr}, 1)$ was then applied to combine together the three tensors, obtaining a latent representation of MS patients (factor components) which was then exploited for classification using k-means clustering algorithm (Fig. 1).

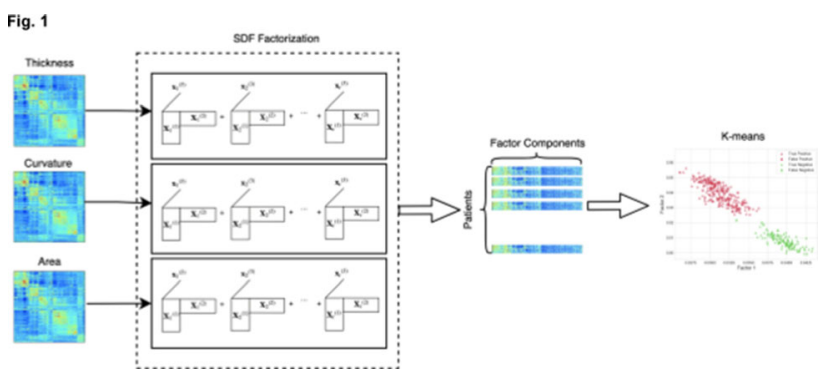

Results: Good levels of performances were observed using our approach compared to the more classical Canonical Polyadic 
Decomposition (CPD) (Table 1). In order to compare the two approaches, a non-parametric Mann-Whitney U test was applied using a standard threshold of significance $(\mathrm{p}=0.05)$ as shown by the numbers in Table 1 in bold.

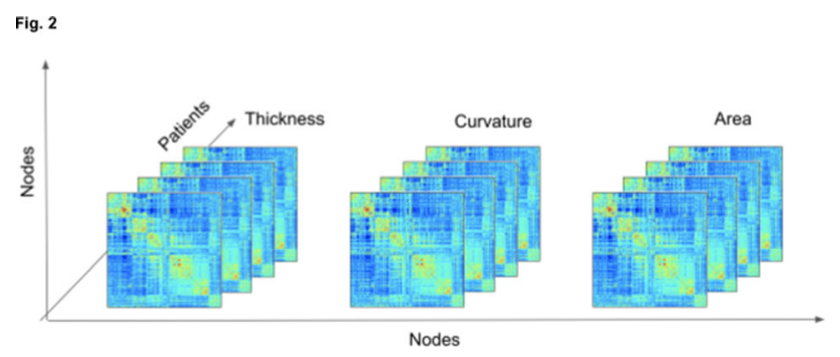

Fig. 3

\begin{tabular}{|c|c|c|c|c|c|c|}
\hline & \multicolumn{2}{|c|}{ F1 } & \multicolumn{2}{|c|}{ Precision } & \multicolumn{2}{|c|}{ Recall } \\
\hline & LL1 & CPD & LL1 & CPD & LL1 & CPD \\
\hline CIS-RR & $0.70 \pm 0.02$ & $0.62 \pm 0.08$ & $0.73 \pm 0.02$ & $0.72 \pm 0.04$ & $0.70 \pm 0.03$ & $0.59 \pm 0.14$ \\
\hline CIS-PP & $0.66 \pm 0.05$ & $0.57 \pm 0.06$ & $0.67 \pm 0.04$ & $0.62 \pm 0.04$ & $0.65 \pm 0.09$ & $0.58 \pm 0.12$ \\
\hline CIS-SP & $0.77 \pm 0.07$ & $0.75 \div 0.10$ & $0.82 \pm 0.06$ & $0.83 \pm 0.03$ & $0.76 \pm 0.11$ & $0.70 \pm 0.16$ \\
\hline RR-PP & $0.64 \pm 0.07$ & $0.63 \pm 0.05$ & $0.62 \pm 0.03$ & $0.62 \pm 0.02$ & $0.68 \pm 0.13$ & $0.65 \pm 0.10$ \\
\hline RR-SP & $0.70 \pm 0.04$ & $0.46 \pm 0.19$ & $0.68 \pm 0.05$ & $0.65 \pm 0.17$ & $0.72 \pm 0.08$ & $0.44 \pm 0.23$ \\
\hline PP-SP & $0.72 \pm 0.01$ & $0.58 \pm 0.08$ & $0.64 \pm 0.01$ & $0.61 \pm 0.06$ & $0.83 \pm 0.02$ & $0.58 \pm 0.12$ \\
\hline
\end{tabular}

Note: Mann-Whitney $\mathrm{U}$ test was performed to assess statistical differences between performances of SDF-LL1 and SDF-CPD. Best values are repotent in bold

Discussion: In this study, a new approach for multiparametric blind source separation using the SDF paradigm was proposed. Higher levels of performances were observed using multilinear rank-(Lr, Lr, 1) compared to the more classical and more rigid CPD model, showing the capability of the GM tissue to discriminate MS clinical profiles. Single factorization of each morphological tensor was also investigated obtaining pour results, demonstrating the usefulness of the fusion approach.

\section{References:}

[1] E. Fisher et al. 2008, 64(3):255-65.

[2] A. Kuceyeski et al. 2018, 19:417-424.

[3] L. Sorber et al. 2015, 9(4):586-600.

[4] L. De Lathauwer 2011, 32(4):1451-1474.

[5] M. Rosenke et al. 2017.

\section{S6.P15.}

\section{Cortical and subcortical volumes changes predict tau- PET positivity in beta-amyloid positive patients}

\author{
${ }^{*}$ H. Pereira ${ }^{1,2}$, H. Teixeira ${ }^{1}$ \\ ${ }^{1}$ University of Lisbon, Faculty of Sciences, Lisbon, PT; \\ ${ }^{2}$ NOVA University Lisbon, Faculty of Science and Technology, \\ Lisbon, $P T$
}

Introduction: Abnormal concentrations of tau, beta-amyloid (AB) proteins, and neurodegeneration are biomarkers of Alzheimer"s Disease (AD), but less is known how they interact. Therefore, we aimed to predict tau positivity $(\mathrm{Tau}+)$ using magnetic resonance imaging (MRI) biomarkers in $\mathrm{AB}$ positive $(\mathrm{AB}+)$ sample of $\mathrm{AD}$ and mild cognitive impairment (MCI) subjects.

Methods: We selected an age-and gender-matched sample from the ADNI database: $\mathrm{AD} \mathrm{AB}+(\mathrm{n}=12$, age $=74.2,50 \%$ female $), \mathrm{MCI}$ $\mathrm{AB}+(\mathrm{n}=26$, age $=71.3 \pm 5.8,54 \%$ female $)$ and Controls $(\mathrm{CL})$ $(\mathrm{n}=35$, age $=71.2 \pm 6.1,43 \%$ female $)-\mathrm{AB}$ negative $(\mathrm{AB}-)$ subjects. Tau positivity was obtained using composites of rSUV computed from Flortaucipir PET for each Braak stage [1] and a global composite tau-rSUV (regions showing statically significant differences $(p<0.05)$ in $\mathrm{NL} \mathrm{AB}-\mathrm{vs}(\mathrm{MCI}+\mathrm{AD}) \mathrm{AB}+)$. The cutoff value of each composite was the one that best discriminates NL ABfrom $(\mathrm{MCI}+\mathrm{AD}) \mathrm{AB}+$.i.e., the value that maximizes the chisquare statistic value with the highest significance. We used machine learning models (voting classifier) to predict Tau + with demographic (age and gender), clinical (Geriatric Depression Score (GDS) and Mini-Mental State Exam (MMSE) score), and brain volumes from MRI. The best features were selected using Spearman correlation or chi-square statistics.

Results: The voting classifier was able to classify Tau + with accuracies above $70.0 \%$ using the cutoff values of Braak1, Braak34 [the respective areas, please see [1] and the composite tau-rSuv (Table 1). The best performance was achieved using the composite Braak1. The right middletemporal, right parahippocampal, left accumbens area, left amygdala, left lateral ventricle and right accumbens volumes, and the GDS score were selected to classify the three rSUV composites.

Fig. 1

Table 1: Tau positivity classification results of Braak1, Braak34 and composite tau-rSUV.

\begin{tabular}{|c|c|c|c|}
\hline & BRAAK1 rSUV & BRAAK34 rSUV & Composite tau-rSUV \\
\hline \begin{tabular}{|l} 
Balanced \\
Accuracy
\end{tabular} & 77.3 & 72.7 & 75.2 \\
\hline $\begin{array}{l}\text { ROC-AUC } \\
\end{array}$ & 0.883 & 0.775 & 0.744 \\
\hline $\begin{array}{l}\text { Cohen's } \\
\text { Kappa }\end{array}$ & 0.545 & 0.468 & 0.472 \\
\hline PPV & 81.8 & 87.5 & 88.9 \\
\hline NPV & 72.7 & 64.3 & 61.5 \\
\hline \begin{tabular}{|l|} 
Sensitivity \\
\end{tabular} & 75.0 & 58.3 & 61.5 \\
\hline \begin{tabular}{|l|} 
Specificity \\
\end{tabular} & 80.0 & 90.0 & 88.9 \\
\hline $\begin{array}{l}\text { Selected } \\
\text { Features }\end{array}$ & \begin{tabular}{|c|} 
left banks of the superior temporal \\
sulcus, left entorhinal, left middle \\
temporal, right entorhinal, right \\
middletemporal, right \\
parahippocampal, GDS, \\
left accumbens area, \\
left amygdala, left lateral ventricle, \\
right accumbens area
\end{tabular} & \begin{tabular}{|c|} 
left inferior parietal, left inferior \\
temporal, left middle temporal, right \\
entorhinal, right middle temporal, \\
right parahippocampal, right \\
pericalcarine, GDS, left amygdala, \\
left hippocampus, MMSE score, \\
right accumbens area, right \\
amygdala \\
\end{tabular} & $\begin{array}{c}\text { left inferior temporal, } \\
\text { right cuneus, right middle temporal } \\
\text { right parahippocampal, right } \\
\text { pericalcarine, GDS, left taccumbens } \\
\text { area, left amygdala, MMSE score, } \\
\text { right accumbens area, } \\
\text { right amygdala } \\
\end{array}$ \\
\hline
\end{tabular}

Discussion: Our results show that cortical and subcortical volume changes are potential biomarkers to predict tau-PET in an $\mathrm{AB}+$ sample. The fact that only Braak1, Braak34 and composite taurSUV could predict tau-PET may be associated with the fact that the cutoffs values were obtained from $\mathrm{CL} A B-$ vs $(\mathrm{MCI}+\mathrm{AD}) \mathrm{AB}+$, as $\mathrm{MCI} A B+$ may present less atrophy in the brain than $\mathrm{AD}$. The best three classifications shared volumes supporting the Braak et al. hypothesis, wherein the severity of brain atrophy increases with tau protein progression [2]. Further studies should be conducted to confirm that the selected regions are only characteristic of $\mathrm{AD}$ and $\mathrm{MCI}$ tau + and $\mathrm{AB}+$ patients.

\section{References:}

[1] M. Schöll et al., "PET Imaging of Tau Deposition in the Aging Human Brain,“ Neuron, vol. 89, no. 5, pp. 971-982, Mar. 2016, doi: 10.1016/j.neuron.2016.01.028.

[2] H. Braak and K. Del Tredici, "Potential pathways of abnormal tau and $\alpha$-synuclein dissemination in sporadic Alzheimer"s and Parkinson"s diseases, " Cold Spring Harb. Perspect. Biol., vol. 8, no. 11, pp. 1-23, 2016, doi: 10.1101/cshperspect.a023630. 


\section{S6.P16.}

\section{Automatic identification of imaging errors in large epidemiological cohort studies}

\author{
*T. Haueise ${ }^{1,2}$, C. L. Schlett ${ }^{3}$, F. Bamberg ${ }^{3}$, F. Schick ${ }^{1,2}$, \\ J. Machann ${ }^{1,2}$ \\ ${ }^{1}$ University Hospital Tübingen, Section on Experimental Radiology, \\ Tübingen, $D E$; \\ ${ }^{2}$ Helmholtz Center Munich, Institute for Diabetes Research \\ and Metabolic Diseases, Munich, DE; \\ ${ }^{3}$ Albert-Ludwig-University of Freiburg, Center for Diagnostic \\ and Therapeutic Radiology, Freiburg im Breisgau, DE
}

Introduction: Abdominal obesity, as manifested by increased visceral adipose tissue (VAT), shows a strong correlation to insulin resistance which is associated with the risk of developing type 2 diabetes $^{1}$. The German National Cohort $(\mathrm{GNC})^{2}$ contains a wholebody magnetic resonance imaging (MRI) sub-study ${ }^{3}$. Automated segmentation of abdominal fat compartments, i.e. VAT and subcutaneous adipose tissue (SAT) was performed using a U-Net model $\left(n n U-N e t^{4}\right)$. Manual inspection to identify imaging errors is timeconsuming, costly and therefore not feasible in a large cohort study. Using nnU-Net, faulty segmentations are most likely to occur due to imaging errors in the regions of interest, e.g. phase swaps.

Methods: Standardized 3D 2-point VIBE Dixon images were acquired on $3 \mathrm{~T}$ whole-body imagers (Magnetom Skyra, Siemens Healthineers, Germany) at multiple sites with $3 \mathrm{~mm}$ section thickness, $1.4 \times 1.4 \mathrm{~mm}$ in-plane voxel size and a repetition time of $4.36 \mathrm{~ms}$. nnU-Net was trained using $\mathrm{n}=30$ training cases in a fivefold crossvalidation scheme yielding an ensemble of five separate U-Nets. Each model was used to segment $n=11,191$ data sets from the GNC. As the models were randomly initialized and different training cases were used during the training, resulting segmentations are slightly different. Taking advantage, the mean pairwise Dice scores of different ensemble members can be used as a measure for segmentation uncertainty per fat compartment ${ }^{5}$. Statistical measures for outliers are applied to identify faulty segmentations.

Results: Figure 1 shows the boxplot of the resulting uncertainty scores for both fat compartments. A total of $n=217$ candidates was identified, reducing datasets for manual inspection by about $98 \%$. After manual inspection, $\mathrm{n}=15$ (about 7\%) of the candidates had to be excluded (see Fig. 2). Figure 3 shows an example of a misidentified candidate.

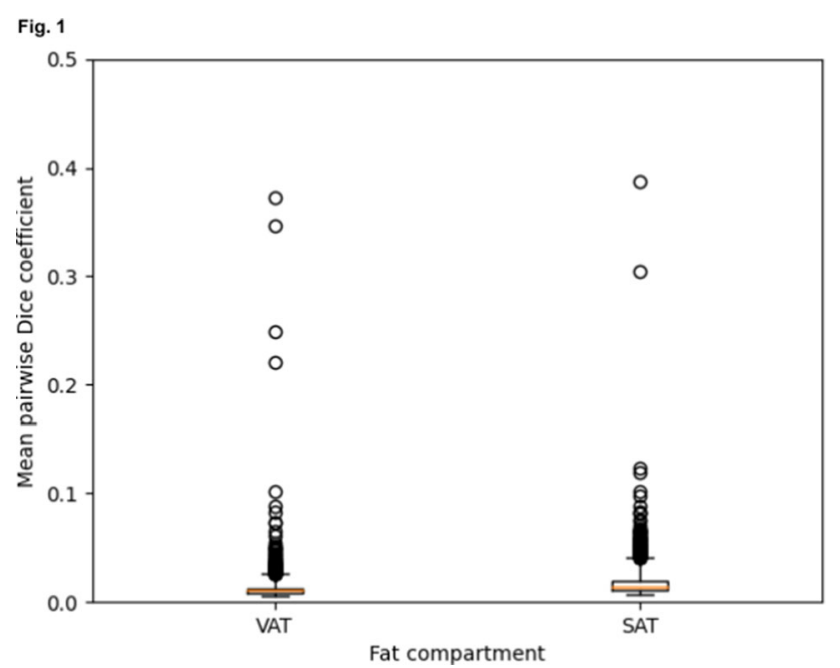

Fig. 2

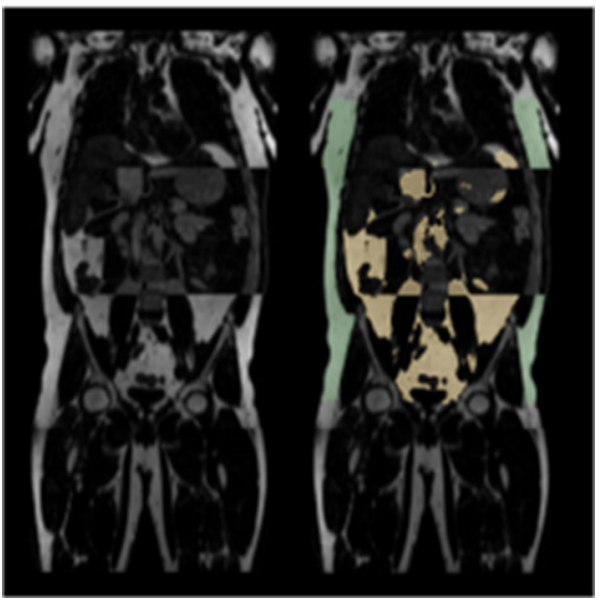

Fig. 3

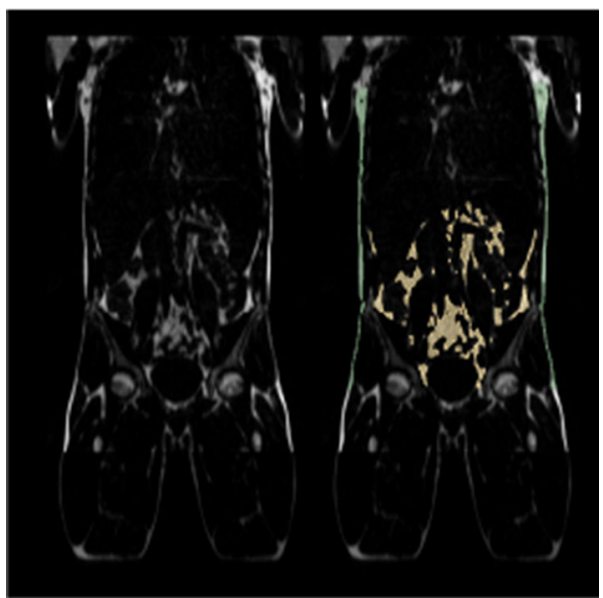

Discussion: Due to quality control of the GNC data, imaging errors were expected to be rarely found. The application of mean pairwise Dice score as a value of segmentation uncertainty significantly reduced the number of data sets for manual inspection. In a small number of data sets, imaging errors in the regions of interest could be identified, and the data sets are excluded from further analysis.

Mean pairwise Dice score is a feasible measure for the identification of imaging errors in large cohorts with very low computational overhead.

\section{References:}

1. Després JP et al. Abdominal obesity and metabolic syndrome. Nature 2006.

2. Ahrens W et al. Das Design der Machbarkeitsstudien für eine bundesweite Kohortenstudie in Deutschland. Bundesgesundheitsbl. 2014.

3. Bamberg F et al. Whole-Body MR Imaging in the German National Cohort: Rationale, Design, and Technical Background. Radiology 2015.

4. Isensee $F$ et al. nnU-Net: a self -conFiguring method for deep learning-based medical image segmentation. Nature Methods 2021. 
5. Roy AG et al. Inherent Brain Segmentation Quality Control from Fully ConvNet Monte Carlo Sampling. MICCAI 2018.

\section{S6.P17.}

\section{Prediction of head motion parameters by measuring extracranial magnetic field changes during multi-slice EPI acquisition}

\section{*L. Bortolotti ${ }^{1}$, R. Bowtell ${ }^{1}$ \\ ${ }^{1}$ University of Nottingham, Sir Peter Mansfield Imaging Centre, Nottingham, GB}

Introduction: Previously, head motion parameters were predicted from NMR field camera measurements without concurrent image acquisition ${ }^{1}$. Here, prediction was performed by making measurements of extra-cranial field changes in the quiet periods of a standard multi-slice EPI acquisition. The results represent a step forward in integrating standard imaging with a marker-less motion monitoring technique.

Methods: The field probes were sited between the transmit and receiver RF head coils using a customized probe holder. An MPT camera was also sited in the magnet bore to allow simultaneous measurements of head position and extracranial magnetic field to be made during the training phase (Fig. 1a). Multi-slice EPI sequence was performed with a slice TR of $75 \mathrm{~ms}$ (48 slices, $3 \mathrm{~mm}$ isotropic resolution, $\mathrm{TR}=3.6 \mathrm{~s}$, TE $=20 \mathrm{~ms}$ ). The field camera acquired field measurements every $150 \mathrm{~ms}$ (synchronised with every 2nd slice acquisition), with the $10 \mathrm{~m} \mathrm{~s}$ acquisition commencing $30 \mathrm{~ms}$ after the slice excitation, so limiting the effect of eddy currents from the applied imaging gradients. Measurements of extracranial magnetic field changes and head motion parameters were acquired with and without simultaneous scanning with the subject executing a range of different head movements (head shaking/nodding, foot wiggle, rest). Head motion parameters were predicted from the extra-cranial field measurements by using a 1-hidden layer delayed feedback neural network (NARX) ${ }^{\mathbf{1}}$.

Results: The magnetic field changes measured with and without simultaneous scanning have similar magnitude and temporal variation for comparable head movements (Fig. 2). Prediction of motion parameters was successfully performed (Fig. 3). The best results were produced when the training dataset was formed from the movement conditions (rest and foot wiggle) involving smaller head displacements. The Table (Fig. 3) compares predicted motion parameters to the actual values.

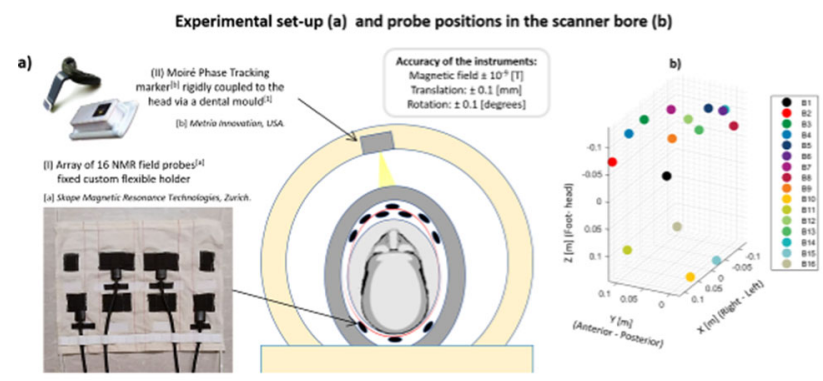

filoure 2: The NMR field probes were placed between the transmit and receiver Rf coils scanner. The optical camera is fixed to the inside of the the
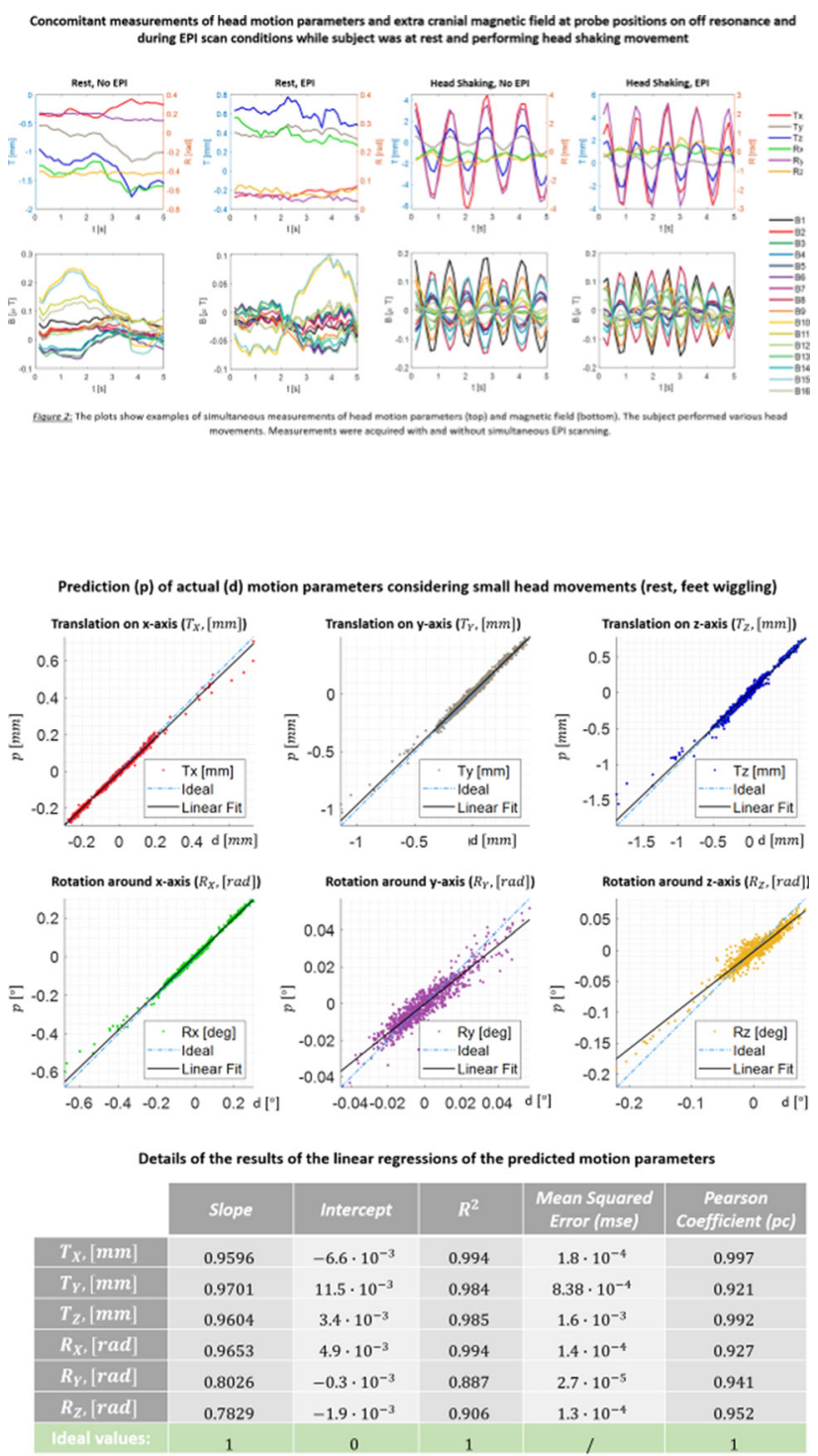

Eiqure 3: Plots show the predictions on small head movements acquired during EPI scanning, the linear fit (black line) and the ideal prediction (blue dashed line) to the data. Details of the fit are reported in the rable.

Discussion: Head motion during EPI scan acquisition can be characterised in a $7 \mathrm{~T}$ MRI scanner by measuring the extra-cranial magnetic field changes using a field camera. Prediction accuracy was improved by forming a training set from data acquired with only small head movements. The acquisition of less than 5 min of training data is then required during which time head movement parameters need to be simultaneously measured using a different motion monitoring approach. Here, we used an MPT camera for concurrent measurements, but in the future, training could be based on analysis of recorded image data. This work represents a step towards the full development of a marker-less technique for head motion tracking that doesn"t not require modification of the image acquisition sequence.

\section{References:}

(1) Bortolotti L. et al., 2020, https://doi.org/10.1007/s10334-02000876-y.

(2) Aranovitch A et al., 2019, https://doi.org/10.1002/mrm.28094.

(3) Eschelbach M. et al., 2018, https://doi.org/10.1002/mrm.27343. 


\section{S6.P18.}

\section{Vessel distance mapping of the aging subcortical venous vasculature}

\section{*H. Mattern ${ }^{1}$}

${ }^{1}$ Otto von Guericke University, Department of Biomedical Magnetic Resonance, Magdeburg, DE

Introduction: The effect of aging on the volume, T1, T2*, and susceptibility of subcortical structures has been studied ${ }^{1}$. Although venous atlases gained interest recently ${ }^{2,3}$, venous assessment in subcortical regions is challenging due to surrounding iron-rich structures $^{2}$. Further, MR-based assessment of aging-induced changes in the venous vasculature remains understudied. Here, the recently introduced vessel distance mapping $(\mathrm{VDM})^{4}$ along with ROI-based vessel densities was applied to assess if the subcortical venous vasculature declines with aging.

Methods: The openly available ATAG study was re-used. It provides 7 T GRE and MP2RAGE data of 30 young, 14 middle-aged, and 9 elderly subjects ${ }^{5,6}$. From the $0.5 \mathrm{~mm}$ isotropic GRE, a high-passed, QSM-based SWI was computed with QSMbox ${ }^{7}$. The high-pass minimizes enhancement of non-vessel structures. Vessel segmentation was done with OMELETTE ${ }^{8}$. Vessel densities were computed per ROI as the ratio of detected vessel volume to total ROI volume. From the segmentation, VDM computes for each non-vessel voxel the distance to the closest vessel using the Euclidian distance transform. The workflow is shown in Fig. 1. To build age-specific atlases, the GRE data was registered nonlinearly to MNI space with ANTs $^{9}$ via the additionally provided MP2RAGE slab and whole brain volumes. For each age group, the respective ATAG atlas was used to compute group statistics of the VDM and vessel densities. KolmogorowSmirnow tests were used to detect statistically significant differences.

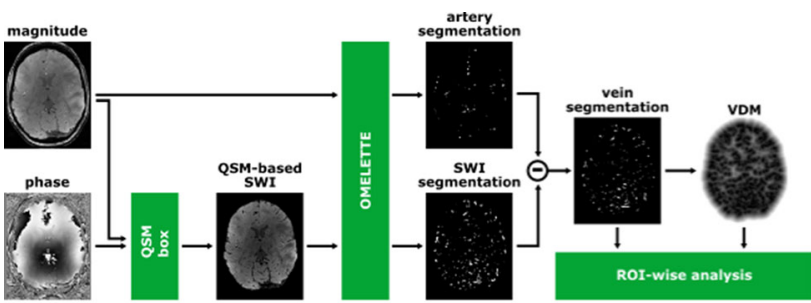

Figure 1: Proposed pipeline to estimate venous densities and vessel distance maps (VDM). Based on the magnitude and phase information of the GRE, QSM-based SWI is computed using QSMbox to enhance veins. With OMELETTE veins and arteries are segmented from the SWI and GRE magnitude, respectively. To prevent arterial contamination (i.e. due to flow artifacts in the phase data), vessels identified in arterial and SWI segmentation are removed to create the final vein segmentation. From this segmentation, VDM and vessel densities are computed and analyzed per ROI.

Results: The averages per age group are shown in Fig. 2. The sparse vessel information is interpolated by VDM, yielding distance maps resembling the underlying structure (distances increasing from CSF to white matter) and providing non-zero values even if no vessel is detected within the ROI. Figure 3 shows the group results for VDM and vessel densities for subcortical regions. Only the striatum showed the expected aging-related decrease in vasculature as lower densities and large distances, respectively. Other regions show an unexpected U-shape with decreased vessel distances in the middle-aged group. Significant differences were found for VDM in the internal globus pallidus and for densities in the striatum (see Fig. 3).

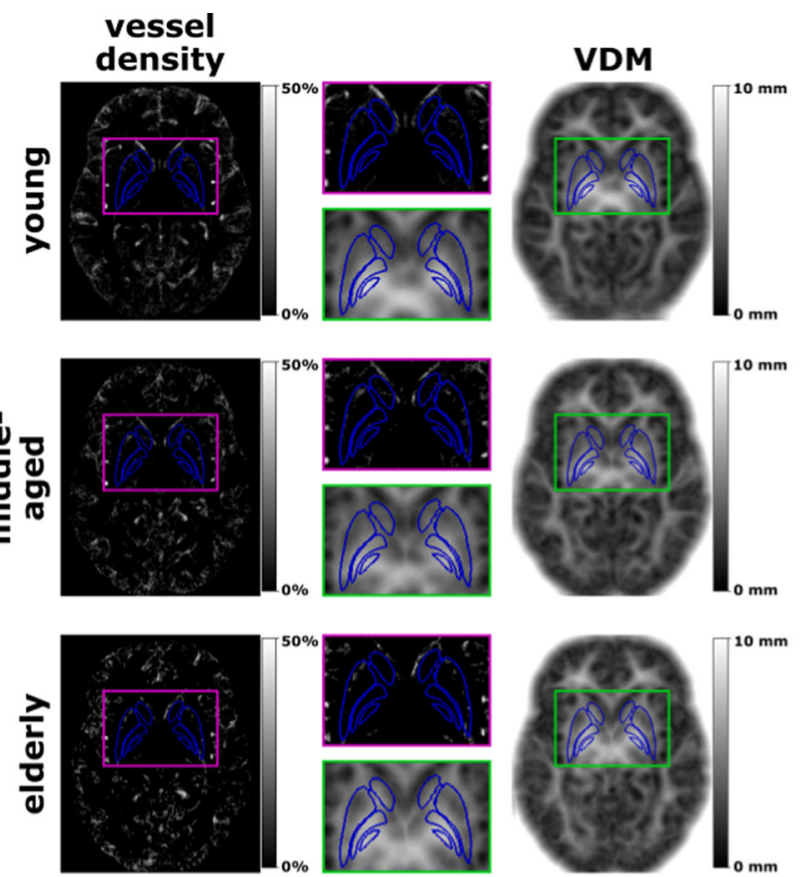

Figure 2: Average vessel densities and vessel distance maps (VDM) per age group in MNI space. For each group, the respective ATAG atlas is used. Outlines for striatum and globus pallidum are shown in blue.
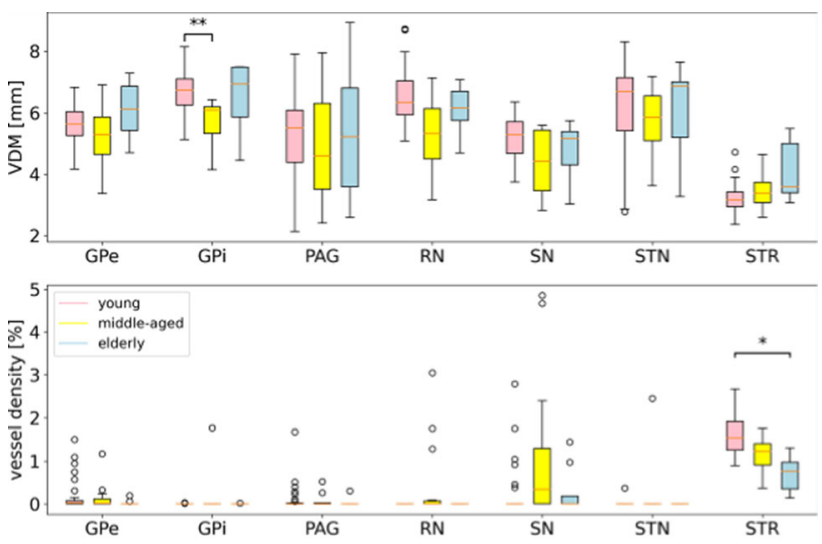

Figure 3: Box plots for vessel distance maps (VDM) and densities per ROI. Statistical significance is indicated with $*$ for $\mathrm{p}<0.05$ and $* *$ for $\mathrm{p}<0.01$

(Bonferroni corrected). GPe - globus pallidus external; GPi - globus pallidus internal; PAG - periaqueductal grey; RN - red nucleus; SN - substantia nigra; STN - subthalamic nucleus, STR - striatum

Discussion: Assessment of aging-induced changes in the subcortical vasculature is feasible with the presented pipeline. Except for the striatum, the results show an unexpected aging-vasculature-relation, requiring future investigations to assess if these are due to bias in the data acquisition/analysis or actual changes in the venous vasculature. Acknowledgment: This work was funded by the DFG-MA 9235/1-1

\section{References}

1. Keuken et al. Brain Struct Funct 2017.

2. Huck et al. Brain Struct Funct 2019.

3. Bernier et al. HBM 2018. 
4. Mattern et al. ESMRMB 2020.

5. Frostmann et al. Sci.Data 2014.

6. Keuken et al. NIMG 2014.

7. Acosta-Cabronero et al. NeuroImage 2018.

8. Mattern et al. ISMRM 2021.

9. Avants et al. Medical Image Analysis 2008.

\section{S6.P19.}

\section{Repeatability measurements of biomarkers derived from whole-body diffusion weighted imaging (WBDWI) to assess response to treatment on patients with metastatic bone disease using a fully automated software solution}

*A. Candito ${ }^{1}$, R. Holbrey ${ }^{1}$, S. Schäfer ${ }^{2}$, M. R. Orton ${ }^{1}$, A. Ribeiro ${ }^{3}$, M. Baumhauer ${ }^{2}$, N. Tunariu ${ }^{1}$, D. M. Koh ${ }^{1}$, M. Blackledge ${ }^{1}$

${ }^{1}$ The Institute of Cancer Research, Sutton, GB;

${ }^{2}$ Mint Medical GmbH, Heidelberg, DE;

${ }^{3}$ The Royal Marsden NHS Foundation Trust, Sutton, GB

Introduction: Whole-Body Diffusion Weighted Imaging (WBDWI) demonstrates high sensitivity for detecting metastatic bone disease in patients with advanced prostate cancer (APC) [1]. WBDWI is a quantitative technique that provides non-invasive measurement of the tumour diffusion volume (tDV) and the global Apparent Diffusion Coefficient (gADC), a surrogate of tumour cellularity. However, manual delineation of regions of interest (ROIs) within these datasets is too cumbersome to be clinically viable. Therefore, we have assessed the repeatability of quantitative ADC-based biomarkers derived from automated delineation of metastatic bone lesions on WBDWI.

Methods: Nine APC patients were scanned twice using WBDWI prior to initiation of anti-cancer treatment. WBDWI may vary depending on acquisition date, imaging protocol or scanner manufacturer. Therefore, signal on $b=900 \mathrm{~s} / \mathrm{mm}^{2}$ images was normalized using a Noise-Corrected, Exponentially Diffusion Weighted MRI (niceDWI), which synthesizes new contrast using a weighted combination of voxel-wise ADC and ADC uncertainty,, estimated using a recently developed algorithm [2]:

$\mathrm{S}_{\text {niceDWI }},=\mathrm{e}_{\mathrm{c}}^{-\mathrm{a}} \cdot \sigma_{\mathrm{ADC}} \times \mathrm{e}_{\mathrm{c}}^{-\mathrm{b}} \cdot \mathrm{ADC}$

with $\mathrm{a}_{\mathrm{c}}=20,000 \mathrm{~s} / \mathrm{mm}^{2}$, and $\mathrm{b}_{\mathrm{c}}=900 \mathrm{~s} / \mathrm{mm}^{2}$.

Signal images were used as inputs to an atlas-based pipeline to automatically delineate ROIs in all repeatability studies. ROIs were transferred onto estimated ADC maps, from which 5 first-order global ADC (gADC) histogram parameters (mean, median, variance, skew and kurtosis) and one size/shape-based parameter (logarithm of tumour diffusion volume, tDV) were derived. Repeatability of these parameters was calculated using Bland-Altman analysis. The intraclass correlation coefficient (ICC), Coefficient of Variation (CoV), repeatability coefficient (RC), and percentage repeatability (\%RC) were calculated for each of the WBDWI parameters.

Results: Table 1 summarises the values estimated for $\mathrm{CoV}, \mathrm{RC}$ and $\%$ RC. Mean/median/variance gADC and log-tDV showed CoV lower than $10 \%$ and ICC higher than 0.9. Figure 1 shows the Bland-Altman plots for each of the WBDWI parameters investigated. Figure 2 demonstrates the best performing case-study of automatic ROI delineation.
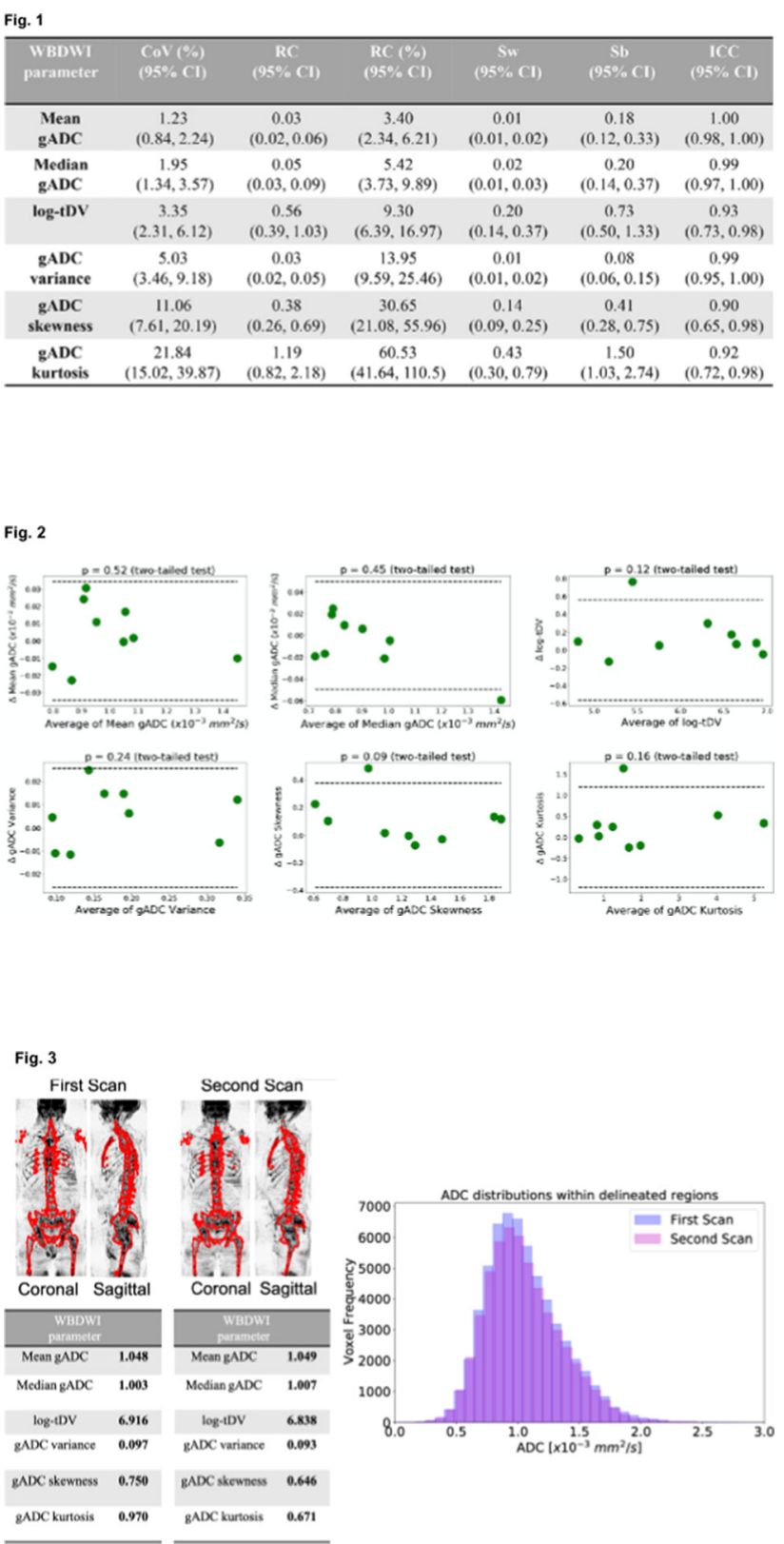

Discussion: The repeatability of WBDWI parameters derived from an automated software solution showed high repeatability for mean/median/variance metrics derived from histograms of gADC and $\log$-tDV. A previous study indicated a variation of $17 \%$ for Median $\mathrm{gADC}$ and from $-50 \%$ to $+26 \%$ for $\log$-tDV after treatment [3]. A variation of this magnitude would be outside the limits of agreement derived in this study. Therefore, DWI-based biomarkers derived from an automated segmentation algorithm with signal normalization using the niceDWI technique may be able to assess successful response to treatment in clinical practice.

\section{References:}

[1] D. M. Koh et al., Am. J. Roentgenol., 2012.

[2] M. D. Blackledge et al., Front. Oncol., 2020.

[3] M. D. Blackledge et al., PLoS One, 2014. 


\section{S6.P20.}

\section{Investigation of $\mathbf{R} 1$ white matter fiber orientation dependency in in situ post mortem conditions}

\author{
${ }^{*}$ C. Berger ${ }^{1}$, M. Bauer ${ }^{1}$, E. Scheurer ${ }^{1}$, C. Birkl ${ }^{2}$, C. Lenz ${ }^{1}$ \\ ${ }^{1}$ University of Basel, Institute of Forensic Medicine, Basel, CH; \\ ${ }^{2}$ Medical University of Innsbruck, Department of Neuroradiology, \\ Innsbruck, AT
}

Introduction: Prior studies proposed that the longitudinal relaxation rate $R_{1}$ depends on the orientation of the nerve fibers with respect to the main magnetic field based on molecular dynamics (MD) simulations [1, 2]. The MD simulations proposed that orientation dependency may be mainly attributed to anisotropic dipole-dipole interactions due to structural polarization of water molecules occurring within the myelin sheath [2]. Further, the hydrogen nuclei in the lipid membrane of the myelin sheath reveal a structural conFiguration, which lead to a spatially and temporally asymmetric spin environment causing anisotropic dipole-dipole interactions with neighboring protons [2]. This would result in an orientation dependent relaxation rate $R_{1}$ observed in the MD simulations. $R_{1}$ orientation dependency has only been examined in a few studies but may be crucial for the extraction of more precise tissue features $[1,2]$. The goal of this study was to investigate the behavior of the orientation dependency of $R_{1}$ in in situ post mortem conditions.

Methods: 13 deceased subjects were examined and stored in a cooling chamber at $4{ }^{\circ} \mathrm{C}$ prior to study procedures. The in situ brain temperature was measured transethmoidally prior to the MRI scan. In order to determine $\mathrm{R}_{1}$ orientation dependency, post mortem in situ MRI scans were conducted at $3 \mathrm{~T}$. $\mathrm{R}_{1}$ was acquired using an inversion-recovery spin-echo sequence [6 TIs: 30-1200 ms, TE/TR: $12 / 7060 \mathrm{~ms}$, 40 slices, slice thickness $4 \mathrm{~mm}$, in-plane resolution $\left.1 \times 1 \mathrm{~mm}^{2}\right]$ and subsequent fitting of a mono-exponential decay signal model. The orientation of white matter fibers were computed using diffusion-weighted single-shot echo-planar imaging [64 isotropically distributed diffusion directions, TE/TR: 109/18700 ms, 100 slices, isotropic resolution of $\left.1.8 \mathrm{~mm}^{3}, \mathrm{~b}=2000 \mathrm{~s} / \mathrm{mm}^{2}\right]$.

Results: The Figure reveals the averaged post mortem orientation dependent $R_{1}$ over all subjects. $R_{1}$ relaxation rate increases for an increasing fiber angle with respect to the main magnetic field between fiber angles of $\theta=0-50^{\circ}$, while $\mathrm{R}_{1}$ slightly decreases for the fiber angles between $\theta=55-90^{\circ}$.

Discussion: In situ post mortem $\mathrm{R}_{1}$ reveals a similar orientation dependency compared to the MD simulations of Schyboll et al. [1, 2]. The behavior found in this study of the post mortem $R_{1}$ orientation dependency reflects the orientation dependency of the averaged correlation time (reaches maximum at $\theta=50^{\circ}$ ) proposed by Schyboll et al. [1]. These post mortem findings would enable to investigate the biophysical meaning of the orientation dependency of $\mathrm{R}_{1}$ based on brain tissue histology.
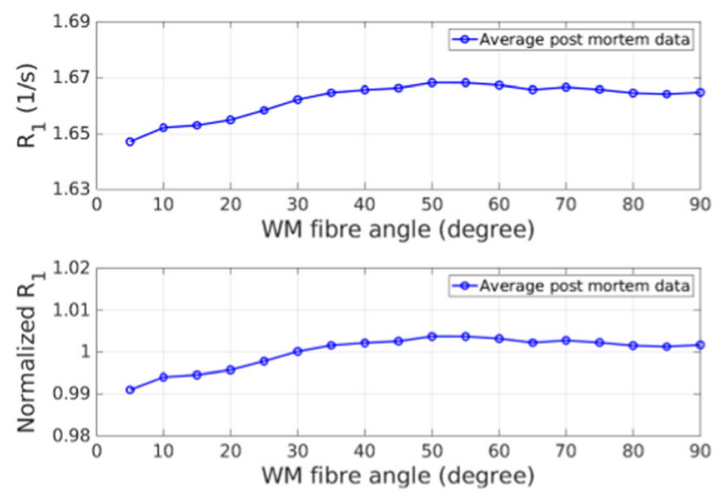

Figure 1: Absolute $R_{1}$ (upper row) and normalized $R_{1}$ to the mean $R_{1}$ of $W M$ (bottom row) as a function of the white matter fiber angle averaged over the post mortem subjects (blue).

\section{References:}

1. Schyboll F, Jaekel U, Petruccione F, Neeb H (2019) Fibre-orientation dependent $\mathrm{R} 1(=1 / \mathrm{T} 1)$ relaxation in the brain: The role of susceptibility induced spin-lattice relaxation in the myelin water compartment. J Magn Reson 300:135-141.

2. Schyboll F, Jaekel U, Petruccione F, Neeb H (2020) Origin of orientation-dependent R1 $(=1 / \mathrm{T} 1)$ relaxation in white matter. Magn Reson Med 84 (5):2713-2723.

\section{S6.P21.}

Predicting glioma genotype using n-component gaussian distributions of diffusion anisotropy distributions of normal-appearing white matter

*H. Halilibrahimoğlu ${ }^{1}$, K. Polat ${ }^{2}$, S. Keskin ${ }^{3}$, K. Özduman ${ }^{4,5}$, C. Yakıcier ${ }^{6}$, A. Ersen Danyeli ${ }^{5,7}$, M. N. Pamir ${ }^{5}$, A. Dinçer ${ }^{5,8}$, E. Öztürk-Iş1k ${ }^{1,5}$, A. Özcan ${ }^{2,5}$

${ }^{I}$ Bogazici University, Institute of Biomedical Engineering, Istanbul, $T R$;

${ }^{2}$ Boğaziçi University, Department of Electrical and Electronics Engineering, Istanbul, TR;

${ }^{3}$ Istanbul Technical University, Istanbul, TR;

${ }^{4}$ Acıbadem Mehmet Ali Aydinlar University, Department of Neurosurgery, Istanbul, TR;

${ }^{5}$ Aclbadem Mehmet Ali Aydinlar University, Center

for Neuroradiological Applications and Research, Istanbul, TR;

${ }^{6}$ YoctoSensum Biotechnoogy, Istanbul, TR;

${ }^{7}$ Acıbadem Mehmet Ali Aydinlar University, Department of Medical Pathology, Istanbul, TR;

${ }^{8}$ Aclbadem Mehmet Ali Aydinlar University, Department

of Radiology, Istanbul, TR

Introduction: In glioma patients, alterations of diffusion anisotropy indices (DAIs; apparent diffusion coefficient (ADC), fractional (FA), relative (RA) anisotropy) in NAWM may detect differences in varying tumor genotype [1]. However, multimodal Gaussian fitting (MGF) parameters may better model the competing effects of infiltrating cells, in contrast to standard summary statistics [2]. This study aims to investigate the predictive power of MGF parameters of DAIs and diffusion tensor eigenvalues (DTEs; E1, E2, E3) in the NAWM for glioma genotype prediction. 
Methods: In this IRB-approved study, 70 patients (age: $43.72 \pm 15.32$, F/M: 30/40) with written informed consent were included. T2-weighted (turbo spin echo, TE/TR $=107 / 3470 \mathrm{~ms}$, $0.26 \times 0.26 \times 0.26 \mathrm{~mm}$, axial, 20 slices) and diffusion-tensor imaging $(\mathrm{TE} / \mathrm{TR}=89.1 / 7733 \mathrm{~ms}, 1.8 \times 1.8 \times 1.8 \mathrm{~mm}, 60$ slices, $\mathrm{b}=1000 \mathrm{~s} / \mathrm{mm}^{2}, 20$ directions) were acquired on a $3 \mathrm{~T}$ TimTrio scanner (Siemens Healthineers, Erlangen, Germany). IDH (41 MUT/ $29 \mathrm{WT}$ ) and TERT (37 MUT/33 WT) mutation status were obtained using Sanger sequencing and defined 4 molecular subgroups: double negative (-), IDHonly $(+-)$, TERTonly $(-+)$, double positive $(++)$ with $9,24,20,17$ patients in each group, respectively. NAWM was semi-automatically segmented[3] and registered to diffusion space[4]. An in-house MATLAB ${ }^{\circledR}$ program separated NAWM masks into hemispheres and calculated DAIs from DTEs[5]. 1000-bin DAI and DTE distributions were fitted with $\mathrm{n}=1-6-$ component Gaussian functions (Fig. 1). To determine the number best-fitting Gaussians, root mean square error (RMSE) of each fit was averaged over all patients, yielding the mean RMSE (mRMSE) of n-component Gaussians. Parameters of the n-component Gaussians with the lowest mRMSE were chosen and mean-centered for machine learning algorithms (MLAs). MLAs available in MATLAB ${ }^{\circledR}$ were run using leave-one-out cross-validation with 70 times execution.
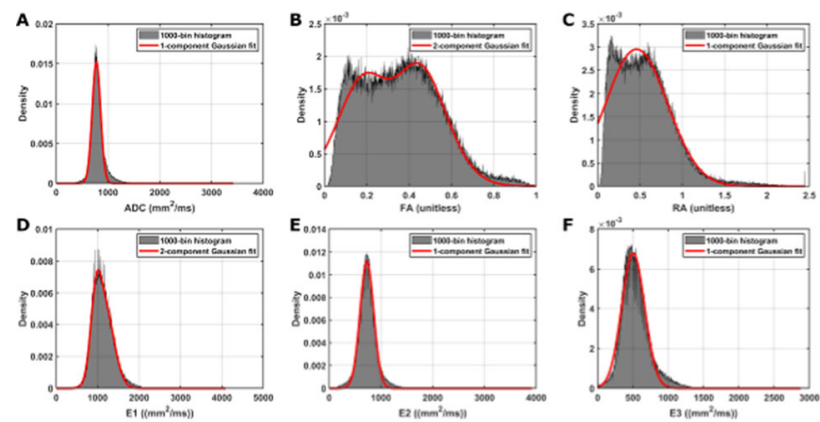
Figure 1: Whole brain NAWM (A) ADC, (B) FA, (C) RA, (D) E1, (E) E2, (F) E3 distributions of an example

Results: The number of best-fitting Gaussians and corresponding mRMSE are shown in Table 1. FA parameters in ipsilateral NAWM predicted IDH-TERT subgroups the best with $51.4 \%$ accuracy (Table 2). Ipsilateral E1 parameters predicted IDH mutation with $80.0 \%$ accuracy, while contralateral E3 parameters predicted TERT with $75.7 \%$.

\begin{tabular}{|c|c|c|c|c|c|c|c|}
\hline \multirow{2}{*}{$\begin{array}{c}\text { NAWM } \\
\text { Coverage }\end{array}$} & \multirow{2}{*}{ Diffusion Map } & \multicolumn{6}{|c|}{$\min$ mRMSE } \\
\hline & & \begin{tabular}{|l|} 
gauss1 \\
\end{tabular} & gouss 2 & gauss 3 & gauss 4 & gauss 5 & \begin{tabular}{|l|l|} 
gauss 6 \\
\end{tabular} \\
\hline \multirow{6}{*}{ Whole brain } & $A D C$ & 0.00032 & 0.00045 & 0.00040 & 0.00034 & 0.00035 & 0.00033 \\
\hline & FA & 0.00018 & 0.00017 & 0.00037 & 0.00031 & 0.00030 & 0.00023 \\
\hline & RA & 0.00020 & 0.00022 & 0.00039 & 0.00038 & 0.00034 & 0.00029 \\
\hline & $\mathrm{E} 1$ & 0.00032 & 0.00030 & 0.00059 & 0.00089 & 0.00093 & 0.00077 \\
\hline & E2 & 0026 & 0.00048 & 0.00072 & 0.00066 & 0.00061 & 0.00060 \\
\hline & E3 & 0.00032 & 0.00064 & 0.00064 & 0.00070 & 0.00068 & 0.00064 \\
\hline \multirow{6}{*}{ Contralateral } & $A D C$ & 0.00029 & 0.00039 & 0.00036 & 0.00033 & 0.00032 & 0.00031 \\
\hline & FA & 0.00019 & 0.00019 & 0.00037 & 0.00029 & 0.00029 & 0.00024 \\
\hline & $\mathrm{R}$ & 0.00021 & 0.00023 & 0.00037 & 0.00027 & 0.00033 & 0.00026 \\
\hline & E1 & 0.00032 & 0.00035 & 0.00053 & 0.00087 & 0.00091 & 0.00077 \\
\hline & E2 & 0.00032 & 0.00062 & 0.00069 & 0.00069 & 0.00063 & 0.00061 \\
\hline & $f$ & 0.00040 & 0.00061 & 0.00076 & 0.00069 & 0.00067 & 0.00063 \\
\hline \multirow{6}{*}{ Ipsilateral } & $A D C$ & 0.00035 & 0.00047 & 0.00042 & 0.00036 & 0.00036 & 0.00035 \\
\hline & FA & 0.00023 & 0.00024 & 0.00034 & 0.00026 & 0.00032 & 0.00026 \\
\hline & RA & 0.00023 & 0.00026 & 0.00038 & 0.00028 & 0.00028 & 0.00024 \\
\hline & E1 & & 0.00033 & 0.00067 & 0.00084 & 0.00087 & 0.0007 \\
\hline & E2 & 0.00043 & 0.00063 & 0.00078 & 0.00071 & 0.00068 & \\
\hline & & 0.00034 & .00061 & 0.00069 & 0.00070 & 0.00066 & 0.00062 \\
\hline
\end{tabular}

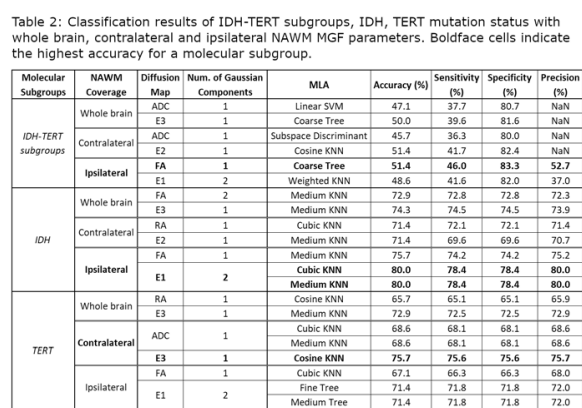

Discussion: This study demonstrates the potential of MGF parameters in NAWM for predicting tumor genotype. Use of resampling techniques may increase the performance of IDH-TERT subgroups classification, where there is class imbalance [6].

Acknowledgement: This work was funded by The Scientific and Technological Research Council of Turkey (TUBITAK) 1003 Grant No: 216 S432.

\section{References:}

[1] Jütten et al. Front Oncol. 2019; 9: 536.

[2] Pope et al. Radiology. 2009 Jul;252(1):182-9.

[3] Bazin et al. J Neurosci Methods. 2007 Sep 15; 165(1): 111-121.

[4] Smith et al. Neuroimage. 2004;23 Suppl 1:S208-19.

[5] Basser et al. J Magn Reson. 2011 Dec;213(2):560-70.

[6] Vabalas et al. PLoS One. 2019 Nov 7;14(11):e0224365.

\section{S6.P22.}

Complexity analysis of resting state functional magnetic resonance imaging (rsfMRI) data as an early diagnostic tool for Alzheimer's disease (AD)

*E. Samson ${ }^{1}$, S. Ulmer ${ }^{2}$, N. Simard ${ }^{1}$, M. Warsi ${ }^{1}$, K. Zukotynski ${ }^{1}$, M. D. Noseworthy ${ }^{1}$

${ }^{I}$ McMaster University, Hamilton, CA;

${ }^{2}$ University Medical Center Schleswig Holstein, Department of Radiology and Neuroradiology, Kiel, DE

Alzheimer's Disease (AD) is a neurodegenerative disorder associated with amyloid $(A \beta)$ deposition in the brain leading to cognitive impairment. While the diagnosis is typically based on clinical findings, imaging plays a key supportive role [i]. A tool for early diagnosis could improve understanding and provide better patient outcome.

Biological time-varying signals can be classified as statistical fractals [ii]. The brain regional fractal dimension (FD), or temporal complexity, can be determined using resting state functional magnetic resonance imaging (rsfMRI). Once the FD is known, the Hurst exponent can be determined. In $\mathrm{AD}$ there is reduced time domain complexity [iii]. Our hypothesis is that AD impacts specific brain regions creating a unique signature, evident in $\mathrm{H}$ frequency domain variants, which could serve as a metric for early diagnosis/establishment of disease severity. Specifically, rsfMRI may serve as a tool for the early diagnosis of AD and could improve our understanding of the underlying pathophysiology.

Patients with clinically suspected AD had a 3 T MRI with 32-channel head coil. Imaging included high resolution T1-weighted and resting state BOLD data. MR images of all patients was reviewed by a neuroradiologist to remove any patients from this data base, whose findings might also impair cognitive function (like amyloid angiopathy/strokes/...). Post-scan analysis with FSL [iv,v] included 
motion/eddy current correction/spatial warping to MNI152 atlas. Conversion of BOLD signals from time to power spectral density, detrended fluctuation analysis, and FD calculation was done with Matlab. Using normative age/sex matched controls, brain regional Z-score (ZFD) was calculated. ROI-based analysis was used to compare 91 regions from each brain with $\mathrm{ZFD} \leq-1.68$ defined as clinically important. Overall disease burden (ODB) was defined as the sum of all regions with significantly lower Z-scores (i.e., $<1.68$ standard deviations lower than the mean). Personalized regional brain FD anomalies were compared against regional brain hippocampal volumes determined using Neuroquant [vi].

The most affected brain regions were the secondary somatosensory cortex (74\%), Broca's area (63\%), the amygdala (53\%), insula (42\%), and hippocampus $(37 \%)$. For predicting ODB, significant metrics were the hippocampal volume $(\mathrm{P}<0.004)$, sex $(\mathrm{P}<0.04)$, and age $(\mathrm{P}<0.0005)$, with interaction terms of age:sex $(\mathrm{P}<0.02)$ and age:hippocampal volume $(\mathrm{P}<0.002)$. FD may thus be a useful metric in the assessment of clinically suspected AD. Future analysis will include neurocognitive scores and additional brain measures.
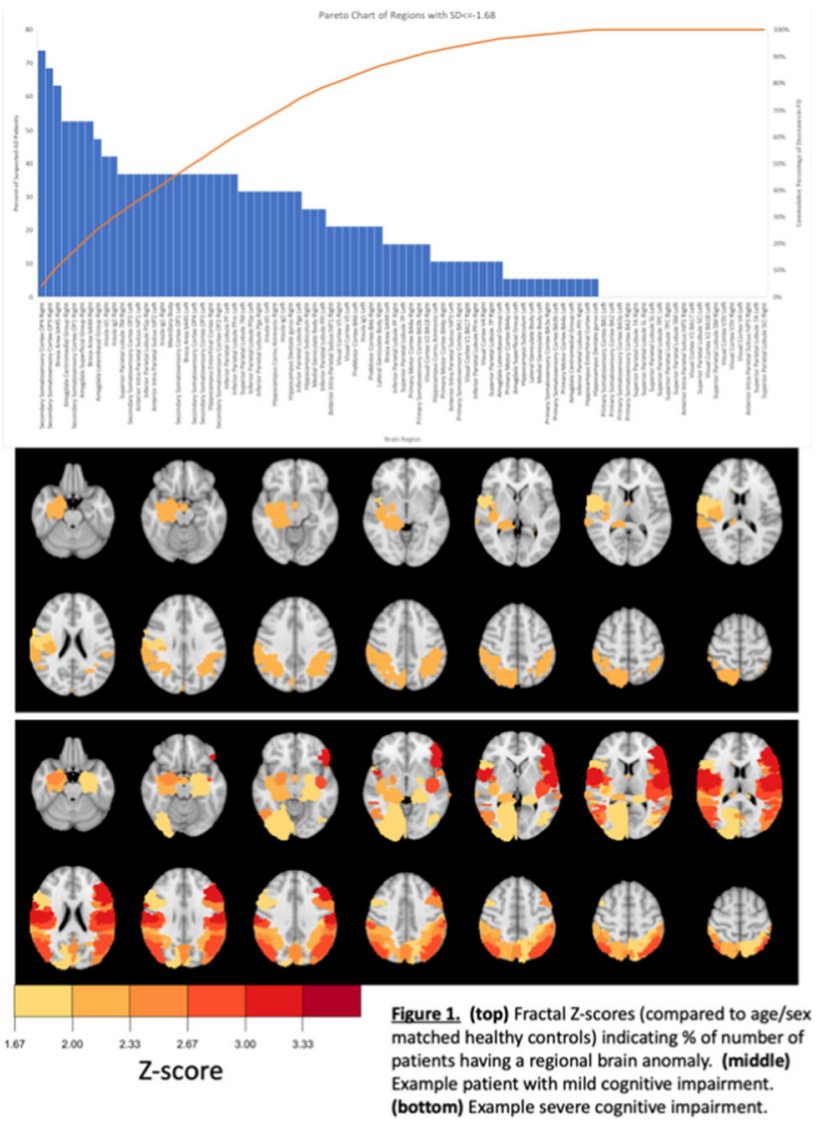

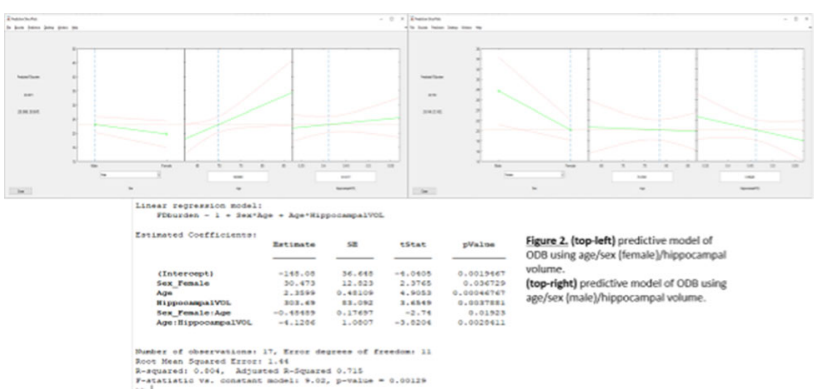

References:

[i] Smith SM, et al. (2004) NeuroImage, 23(S1):208-219.

[ii] Jenkinson M, et al. (2012) NeuroImage, 62:782-790.

[iii] https://www.cortechs.ai/products/neuroquant/.

[iv] Iliff J, et al. (2012) Sci Transl Med. 4(147):147ra111.

[v] Eke A., et al. (2000) Eur J Physiol. 439:403-415.

[vi] Warsi MA, et al. (2012) Magn. Reson. Matls Phys. Biol. Med. (MAGMA). 25(5):335-344.

\section{S6.P23.}

Reproducibility of $7 \mathrm{~T}$ measurements of the susceptibility and volume of hippocampal subfields

*O. Adeyemi ${ }^{1,2}$, O. Mougin ${ }^{1}$, P. Gowland ${ }^{1}$, R. Bowtell ${ }^{1}$, A. Hosseini ${ }^{1}$

${ }^{1}$ University of Nottingham, Department of Physics, Nottingham, GB;

${ }^{2}$ University of Abuja, Department of Physics, Abuja, GB

Introduction: Quantitative susceptibility mapping (QSM) at $7 \mathrm{~T}$ provides a powerful approach for characterising brain iron concentration at the sub-millimetre level1,2. Changes in tissue iron have been linked to a range of neurological conditions. In preparation for measurements of longitudinal changes in the hippocampal sub-fields in Alzheimer"s disease, we have characterised the reproducibility of high-resolution $7 \mathrm{~T}$ susceptibility measurements in the hippocampus. Methods: Ten healthy volunteers (age $32 \pm 6$ years) were scanned five times using a single-echo gradient echo (GE) sequence $\left(0.7 \times 0.7 \times 0.7 \mathrm{~mm}^{3}\right.$ resolution $\left.\mathrm{TE} / \mathrm{TR}=20 / 31 \mathrm{~ms} ; \mathrm{FA}=15 \mathrm{o}\right)$, along with similar resolution T1- and T2-weighted sequences 2, 3 . Susceptibility maps of the brain were created from the GE data using the multi-scale dipole inversion (MSDI) approach implemented in QSMbox4.

Hippocampal segmentation involved applying the open-source, ASHS software 5 to the $\mathrm{T} 1$ - and $\mathrm{T} 2$-weighted images using a pipeline that combines multi-atlas6 label fusion and learning-based error correction. Regions of interest (ROIs) delineated using this approach are: Cornu ammonis (CA) areas: CA1, 2 and 3, hippocampal tail (TAIL), dentate gyrus (DG), subiculum (SUB) \& entorhinal cortex (ERC). The average value of the susceptibility and volume of each of ROI was evaluated for each scan. Volumes of CA2 \& CA3 were too low to produce reliable susceptibility values, so CA1-3 values were combined into a single CA measurement.

Results: Figure 1 shows a susceptibility map, with overlaid hippocampus subfield classification for one subject. Figure 2 shows the average susceptibility and volumes in the 10 subjects, along with the standard deviation over repeated measurements. Figure 3 shows the 
average and standard deviation over subjects of the measured volumes and susceptibility.

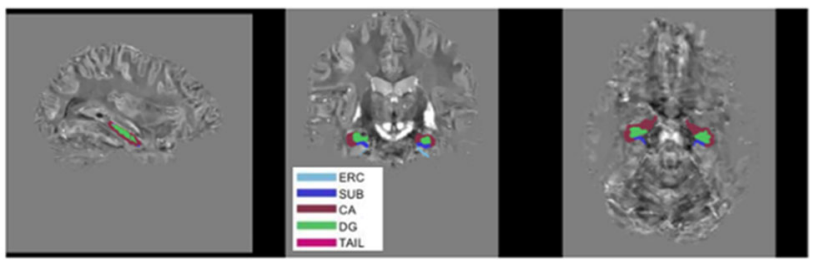

Fig. 1 Three-plane view of $0.7 \mathrm{~mm}$ resolution susceptibility map of one the subject with an overlay of the ASHS segmentation of the hippocampus.
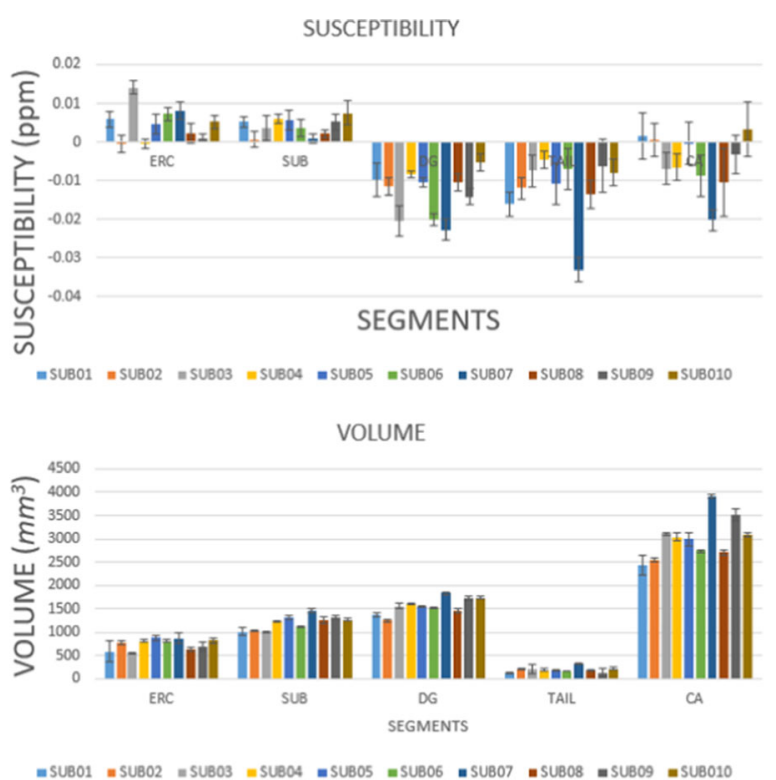

Fig. 2 Graph showing the volume ( $\mathrm{mm}^{3}$ ) and susceptibility (ppm) of the subfields of the hippocampus for the 10 subjects. Error bars show the standard deviation across repeated measwements.
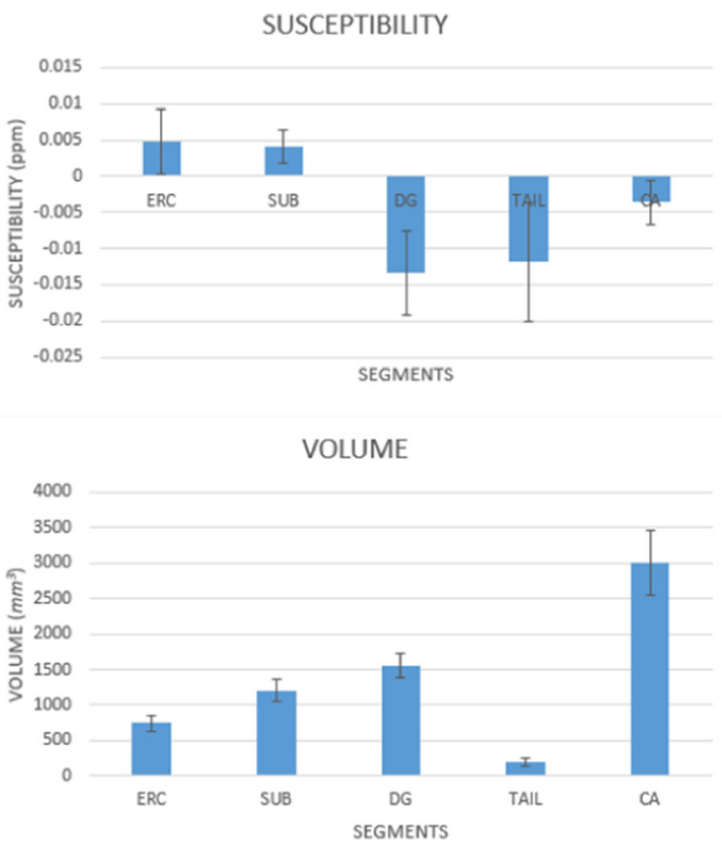

Fig. 3 Variation of the average values of the susceptibility and volume in the sub-fields across the ten participants. Error bars show the standard deviation across participants.

Discussion: Individual and average sub-field volumes (Figs. 2 and 3) are in good agreement with previously reported $7 \mathrm{~T}$ measurements6. The susceptibility values show a consistent pattern of variation across sub-regions in the range of -33 to $14 \mathrm{ppb}$ (parts per billion), with the ERC \& SUB/ DG \& TAIL showing consistently positive/negative values, while CA is generally slightly negative. The average standard deviation across the 5 repeated scans varied from 4 (TAIL) to 2 (ERC) ppb, while the standard deviation of average values across subjects was on average twice as large. These values were obtained with no additional susceptibility referencing; referencing to ROIs in CSF increased the variability of susceptibility measurements. These measurements provide an assessment of the level of susceptibility change that can be reliably characterised using high-resolution QSM in healthy subjects.

\section{S6.P24.}

Implementation of network metrics in neural connectivity analysis based on MRI for the assessment of idiopathic epilepsy patients

*C. Lisazo ${ }^{1,2,3}$, D. Fino ${ }^{1,2,4}$, N. Moyano Brandi ${ }^{1,2,3}$, S. Moguilner ${ }^{1,5}$, J. Capó Vera ${ }^{1,2,3}$, N. N. Massaccesi Bove ${ }^{1,2,3}$, F. González N. ${ }^{1,4,5}$, R. Otoya ${ }^{6}$, P. Ariza ${ }^{1,2}$

${ }^{l}$ Fundación Escuela de Medicina Nuclear, Department of Magnetic Resonance Imaging, Mendoza, AR;

${ }^{2}$ Fundación Argentina para el Desarrollo en Salud, Department of Magnetic Resonance Imaging, Mendoza, AR;

${ }^{3}$ University of Mendoza, Faculty of Engineering, Mendoza, AR;

${ }^{4}$ National University of Cuyo, Balseiro Institute, San Carlos de Bariloche, AR;

${ }^{5}$ Comisión Nacional de Energía Atómica, GQNYCS, Buenos Aires, $A R$;

${ }^{6}$ Neuromed, Mendoza, AR 
Introduction: Epilepsy is a disease characterized by abnormal structural and functional properties of brain networks. Structural and functional connectivity (SC and FC) based on magnetic resonance imaging (MRI) and their network metrics can be used to detect features of functional integration and segregation and identify certain patterns of local networks [1]. We aim to study the behaviour of connectivity matrices/metrics to recognize hypo or hyperconnected areas that could be associated with epileptogenic zones.

Methods: This study was approved by the Institutional Review Board, and it comprised 10 patients ( 3 women, 7 men; mean age $31 \pm 10$ years; range $21-50$ years) with refractory idiopathic epilepsy. In addition to a standard protocol, resting-state functional MRI (rs-fMRI) and diffusion-weighted images (DWI) in 64 directions were acquired on a $3.0 \mathrm{~T}$ Signa PET/MRI system (General Electric HC, Fig. 1). FC was obtained using the CONN toolbox (Matlab R2019b), to estimate seed-based connectivity measures. On the other hand, SC was quantified with probabilistic tractography based on the ball and stick model, using FSL Software. Network measures, including betweenness centrality (BC), cluster coefficient (CC), degree, local efficiency (LE), strength, and edges distance (ED), were calculated for SC and FC with the Brain Connectivity Toolbox. Significant differences in the connections and metrics between a single patient and a group of healthy controls $(n=70$ [2]) were found using a Crawford-Howell modified $t$ test. Each $p$ value was then transformed to its multiplicative inverse ( $\mathrm{h}$ value), obtaining contrast matrices and metrics (Fig. 2). Clusters of 4 or more regions with significant differences (above a threshold of an $\mathrm{h}$ value) were identified and compared with the location of the epileptogenic zone according to the patient"s clinical diagnosis. Sensitivity and specificity were calculated for different thresholds (ROC curves), and the threshold cut-off value was quantified for each matrix and metric to achieve the best performance.

\begin{tabular}{|c|c|c|c|c|}
\hline & & DWI-Tractography & RS-fMRI & $\begin{array}{l}\text { Tractography + } \\
\text { RS-fMRI }\end{array}$ \\
\hline \multirow{5}{*}{ 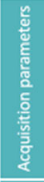 } & Scanning sequence & EPI_SE & EPI_GRE & - \\
\hline & $\begin{array}{l}\text { Slice thickness [mm] / } \\
\text { Gap [mm] }\end{array}$ & $3 / 0$ & $4 / 0$ & $\cdot$ \\
\hline & Matrix size & $80 \times 80$ & $96 \times 96$ & $\cdot$ \\
\hline & $\mathrm{TE}[\mathrm{ms}] / \mathrm{TR}[\mathrm{ms}]$ & $92.7 / 12000$ & $23 / 2800$ & - \\
\hline & Flip angle & 90 & 90 & $\cdot$ \\
\hline \multirow{3}{*}{ 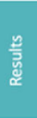 } & $\begin{array}{l}\text { Threshold cut-off } \\
\text { h-value }\end{array}$ & 9 & 6 & 50 \\
\hline & Sensitivity & $99.8 \%$ & $99.7 \%$ & $99.9 \%$ \\
\hline & Specificity & $93 \%$ & $96 \%$ & $93.6 \%$ \\
\hline
\end{tabular}

FIGURE 1: Acquistion porometers and results

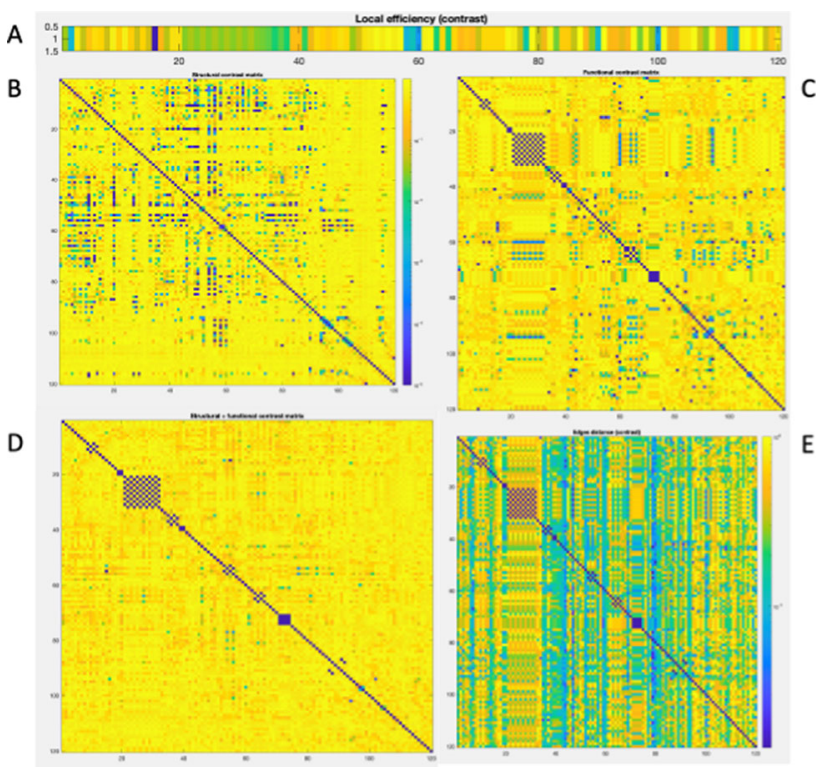

FIGURE 2: (A) Local efficiency contrast measures; (B) Structural contrast matrix; (C) Functional contrast matrix; (D) Structural + functional contrast matrix; (E) Edges distances contrast measures

Results: Results are summarised in Figs. 1 and 3. SC, FC and $\mathrm{SC}+\mathrm{FC}$ matrices showed high sensitivity and specificity values. The specificity of FC is higher than that of SC. The ED and CC metrics showed better performance than $\mathrm{LE}, \mathrm{BC}$, degree and strength.

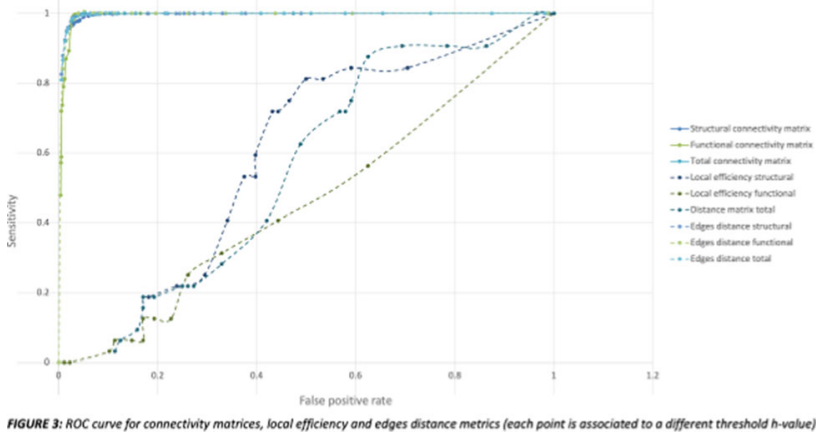

Discussion: The analysis of contrast FC and SC matrices, together with the ED and CC metrics, could be a possible method to implement in the evaluation of individual patients. Sensitivity and specificity values were high because the considered epileptogenic zones were up to $8 \mathrm{~cm}^{2}$; in the future, we aim to add information provided by EEG and PET to reduce the areas up to $2 \mathrm{~cm}^{2}$.

\section{References}

[1] Luo, D. An, D. Yao, and J. Gotman, "Patient-specific connectivity pattern of epileptic network in frontal lobe epilepsy," NeuroImage: Clinical, vol. 4, pp. 668-675, 2014.

[2] "OpenNeuro." https://openneuro.org/datasets/ds000030 (accessed Jan. 02, 2021). 


\section{S6.P25.}

Automated cell motion pattern categorization of single iron-labelled monocytes in time-lapse MRI using an adapted cell tracking tool

\author{
*I. Fredrich ${ }^{1}$, E. Wilken ${ }^{1}$, M. Masthoff ${ }^{1}$, A. Helfen ${ }^{1}$, C. Faber ${ }^{1}$ \\ ${ }^{1}$ University Hospital Münster, Münster, DE
}

Introduction: Time-lapse MRI provides a non-invasive tool for three-dimensional tracking of single labelled immune cells with whole-brain coverage in vivo. ${ }^{1,2}$ However, manual cell counting and pattern categorizing is time-consuming and elaborate. We aimed to improve analysis of time-lapse MRI data via an adapted cell tracking tool that links cell positions to trajectories allowing for automated motion pattern categorization.

Methods: In vivo time-lapse MRI using a repeated T2* gradient echo sequence $^{1}$ of 15 mouse brains was performed on a $9.4 \mathrm{~T}$ Biospec (Bruker Biospin) small animal MRI. Monocytes were labelled in vivo by i.v. injection of Ferucarbotran (Resovist, Bayer AG) $24 \mathrm{~h}$ before MRI.

First, detected cells were counted manually and were categorized in short-term short-range (1-2 consecutive time frames), long-term short-range ( $>2$ consecutive time frames), or long-term long-range ( $>2$ consecutive time frames and cell movement) motion patterns. Second, for automated categorization, cells were tagged in Image J and coordinates were linked to trajectories (Fig. 1) using the corresponding part of the MATLAB implementation by D. Blair and E. Dufresne $^{3}$ of the IDL particle tracking code of J. Crocker, D. Grier, E. Weeks ${ }^{4,5}$. Hereby, patterns were classified as before with a defined minimum travel distance of 1.2 pixels for a long-term long-range pattern. Mean velocity of moving cells was also calculated.
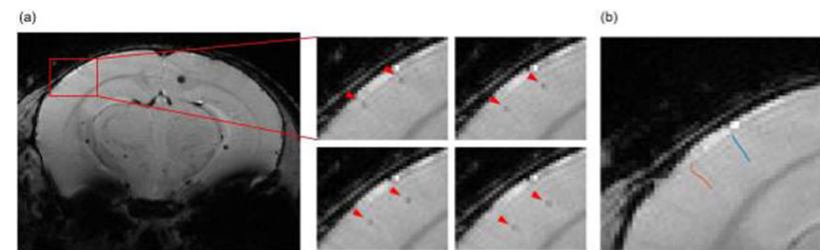

Figure 1: Reprosentative oxarnole of one slice of a mouse brain dermonstrating in viro dotected cells in motion. a) Image details, indicated by the red rectangle, of tour sequental time frames showing wo migrating cells (red arrowheac) in the brain contex as hypointense spols.

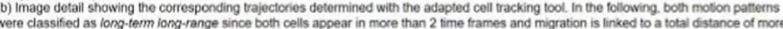
than 1.2 pixels

Results: For comparison of manual categorization versus the automated tool, Bland-Altman analysis was performed resulting in a positive bias for the total number of detected cells $(2.53 \pm 7.23)$ as well as for the short-term short-range $(2.33 \pm 7.84)$ and long-term short-range $(2.47 \pm 3.50)$, and a negative bias for long-term longrange $(-2.27 \pm 3.08)$ motion pattern (Fig. 2). Overall, reliability of the automated approach seems to be higher when fewer cells are tagged.
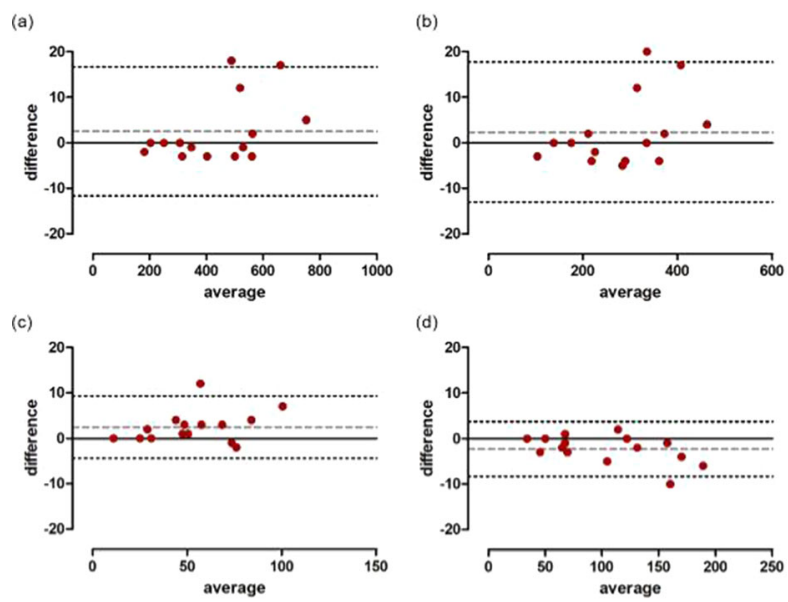

Figure 2: Bland-Altman plots comparing manually counted cells and cells categorized by the adapted cell tracking tool for 15 analyzed mice. The dashed grey line represents the mean bias, the dotted black lines represent the $95 \%$ limits of agreement, respectively.

(a) total number of cells (bias of $2.53 \pm 7.23,95 \%$ limits of agreement between -11.64 and 16.70 average of $437 \pm 167$ manually counted compared to $440 \pm 170$ automatically counted cells) (b) short-term short-range pattern (bias of $2.33 \pm 7.84,95 \%$ limits of agreement between -13.04 and 17.70 , manual $281 \pm 100$ vs. automated $283 \pm 104)$

(c) long-term short-range pattern (bias of $2.47 \pm 3.50,95 \%$ limits of agreement between -4.40 and 9.33 , manual $52 \pm 24$ vs. automated $55 \pm 25$ )

(d) long-term long-range pattern (bias of $-2.27 \pm 3.08,95 \%$ limits of agreement between -8.31 and 3.77 , manual $104 \pm 51$ vs. automated $102 \pm 49$ )

Furthermore, the mean velocity of moving cells was determined to be $0.20 \pm 0.06 \mu \mathrm{m} / \mathrm{s}$ (Fig. 3), matching the speed of patrolling monocytes. $^{6}$

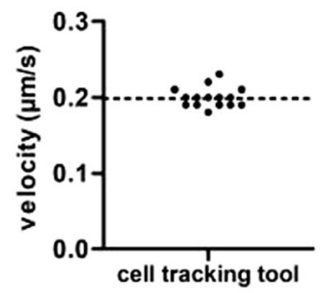

Figure 3: Mean velocity of all cells moving across at least 3 pixels was determined to be $0.20 \pm 0.06 \mu \mathrm{m} / \mathrm{s}$. For 15 analyzed mice, dots show the mean velocity of moving cells and the dotted line represents the overall mean.

Discussion: The automated cell tracking tool allows for a reliable analysis regarding motion pattern categorization of iron-labelled monocytes in time-lapse MRI of mouse brains and can therefore be used to analyze data more efficiently. To further improve observer dependence of time-lapse MRI data analysis, we aim at developing a tool that automatically detects labelled cells too.

\section{References}

1. PMID: 29934611

2. PMID: 25385430

3. Blair, D. \& Dufresne, E. "The Matlab Particle Tracking Code Repository", http://site.physics.georgetown.edu/matlab/ (accessed $04 / 30 / 21)$

4. Crocker, J. \& Grier, D. "Methods of Digital Video Microscopy for Colloidal Studies." (1996).

5. Crocker, J. \& Weeks, E. R. "Particle tracking using IDL", http:// www.physics.emory.edu/faculty/weeks//idl/ (accessed 04/30/21)

6. PMID: 17673663 


\section{S6.P26.}

\section{Optimizing T2 mapping of knee cartilage with dictionary-based methods}

\author{
*Â. T. Nunes ${ }^{1,2}$, A. C. Freitas ${ }^{2}$, J. M. Coelho ${ }^{3,4}$, L. Nogueira ${ }^{3,5,6}$, \\ R. G. Nunes ${ }^{2}$ \\ ${ }^{1}$ University of Lisbon, Department of Physics, Lisbon, PT; \\ ${ }^{2}$ University of Lisbon, Institute for Systems and Robotics / Department \\ of Bioengineering, Lisbon, PT; \\ ${ }^{3}$ Escola Superior de Saúde do Politécnico do Porto, Oporto, PT; \\ ${ }^{4}$ University of Porto, Radiology Department, Oporto, PT; \\ ${ }^{5}$ University of Porto, EPIUnit, Oporto, PT; \\ ${ }^{6}$ Laboratory for Integrative and Translational Research in Population \\ Health (ITR), Oporto, PT
}

Introduction: $\mathrm{T}_{2}$ mapping allows early detection of osteoarthritis(OA) in knee cartilage. OA is characterized by loss of collagen integrity and increased water content resulting in longer $\mathrm{T}_{2}$ relaxation times [1]. Previous studies have reported mean population $T_{2}$ values for healthy and OA cartilage of 35 and $40 \mathrm{~ms}$, respectively [2]. Due to time constraints, clinical $T_{2}$ mapping is usually performed with a multi-spin-echo (MSE) sequence and mono-exponential fitting. Recently, dictionary-based methods have enabled more accurate $\mathrm{T}_{2}$ estimates by matching the MSE data to a dictionary of pre-computed echo modulation curves (EMC) [3]. Our aim is to compare different MSE protocols for knee cartilage $T_{2}$ mapping, varying the refocusing pulse as it impacts RF power tissue deposition (SAR). Protocols were applied to the ISMRM phantom and initial tests were performed in vivo.

Methods: Several MSE sequences were acquired in the ISMRM phantom [4] (Fig. 1a) on a 3 T Philips Achieva, with refocusing flip angles (FA) of $[90,120: 5: 145,180]^{\circ}, 90^{\circ}$ excitation pulse, echo train length $=10, \mathrm{FOV}=210 \times 210 \mathrm{~mm}^{2}$, in-plane reconstructed resolution $=0.29 \times 0.29 \mathrm{~mm}^{2}$ and $2.5 \mathrm{~mm}$ slice thickness. An inter echo spacing of $5.8 \mathrm{~ms}$ (the smallest allowed by the software) was chosen to potentiate the detection of short $\mathrm{T}_{2}$ values [5]. To create the EMCs, $\mathrm{T}_{1}$ was fixed at $1000 \mathrm{~ms}$ (a prior simulation study revealed very low sensitivity to $\mathrm{T}_{1}$ ); transmit field $\mathrm{B}_{1+}=0.6: 0.01: 1.4$ and $\mathrm{T}_{2}=[1: 0.2: 10,10: 0.5: 50,50: 1: 100] \mathrm{ms}$ values were considered [3]. $\mathrm{T}_{2}$ maps were generated for each protocol with both methods (EMC and mono-exponential fit) and compared to the ISMRM phantom values. The best candidate protocol was applied in vivo in a preliminary validation test in an asymptomatic subject.

Results: The $\mathrm{T}_{2}$ map and mean values estimated with the EMC and mono-exponential fit methods for the different protocols in the phantom vials with $T_{2}$ values close to those previously reported for the knee cartilage are shown in Figs. $1 \mathrm{~b}$ and 2. $\mathrm{T}_{2}$ mapping of the cartilage is shown in Fig. 3. (a) Phantom T2 Weighted

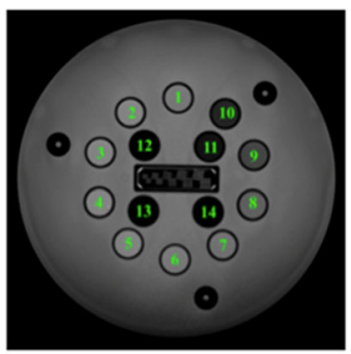

(b) Phantom T2 map (ms) with ROI mask

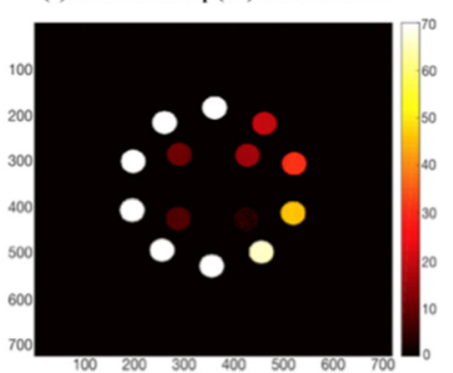

Figure 1 - (a) $T_{2}$ weighted image of the ISMRM quantitative MRI $T_{2} / T_{2}$ phantom, numbers indicate the different $T_{2}$ vials in the phantom. (b) $T_{2}$ map (ms) obtained with EMC $\mathrm{H}_{\text {, showing only the vials with the background set to }}$
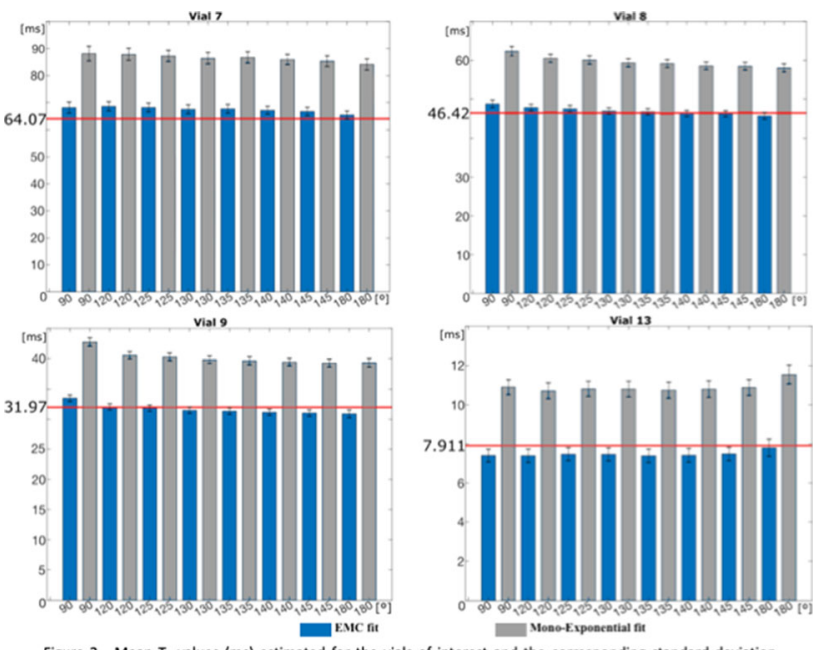

sing different RF refocussing ( estimated for the vials of interest and the corresponding standard deviation red line indicates the reference value for each vial [4].

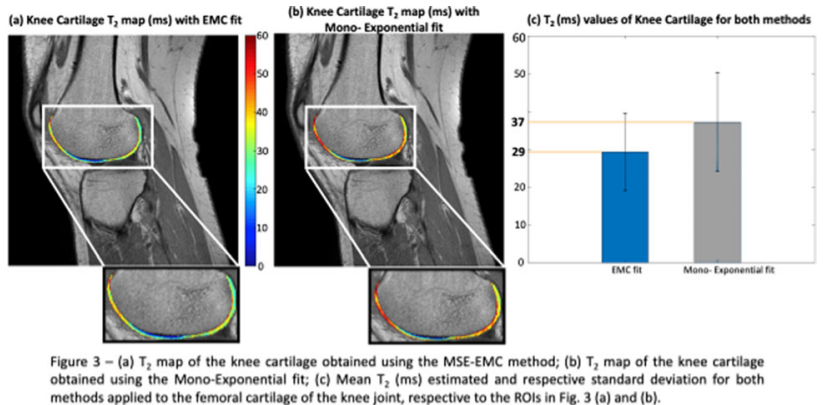

Discussion: Higher accuracy of the EMC method was confirmed at both short and long $T_{2}$ values. For short $T_{2}$ values (vials 8,9 and 13) the use of a $90^{\circ}$ refocusing FA resulted in poorer accuracy, while for longer $\mathrm{T}_{2}$ (vial 7) more accurate estimates were obtained for the tested protocols. By using a refocusing FA of at least $120^{\circ}$, accurate estimates could be obtained. However, to limit SAR, the use of a $120 / 125^{\circ}$ refocusing FA may be preferred in vivo. Regarding the in vivo preliminary test, the $T_{2}$ value of the cartilage agreed with previous studies, as the EMC fit $(27 \pm 13 \mathrm{~ms})$ has lower values compared to the mono-exponential fit $(38 \pm 14 \mathrm{~ms})$. Future work will be to evaluate the in vivo reproducibility.

\section{References}

[1] Joseph, Osteoarthritis and Cartilage, 2012,20(7),727-35.

[2] Li, Osteoarthritis and Cartilage, 2007,15(7),789-97.

[3] Ben-Eliezer, Magn Reson Med, 2015,73(2),809-17.

[4] Jiang, Magn Reson Med. 78(2017),1452-57.

[5] Sharafi, JMRI. 2017;47:809-19. 


\section{Author Index}

A

Aamir, F. S3.P13, S3.P17

Abdulrazaq, Z. S5.O3

Abreu, R. S4.P12

Adema, G.J. S1.P9

Adeyemi, O. S6.P23

Afshari, R. S3.O3

Ahangari, S. C2.O4

Aime, S. S4.P20

Akhadov, T. S2.O6, S2.P20, S2.P21, S2.P27, S4.P17, S4.P19

Akl, P. S6.O2

Alberola-López, C. S3.P10

Alecci, M. S5.O4

Algarín, J.M. S3.P18, S5.O6, S5.P14, S5.P15

Allelein, A. S4.P15

Allen, S. S1.O1

Alonso, J. S5.O6, S3.P18, S5.P14, S5.P15

Alves, L. S4.P10

Anania, V. C1.O5

Anazodo, U. S2.P9

Andersen, F.L. C2.O4

Anzalone, N. S6.P9

Arbabi, A. S3.P20

Arias-Ramos, N. S5.P7, S1.P10, S2.P14, S2.P19, S2.P7

Ariza, P. S1.P2, S1.P4, S1.P6, S6.P24

Arpak, A. S6.P8

Arribarat, G. S4.P27

Arslan, D.B. S2.P24, S2.P25

Arzanforoosh, F. S4.P25, S4.O3

Aslam, I. S3.P13, S3.P17

Assili, S. S1.O5

Auer, D. S5.P9

Ayres-Ribeiro, F. S4.P12, S4.O9

Azamat, S. S2.P24, S2.P25

Azilinon, M. S2.O1

\section{B}

Baas, K.P.A. S4.P3

Backhaus, P. S4.P21

Baete, K. C2.O6

Bamberg, F. S6.P16

Bao, Q. S3.P21

Barbier, E.L. S4.O4

Bardin, S. S3.P22

Barile, B. S6.P14

Barkhof, F. S4.P2, S4.P8

Bartolomei, F. S2.O1

Bas, A. S6.P4

Battini, R. S3.P25

Baudin, P.-Y. S6.P5

Bauer, M. S2.P6, S6.P20

Bauman, G. S1.O2, S2.09, S3.O3, S3.P1, S3.P2, S3.P3

Baumhauer, M. S6.P19

Baş, A. S6.P10

Beenakker, J.-W. S1.P14

Beirinckx, Q. C1.O3, C1.O7

Belousov, M.V. S1.O6
Belyanin, M.L. S1.O6

Benlloch, J.M. S5.O6, S5.P14

Berg, R. S3.06

Bergen, T.A. S1.P7

Berger, C. S6.P20, S2.P6

Bernard, M. S2.P18

Berthelsen, A.K. C2.O4

Besson, F. C2.O2

Beyer, T. C2.O1

Biagi, L. S3.P25

Biemond, B. S2.P12

Bieri, O. S2.09, S1.O2, S3.O3, S3.P1, S3.P2, S3.P3, S5.P10, S5.P16

Bilgic, B. S2.P24, S2.P25

Billiet, T. C1.O5, C1.O8

Birkl, C. S6.P20

Blackledge, M. S1.O1, S6.P19

Blaimer, M. S3.O8

Blockley, N. S4.06, S4.O7

Boccalini, S. S1.O3

Bock, M. S3.O4

Bock, N.A. S4.P24

Bogomyakova, O. S2.O5, S2.P1, S2.P11

Boitard, C. S1.P11

Bonvento, G. S6.O7

Boon, M.R. S1.O4

Borodin, O.Y. S1.O6

Borreguero, J. S3.P18, S5.O6, S5.P14

Bortolotti, L. S6.P17

Bosch, R. S5.O6, S5.P14

Boschi, R. S1.P6

Bosco, P. S3.P25

Botnar, R. C2.O5

Boulant, N. S3.P9

Boumezbeur, F. S2.07

Boussel, L. S3.P15

Bouzier-Sore, A.-K. S2.P16

Bowtell, R. S5.P9, S6.P17, S6.P23

Bozhko, O. S2.P20, S2.P23, S4.P19

Brackenier, Y. S3.P7

Breuer, F. S3.O8

Bright, M. S2.O4

Brill, R. S6.O1

Broche, L. S2.O8

Brook, M. S5.P9

Buck, M.A. S4.O5, S4.P1

Budde, T. S2.O2

Buikema, A.E. C1.O5

Bulanov, P. S1.P5, S2.P21, S2.P20

Bulte, D.P. S4.O7

Buonincontri, G. S3.P25

Burd, P.R. S3.O8

Burhan, A. S2.P9

Buz Yaluğ, B. S6.P11

Byanju, R. C1.O6

Bödenler, M. S2.O8

Büther, F. S4.P21

C

Caballero-Gaudes, C. S2.O4

Cachado, F. S3.P11, S3.P12

Cakmak, J. S2.P9
Calado, S. S4.P10

Caldas de Almeida Araujo, E. S6.P5

Caligiuri, M.E. S2.P8

Calimeri, F. S6.P9

Calimeri, T. S6.P9

Candito, A. S6.P19

Capela, C. S2.P4

Capobianco, N. C2.O7

Capó Vera, J. S1.P2, S1.P4, S1.P6, S6.P24

Carril Garcia, M. C3.O1, C3.O3

Carvalho, J. S4.P26

Cascini, G.L. S2.P8

Casula, V. S1.O5

Cavallotti, C. S4.P20

Caverzán, M.D. S5.P7

Cebi, M. S2.P25

Cecchetti, L. S4.O1

Cecchi, P. S3.P25

Cencini, M. S3.P25

Cengiz, S. S2.P24, S2.P25

Cerdán, S. S2.P14, S2.P19

Cerqueira, L. S2.P4

Chalampalakis, Z. C2.O2

Chang, C.-K. S3.P14

Chang, H.-C. S3.P14

Channaveerappa, M. S4.P7

Chantadisai, M. C2.O7

Chao, Y.-C. S3.P14, S6.P12

Chatterjee, S. S6.O3

Chavarrias, C. S4.P26

Chen, C.-C. S3.P14

Chen, G. S2.O4

Chen, H.-C. S3.P14, S6.P12

Chen, H.-F. S4.P15

Chesta, C.A. S5.P7

Chevalier, P. S1.O3

Chiaffarelli, R. S3.O1

Ciobanu, L. S3.P22

Cirillo, C. S4.O4

Claesener, M. S4.P21

Clement, J. S5.P6

Clement, P. S4.P2, S6.08

Cloos, M.A. S1.O5

Clouchoux, C. S6.O7

Cocking, D. S5.P9

Coelho, J.M. S6.P26

Comino Garcí-Muñoz, A. S2.P18

Comtat, C. C2.O2

Conceição, R. S1.P3

Correia, M. C1.O4

Cosottini, M. S3.P25

Costagli, M. S3.P25, S4.O1

Coudyzer, W. C2.O6

Couture, O. S1.P11

Craven-Brightman, L. S5.P15

Cristobal-Huerta, A. C1.O6

Cruz, G. S3.P8

Cruz, L.J. C3.O2

D

D'Amelio, C. S3.P25

da Silva, N.A. S1.P3 
Damion, R. S5.P9

Danielli, E. S2.P10

Dawood, P. S3.O8

de Bresser, J. S4.O2

de Bruin, P. S4.P4

de la Rosa, E. C1.O8

De Momi, E. S6.P9

de Vos, B. S5.O1

Debruin, H. S4.P14

Deistung, A. S6.O1

Del Pópolo, M. S1.P2, S1.P4

Deligianni, X. S5.P10, S5.P16

Delzescaux, T. S6.O7

Demiralp, T. S2.P24, S2.P25

den Dekker, A.J. C1.O2, C1.O3, C1.O4, C1.05, C1.O7

Derks, S. S2.P26

Deroose, C. C2.O6

Desmoulin, F. S4.O4

Destito, M. S6.P9

Di Gregorio, E. S4.P20

Dias, J.M. S3.P12

Dijsselhof, M. S4.P2, S4.P3, S4.P8, S6.O8

Dincer, A. S6.P4

Dinçer, A. S6.P10, S6.P11, S6.P21, S6.P7, S6.P8

Dirven, L. S4.O2

Dive, D. C1.O8

Doblas, S. S1.P11

Doganay, O. S3.P24

Domingos, C. S4.P9

Domínguez, A. S1.P6

Donatelli, G. S3.P25

Donnay Pérez, M. S1.P10

Douek, P. S1.O3, S3.P15

Doughty, M. S2.P10

Downey, K. S1.O1

Driessen, D. S1.P9

Dubovaya, A.V. S1.P7

Dumoulin, S. S4.P16, S6.O4

Durand-Dubief, F. C1.08, S6.P14

Duvivier, V. S1.P11

Dziadosz, M. S6.P2, S6.P3

Dzyubachyk, O. S1.O4

Díaz, E. S5.06

\section{$\mathbf{E}$}

Echternach, M. S3.O4

Edwards, L. S6.O5, S3.O2

Eggenreich, S. S6.O6

Ehses, M. S5.O2

Eiber, M. C2.O7

Ekşi, M.Ş. S6.P11

El-Mendili, M. S2.O1

Ercan, E. S4.O2

Erdoğdu, E. S2.P24, S2.P25

Ernemann, U. S4.P22

Ersen Danyeli, A. S6.P10, S6.P11, S6.P21, S6.P4

Esteves, I. S4.P11, S4.O9, S4.P12, S4.P9

\section{F}

Faber, C. S2.O2, S2.O3, S3.P22, S3.P5, S4.O8, S4.P15, S4.P21, S6.P25
Fasano, L. S2.P18

Fave, Y. S4.P27

Fedorova, Y. S2.P23

Fernandes, F. S4.P26

Fernandes, T.T. S3.P23

Fernández-Cabello, S. S4.P18

Ferreira, H. S2.P4, S4.P13

Ferreira, T. S1.P14

Ferreri, A.J.M. S6.P9

Fierstra, J. S4.P23

Figueiredo, P. S2.P2, S4.O9, S4.P10, S4.P11, S4.P12, S4.P9

Filimonov, V.D. S1.O6

Finger, E. S2.P9

Fino, D. S1.P2, S1.P4, S1.P6, S6.P24

Fischbach, F. S1.P12

Fischer, B.M. C2.O4

Fischer, J. S3.O4

Flament, J. S6.O7

Fomin, I. S1.P12

Fortunati, V. C1.O14

Fouto, A. S4.P10, S4.P11, S4.P9

Francisco, A. S4.P12

Frangi, A. C1.O11, C1.O12

Franks, H. S5.P9

Fredrich, I. S6.P25

Freitag, D. S2.P15

Freitas, A.C. S3.P12, S3.P23, S6.P26

Freppon, F. S3.P5

Frey, B.N. S4.P24

Fritz, F.J. S5.P8

Frouin, F. S6.O2

Frydman, L. S3.P21

Fuchs, P. S3.O5

Furlan, C. S1.P13

\section{G}

Gafita, A. C2.O7

Galante, A. S5.O4

Galguera, D. S2.P19

Galve, F. S3.P18, S5.O6, S5.P14

Garello, F. S1.P13

Garipov, R. C1.O10

Garteiser, P. S1.P11, S1.P8

Gaspar, A. S3.P16, S3.P11, S3.P12, S3.P23

Gatidis, S. S3.O9

Gaudin, M. S6.O7

Geethanath, S. S3.P23

Gianolio, E. S4.P20

Gierse, F. S4.P21

Gil-Gouveia, R. S2.P2, S4.P11

Gilbank, A. S4.P24

Giraud, A.-L. S4.O9

Gleißner, C. S6.P13

Glocker, B. C1.O4

Goffin, K. C2.O3

GOKPEK, Y. S3.P24

Golay, X. S4.P2, S4.P8

Golshani, S. C1.O12

Gomolka, R. S2.P13

González, J.M. S5.O6, S5.P14

González N., F. S1.P2, S1.P4, S1.P6, S6.P24

González Padin, T. S1.P6, S1.P2

Gonçalves, C. S4.P10

Gonçalves, S. S3.P21
Gooding, M. C2.O6

Gordon, K. S1.P1

Gothard, L. S1.O1

Gouveia Nunes, R. S4.P10, S4.P9

Gowland, P. S6.P23

Gramage, C. S5.O6, S5.P14

Grau-Ruiz, D. S5.O6, S5.P14

Gregori, J. S4.P2, S4.P8

Gritskova, A. S2.P27

Gros-Dagnac, H. S4.P27

Guadilla Gómez, I. S2.P14

Guerra, A. S1.P3

Gufler, H. S2.P15

Guillén Gómez, M.J. S2.P7, S5.P7

Gulyaev, V.M. S1.P7

Guns, P.-J. C1.O4

Gussew, A. S6.O1

Guye, M. S2.O1

Göttler, J. S4.P6, S6.P13

Günther, M. S3.P27, S4.O5, S4.P1, S4.P2, S4.P5, S4.P7, S4.P8

Gürvit, H. S2.P24, S2.P25

\section{H}

Haast, R.A.M. S5.P8

Habel, U. S2.P3

Had, L. S2.P18

Halilibrahimoğlu, H. S6.P10, S6.P11, S6.P21

Hammernik, K. S3.O9, S3.P8

Hanagasi, H. S2.P24, S2.P25

Handjaras, G. S4.O1

Hansen, A.E. C2.O4

Hansen, N.L. C2.O4

Harkin, J.W. S4.O7

Harrevelt, S. S3.P14

Harris, E. S1.O1

Haueise, T. S6.P16

Hauser, T.-K. S4.P22

Hebbelmann, L. S2.O2

Heij, J. S4.P16, S6.O4

Helfen, A. S6.P25

Helms, G. S3.O2, S6.O5

Hensen, B. S1.P12

Hermann, S. S4.P21

Hernandez-Tamames, J.A. C1.O6, S4.O3

Herrmann, K.-H. S2.P15

Hicks, J. S2.P9

Hirschler, L. S4.O4

H.J.Poot, D. C1.O1

Ho, B. S1.P1

Hoerr, V. S3.P22

Hoffmann, G. S4.P6

Hoffmann, S.H.L. S3.O1

Hoinkiss, D. S4.P5, S4.P7

Hoinkiss, D.C. S3.P27

Holbrey, R. S6.P19

Holly, S. S2.P22

Hopkinson, G. S1.O1

Hosseini, A. S6.P23

Howe, F.A. S1.P1

Huber, J. S4.P5, S4.P7, S4.O5, S4.P1

Huber, L. S3.P19

Huber, R. S4.P18

Hussain, M. S5.P11 
Hwang, F.-N. S3.P14

I

Ianus, A. S4.P26

Ibarra, L.E. S5.P7

Ibrahim, T. C2.O5

Ignatenko, G.A. S1.P7

Ilbey, S. S3.O4

Ipek, O. S5.P6

Isoardi, R. S1.P2, S1.P6

Ivanov, D. S3.P19, S5.P8

\section{$\mathbf{J}$}

Jaarsma, M. S1.P14

Jafargholi Rangraz, E. C2.O6

Jakob, P.M. S3.O8

Jan, C. S6.O7

Janssens, T. C1.O3

Jeurissen, B. C1.O2, C1.O3, C1.O5, C1.O7, $\mathrm{C} 1 . \mathrm{O} 8$

Jirsa, V. S2.O1

Jorge, J. S4.P12

Julea, F. S1.P11

Juravleva, K. S2.P1

Jurga, S. S1.P15

Juskanic, D. S2.P22

\section{K}

Kaanders, J.H. S1.P9

Kaczmarz, S. S4.P6, S6.P13

Kaijzel, E.L. C3.O2

Kallmayer, M. S6.P13

Kan, H.E. S1.O4

Karmazanovsky, G.G. S1.P7

Kartashov, S. S2.O6

Kashyap, S. S5.P8, S3.P19

Kaykayoğlu, A. S6.P10, S6.P11

Keil, V. S6.08

Keith, G.A. S3.P27

Kerkering, K.M. S3.P26

Kers-Rebel, E.D. S1.P9

Keskin, S. S6.P21

Khalfallah, M. S1.P11

Khan, N. S4.P22

Kicik, A. S2.P24, S2.P25

King, A.P. S3.P8

Kiryanova, A. S2.P23

Kjaer, A. C2.O4

Klaassen, L. S1.P14

Klaver, Y. S1.P14

Klein, S. C1.O1, C1.O6

Klimaszyk, A. S1.P15

Klose, U. S4.P22

Knapen, T. S4.P16, S6.O4

Knutsson, L. S2.P26

Koekkoek, J.A.F. S4.O2

Koh, D.-M. S6.P19

Kohn, N. S2.P3

Kolbitsch, C. S3.P26

Konyer, N. S3.O7

Koole, M. C2.O3

Koolstra, K. S4.P4

Kornaropoulos, E. C1.O4
Kowal, R. S5.O2

Kreis, R. S6.P2, S6.P3

Kronbichler, L. S4.P18

Kronbichler, M. S4.P18

Kuestner, T. C2.O5

Kufer, J. S6.P13

Kupriyanov, D. S1.P5, S2.O6

Kurban, D. S3.P19, S5.P8

Kurzawski, J. S3.P25

Kyathanahally, S.P. S6.P2, S6.P3

Küstner, T. S3.O9

\section{$\mathbf{L}$}

Labriji, W. S4.O4

Lafon, C. S4.O4

Lamb, H. C3.O1, C3.O2

Lambers, H. S2.O3, S2.O2, S4.P15

Lancione, M. S4.O1

Langbein, T. C2.O7

Larreina, N. C3.O1

Laser, R. S5.P12

Lashgari, M. C1.O11

Lattanzi, R. S1.O5

Leite, F. S1.P3

Lenz, C. S2.P6, S6.P20

Leone, R. S6.P9

Leutritz, T. S3.O6

Levardon, M. S4.P27

Lima, B. S1.P4

Lin, J.-M. S3.P14, S6.P12

Lisazo, C. S6.P24, S1.P2

Liu, L. S2.P9

Lizarbe, B. S2.P19, S1.P10, S2.P14

Loft, A. C2.O4

Lokossou, A. S2.P18

Lotterie, J.-A. S4.P27

Loubinoux, I. S4.O4

Lucy, C. S1.O1

Luyten, G.P.M. S1.P14

López, G. S5.O6

López-Gil, X. S2.P17

López-Larrubia, P. S1.P10, S2.P14, S2.P19, S2.P7, S5.P7

Lütjohann, A. S2.O2

M

Ma, R.E. S3.P19

Machado, I. S3.P8

Machann, J. S6.P16

MacLeod, M.-J. S2.O8

Madörin, P. S5.P10

Mahroo, A. S4.P1, S4.O5

Maier, O. S2.O8

Malhaire, C. S6.O2

Mali, A. C3.O2

Mallikourti, V. S2.O8

Manzhurtsev, A. S2.P20, S2.O6, S2.P21, S2.P23, S2.P27, S4.P17, S4.P19

Manzhurtseva, E. S1.P5

Marjanska, M. S2.P22

Marrufo, O. S5.P1, S5.P2, S5.P3

Martin, R. S5.P1, S5.P2, S5.P3

Martinez-Tellez, B. S1.O4

Martins, A.F. S3.O1
Marty, B. S6.P5

Martín-González, E. S3.P10

Marzullo, A. S6.P14, S6.P9

Massaccesi Bove, N.N. S1.P4, S1.P2, S6.P24

Masthoff, M. S3.P5, S6.P25

Mattern, H. S6.P18

Matuszewska, J. S1.P15

Mauconduit, F. S2.O7, S3.P9

Maus, B. S4.P21

McCabe, A. S4.06

McGillivray, J.E. S4.P14

McGrath, D. S4.O6

Melnikov, I. S2.P20

Mengel, A. S4.P22

Menküc, B. S5.P15

Menon, D. C1.O4

Menshchikov, P. S2.O6, S1.P5, S2.P20, S2.P21

Menze, B. S3.P25

Menzel, M. S3.P25

Meyer, P.T. S4.P22

Migaleddu, G. S3.P25

Mills, M. S1.P1

Moguilner, S. S6.P24

Moia, S. S2.O4

Monreal Madrigal, A. S3.P9

Morez, J. C1.O5

Mori, Y. S2.P13

Morris, M. S1.O1

Mortimer, P. S1.P1

Moseley, M. S2.P5

Mougin, O. S6.P23

Mousseaux, M. S1.O3

Moyano Brandi, N. S1.P2, S1.P4, S1.P6, S6.P24

Mueller, S. S3.O1

Munoz, C. C2.O5

Mutsaerts, H.-J. S2.P10, S4.P2, S4.P3, S4.P8, S6.08

Muñoz-Moreno, E. S2.P17, S6.P6

\section{$\mathbf{N}$}

Nabarro, C. S1.P14

Nagelmann, N. S4.O8, S4.P15

Nair, P. S3.O7

Navab, N. C2.O7

Nedergaard, M. S2.P13

Nederveen, A. S2.P12

Negnevitsky, V. S5.P15

Nekolla, S.G. C2.O5, C2.O7

Newcombe, V. C1.O4

Nicastro, M. C1.O7

Nickel, D. S3.O9

Niella, M. S1.P4

Niella, N. S1.P4

Nieminen, M.T. S1.O5

Niendorf, T. S6.O9

Noceti, M. S1.P6

Nogueira, L. S6.P26

Norris, D.G. S3.P20

Noseworthy, M.D. S2.P10, S3.O7, S3.P4, S4.P14, S4.P24, S6.P22

Novichkova, G. S1.P5

Nudnov, N.V. S1.P7 
Nunes, R.G. S3.P11, S3.P12, S3.P16, S3.P23, S4.P11, S6.P26

Nunes, Â.T. S6.P26

Nuyts, J. C2.O6

Nøttrup, T.J. C2.O4

Nürnberger, A. S6.O3

\section{$\mathbf{O}$}

O'Reilly, T. S5.O3, S5.P15

Oberberger, J. S3.08

Odenbach, R. S1.P12

Oksuz, I. S3.P8

Olin, A.B. C2.O4

Oliver-Taylor, A. S4.P2, S4.P3, S4.P8

Omer, H. S3.P13, S3.P17

Orlhac, F. S6.O2

Oros-Peusquens, A.-M. C1.O9

Orton, M.R. S6.P19

Ostergaard, P. S1.P1

Otoya, R. S6.P24

Ozduman, K. S6.P4

Ozturk-Isik, E. S2.P24, S2.P25, S6.P4, S6.P7, S6.P8

$\mathbf{P}$

P. Monteiro, S. S4.P10

Padrela, B. S2.P10, S4.P2, S4.P8

Pagé, G. S1.P8

Palacios, R.E. S5.P7

Palagi, L. S4.P20

Palange, E. S5.O4

Palaniyappan, L. S2.P9

Pallás, E. S5.O6, S5.P14

Palombo, M. S4.P26

Pamir, M.N. S6.P10, S6.P11, S6.P21, S6.P4, S6.P7, S6.P8

Panek, R. S4.06

Panfilov, E. S1.O5

Pannicke, E. S1.P12, S5.O2

Paolella, R. C1.O8

Paradis, V. S1.P11, S1.P8

Parsa, J. C3.O4, S5.O1

Pasquariello, R. S3.P25

Passarinho, C. S6.O4

Pasternak, M. S6.08

Patrovic, L. S2.P22

Patrucco, D. S1.P13

Pavlova, I. S6.P1

Pavão, I. S2.P2

Pearce, J. S1.P1

Pellerin, L. S2.P16

Pellicer, R. S5.O6, S5.P14

Pellicer-Guridi, R. S5.P15

Pereira, H. S6.P15

Pereira, N. S2.P4

Perles-Barbacaru, T.-A. S2.P18

Pervak, M.B. S1.P7

Petr, J. S2.P10, S4.P2, S4.P3, S4.P8, S6.O8

Piella, G. S6.P6

Pietrini, P. S4.O1

Pietro, C. S3.P8

Piluso, S. S6.O7

Pine, K. S3.O2, S6.O5
Pinter, N. S6.08

Pinto, J. S4.O7, S2.P2, S4.P10

Pirkl, C. S3.P25

Pirpamer, L. S6.O6

Platsch, G. C2.O7

Plaza, L. S1.P6

Podyablonskii, A.S. S1.O6

Pogatzki-Zahn, E. S4.O8

Pohmann, R. S3.O1

Poirier, S. S2.P9

Polak, P. S3.P4

Polakova Mistinova, J. S2.P22

Polat, K. S6.P21

Poolacherla, R. S2.P9

Poot, D.H.J. C1.O2, C1.O6, C1.O7, C1.O9, $\mathrm{S} 4 . \mathrm{O} 3$

Porciuncula Baptista, R. S2.O7

Porter, D.A. S3.P27

Poser, B.A. S3.P19, S3.P9, S4.P18, S5.P8

Postic, C. S1.P11

Postigo Fliquete, M. C1.O14

Pradier, B. S4.08, S4.P15

Preibisch, C. S3.O6, S4.P6, S6.P13

Prier, M. S5.P15

Prieto, C. C2.O5

Pruessmann, K.P. S5.P5

Prysiazhniuk, Y. S4.P3

Pusterla, O. S3.P2, S3.P3, S5.P16

Puyol-Antón, E. S3.P8

Péran, P. S4.P27

\section{$\mathbf{R}$}

Rabrait-Lerman, C. S2.O7

Rahimpour, M. C2.O3

Raimondo, L. S4.P16, S6.O4

Ranjeva, J.-P. S2.O1

Rasch, C. S1.P14

Rausch, I. C2.O8, C2.O1

Ravi, K.S. S3.P23

Ravikumar, N. C1.O11, C1.O12

Rebolo, M. S4.P10

Regli, L. S4.P23

Rensen, P.C.N. S1.O4

Reyngoudt, H. S6.P5

Ribeiro, A. S6.P19

Ribeiro Sabidussi, E. C1.O1

Ricciardi, E. S4.O1

Richter, B. S3.O4

Richter, S. C1.O4

Rigla, J.P. S5.P14, S5.O6

Rizza, C. S5.O4

Rizzo, R. S6.P3, S6.P2

Roder, C. S4.P22

Rodrigues, M. S1.P14

Rodriguez, A. S5.P1, S5.P2, S5.P3

Rodríguez-Galván, J.R. S3.P10

Roebroeck, A. S5.P8

Roos, T. S3.P6

Ropele, S. S6.O6

Rosa, A. S4.P11

Rose, G. S1.P12, S5.O2

Ross, J. S2.O8

Rothe, M. S6.O1

Roumes, H. S2.P16
Roussel, T. S2.O1

Rueckert, D. S3.O9

Ruijsink, B. S3.P8

Ruiz, R. S5.P3

Ruiz-Cabello, J. C3.O1, C3.O3

Ruiz-Tagle, A. S2.P2, S4.P11, S4.P9

Ríos, A. S5.O6, S5.P14

\section{$\mathbf{S}$}

Sabatini, U. S2.P8

Sabesan, P. S2.P9

Sacli-Bilmez, B. S6.P4

Sadaghiani, S. S4.O9

Saint Martin, M.-J. S6.O2

Salameh, N. S5.P4

Samson, E. S6.P22

Sanchez, S. S2.P16

Sanchez-Andres, J.V. S5.P14

Santini, F. S5.P16, S5.P10

Sappey-Marinier, D. C1.O8, S6.P14

Saraiva, D. S4.P13

Sarasaen, C. S6.O3

Sardjoe Mishre, A.S.D. S1.O4

Sarracanie, M. S5.P4

Savelov, A. S2.O5, S2.P1

Scanu, N. C3.O3, C3.O1

Schache, D. S3.P22

Schaefer, B. S2.P9

Schaefers, G. S5.P11, S5.P12

Schaeffter, T. S3.P26

Scharfetter, H. S2.O8

Scheenen, T.W. S1.P9

Scheffler, K. S3.O1

Scheurer, E. S2.P6, S6.P20

Schick, F. S6.P16

Schildknecht, C.M. S5.P5

Schlett, C.L. S6.P16

Schmitz Abecassis, B. S4.O2

Schmitzer, L. S6.P13

Schnabel, J.A. S3.P8

Schneider, J. C1.O11, C1.O12

Scholly, J. S2.O1

Scholten, M. S5.P12

Schote, D. S5.P15

Schäfer, S. S6.P19

Schäfers, M. S4.P21

Schäper, J. S3.P1

Scurr, E. S1.O1

Sebbag Sfez, D. S6.O2

Sebök, M. S4.P23

Segelcke, D. S4.O8

Semenova, N. S2.O6, S2.P20, S2.P21, S2.P23, S2.P27, S4.P17, S4.P19

Serrano-Torres, M. S5.P7

Seystahl, K. S4.P23

Shafieizargar, B. C1.O2

Shah, N.J. C1.O13, C1.O9

Shah, V. C2.O7

Shamaei, A. S6.P1

Shemesh, N. S3.P21, S4.P26

Shimanovskii, N.L. S1.O6

Shin, S. C1.O13

Shmueli, K. S3.O5

Shymanskaya, A. S2.P3 
Sibille, L. C2.O7

Siero, J. S3.P6, S4.P16, S6.O4

Sigovan, M. S1.O3, S3.P15

Sijbers, J. C1.O10, C1.O2, C1.O3, C1.O4, C1.O5, C1.O7, C1.08

Silva, N.A. S3.P12, S3.P16, S3.P23, S4.P11, S4.P9

Sima, D.M. C1.O8

Simard, N. S6.P22

Simoncini, C. S4.O4

Sinkus, R. S1.P8

Sinnett, V. S1.O1

Siqueira Pinto, M. C1.O4

Skalej, M. S2.P15

Sliwowska, J.H. S1.P15

Smekens, C. C1.O3, C1.O7

Smits, M. S2.P26, S4.O3, S4.P25

Solari, E.L. C2.O5, C2.O7

Solis-Najera, S. S5.P1, S5.P2, S5.P3

Sollmann, N. S4.P6

Somaiah, N. S1.O1

Soria, G. S2.P17, S6.P6

Sorribes Torrent, E. S6.P6

Souedet, N. S6.O7

Sourdon, J. S2.P18

Spadea, M.F. S6.P9

Speck, O. S5.O2, S6.O3

Spottiswoode, B. C2.O7

Squitieri, F. S2.P8

Srinivas, M. C3.O1

St. Lawrence, K. S2.P9

Stamile, C. S6.P14

Stankevich, Y. S2.P1, S2.O5, S2.P11

Staring, M. S4.P4

Starke, L. S6.09

Starčuk Jr, Z. S6.P1

Starčuková, J. S6.P1

Stefania, R. S4.P20

Steffanoni, S. S6.P9

Steinberg, G. S2.P5

Stickland, R. S2.O4

Stirnberg, R. S4.P18

Stockmann, J. S5.P15

Stollberger, R. S2.O8

Stöcker, T. S4.P18

Straat, M.E. S1.O4

Stritt, M. S4.P2, S4.P8, S6.O8

Stumpo, V. S4.P23

Stute, S. C2.O2

Svenningsen, S. S3.O7

Sümer, E. S6.P7, S6.P8

\section{$\mathbf{T}$}

Tang, M. S1.O1

Tang, X. C2.O6

Taphoorn, M.J.B. S4.O2

Tatagiba, M. S4.P22

Tavakkoli, M. S3.O7

Tavares, V. S2.P4

Teh, I. C1.O11, C1.O12

Teixeira, H. S6.P15

Tereshchenko, G. S1.P5

Termenon, M. S2.O4

Terreno, E. S1.P13
Tewari, S. S5.P13

Theberge, J. S2.P9

Thomas, D.L. S4.P3

Thomas, D. C1.O9

Thurow, J. S4.P22

Ticci, C. S3.P25

Tol, J. C1.O14

Tosetti, M. S3.P25, S4.O1

Toulemonde, M. S1.O1

Touquet, G. S3.P15

Tourais, J. S5.O5

Tournier, N. C2.O2

Traser, L. S3.O4

Tudela, R. S2.P17

Tufekcioglu, Z. S2.P24

Tulupov, A. S2.O5, S2.P1, S2.P11

Tunariu, N. S1.O1, S6.P19

$\mathbf{U}$

Ublinskiy, M. S4.P17, S2.O6, S2.P20, S2.P21, S2.P27, S4.P19

Ugurlu, D. S3.P8

Ulmer, S. S6.P22

Uruñuela, E. S2.O4

Ussov, W.-Y. S1.P7, S1.O6

V

V. Batista, M. S4.P10

Vaclavu, L. S2.P12

Valla, D. S1.P11

Valladares, A. C2.O1

van Asten, J.J. S1.P9

Van Audekerke, J. C1.O2

Van Beers, B. S1.P11, S1.P8

van de Ven, K.C.C. S4.P3

van der Veldt, A. S2.P26

van der Voort, S. S4.P25

van der Zwaag, W. S4.P16, S6.O4

van Dorth, D. S4.O3

Van Dyck, P. C1.O3, C1.O4

van Engelen, A. C1.O14

van Herk, M. S1.P14

van Niftrik, C.H.B. S4.P23

van Ooij, P. S2.P12

van Osch, M.J.P. S2.P12, S4.O2, S4.O3, S4.P4

Van Zanten, M. S1.P1

Vanhevel, F. C1.O3

Varlet, I. S2.P18

Vasilkiv, L. S2.P11, S2.O5, S2.P1

Vazquez, F. S5.P1, S5.P2, S5.P3

Veltri, P. S2.P8

Venancio, L.M. S1.P3

Venugopal, K. S4.O3

Verbist, B. S1.P14

Verhoye, M. C1.O10, C1.O2

Versteeg, E. S3.P6, S3.P9

Veríssimo Cabete, I.J. S2.P7

Vignaud, A. S2.07, S3.P9

Vilela, P. S2.P2, S4.P10, S4.P9

Vilgrain, V. S1.P8

Villagran Asiares, A. C2.O5

Villareal, A. S5.P2
Viola, A. S2.P18

Vioult, P. S2.O1

Vitadello, T. C2.O5

Vives-Gilabert, Y. S5.P15

Vizza, P. S2.P8

Vogelius, I.R. C2.O4

Vorobyeva, V.O. S1.P7

Vourvopoulos, A. S4.P11

Vu, T.H.K. S1.P14

W

Wachsmuth, L. S2.O2, S2.O3, S4.O8

Wacker, F. S1.P12

Wagels, L. S2.P3

Wagner, S. S2.P15

Waiczies, S. S6.09

Warnert, E. S2.P26, S4.O3, S4.P25

Warnking, J. S4.O4

Warsi, M. S6.P22

Webb, A.G. C3.O4, S1.O4, S5.O1, S5.O3, S5.P13, S5.P15

Weber, W. C2.O7

Weiger, M. S5.P5

Weingärtner, S. S5.O5

Weiskopf, N. S3.O2, S3.O6, S6.O5

Weller, M. S4.P23

Wiestler, B. S6.P13

Wilken, E. S3.P5, S6.P25

Wilkinson, D. S5.P9

Winata, S. S3.P27

Winzeck, S. C1.O4

Wirsich, J. S4.O9

Witzel, T. S5.P15

Wohlgemuth, W. S2.P15, S6.O1

Wood, T. S3.P7

Wu, Y. S2.P26

$\mathbf{X}$

Xavier, M. S4.O9, S4.P11, S4.P12

Y

Yagüe Jiménez, B. S1.P10, S2.P14

Yakovlev, A. S4.P19, S2.O6, S2.P20, S2.P21, S2.P27, S4.P17

Yakıcier, C. S6.P10, S6.P11, S6.P21, S6.P4

Yang, H.C. S3.P14, S6.P12

Yon, M. S3.P21

Young, A. S3.P8

Yousefi, S. S5.P13

Yu, W.-H. S3.P14

Yun, S.D. C1.O13

YURDAKOS, O.B. S3.P24

Yushchenko, M. S5.P4

$\mathbf{Z}$

Zaaraoui, W. S2.O1

Zaffino, P. S6.P9

Zaharchuk, G. S2.P5

Zaiss, M. S3.O1

Zalewski, T. S1.P15

Zampini, M.A. C1.O10 
Zerweck, L. S4.P22

Zhang, J.H. S3.P26

Zhao, M. S2.P5

Zimmer, C. S4.P6, S6.P13

Zukotynski, K. S6.P22
Özcan, A. S6.P10, S6.P11, S6.P21

Özduman, K. S6.P10, S6.P11, S6.P21, S6.P7, S6.P8

Özen, A.C. S3.O4

Öztürk-Iş1k, E. S6.P10, S6.P11, S6.P21
Šiuryte, P. S5.O5

's Heeren, R. C2.O6

Publisher's Note Springer Nature remains neutral with regard to jurisdictional claims in published maps and institutional affiliations. 